
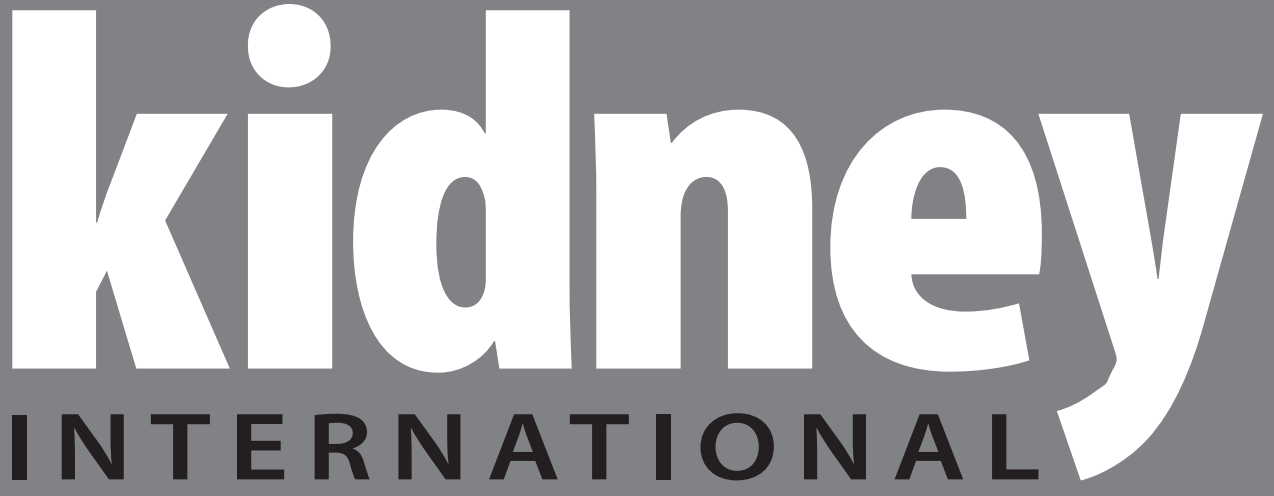

INTER N

NATIONALI

SUPDlements

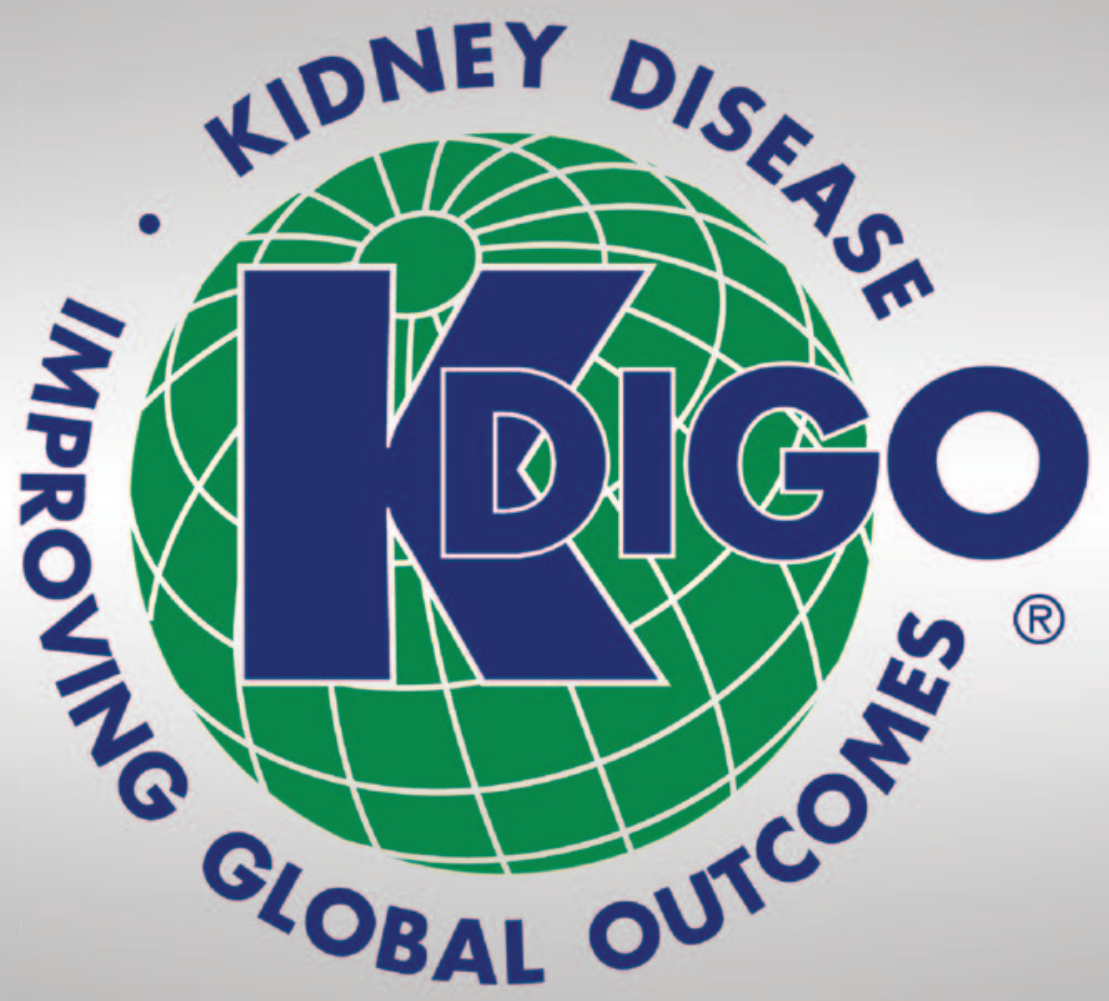

KDIGO 2012 Clinical Practice Guideline for the Evaluation and Management of Chronic Kidney Disease

VOLUME 3 | ISSUE 1 | JANUARY 2013

http://www.kidney-international.org 


\section{KDIGO 2012 Clinical Practice Guideline for the Evaluation and Management of Chronic Kidney Disease}

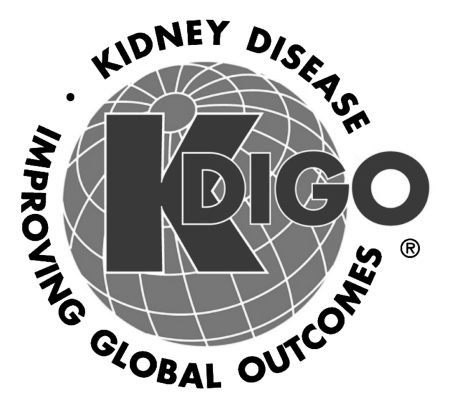

KDIGO gratefully acknowledges the following consortium of sponsors that make our initiatives possible: Abbott, Amgen, Bayer Schering Pharma, Belo Foundation, Bristol-Myers Squibb, Chugai Pharmaceutical, Coca-Cola Company, Dole Food Company, Fresenius Medical Care, Genzyme, Hoffmann-LaRoche, JC Penney, Kyowa Hakko Kirin, NATCO-The Organization for Transplant Professionals, NKF-Board of Directors, Novartis, Pharmacosmos, PUMC Pharmaceutical, Robert and Jane Cizik Foundation, Shire, Takeda Pharmaceutical, Transwestern Commercial Services, Vifor Pharma, and Wyeth.

Sponsorship Statement: KDIGO is supported by a consortium of sponsors and no funding is accepted for the development of specific guidelines. 


\section{KDIGO 2012 Clinical Practice Guideline for the Evaluation and Management of Chronic Kidney Disease}

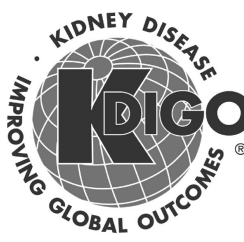

v Tables and Figures

vii KDIGO Board Members

viii Reference Keys

$x \quad$ CKD Nomenclature

xi Conversion Factors \& $\mathrm{HbA}_{1 \mathrm{c}}$ Conversion

xii Abbreviations and Acronyms

$1 \quad$ Notice

2 Foreword

3 Work Group Membership

4 Abstract

5 Summary of Recommendation Statements

15 Introduction: The case for updating and context

19 Chapter 1: Definition, and classification of CKD

63 Chapter 2: Definition, identification, and prediction of CKD progression

73 Chapter 3: Management of progression and complications of CKD

91 Chapter 4: Other complications of CKD: CVD, medication dosage, patient safety, infections, hospitalizations, and caveats for investigating complications of CKD

112 Chapter 5: Referral to specialists and models of care

120 Methods for Guideline Development

128 Biographic and Disclosure Information

134 Acknowledgments

136 References 


\section{TABLES}

Table 1. KDIGO nomenclature and description for grading recommendations

Table 2. Criteria for CKD

Table 3. Criteria for definition of CKD

Table 4. Classification of CKD based on presence or absence of systemic disease and location within the kidney of pathologic-anatomic findings

Table 5. GFR categories in CKD

Table 6. Albuminuria categories in CKD

Table 7. Relationship among categories for albuminuria and proteinuria

Table 8. CGA staging of CKD: examples of nomenclature and comments

Table 9. Prognosis of CKD: Relationship of outcomes and strength of relationship to Cause (C), GFR (G), Albuminuria (A) and other measures

Table 10. Annual percentage change in GFR across diagnosis categories

Table 11. Sources of error in GFR estimating using creatinine

Table 12. Equations based on serum creatinine assays in adults that are traceable to the standard reference material

Table 13. Performance comparison of creatinine-based GFR estimating equations in North America, Europe, and Australia

Table 14. Performance comparison of creatinine-based GFR estimating equations outside of North America, Europe, and Australia

Table 15. Sources of error in GFR estimating using cystatin C

Table 16. Equations based on IDMS traceable creatinine and IFCC traceable cystatin C assays

Table 17. Performance comparison of cystatin C-based estimating equations in North American and European populations

Table 18. Strengths and limitations of GFR measurement methods and markers

Table 19. Factors affecting urinary ACR

Table 20. Decline in kidney function in various populations (longitudinal studies only)

Table 21. Decline in kidney function in CKD populations

Table 22. Studies evaluating rapid progression (general population studies only)

Table 23. CKD progression and risk of all-cause mortality and ESRD using baseline (first) eGFR

Table 24. Association between absolute and percentage change in kidney function and risk of ESRD, based on adjustment for eGFR at the first and last measurement

Table 25. Intensive versus normal glycemic control and albuminuria outcome

Table 26. Recommended Daily Intake of sodium for healthy children

Table 27. Prevalence of CKD complications by GFR category derived from CKD cohorts

Table 28. Hemoglobin cutoffs for people living at sea level

Table 29. Phosphate binding agents in routine clinical practice and their ranked cost

Table 30. Summary data for bisphosphonates and CKD

Table 31. Peripheral arterial disease and CKD

Table 32. Cautionary notes for prescribing in people with CKD

Table 33. Risk factors for infection in people with CKD

Table 34. Components of community CKD management programs

Table 35. Early versus late referral: consequences and benefits

Table 36. Outcomes of early versus late referral

Table 37. Topics of interest for the management of CKD guideline

Table 38. Literature yield of primary articles for all topics

Table 39. Classification of study quality

Table 40. GRADE system for grading quality of evidence

Table 41. Final grade for overall quality of evidence

Table 42. Balance of benefits and harm

Table 43. KDIGO nomenclature and description for grading recommendations

Table 44. Determinants of strength of recommendation

Table 45. The Conference on Guideline Standardization checklist for reporting clinical practice guidelines 


\section{FIGURES}

Figure 1. Conceptual model of CKD

Figure 2. Normal values for GFR by age

Figure 3. Relationship of eGFR with mortality

Figure 4. Relationship of albuminuria with mortality

Figure 5. Age-standardized rates of death from any cause, cardiovascular events, and hospitalization, according to the eGFR among 1,120,295 ambulatory adults

Figure 6. Summary of continuous meta-analysis for general population cohorts with ACR

Figure 7. Summary of categorical meta-analysis for general population cohorts with ACR

Figure 8. Prevalence of CKD in the USA by GFR and albuminuria

Figure 9. Prognosis of CKD by GFR and albuminuria category

Figure 10. Determinants of the serum level of endogenous filtration markers

Figure 11. Performance of the CKD-EPI and MDRD Study equations in estimating measured GFR in the external validation data set

Figure 12. Comparison of distribution of GFR and CKD prevalence by age (NHANES 1999-2004)

Figure 13. Meta-analysis of NRI for all-cause mortality, CVD mortality, and ESRD

Figure 14. Association of CKD definitions with all-cause mortality and ESRD

Figure 15. Performance of three equations for estimating GFR

Figure 16. Suggested protocol for the further investigation of an individual demonstrating a positive reagent strip test for albuminuria/proteinuria or quantitative albuminuria/proteinuria test

Figure 17. GFR and albuminuria grid to reflect the risk of progression

Figure 18. Distribution of the probability of nonlinearity with three example trajectories demonstrating different probabilities of nonlinearity

Figure 19. Summary estimates for risks of all-cause mortality and cardiovascular mortality associated with levels of serum phosphorus, PTH, and calcium

Figure 20. Prevalence of deficiency of $1,25(\mathrm{OH})_{2} \mathrm{D}_{3}, 25(\mathrm{OH}) \mathrm{D}_{3}$, and secondary hyperparathyroidism by GFR intervals

Figure 21. Referral decision making by GFR and albuminuria

Figure 22. The CKD chronic care model 


\title{
KDIGO Board Members
}

\author{
Garabed Eknoyan, MD \\ Norbert Lameire, MD, PhD \\ Founding KDIGO Co-Chairs \\ Kai-Uwe Eckardt, MD \\ Immediate Past Co-Chair
}

Bertram L Kasiske, MD

KDIGO Co-Chair

Omar I Abboud, MD, FRCP

Sharon Adler, MD, FASN

Rajiv Agarwal, MD

Sharon P Andreoli, MD

Gavin J Becker, MD, FRACP

Fred Brown, MBA, FACHE

Daniel C Cattran, MD, FRCPC

Allan J Collins, MD, FACP

Rosanna Coppo, MD

Josef Coresh, MD, PhD

Ricardo Correa-Rotter, MD

Adrian Covic, $\mathrm{MD}, \mathrm{PhD}$

Jonathan C Craig, MBChB, MM (Clin Epi), DCH, FRACP, PhD

Angel LM de Francisco, MD

Paul E de Jong, MD, PhD

Ana Figueiredo, RN, MSc, PhD

Mohammed Benghanem Gharbi, MD

Gordon Guyatt, MD, MSc, BSc, FRCPC

David Harris, MD

Lai Seong Hooi, MD

Enyu Imai, MD, $\mathrm{PhD}$

Lesley A Inker, MD, MS, FRCP
David C Wheeler, MD, FRCP

KDIGO Co-Chair

Michel Jadoul, MD

Simon Jenkins, MBE, FRCGP

Suhnggwon Kim, MD, PhD

Martin K Kuhlmann, MD

Nathan W Levin, MD, FACP

Philip K-T Li, MD, FRCP, FACP

Zhi-Hong Liu, MD

Pablo Massari, MD

Peter A McCullough, MD, MPH, FACC, FACP

Rafique Moosa, MD

Miguel C Riella, MD

Adibul Hasan Rizvi, MBBS, FRCP

Bernardo Rodriquez-Iturbe, MD

Robert Schrier, MD

Justin Silver, MD, PhD

Marcello Tonelli, MD, SM, FRCPC

Yusuke Tsukamoto, MD

Theodor Vogels, MSW

Angela Yee-Moon Wang, MD, PhD, FRCP

Christoph Wanner, MD

Elena Zakharova, MD, PhD

\section{NKF-KDIGO GUIDELINE DEVELOPMENT STAFF}

Kerry Willis, PhD, Senior Vice-President for Scientific Activities

Michael Cheung, MA, Guideline Development Director

Sean Slifer, BA, Guideline Development Manager 


\section{Reference Keys}

\section{NOMENCLATURE AND DESCRIPTION FOR RATING GUIDELINE RECOMMENDATIONS}

Within each recommendation, the strength of recommendation is indicated as Level 1, Level 2, or Not Graded, and the quality of the supporting evidence is shown as $\mathbf{A}, \mathbf{B}, \mathbf{C}$, or $\mathbf{D}$.

\begin{tabular}{|c|c|c|c|}
\hline \multirow[b]{2}{*}{ Grade* } & \multicolumn{3}{|c|}{ Implications } \\
\hline & Patients & Clinicians & Policy \\
\hline $\begin{array}{l}\text { Level } 1 \\
\text { 'We recommend' }\end{array}$ & $\begin{array}{l}\text { Most people in your situation would want } \\
\text { the recommended course of action and only } \\
\text { a small proportion would not. }\end{array}$ & $\begin{array}{l}\text { Most patients should receive the } \\
\text { recommended course of action. }\end{array}$ & $\begin{array}{l}\text { The recommendation can be evaluated as } \\
\text { candidate for developing a policy or a } \\
\text { performance measure. }\end{array}$ \\
\hline $\begin{array}{l}\text { Level } 2 \\
\text { 'We suggest' }\end{array}$ & $\begin{array}{l}\text { The majority of people in your situation } \\
\text { would want the recommended course of } \\
\text { action, but many would not. }\end{array}$ & $\begin{array}{l}\text { Different choices will be appropriate for } \\
\text { different patients. Each patient needs help to } \\
\text { arrive at a management decision consistent } \\
\text { with her or his values and preferences. }\end{array}$ & $\begin{array}{l}\text { The recommendation is likely to require } \\
\text { substantial debate and involvement of } \\
\text { stakeholders before policy can be } \\
\text { determined. }\end{array}$ \\
\hline
\end{tabular}

*The additional category 'Not Graded' was used, typically, to provide guidance based on common sense or where the topic does not allow adequate application of evidence. The most common examples include recommendations regarding monitoring intervals, counseling, and referral to other clinical specialists. The ungraded recommendations are generally written as simple declarative statements, but are not meant to be interpreted as being stronger recommendations than Level 1 or 2 recommendations.

\begin{tabular}{|c|c|c|}
\hline Grade & Quality of evidence & Meaning \\
\hline A & High & We are confident that the true effect lies close to that of the estimate of the effect. \\
\hline B & Moderate & The true effect is likely to be close to the estimate of the effect, but there is a possibility that it is substantially different. \\
\hline C & Low & The true effect may be substantially different from the estimate of the effect. \\
\hline D & Very low & The estimate of effect is very uncertain, and often will be far from the truth. \\
\hline
\end{tabular}

\section{ADULT GFR ESTIMATING EQUATIONS}

2009 CKD-EPI creatinine equation: $141 \times \min (\mathrm{SCr} / \kappa, 1)^{\alpha} \times \max (\mathrm{SCr} / \kappa, 1)^{-1.209} \times 0.993^{\text {Age }}[\times 1.018$ if female] [ $\times 1.159$ if black], where $\mathrm{SCr}$ is serum creatinine (in $\mathrm{mg} / \mathrm{dl}$ ), $\kappa$ is 0.7 for females and 0.9 for males, $\alpha$ is -0.329 for females and -0.411 for males, min is the minimum of $\mathrm{SCr} / \kappa$ or 1 , and max is the maximum of $\mathrm{SCr} / \kappa$ or 1 .

Equations expressed for specified sex and serum creatinine level

\begin{tabular}{|c|c|c|}
\hline Gender & Serum creatinine & Equation for estimating GFR \\
\hline Female & $\leqslant 0.7 \mathrm{mg} / \mathrm{dl}(\leqslant 62 \mu \mathrm{mol} / \mathrm{l})$ & $144 \times(\mathrm{SCr} / 0.7)^{-0.329} \times 0.993^{\text {Age }}[\times 1.159$ if black $]$ \\
\hline Female & $>0.7 \mathrm{mg} / \mathrm{dl}(>62 \mu \mathrm{mol} / \mathrm{l})$ & $144 \times(\mathrm{SCr} / 0.7)^{-1.209} \times 0.993^{\text {Age }}[\times 1.159$ if black $]$ \\
\hline Male & $\leqslant 0.9 \mathrm{mg} / \mathrm{dl}(\leqslant 80 \mu \mathrm{mol} / \mathrm{l})$ & $141 \times(\mathrm{SCr} / 0.9)^{-0.411} \times 0.993^{\mathrm{Age}}[\times 1.159$ if black $]$ \\
\hline Male & $>0.9 \mathrm{mg} / \mathrm{dl}(>80 \mu \mathrm{mol} / \mathrm{l})$ & $141 \times(\mathrm{SCr} / 0.9)^{-1.209} \times 0.993^{\text {Age }}[\times 1.159$ if black $]$ \\
\hline
\end{tabular}

2012 CKD-EPI cystatin C equation: $133 \times \min (\mathrm{SCysC} / 0.8,1)^{-0.499} \times \max (\mathrm{SCys} / 0.8,1)^{-1.328} \times 0.996^{\mathrm{Age}}[\times 0.932$ if female], where SCysC is serum cystatin C (in mg/l), min indicates the minimum of SCysC/0.8 or 1 , and max indicates the maximum of SCysC/0.8 or 1 .

Equations expressed for serum cystatin C level

\begin{tabular}{llr}
\hline & Serum cystatin C & Equation for estimating GFR \\
\hline Female or male & $\leqslant 0.8 \mathrm{mg} / \mathrm{l}$ & $133 \times(\mathrm{SCysC} / 0.8)^{-0.499} \times 0.996^{\text {Age }}[\times 0.932$ if female $]$ \\
Female or male & $>0.8 \mathrm{mg} / \mathrm{l}$ & $133 \times(\mathrm{SCys} / 0.8)^{-1.328} \times 0.996^{\text {Age }}[\times 0.932$ if female $]$ \\
\hline
\end{tabular}


(c) 2013 KDIGO

2012 CKD-EPI creatinine-cystatin C equation: $135 \times \min (\mathrm{SCr} / \kappa, 1)^{\alpha} \times \max (\mathrm{SCr} / \kappa, 1)^{-0.601} \times \min (\mathrm{SCySC} / 0.8,1)^{-0.375} \times \max (\mathrm{SCysC} /$ $0.8,1)^{-0.711} \times 0.995^{\mathrm{Age}}[\times 0.969$ if female] [ $\times 1.08$ if black], where $\mathrm{SCr}$ is serum creatinine (in mg/dl), SCysC is serum cystatin C (in mg/l), $\kappa$ is 0.7 for females and 0.9 for males, $\alpha$ is -0.248 for females and -0.207 for males, $\mathrm{min}(\mathrm{SCr} / \kappa, 1)$ indicates the minimum of $\mathrm{SCr} / \kappa$ or 1 , and $\max (\mathrm{SCr} / \kappa, 1)$ indicates the maximum of $\mathrm{SCr} / \kappa$ or $1 ; \min (\mathrm{SCysC} / 0.8,1)$ indicates the minimum of $\mathrm{SCysC} / 0.8$ or 1 and $\max (\mathrm{SCysC} /$ $0.8,1)$ indicates the maximum of $\mathrm{SCysC} / 0.8$ or 1 .

Equations expressed for specified sex, serum creatinine, and serum cystatin $C$ level

\begin{tabular}{|c|c|c|c|}
\hline Gender & Serum creatinine & Serum cystatin $\mathrm{C}$ & Equation for estimating GFR \\
\hline \multirow[t]{2}{*}{ Female } & \multirow[t]{2}{*}{$\leqslant 0.7 \mathrm{mg} / \mathrm{dl}(\leqslant 62 \mu \mathrm{mol} / \mathrm{l})$} & $\leqslant 0.8 \mathrm{mg} / \mathrm{l}$ & $130 \times(\mathrm{SCr} / 0.7)^{-0.248} \times(\mathrm{SCysC} / 0.8)^{-0.375} \times 0.995^{\mathrm{Age}}[\times 1.08$ if black $]$ \\
\hline & & $>0.8 \mathrm{mg} / \mathrm{l}$ & $130 \times(\mathrm{SCr} / 0.7)^{-0.248} \times(\mathrm{SCysC} / 0.8)^{-0.711} \times 0.995^{\mathrm{Age}}[\times 1.08$ if black $]$ \\
\hline \multirow[t]{2}{*}{ Female } & \multirow[t]{2}{*}{$>0.7 \mathrm{mg} / \mathrm{dl}(>62 \mu \mathrm{mol} / \mathrm{l})$} & $\leqslant 0.8 \mathrm{mg} / \mathrm{l}$ & $130 \times(\mathrm{SCr} / 0.7)^{-0.601} \times(\mathrm{SCysC} / 0.8)^{-0.375} \times 0.995^{\text {Age }}[\times 1.08$ if black $]$ \\
\hline & & $>0.8 \mathrm{mg} / \mathrm{l}$ & $130 \times(\mathrm{SCr} / 0.7)^{-0.601} \times(\mathrm{SCysC} / 0.8)^{-0.711} \times 0.995^{\mathrm{Age}}[\times 1.08$ if black $]$ \\
\hline \multirow[t]{2}{*}{ Male } & \multirow[t]{2}{*}{$\leqslant 0.9 \mathrm{mg} / \mathrm{dl}(\leqslant 80 \mu \mathrm{mol} / \mathrm{l})$} & $\leqslant 0.8 \mathrm{mg} / \mathrm{l}$ & $135 \times(\mathrm{SCr} / 0.9)^{-0.207} \times(\mathrm{SCysC} / 0.8)^{-0.375} \times 0.995^{\mathrm{Age}}[\times 1.08$ if black $]$ \\
\hline & & $>0.8 \mathrm{mg} / \mathrm{l}$ & $135 \times(\mathrm{SCr} / 0.9)^{-0.207} \times(\mathrm{SCysC} / 0.8)^{-0.711} \times 0.995^{\text {Age }}[\times 1.08$ if black $]$ \\
\hline \multirow[t]{2}{*}{ Male } & \multirow[t]{2}{*}{$>0.9 \mathrm{mg} / \mathrm{dl}(>80 \mu \mathrm{mol} / \mathrm{l})$} & $\leqslant 0.8 \mathrm{mg} / \mathrm{l}$ & $135 \times(\mathrm{SCr} / 0.9)^{-0.601} \times(\mathrm{SCysC} / 0.8)^{-0.375} \times 0.995^{\mathrm{Age}}[\times 1.08$ if black $]$ \\
\hline & & $>0.8 \mathrm{mg} / \mathrm{l}$ & $135 \times(\mathrm{SCr} / 0.9)^{-0.601} \times(\mathrm{SCysC} / 0.8)^{-0.711} \times 0.995^{\mathrm{Age}}[\times 1.08$ if black $]$ \\
\hline
\end{tabular}

PEDIATRIC GFR ESTIMATING EQUATIONS

\section{Creatinine-based equations}

\begin{tabular}{c}
$\begin{array}{c}41.3 \times(\text { height } / \mathrm{SCr}) \\
40.7 \times(\text { height } / \mathrm{SCr})^{0.64} \times(30 / \mathrm{BUN})^{0.202}\end{array}$ \\
\hline BUN, blood urea nitrogen in mg/dl; height in meters; $\mathrm{SCr}$, serum creatinine in mg/dl \\
Cystatin C-based equations \\
$70.69 \times(\mathrm{SCySC})^{-0.931}$
\end{tabular}

SCysC, serum cystatin $\mathrm{C}$ in $\mathrm{mg} / \mathrm{l}$. 


\section{CURRENT CHRONIC KIDNEY DISEASE (CKD) NOMENCLATURE USED BY KDIGO}

CKD is defined as abnormalities of kidney structure or function, present for $>3$ months, with implications for health and CKD is classified based on cause, GFR category, and albuminuria category (CGA).

Prognosis of CKD by GFR and albuminuria category

\begin{tabular}{|c|c|c|c|c|c|c|}
\hline & & & & \multicolumn{3}{|c|}{$\begin{array}{l}\text { Persistent albuminuria categories } \\
\text { Description and range }\end{array}$} \\
\hline \multirow{2}{*}{\multicolumn{4}{|c|}{$\begin{array}{l}\text { Prognosis of CKD by GFR } \\
\text { and Albuminuria Categories: } \\
\text { KDIGO } 2012\end{array}$}} & A1 & A2 & A3 \\
\hline & & & & $\begin{array}{l}\text { Normal to } \\
\text { mildly } \\
\text { increased }\end{array}$ & $\begin{array}{l}\text { Moderately } \\
\text { increased }\end{array}$ & $\begin{array}{l}\text { Severely } \\
\text { increased }\end{array}$ \\
\hline & & & & $\begin{array}{c}<30 \mathrm{mg} / \mathrm{g} \\
<3 \mathrm{mg} / \mathrm{mmol}\end{array}$ & $\begin{array}{c}30-300 \mathrm{mg} / \mathrm{g} \\
3-30 \mathrm{mg} / \mathrm{mmol}\end{array}$ & $\begin{aligned} & >300 \mathrm{mg} / \mathrm{g} \\
> & 30 \mathrm{mg} / \mathrm{mmol}\end{aligned}$ \\
\hline \multirow{6}{*}{ 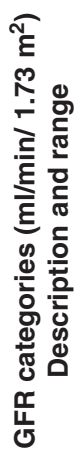 } & G1 & Normal or high & $\geq 90$ & & & \\
\hline & G2 & Mildly decreased & $60-89$ & & & \\
\hline & G3a & $\begin{array}{l}\text { Mildly to moderately } \\
\text { decreased }\end{array}$ & $45-59$ & & & \\
\hline & G3b & $\begin{array}{l}\text { Moderately to } \\
\text { severely decreased }\end{array}$ & $30-44$ & & & \\
\hline & G4 & Severely decreased & $15-29$ & & & \\
\hline & G5 & Kidney failure & $<15$ & & & \\
\hline
\end{tabular}

Green: low risk (if no other markers of kidney disease, no CKD); Yellow: moderately increased risk; Orange: high risk; Red, very high risk. 
CONVERSION FACTORS OF METRIC UNITS TO SI UNITS

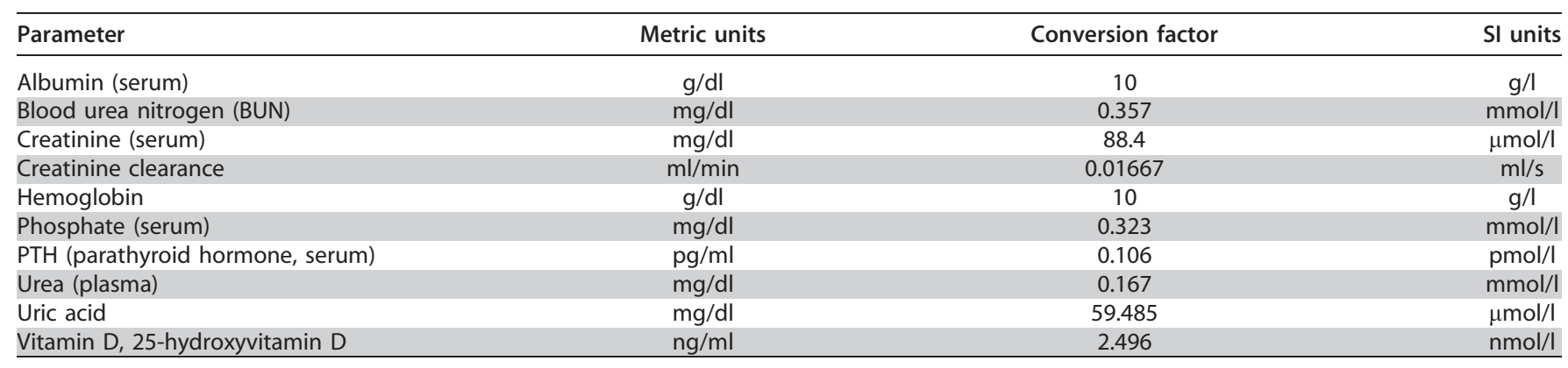

Note: Metric unit $\times$ conversion factor $=$ SI unit.

HbA $_{1 C}$ CONVERSION CHART

\begin{tabular}{|c|c|c|c|c|c|c|c|c|c|}
\hline $\begin{array}{l}\text { DCCT } \\
(\%)\end{array}$ & $\begin{array}{c}\text { IFCC } \\
(\mathrm{mmol} / \mathrm{mol})\end{array}$ & $\begin{array}{c}\text { DCCT } \\
(\%)\end{array}$ & $\begin{array}{c}\text { IFCC } \\
(\mathrm{mmol} / \mathrm{mol})\end{array}$ & $\begin{array}{c}\text { DCCT } \\
(\%)\end{array}$ & $\begin{array}{c}\text { IFCC } \\
(\mathrm{mmol} / \mathrm{mol})\end{array}$ & $\begin{array}{c}\text { DCCT } \\
(\%)\end{array}$ & $\begin{array}{c}\text { IFCC } \\
(\mathrm{mmol} / \mathrm{mol})\end{array}$ & $\begin{array}{c}\text { DCCT } \\
(\%)\end{array}$ & $\begin{array}{c}\text { IFCC } \\
(\mathrm{mmol} / \mathrm{mol})\end{array}$ \\
\hline 5.0 & 31 & 6.0 & 42 & 7.0 & 53 & 8.0 & 64 & 9.0 & 75 \\
\hline 5.1 & 32 & 6.1 & 43 & 7.1 & 54 & 8.1 & 65 & 9.1 & 76 \\
\hline 5.2 & 33 & 6.2 & 44 & 7.2 & 55 & 8.2 & 66 & 9.2 & 77 \\
\hline 5.3 & 34 & 6.3 & 45 & 7.3 & 56 & 8.3 & 67 & 9.3 & 78 \\
\hline 5.4 & 36 & 6.4 & 46 & 7.4 & 57 & 8.4 & 68 & 9.4 & 79 \\
\hline 5.5 & 37 & 6.5 & 48 & 7.5 & 58 & 8.5 & 69 & 9.5 & 80 \\
\hline 5.6 & 38 & 6.6 & 49 & 7.6 & 60 & 8.6 & 70 & 9.6 & 81 \\
\hline 5.7 & 39 & 6.7 & 50 & 7.7 & 61 & 8.7 & 72 & 9.7 & 83 \\
\hline 5.8 & 40 & 6.8 & 51 & 7.8 & 62 & 8.8 & 73 & 9.8 & 84 \\
\hline 5.9 & 41 & 6.9 & 52 & 7.9 & 63 & 8.9 & 74 & 9.9 & 85 \\
\hline $\begin{array}{l}\text { DCCT } \\
(\%)\end{array}$ & $\begin{array}{c}\text { IFCC } \\
(\mathrm{mmol} / \mathrm{mol})\end{array}$ & $\begin{array}{c}\text { DCCT } \\
(\%)\end{array}$ & $\begin{array}{c}\text { IFCC } \\
(\mathrm{mmol} / \mathrm{mol})\end{array}$ & $\begin{array}{c}\text { DCCT } \\
(\%)\end{array}$ & $\begin{array}{c}\text { IFCC } \\
(\mathrm{mmol} / \mathrm{mol})\end{array}$ & $\begin{array}{c}\text { DCCT } \\
(\%)\end{array}$ & $\begin{array}{c}\text { IFCC } \\
(\mathrm{mmol} / \mathrm{mol})\end{array}$ & $\begin{array}{c}\text { DCCT } \\
(\%)\end{array}$ & $\begin{array}{c}\text { IFCC } \\
(\mathrm{mmol} / \mathrm{mol})\end{array}$ \\
\hline 10.0 & 86 & 11.0 & 97 & 12.0 & 108 & 13.0 & 119 & 14.0 & 130 \\
\hline 10.1 & 87 & 11.1 & 98 & 12.1 & 109 & 13.1 & 120 & 14.1 & 131 \\
\hline 10.2 & 88 & 11.2 & 99 & 12.2 & 110 & 13.2 & 121 & 14.2 & 132 \\
\hline 10.3 & 89 & 11.3 & 100 & 12.3 & 111 & 13.3 & 122 & 14.3 & 133 \\
\hline 10.4 & 90 & 11.4 & 101 & 12.4 & 112 & 13.4 & 123 & 14.4 & 134 \\
\hline 10.5 & 91 & 11.5 & 102 & 12.5 & 113 & 13.5 & 124 & 14.5 & 135 \\
\hline 10.6 & 92 & 11.6 & 103 & 12.6 & 114 & 13.6 & 125 & 14.6 & 136 \\
\hline 10.7 & 93 & 11.7 & 104 & 12.7 & 115 & 13.7 & 126 & 14.7 & 137 \\
\hline 10.8 & 95 & 11.8 & 105 & 12.8 & 116 & 13.8 & 127 & 14.8 & 138 \\
\hline 10.9 & 96 & 11.9 & 107 & 12.9 & 117 & 13.9 & 128 & 14.9 & 139 \\
\hline
\end{tabular}

IFCC-HbA $1 c(\mathrm{mmol} / \mathrm{mol})=\left[\mathrm{DCCT}-\mathrm{HbA}_{1 \mathrm{c}}(\%)-2.15\right] \times 10.929$

Abbreviations: DCCT, Diabetes Control and Complications Trial; IFCC, International Federation of Clinical Chemistry and Laboratory Medicine.

Source: Diabetes UK, www.diabetes.org.uk. 


\section{Abbreviations and Acronyms}

4C
AASK
ABPM
ACCORD
ACE-I
ACR
ACS

AER

AGREE

$\mathrm{AKD}$

AKDN

AKI

ANP

APPROACH

AusDiab

ARB

BMD

BMI

BNP

BP

BSA

BUN

CAD

CAPRICORN

CGA

CHARM

CHF

CHS

CI

CIBIS II

CKD

CKD-EPI

CKD-MBD

CKiD

COGS

COPERNICUS

COX-2

Cr-EDTA

CREATE

CREDO

$\mathrm{CrCl}$

CRIC

CRP
Cardiovascular Comorbidity in Children with CKD African American Study of Kidney Disease and Hypertension

Ambulatory blood pressure monitoring

Action to Control Cardiovascular Risk in Diabetes

Angiotensin-converting enzyme inhibitor

Albumin-to-creatinine ratio

Acute coronary syndrome

Action in Diabetes and Vascular Disease: Preterax and Diamicron Modified Release Controlled Evaluation

Albumin excretion rate

Appraisal of Guidelines for Research and Evaluation

Acute kidney disease

Alberta Kidney Disease Network

Acute kidney injury

Atrial natriuretic peptide

Alberta Provincial Project for Outcome Assess-

ment in Coronary Heart Disease

Australian Diabetes, Obesity and Lifestyle study

Angiotensin-receptor blocker

Bone mineral density

Body mass index

B-type natriuretic peptide

Blood pressure

Body surface area

Blood urea nitrogen

Coronary artery disease

Carvedilol Post-Infarct Survival Control in Left

Ventricular Dysfunction

Cause, GFR and Albuminuria categories

Candesartan in Heart Failure-Assessment of

Reduction in Mortality and Morbidity

Congestive heart failure

Cardiovascular Health Study

Confidence interval

Cardiac Insufficiency Bisoprolol Study II

Chronic kidney disease

CKD Epidemiology Collaboration

Chronic Kidney Disease-Mineral and Bone

Disorder

Chronic Kidney Disease in Children

Conference on Guideline Standardization

Carvedilol Prospective Randomized Cumulative

Survival

Cyclooxygenase-2

Chromium-ethylenediamine tetraacetic acid

Cardiovascular Risk Reduction by Early Anemia

Treatment with Epoetin Beta Trial

Clopidogrel for Reduction of Events During

Observation

Creatinine clearance

Chronic Renal Insufficiency Cohort

C-reactive protein
cTnI

cTnT

CVD

DCCT/EDIC

DIG

DPI

DXA

ECG

eGFR

EMU

ERT

ESA

ESCAPE

ESRD

ESUR

FGF-23

GBCA

GFR

GN

GRADE

$\mathrm{Hb}$

$\mathrm{HbA}_{1 \mathrm{c}}$

HBV

HDL-C

HOPE

HOT

HR

HR-pQCT

HUNT 2

ICD

ICU

IDF

IDMS

IQR

IRMM

ItalKid

JCTLM

KDIGO

KDOQI

LDL-C

LIFE

LPD

LVH

MAP

MDRD

MESA
Cardiac troponin I

Cardiac troponin $\mathrm{T}$

Cardiovascular disease

Diabetes Control and Complications Trial/ Epidemiology of Diabetes Interventions and Complications

Digitalis Intervention Group

Dietary protein intake

Dual-energy x-ray absorptiometry

Electrocardiography

Estimated GFR

Early morning urine

Evidence review team

Erythropoiesis-stimulating agent

Effect of Strict Blood Pressure Control and ACE-Inhibition on Progression of Chronic Renal Failure in Pediatric Patients

End-Stage Renal Disease

European Society of Urogenital Radiology

Fibroblast growth factor-23

Gadolinium-based contrast agent

Glomerular filtration rate

Glomerulonephritis

Grading of Recommendations Assessment, Development and Evaluation

Hemoglobin

Hemoglobin A1c

Hepatitis B virus

High-density lipoprotein cholesterol

Heart Outcomes Prevention Evaluation

Hypertension Optimal Treatment

Hazard ratio

High-resolution peripheral quantitative computed tomography

Nord-Trøndelag Health Study (1995-1997)

International Classification of Diseases

Intensive-care unit

International Diabetes Federation

Isotope-dilution mass spectrometry

Interquartile range

Institute for Reference Materials and Measurements

Italian Pediatric Registry of Chronic Renal Failure Joint Committee for Traceability in Laboratory Medicine

Kidney Disease: Improving Global Outcomes

Kidney Disease Outcomes Quality Initiative

Low-density lipoprotein cholesterol

Losartan Intervention For Endpoint Reduction in Hypertension

Low-protein diet

Left ventricular hypertrophy

Mean arterial pressure

Modification of Diet in Renal Disease

Multi-Ethnic Study of Atherosclerosis 
MI

MRI

NAPRTCS

NCEP III

NECOSAD

NHANES

NICE

$\mathrm{NIH}$

NKDEP

NKF

NSAID

NSF

NT-proBNP

ONTARGET

OR

PAD

PCR

PER

PICARD

PICODD

PREVEND
Myocardial infarction

Magnetic resonance imaging

North American Pediatric Renal Trials and Collaborative Studies

Third Report of the National Cholesterol Education Program

Netherlands Cooperative Study on the Adequacy of Dialysis Study Group

National Health and Nutrition Examination Survey

National Institute for Health and Clinical Excellence

National Institutes of Health

National Kidney Disease Education Program

National Kidney Foundation

Nonsteroidal anti-inflammatory drug

Nephrogenic systemic fibrosis

$\mathrm{N}$-terminal pro-BNP

Ongoing Telmisartan Alone and in Combination with Ramipril Global Endpoint trial

Odds ratio

Peripheral arterial disease

Protein-to-creatinine ratio

Protein excretion rate

Program to Improve Care in Acute Renal Disease

Population, Intervention or Predictor, Comparator, Outcome, study Design, and Duration of follow-up

Prevention of Renal and Vascular Endstage Disease
PTH Parathyroid hormone

QOL Quality of life

RAAS

RBC

RCT

RENAAL

RR

RRT

SCORE

$\mathrm{SCr}$

SCysC

$\mathrm{SD}$

SEEK

SHARP

SPECT

SUA

TREAT

UKPDS

USA-PRC

USRDS

VADT

Val-HeFT

VLPD

WBC

WHO

Red blood cell

Relative risk

Serum creatinine

Serum cystatin C

Standard deviation Serum uric acid Aranesp Therapy Epidemiology

Very low-protein diet

White blood cell
Renin-angiotensin-aldosterone system

Randomized controlled trial

Reduction of Endpoints in NIDDM with the Angiotensin II Antagonist Losartan

Renal replacement therapy

Systematic COronary Risk Evaluation

Study for the Evaluation of Early Kidney disease Study of Heart and Renal Protection

Single photon emission computed tomography

Trial to Reduce Cardiovascular Events with

United Kingdom Prospective Diabetes Study

USA-People's Republic of China Collaborative Study of Cardiovascular and Cardiopulmonary

US Renal Data System

Veterans Affairs Diabetes Trial

Valsartan Heart Failure Trial

World Health Organization 



\section{Notice}

Kidney International Supplements (2013) 3, 1; doi:10.1038/kisup.2012.73

\section{SECTION I: USE OF THE CLINICAL PRACTICE GUIDELINE}

This Clinical Practice Guideline document is based upon systematic literature searches last conducted in June 2011, supplemented with additional evidence through November 2012. It is designed to provide information and assist decision making. It is not intended to define a standard of care, and should not be construed as one, nor should it be interpreted as prescribing an exclusive course of management. Variations in practice will inevitably and appropriately occur when clinicians take into account the needs of individual patients, available resources, and limitations unique to an institution or type of practice. Every health-care professional making use of these recommendations is responsible for evaluating the appropriateness of applying them in any particular clinical situation. The recommendations for research contained within this document are general and do not imply a specific protocol.

\section{SECTION II: DISCLOSURE}

Kidney Disease: Improving Global Outcomes (KDIGO) makes every effort to avoid any actual or reasonably perceived conflicts of interest that may arise as a result of an outside relationship or a personal, professional, or business interest of a member of the Work Group. All members of the Work Group are required to complete, sign, and submit a disclosure and attestation form showing all such relationships that might be perceived as or are actual conflicts of interest. This document is updated annually and information is adjusted accordingly. All reported information is published in its entirety at the end of this document in the Work Group members' Biographic and Disclosure Information section, and is kept on file at the National Kidney Foundation (NKF), former Managing Agent for KDIGO.

Copyright (c) 2012 by KDIGO. All rights reserved.

Single photocopies may be made for personal use as allowed by national copyright laws. Special rates are available for educational institutions that wish to make photocopies for non-profit educational use. No part of this publication may be reproduced, amended, or transmitted in any form or by any means, electronic or mechanical, including photocopying, recording, or any information storage and retrieval system, without explicit permission in writing from KDIGO. Details on how to seek permission for reproduction or translation, and further information about KDIGO's permissions policies can be obtained by contacting Danielle Green, Managing Director, at danielle.green@kdigo.org

To the fullest extent of the law, neither KDIGO, Kidney International Supplements, National Kidney Foundation (KDIGO's former Managing Agent) nor the authors, contributors, or editors, assume any liability for any injury and/or damage to persons or property as a matter of products liability, negligence or otherwise, or from any use or operation of any methods, products, instructions, or ideas contained in the material herein. 


\section{Foreword}

Kidney International Supplements (2013) 3, 2; doi:10.1038/kisup.2012.74

It is our hope that this document will serve several useful purposes. Our primary goal is to improve patient care. We hope to accomplish this, in the short term, by helping clinicians know and better understand the evidence (or lack of evidence) that determines current practice. By providing comprehensive evidence-based recommendations, this guideline will also help define areas where evidence is lacking and research is needed. Helping to define a research agenda is an often neglected, but very important, function of clinical practice guideline development.

We used the Grading of Recommendations Assessment, Development and Evaluation (GRADE) system to rate the quality of evidence and the strength of recommendations. In all, there were $12(17.1 \%)$ recommendations in this guideline for which the overall quality of evidence was graded 'A,' whereas 36 (51.4\%) were graded 'B', 17 (24.3\%) were graded 'C', and 5 (7.1\%) were graded 'D.' Although there are reasons other than quality of evidence to make a grade 1 or 2 recommendation, in general, there is a correlation between the quality of overall evidence and the strength of the recommendation. Thus, there were 43 $(62.3 \%)$ recommendations graded ' 1 ' and $26(37.7 \%)$ graded '2.' There were $9(13.0 \%)$ recommendations graded ' $1 A$,' 23 (33.3\%) were ' $1 \mathrm{~B}$ ', $10(14.5 \%)$ were ' $1 \mathrm{C}$ ', and $1(1.4 \%)$ was '1D.' There were $2(2.9 \%)$ recommendations graded ' $2 \mathrm{~A}$,' $13(18.8 \%)$ were '2B,' 7 (10.1\%) were ' $2 \mathrm{C}$ ', and $4(5.8 \%)$ were '2D.' There were $41(37.3 \%)$ statements that were not graded.

Some argue that recommendations should not be made when evidence is weak. However, clinicians still need to make decisions in their daily practice, and they often ask, "What do the experts do in this setting?" We opted to give guidance, rather than remain silent. These recommendations are often rated with a low strength of recommendation and a low quality of evidence, or were not graded. It is important for the users of this guideline to be cognizant of this (see Notice). In every case these recommendations are meant to be a place for clinicians to start, not stop, their inquiries into specific management questions pertinent to the patients they see in daily practice.

We wish to thank the Work Group Co-Chairs, Drs. Adeera Levin and Paul Stevens, along with all of the Work Group members who volunteered countless hours of their time developing this guideline. We also thank the Evidence Review Team members and staff of the National Kidney Foundation who made this project possible. Finally, we owe a special debt of gratitude to the many KDIGO Board members and individuals who volunteered time reviewing the guideline, and making very helpful suggestions.

Bertram L Kasiske, MD KDIGO Co-Chair
David C Wheeler, MD, FRCP KDIGO Co-Chair 


\section{Work Group Membership}

Kidney International Supplements (2013) 3, 3; doi:10.1038/kisup.2012.75

\section{WORK GROUP CO-CHAIRS}

Adeera Levin, MD, FRCPC

University of British Columbia

Vancouver, Canada
Paul E Stevens, MB, FRCP

East Kent Hospitals University

NHS Foundation Trust

Canterbury, United Kingdom

\section{WORK GROUP}

Rudy W Bilous, MD

Newcastle University and James Cook University Hospital

Middlesbrough, United Kingdom

Josef Coresh, MD, PhD, MHS

Johns Hopkins University

Baltimore, USA

Angel LM de Francisco, MD, PhD

Hospital Universitario Valdecilla

Santander, Spain

Paul E de Jong, MD, PhD

University Medical Center Groningen

Groningen, The Netherlands

Kathryn E Griffith, BM, BS, MSc, MRCP, MRCGP

University Health Centre, York University

York, United Kingdom

Brenda R Hemmelgarn, MD, PhD, FRCP(C)

University of Calgary

Alberta, Canada

Kunitoshi Iseki, MD

University Hospital of the Ryukyus

Nishihara, Okinawa, Japan

\author{
Edmund J Lamb, PhD, FRCPath \\ East Kent Hospitals University \\ NHS Foundation Trust \\ Canterbury, United Kingdom \\ Andrew S Levey, MD \\ Tufts Medical Center \\ Boston, USA \\ Miguel C Riella, MD, PhD, FACP \\ Evangelic University Hospital \\ Curitiba, Brazil \\ Michael G Shlipak, MD, MPH \\ VA Medical Center, UCSF \\ San Francisco, USA \\ Haiyan Wang, MD \\ Peking University First Hospital \\ Beijing, China \\ Colin T White, MD, FRCPC \\ University of British Columbia \\ Vancouver, Canada \\ Christopher G Winearls, MB, DPhil, FRCP \\ Oxford Radcliffe Hospitals NHS Trust \\ Oxford, United Kingdom
}

\section{EVIDENCE REVIEW TEAM}

Tufts Center for Kidney Disease Guideline Development and Implementation

Tufts Medical Center, Boston, MA, USA:

Katrin Uhlig, MD, MS, Project Director; Director, Guideline Development

Dana Miskulin, MD, MS, Staff Nephrologist

Amy Earley, BS, Project Coordinator

Shana Haynes, MS, DHSc, Research Assistant

Jenny Lamont, MS, Project Manager

In addition, support and supervision were provided by:

Ethan M Balk, MD, MPH; Program Director, Evidence Based Medicine 


\section{Abstract}

Kidney International Supplements (2013) 3, 4; doi:10.1038/kisup.2012.76

The Kidney Disease: Improving Global Outcomes (KDIGO) 2012 Clinical Practice Guideline for the Evaluation and Management of Chronic Kidney Disease (CKD) serves to update the 2002 KDOQI Clinical Practice Guidelines for Chronic Kidney Disease: Evaluation, Classification, and Stratification following a decade of focused research and clinical practice in CKD. The document aims to provide state-of-the-art guidance on the evaluation, management and treatment for all patients with CKD. Specifically, the guideline retains the definition of CKD but presents an enhanced classification framework for CKD; elaborates on the identification and prognosis of CKD; discusses the management of progression and complications of CKD; and expands on the continuum of CKD care: timing of specialist referral, ongoing management of people with progressive CKD, timing of the initiation of dialysis, and finally the implementation of a treatment program which includes comprehensive conservative management. The development of the guideline followed an explicit process of evidence review and appraisal. Treatment approaches are addressed in each chapter and guideline recommendations are based on systematic reviews of relevant trials. Practical comments or statements which serve as educational purposes are ungraded, but included as important information for the readership. Appraisal of the quality of the evidence and the strength of recommendations followed the GRADE approach. Ongoing areas of controversies, limitations of the evidence, and international relevance are discussed and additional suggestions are provided for future research.

Keywords: Albuminuria; Chronic kidney disease; Classification; Clinical practice guideline; Evidence-based recommendation; GFR; Glomerular filtration rate; KDIGO; Proteinuria; Systematic review

\section{CITATION}

In citing this document, the following format should be used: Kidney Disease: Improving Global Outcomes (KDIGO) CKD Work Group. KDIGO 2012 Clinical Practice Guideline for the Evaluation and Management of Chronic Kidney Disease. Kidney inter., Suppl. 2013; 3: 1-150. 


\section{Summary of Recommendation Statements}

Kidney International Supplements (2013) 3, 5-14; doi:10.1038/kisup.2012.77

\section{Chapter 1: Definition and classification of CKD}

\section{1: DEFINITION OF CKD}

1.1.1: CKD is defined as abnormalities of kidney structure or function, present for $>3$ months, with implications for health. (Not Graded)

Criteria for CKD (either of the following present for $>\mathbf{3}$ months)

\begin{tabular}{ll}
\hline Markers of kidney damage (one or more) & Albuminuria (AER $\geq 30 \mathrm{mg} / 24$ hours; ACR $\geq 30 \mathrm{mg} / \mathrm{g}[\geq 3 \mathrm{mg} / \mathrm{mmol}]$ ) \\
& Urine sediment abnormalities \\
& Electrolyte and other abnormalities due to tubular disorders \\
& $\begin{array}{l}\text { Abnormalities detected by histology } \\
\text { Structural abnormalities detected by imaging } \\
\text { History of kidney transplantation }\end{array}$ \\
\hline Decreased GFR & GFR $<60 \mathrm{ml} / \mathrm{min} / 1.73 \mathrm{~m}^{2}$ (GFR categories G3a-G5) \\
\hline
\end{tabular}

Abbreviations: CKD, chronic kidney disease; GFR, glomerular filtration rate.

\section{2: STAGING OF CKD}

1.2.1: We recommend that CKD is classified based on cause, GFR category, and albuminuria category (CGA). (1B)

1.2.2: Assign cause of CKD based on presence or absence of systemic disease and the location within the kidney of observed or presumed pathologic-anatomic findings. (Not Graded)

1.2.3: Assign GFR categories as follows (Not Graded):

GFR categories in CKD

\begin{tabular}{lll}
\hline GFR category & GFR $\left(\mathrm{ml} / \mathrm{min} / 1.73 \mathrm{~m}^{2}\right)$ & Terms \\
\hline G1 & $\geq 90$ & Normal or high \\
G2 & $60-89$ & Mildly decreased* \\
G3a & $45-59$ & Mildly to moderately decreased \\
G3b & $30-44$ & Moderately to severely decreased \\
G4 & $15-29$ & Severely decreased \\
G5 & $<15$ & Kidney failure \\
\hline
\end{tabular}

Abbreviations: CKD, chronic kidney disease; GFR, glomerular filtration rate.

*Relative to young adult level

In the absence of evidence of kidney damage, neither GFR category G1 nor G2 fulfill the criteria for CKD.

1.2.4: Assign albuminuria ${ }^{\star}$ categories as follows (Not Graded):

${ }^{*}$ note that where albuminuria measurement is not available, urine reagent strip results can be substituted (Table 7)

Albuminuria categories in CKD

\begin{tabular}{|c|c|c|c|c|}
\hline \multirow[b]{2}{*}{ Category } & \multirow{2}{*}{$\begin{array}{c}\text { AER } \\
\text { (mg/24 hours) }\end{array}$} & \multicolumn{2}{|c|}{ ACR (approximate equivalent) } & \multirow[b]{2}{*}{ Terms } \\
\hline & & (mg/mmol) & $(\mathrm{mg} / \mathrm{g})$ & \\
\hline $\mathrm{A} 1$ & $<30$ & $<3$ & $<30$ & Normal to mildly increased \\
\hline A2 & $30-300$ & $3-30$ & $30-300$ & Moderately increased* \\
\hline A3 & $>300$ & $>30$ & $>300$ & Severely increased ${ }^{* *}$ \\
\hline
\end{tabular}

Abbreviations: AER, albumin excretion rate; $A C R$, albumin-to-creatinine ratio; CKD, chronic kidney disease.

*Relative to young adult level.

**Including nephrotic syndrome (albumin excretion usually $>2200 \mathrm{mg} / 24$ hours $[A C R>2220 \mathrm{mg} / \mathrm{g} ;>220 \mathrm{mg} / \mathrm{mmol}$ ).

\section{3: PREDICTING PROGNOSIS OF CKD}

1.3.1: In predicting risk for outcome of CKD, identify the following variables: 1) cause of CKD; 2) GFR category; 3) albuminuria category; 4) other risk factors and comorbid conditions. (Not Graded) 
1.3.2: In people with $\mathrm{CKD}$, use estimated risk of concurrent complications and future outcomes to guide decisions for testing and treatment for CKD complications. (Not Graded)

1.3.3: In populations with CKD, group GFR and albuminuria categories with similar relative risk for CKD outcomes into risk categories. (Not Graded)

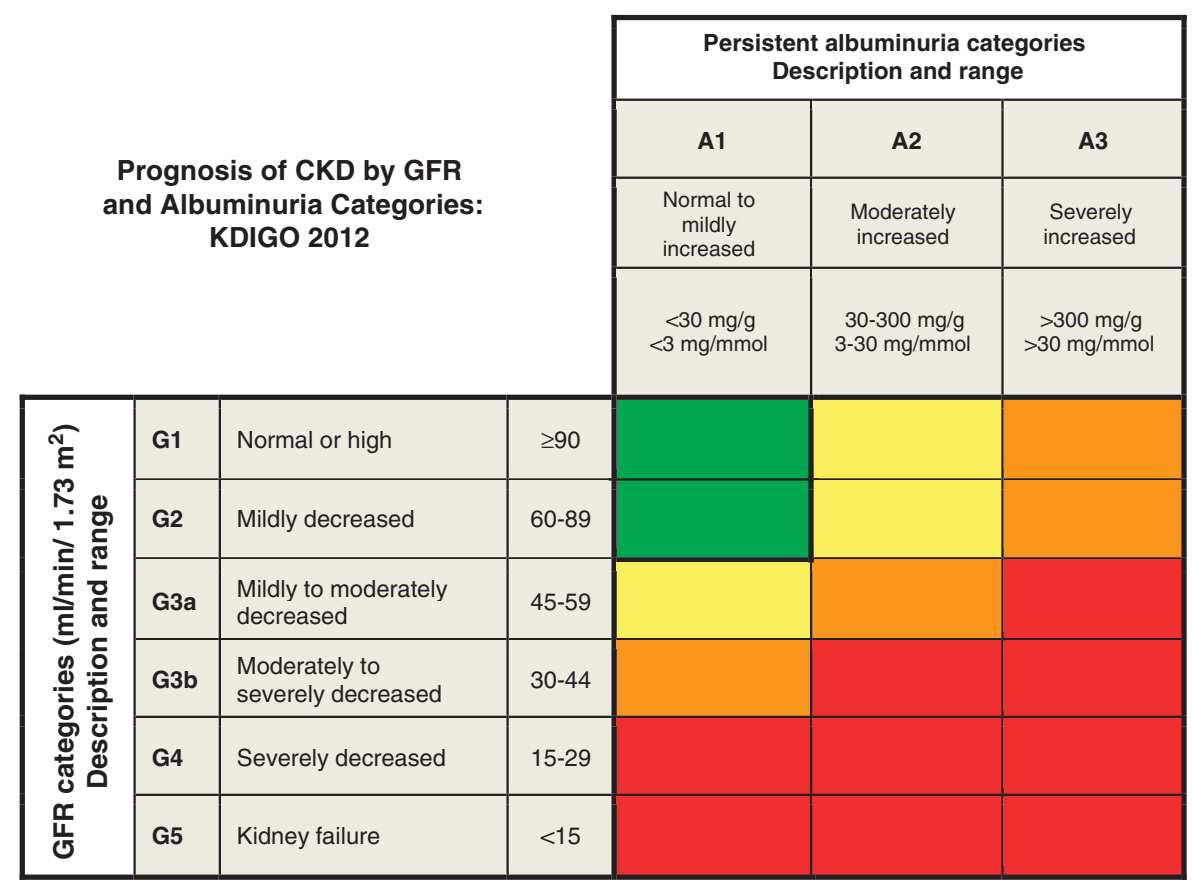

Green: low risk (if no other markers of kidney disease, no CKD); Yellow: moderately increased risk; Orange: high risk; Red, very high risk.

\section{4: EVALUATION OF CKD}

\subsection{1: Evaluation of chronicity}

1.4.1.1: In people with GFR $<60 \mathrm{ml} / \mathrm{min} / 1.73 \mathrm{~m}^{2}$ (GFR categories G3a-G5) or markers of kidney damage, review past history and previous measurements to determine duration of kidney disease. (Not Graded)

- If duration is $>3$ months, CKD is confirmed. Follow recommendations for CKD.

- If duration is not $>3$ months or unclear, CKD is not confirmed. Patients may have CKD or acute kidney diseases (including AKI) or both and tests should be repeated accordingly.

\subsection{2: Evaluation of cause}

1.4.2.1: Evaluate the clinical context, including personal and family history, social and environmental factors, medications, physical examination, laboratory measures, imaging, and pathologic diagnosis to determine the causes of kidney disease. (Not Graded)

\subsection{3: Evaluation of GFR}

1.4.3.1: We recommend using serum creatinine and a GFR estimating equation for initial assessment. (1A)

1.4.3.2: We suggest using additional tests (such as cystatin $\mathrm{C}$ or a clearance measurement) for confirmatory testing in specific circumstances when eGFR based on serum creatinine is less accurate. (2B)

1.4.3.3: We recommend that clinicians $(1 B)$ :

- use a GFR estimating equation to derive GFR from serum creatinine $\left(e G F R_{\text {creat }}\right)$ rather than relying on the serum creatinine concentration alone.

- understand clinical settings in which eGFR $_{\text {creat }}$ is less accurate.

1.4.3.4: We recommend that clinical laboratories should $(1 B)$ :

- measure serum creatinine using a specific assay with calibration traceable to the international standard reference materials and minimal bias compared to isotope-dilution mass spectrometry (IDMS) reference methodology.

- report $\mathrm{eGFR}_{\text {creat }}$ in addition to the serum creatinine concentration in adults and specify the equation used whenever reporting $\mathrm{eGFR}_{\text {creat }}$. 


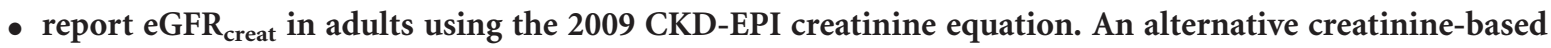
GFR estimating equation is acceptable if it has been shown to improve accuracy of GFR estimates compared to the 2009 CKD-EPI creatinine equation.

When reporting serum creatinine:

- We recommend that serum creatinine concentration be reported and rounded to the nearest whole number when expressed as standard international units $(\mu \mathrm{mol} / \mathrm{l})$ and rounded to the nearest $100^{\text {th }}$ of a whole number when expressed as conventional units $(\mathrm{mg} / \mathrm{dl})$.

When reporting eGFR creat:

- We recommend that eGFR $_{\text {creat }}$ should be reported and rounded to the nearest whole number and relative to a body surface area of $1.73 \mathrm{~m}^{2}$ in adults using the units $\mathrm{ml} / \mathrm{min} / 1.73 \mathrm{~m}^{2}$.

- We recommend eGFR creat levels less than $60 \mathrm{ml} / \mathrm{min} / 1.73 \mathrm{~m}^{2}$ should be reported as "decreased."

1.4.3.5: We suggest measuring cystatin $C$ in adults with eGFR $_{\text {creat }} 45-59 \mathrm{ml} / \mathrm{min} / 1.73 \mathrm{~m}^{2}$ who do not have markers of kidney damage if confirmation of CKD is required. (2C)

- If $\mathrm{GGFR}_{\text {cys }} / \mathrm{eGFR}_{\text {creat-cys }}$ is also $<60 \mathrm{ml} / \mathrm{min} / 1.73 \mathrm{~m}^{2}$, the diagnosis of CKD is confirmed.

- If $e G_{\text {cys }} / \mathrm{eGFR}_{\text {creat-cys }}$ is $\geq 60 \mathrm{ml} / \mathrm{min} / 1.73 \mathrm{~m}^{2}$, the diagnosis of $\mathrm{CKD}$ is not confirmed.

1.4.3.6: If cystatin $C$ is measured, we suggest that health professionals $(2 C)$ :

- use a GFR estimating equation to derive GFR from serum cystatin $C$ rather than relying on the serum cystatin $\mathrm{C}$ concentration alone.

- understand clinical settings in which $\mathrm{eGFR}_{\text {cys }}$ and $\mathrm{eGFR}_{\text {creat-cys }}$ are less accurate.

1.4.3.7: We recommend that clinical laboratories that measure cystatin $C$ should (1B):

- measure serum cystatin $C$ using an assay with calibration traceable to the international standard reference material.

- report eGFR from serum cystatin $\mathrm{C}$ in addition to the serum cystatin $\mathrm{C}$ concentration in adults and specify the equation used whenever reporting eGFR $\mathrm{cys}_{\text {s }}$ and $\mathrm{GGFR}_{\text {creat-cys. }}$

- report eGFR $\mathrm{Gys}_{\text {s }}$ and $\mathrm{GGFR}_{\text {creat-cys }}$ in adults using the 2012 CKD-EPI cystatin C and 2012 CKD-EPI creatinine-cystatin C equations, respectively, or alternative cystatin C-based GFR estimating equations if they have been shown to improve accuracy of GFR estimates compared to the 2012 CKD-EPI cystatin C and 2012 CKD-EPI creatinine-cystatin C equations.

When reporting serum cystatin $\mathrm{C}$ :

- We recommend reporting serum cystatin C concentration rounded to the nearest $100^{\text {th }}$ of a whole number when expressed as conventional units $(\mathrm{mg} / \mathrm{l})$.

When reporting eGFR $\mathrm{Gys}_{\text {s }}$ and $\mathrm{eGFR}_{\text {creat-cys: }}$

- We recommend that $\mathrm{GFR}_{\text {cys }}$ and $\mathrm{GGFR}_{\text {creat-cys }}$ be reported and rounded to the nearest whole number and relative to a body surface area of $1.73 \mathrm{~m}^{2}$ in adults using the units $\mathrm{ml} / \mathrm{min} / 1.73 \mathrm{~m}^{2}$.

- We recommend eGFR ${ }_{\text {cys }}$ and eGFR $_{\text {creat-cys }}$ levels less than $60 \mathrm{ml} / \mathrm{min} / 1.73 \mathrm{~m}^{2}$ should be reported as "decreased."

1.4.3.8: We suggest measuring GFR using an exogenous filtration marker under circumstances where more accurate ascertainment of GFR will impact on treatment decisions. (2B)

\subsection{4: Evaluation of albuminuria}

1.4.4.1: We suggest using the following measurements for initial testing of proteinuria (in descending order of preference, in all cases an early morning urine sample is preferred) $(2 B)$ :

1) urine albumin-to-creatinine ratio (ACR);

2) urine protein-to-creatinine ratio (PCR);

3) reagent strip urinalysis for total protein with automated reading;

4) reagent strip urinalysis for total protein with manual reading.

1.4.4.2: We recommend that clinical laboratories report ACR and PCR in untimed urine samples in addition to albumin concentration or proteinuria concentrations rather than the concentrations alone. (1B)

1.4.4.2.1: The term microalbuminuria should no longer be used by laboratories. (Not Graded) 
1.4.4.3: Clinicians need to understand settings that may affect interpretation of measurements of albuminuria and order confirmatory tests as indicated (Not Graded):

- Confirm reagent strip positive albuminuria and proteinuria by quantitative laboratory measurement and express as a ratio to creatinine wherever possible.

- Confirm ACR $\geq 30 \mathrm{mg} / \mathrm{g}$ ( $\geq 3 \mathrm{mg} / \mathrm{mmol}$ ) on a random untimed urine with a subsequent early morning urine sample.

- If a more accurate estimate of albuminuria or total proteinuria is required, measure albumin excretion rate or total protein excretion rate in a timed urine sample.

1.4.4.4: If significant non-albumin proteinuria is suspected, use assays for specific urine proteins (e.g., $\alpha_{1}-\mathrm{micro}$ globulin, monoclonal heavy or light chains, [known in some countries as "Bence Jones" proteins]). (Not Graded)

\section{Chapter 2: Definition, identification, and prediction of CKD progression}

\section{1: DEFINITION AND IDENTIFICATION OF CKD PROGRESSION}

2.1.1: Assess GFR and albuminuria at least annually in people with CKD. Assess GFR and albuminuria more often for individuals at higher risk of progression, and/or where measurement will impact therapeutic decisions (see figure below). (Not Graded)

2.1.2: Recognize that small fluctuations in GFR are common and are not necessarily indicative of progression. (Not Graded)

2.1.3: Define CKD progression based on one of more of the following (Not Graded):

- Decline in GFR category ( $\geq 90$ [G1], 60-89 [G2], 45-59 [G3a], 30-44 [G3b], 15-29 [G4], <15 [G5] ml/min/ $1.73 \mathrm{~m}^{2}$ ). A certain drop in eGFR is defined as a drop in GFR category accompanied by a $25 \%$ or greater drop in eGFR from baseline.

- Rapid progression is defined as a sustained decline in eGFR of more than $5 \mathrm{ml} / \mathrm{min} / 1.73 \mathrm{~m}^{2} / \mathrm{yr}$.

- The confidence in assessing progression is increased with increasing number of serum creatinine measurements and duration of follow-up.

2.1.4: In people with CKD progression, as defined in Recommendation 2.1.3, review current management, examine for reversible causes of progression, and consider referral to a specialist. (Not Graded)

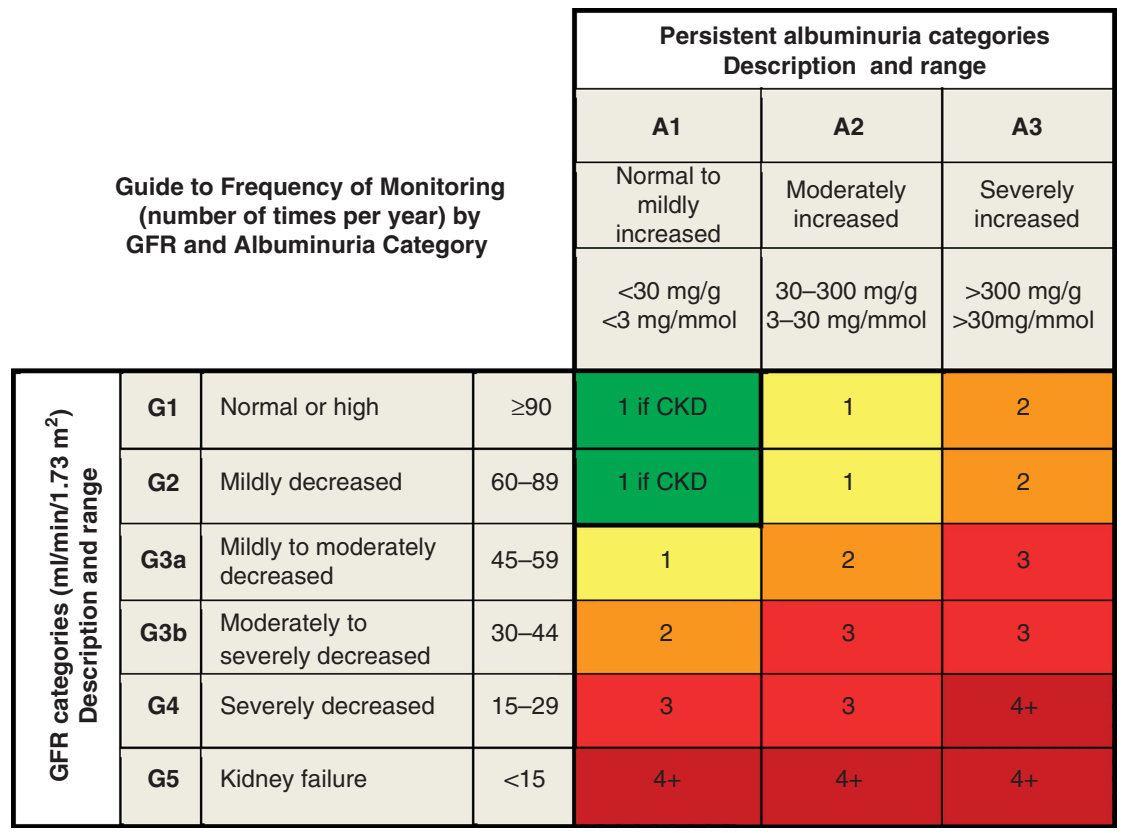

GFR and albuminuria grid to reflect the risk of progression by intensity of coloring (green, yellow, orange, red, deep red). The numbers in the boxes are a guide to the frequency of monitoring (number of times per year). 


\section{2: PREDICTORS OF PROGRESSION}

2.2.1: Identify factors associated with CKD progression to inform prognosis. These include cause of CKD, level of GFR, level of albuminuria, age, sex, race/ethnicity, elevated BP, hyperglycemia, dyslipidemia, smoking, obesity, history of cardiovascular disease, ongoing exposure to nephrotoxic agents, and others. (Not Graded)

\section{Chapter 3: Management of progression and complications of CKD}

\section{1: PREVENTION OF CKD PROGRESSION}

\section{$B P$ and RAAS interruption}

3.1.1: Individualize BP targets and agents according to age, coexistent cardiovascular disease and other comorbidities, risk of progression of $\mathrm{CKD}$, presence or absence of retinopathy (in CKD patients with diabetes), and tolerance of treatment as described in the KDIGO 2012 Blood Pressure Guideline. (Not Graded)

3.1.2: Inquire about postural dizziness and check for postural hypotension regularly when treating CKD patients with BP-lowering drugs. (Not Graded)

3.1.3: Tailor BP treatment regimens in elderly patients with CKD by carefully considering age, comorbidities and other therapies, with gradual escalation of treatment and close attention to adverse events related to $\mathrm{BP}$ treatment, including electrolyte disorders, acute deterioration in kidney function, orthostatic hypotension and drug side effects. (Not Graded)

3.1.4: We recommend that in both diabetic and non-diabetic adults with CKD and urine albumin excretion $<30 \mathrm{mg} /$ 24 hours (or equivalent ${ }^{\star}$ ) whose office BP is consistently $>140 \mathrm{~mm} \mathrm{Hg}$ systolic or $>90 \mathrm{~mm} \mathrm{Hg}$ diastolic be treated with BP-lowering drugs to maintain a BP that is consistently $\leq 140 \mathrm{~mm} \mathrm{Hg}$ systolic and $\leq 90 \mathrm{~mm} \mathrm{Hg}$ diastolic. (1B)

3.1.5: We suggest that in both diabetic and non-diabetic adults with CKD and with urine albumin excretion of $\geq 30 \mathrm{mg} / 24$ hours (or equivalent ${ }^{\star}$ ) whose office BP is consistently $>130 \mathrm{~mm} \mathrm{Hg}$ systolic or $>80 \mathrm{~mm} \mathrm{Hg}$ diastolic be treated with BP-lowering drugs to maintain a BP that is consistently $\leq 130 \mathrm{~mm} \mathrm{Hg}$ systolic and $\leq 80 \mathrm{~mm} \mathrm{Hg}$ diastolic. (2D)

3.1.6: We suggest that an ARB or ACE-I be used in diabetic adults with CKD and urine albumin excretion 30-300 mg/ 24 hours (or equivalent ${ }^{\star}$ ). (2D)

3.1.7: We recommend that an ARB or ACE-I be used in both diabetic and non-diabetic adults with CKD and urine albumin excretion $>300 \mathrm{mg} / 24$ hours $\left(\right.$ or equivalent $^{\star}$ ). (1B)

3.1.8: There is insufficient evidence to recommend combining an ACE-I with ARBs to prevent progression of CKD. (Not Graded)

3.1.9: We recommend that in children with CKD, BP-lowering treatment is started when $B P$ is consistently above the $90^{\text {th }}$ percentile for age, sex, and height. (1C)

3.1.10: We suggest that in children with CKD (particularly those with proteinuria), BP is lowered to consistently achieve systolic and diastolic readings less than or equal to the $50^{\text {th }}$ percentile for age, sex, and height, unless achieving these targets is limited by signs or symptoms of hypotension. (2D)

3.1.11: We suggest that an ARB or ACE-I be used in children with CKD in whom treatment with BP-lowering drugs is indicated, irrespective of the level of proteinuria. (2D)

*Approximate equivalents for albumin excretion rate per 24 hours-expressed as protein excretion rate per 24 hours, albumin-to-creatinine ratio, protein-tocreatinine ratio, and protein reagent strip results- are given in Table 7 , Chapter 1.

\section{CKD and risk of AKI}

3.1.12: We recommend that all people with CKD are considered to be at increased risk of AKI. (1A)

3.1.12.1: In people with CKD, the recommendations detailed in the KDIGO AKI Guideline should be followed for management of those at risk of AKI during intercurrent illness, or when undergoing investigation and procedures that are likely to increase the risk of AKI. (Not Graded) 


\section{Protein intake}

3.1.13: We suggest lowering protein intake to $0.8 \mathrm{~g} / \mathrm{kg} / \mathrm{day}$ in adults with diabetes $(2 C)$ or without diabetes $(2 B)$ and GFR $<30 \mathrm{ml} / \mathrm{min} / 1.73 \mathrm{~m}^{2}$ (GFR categories G4-G5), with appropriate education.

3.1.14: We suggest avoiding high protein intake $(>1.3 \mathrm{~g} / \mathrm{kg} /$ day $)$ in adults with CKD at risk of progression. $(2 C)$

\section{Glycemic control}

3.1.15: We recommend a target hemoglobin $\mathrm{A}_{1 \mathrm{c}}\left(\mathrm{HbA}_{1 \mathrm{c}}\right)$ of $\sim 7.0 \%(53 \mathrm{mmol} / \mathrm{mol})$ to prevent or delay progression of the microvascular complications of diabetes, including diabetic kidney disease. $(1 \mathrm{~A})$

3.1.16: We recommend not treating to an $\mathrm{HbA}_{1 \mathrm{c}}$ target of $<7.0 \%(<53 \mathrm{mmol} / \mathrm{mol})$ in patients at risk of hypoglycemia. (1B)

3.1.17: We suggest that target $\mathrm{HbA}_{1 \mathrm{c}}$ be extended above $7.0 \%(53 \mathrm{mmol} / \mathrm{mol})$ in individuals with comorbidities or limited life expectancy and risk of hypoglycemia. (2C)

3.1.18: In people with CKD and diabetes, glycemic control should be part of a multifactorial intervention strategy addressing blood pressure control and cardiovascular risk, promoting the use of angiotensin-converting enzyme inhibition or angiotensin receptor blockade, statins, and antiplatelet therapy where clinically indicated. (Not Graded)

Salt intake

3.1.19: We recommend lowering salt intake to $<90 \mathrm{mmol}(<2 \mathrm{~g})$ per day of sodium (corresponding to $5 \mathrm{~g}$ of sodium chloride) in adults, unless contraindicated (see rationale). (1C)

3.1.19.1: We recommend restriction of sodium intake for children with CKD who have hypertension (systolic and/ or diastolic blood pressure $>95^{\text {th }}$ percentile) or prehypertension (systolic and/or diastolic blood pressure $>90^{\text {th }}$ percentile and $<95^{\text {th }}$ percentile), following the age-based Recommended Daily Intake. (1C)

3.1.19.2: We recommend supplemental free water and sodium supplements for children with CKD and polyuria to avoid chronic intravascular depletion and to promote optimal growth. (1C)

\section{Hyperuricemia}

3.1.20: There is insufficient evidence to support or refute the use of agents to lower serum uric acid concentrations in people with CKD and either symptomatic or asymptomatic hyperuricemia in order to delay progression of CKD. (Not Graded)

\section{Lifestyle}

3.1.21: We recommend that people with CKD be encouraged to undertake physical activity compatible with cardiovascular health and tolerance (aiming for at least 30 minutes 5 times per week), achieve a healthy weight (BMI 20 to 25, according to country specific demographics), and stop smoking. (1D)

\section{Additional dietary advice}

3.1.22: We recommend that individuals with CKD receive expert dietary advice and information in the context of an education program, tailored to severity of CKD and the need to intervene on salt, phosphate, potassium, and protein intake where indicated. $(1 B)$

\section{2: COMPLICATIONS ASSOCIATED WITH LOSS OF KIDNEY FUNCTION}

\section{Definition and identification of anemia in CKD}

3.2.1: Diagnose anemia in adults and children $>15$ years with CKD when the $\mathrm{Hb}$ concentration is $<13.0 \mathrm{~g} / \mathrm{dl}$ $(<130 \mathrm{~g} / \mathrm{l})$ in males and $<12.0 \mathrm{~g} / \mathrm{dl}(<120 \mathrm{~g} / \mathrm{l})$ in females. (Not Graded)

3.2.2: Diagnose anemia in children with $\mathrm{CKD}$ if $\mathrm{Hb}$ concentration is $<11.0 \mathrm{~g} / \mathrm{dl}(<110 \mathrm{~g} / \mathrm{l})$ in children $0.5-5$ years, $<11.5 \mathrm{~g} / \mathrm{dl}(115 \mathrm{~g} / \mathrm{l})$ in children $5-12$ years, and $<12.0 \mathrm{~g} / \mathrm{dl}(120 \mathrm{~g} / \mathrm{l})$ in children $12-15$ years. (Not Graded)

Evaluation of anemia in people with CKD

3.2.3: To identify anemia in people with CKD measure $\mathrm{Hb}$ concentration (Not Graded):

- when clinically indicated in people with GFR $\geq 60 \mathrm{ml} / \mathrm{min} / 1.73 \mathrm{~m}^{2}$ (GFR categories G1-G2);

- at least annually in people with GFR $30-59 \mathrm{ml} / \mathrm{min} / 1.73 \mathrm{~m}^{2}$ (GFR categories G3a-G3b);

- at least twice per year in people with GFR $<30 \mathrm{ml} / \mathrm{min} / 1.73 \mathrm{~m}^{2}$ (GFR categories G4-G5). 


\section{3: CKD METABOLIC BONE DISEASE INCLUDING LABORATORY ABNORMALITIES}

3.3.1: We recommend measuring serum levels of calcium, phosphate, PTH, and alkaline phosphatase activity at least once in adults with GFR $<45 \mathrm{ml} / \mathrm{min} / 1.73 \mathrm{~m}^{2}$ (GFR categories G3b-G5) in order to determine baseline values and inform prediction equations if used. (1C)

3.3.2: We suggest not to perform bone mineral density testing routinely in those with eGFR $<45 \mathrm{ml} / \mathrm{min} / 1.73 \mathrm{~m}^{2}$ (GFR categories G3b-G5), as information may be misleading or unhelpful. (2B)

3.3.3: In people with GFR $<45 \mathrm{ml} / \mathrm{min} / 1.73 \mathrm{~m}^{2}$ (GFR categories G3b-G5), we suggest maintaining serum phosphate concentrations in the normal range according to local laboratory reference values. $(2 C)$

3.3.4: In people with GFR $<45 \mathrm{ml} / \mathrm{min} / 1.73 \mathrm{~m}^{2}$ (GFR categories G3b-G5) the optimal PTH level is not known. We suggest that people with levels of intact PTH above the upper normal limit of the assay are first evaluated for hyperphosphatemia, hypocalcemia, and vitamin $\mathrm{D}$ deficiency. $(2 C)$

Vitamin D supplementation and bisphosphonates in people with CKD

3.3.5: We suggest not to routinely prescribe vitamin $D$ supplements or vitamin $D$ analogs, in the absence of suspected or documented deficiency, to suppress elevated PTH concentrations in people with CKD not on dialysis. (2B)

3.3.6: We suggest not to prescribe bisphosphonate treatment in people with GFR $<30 \mathrm{ml} / \mathrm{min} / 1.73 \mathrm{~m}^{2}$ (GFR categories G4-G5) without a strong clinical rationale. (2B)

\section{4: ACIDOSIS}

3.4.1: We suggest that in people with CKD and serum bicarbonate concentrations $<22 \mathrm{mmol} / \mathrm{l}$ treatment with oral bicarbonate supplementation be given to maintain serum bicarbonate within the normal range, unless contraindicated. $(2 B)$

\section{Chapter 4: Other complications of CKD: CVD, medication dosage, patient safety, infections, hospitalizations, and caveats for investigating complications of CKD}

\section{1:CKD AND CVD}

4.1.1: We recommend that all people with CKD be considered at increased risk for cardiovascular disease. $(1 A)$

4.1.2: We recommend that the level of care for ischemic heart disease offered to people with CKD should not be prejudiced by their CKD. $(1 A)$

4.1.3: We suggest that adults with CKD at risk for atherosclerotic events be offered treatment with antiplatelet agents unless there is an increased bleeding risk that needs to be balanced against the possible cardiovascular benefits. (2B)

4.1.4: We suggest that the level of care for heart failure offered to people with CKD should be the same as is offered to those without CKD. $(2 A)$

4.1.5: In people with CKD and heart failure, any escalation in therapy and/or clinical deterioration should prompt monitoring of eGFR and serum potassium concentration. (Not Graded)

\section{2: CAVEATS WHEN INTERPRETING TESTS FOR CVD IN PEOPLE WITH CKD}

BNP/N-terminal-proBNP (NT-proBNP)

4.2.1: In people with GFR $<60 \mathrm{ml} / \mathrm{min} / 1.73 \mathrm{~m}^{2}$ (GFR categories G3a-G5), we recommend that serum concentrations of BNP/NT-proBNP be interpreted with caution and in relation to GFR with respect to diagnosis of heart failure and assessment of volume status. (1B)

Troponins

4.2.2: In people with GFR $<60 \mathrm{ml} / \mathrm{min} / 1.73 \mathrm{~m}^{2}$ (GFR categories G3a-G5), we recommend that serum concentrations of troponin be interpreted with caution with respect to diagnosis of acute coronary syndrome. (1B) 


\section{Non-invasive testing}

4.2.3: We recommend that people with CKD presenting with chest pain should be investigated for underlying cardiac disease and other disorders according to the same local practice for people without CKD (and subsequent treatment should be initiated similarly). (1B)

4.2.4: We suggest that clinicians are familiar with the limitations of non-invasive cardiac tests (e.g., exercise electrocardiography [ECG], nuclear imaging, echocardiography, etc.) in adults with CKD and interpret the results accordingly. $(2 B)$

\section{3: CKD AND PERIPHERAL ARTERIAL DISEASE}

4.3.1: We recommend that adults with CKD be regularly examined for signs of peripheral arterial disease and be considered for usual approaches to therapy. (1B)

4.3.2: We suggest that adults with CKD and diabetes are offered regular podiatric assessment. $(2 A)$

\section{4: MEDICATION MANAGEMENT AND PATIENT SAFETY IN CKD}

4.4.1: We recommend that prescribers should take GFR into account when drug dosing. $(1 A)$

4.4.2: Where precision is required for dosing (due to narrow therapeutic or toxic range) and/or estimates may be unreliable (e.g., due to low muscle mass), we recommend methods based upon cystatin C or direct measurement of GFR. (1C)

4.4.3: We recommend temporary discontinuation of potentially nephrotoxic and renally excreted drugs in people with a GFR $<60 \mathrm{ml} / \mathrm{min} / 1.73 \mathrm{~m}^{2}$ (GFR categories G3a-G5) who have serious intercurrent illness that increases the risk of AKI. These agents include, but are not limited to: RAAS blockers (including ACE-Is, ARBs, aldosterone inhibitors, direct renin inhibitors), diuretics, NSAIDs, metformin, lithium, and digoxin. (1C)

4.4.4: We recommend that adults with CKD seek medical or pharmacist advice before using over-the-counter medicines or nutritional protein supplements. (1B)

4.4.5: We recommend not using herbal remedies in people with CKD. (1B)

4.4.6: We recommend that metformin be continued in people with GFR $\geq 45 \mathrm{ml} / \mathrm{min} / 1.73 \mathrm{~m}^{2}$ (GFR categories G1-G3a); its use should be reviewed in those with GFR $30-44 \mathrm{ml} / \mathrm{min} / 1.73 \mathrm{~m}^{2}$ (GFR category G3b); and it should be discontinued in people with GFR $<30 \mathrm{ml} / \mathrm{min} / 1.73 \mathrm{~m}^{2}$ (GFR categories G4-G5). (1C)

4.4.7: We recommend that all people taking potentially nephrotoxic agents such as lithium and calcineurin inhibitors should have their GFR, electrolytes and drug levels regularly monitored. $(1 \mathrm{~A})$

4.4.8: People with CKD should not be denied therapies for other conditions such as cancer but there should be appropriate dose adjustment of cytotoxic drugs according to knowledge of GFR. (Not Graded)

\section{5: IMAGING STUDIES}

4.5.1: Balance the risk of acute impairment in kidney function due to contrast agent use against the diagnostic value and therapeutic implications of the investigation. (Not Graded)

\section{Radiocontrast}

4.5.2: We recommend that all people with GFR $<60 \mathrm{ml} / \mathrm{min} / 1.73 \mathrm{~m}^{2}$ (GFR categories G3a-G5) undergoing elective investigation involving the intravascular administration of iodinated radiocontrast media should be managed according to the KDIGO Clinical Practice Guideline for AKI including:

- Avoidance of high osmolar agents (1B);

- Use of lowest possible radiocontrast dose (Not Graded);

- Withdrawal of potentially nephrotoxic agents before and after the procedure (1C);

- Adequate hydration with saline before, during, and after the procedure (1A);

- Measurement of GFR 48-96 hours after the procedure (1C).

\section{Gadolinium-based contrast media}

4.5.3: We recommend not using gadolinium-containing contrast media in people with $\mathrm{GFR}<15 \mathrm{ml} / \mathrm{min} / 1.73 \mathrm{~m}^{2}$ (GFR category G5) unless there is no alternative appropriate test. (1B)

4.5.4: We suggest that people with a GFR $<30 \mathrm{ml} / \mathrm{min} / 1.73 \mathrm{~m}^{2}$ (GFR categories G4-G5) who require gadoliniumcontaining contrast media are preferentially offered a macrocyclic chelate preparation. (2B)

\section{Bowel preparation}

4.5.5: We recommend not to use oral phosphate-containing bowel preparations in people with a $\mathrm{GFR}<60 \mathrm{ml} / \mathrm{min} /$ $1.73 \mathrm{~m}^{2}$ (GFR categories G3a-G5) or in those known to be at risk of phosphate nephropathy. (1A) 


\section{6: CKD AND RISKS FOR INFECTIONS, AKI, HOSPITALIZATIONS, AND MORTALITY}

\section{CKD and risk of infections}

4.6.1: We recommend that all adults with CKD are offered annual vaccination with influenza vaccine, unless contraindicated. $(1 B)$

4.6.2: We recommend that all adults with eGFR $<30 \mathrm{ml} / \mathrm{min} / 1.73 \mathrm{~m}^{2}$ (GFR categories G4-G5) and those at high risk of pneumococcal infection (e.g., nephrotic syndrome, diabetes, or those receiving immunosuppression) receive vaccination with polyvalent pneumococcal vaccine unless contraindicated. $(1 B)$

4.6.3: We recommend that all adults with CKD who have received pneumococcal vaccination are offered revaccination within 5 years. $(1 B)$

4.6.4: We recommend that all adults who are at high risk of progression of CKD and have GFR $<30 \mathrm{ml} / \mathrm{min} / 1.73 \mathrm{~m}^{2}$ (GFR categories G4-G5) be immunized against hepatitis B and the response confirmed by appropriate serological testing. (1B)

4.6.5: Consideration of live vaccine should include an appreciation of the patient's immune status and should be in line with recommendations from official or governmental bodies. (Not Graded)

4.6.6: Pediatric immunization schedules should be followed according to official international and regional recommedations for children with CKD. (Not Graded)

CKD and risk of $A K I$

4.6.7: We recommend that all people with CKD are considered to be at increased risk of AKI. (1A)

4.6.7.1: In people with CKD, the recommendations detailed in the KDIGO AKI Guideline should be followed for management of those at risk of AKI during intercurrent illness, or when undergoing investigation and procedures that are likely to increase the risk of AKI. (Not Graded)

\section{CKD and risk of hospitalization and mortality}

4.6.8: CKD disease management programs should be developed in order to optimize the community management of people with CKD and reduce the risk of hospital admission. (Not Graded)

4.6.9: Interventions to reduce hospitalization and mortality for people with CKD should pay close attention to the management of associated comorbid conditions and cardiovascular disease in particular. (Not Graded)

\section{Chapter 5: Referral to specialists and models of care}

\section{1: REFERRAL TO SPECIALIST SERVICES}

5.1.1: We recommend referral to specialist kidney care services for people with CKD in the following circumstances (1B):

- AKI or abrupt sustained fall in GFR;

- GFR $<30 \mathrm{ml} / \mathrm{min} / 1.73 \mathrm{~m}^{2}$ (GFR categories G4-G5)*;

- a consistent finding of significant albuminuria (ACR $\geq 300 \mathrm{mg} / \mathrm{g}$ [ $\geq 30 \mathrm{mg} / \mathrm{mmol}]$ or AER $\geq 300 \mathrm{mg}$ / 24 hours, approximately equivalent to PCR $\geq 500 \mathrm{mg} / \mathrm{g}$ [ $\geq 50 \mathrm{mg} / \mathrm{mmol}]$ or PER $\geq 500 \mathrm{mg} / 24$ hours);

- progression of CKD (see Recommendation 2.1.3 for definition);

- urinary red cell casts, $\mathrm{RBC}>20$ per high power field sustained and not readily explained;

- CKD and hypertension refractory to treatment with 4 or more antihypertensive agents;

- persistent abnormalities of serum potassium;

- recurrent or extensive nephrolithiasis;

- hereditary kidney disease.

5.1.2: We recommend timely referral for planning renal replacement therapy (RRT) in people with progressive CKD in whom the risk of kidney failure within 1 year is $10-20 \%$ or higher ${ }^{\dagger}$, as determined by validated risk prediction tools. (1B)

${ }^{\star}$ If this is a stable isolated finding, formal referral (i.e., formal consultation and ongoing care management) may not be necessary and advice from specialist services may be all that is required to facilitate best care for the patients. This will be health-care system dependent.

${ }^{\dagger}$ The aim is to avoid late referral, defined here as referral to specialist services less than 1 year before start of RRT. 


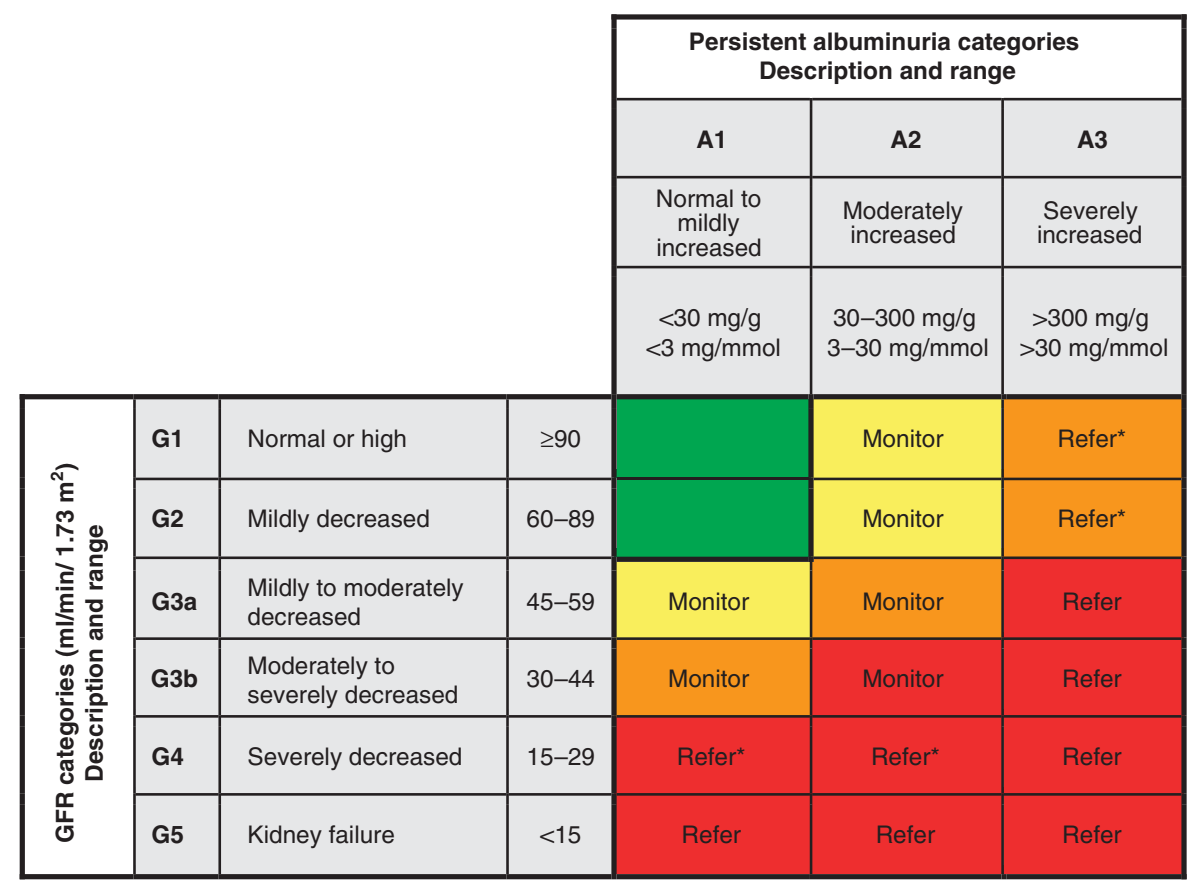

Referral decision making by GFR and albuminuria. *Referring clinicians may wish to discuss with their nephrology service depending on local arrangements regarding monitoring or referring.

\section{2: CARE OF THE PATIENT WITH PROGRESSIVE CKD}

5.2.1: We suggest that people with progressive CKD should be managed in a multidisciplinary care setting. (2B)

5.2.2: The multidisciplinary team should include or have access to dietary counseling, education and counseling about different RRT modalities, transplant options, vascular access surgery, and ethical, psychological, and social care. (Not Graded)

\section{3: TIMING THE INITIATION OF RRT}

5.3.1: We suggest that dialysis be initiated when one or more of the following are present: symptoms or signs attributable to kidney failure (serositis, acid-base or electrolyte abnormalities, pruritus); inability to control volume status or blood pressure; a progressive deterioration in nutritional status refractory to dietary intervention; or cognitive impairment. This often but not invariably occurs in the GFR range between 5 and $10 \mathrm{ml} / \mathrm{min} / 1.73 \mathrm{~m}^{2} .(2 B)$

5.3.2: Living donor preemptive renal transplantation in adults should be considered when the GFR is $<20 \mathrm{ml} / \mathrm{min} /$ $1.73 \mathrm{~m}^{2}$, and there is evidence of progressive and irreversible CKD over the preceding 6-12 months. (Not Graded)

\section{4: STRUCTURE AND PROCESS OF COMPREHENSIVE CONSERVATIVE MANAGEMENT}

5.4.1: Conservative management should be an option in people who choose not to pursue RRT and this should be supported by a comprehensive management program. (Not Graded)

5.4.2: All CKD programs and care providers should be able to deliver advance care planning for people with a recognized need for end-of-life care, including those people undergoing conservative kidney care. (Not Graded)

5.4.3: Coordinated end-of-life care should be available to people and families through either primary care or specialist care as local circumstances dictate. (Not Graded)

5.4.4: The comprehensive conservative management program should include protocols for symptom and pain management, psychological care, spiritual care, and culturally sensitive care for the dying patient and their family (whether at home, in a hospice or a hospital setting), followed by the provision of culturally appropriate bereavement support. (Not Graded) 


\section{Introduction: The case for updating and context}

Kidney International Supplements (2013) 3, 15-18; doi:10.1038/kisup.2012.63

Justification for updating the guideline for CKD Definition, Evaluation, Classification, and Stratification from an international perspective

In 2002, the US-based Kidney Disease Outcomes Quality Initiative (KDOQI) group published a guideline on definition, classification and evaluation of chronic kidney disease (CKD). The guideline proposed uniform definitions of CKD together with a staging system ${ }^{1}$ and described issues related to measurement of kidney function that had not previously been identified by the clinical community. This publication revolutionized the concept and management of CKD, generating substantial research and controversy, stimulating discussion, and influencing public policy and laboratory practice. The research generated has led to new insights which require contextualizing in the current era, providing the evidence drivers for updating guidance for defining, diagnosing, staging and managing $\mathrm{CKD}$, and promoting improved care of those with early CKD. Successive international controversies conferences, under the direction of Kidney Disease: Improving Global Outcomes (KDIGO), have shaped the scope of this international update through:

1. Facilitating global implementation of the definition and classification of $\mathrm{CKD}$, identifying areas of uncertainty, and developing a collaborative research agenda to improve the evidence base and facilitate implementation (November 2004).

2. Evaluating the definition and classification of CKD from a global public health perspective (October 2006).

3. Reviewing the definition and classification of CKD based on data on patient prognosis derived from a unique research collaboration on prognosis (October 2009).

Given the international interest in understanding and improving the outcomes of people living with kidney disease and the tremendous amount of data generated since 2002, a need was identified to review, revise, and update the original 2002 KDOQI guideline.

There has been a wealth of published data highlighting the risk of adverse consequences and outcomes in people with albumin excretion rate (AER) $>30 \mathrm{mg} / 24$ hours and/or glomerular filtration rate (GFR) $<60 \mathrm{ml} / \mathrm{min} / 1.73 \mathrm{~m}^{2}$ (GFR categories G3a-G5), irrespective of the etiology or duration of reduced kidney function. Description of the relationship between GFR, albuminuria and prognosis has significantly improved the understanding of CKD in multiple populations. $^{2-5}$ Internationally, the widespread use of albumin-tocreatinine ratio (ACR) and reagent strip urine testing to detect elevated albuminuria together with reporting of estimated GFR (eGFR) has led to easier identification of people with CKD. However elevated albuminuria or reduced GFR alone are not necessarily indicators of need for specialist referral. Clinicians and medical systems are still adjusting to the improved "identification" of CKD and guidance about appropriate stratification of risk and modified action plans for different subgroups of individuals regarding further evaluation, referral, or treatment is needed.

The goal of this guideline is to clarify the definition and classification system of $C K D$, and to develop appropriate guidance as to the management and care of people with CKD. In addition, we present a framework which should foster an extended collaborative research agenda over the next decade and inform guidelines in the future.

\section{Kidney disease is an important problem worldwide}

Kidney disease is defined as an abnormality of kidney structure or function with implications for the health of an individual, which can occur abruptly, and either resolve or become chronic. CKD is a general term for heterogeneous disorders affecting kidney structure and function with variable clinical presentation, in part related to cause, severity and the rate of progression. The concept of CKD evolved after the recognition of the contribution of disordered kidney structure and function on the health of individuals across a wide range of severity. ${ }^{1}$ The utility of the concept is that recognition of CKD will have implications for the individual and their care. Kidney failure is traditionally considered as the most serious outcome of CKD. Symptoms are usually due to complications of decreased kidney function and when severe, they can be treated only by dialysis or transplantation. Earlier stages of kidney disease are often asymptomatic, are detected during the evaluation of comorbid conditions, and may be reversible. Rapidly progressive diseases may lead to kidney failure within months but most diseases evolve over decades, and some patients do not progress during many years of follow-up.

Figure 1 shows a conceptual model for the development, progression, and complications of $\mathrm{CKD}^{1,6}$ The model includes antecedents associated with increased risk for development of CKD, stages of disease, and complications including death. Risks for development of CKD may be categorized either as susceptibility to kidney disease due to sociodemographic and genetic factors or exposure to factors that can initiate kidney disease. Abnormalities in kidney structure (damage) usually precede abnormalities in function. Outcomes of CKD may be progression, as shown by the horizontal arrows, and complications, as shown by the diagonal arrows, or both. 


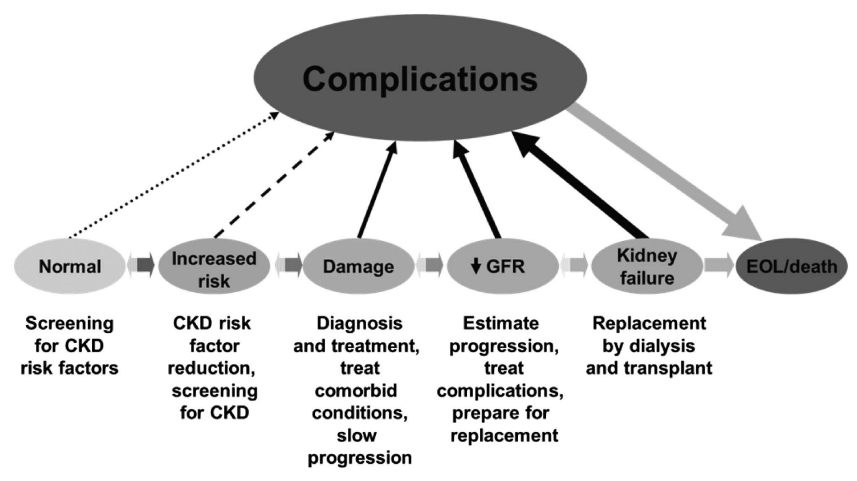

Figure 1 | Conceptual model of CKD. Continuum of development, progression, and complications of CKD and strategies to improve outcomes. Horizontal arrows between circles represent development, progression, and remission of CKD. Left-pointing horizontal arrowheads signify that remission is less frequent than progression. Diagonal arrows represent occurrence of complications of CKD, including drug toxicity, endocrine and metabolic complications, cardiovascular disease, and others such as infection, cognitive impairment, and frailty. Complications might also arise from adverse effects of interventions to prevent or treat the disease. CKD, chronic kidney disease; EOL, end-of-life care and/or conservative management; GFR, glomerular filtration rate. Adapted from Levey AS, Stevens LA, Coresh J. ${ }^{6}$ Conceptual model of CKD: applications and implications.

Am J Kidney Dis. 2009; 53:S4-16 with permission from the National Kidney Foundation; accessed http://download.journals. elsevierhealth.com/pdfs/journals/0272-6386/PIIS02726386080 17186.pdf

Although the need for treatment of chronic kidney failure with dialysis and/or kidney transplantation arises in only $1 \%$ of people with CKD, it remains the most expensive of chronic diseases and reduces lifespan significantly. The costs of dialysis and transplantation consume disproportionate amounts within the health-care budgets in all jurisdictions (5\% of annual budgets consumed by less than $1 \%$ of the population). Failure to recognize CKD results in neglect of its consequences and complications, and late referral of people with advanced CKD resulting in worse renal replacement therapy (RRT) outcomes. In addition, there is a growing body of evidence that indicates people with CKD are at increased risk of acute kidney injury (AKI), which is also associated with poor outcomes and may accelerate progression of CKD. Therefore, identification of people at earlier time points in the trajectory of $\mathrm{CKD}$, with appropriate management and earlier referral of those who would benefit from specialist kidney services, should lead to both economic and clinical benefits.

In those countries where access to dialysis and transplantation services may be limited or unavailable, the final consequence of progressive CKD is death. In all locations, irrespective of availability of dialysis and transplantation, early identification of CKD therefore assumes great importance, as delay or prevention of progression has the potential to prolong health and save lives for much lower cost than RRT. Although etiologies vary in frequency or absolute numbers in different countries, the proportion of people with important antecedents to CKD, such as diabetes, is growing alarmingly worldwide in both developed and developing countries.

Complications of CKD affect all organ systems. Kidney failure leads to the commonly recognized symptoms of uremia. Less severe CKD has been recognized as an independent risk factor for cardiovascular disease (CVD) and other common conditions affecting the elderly, such as infection and impairments in physical function and cognition. In addition, CKD is associated with increased risk from adverse effects of drugs, intravascular radiocontrast administration, surgery and other invasive procedures. Altogether, these complications are associated with higher morbidity, mortality and cost. If CKD is detected early, the associated complications and the progression to kidney failure can be delayed or even prevented through appropriate interventions. Regular testing of high-risk groups (i.e., people with diabetes, hypertension, CVD, structural renal tract disease, multisystem diseases with potential kidney involvement such as systemic lupus erythematosus, family history of kidney failure, hereditary kidney disease, the elderly, those receiving potential nephrotoxic drugs or those opportunistically found to have hematuria or proteinuria) can give an early indication of kidney damage, thus permitting the introduction of available interventions at an early stage, and the testing of novel interventions with potential added value.

Factors associated with progression of CKD and with increased cardiovascular risk are overlapping to a large extent. Thus targeting of those risk factors that are modifiable may both reduce CVD in people with CKD and reduce progression of CKD to end-stage renal disease (ESRD). There is strong evidence that blockade of the renin-angiotensinaldosterone system (RAAS) is a blood pressure (BP) lowering strategy which is more effective in reducing risk of kidney and cardiovascular disease in the presence of albuminuria.

The development of guidance for health-care providers will provide opportunities to improve the care of people with kidney disease. We hope that this publication serves to stimulate strategic research initiatives from basic, translational, clinical and health outcome perspectives.

\section{General summary for the reader: what you will and will not find in this guideline}

1. The guideline will offer best practice and evidence-based advice on the evaluation and approach to management of CKD.

a. The target population for the guideline is all people identified with CKD who are not on RRT (i.e., not on dialysis or have not received a kidney transplant).

b. The target population includes adults and children. The guideline will cover the spectrum of individuals with CKD, from children to the elderly who form important subgroups, underscoring current issues at the extremes of age with respect to the evidence base, especially in relation to implementation and management issues. Where the guideline does not apply to 
children, statements to that effect will be made. It is beyond the scope of this guideline to address all issues related to children with $\mathrm{CKD}$, given the heterogeneous nature of this group of individuals who range from newborn to post-adolescents, with specific physiological differences within each of those groups. Specific evidence and rationale will be articulated as appropriate in each section.

c. The target condition is CKD of any or unknown etiology. Identifying the cause of the CKD is strongly encouraged, both because treatment may need to be adjusted according to etiology and because it influences the prognosis and relative importance of risk factors associated with CKD. A comprehensive list of possible etiologies is not practical and guidance on detailed work-up for specific causes of CKD is beyond the scope of this document (readers will be referred to other pertinent sources). We will describe how knowledge of the etiology of CKD in an individual may be important in prognostication and management.

d. The target audience of the guideline includes nephrologists, primary care physicians, non-nephrology specialists (e.g., cardiologists, diabetologists, etc), clinical chemists and other practitioners caring for adults and children with CKD. The guideline is also expected to be suitable for use in public policy and other health-care arenas.

e. As a global guideline it is written for use in different health-care settings, but unavoidably its full implementation relies on health-care resources that are not universally available. We recognize this overtly in some of the discussion sections within the guideline.

f. The target health-care settings include primary, secondary, and tertiary care.

2. The guideline will provide information, advice, and education to support self-management for people with $\mathrm{CKD}$ and aid caregivers with the diagnosis and management of CKD. To avoid redundancy and potential for becoming outdated, the reader is asked to refer to existing KDIGO guidance on anemia, metabolic bone disease, BP, AKI, hepatitis C, lipid management, glomerulonephritis (GN) and other pertinent guidelines.

3. The guideline will provide a blueprint for an approach to $\mathrm{CKD}$ care in an international context. While the guideline will be sensitive to issues related to ethnicity and also geographical considerations, it is expected that subsequent regional adaptation will be required for specific healthcare settings or contexts.

4. Research recommendations in general are described to inform a framework for ongoing research agendas in the international community. We have attempted to identify important study questions in need of answers. Through identification of gaps in knowledge, the reader will be better able to define methodologies, definitions of populations, and outcome measures of relevance to study designs in the future.

\section{Topics that will not be covered}

This document is not intended to provide enough detail to replace training and education in nephrology, nor is it intended to serve as a textbook of medicine or nephrology.

Thus, there are some specific topics that will not be covered. Specifically we will not discuss:

1. Evaluation and management of people receiving RRT (management of kidney failure by dialysis or kidney transplantation).

2. Specific approaches to the diagnosis of people with AKI and other acute kidney diseases. This topic has been extensively reviewed in KDIGO Clinical Practice Guideline for Acute Kidney Injury. ${ }^{7}$

3. Diagnostic work-up or treatment of specific causes of $\mathrm{CKD}$, including $\mathrm{GN} .^{8}$

4. Management of pregnancy in women with CKD or of pregnant women who develop kidney disease.

5. Detailed management of endocrine and metabolic complications of CKD. These are reviewed extensively by recent KDIGO guidelines on CKD-MBD, ${ }^{9}$ management of $\mathrm{BP}^{10}$ and anemia ${ }^{11}$ in CKD.

6. Detailed management of CVD and CVD risk factors in CKD. This is reviewed in a recent KDIGO publication. ${ }^{12}$

7. Drug dosing in CKD. This topic has been addressed in a recent KDIGO publication. ${ }^{13}$

8. Details of resource implications and barriers to implementation are beyond the scope of this guideline. By virtue of its being international, the variability in these aspects by country, region, and even jurisdiction is vast. We look to the individual commentaries from around the world to inform those aspects more fully.

\section{Brief overview on methodology}

The Work Group included an international group of kidney specialists, primary care physicians, diabetologists, epidemiologists, clinical chemists, administrators, and a professional Evidence Review Team (ERT) who provided support and guidance to the group. Details of the methods used by the ERT are described in Methods for Guideline Development, along with the systematic searches for areas identified by Work Group members and performed by the ERT.

The recommendations and statements created herein will serve to direct both care and research in the next decade. Importantly, we expect the renewed classification system and risk stratification concepts to direct research and enrollment into trials which address test therapies to improve patient outcomes.

Statement grading and wording. The methods for formulating recommendations were based on modified Grading of Recommendations Assessment, Development, and Evaluation (GRADE) system, and have used the words 
Table 1 | KDIGO nomenclature and description for grading recommendations

\begin{tabular}{llll}
\hline Grade* & & \multicolumn{1}{c}{ Implications } & Policy \\
\cline { 2 - 5 } $\begin{array}{l}\text { Level } 1 \\
\text { "We } \\
\text { recommend" }\end{array}$ & $\begin{array}{l}\text { Post people in your situation would } \\
\text { want the recommended course of } \\
\text { action and only a small proportion } \\
\text { would not. }\end{array}$ & $\begin{array}{l}\text { Most patients should receive the } \\
\text { recommended course of action. }\end{array}$ & $\begin{array}{l}\text { The recommendation can be evaluated } \\
\text { as a candidate for developing a policy } \\
\text { or a performance measure. }\end{array}$ \\
$\begin{array}{llll}\text { Level } 2 \\
\text { "We suggest" }\end{array}$ & $\begin{array}{l}\text { The majority of people in your situation } \\
\text { would want the recommended course } \\
\text { of action, but many would not. }\end{array}$ & $\begin{array}{l}\text { Different choices will be appropriate } \\
\text { for different patients. Each patient } \\
\text { needs help to arrive at a management } \\
\text { decision consistent with her or his } \\
\text { values and preferences. }\end{array}$ & $\begin{array}{l}\text { The recommendation is likely to } \\
\text { require substantial debate and } \\
\text { involvement of stakeholders before } \\
\text { policy can be determined. }\end{array}$ \\
\end{tabular}

*The additional category "Not Graded" was used, typically, to provide guidance based on common sense or where the topic does not allow adequate application of evidence. The most common examples include recommendations regarding monitoring intervals, counseling, and referral to other clinical specialists. The ungraded recommendations are generally written as simple declarative statements, but are not meant to be interpreted as being stronger recommendations than Level 1 or 2 recommendations.

'recommend' when the balance of evidence would support the notion that the majority of patients would benefit from the implementation of this recommendation. The words 'suggest' are used when the balance of evidence would support the notion that some patients would benefit from the implementation of the recommendation, but that individual patient, physician and health-care system considerations would be necessary to adopt the practice. There are also ungraded statements many of which are often key practice points or educational issues (Table 1). The Work Group had struggled whether to organize them differently or move them to the rationale section. Ultimately they remain here in the guideline statement format so that they are not overlooked by those wishing to understand the condition better.

A significant proportion of statements in this guideline are ungraded because the grading system is most appropriate for statements of intervention. The international system, GRADE, allows for such statements which guide thoughts and attitude, and not specific actions. In the descriptive statements identifying, classifying, and defining the condition of CKD, grading is not possible. Since few studies have compared different methods of evaluation or care models, those statements too are difficult to grade. Thus, grading of specific statements is reserved for interventions or alternative diagnostic test strategies for which there is a substantial body of evidence.
Consideration of health benefits, side effects, and risks. These have been considered when formulating the recommendations but given the paucity of data in many of the areas reviewed, this has been less consistent than the Work Group would have liked. We see this as an area of research and future study that will inform future updates.

Review process. As with all KDIGO guidelines a two step process was used. This included a review by the Board of Directors, with feedback to the Work Group Chairs followed by revisions to the document. The public review, consisting of interested stakeholders from international communities, organizations and individuals, was then undertaken. The draft document was sent to a total of 2320 external reviewers, with 293 responses received and tabulated. The feedback was carefully reviewed and where appropriate, suggested changes were incorporated into the final document. In the interest of transparency, the Work Group prepared individual responses to each reviewer comment and these will be posted on the KDIGO website.

Planned update. At the current time there is no official plan to update the guideline in its entirety. Given the breadth and depth of the current undertaking and with knowledge of new studies and applications of some of these recommendations, the Work Group recommends that individual sections of this guideline be updated every 3-5 years as new evidence becomes available. We believe that this will be more practical for the readership. 


\section{Chapter 1: Definition and classification of CKD}

Kidney International Supplements (2013) 3, 19-62; doi:10.1038/kisup.2012.64

\section{1: DEFINITION OF CKD}

1.1.1: CKD is defined as abnormalities of kidney structure or function, present for $>3$ months, with implications for health (Table 2). (Not Graded)

\section{RATIONALE}

The definition of CKD remains intact, but we have clarified the classification and risk stratification as indicated below. The addition of 'with implications for health' is intended to reflect the notion that a variety of abnormalities of kidney structure or function may exist, but not all have implications for health of individuals, and therefore need to be contextualized.

Kidney damage refers to a broad range of abnormalities observed during clinical assessment, which may be insensitive and non-specific for the cause of disease but may precede reduction in kidney function (Table 2). Excretory, endocrine and metabolic functions decline together in most chronic kidney diseases. GFR is generally accepted as the best overall index of kidney function. We refer to a GFR $<60 \mathrm{ml} / \mathrm{min} /$ $1.73 \mathrm{~m}^{2}$ as decreased GFR (Table 2) and a GFR $<15 \mathrm{ml} / \mathrm{min} /$ $1.73 \mathrm{~m}^{2}$ as kidney failure. AKI may occur in patients with CKD and hasten the progression to kidney failure. ${ }^{14}$

Complications include drug toxicity, metabolic and endocrine complications, increased risk for CVD, and a variety of other recently recognized complications, including infections, frailty, and cognitive impairment. ${ }^{15-18}$ Complications may occur at any stage, often leading to death without progression to kidney failure. Complications may also arise from adverse effects of interventions to prevent or treat the disease and associated comorbidity.

\section{Criteria for CKD}

Defining terms: The following section aims to define specific terms and concepts so as to ensure clarity among all users. In addition, the rationale for including these terms is included. Table 3 provides a justification for the criteria for CKD. The criteria for definition of CKD are objective and can be ascertained by means of simple laboratory tests without identification of the cause of disease, thereby enabling detection of CKD by non-nephrologist physicians and other health professionals.

\section{Duration $>\mathbf{3}$ Months}

Kidney diseases may be acute or chronic. We explicitly but arbitrarily define duration of $>3$ months ( $>90$ days) as delineating "chronic" kidney disease. The rationale for defining chronicity is to differentiate CKD from acute kidney diseases (such as acute GN), including AKI, which may require different interventions, and have different etiologies and outcomes. ${ }^{7}$ We did not define acute kidney disease (AKD) because there does not appear be an evidence base for a precise definition.

The duration of kidney disease may be documented or inferred based on the clinical context. For example, a patient with decreased kidney function or kidney damage in the midst of an acute illness, without prior documentation of kidney disease, may be inferred to have AKI. Resolution over days to weeks would confirm the diagnosis of AKI. A patient with similar findings in the absence of an acute illness may be inferred to have CKD, and if followed over time would be confirmed to have CKD. In both cases, repeat ascertainment of kidney function and kidney damage is recommended for accurate diagnosis. The timing of the evaluation depends on clinical judgment, with earlier evaluation for the patients suspected of having AKI and later evaluation for the patient suspected of having CKD. For further details on the Evaluation of CKD, see Chapter 1.4.

Reversibility. Most kidney diseases do not have symptoms or findings until later in their course and are detected only when they are chronic. Most causes of CKD are irreversible with a life-long course, and treatment aimed at slowing progression to kidney failure. However, chronicity is not synonymous with irreversibility. In some cases, CKD is entirely reversible, either spontaneously or with treatment, and in other cases, treatment can cause partial regression of kidney damage and improvement in function (e.g., immunosuppressive therapies for GN). Even kidney failure may be reversed with transplantation. Because of the long course of most cases of CKD, patients often have one or more episodes of AKI, superimposed upon CKD.

\section{Decreased GFR}

The kidney has many functions, including excretory, endocrine and metabolic functions. The GFR is one component of excretory function, but is widely accepted as the best overall index of kidney function because it is generally reduced after widespread structural damage and most other kidney functions decline in parallel with GFR in CKD.

We chose a threshold of GFR $<60 \mathrm{ml} / \mathrm{min} / 1.73 \mathrm{~m}^{2}$ (GFR categories G3a-G5) for $>3$ months to indicate CKD. A GFR $<60 \mathrm{ml} / \mathrm{min} / 1.73 \mathrm{~m}^{2}$ is less than half of the normal value in young adult men and women of approximately $125 \mathrm{ml} / \mathrm{min} /$ $1.73 \mathrm{~m}^{2}$. Figure 2 shows a compilation of GFR measurements in apparently healthy men and women in the US and Europe by age from more than 40 years ago. ${ }^{20}$ The age-associated GFR decline is observed in longitudinal as well as cross 
Table 2 | Criteria for CKD (either of the following present for $>\mathbf{3}$ months)

\begin{tabular}{ll}
\hline Markers of kidney damage (one or more) & Albuminuria (AER $\geq 30 \mathrm{mg} / 24$ hours; ACR $\geq 30 \mathrm{mg} / \mathrm{g}[\geq 3 \mathrm{mg} / \mathrm{mmol}])$ \\
& Urine sediment abnormalities \\
& Electrolyte and other abnormalities due to tubular disorders \\
& Abnormalities detected by histology \\
& Structural abnormalities detected by imaging \\
& History of kidney transplantation
\end{tabular}

Decreased GFR

GFR $<60 \mathrm{ml} / \mathrm{min} / 1.73 \mathrm{~m}^{2}$ (GFR categories G3a-G5)

Abbreviations: CKD, chronic kidney disease; GFR, glomerular filtration rate.

Table 3 | Criteria for definition of CKD ${ }^{19}$

\begin{tabular}{|c|c|}
\hline Criteria & Comment \\
\hline $\begin{array}{l}\text { Duration }>3 \text { months, based on documentation or } \\
\text { inference }\end{array}$ & $\begin{array}{l}\text { Duration is necessary to distinguish chronic from acute kidney diseases } \\
\text { - Clinical evaluation will often enable documentation or inference of duration } \\
\text { - Documentation of duration is usually not declared in epidemiologic studies }\end{array}$ \\
\hline GFR $<60 \mathrm{ml} / \mathrm{min} / 1.73 \mathrm{~m}^{2}$ (GFR categories G3a-G5) & $\begin{array}{l}\text { GFR is the best overall index of kidney function in health and disease } \\
\text { - The normal GFR in young adults is approximately } 125 \mathrm{ml} / \mathrm{min} / 1.73 \mathrm{~m}^{2} \text {. GFR } \\
<15 \mathrm{ml} / \mathrm{min} / 1.73 \mathrm{~m}^{2} \text { (GFR category G5) is defined as kidney failure } \\
\text { - Decreased GFR can be detected by current estimating equations for GFR based } \\
\text { on } \mathrm{SCr} \text { or cystatin C but not by SCr or cystatin C alone } \\
\text { - Decreased eGFR can be confirmed by measured GFR, if required }\end{array}$ \\
\hline
\end{tabular}

Kidney damage as defined by structural abnormalities or functional abnormalities other than decreased GFR
Albuminuria as a marker of kidney damage [increased glomerular permeability], urine $A E R \geq 30 \mathrm{mg} / 24$ hours, approximately equivalent to urine $A C R \geq 30 \mathrm{mg} / \mathrm{g}$ $(\geq 3 \mathrm{mg} / \mathrm{mmol})^{*}$

- The normal urine ACR in young adults is $<10 \mathrm{mg} / \mathrm{g}(<1 \mathrm{mg} / \mathrm{mmol})$

- Urine ACR $30-300 \mathrm{mg} / \mathrm{g}$ (3-30 mg/mmol; category A2) generally corresponds to "microalbuminuria," now referred to as "moderately increased"

- Urine ACR $>300 \mathrm{mg} / \mathrm{g}$ (>30 mg/mmol; category A3) generally corresponds to "macroalbuminuria," now termed "severely increased"

- Urine ACR $>2200 \mathrm{mg} / \mathrm{g}(220 \mathrm{mg} / \mathrm{mmol})$ may be accompanied by signs and symptoms of nephrotic syndrome (e.g., low serum albumin, edema, and high serum cholesterol)

- Threshold value corresponds approximately to urine reagent strip values of trace or + , depending on urine concentration.

- High urine ACR can be confirmed by urine albumin excretion in a timed urine collection expressed as AER

Urinary sediment abnormalities as markers of kidney damage

- Isolated non-visible (microscopic) hematuria with abnormal RBC morphology (anisocytosis) in GBM disorders

- $\mathrm{RBC}$ casts in proliferative glomerulonephritis

- WBC casts in pyelonephritis or interstitial nephritis

- Oval fat bodies or fatty casts in diseases with proteinuria

- Granular casts and renal tubular epithelial cells in many parenchymal diseases (non-specific)

Renal tubular disorders

- Renal tubular acidosis

- Nephrogenic diabetes insipidus

- Renal potassium wasting

- Renal magnesium wasting

- Fanconi syndrome

- Non-albumin proteinuria

- Cystinuria

Pathologic abnormalities detected by histology or inferred (examples of causes)

- Glomerular diseases (diabetes, autoimmune diseases, systemic infections, drugs, neoplasia)

- Vascular diseases (atherosclerosis, hypertension, ischemia, vasculitis, thrombotic microangiopathy)

- Tubulointerstitial diseases (urinary tract infections, stones, obstruction, drug toxicity)

- Cystic and congenital diseases 


\begin{tabular}{|c|c|}
\hline Criteria & Comment \\
\hline & $\begin{array}{l}\text { Structural abnormalities as markers of kidney damage detected by imaging } \\
\text { (ultrasound, computed tomography and magnetic resonance with or without contrast, } \\
\text { isotope scans, angiography) } \\
\text { - Polycystic kidneys } \\
\text { - Hysplastic kidneys } \\
\text { - Cortical scarring due to infarcts, pyelonephritis or associated with vesicoureteral } \\
\text { reflux } \\
\text { - Renal masses or enlarged kidneys due to infiltrative diseases } \\
\text { - Renal artery stenosis } \\
\text { - Small and hyperechoic kidneys (common in more severe CKD due to many } \\
\text { History of kidney transplantation } \\
\text { Kidney biopsies in most kidney transplant recipients have histopathologic } \\
\text { abnormalities even if GFR is }>60 \text { ml/min/1.73 } \text { m }^{2} \text { (GFR categories G1-G2) and } \\
\text { ACR is < } 30 \text { mg/g (<3 mg/mmol) } \\
\text { Kidney transplant recipients have an increased risk for mortality and kidney } \\
\text { failure compared to populations without kidney disease } \\
\text { Kidney transplant recipients routinely receive subspecialty care }\end{array}$ \\
\hline
\end{tabular}

Abbreviations: ACR, albumin-to-creatinine ratio; AER, albumin excretion rate; CKD, chronic kidney disease; eGFR, estimated glomerular filtration rate; GBM, glomerular basement membrane; GFR, glomerular filtration rate; RBC, red blood cell; SCr, serum creatinine; WBC, white blood cell.

*For conversion, see Table 7, Chapter 1

sectional studies, but varies substantially among individuals within the population. ${ }^{21}$ More recent data in kidney donors confirm these general trends. ${ }^{22,23}$ Limited data are available for non-whites in the US and Europe or in other countries, although data suggest that the normal range for measured GFR and the age-associated decline is similar. ${ }^{24-26}$

A GFR $<60 \mathrm{ml} / \mathrm{min} / 1.73 \mathrm{~m}^{2}$ can be detected by routine laboratory testing. Current estimating equations for GFR (eGFR) based on serum creatinine (SCr), but not $\mathrm{SCr}$ alone, are sensitive for detecting measured GFR $<60 \mathrm{ml} / \mathrm{min} /$ $1.73 \mathrm{~m}^{2} \cdot{ }^{27}$ A decreased eGFR using $\mathrm{SCr}$ can be confirmed by GFR estimation using an alternative filtration marker (cystatin C) or GFR measurement, as necessary.

A GFR $<60 \mathrm{ml} / \mathrm{min} / 1.73 \mathrm{~m}^{2}$ is associated with a higher risk of complications of CKD than in subjects with CKD and conserved GFR. The causal mechanisms underlying these associations are not fully understood. We consider three main types of complications, which are of relevance to all patients with CKD and reduced GFR, irrespective of country, age or etiology:

Drug toxicity. Altered pharmacokinetics of drugs excreted by the kidney and an increased risk of drug-interactions are common and require adjustment in the dosage of many drugs (see Chapter 4.4). ${ }^{13}$ At lower GFR, altered pharmacokinetics and pharmacodynamics of drugs not excreted by the kidney may also be observed. Errors in drug dosing are common in patients with CKD and may be associated with toxicity to the kidney (resulting in AKI) or systemic toxicity, resulting in threats to patient safety.

Metabolic and endocrine complications. As GFR declines a variety of complications reflecting loss of endocrine or exocrine function of the kidneys develop including anemia, acidosis, malnutrition, bone and mineral disorders (described in Chapters 3 and 4).
Risk of CVD and death. A meta-analysis by the CKD Prognosis Consortium demonstrated associations of eGFR $<60 \mathrm{ml} /$ $\min / 1.73 \mathrm{~m}^{2}$ with subsequent risk of all-cause and cardiovascular mortality, kidney failure, AKI, and CKD progression in the general population and in populations with increased risk for CVD. ${ }^{3-5}$ Figure 3 shows the relationship for total and cardiovascular mortality in general population cohorts. The risk for all outcomes was relatively constant between eGFR of $75-105 \mathrm{ml} / \mathrm{min} / 1.73 \mathrm{~m}^{2}$, with a suggestion of a U-shaped curve for total mortality. The increased relative risk (RR) for all outcomes was significant for eGFR of $<60 \mathrm{ml} / \mathrm{min} / 1.73 \mathrm{~m}^{2}$.

\section{Kidney Damage}

Damage to the kidney can be within the parenchyma, large blood vessels or collecting systems, and is most often inferred from markers rather than direct examination of kidney tissue. The markers of kidney damage often provide a clue to the likely site of damage within the kidney and in association with other clinical findings, the cause of kidney disease.

Proteinuria. Proteinuria is a general term for the presence of increased amounts of protein in the urine. Proteinuria may reflect abnormal loss of plasma proteins due to a) increased glomerular permeability to large molecular weight proteins (albuminuria or glomerular proteinuria), b) incomplete tubular reabsorption of normally filtered low-molecularweight proteins (tubular proteinuria), or c) increased plasma concentration of low-molecular-weight proteins (overproduction proteinuria, such as immunoglobulin light chains). Proteinuria may also reflect abnormal loss of proteins derived from the kidney (renal tubular cell constituents due to tubular damage) and lower urinary tract. Albuminuria, tubular proteinuria and renal tubular cell constituents are pathognomonic of kidney damage. In addition, findings 


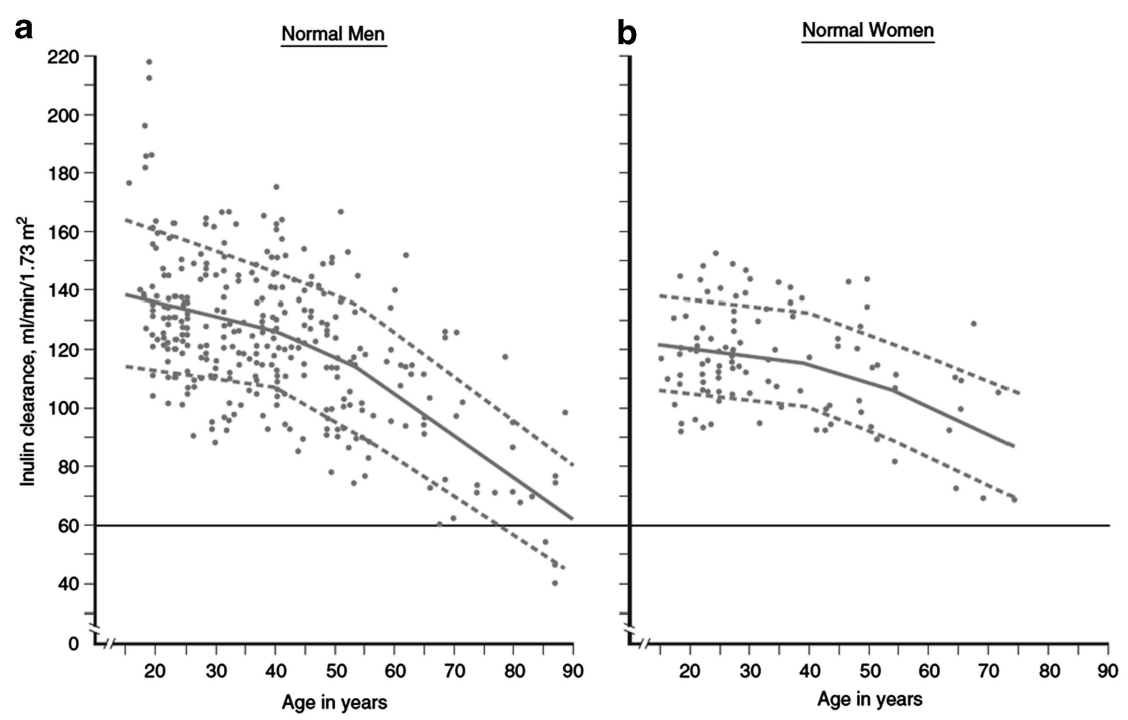

Figure $\mathbf{2} \mid$ Normal values for GFR by age. GFR is shown for men (Panel a) and women (Panel b) of various ages, with the GFR measured as the urinary clearance of inulin. The horizontal line indicates a GFR value of $60 \mathrm{ml} / \mathrm{min} / 1.73 \mathrm{~m}^{2}$, which is the threshold for the definition of CKD. Solid lines represent the mean value of GFR per decade of age, and dashed lines represent the value $1 \mathrm{SD}$ from the mean value of GFR per decade of age. CKD, chronic kidney disease; GFR, glomerular filtration rate; SD, standard deviation. Adapted with permission from Wesson L. ${ }^{20}$ Physiology of the Human Kidney. Grune \& Stratton: New York, 1969.

from experimental and clinical studies have suggested an important role for proteinuria in the pathogenesis of disease progression of CKD. ${ }^{28}$

Albuminuria. Albuminuria refers to abnormal loss of albumin in the urine. Albumin is one type of plasma protein found in the urine in normal subjects and in larger quantity in patients with kidney disease.

For a number of reasons, clinical terminology is changing to focus on albuminuria rather than proteinuria: a) albumin is the principal component of urinary protein in most kidney diseases; recent recommendations for measurement of urine proteins emphasize quantification of albuminuria rather than total protein; b) recent epidemiologic data from studies around the world demonstrate a strong graded relationship of the quantity of urine albumin with both kidney and CVD risk; and c) later recommendations in these guidelines classify kidney disease by level of albuminuria. In this guideline, we will refer to proteinuria when discussing general concepts and will refer either to total protein, albumin or other specific proteins when discussing measurements, patterns, and interpretation of proteinuria.

Albuminuria is a common but not uniform finding in CKD. It is the earliest marker of glomerular diseases, including diabetic glomerulosclerosis, where it generally appears before the reduction in GFR. It is a marker of hypertensive nephrosclerosis but may not appear until after the reduction in GFR. It is often associated with underlying hypertension, obesity, and vascular disease, where the underlying renal pathology is not known.

Normative values for albuminuria and proteinuria are generally expressed as the urinary loss rate. The urinary loss rate of albumin and protein has commonly been referred to as AER and protein excretion rate (PER), respectively, although in the strict physiological sense they are not excreted. The terms AER and PER will be retained herein.

We chose a threshold for urinary AER of $\geq 30 \mathrm{mg} /$ 24 hours sustained for $>3$ months to indicate CKD. This value is considered to be approximately equivalent to an ACR in a random untimed urine sample of $\geq 30 \mathrm{mg} / \mathrm{g}$ or $\geq 3 \mathrm{mg} / \mathrm{mmol}$. The rationale for this threshold is as follows:

- An AER of $\geq 30 \mathrm{mg} / 24$ hours (ACR $\geq 30 \mathrm{mg} / \mathrm{g}$ [ $\geq 3 \mathrm{mg} /$ $\mathrm{mmol}]$ ) is greater than 3 times the normal value in young adult men and women of approximately $10 \mathrm{mg} / 24$ hours (ACR $10 \mathrm{mg} / \mathrm{g}$ or $1 \mathrm{mg} / \mathrm{mmol}$ ).

- An AER of $\geq 30 \mathrm{mg} / 24$ hours (ACR $\geq 30 \mathrm{mg} / \mathrm{g}$ [ $\geq 3 \mathrm{mg}$ / mmol]) may sometimes be detectable as 'trace' using a urine reagent strip, depending on urine concentration, but this is not a consistent finding until AER exceeds approximately $300 \mathrm{mg} / 24$ hours (ACR $\geq 300 \mathrm{mg} / \mathrm{g}[\geq 30 \mathrm{mg} / \mathrm{mmol}]$ ). As described later, trace or positive reagent strip values/readings can be confirmed by ACR, and an elevated ACR can be confirmed by urine AER in a timed urine collection, as necessary.

- An $\mathrm{AER} \geq 30 \mathrm{mg} / 24$ hours (ACR $\geq 30 \mathrm{mg} / \mathrm{g}$ [ $\geq 3 \mathrm{mg} /$ $\mathrm{mmol}]$ ) is associated with an increased risk for complications of CKD. A meta-analysis by the CKD Prognosis Consortium demonstrated associations of an ACR $\geq 30 \mathrm{mg} / \mathrm{g} \quad(\geq 3 \mathrm{mg} / \mathrm{mmol})$ or reagent strip $1+$ protein with subsequent risk of all-cause and cardiovascular mortality, kidney failure, $\mathrm{AKI}$, and $\mathrm{CKD}$ progression in the general population and in populations with increased risk for $\mathrm{CVD}^{3-5}$ (Figure 4). 

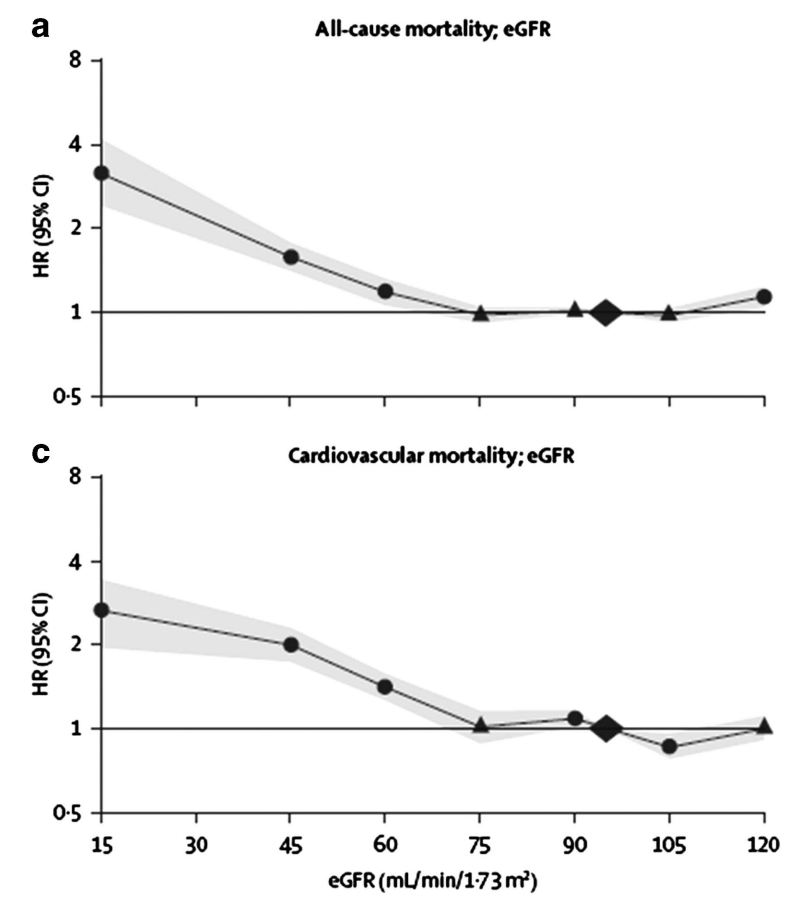

Figure 3 | Relationship of eGFR with mortality. HRs and $95 \% \mathrm{Cls}$ for all-cause (a) and cardiovascular mortality (c) according to spline eGFR. HRs and 95\% Cls (shaded areas) are adjusted for ACR, age, sex, ethnic origin, history of CVD, systolic BP, diabetes, smoking, and total cholesterol. The reference (diamond) was eGFR $95 \mathrm{ml} / \mathrm{min} / 1.73 \mathrm{~m}^{2}$ and ACR $5 \mathrm{mg} / \mathrm{g}(0.6 \mathrm{mg} / \mathrm{mmol})$, respectively. Circles represent statistically significant and triangles represent not significant. ACR, albumin-to-creatinine ratio; $\mathrm{BP}$, blood pressure; $\mathrm{Cl}$, confidence interval; $\mathrm{CVD}$, cardiovascular disease; eGFR, estimated glomerular filtration rate; HR, hazard ratio. Reprinted from The Lancet, vol 375, Matshushita K, van de Velde $\mathrm{M}$, Astor BC, et al. ${ }^{4}$ Association of estimated glomerular filtration rate and albuminuria with all-cause and cardiovascular mortality in general population cohorts: a collaborative meta-analysis, $\mathrm{p}$. 2073-2081, 2010, with permission from Elsevier; accessed http:// download.thelancet.com/pdfs/journals/lancet/ PIIS0140673610606745.pdf
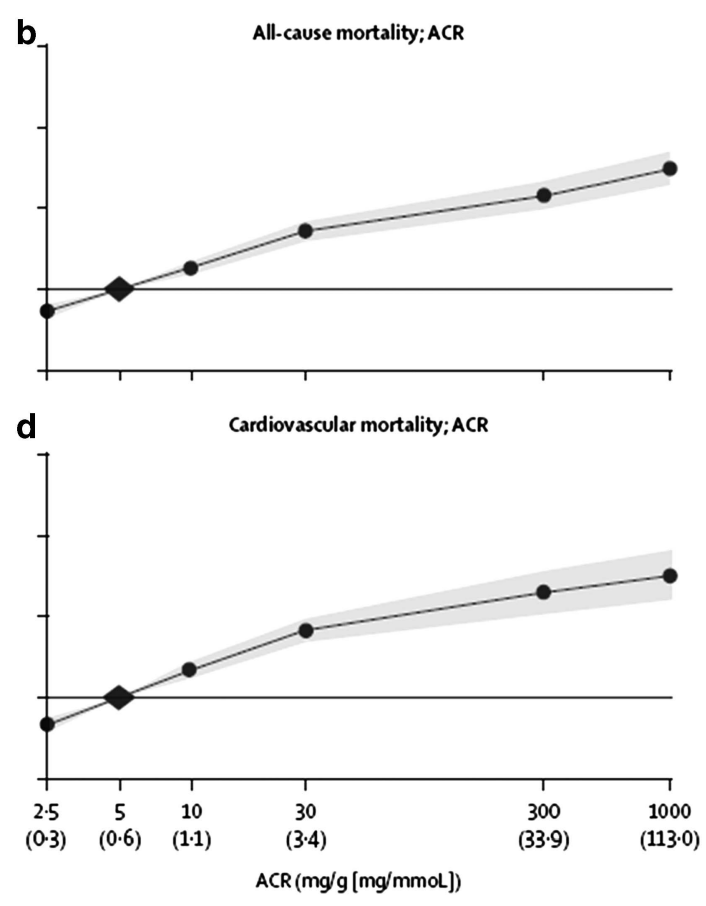

Figure 4 |Relationship of albuminuria with mortality. HRs and 95\% Cls for all-cause (b) and cardiovascular mortality (d) according to ACR. HRs and $95 \% \mathrm{Cls}$ (shaded areas) are adjusted for age, sex, ethnic origin, history of CVD, systolic BP, diabetes, smoking, and total cholesterol and spline eGFR. The reference (diamond) was ACR $5 \mathrm{mg} / \mathrm{g}(0.6 \mathrm{mg} / \mathrm{mmol})$ and eGFR $95 \mathrm{ml} / \mathrm{min} /$ $1.73 \mathrm{~m}^{2}$, respectively. Circles represent statistically significant and triangles represent not significant. ACR plotted in $\mathrm{mg} / \mathrm{g}$. To convert $\mathrm{ACR}$ in $\mathrm{mg} / \mathrm{g}$ to $\mathrm{mg} / \mathrm{mmol}$ multiply by 0.113 . Approximate conversions to $\mathrm{mg} / \mathrm{mmol}$ are shown in parentheses. ACR, albumin-to-creatinine ratio; $\mathrm{BP}$, blood pressure; $\mathrm{Cl}$, confidence interval; CVD, cardiovascular disease; eGFR, estimated glomerular filtration rate; $H R$, hazard ratio. Reprinted from The Lancet, vol 375, Matshushita K, van de Velde M, Astor BC, et al. ${ }^{4}$ Association of estimated glomerular filtration rate and albuminuria with all-cause and cardiovascular mortality in general population cohorts: a collaborative meta-analysis, p. 2073-2081, 2010, with permission from Elsevier; accessed http://download.thelancet.com/pdfs/ journals/lancet/PIIS0140673610606745.pdf

parenchyma in kidney biopsies irrespective of eGFR or other markers of kidney damage must be acknowledged as an important parameter in defining kidney damage. The pathologic classification of diseases of the renal parenchyma reflects the localization of the disease to glomeruli, vessels, tubules and interstitium, or cysts. Renal biopsies are performed in the minority of CKD patients.

Imaging abnormalities. Imaging techniques allow the diagnosis of diseases of the renal structure, vessels and/or collecting systems. Thus, patients with significant structural abnormalities are considered to have CKD if the abnormality persists for greater than 3 months (note that this does not include simple cysts and clinical context is required for action).

History of kidney transplantation. Kidney transplant recipients are defined as having CKD, irrespective of the level of GFR or presence of markers of kidney damage. The rationale 
for this designation is that biopsies in kidney transplant recipients reveal pathologic abnormalities even in patients without decreased GFR or albuminuria. Kidney transplant recipients have an increased risk of mortality and kidney outcomes compared to the general population and they require specialized medical management. ${ }^{29}$

\section{Implications for Health}

$\mathrm{CKD}$ is associated with a wide range of complications leading to adverse health outcomes. For some complications, the causal pathway between kidney disease and adverse outcomes is well-known. For these complications, there are clinical practice guidelines for testing and treatment for modifiable factors to prevent adverse outcomes. Since 2002 a large number of epidemiologic studies have linked decreased GFR and albuminuria to the risk of adverse health outcomes not previously identified as CKD complications. The exploration of the mechanisms for the relationships of CKD with these complications is a rapidly growing topic for basic and clinical research. Because of the high prevalence, adverse outcomes, and high cost of CKD, especially kidney failure, some countries have developed public health programs for early identification and treatment of CKD and its complications. The effectiveness of these programs is being evaluated.

\section{Implications for Clinical Practice and Public Policy}

CKD was first defined in the $2002 \mathrm{KDOQI}$ Guidelines and endorsed at subsequent KDIGO Controversies Conferences with minor modifications. ${ }^{30,31}$ The definition of CKD proposed here is intended for use in clinical practice, research and public health, and has not changed. Thus, the updated version does not change any of the initiatives that have been commenced with respect to public policy. We recognize the variation around the world regarding measurement of urine albumin versus total protein in clinical practice, and we anticipate variation in implementation of the guideline until more widespread dissemination of the guideline has occurred. For additional discussion about methods for ascertainment of urine albumin versus total protein, see Recommendation 1.4.4 (Evaluation of albuminuria). The implications of highlighting the importance of albuminuria for general practitioners in evaluation and prognostication may help with identification and care planning. Nonetheless, a number of concerns about the definition remain, which are clarified below. ${ }^{30,32-36}$

\section{Areas of Controversy, Confusion, or Non-consensus and Clarification of Issues and Key Points \\ General concerns:}

a) The use of single thresholds without consideration of patient specific factors

The use of single thresholds to define decreased GFR and increased AER, without consideration for cause of disease, age, sex, race-ethnicity and clinical context is consistent with the use of single thresholds for disease markers to define other chronic non-communicable diseases, such as hyperten- sion, diabetes, and hypercholesterolemia, that primarily affect the elderly and are associated with an increased risk for cardiovascular mortality. Biologic variability and error in ascertainment of GFR and AER can lead to misclassification and false negative and false positive diagnosis. Furthermore, these single thresholds appear to differentiate groups of individuals and outcomes, irrespective of specific patient characteristics in a multitude of studies. However, they correspond to thresholds for RRs for complications, rather than predictions of absolute risk. Furthermore, as with any diagnostic tests, findings must be interpreted with considerations of likelihood of disease based on the clinical context but this should not negate the application of a standard definition for CKD.

\section{Specific concerns:}

\section{b) Relationship of CKD criteria to aging}

Epidemiologic studies show an increased prevalence of decreased eGFR and increased ACR in older subjects. There has been vigorous debate as to whether decreased GFR or increased ACR in older people represent a disease or "normal aging." Numerous studies show pathologic abnormalities associated with aging, including glomerular sclerosis, tubular atrophy and vascular sclerosis. The cause for this association is not clear but has been hypothesized to reflect disparate processes, such as vascular disease or senescence. ${ }^{37-39}$ Irrespective of cause, there appears to be increased risk associated with decreased eGFR or increased ACR in older people, and for this reason, we consider all individuals with persistently decreased GFR or increased albuminuria to have CKD. Comparison of the magnitude of risk to younger individuals is complicated. As with other CVD risk factors, absolute risk appears to be higher in older than in younger individuals, but RR appears to be lower. ${ }^{3-5}$ Note is also made that healthy older individuals do not necessarily have decreased GFR, so that while one may expect some decline, levels below $60 \mathrm{ml} / \mathrm{min} / 1.73 \mathrm{~m}^{2}$ in individuals without comorbidity is the exception. ${ }^{20}$

\section{c) Isolated decreased GFR without markers of kidney damage}

A variety of clinical circumstances are associated with GFR $<60 \mathrm{ml} / \mathrm{min} / 1.73 \mathrm{~m}^{2}$ for $>3$ months in the absence of known structural alterations. Below are examples of these conditions and the rationale for considering them as CKD:

- Heart failure, cirrhosis of the liver, and hypothyroidism. Decreased GFR complicates the management of the primary disease and patients with these disorders with decreased GFR have a worse prognosis than those without decreased GFR. In addition, renal biopsy in these patients may reveal renal parenchymal lesions.

- Kidney donors. The usual level of GFR in kidney donors after transplantation is approximately $70 \%$ of the predonation level, in the range of $60-90 \mathrm{ml} / \mathrm{min} / 1.73 \mathrm{~m}^{2}$ in most donors. However, a minority of donors have GFR $<60 \mathrm{ml} / \mathrm{min} / 1.73 \mathrm{~m}^{2}$. The prognosis of these donors 
compared to those with higher GFR has not been carefully studied. However, as with decreased GFR due to recognized kidney diseases, donors with decreased GFR require closer follow-up for adjustment of drug doses.

- Malnutrition. The level of GFR is affected by habitual protein intake. ${ }^{40}$ Healthy adults with lower protein intake may have lower mean GFR, but usually do not have GFR $<60 \mathrm{ml} / \mathrm{min} / 1.73 \mathrm{~m}^{2}$. Older studies of patients with protein-calorie malnutrition and more recent studies of subjects with anorexia nervosa have documented reduced measured GFR that can improve following restoration of nutritional status. However, renal biopsies may reveal structural abnormalities in these conditions and decreased GFR can complicate their management.

\section{d) Isolated albuminuria without decreased GFR}

As described later, transient ACR $\geq 30 \mathrm{mg} / \mathrm{g}$ ( $\geq 3 \mathrm{mg} /$ $\mathrm{mmol}$ ) can occur in disorders other than CKD. Remission of albuminuria within 3 months in association with recovery from these disorders is not defined as CKD. Patients with persistent albuminuria would be considered to have CKD. Below are examples of these conditions and the rationale for considering them as CKD:

- Obesity and metabolic syndrome. Albuminuria can be associated with obesity and metabolic syndrome, and can remit during weight loss. The mechanism of albuminuria in these conditions is not known but renal biopsies may reveal prominent vascular lesions. Patients with obesity and metabolic syndrome are at increased risk for development of diabetes and hypertension. The risk of persistent albuminuria in this condition has not been carefully studied.

- Orthostatic (postural) proteinuria. ${ }^{41}$ Albuminuria may rarely be observed in the upright but not recumbent posture in patients with the syndrome of postural proteinuria. This condition is not associated with an increased risk of long-term adverse outcomes but a thorough evaluation is required to exclude other causes of CKD. Exclusion is generally possible by studying a first pass early morning urine (EMU) after overnight recumbency: total protein loss of $>1000 \mathrm{mg} / 24$ hours is unlikely to be explained by orthostatic proteinuria.

\section{e) Remission of decreased GFR or markers of kidney damage}

If decreased GFR and markers of kidney damage resolve while on treatment, the patient would be considered to have treated CKD, consistent with nomenclature for treated hypertension, treated diabetes, or treated hypercholesterolemia if blood pressure, blood glucose and blood cholesterol are within normal range while on medications. If resolution of decreased GFR and markers of kidney damage is sustained after withdrawal of treatment, the patient would be considered to have a history of CKD. f) Kidney disease in the absence of decreased GFR and markers of kidney damage

A GFR $\geq 60 \mathrm{ml} / \mathrm{min} / 1.73 \mathrm{~m}^{2}$ may reflect a decline from a higher value, and an AER of $<30 \mathrm{mg} / 24$ hours (ACR $<30 \mathrm{mg} / \mathrm{g}$ or $<3 \mathrm{mg} / \mathrm{mmol}$ ) may reflect a rise from a lower value. Both findings may be associated with a pathologic process, even in the absence of other markers of kidney damage. Although such patients do not fulfill the criteria for CKD, a clinician's high index of suspicion may warrant additional diagnostic testing or close follow-up to detect the onset of CKD.

\section{Pediatric Considerations}

In general the definition of CKD in adults applies to children (birth-18 years) with the following exceptions or allowances:

- the criteria for duration $>3$ months does not apply to newborns or infants $\leq 3$ months of age.

- the criteria of a GFR $<60 \mathrm{ml} / \mathrm{min} / 1.73 \mathrm{~m}^{2}$ does not apply to children $<2$ years of age in whom an age appropriate value should be applied.

- a urinary total protein or albumin excretion rate above the normal value for age may be substituted for albuminuria $\geq 30 \mathrm{mg} / 24$ hours.

- all electrolyte abnormalities are to be defined in light of age normative values.

Developmental renal abnormalities account for as many as $30-50 \%$ of the children with CKD or ESRD. ${ }^{42}$ As such many infants while born with normal SCr for age will in fact meet the definition of CKD based on structural abnormalities despite the appearance of a normal GFR and may be classified as such within the first few days of life.

Normal GFR in newborns is less than $60 \mathrm{ml} / \mathrm{min} / 1.73 \mathrm{~m}^{2}$, and it is not until approximately 2 years of age that one expects to see body surface area (BSA) adjusted GFR values comparable to those seen in the adult. ${ }^{43,44}$ The expected increases in GFR that occur in the first months of life are due to increases in mean arterial pressure (MAP), decrease in renal vascular resistance, and redistribution of intrarenal blood flows to the superficial cortical nephrons in the newborn and increases in glomerular size and capillary permeability in the infant. ${ }^{45-48}$ As such direct application of the GFR threshold values in the current CKD definition would not be appropriate in children less than 2 years of age as their normative maximal values would be below those of the adult or older child; hence most neonates and infants would be classified $a$ priori at a decreased GFR based not on a reduction in GFR from a higher value, but rather failure of maturity of the kidney.

Numerous references exist for fetal, ${ }^{49}$ neonatal term, ${ }^{44,48}$ pre-term, ${ }^{46,50,51}$ infant, child and adolescent GFR values ${ }^{43,44}$ and the reader is strongly encouraged to use such references when comparison to a normative range is required for approximating the reduction in renal clearance of the individual child. It should be noted that across these ages the method of GFR measurement has often varied with the 
majority of such measurements in the neonate (term or preterm) or infant being derived from urinary collections and creatinine clearance $(\mathrm{CrCl})$ measurements, whereas the older children and adolescents are often investigated with exogenous markers including inulin, radionuclides, and other markers such as iohexol or iothalamate.

The most comprehensive list of GFR based on the gold standard of inulin clearance and stratified by age for both term and preterm babies and children up to the age of young adults can be found in Schwartz and Furth's review on GFR measurements and estimation in pediatric $\mathrm{CKD}^{52}$

Similarly, age relevant normative values should be utilized when interpreting urinary protein (albumin) excretions as well as other important urinary and serum laboratory values. Such values may be found in a number of pediatric nephrology texts. For neonates and infants this includes Waters ${ }^{53}$ and for post-neonate to young adults, more comprehensive values can be found in Langlois. ${ }^{54}$

\subsection{STAGING OF CKD}

\subsection{1: We recommend that CKD is classified based on cause, GFR category, and albuminuria category (CGA). (1B)}

\section{RATIONALE}

This statement is worded in this way because a classification encompassing cause and severity, as expressed by the level of GFR and the level of albuminuria, links to risks of adverse outcomes including mortality and kidney outcomes. These factors will therefore guide management of CKD and this recommended classification is consistent with other classification systems of disease which are based on the general domains of cause, duration and severity which provide a guide to prognosis. We included only kidney measures as factors in the classification of kidney disease, although we acknowledge that factors other than kidney measures, such as level of BP, also affect prognosis in CKD.

This recommended staging with inclusion of two additional domains represents a revision of the previous CKD guidelines, which included staging only by level of GFR. Cause of disease is included because of its fundamental importance in predicting the outcome of CKD and choice of cause-specific treatments. With inclusion of cause of kidney disease in the classification, we considered that it was no longer necessary to retain the use of the letter " $\mathrm{T}$ " to refer to kidney transplant recipients. Albuminuria is included as an additional expression of severity of disease not only because it is a marker of the severity of injury but also because albuminuria itself strongly associates with progression of kidney disease. Numerous studies have identified the adverse prognostic implication of albuminuria irrespective of level of kidney function.

We propose that this classification of CKD by Cause, GFR and Albuminuria, respectively be referred to as CGA staging. It can be used to inform the need for specialist referral, general medical management, and indications for investigation and therapeutic interventions. It will also be a tool for the study of the epidemiology, natural history, and prognosis of CKD.

\section{Pediatric Considerations}

The principles inherent in this guideline are fully applicable to children.

While large scale trials in children relating cause, GFR and albuminuria or proteinuria are rare, the principles of a multimodal classification in these three spheres should apply to children.

To date the only large scale trial utilizing a validated exogenously measured GFR (iohexol) and urinary protein excretion in a well-described cohort of children with renal disease is the Chronic Kidney Disease in Children (CKiD) trial. ${ }^{55}$ They have enrolled over 600 children aged 1-16 years and have described GFR and urinary proteinuria related outcomes in the areas of neurodevelopment, cognition, behavior, cardiovascular health and risk, and somatic growth. They have also collected samples for ongoing and future genetic study. While these data are sparse in relation to overall adult numbers, this represents one of the largest pediatric nephrology trials. The use of true measured GFR, the quality and completeness of the data, and the long term longitudinal follow-up will form the basis for the best evidence-based outcomes in children with CKD for the foreseeable future. A recent review article by Copelovitch et al. $^{56}$ summarizes the major findings of the trial up to the present time.

\subsection{2: Assign cause of CKD based on presence or absence of systemic disease and the location within the kidney of observed or presumed pathologic-anatomic findings. (Not Graded)}

\section{RATIONALE}

This statement has been included so as to ensure that clinicians are alerted to the fact that CKD is not a diagnosis in and of itself, and that the assignment of cause is important for prognostication and treatment.

The cause of CKD has been traditionally assigned based on presence or absence of underlying systemic diseases and location of known or presumed pathologicanatomic abnormalities. The distinction between systemic diseases affecting the kidney and primary kidney diseases is based on the origin and locus of the disease process. In primary kidney disease the process arises and is confined to the kidney whereas in systemic diseases the kidney is only one victim of a specific process, for example diabetes mellitus. Certain genetic diseases cross this boundary by affecting different tissues, e.g., adult polycystic kidney disease. The location of pathologic-anatomic findings is based on the magnitude of proteinuria, findings from the urine sediment examination, imaging, and renal pathology. 
Table 4 | Classification* of CKD based on presence or absence of systemic disease and location within the kidney of pathologicanatomic findings

\begin{tabular}{|c|c|c|}
\hline & $\begin{array}{l}\text { Examples of systemic diseases } \\
\text { affecting the kidney }\end{array}$ & $\begin{array}{l}\text { Examples of primary kidney diseases (absence of } \\
\text { systemic diseases affecting the kidney) }\end{array}$ \\
\hline Glomerular diseases & $\begin{array}{l}\text { Diabetes, systemic autoimmune diseases, systemic } \\
\text { infections, drugs, neoplasia (including amyloidosis) }\end{array}$ & $\begin{array}{l}\text { Diffuse, focal or crescentic proliferative GN; focal and } \\
\text { segmental glomerulosclerosis, membranous nephropathy, } \\
\text { minimal change disease }\end{array}$ \\
\hline $\begin{array}{l}\text { Tubulointerstitial } \\
\text { diseases }\end{array}$ & $\begin{array}{l}\text { Systemic infections, autoimmune, sarcoidosis, } \\
\text { drugs, urate, environmental toxins } \\
\text { (lead, aristolochic acid), neoplasia (myeloma) }\end{array}$ & Urinary-tract infections, stones, obstruction \\
\hline Vascular diseases & $\begin{array}{l}\text { Atherosclerosis, hypertension, ischemia, cholesterol } \\
\text { emboli, systemic vasculitis, thrombotic } \\
\text { microangiopathy, systemic sclerosis }\end{array}$ & $\begin{array}{l}\text { ANCA-associated renal limited vasculitis, fibromuscular } \\
\text { dysplasia }\end{array}$ \\
\hline $\begin{array}{l}\text { Cystic and congenital } \\
\text { diseases }\end{array}$ & $\begin{array}{l}\text { Polycystic kidney disease, Alport syndrome, } \\
\text { Fabry disease }\end{array}$ & Renal dysplasia, medullary cystic disease, podocytopathies \\
\hline
\end{tabular}

Abbreviations: ANCA, antineutrophil cytoplasmic antibody; CKD, chronic kidney disease, GN, glomerulonephritis

Genetic diseases are not considered separately because some diseases in each category are now recognized as having genetic determinants.

* Note that there are many different ways in which to classify CKD. This method of separating systemic diseases and primary kidney diseases is only one, proposed by the Work Group, to aid in the conceptual approach.

Table 4 represents an example of a classification of causes of kidney diseases based on these two domains.

There is wide geographic variation in the cause of kidney disease. In developed countries, hypertension and diabetes are the most frequent causes of CKD, especially in the elderly. In populations with a high prevalence of diabetes and hypertension, it can be difficult to distinguish CKD due to hypertension and diabetes from CKD due to other disorders. In other countries, other causes of CKD may be as frequent as hypertension and diabetes (e.g., glomerular disease in East Asia) or coexist with them. Specialized diagnostic testing, such as kidney biopsy or invasive imaging studies are performed only when it is essential to confirm some diagnoses and the benefits justify the risks and cost. It is anticipated that cause of disease will not be known with certainty for many patients with CKD but can be either inferred or not known.

\section{Pediatric Considerations}

The principles inherent in this guideline are fully applicable to children.

\subsection{3: Assign GFR categories as follows [Table 5] (Not Graded):}

Table 5 | GFR categories in CKD

\begin{tabular}{lcl}
\hline GFR category & GFR $\left(\mathrm{ml} / \mathrm{min} / \mathbf{1 . 7 3} \mathrm{m}^{2}\right)$ & Terms \\
\hline G1 & $\geq 90$ & Normal or high \\
G2 & $60-89$ & Mildly decreased* \\
G3a & $45-59$ & Mildly to moderately decreased \\
G3b & $30-44$ & Moderately to severely decreased \\
G4 & $15-29$ & Severely decreased \\
G5 & $<15$ & Kidney failure \\
\hline
\end{tabular}

Abbreviations: CKD, chronic kidney disease; GFR, glomerular filtration rate.

*Relative to young adult level

In the absence of evidence of kidney damage, neither GFR category G1 nor G2 fulfill the criteria for CKD.

\section{RATIONALE}

The purpose of this statement is to ensure clarity in communication. The terms associated with each of the GFR categories are descriptors which need to be taken in the context of the individual and are all references to normal young adults. Note that mildly decreased kidney function (G2) in the absence of other markers, does not constitute CKD.

The associations of lower categories of GFR and risks of metabolic and endocrine complications formed the basis of the previous stratification into 5 stages. This current classification further acknowledges the importance of dividing Stage 3 based on data supporting different outcomes and risk profiles into categories G3a and G3b (Figure 5). A number of other concurrent complications are associated with decreased categories of GFR including infection, impaired cognitive and physical function, and threats to patient safety. ${ }^{57}$

Figures 6 and 7 detail the RRs of decreased eGFR and increasing ACR with future complications, including mortality and kidney outcomes. ${ }^{30}$ Even for the group with the lowest value of albuminuria, the increased RR for all outcomes is significant for eGFRs below $60 \mathrm{ml} / \mathrm{min} / 1.73 \mathrm{~m}^{2}$ in the continuous analysis and in the range of $45-59 \mathrm{ml} / \mathrm{min} /$ $1.73 \mathrm{~m}^{2}$ for the categorical analysis.

\section{Pediatric Considerations}

In children $<2$ years of age with CKD, the GFR categories as per the adult in Table 5 do not apply; these children should be categorized as having normal, moderately reduced, or severely reduced age-adjusted GFR.

No currently agreed upon set of international normative values or categories exist for GFR in children under the age of 1-2 years. However, the international pediatric nephrology community has embraced the adult CKD staging system as per the 2002 KDOQI guidelines in children over the age of 2 years, as suggested by Hogg et al. ${ }^{43}$ 


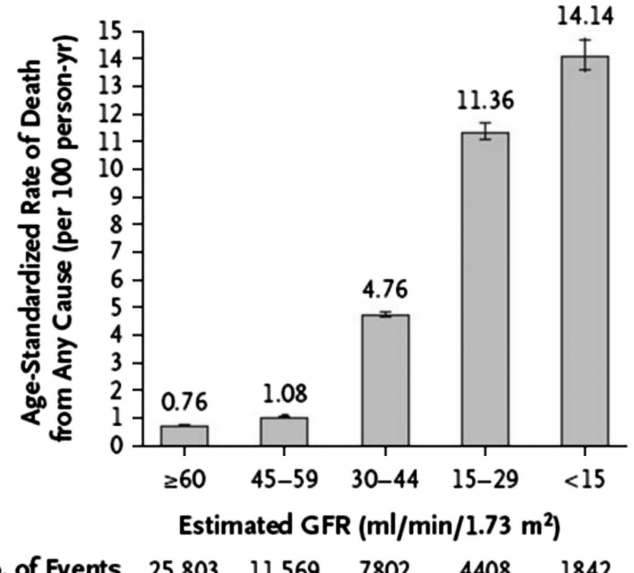

$\begin{array}{lllll}\text { No. of Events } \quad 25,803 & 11,569 & 7802 & 4408 & 1842\end{array}$

b

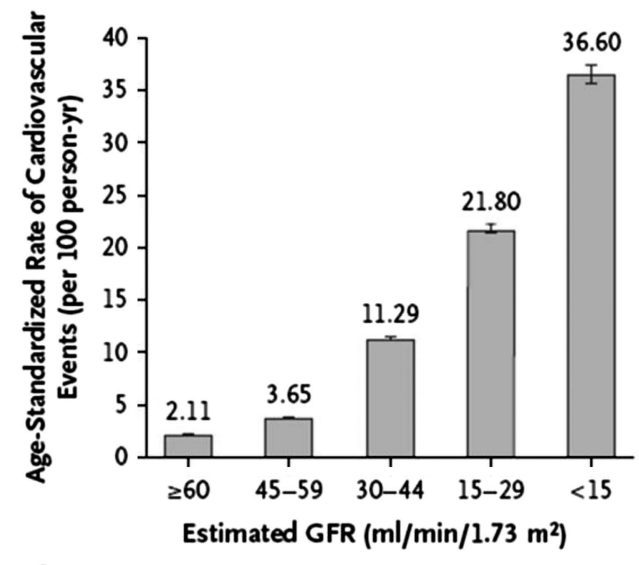

No. of Events $\quad \begin{array}{lllll}73,108 & 34,690 & 18,580 & 8809 & 3824\end{array}$

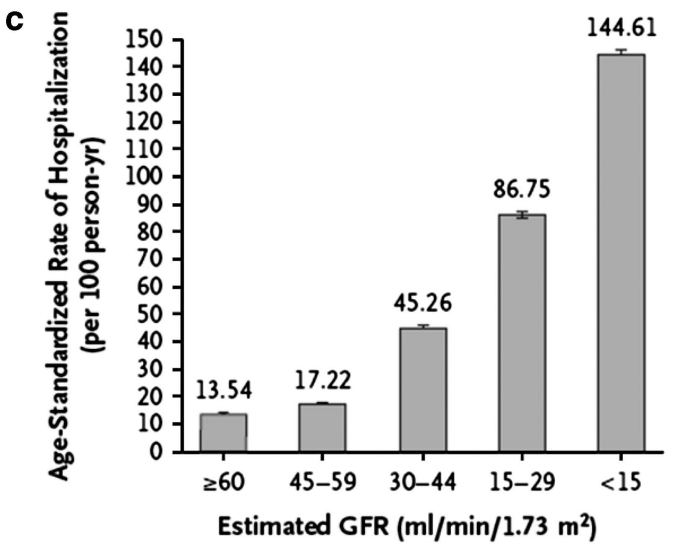

$\begin{array}{lllll}\text { No. of Events } \quad 366,757 & 106,543 & 49,177 & 20,581 & 11,593\end{array}$

Figure 5 Age-standardized rates of death from any cause (panel a), cardiovascular events (panel b), and hospitalization (panel c), according to the eGFR among 1,120,295 ambulatory adults. eGFR, estimated glomerular filtration rate. From $\mathrm{N}$ Engl J Med, Go AS, Chertow GM, Fan D, et al..$^{58}$ Chronic kidney disease and the risks of death, cardiovascular events, and hospitalization, 351: 1296-1305. Copyright (c) (2004) Massachusetts Medical Society. Reprinted with permission from Massachusetts Medical Society; accessed http://www.nejm.org/doi/pdf/10.1056/NEJMoa041031

As indicated in Pediatric Considerations for Guideline 1.1, the normative GFR values for children less than 2 years vary quite widely by both age and method of measurement. More importantly these values are expected to increase in a nonlinear fashion over the first 2 years of life with significant changes seen in the first few months post-birth and no current evidence of presence of comorbid conditions at any given level of measured or estimated GFR in this population. As such, specific categorization of G1-5 as suggested in this Recommendation would seem not be of value, and might be misleading if applied to a child less than 2 years of age.

With this in mind, it is suggested that based on the chosen method of GFR measurement or comparison for the individual (i.e., $\mathrm{CrCl}$, radioactive or cold exogenous serum markers, or estimating formula), that one should attempt to classify the child under the age of 2 years as having normal, moderate or severe reductions in GFR based on the normative range and standard deviations (SDs) for the method. No evidence exists for this recommendation but recognition that values of GFR more than 1 SD below the mean would seem likely to raise concern of the clinician and foster the need for closer monitoring. For drug dosing adjustments it is suggested that those children with GFRs below the mean by $>1$ but $<2$ SD be classified as having a moderate reduction in GFR whereas those more than $2 \mathrm{SD}$ below the mean for the method be classified as having a severe reduction in GFR.

1.2.4: Assign albuminuria ${ }^{\star}$ categories as follows [Table 6] (Not Graded):

${ }^{\star}$ note that where albuminuria measurement is not available, urine reagent strip results can be substituted (Table 7)

Table 6| Albuminuria categories in CKD

\begin{tabular}{|c|c|c|c|c|}
\hline \multirow{2}{*}{ Category } & \multirow{2}{*}{$\begin{array}{c}\text { AER } \\
\text { (mg/24 hours) }\end{array}$} & \multicolumn{2}{|c|}{$\begin{array}{c}\text { ACR (approximate } \\
\text { equivalent) }\end{array}$} & \multirow{2}{*}{ Terms } \\
\hline & & $(\mathrm{mg} / \mathrm{mmol})$ & $(\mathrm{mg} / \mathrm{g})$ & \\
\hline A1 & $<30$ & $<3$ & $<30$ & $\begin{array}{l}\text { Normal to mildly } \\
\text { increased }\end{array}$ \\
\hline A2 & $30-300$ & $3-30$ & $30-300$ & Moderately increased* \\
\hline A3 & $>300$ & $>30$ & $>300$ & Severely increased** \\
\hline
\end{tabular}

Abbreviations: AER, albumin excretion rate; $A C R$, albumin-to-creatinine ratio; CKD, chronic kidney disease.

* Relative to young adult level.

**Including nephrotic syndrome (albumin excretion usually $>2200 \mathrm{mg} / 24$ hours [ACR $>2220 \mathrm{mg} / \mathrm{g} ;>220 \mathrm{mg} / \mathrm{mmol}]$ ).

\section{RATIONALE}

The purpose of this statement is to ensure communication and to reflect that albuminuria category is an important predictor of outcomes. The association of high levels of proteinuria with signs and symptoms of nephrotic syndrome is well known. The detection and evaluation of lesser quantities of proteinuria have gained additional significance as multiple studies have demonstrated its diagnostic, pathogenic, and prognostic importance. There is a continuous risk associated with albuminuria but the use of a simple categorical approach was selected to simplify the concept for 


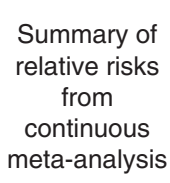

continuous
meta-analysis

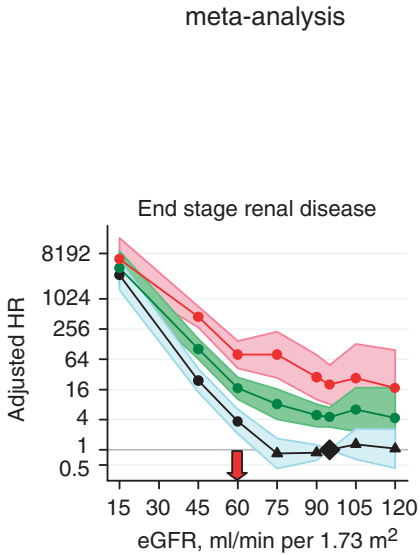

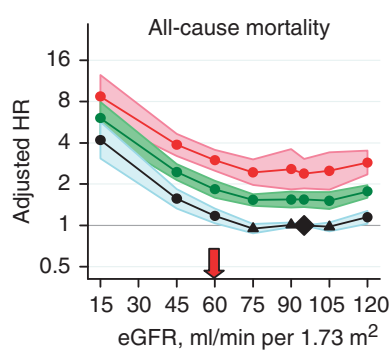

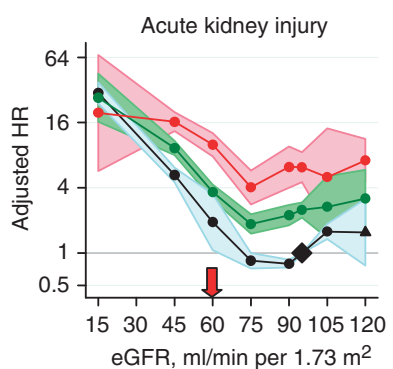

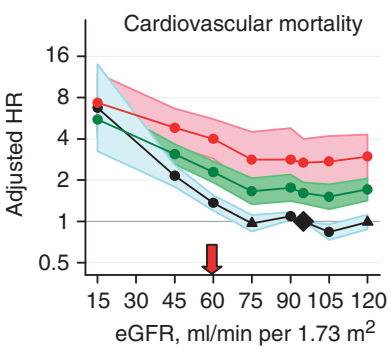

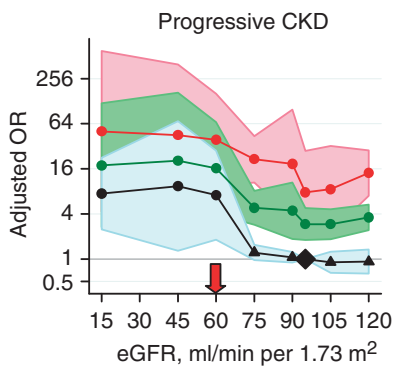

Figure 6 | Summary of continuous meta-analysis (adjusted RRs) for general population cohorts with ACR. Mortality is reported for general population cohorts assessing albuminuria as urine ACR. Kidney outcomes are reported for general population cohorts assessing albuminuria as either urine ACR or reagent strip. eGFR is expressed as a continuous variable. The three lines represent urine ACR of $<30$, $30-299$ and $\geq 300 \mathrm{mg} / \mathrm{g}(<3,3-29$, and $\geq 30 \mathrm{mg} / \mathrm{mmol}$, respectively) or reagent strip negative and trace, $1+$ positive, $\geq 2+$ positive. All results are adjusted for covariates and compared to reference point of eGFR of $95 \mathrm{ml} / \mathrm{min} / 1.73 \mathrm{~m}^{2}$ and ACR of $<30 \mathrm{mg} / \mathrm{g}$ ( $<3 \mathrm{mg} / \mathrm{mmol}$ ) or reagent strip negative (diamond). Each point represents the pooled RR from a meta-analysis. Solid circles indicate statistical significance compared to the reference point $(P<0.05)$; triangles indicate non-significance. Red arrows indicate eGFR of $60 \mathrm{ml} / \mathrm{min} / 1.73 \mathrm{~m}{ }^{2}$, threshold value of eGFR for the current definition of CKD. ACR, albumin-to-creatinine ratio; CKD, chronic kidney disease; eGFR, estimated glomerular filtration rate; $\mathrm{HR}$, hazard ratio; $\mathrm{OR}$, odds ratio, RR, relative risk. Reprinted with permission from Macmillan Publishers Ltd: Kidney International. Levey AS, de Jong PE, Coresh J, et al. The definition, classification, and prognosis of chronic kidney disease: a KDIGO controversies conference report. Kidney Int 2011; 80: 17-2830; accessed http://www.nature.com/ki/journal/v80/n1/full/ki2010483a.html

clinical practice. Several groups had suggested subdividing one or more GFR categories based on albuminuria category.

For the detection of diabetic nephropathy some guidelines recommend the use of different ACR thresholds for males and females $(>25 \mathrm{mg} / \mathrm{g}[>2.5 \mathrm{mg} / \mathrm{mmol}]$ and $>35 \mathrm{mg} / \mathrm{g}$ $[>3.5 \mathrm{mg} / \mathrm{mmol}]$, respectively) to take into account variations in creatinine excretion. A single threshold is used in North America $(30 \mathrm{mg} / \mathrm{g}$ or $3.4 \mathrm{mg} / \mathrm{mmol})$. Earlier KDIGO guidance was reluctant to adopt gender-specific thresholds due to greater complexity, uncertainty about assay precision, and effects of race, ethnicity, diet and measures of body size on creatinine and this stance is maintained here. For simplicity, and to reflect the fact that it is an approximation, $3.4 \mathrm{mg} / \mathrm{mmol}$ as the current guideline threshold has been rounded to $3.0 \mathrm{mg} / \mathrm{mmol}$.

There is a graded increase in risk for higher albuminuria categories, at all GFR categories, without any clear threshold value. Even for subjects with GFR $>60 \mathrm{ml} / \mathrm{min} / 1.73 \mathrm{~m}^{2}$, the increased RR is statistically significant for urine ACR $\geq 30 \mathrm{mg} / \mathrm{g}$ ( $\geq 3 \mathrm{mg} / \mathrm{mmol}$ ) for mortality and kidney outcomes (Figures 6 and 7). The predictive ability of albuminuria at all categories of GFR supports the suggestion to add albuminuria categories to all GFR categories. Since the relationship with albuminuria is continuous, the selection of the number of categories and the cutoff values appears arbitrary. The Work Group has recommended the classification of albuminuria into only 3 categories, based on practical considerations, but recognized that further subdivisions within the category of $<30 \mathrm{mg} / 24$ hours $(\mathrm{ACR}<30 \mathrm{mg} / \mathrm{g}$ or $<3 \mathrm{mg} / \mathrm{mmol}$ ) may be useful for risk stratification, and that subdivisions within the category of $>300 \mathrm{mg} /$ 24 hours (ACR $>300 \mathrm{mg} / \mathrm{g}$ or $>30 \mathrm{mg} / \mathrm{mmol}$ ) may be useful for diagnosis and management. Specifically there is a recognition that nephrotic range proteinuria (AER $>2200 \mathrm{mg} / 24$ hours [ACR $>2200 \mathrm{mg} / \mathrm{g} ;>220 \mathrm{mg} /$ $\mathrm{mmol}$ ]; PER $>3000 \mathrm{mg} / 24$ hours [ $>3000 \mathrm{mg} / \mathrm{g} ;>300 \mathrm{mg} /$ $\mathrm{mmol}]$ ) confers unique additional risks and is usually associated with specific conditions (such as GN). As these are relatively rare in general practices, the simplicity of the AER categorization was preferred. Table 7 shows the approximate relationships of categories of AER to other measures of albuminuria and proteinuria.

\section{Implications for Clinical Practice and Public Policy}

Data from around the world suggest that CKD prevalence is between $10-16 \%$ but information concerning population prevalence by category of GFR and ACR is scant. Figure 8 shows the proportion of adults in the US by categories of GFR and albuminuria. ${ }^{19}$ While CKD is common, few individuals have severely reduced GFR or kidney failure or severely increased albuminuria. 


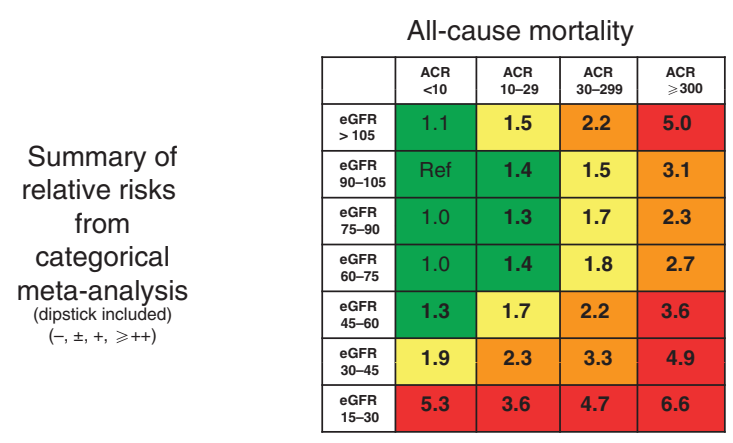

Kidney failure (ESRD)

\begin{tabular}{|c|c|c|c|c|}
\hline & $\begin{array}{c}\text { ACR } \\
<10\end{array}$ & $\begin{array}{c}\text { ACR } \\
10-29\end{array}$ & $\begin{array}{c}\text { ACR } \\
30-299\end{array}$ & $\begin{array}{c}\text { ACR } \\
\geqslant 300\end{array}$ \\
\hline $\begin{array}{c}\text { eGFR } \\
>105\end{array}$ & Ref & Ref & 7.8 & 18 \\
\hline $\begin{array}{c}\text { eGFR } \\
90-105\end{array}$ & Ref & Ref & 11 & 20 \\
\hline $\begin{array}{c}\text { eGFR } \\
75-90\end{array}$ & Ref & Ref & 3.8 & 48 \\
\hline $\begin{array}{c}\text { eGFR } \\
60-75\end{array}$ & Ref & Ref & 7.4 & 67 \\
\hline $\begin{array}{c}\text { eGFR } \\
45-60\end{array}$ & 5.2 & 22 & 40 & 147 \\
\hline $\begin{array}{l}\text { eGFR } \\
30-45\end{array}$ & 56 & 74 & 294 & 763 \\
\hline $\begin{array}{l}\text { eGFR } \\
15-30\end{array}$ & 433 & 1044 & 1056 & 2286 \\
\hline
\end{tabular}

Acute kidney injury (AKI)

\begin{tabular}{|c|c|c|c|c|}
\hline & $\begin{array}{c}\text { ACR } \\
<10\end{array}$ & $\begin{array}{c}\text { ACR } \\
10-29\end{array}$ & $\begin{array}{c}\text { ACR } \\
30-299\end{array}$ & $\begin{array}{c}\text { ACR } \\
\geqslant 300\end{array}$ \\
\hline $\begin{array}{c}\text { eGFR } \\
>105\end{array}$ & Ref & Ref & 2.7 & 8.4 \\
\hline $\begin{array}{c}\text { eGFR } \\
90-105\end{array}$ & Ref & Ref & 2.4 & 5.8 \\
\hline $\begin{array}{c}\text { eGFR } \\
75-90\end{array}$ & Ref & Ref & 2.5 & 4.1 \\
\hline $\begin{array}{c}\text { eGFR } \\
60-75\end{array}$ & Ref & Ref & 3.3 & 6.4 \\
\hline $\begin{array}{c}\text { eGFR } \\
45-60\end{array}$ & 2.2 & 4.9 & 6.4 & 5.9 \\
\hline $\begin{array}{c}\text { eGFR } \\
30-45\end{array}$ & 7.3 & 10 & 12 & 20 \\
\hline $\begin{array}{c}\text { eGFR } \\
15-30\end{array}$ & 17 & 17 & 21 & 29 \\
\hline
\end{tabular}

Cardiovascular mortality

\begin{tabular}{|c|c|c|c|c|}
\hline & $\begin{array}{c}\text { ACR } \\
<10\end{array}$ & $\begin{array}{c}\text { ACR } \\
10-29\end{array}$ & $\begin{array}{c}\text { ACR } \\
30-299\end{array}$ & $\begin{array}{c}\text { ACR } \\
\geqslant 300\end{array}$ \\
\hline $\begin{array}{c}\text { eGFR } \\
>105\end{array}$ & 0.9 & 1.3 & 2.3 & 2.1 \\
\hline $\begin{array}{c}\text { eGFR } \\
90-105\end{array}$ & Ref & 1.5 & 1.7 & 3.7 \\
\hline $\begin{array}{c}\text { eGFR } \\
75-90\end{array}$ & 1.0 & 1.3 & 1.6 & 3.7 \\
\hline $\begin{array}{c}\text { eGFR } \\
\text { 60-75 }\end{array}$ & 1.1 & 1.4 & 2.0 & 4.1 \\
\hline $\begin{array}{c}\text { eGFR } \\
45-60\end{array}$ & 1.5 & 2.2 & 2.8 & 4.3 \\
\hline $\begin{array}{c}\text { eGFR } \\
30-45\end{array}$ & 2.2 & 2.7 & 3.4 & 5.2 \\
\hline $\begin{array}{c}\text { eGFR } \\
15-30\end{array}$ & 14 & 7.9 & 4.8 & 8.1 \\
\hline
\end{tabular}

Progressive CKD

\begin{tabular}{|c|c|c|c|c|}
\hline & $\begin{array}{c}\text { ACR } \\
<10\end{array}$ & $\begin{array}{c}\text { ACR } \\
10-29\end{array}$ & $\begin{array}{c}\text { ACR } \\
30-299\end{array}$ & $\begin{array}{c}\text { ACR } \\
\geqslant 300\end{array}$ \\
\hline $\begin{array}{c}\text { eGFR } \\
>105\end{array}$ & Ref & Ref & 0.4 & 3.0 \\
\hline $\begin{array}{c}\text { eGFR } \\
90-105\end{array}$ & Ref & Ref & 0.9 & 3.3 \\
\hline $\begin{array}{c}\text { eGF } \\
75-90\end{array}$ & Ref & Ref & 1.9 & 5.0 \\
\hline $\begin{array}{c}\text { eGFR } \\
60-75\end{array}$ & Ref & Ref & 3.2 & 8.1 \\
\hline $\begin{array}{c}\text { eGF } \\
\text { 45-60 }\end{array}$ & 3.1 & 4.0 & 9.4 & 57 \\
\hline $\begin{array}{l}\text { eGFR } \\
30-45\end{array}$ & 3.0 & 19 & 15 & 22 \\
\hline $\begin{array}{c}\text { eGR } \\
15-30\end{array}$ & 4.0 & 12 & 21 & 7.7 \\
\hline
\end{tabular}

Figure 7| Summary of categorical meta-analysis (adjusted RRs) for general population cohorts with ACR. Mortality is reported for general population cohorts assessing albuminuria as urine ACR. Kidney outcomes are reported for general population cohorts assessing albuminuria as either urine ACR or reagent strip. eGFR and albuminuria are expressed as categorical variables. All results are adjusted for covariates and compared to the reference cell (Ref). Each cell represents a pooled RR from a meta-analysis; bold numbers indicate statistical significance at $\mathrm{P}<0.05$. Incidence rates per 1000 person-years for the reference cells are 7.0 for all-cause mortality, 4.5 for CVD mortality, 0.04 for kidney failure, 0.98 for AKI, and 2.02 for CKD progression. Colors reflect the ranking of adjusted RR. The point estimates for each cell were ranked from 1 to 28 (the lowest RR having rank number 1, and the highest number 28). The categories with a rank number 1-8 are green, rank numbers 9-14 are yellow, the rank numbers 15-21 are orange and the rank numbers 22-28 are colored red. (For the outcome of CKD progression, two cells with $\mathrm{RR}<1.0$ are also green, leaving fewer cells as yellow, orange and red). ACR, albumin-to-creatinine ratio; AKI, acute kidney injury; CKD, chronic kidney disease; CVD, cardiovascular disease; eGFR, estimated glomerular filtration rate; ESRD, end-stage renal disease; RR, relative risk. Reprinted with permission from Macmillan Publishers Ltd: Kidney International. Levey AS, de Jong PE, Coresh J, et al. $^{30}$ The definition, classification, and prognosis of chronic kidney disease: a KDIGO controversies conference report. Kidney Int 2011; 80: 17-28; accessed http://www.nature.com/ki/journal/v80/n1/full/ki2010483a.html

The classification of kidney disease by cause, category of GFR and category of albuminuria does not conform to the International Classification of Diseases (ICD) maintained by the World Health Organization (WHO). Currently the WHO is developing an update (ICD 11). It will be important to communicate and coordinate efforts with the kidney disease subgroup for ICD 11. However, the proposed current classification does address the need in clinical practice to acknowledge the multiple dimensions and variables by which individual patients are assessed. Table 8 gives examples of the use of CGA nomenclature.

Definition of GFR categories have been deliberately based upon the concept of "true" GFR, whereas clinical practice and research has predominantly used creatinine-based estimates of GFR. The belief of the Work Group is that the non-GFR determinants of creatinine and the imprecision of creatinine-based GFR estimates have resulted in the absence of strong dose-dependent association of eGFR with clinical outcomes in the GFR range of $>60 \mathrm{ml} / \mathrm{min} / 1.73 \mathrm{~m}^{2}$. The Work Group felt confident that GFR levels of $\geq 90 \mathrm{ml} / \mathrm{min} /$ $1.73 \mathrm{~m}^{2}$ portend better prognosis than GFR levels $60-89 \mathrm{ml} /$ $\min / 1.73 \mathrm{~m}^{2}$, if they could be estimated accurately. Therefore, the GFR categories include separate G1 $(\geq 90 \mathrm{ml} / \mathrm{min} /$ $\left.1.73 \mathrm{~m}^{2}\right)$ and $\mathrm{G} 2\left(60-89 \mathrm{ml} / \mathrm{min} / 1.73 \mathrm{~m}^{2}\right)$ designations despite limited data from creatinine-based estimates that prognosis differs between these two categories. It is also an acknowledgement that the degree of precision of some of our measurements may not be able to differentiate between these 2 categories reliably. As described later, studies that have used cystatin $\mathrm{C}$ have found gradients in prognosis at eGFR levels above $60 \mathrm{ml} / \mathrm{min} / 1.73 \mathrm{~m}^{2}$, which supports the belief of the committee that separating these 2 GFR categories is appropriate for CKD classification.

Albuminuria categories are "wide" with respect to risk, with significant gradients within each category. The decision to propose only 3 categories is based on the perceived need for simplification in clinical practice. In specialized clinical nephrology centers, A3 (>300 mg/g or $>30 \mathrm{mg} / \mathrm{mmol}$ ) is often more precisely assessed and divided into additional categories. For example, nephrotic range proteinuria is defined as PER $>3500 \mathrm{mg} / 24$ hours or PCR (proteinto-creatinine ratio) $>3500 \mathrm{mg} / \mathrm{g}[>350 \mathrm{mg} / \mathrm{mmol}]$ which is approximately equivalent to AER $>2200 \mathrm{mg} / 24$ hours or ACR $>2200 \mathrm{mg} / \mathrm{g}$ [220 mg/mmol]. It is clearly recognized 
Table $7 \mid$ Relationship among categories for albuminuria and proteinuria

\begin{tabular}{|c|c|c|c|}
\hline \multirow[b]{2}{*}{ Measure } & \multicolumn{3}{|c|}{ Categories } \\
\hline & Normal to mildly increased (A1) & Moderately increased (A2) & Severely increased (A3) \\
\hline AER (mg/24 hours) & $<30$ & $30-300$ & $>300$ \\
\hline PER (mg/24 hours) & $<150$ & $150-500$ & $>500$ \\
\hline \multicolumn{4}{|l|}{ ACR } \\
\hline (mg/mmol) & $<3$ & $3-30$ & $>30$ \\
\hline$(\mathrm{mg} / \mathrm{g})$ & $<30$ & $30-300$ & $>300$ \\
\hline \multicolumn{4}{|l|}{ PCR } \\
\hline (mg/mmol) & $<15$ & $15-50$ & $>50$ \\
\hline$(\mathrm{mg} / \mathrm{g})$ & $<150$ & $150-500$ & $>500$ \\
\hline Protein reagent strip & Negative to trace & Trace to + & + or greater \\
\hline
\end{tabular}

Abbreviations: ACR, albumin-to-creatinine ratio; $A E R$, albumin excretion rate; $P C R$, protein-to-creatinine ratio; PER, protein excretion rate.

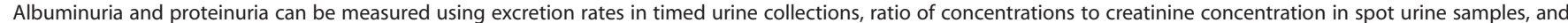

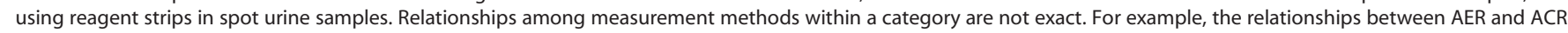

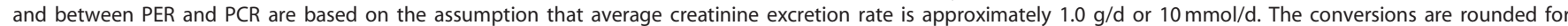
pragmatic reasons. (For an exact conversion from $\mathrm{mg} / \mathrm{g}$ of creatinine to $\mathrm{mg} / \mathrm{mmol}$ of creatinine, multiply by 0.113 .) Creatinine excretion varies with age, sex, race and diet; therefore the relationship among these categories is approximate only. ACR $<10 \mathrm{mg} / \mathrm{g}(<1 \mathrm{mg} / \mathrm{mmol})$ is considered normal; ACR $10-30 \mathrm{mg} / \mathrm{g}(1-3 \mathrm{mg} / \mathrm{mmol})$ is considered "high normal." ACR $>2200 \mathrm{mg} / \mathrm{g}$ (>220 mg/mmol) is considered "nephrotic range." The relationship between urine reagent strip results and other measures depends on urine concentration.

\begin{tabular}{|c|c|c|c|c|c|c|c|}
\hline & & & & \multicolumn{3}{|c|}{$\begin{array}{l}\text { Persistent albuminuria categories } \\
\text { Description and range }\end{array}$} & \\
\hline \multirow{2}{*}{\multicolumn{4}{|c|}{$\begin{array}{l}\text { Percentage of US Population by } \\
\text { eGFR and Albuminuria } \\
\text { Category: KDIGO } 2012 \text { and } \\
\text { NHANES 1999-2006 }\end{array}$}} & A1 & A2 & A3 & \\
\hline & & & & $\begin{array}{l}\text { Normal to } \\
\text { mildly }\end{array}$ & $\begin{array}{l}\text { Moderately } \\
\text { increased }\end{array}$ & $\begin{array}{l}\text { Severely } \\
\text { increased }\end{array}$ & \\
\hline & & & & $\begin{aligned} &<30 \mathrm{mg} / \mathrm{g} \\
&<3 \mathrm{mg} / \mathrm{mmol}\end{aligned}$ & $\begin{array}{c}30-300 \mathrm{mg} / \mathrm{g} \\
3-30 \mathrm{mg} / \mathrm{mmol}\end{array}$ & $\begin{array}{c}>300 \mathrm{mg} / \mathrm{g} \\
>30 \mathrm{mg} / \mathrm{mmol}\end{array}$ & \\
\hline \multirow{6}{*}{ 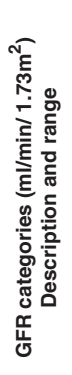 } & G1 & Normal or high & $\geq 90$ & 55.6 & 1.9 & 0.4 & 57.9 \\
\hline & G2 & Mildly decreased & $60-89$ & 32.9 & 2.2 & 0.3 & 35.4 \\
\hline & G3a & $\begin{array}{l}\text { Mildly to moderately } \\
\text { decreased }\end{array}$ & $45-59$ & 3.6 & 0.8 & 0.2 & 4.6 \\
\hline & G3b & $\begin{array}{l}\text { Moderately to } \\
\text { severely decreased }\end{array}$ & $30-44$ & 1.0 & 0.4 & 0.2 & 1.6 \\
\hline & G4 & Severely decreased & $15-29$ & 0.2 & 0.1 & 0.1 & 0.4 \\
\hline & G5 & Kidney failure & $<15$ & 0.0 & 0.0 & 0.1 & 0.1 \\
\hline & & & & 93.2 & 5.4 & 1.3 & 100.0 \\
\hline
\end{tabular}

Figure $8 \mid$ Prevalence of CKD in the USA by GFR and albuminuria. Cells show the proportion of adult population in the USA. Data from the NHANES 1999-2006, $\mathrm{N}=18,026$. GFR is estimated with the CKD-EPI equation and standardized serum creatinine. ${ }^{19}$ Albuminuria is determined by one measurement of ACR and persistence is estimated as described elsewhere. ${ }^{59}$ Values in cells do not total to values in margins because of rounding. Category of very high albuminuria includes nephrotic range. Green, low risk (if no other markers of kidney disease, no CKD); Yellow, moderately increased risk; Orange, high risk; Red, very high risk. ACR, albumin-to-creatinine ratio; CKD, chronic kidney disease; CKD-EPI, CKD Epidemiology Collaboration; GFR, glomerular filtration rate; NHANES, National Health and Nutrition Examination Survey. Modified with permission from Macmillan Publishers Ltd: Kidney International. Levey AS, de Jong PE, Coresh J, et al. ${ }^{30}$ The definition, classification, and prognosis of chronic kidney disease: a KDIGO controversies conference report. Kidney Int 2011; 80: 17-28; accessed http://www.nature.com/ki/journal/v80/n1/full/ki2010483a.html

that these very high levels of proteinuria carry a different risk than lower values within the same category. Further differentiation after quantification and evaluation would inform treatment decisions for an individual patient. These categories serve as an initial assessment and prognostication tool; further classification is appropriate for specific circum- stances and is not limited by the initial classification into only 3 categories.

Note that the term 'microalbuminuria' is not used and is discouraged in this classification system. This will require a formal education program and review of existing guidelines in other disciplines so that consistency of terminology and 
Table 8|CGA staging of CKD: examples of nomenclature and comments

\begin{tabular}{|c|c|c|c|c|}
\hline Cause & $\begin{array}{l}\text { GFR } \\
\text { category }\end{array}$ & $\begin{array}{l}\text { Albuminuria } \\
\text { category }\end{array}$ & Criterion for CKD & Comment \\
\hline Diabetic kidney disease & G5 & A3 & Decreased GFR, Albuminuria & Most common patient in the low clearance clinic \\
\hline Idiopathic focal sclerosis & G2 & A3 & Albuminuria & Common cause of nephrotic syndrome in childhood \\
\hline $\begin{array}{l}\text { Kidney transplant } \\
\text { recipient }\end{array}$ & G2 & A1 & History of kidney transplantation & Best outcome after kidney transplantation \\
\hline Polycystic kidney disease & G2 & A1 & Imaging abnormality & $\begin{array}{l}\text { Most common disease caused by a mutation in a single } \\
\text { gene }\end{array}$ \\
\hline Vesicoureteral reflex & G1 & A1 & Imaging abnormality & Common condition in children \\
\hline $\begin{array}{l}\text { Distal renal tubular } \\
\text { acidosis }\end{array}$ & G1 & A1 & Electrolyte abnormalities & Rare genetic disorder \\
\hline $\begin{array}{l}\text { Hypertensive kidney } \\
\text { disease }\end{array}$ & G4 & $\mathrm{A} 2$ & Decreased GFR and albuminuria & $\begin{array}{l}\text { Usually due to long-standing poorly controlled } \\
\text { hypertension, likely to include patients with genetic } \\
\text { predisposition- more common in blacks- who should } \\
\text { be referred to nephrologist because of severely } \\
\text { decreased GFR }\end{array}$ \\
\hline $\begin{array}{l}\text { CKD presumed due to } \\
\text { diabetes and hypertension }\end{array}$ & G4 & A1 & Decreased GFR & $\begin{array}{l}\text { Should be referred to nephrologist because of severely } \\
\text { decreased GFR }\end{array}$ \\
\hline $\begin{array}{l}\text { CKD presumed due to } \\
\text { diabetes and hypertension }\end{array}$ & $\mathrm{G} 2$ & A3 & Albuminuria & $\begin{array}{l}\text { Should be referred to nephrologist because of } \\
\text { albuminuria }\end{array}$ \\
\hline $\begin{array}{l}\text { CKD presumed due to } \\
\text { diabetes and hypertension }\end{array}$ & G3a & A1 & Decreased GFR & Very common, may not require referral to nephrologist \\
\hline CKD cause unknown & G3a & A1 & Decreased GFR & May be the same patient as above \\
\hline
\end{tabular}

Abbreviations: CGA, Cause, GFR category and albuminuria category; CKD, chronic kidney disease; GFR, glomerular filtration rate.

Note: Patients above the thick horizontal line are likely to be encountered in nephrology practice. Patients below the thick horizontal line are likely to be encountered in primary care practice and in nephrology practice.

understanding of the changes are universal (see Recommendation 1.4.4.2.1).

\section{Pediatric Considerations}

This statement would need to be altered for application in pediatric practice in the following way. In children with CKD any expression of abnormal urinary protein excretion, irrespective of the marker:

- must account for variation in that measurement as seen across age, sex, puberty and or body size (height, weight, body mass index $[\mathrm{BMI}]$ ).

- should account for the possibility of tubular versus glomerular proteinuria dominance dependent on the underlying disease.

- may utilize proteinuria in place of albuminuria.

There is no set standard encompassing all children with respect to the normal range of urinary protein (or albumin) excretion. Values vary across age, sex, race, pubertal status, the presence of obesity (high BMI) and may be modified by exercise, fever, and posture. ${ }^{60-63}$

In general, neonates and young infants/ children are both expected and allowed to have higher urinary losses of both glomerular and tubular proteinuria due to lack of maturation in the proximal tubular reabsorption of proteins. The rough equivalences for ACR and PCR quoted in the pediatric literature are similar, but not identical to those quoted in the adult literature. Normal ranges vary but at least one reference suggests as much as $6-8 \mathrm{mg} / \mathrm{m}^{2} / \mathrm{hr}$ or $>240 \mathrm{mg} / \mathrm{m}^{2} /$ day of proteinuria as being acceptable at $<6$ months of age; ${ }^{64}$ normal ranges for urinary albumin losses are not known at this age.
The normal range of protein excretion for children 6-24 months of age in a 24-hour urine collection is quoted as being $<4 \mathrm{mg} / \mathrm{m}^{2} / \mathrm{hr}\left(<150 \mathrm{mg} / \mathrm{m}^{2} /\right.$ day $)$, whereas the first morning spot urine protein sample is said to be normal at levels of $<500 \mathrm{mg} / \mathrm{g}$ creatinine $(<50 \mathrm{mg} / \mathrm{mmol})$. In children older than 24 months these values are $<4 \mathrm{mg} / \mathrm{m}^{2} / \mathrm{hr}$ $\left(<150 \mathrm{mg} / \mathrm{m}^{2} /\right.$ day $)$ for the 24-hour collection and PCR $<200 \mathrm{mg} / \mathrm{g}$ creatinine $(<20 \mathrm{mg} / \mathrm{mmol})$ in the first morning urine sample, or a first morning urine ACR $<30 \mathrm{mg} / \mathrm{g}$ $(<3 \mathrm{mg} / \mathrm{mmol}) .{ }^{43,65}$

At all ages, total urinary protein excretion $>40 \mathrm{mg} / \mathrm{m}^{2} / \mathrm{hr}$ ( $>3$ grams $/ 1.73 \mathrm{~m}^{2} /$ day) is considered to represent 'nephrotic range' loss of protein, with intermediate values, i.e., $4-40 \mathrm{mg} / \mathrm{m}^{2} / \mathrm{hr}$ or its equivalent representing abnormal but 'non-nephrotic' losses. ${ }^{43,65}$

Children older than 24 months of age are expected to achieve normal ('adult') urinary protein values with the caveat of an exaggerated postural loss of glomerular proteins (albumin) as can commonly be seen in the $2-5 \%$ of the adolescent population (i.e., orthostatic proteinuria). ${ }^{62}$

Based on National Health and Nutrition Examination Survey III (NHANES III) data from just under 6000 healthy 6-19 year old children using either immunonephelometry or radioimmunoassay, the definition of urinary albumin excretion was determined to be $30-300 \mathrm{mg} / 24 \mathrm{~h}$ collection; $20-200 \mu \mathrm{g} / \mathrm{min}$ in an overnight collection and $30-300 \mathrm{mg} / \mathrm{g}$ creatinine $(3-30 \mathrm{mg} / \mathrm{mmol})$ in a first morning urine sample. ${ }^{66}$

Of note, to date the majority of studies that have examined the effects of urinary protein losses or therapeutic interventions have concentrated on so-called total protein excretion or random or first morning PCRs. The utility of measuring the albumin only fraction, and in particular 
quantitating this at the lower level of detection, i.e., $<30 \mathrm{mg} / \mathrm{g}(<3 \mathrm{mg} / \mathrm{mmol})$ creatinine, is only now being investigated in more detail in large pediatric studies. As such it should be recognized that in children the quantification of total protein, as compared to the albumin only fraction, may be the preferred method for assigning risk as it relates to the presence of urinary protein loss.

In summary, for children older than 2 years of age the assignment of 'proteinuria' categories can be used as per the adult guidelines with the understanding that modification to the upper limit of expected values may be necessary in consideration of the factors outlined above. Although there is a preference for reporting albumin values, currently many clinicians still categorize these children based on total protein and in the child $<2$ years of age or the adolescent with demonstrable orthostatic proteinuria, the current albuminuria categories are unlikely to apply.

\section{3: PREDICTING PROGNOSIS OF CKD}

1.3.1: In predicting risk for outcome of CKD, identify the following variables: 1) cause of CKD; 2) GFR category; 3) albuminuria category; 4) other risk factors and comorbid conditions. (Not Graded)

1.3.2: In people with $C K D$, use estimated risk of concurrent complications and future outcomes to guide decisions for testing and treatment for CKD complications (Figure 9). (Not Graded)

1.3.3: In populations with CKD, group GFR and albuminuria categories with similar relative risk for CKD outcomes into risk categories (Figure 9). (Not Graded)

\section{RATIONALE}

These statements are worded in this way because for all CKD complications, prognosis will vary depending on: 1) cause; 2 )
GFR; 3) degree of albuminuria; and 4) other comorbid conditions. The relative strength of each of these factors will vary for each complication or outcome of interest. Risk for kidney disease end points, such as kidney failure and AKI, is predominately driven by an individual patient's clinical diagnosis, GFR, and the degree of albuminuria or other markers of kidney damage and injury. For CVD, risk will be determined by history of CVD and traditional and nontraditional CVD risk factors. For other conditions, the risk will be determined by risk factors specific for those conditions. For all conditions, the cause of CKD, GFR category, and albuminuria category will still have important influence as "risk multipliers," but will have smaller overall influence on disease prediction than risk factors specific for the condition. All these conditions have an impact on life expectancy and quality of life (QOL) and contribute substantially to predicting the prognosis of CKD. CKD is associated with numerous complications directly or indirectly related to the cause of CKD, decreased GFR, or albuminuria (Table 9).

The risk associations of GFR and albuminuria categories appear to be largely independent of one another. Therefore, neither the category of GFR nor the category of albuminuria alone can fully capture prognosis for a patient with CKD. The magnitude and gradients of risk across categories of GFR and albuminuria will likely differ for each specific adverse event. This heterogeneity across the GFR and ACR grids in RRs for different outcomes makes it impractical to have a simple hierarchical staging of prognosis across all cells. Thus, the staging using CGA should be descriptive, but encompassing the ordered categories of GFR and ACR (Figure 9).

The CGA staging system proposed in this guideline provides a framework for future recommendations on CKD clinical management. At present, much of the evidence on clinical decision making in CKD is based solely on GFR. This recommendation serves to highlight the multidimensional

Table 9|Prognosis of CKD: Relationship of outcomes and strength of relationship to Cause (C), GFR (G), Albuminuria (A) and other measures*67,68

\begin{tabular}{|c|c|c|c|c|}
\hline \multirow[b]{2}{*}{ Outcomes } & \multicolumn{3}{|c|}{ Kidney measures } & \multirow[b]{2}{*}{ Other measures } \\
\hline & Cause & GFR & Albuminuria & \\
\hline \multicolumn{5}{|l|}{ Kidney outcomes } \\
\hline GFR decline & +++ & + & +++ & High $\mathrm{BP}$, male sex, black race, younger age \\
\hline Albuminuria rise & +++ & + & +++ & High $\mathrm{BP}$, diabetes \\
\hline AKI & + & +++ & + & Older age \\
\hline Chronic kidney failure (GFR < $15 \mathrm{ml} / \mathrm{min} / 1.73 \mathrm{~m}^{2}$; category G5) & +++ & +++ & + & Younger age \\
\hline \multicolumn{5}{|l|}{ Complications (current and future) } \\
\hline Drug toxicity & + & +++ & + & Drug exposure, liver disease \\
\hline Endocrine and metabolic & + & +++ & + & Various \\
\hline CVD and mortality & ++ & +++ & +++ & Older age, history of CVD, CVD risk factors \\
\hline Others (infection, cognitive impairment, frailty, etc) & ++ & ++ & ++ & Older age, comorbid conditions \\
\hline \multicolumn{5}{|c|}{$\begin{array}{l}\text { Abbreviations: AKI, acute kidney injury; BP, blood pressure; CVD, cardiovascular disease; GFR, glomerular filtration rate. } \\
\text { Plus signs indicate the strength of the risk relationship between the CKD characteristic and the outcome: }+ \text {, somewhat associated; ++, moderately associated; +++, strongly } \\
\text { associated. } \\
\text { *Note that the + designations refer to strength of relationship not strength of evidence to support, and are based on consensus overview by the Work Group members. } \\
\text { Adapted with permission from Uhlig K, Levey AS. }{ }^{68} \text { Developing guidelines for chronic kidney disease: we should include all of the outcomes. Ann Intern Med } 2012 ; 156(8) \text { : } \\
599-601 .\end{array}$} \\
\hline
\end{tabular}




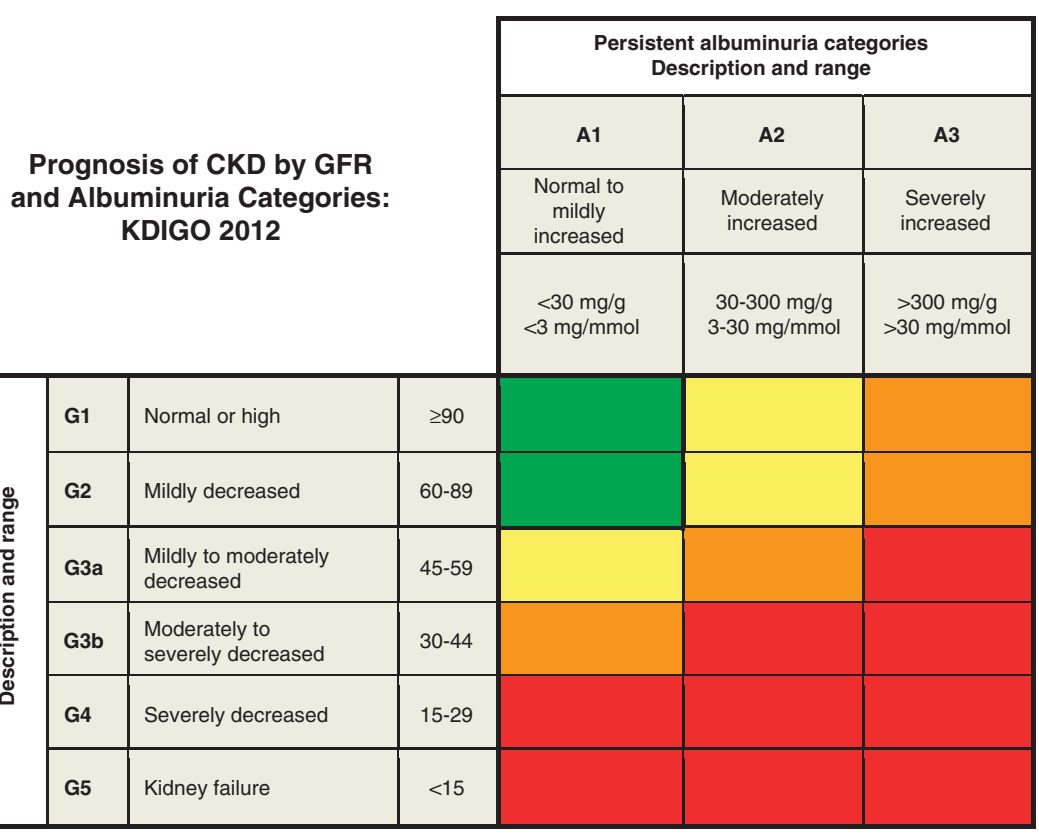

Figure 9 |Prognosis of CKD by GFR and albuminuria category. Green, low risk (if no other markers of kidney disease, no CKD); Yellow, moderately increased risk; Orange, high risk; Red, very high risk. CKD, chronic kidney disease; GFR, glomerular filtration rate; KDIGO, Kidney Disease: Improving Global Outcomes. Modified with permission from Macmillan Publishers Ltd: Kidney International. Levey AS, de Jong PE, Coresh $\mathrm{J}$, et al. $^{30}$ The definition, classification, and prognosis of chronic kidney disease: a KDIGO controversies conference report. Kidney Int 2011; 80: 17-28; accessed http://www.nature.com/ki/journal/v80/n1/full/ki2010483a.html

aspect of CKD so as to ensure appropriate consideration of the complexity of the condition.

\section{Evidence Base}

The evidence base from which these statements are derived includes large observational cohort studies from diverse populations. For some outcomes, including mortality, CVD, and kidney disease progression, metaanalyses have summarized the risk associations. For outcomes that occur predominately in older adults (e.g., dementia, fracture), the evidence is largely limited to cohorts of older people.

Extensive work by the CKD Prognosis Consortium has defined the RRs across GFR and albuminuria categories for several important outcomes, including all-cause mortality, CVD, and kidney failure (Figures 6 and 7). Risk increases incrementally in both directions - down the GFR categories and across the albuminuria categories. Levels of risk can be identified and grouped into categories, but they may differ somewhat for each outcome. Additional research is needed to map these GFR and albuminuria categories and cause of kidney disease to other important outcomes of CKD (Table 9).

\section{International Relevance}

The above statements appear to be robust when applied in North America, Europe and Asia. ${ }^{30}$ Thus, it appears for all methods used to determine GFR and to detect albuminuria, the use of the 3 parameters (cause, category of GFR and category of albuminuria) influences prognosis irrespective of ethnicity or country of origin.

\section{Implications for Clinical Practice and Public Policy}

Providers must incorporate cause of kidney disease, GFR category and albuminuria category in order to better develop an accurate assessment of an individual's prognosis related to CKD. Many providers who are not nephrologists will need guidance in the local methods for requesting and interpreting a urine albumin assessment and an eGFR. Use of risk scores which are being developed and refined is advised.

Public policy and estimates of total burden of illness in a community need to take into account the incidence and prevalence of specific conditions (such as diabetes and congestive heart failure). In addition, knowledge of distribution of levels of eGFR and ACR may be valuable for resource planning. Community or health-system based interventions to reduce the incidence of kidney failure in populations should be targeted and prioritized based on these 3 criteria.

The primary impact on clinical practice will relate to kidney-specific complications of CKD and referral patterns to help prevent and manage them. Decisions related to screening and monitoring CKD disorders will be informed and guided by the CGA system. At present, this evidence for issues such as management of anemia, CKD bone and mineral disorders, and acid-base disorders has not been organized and presented in this way.

Decisions on screening and referral strategies have major impact on the costs and quality of health-care. The value of 
this revised system of classification is that it will allow the evaluation of different referral patterns and the impact of treatment strategies in those with diverse CGA assignment. In this way, we will develop additional evidence which will inform practice patterns. These will necessarily be developed locally and reflect the values and economic realities of each health-care system.

\section{Areas of Controversy, Confusion, or Non-consensus and Clarification of Issues and Key points}

Current clinical practice has not overtly incorporated these 3 variables into all decision making activities. The utility of the system will need to be vetted by those referring and those to whom patients are referred. The overt description of the 3 dimensions of diagnosis and staging of kidney disease which include the cause, the category of GFR and the category of albuminuria, should help to inform referral and treatment patterns of large groups of individuals. Risk calculators for specific events are under development.

- The CGA classification system will be useful for quantifying risk for specific outcomes of CKD but its utility has not been fully assessed in clinical practice and research studies.

- Additional evidence is required before decisions on screening, monitoring, and referral patterns can be fully informed.

\section{Pediatric Considerations}

For Recommendation 1.3.1 the rationale and principles behind this statement would apply to pediatrics though the data are not available.

Unlike in adults, the knowledge of risk of progression or outcomes of CKD is less robust in children, with the majority of such information gleaned from either registry datasets or longitudinal trials. In a 2008 report of a select group of patients enrolled by various North American pediatric nephrology centers in the North American Pediatric Renal Trials and Collaborative Studies (NAPRTCS) registry, $46 \%$ of nearly 7100 cases had reached a final 'end point' with $86 \%$ progressing to ESRD over their time in the registry. ${ }^{69}$ Data from the prospective registry and population-based Italian Pediatric Registry of Chronic Renal Failure (ItalKid) study demonstrated a risk of progression to ESRD of $\sim 68 \%$ by age 20 years. $^{70}$

Cause of CKD. Specific information related to rate of progression for all pediatric causes of CKD is not easily available. However data from the prospective longitudinal CKiD trial has demonstrated a more rapid decline in renal function in children whose underlying cause of CKD is classified as glomerular with an annualized rate of change in iohexol GFR of $-10.5 \%$ as compared to those with a non-glomerular cause in whom the annualized rate of change is only $-3.9 \% .{ }^{71}$ In terms of absolute rates of change in measured iohexol GFR this translated, in a separate analysis from the same dataset, into a median change of GFR of $-4.3 \mathrm{ml} / \mathrm{min} / 1.73 \mathrm{~m}^{2}$ versus $-1.5 \mathrm{ml} / \mathrm{min} /$ $1.73 \mathrm{~m}^{2}$ in the glomerular versus non-glomerular groups, respectively. ${ }^{72}$ This paper also provides the only current individual disease-specific estimate of annual decline in a pediatric population. Table 10 illustrates that the median values for annualized change in GFR for various diagnosis categories.

Similarly, a randomized controlled trial (RCT) from Europe $^{73}$ examining the effects of diet on rate of progression demonstrated a statistically significant difference in $\mathrm{CrCl}$ between their glomerular and non-glomerular cohorts at 2 years of follow-up; with the mean decline $[\mathrm{SD}]$ in the glomerular group being -10.7 [11.3] versus $-8.4[13.5] \mathrm{ml} /$ $\mathrm{min} / 1.73 \mathrm{~m}^{2}$ in the non-glomerular patients $(\mathrm{P}=0.048)$.

GFR category. It is also well recognized that there is an inverse relationship between the rates of progression of kidney disease to the level of kidney function present at that presentation with more rapid decline seen in patients with lower initial levels of GFR. Staples et al $^{74}$ in their retrospective review of the NAPRTCS CKD database involving nearly 4200 children registered with GFR categories G2-G4 (GFR $15-89 \mathrm{ml} / \mathrm{min} / 1.73 \mathrm{~m}^{2}$ ) demonstrated significantly higher rates of progression, defined by progression to GFR category G5 (GFR $<15 \mathrm{ml} / \mathrm{min} / 1.73 \mathrm{~m}^{2}$ ) or initiation of dialysis or transplant, for children in GFR categories G3a-G4 (GFR $15-59 \mathrm{ml} / \mathrm{min} / 1.73 \mathrm{~m}^{2}$ ) as compared to those with CKD and GFR category G2 (GFR $60-89 \mathrm{ml} / \mathrm{min} / 1.73 \mathrm{~m}^{2}$ ) at time of enrollment: hazard ratio (HR) of GFR categories 3a and $3 \mathrm{~b}$ (GFR $30-59 \mathrm{ml} / \mathrm{min} / 1.73 \mathrm{~m}^{2}$ ) (GFR category 2 (GFR $\left.60-89 \mathrm{ml} / \mathrm{min} / 1.73 \mathrm{~m}^{2}\right)=1.00$ as referent): $2.00 ; 95 \%$ confidence interval (CI) 1.64-2.42; $\mathrm{P}<0.0001$ and $\mathrm{HR}$ of GFR category $4\left(\right.$ GFR $\left.15-29 \mathrm{ml} / \mathrm{min} / 1.73 \mathrm{~m}^{2}\right): 6.68 ; 95 \%$ CI 5.46-8.18; $\mathrm{P}<0.0001$.

Albuminuria (proteinuria). Several studies have also demonstrated the effect of proteinuria on rate of progression of CKD in children. Using registry data, and in non-glomerular conditions the ItalKids trial $^{75}$ demonstrated a significantly slower decline in $\mathrm{CrCl}$ in patients with baseline PCRs of $<200 \mathrm{mg} / \mathrm{g} \quad(20 \mathrm{mg} / \mathrm{mmol})$ and $200-900 \mathrm{mg} / \mathrm{g} \quad(20-90 \mathrm{mg} /$ mmol) when compared to those patients with a PCR of $>900 \mathrm{mg} / \mathrm{g} \quad(>90 \mathrm{mg} / \mathrm{mmol}) ;$ slope $+0.16 \pm 3.64$ and

\begin{tabular}{lc}
$\begin{array}{l}\text { Table } 10 \mid \text { Annual percentage change in GFR across diagnosis } \\
\text { categories }\end{array}$ & $\begin{array}{c}\text { Annualized percentage change } \\
\text { [number of patients] }\end{array}$ \\
\hline Disease & $-13.3 \%[\mathrm{~N}=34]$ \\
\hline $\begin{array}{l}\text { Focal and segmental } \\
\text { glomerulosclerosis }\end{array}$ & $-1.3 \%[\mathrm{~N}=27]$ \\
Hemolytic uremic syndrome & $-15.5 \%[\mathrm{~N}=51]$ \\
Other glomerular & $-4.6 \%[\mathrm{~N}=109]$ \\
Obstructive uropathy & $-3.3 \%[\mathrm{~N}=96]$ \\
$\begin{array}{l}\text { Aplastic/hypoplastic/dysplastic } \\
\text { kidneys }\end{array}$ & $-3.8 \%[\mathrm{~N}=82]$ \\
Reflux nephropathy & $-4.4 \%[\mathrm{~N}=18]$ \\
Autosomal recessive polycystic & $-2.5 \%[\mathrm{~N}=119]$ \\
kidney disease &
\end{tabular}

Abbreviation: GFR, glomerular filtration rate. Data from Furth et al. ${ }^{72}$ 
$-0.54 \pm 3.67$ versus $-3.61 \pm 5.47 \quad(\mathrm{P}<0.0001)$. This translated to higher rates of kidney survival over 5 years in the lower proteinuria groups, $96.7 \%$ and $94.1 \%$ versus $44.9 \%$, $(\mathrm{P}<0.01)$. Multivariate analysis confirmed that the baseline PCR correlated with a more rapid decline in $\mathrm{CrCl}$ for any given level of baseline function.

In a prospective multicenter randomized trial of protein intake on rates of progression in children aged 2-18 years of age, Wingen et al. employed the Schwartz equation to estimate $\mathrm{CrCl}$ and demonstrated that baseline proteinuria in multivariate analysis was the most important independent predictor of change in $\mathrm{CrCl}$. The authors reported a partial $\mathrm{R}^{2}$ of 0.259 at 2 years follow-up and similar results were found after the study was extended for a third year. ${ }^{73}$ Lifetable analysis in this study also suggested a cutoff value of $50 \mathrm{mg} / \mathrm{kg} /$ day of proteinuria as a strong predictor of time to a decline in $\mathrm{CrCl}>10 \mathrm{ml} / \mathrm{min} / 1.73 \mathrm{~m}^{2}$ and found a risk ratio of 4.01 (95\% CI 2.23-7.25; P<0.001).

Finally Wong et al. ${ }^{76}$ used cross sectional data from the prospective longitudinal CKiD trial to demonstrate that even after controlling for age, race, BMI, cause of CKD and use of RAAS antagonists they could expect an average decline in measured GFR of $10 \%$ for every increase in urinary PCR of $14 \%$ (95\% CI 10-18\%).

Other risk factors and comorbid conditions. Many other risk factors and comorbid conditions have also been associated with greater risk of progression of CKD in adults but only a few of these have been convincingly proven in children due to lack of pediatric prospective trials.

Hypertension is by far the best studied of these risk factors in children, with clear evidence from multiple sources to document the value of aggressive BP control on slowing the rate of progression of CKD. Wingen et al. ${ }^{73}$ demonstrated the importance of systolic BP in rate of progression in both univariate and multivariate models. In this study Cox proportional hazards analysis demonstrated a systolic BP $>120 \mathrm{~mm} \mathrm{Hg}$ was an independent risk for decline in $\mathrm{CrCl}$ by $>10 \mathrm{ml} / \mathrm{min} / 1.73 \mathrm{~m}^{2}$; risk ratio was 3.1 (95\% CI 1.74-5.53; $\mathrm{P}<0.001)$.

The most important prospective pediatric BP trial to date, the Effect of Strict Blood Pressure Control and ACEInhibition on Progression of Chronic Renal Failure in Pediatric Patients (ESCAPE) study, used ambulatory BP monitoring (ABPM) and a fixed dose of ramipril plus additional antihypertensive agents that do not target the RAAS to assess (as primary outcomes) the time to decline of $50 \%$ in GFR or development of ESRD. Their results demonstrated a $35 \%$ reduction in the risk of achieving the primary end point in the more intensely treated BP: HR 0.65 ; 95\% CI 0.44-0.94; $\mathrm{P}=0.02$. Further sub-analysis as reported in the KDIGO BP Guideline ${ }^{10}$ demonstrated that kidney survival was $66.1 \%$ at 5 year follow-up in patients with systolic $\mathrm{BP}<90^{\text {th }}$ percentile for age whereas it was $41 \%$ in the patients who did not achieve this level of reduction $(\mathrm{P}=0.0002)$; similar numbers were seen if diastolic BP was the metric considered.
The issue of puberty and its effect on rate of progression has recently been addressed by the ItalKids investigators. ${ }^{77}$ While the methodology of their analysis is less than ideal as they did not determine actual Tanner stages in the majority of their cohort and used estimated rather than measured GFR, they do appear to demonstrate a decrease in kidney survival probability beginning around 10.9 years in girls and 11.6 years in boys with CKD. Of note, the rate of decline in kidney survival, using these age points as 'inflection' or break points, is dramatically increased in both sexes based on their evidence provided in graphical form, although more precise analyses are not possible from the data provided.

As in adults, other factors for consideration and value in monitoring in children with respect to risk of progression include obesity, metabolic acidosis, anemia, calcium-phosphate metabolism, chronic inflammation, diabetes, hyperuricemia, dyslipidemia, and smoking.

The most comprehensive review of many of these factors in children comes from a retrospective study of the NAPRTCS CKD database. Staples et al. ${ }^{74}$ demonstrated that in a multivariate analysis of nearly 4200 children registered with CKD and GFR categories G2-G4 (GFR 15-89 ml/min/ $1.73 \mathrm{~m}^{2}$ ), the following factors were significantly associated with the risk of CKD progression (defined by progression to GFR category G5 (GFR $\left.<15 \mathrm{ml} / \mathrm{min} / 1.73 \mathrm{~m}^{2}\right)$ or initiation of dialysis or transplant): age; primary disease; GFR category; registration year; hypertension; corrected calcium, phosphorus, albumin, and hematocrit; and as proxies, the use of medications for anemia and short stature. The ability of this paper to prove causation or value in treating any of these conditions in hopes of delaying CKD progression is limited by its retrospective nature, and the fact that data were accrued from a voluntary registry.

There is optimism that prospective data from current large pediatric trials such as $\mathrm{CKiD}^{55}$ and the European Cardiovascular Comorbidity in Children with CKD (4C) trial $^{78}$ will lead to a better understanding of how risk factors may be influencing the rate of progression of CKD in children.

For Recommendation 1.3.2 the rationale and principles behind this statement would apply to pediatrics, though the data are not available. Insufficient evidence currently exists with respect to the predictive value of prevalent risk factors to guide future decisions for testing or treatment for CKD complications in an individual child.

It is hoped that well powered, prospective trials with adequate follow-up, such as the $\mathrm{CKiD}^{55}$ and European $4 \mathrm{C}^{78}$ trials, will gather sufficient numbers of patients, comorbidities, and outcomes to allow for predictive models to be built in pediatric CKD that incorporate traditional and nontraditional cardiac risk factors including dyslipidemia and hypertension, proteinuria (albuminuria), specific diseaserelated issues (e.g., diabetes, tubulopathy), prematurity, and birth weight.

For Recommendation 1.3.3 the rationale and principles behind this statement would apply to pediatrics, though the data are not available. Current evidence and a paucity of 
numbers do not allow for the statistically relevant categorization of RR for CKD outcomes based solely on GFR and albuminuria or proteinuria. Again both the $\mathrm{CKiD}^{55}$ and European $4 \mathrm{C}^{78}$ trials may be able to address these shortcomings.

\section{4: EVALUATION OF CKD}

\subsection{1: Evaluation of chronicity}

\subsubsection{1: In people with GFR $<60 \mathrm{ml} / \mathrm{min} / 1.73 \mathrm{~m}^{2}$ (GFR categories G3a-G5) or markers of kidney damage, review past history and previous measurements to determine duration of kidney disease. (Not Graded) \\ - If duration is $>3$ months, CKD is con- firmed. Follow recommendations for CKD. \\ - If duration is not $>3$ months or unclear, CKD is not confirmed. Patients may have CKD or acute kidney diseases (including AKI) or both and tests should be repeated accordingly.}

\section{RATIONALE}

When evidence of CKD is first ascertained, proof of chronicity can be obtained or confirmed by:

(i) review of past measurements of GFR;

(ii) review of past measurements of albuminuria or proteinuria and urine examinations;

(iii) imaging findings such as reduced kidney size and reduction in cortical thickness;

(iv) pathological findings such as fibrosis and atrophy;

(v) medical history especially duration of disorders known to cause CKD;

(vi) repeat measurements within and beyond the 3 month point.

Chronicity should not be assumed as AKI can present with similar abnormalities.

\section{Pediatric Considerations}

See Pediatric Considerations for next section.

\subsection{2: Evaluation of cause}

1.4.2.1: Evaluate the clinical context, including personal and family history, social and environmental factors, medications, physical examination, laboratory measures, imaging, and pathologic diagnosis to determine the causes of kidney disease. (Not Graded)

\section{RATIONALE}

Once the presence of CKD is proven it is essential to establish a cause for this which will inform specific management and modify risk projections. The diagnosis will be reached by standard clinical method (i.e., history examination) and special investigation, based on knowledge of the common causes of CKD and their manifestations. Not all evaluations are required in all patients, and will be directed by clinical context, and resource availability. For most patients the following evaluations are indicated:

- Reagent strip urinalysis to detect hematuria or pyuria. If positive, use urine microscopy to detect $\mathrm{RBC}$ casts or WBC casts.

- Ultrasound to assess kidney structure (i.e., kidney shape, size, symmetry and evidence of obstruction) as clinically indicated.

- Serum and urine electrolytes to assess renal tubular disorders, as clinically indicated.

Many individuals found to have CKD will not have a primary kidney disease but kidney damage caused by diabetes mellitus, vascular disease, and hypertension. The issue for the clinician will be to decide whether the presence of these is a sufficient explanation and if not, to investigate further. The prevalence of other conditions will vary depending on region, age, and other factors.

It is beyond the scope of this guideline to describe how specific diagnoses are reached but non-nephrologists in the first instance should review the family history, medications, symptoms and signs for manifestations of systemic diseases. Urinalysis should be performed, along with imaging of the kidneys if obstruction of the urinary tract or polycystic kidney disease is considered.

\section{Pediatric Considerations}

For Recommendations 1.4.1.1 and 1.4.2.1, the statements would need to be altered for application in pediatric practice in the following way.

In any child with GFR $<60 \mathrm{ml} / \mathrm{min} / 1.73 \mathrm{~m}^{2}$ (or more than $1 \mathrm{SD}$ below expected for their age and sex) or with markers of kidney damage, a complete review of their past history and previous measurement or estimate of renal function and full consideration of the clinical context, including prenatal history, drug exposures of fetus or mother, genetic conditions, coincident organ abnormalities, physical examination, fetal and post-natal laboratory measures including amniotic fluid, pre- and post-natal imaging and pathologic diagnosis including those of the fetus and placenta should be used to determine the cause(s) of kidney disease.

As noted in Pediatric Considerations for Recommendation 1.1.1, developmental renal abnormalities account for as many as $30-50 \%$ of the children with CKD. ${ }^{42} \mathrm{~A}$ careful review of all fetal or maternal exposures, genetic risks factors, and any relevant information on the intrauterine environment during gestation are all relevant to the determination of the presence of CKD either prior to or present immediately at the time of delivery. An infant may be born with CKD, leading to 
immediate classification within the CGA framework - up to and including that of dialysis dependency.

\subsubsection{Evaluation of GFR}

This section describes the various methods by which GFR can be estimated. We describe laboratory techniques that satisfy the requirements for robust result reporting and we compare the accuracy of available equations for the purpose of reporting eGFR using a single equation where applicable. We emphasize equations based on standardized measurements of SCr, but also consider newly developed equations based on standardized measurements of serum cystatin C (SCysC) because they are being introduced into clinical practice. We encourage practitioners to have a clear understanding of the value and limitations of both filtration markers, the importance of standardization of assays for both, and to understand that when an accurate assessment of kidney function is required, direct measurement should be undertaken.

1.4.3.1: We recommend using serum creatinine and a GFR estimating equation for initial assessment. (1A)

1.4.3.2: We suggest using additional tests (such as cystatin $\mathrm{C}$ or a clearance measurement) for confirmatory testing in specific circumstances when eGFR based on serum creatinine is less accurate. (2B)

\section{RATIONALE}

These statements specifically address the need to ensure that estimating equations are put into routine clinical practice, and that clinicians understand the utility of further evaluation with additional methods if required.

GFR is measured by the clearance of an exogenous or endogenous filtration marker. ${ }^{27}$ All clearance methods are complex so in clinical practice, GFR is estimated from the serum concentration of the endogenous filtration marker creatinine. Cystatin $\mathrm{C}$ is an alternative endogenous filtration marker; other filtration markers are also under evaluation. The principles of GFR estimation are discussed in the rationale for recommendations regarding the use of creatinine as a filtration marker but the concepts apply to GFR estimation from all endogenous filtration markers. Specific comments about GFR estimation using cystatin $\mathrm{C}$ are presented separately.

For most clinical circumstances, estimating GFR from $\mathrm{SCr}$ is appropriate for diagnosis, staging, and tracking the progression of CKD. However, like all diagnostic tests, interpretation is influenced by varying test characteristics in selected clinical circumstances and the prior probability of disease. In particular, an isolated decreased eGFR in otherwise healthy individuals is more likely to be false positive than in individuals with risk factors for kidney disease or markers of kidney damage. Confirmation of decreased eGFR by measurement of an alternative endogenous filtration marker (cystatin C) or a clearance measurement is warranted in specific circumstances when GFR estimates based on SCr are thought to be inaccurate and when decisions depend on more accurate knowledge of GFR, such as confirming a diagnosis of $\mathrm{CKD}$, determining eligibility for kidney donation, or adjusting dosage of toxic drugs that are excreted by the kidneys. ${ }^{79}$ The choice of confirmatory test depends on the clinical circumstance and the availability of methods where the patient is treated.

\section{Pediatric Considerations}

For Recommendation 1.4.3.1, the statements would need to be altered for application in pediatric practice in the following way. The use of $\mathrm{SCr}$ and a recently derived pediatric specific GFR estimating equation, which incorporates a height term, ${ }^{80}$ is preferred over the use of $\mathrm{SCr}$ alone in the initial assessment of pediatric renal function.

For Recommendation 1.4.3.2, this guideline is fully applicable in pediatrics.

\subsubsection{3: We recommend that clinicians (1B):}

- use a GFR estimating equation to derive GFR from serum creatinine (eGFR creat $)$ rather than relying on the serum creatinine concentration alone.

- understand clinical settings in which $\mathrm{eGFR}_{\text {creat }}$ is less accurate.

\section{RATIONALE}

Estimating GFR from the SCr concentration alone requires implicit judgments that are difficult in routine clinical care, including reciprocal transformation, consideration of the non-GFR determinants, and conversion to the GFR scale. Using GFR estimating equations provides a more direct assessment of GFR than SCr alone. The SCr concentration is influenced by GFR and other physiological processes, collectively termed "non-GFR determinants," including creatinine generation by muscle and dietary intake, tubular creatinine secretion by organic anion transporters, and extrarenal creatinine elimination by the gastrointestinal tract (Figure 10).

GFR estimating equations are developed using regression to relate the measured GFR to steady state SCr concentration and a combination of demographic and clinical variables as surrogates of the non-GFR determinants of SCr. By definition, GFR estimates using SCr concentration are more accurate in estimating measured GFR than the SCr concentration alone in the study population in which they were developed. Sources of error in GFR estimation from SCr concentration include nonsteady state conditions, non-GFR determinants of SCr, measurement error at higher GFR, and interferences with the creatinine assays (Table 11). GFR estimates are less precise at higher GFR levels than at lower levels.

The clinician should remain aware of caveats for any estimating equation which may influence the accuracy in a given individual patient. 
Because of the physiologic and statistical considerations in developing GFR estimating equations, GFR estimates are less precise at higher GFR levels than at lower levels. In principle, equations based on multiple endogenous filtration markers

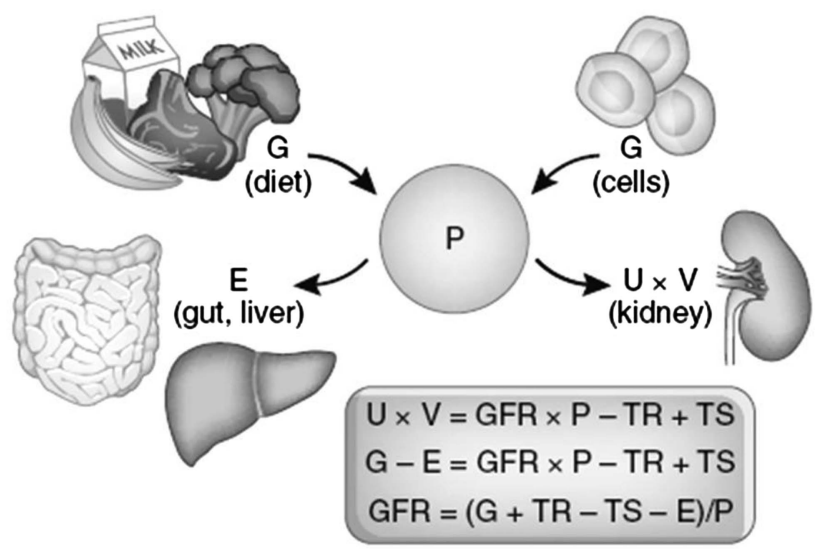

Figure $10 \mid$ Determinants of the serum level of endogenous filtration markers. The plasma level $(P)$ of an endogenous filtration marker is determined by its generation $(G)$ from cells and diet, extrarenal elimination (E) by gut and liver, and urinary excretion (UV) by the kidney. Urinary excretion is the sum of filtered load (GFR X P), tubular secretion (TS), and reabsorption (TR). In the steady state, urinary excretion equals generation and extrarenal elimination. By substitution and rearrangement, GFR can be expressed as the ratio of the non-GFR determinants (G, TS, $\mathrm{TR}$, and $\mathrm{E}$ ) to the plasma level. GFR, glomerular filtration rate. Reprinted with permission of American Society of Nephrology, Measured GFR as a confirmatory test for estimated GFR, Stevens LA, Levey AS. ${ }^{79}$ J Am Soc Nephrol 20: 2305-2313, 2009; permission conveyed through Copyright Clearance Center, Inc.; accessed http://jasn.asnjournals.org/content/20/11/2305.full.pdf can overcome some of the imprecision of GFR estimates at higher levels, due to cancellation of errors from noncorrelated non-GFR determinants.

\section{Pediatric Considerations}

This guideline is fully applicable in pediatrics.

1.4.3.4: We recommend that clinical laboratories should (1B):

- measure serum creatinine using a specific assay with calibration traceable to the international standard reference materials and minimal bias compared to isotope-dilution mass spectrometry (IDMS) reference methodology.

- report $\mathrm{eGFR}_{\text {creat }}$ in addition to the serum creatinine concentration in adults and specify the equation used whenever reporting eGFR $_{\text {creat }}$.

- report $\mathrm{eGFR}_{\text {creat }}$ in adults using the 2009 CKD-EPI creatinine equation. An alternative creatinine-based GFR estimating equation is acceptable if it has been shown to improve accuracy of GFR estimates compared to the 2009 CKD-EPI creatinine equation.

When reporting serum creatinine:

- We recommend that serum creatinine concentration be reported and rounded to the nearest whole number when expressed as standard international units $(\mu \mathrm{mol} / \mathrm{l})$ and rounded to the nearest $100^{\text {th }}$ of a whole number when expressed as conventional units (mg/dl).

Table 11 | Sources of error in GFR estimating using creatinine

\begin{tabular}{ll}
\hline Source of error & Example \\
\hline $\begin{array}{l}\text { Non-steady state } \\
\text { Non-GFR determinants of SCr that differ from study populations }\end{array}$ & $\bullet$ AKI \\
in which equations were developed & \\
Factors affecting creatinine generation & $\bullet$ Race/ethnicity other than US and European black and white \\
& $\bullet$ Extremes of muscle mass \\
& $\bullet$ Diet and nutritional status \\
& $\bullet$ high protein diet \\
Factors affecting tubular secretion of creatinine & $\bullet$ Muscle wasting diseases \\
& $\bullet$ Ingestion of cooked meat \\
Factors affecting extra-renal elimination of creatinine & $\bullet$ Decrease by drug-induced inhibition \\
& $\bullet$ cimetidine \\
Higher GFR & $\bullet$ Dialysis \\
Interferenofibrate
\end{tabular}

Abbreviations: AKI, acute kidney injury; GFR, glomerular filtration rate; SCr, serum creatinine. 
When reporting eGFR creat:

- We recommend that $\mathrm{GGFR}_{\text {creat }}$ should be reported and rounded to the nearest whole number and relative to a body surface area of $1.73 \mathrm{~m}^{2}$ in adults using the units $\mathrm{ml} / \mathrm{min} /$ $1.73 \mathrm{~m}^{2}$.

- We recommend $\mathrm{eGFR}_{\text {creat }}$ levels less than $60 \mathrm{ml} / \mathrm{min} / 1.73 \mathrm{~m}^{2}$ should be reported as "decreased."

\section{RATIONALE}

The statement is worded this way to acknowledge that calibration of assays is essential to interpretation of kidney function measures. This recommendation is directed to laboratories with the intent to clarify the details of such calibration and the use of specific equations so as to facilitate international standardization. ${ }^{81}$

There are numerous assay methods for creatinine for use in clinical laboratories. Variation in assigned values for $\mathrm{SCr}$ concentration among methods is greater at low concentrations, corresponding to high levels of GFR. Variation in assays at low $\mathrm{SCr}$ concentrations contributes to imprecision of GFR estimates at high GFR levels.

Currently available assays fall into two broad categories, the alkaline picrate (Jaffe) assay and enzymatic assays. In general, enzymatic assays are less biased compared to a standardized reference material and less susceptible to interferences. All assays are available on a number of platforms.

We recommend that laboratories use assays that are traceable to pure creatinine standards via a valid calibration hierarchy and that are specific and minimally-biased compared with isotope-dilution mass spectrometry (IDMS) reference method results. Results should be traceable to reference materials and methods listed on the Joint Committee for Traceability in Laboratory Medicine (JCTLM) database. Ideally laboratories should move to enzymatic assays for creatinine measurement: as a minimum, the use of traditional kinetic or end point Jaffe assays should cease and be replaced with IDMS aligned Jaffe methods.

Clinical laboratory information systems generally have access to patient age and sex and thus can report eGFR based on SCr age and sex, thus providing the clinician with the test result in units which are recommended for interpretation. Estimated GFR is now reported together with SCr when creatinine is ordered in more than $75 \%$ of clinical laboratories in the US. ${ }^{82}$ In the UK, $93 \%$ of NHS laboratories report eGFR with $\mathrm{SCr}^{83}$ as is the case in Australia, Canada, and many European countries.

Selection of a single equation for use, where applicable, would facilitate communication among providers, patients, researchers and public health officials. Criteria for selection should be based on accuracy compared to measured GFR and usefulness in clinical care and public health.

The interpretation of measured and eGFR is based on comparison to normative values, which are adjusted for BSA because of the physiologic matching of GFR to kidney size, which is in turn related to BSA. The value of $1.73 \mathrm{~m}^{2}$ reflects the average value of BSA of 25-year old men and women in the USA in $1927 .{ }^{84}$ While it is known that modern populations may have different normal values for BSA, the $1.73 \mathrm{~m}^{2}$ value will be maintained for normalization purposes.

Drug dosing should be based on GFR which is not adjusted for BSA. The effect of drug dosing based on GFR adjusted for BSA compared to GFR unadjusted for BSA has not been studied rigorously and more precise recommendations are not available.

Flagging decreased values for eGFR can alert clinicians to the possibility of AKD or CKD, and may indicate the need for additional investigations or treatments, including adjustment of doses of drugs that are excreted by the kidney. However, values for GFR between 60 and $89 \mathrm{ml} / \mathrm{min} / 1.73 \mathrm{~m}^{2}$ are mildly decreased compared to the usual values in young healthy people. Thus it is important that clinicians appreciate that eGFR values that are not flagged because they are $>60 \mathrm{ml} /$ $\mathrm{min} / 1.73 \mathrm{~m}^{2}$ are not necessarily normal.

\section{Evidence Base}

Numerous equations have been developed to estimate GFR or $\mathrm{CrCl}$ in adults. In general, GFR estimating equations using creatinine include age, sex, race, and body size as surrogates for creatinine generation by muscle. For our review of GFR estimating equations, we only considered equations that were developing using assays that were traceable to reference methods and study populations in which SCr concentration was measured using traceable assays (Supplemental Table 1$).{ }^{85}$

Based on published data, only the Modification of Diet in Renal Disease (MDRD) Study equation, Chronic Kidney Disease Epidemiology Collaboration (CKD-EPI) equation and modifications of these equations were developed using creatinine assays traceable to the international reference material for creatinine (Table 12) ${ }^{86,87}$ The Cockcroft and Gault formula and others were developed before standardization of creatinine assays but cannot be re-expressed for use with standardized creatinine assays (Supplemental Table 2).

The MDRD Study equation was developed in 1999 and is currently recommended for eGFR reporting in adults by the National Kidney Disease Education Program (NKDEP) and by the Department of Health in the UK. It uses standardized SCr, age, sex, and race (black versus white and other) to estimate GFR adjusted for BSA $\left(\mathrm{ml} / \mathrm{min} / 1.73 \mathrm{~m}^{2}\right) .{ }^{86,94}$ Because of imprecision at higher GFR, NKDEP recommends that eGFR $\geq 60 \mathrm{ml} / \mathrm{min} / 1.73 \mathrm{~m}^{2}$ computed using the MDRD Study equation not be reported as a numeric value. For a similar reason, the UK Department of Health recommends not reporting eGFR $>90 \mathrm{ml} / \mathrm{min} / 1.73 \mathrm{~m}^{2}$ using the MDRD Study equation as a numeric value.

The CKD-EPI equation was developed in 2009 and uses the same four variables as the MDRD Study equation. ${ }^{87}$ The CKD-EPI equation had less bias than the MDRD Study equation, especially at $\mathrm{GFR} \geq 60 \mathrm{ml} / \mathrm{min} / 1.73 \mathrm{~m}^{2}$, a small 


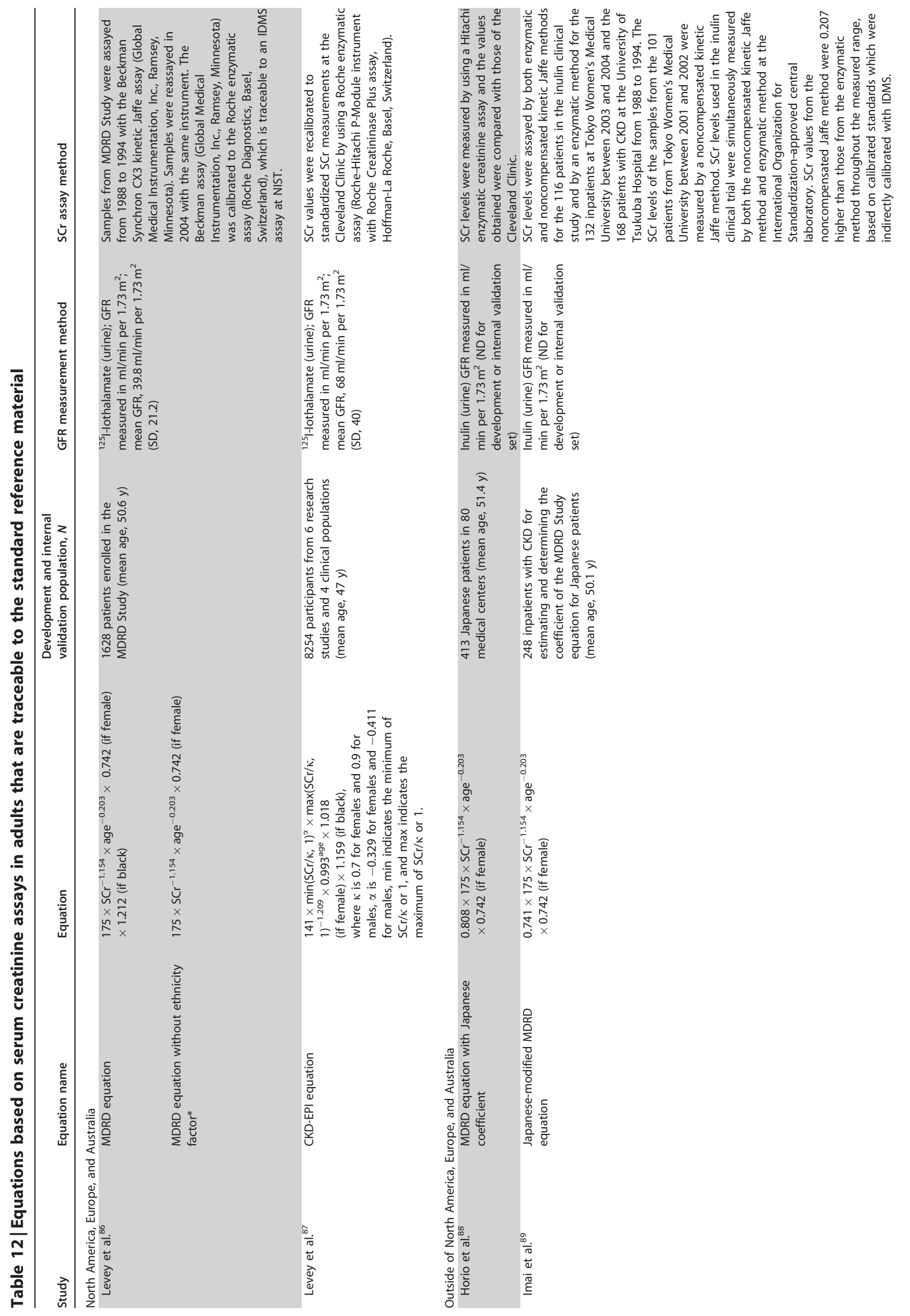




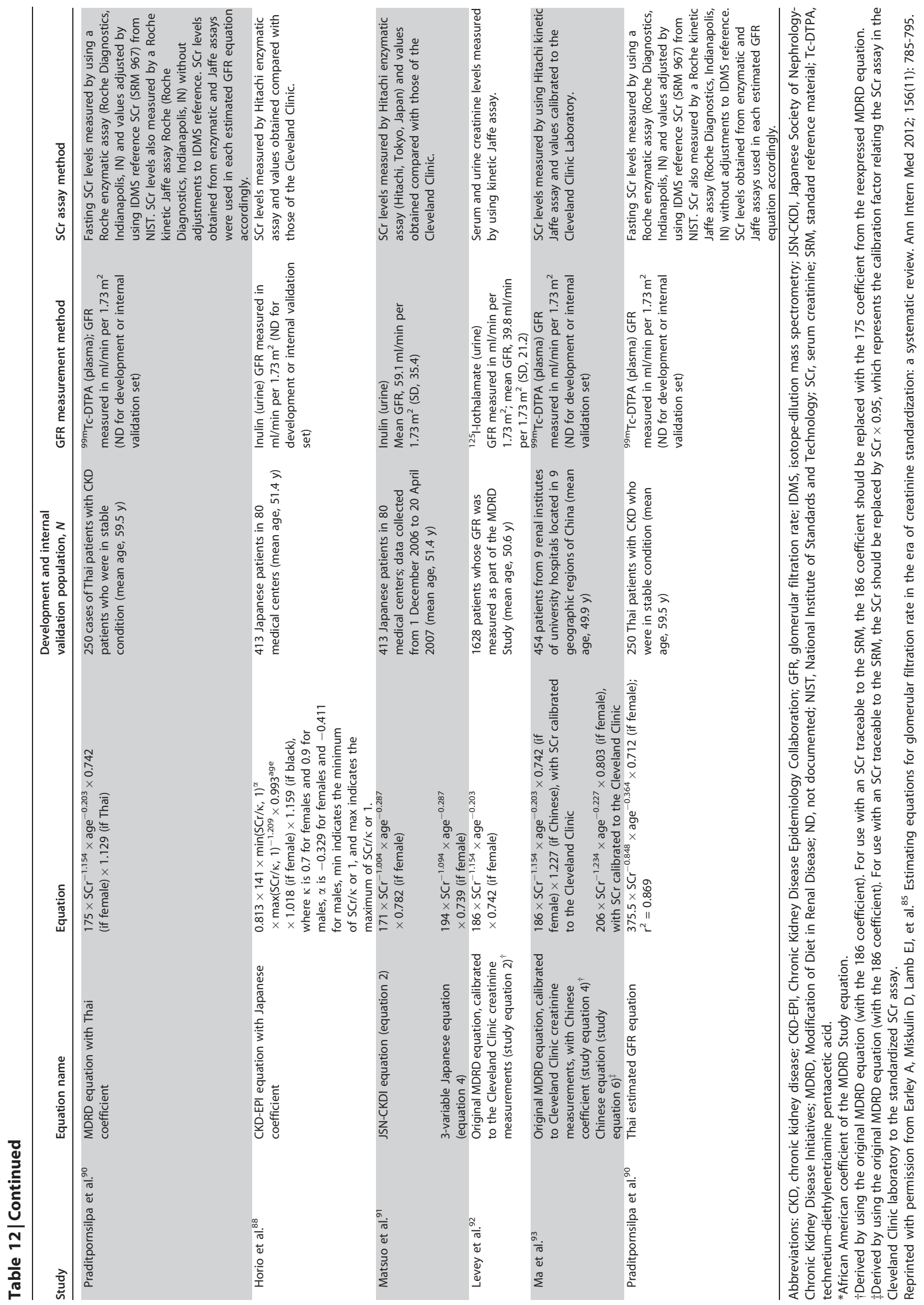




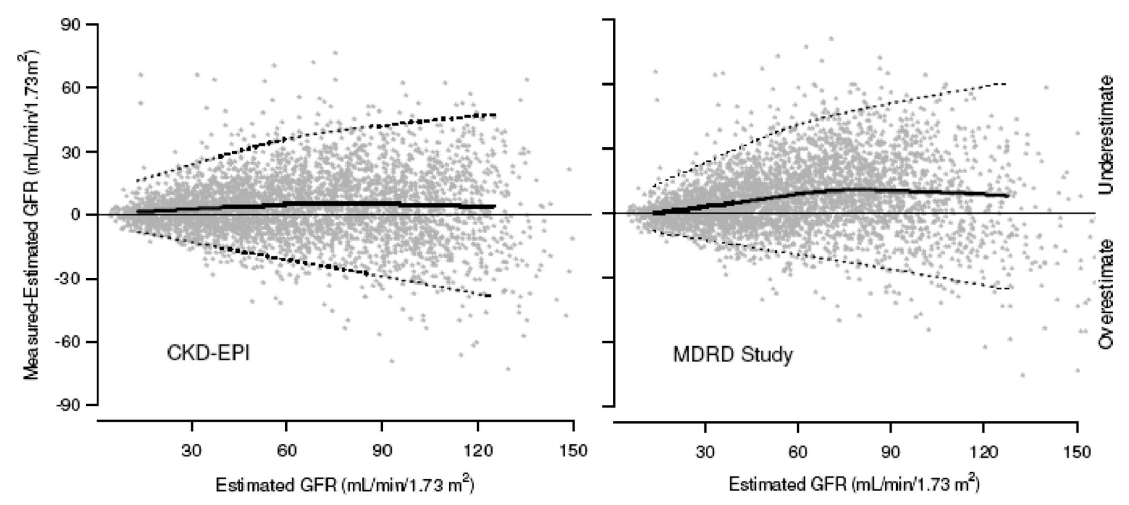

Figure 11 Performance of the CKD-EPI and MDRD Study equations in estimating measured GFR in the external validation data set. Both panels show the difference between measured and estimated versus estimated GFR. A smoothed regression line is shown with the $95 \% \mathrm{Cl}$ (computed by using the lowest smoothing function in R), using quantile regression, excluding the lowest and highest $2.5 \%$ of estimated GFR. To convert GFR from $\mathrm{ml} / \mathrm{min}$ per $1.73 \mathrm{~m}^{2}$ to $\mathrm{ml} / \mathrm{s}$ per $\mathrm{m}^{2}$, multiply by 0.0167 . CKI-EPD, Chronic Kidney Disease Epidemiology Collaboration; Cl, confidence interval; GFR, glomerular filtration rate; MDRD, Modification of Diet in Renal Disease. Reprinted with permission from Levey AS, Stevens LA, Schmid CH, et al. ${ }^{87}$ A new equation to estimate glomerular filtration rate. Ann Intern Med 2009; 150(9): 604-612.

improvement in precision, and greater accuracy (Figure 11). Most but not all studies from North America, Europe and Australia show that the CKD-EPI equation is more accurate than the MDRD Study equation, especially at higher GFR (Table 13), ${ }^{85}$ which enables reporting of numeric values across the range of GFR. At this time, large commercial clinical laboratories in the US have changed from using the MDRD Study equation to the CKD-EPI equation for eGFR reporting.

Lesser bias of the CKD-EPI equation compared to the MDRD Study equation reflects higher eGFR throughout most of the range for age and creatinine, especially in younger individuals, women and whites. Higher eGFR results in lower prevalence estimates for CKD in these groups (Figure 12), with more accurate risk relationships of lower eGFR and adverse outcomes (Figure 13). ${ }^{107}$

To account for possible differences in muscle mass and diet according to race, ethnicity and geographic region, the MDRD Study and CKD-EPI equations have been modified for use in other racial and ethnic groups and in other countries. In some, but not all studies, these modifications are associated with increased accuracy (Table 14), and should be used in preference to unmodified equations. Where tested, the CKD-EPI equation and its modifications were generally more accurate than the MDRD Study and its modifications. In the absence of specific modifications for race, ethnicity, or regional difference, it is reasonable to use the CKD-EPI equation for GFR estimation. Reliance upon $\mathrm{SCr}$ alone is not an appropriate alternative since the uncertainty about the effect of non-GFR determinants affects interpretation of $\mathrm{SCr}$ as much as it affects interpretation of eGFR. More widespread testing of GFR estimating equations is necessary to resolve uncertainties about the need for racial, ethnic, and geographic modifications. ${ }^{108}$

\section{Pediatric Considerations}

This recommendation would need to be altered for application in pediatric practice in the following way.

- Creatinine measurements in all infants and children should be derived from methods that minimize confounders and are calibrated against an international standard.

- $\mathrm{eGFR}_{\text {creat }}$ may only be reported when the height of the child is known by the laboratory.

- If reporting $\mathrm{eGFR}_{\text {creat }}$ laboratories should utilize the most current and accurate pediatric derived equations based on the demographic and laboratory markers available.

In infants or small children the level of creatinine when measured is often below that of the normal 'bottom range' of the adult assay. As such laboratories measuring creatinine in infants or small children must ensure their lower calibration samples include the lowest end of the expected range of values for the group of interest.

As the majority and the most accurate of the published pediatric $\mathrm{eGFR}_{\text {creat }}$ formulas require height, standard laboratory reporting of eGFR $\mathrm{Great}_{\text {is }}$ is neither practical nor recommended in children. In a pediatric CKD population, and using the plasma disappearance of iohexol as the gold standard measure of GFR, Schwartz et al. derived a number of novel GFR prediction equations. ${ }^{80}$ Their analysis demonstrated the importance of the height/SCr variable in the population as it provided the best correlation with the iohexol GFR $\left(\mathrm{R}^{2}=65 \%\right)$. The simplest of such formula, using only height and SCr and a constant of either 41.3 or 0.413 depending on whether height was expressed as meters or centimeters respectively, provided $79 \%$ of estimated GFRs within $30 \%$ of the iohexol values and $37 \%$ of estimated GFRs within $10 \%$ of the iohexol values. 


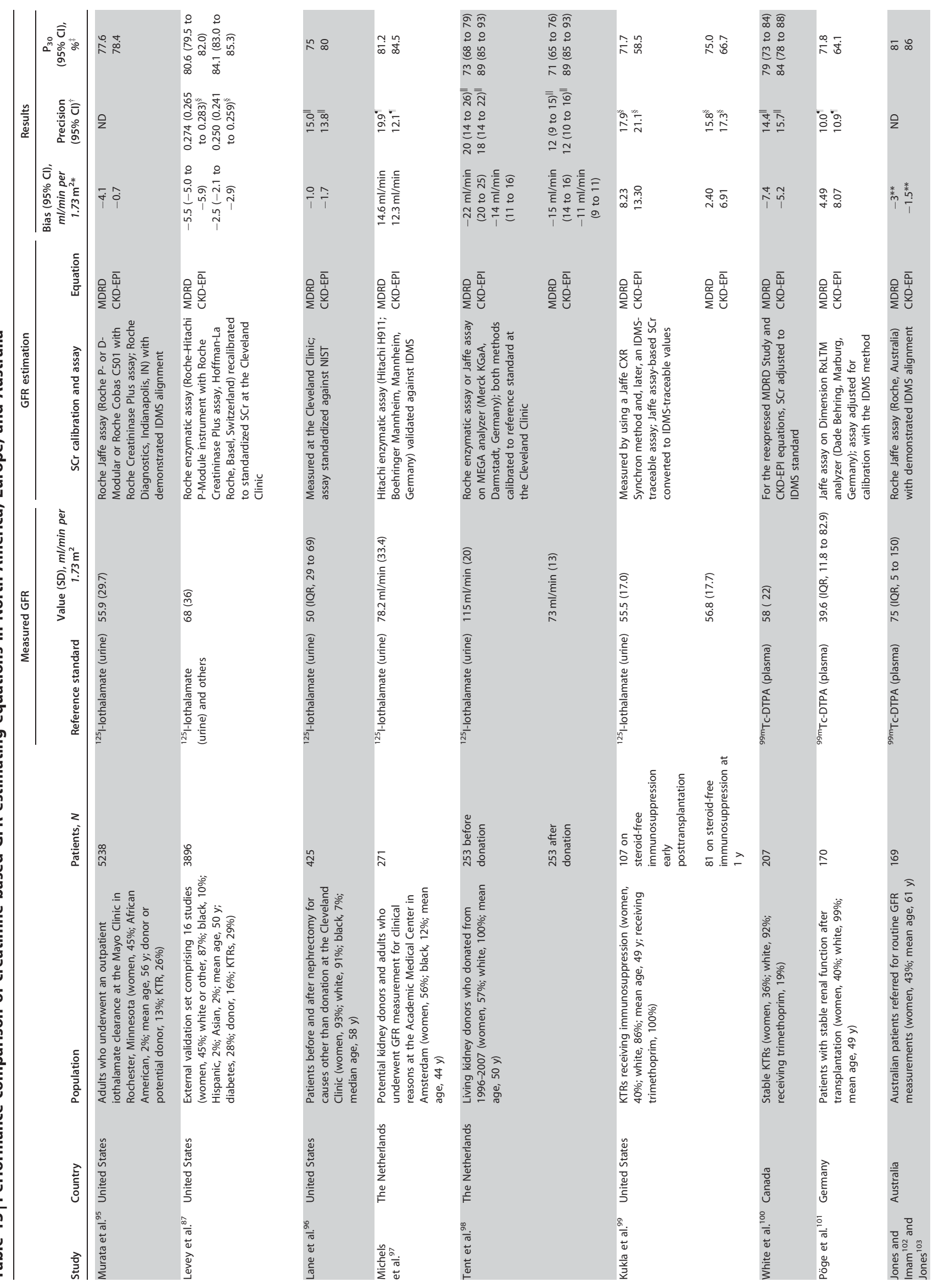




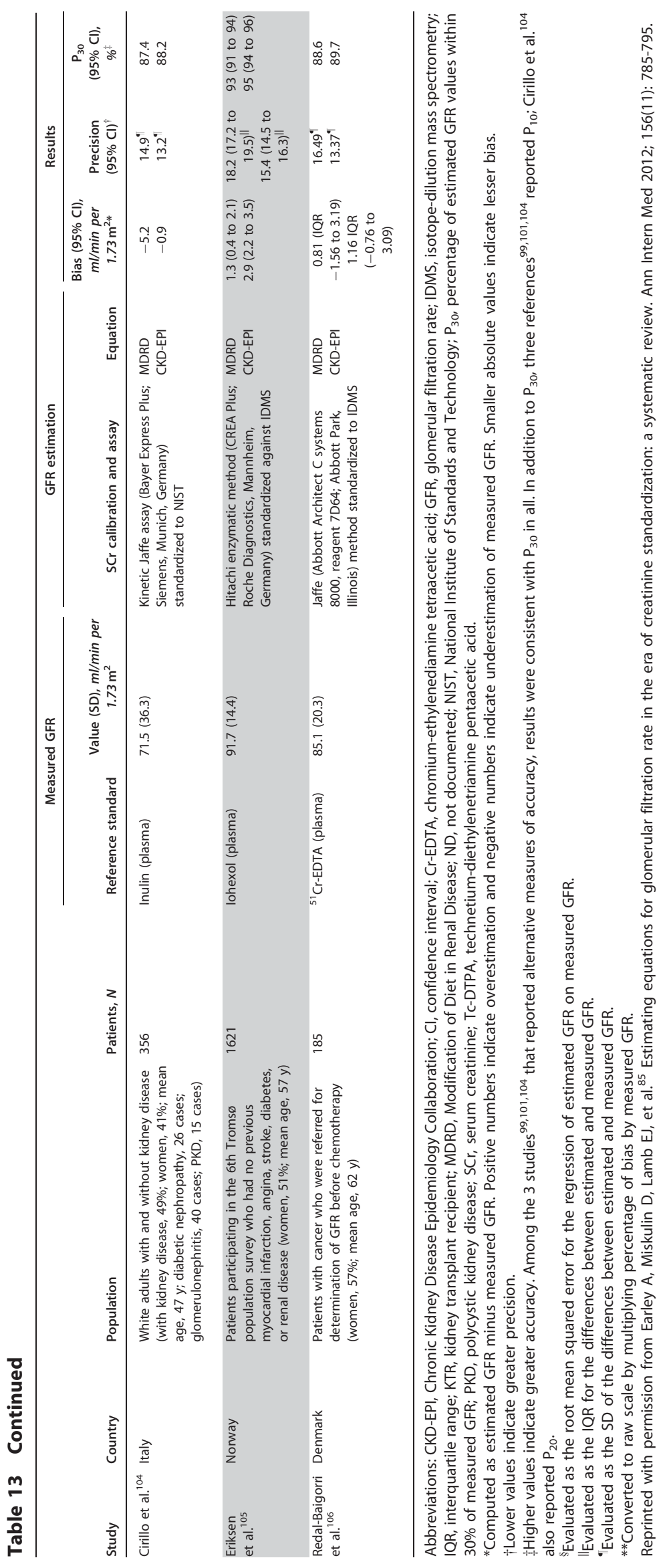



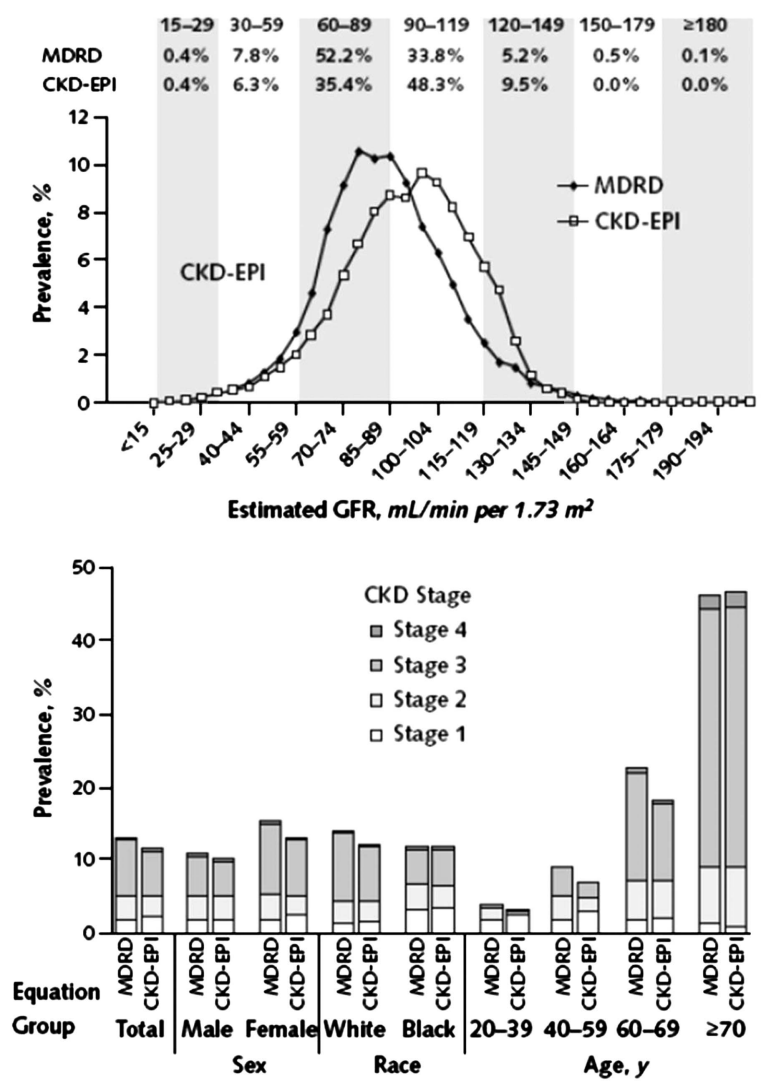

Figure 12 |Comparison of distribution of GFR and CKD prevalence by age (NHANES 1999-2004). GFR was categorized on the basis of the classification system established by the NKF-KDOQI. Top. Distribution of estimated GFR, by $4-\mathrm{ml} / \mathrm{min}$ per $1.73 \mathrm{~m}^{2}$ categories. Values are plotted at the midpoint. Bottom. Prevalence of CKD, by age. CKD, chronic kidney disease; GFR, glomerular filtration rate; NKF-KDOQI, National Kidney Foundation-Kidney Disease Outcomes Quality Initiative; NHANES, National Health and Nutrition Examination Survey. Reprinted with permission from Levey AS, Stevens LA, Schmid CH, et al. ${ }^{87}$ A new equation to estimate glomerular filtration rate. Ann Intern Med 2009; 150(9): 604-612.

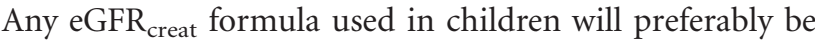
validated at the appropriate age and level of renal function, and the laboratory methods used locally will be calibrated or comparable to those used in the process of developing the formula being applied. Currently the most robust pediatric eGFR formulas, derived using iohexol disappearance and creatinine measurements which were measured centrally and calibrated and traceable to international standards come from the CKiDs study. ${ }^{80}$

The two most common creatinine-based formulas recommended for use in clinical practice include:

Updated "Bedside" Schwartz equation:

eGFR $\left(\mathrm{ml} / \mathrm{min} / 1.73 \mathrm{~m}^{2}\right)=41.3 \times($ height $/ \mathrm{SCr})$, where height is in meters and SCr is in $\mathrm{mg} / \mathrm{dl}$.

"1B" Equation (include blood urea nitrogen [BUN] not cystatin C):
eGFR $\left(\mathrm{ml} / \mathrm{min} / 1.73 \mathrm{~m}^{2}\right)=40.7 \times(\text { height } / \mathrm{SCr})^{0.64} \times(30 /$ BUN $)^{0.202}$, where height is in meters, SCr and BUN are in $\mathrm{mg} / \mathrm{dl}$.

The additional recommendation for laboratory reporting of SCr is fully applicable in pediatrics.

When the individual clinician has information regarding current and accurate height and applies the appropriate pediatric formula, the recommendation to report an individual child's eGFR creat value of less than $60 \mathrm{ml} / \mathrm{min} /$ $1.73 \mathrm{~m}^{2}$ as "decreased," would be applicable in children over the age of 2 years.

1.4.3.5: We suggest measuring cystatin $\mathrm{C}$ in adults with eGFR creat $45-59 \mathrm{ml} / \mathrm{min} / 1.73 \mathrm{~m}^{2}$ who do not have markers of kidney damage if confirmation of CKD is required. $(2 C)$

- If $\mathrm{eGFR}_{\text {cys }} / \mathrm{eGFR}_{\text {creat-cys }}$ is also $<60 \mathrm{ml} / \mathrm{min} /$ $1.73 \mathrm{~m}^{2}$, the diagnosis of CKD is confirmed.

- If $\mathrm{eGFR}_{\text {cys }} / \mathrm{eGFR}_{\text {creat-cys }}$ is $\geq 60 \mathrm{ml} / \mathrm{min} / 1.73 \mathrm{~m}^{2}$, the diagnosis of CKD is not confirmed.

\section{RATIONALE}

A major foundation of this guideline is that CKD classification and staging should be influenced primarily by clinical prognosis. As will be reviewed in the sections below, abundant evidence has shown that GFR estimates based on cystatin $\mathrm{C}$ are more powerful predictors of clinical outcomes than creatinine-based eGFR. These findings have been strongest for mortality and CVD events, and the prognostic advantage of cystatin $\mathrm{C}$ is most apparent among individuals with GFR $>45 \mathrm{ml} / \mathrm{min} / 1.73 \mathrm{~m}^{2}$. In addition, new findings show that using cystatin $\mathrm{C}$ in addition to $\mathrm{SCr}$ can lead to improved accuracy of GFR estimation, including CKD classification. In the opinion of the Work Group, these considerations warrant new recommendations for GFR estimation using cystatin C.

\section{Evidence Base}

Evidence supports the use of cystatin C-based eGFR within the population of persons diagnosed with CKD based on an eGFR creat $45-59 \mathrm{ml} / \mathrm{min} / 1.73 \mathrm{~m}^{2}$ (G3a) but without albuminuria (A1) or other manifestations of kidney damage. This group represents $3.6 \%$ of the US population and $41 \%$ of people in the US estimated to have CKD based on eGFR creat and urine ACR alone (Figure 8), and there has been substantial controversy over whether or not these persons have CKD. Data described below indicate that use of cystatin C to estimate GFR in this population leads to more accurate estimation of GFR and prediction of risk for future adverse events.

In several studies, $\mathrm{eGFR}_{\text {cys }}$ has been measured in populations with and without $\mathrm{eGFR}_{\text {creat }}<60 \mathrm{ml} / \mathrm{min} /$ $1.73 \mathrm{~m}^{2}$, and participants were separated into those with and without eGFR $\mathrm{Gys}_{\mathrm{s}}<60 \mathrm{ml} / \mathrm{min} / 1.73 \mathrm{~m}^{2}$ (Figure 14). Those with both eGFR creat $_{\text {and }}$ eGFR $\mathrm{Gys}_{\text {cys }}<60 \mathrm{ml} / \mathrm{min} / 1.73 \mathrm{~m}^{2}$, about two-thirds of those with eGFR creat $<60 \mathrm{ml} / \mathrm{min} / 1.73 \mathrm{~m}^{2}$, had 

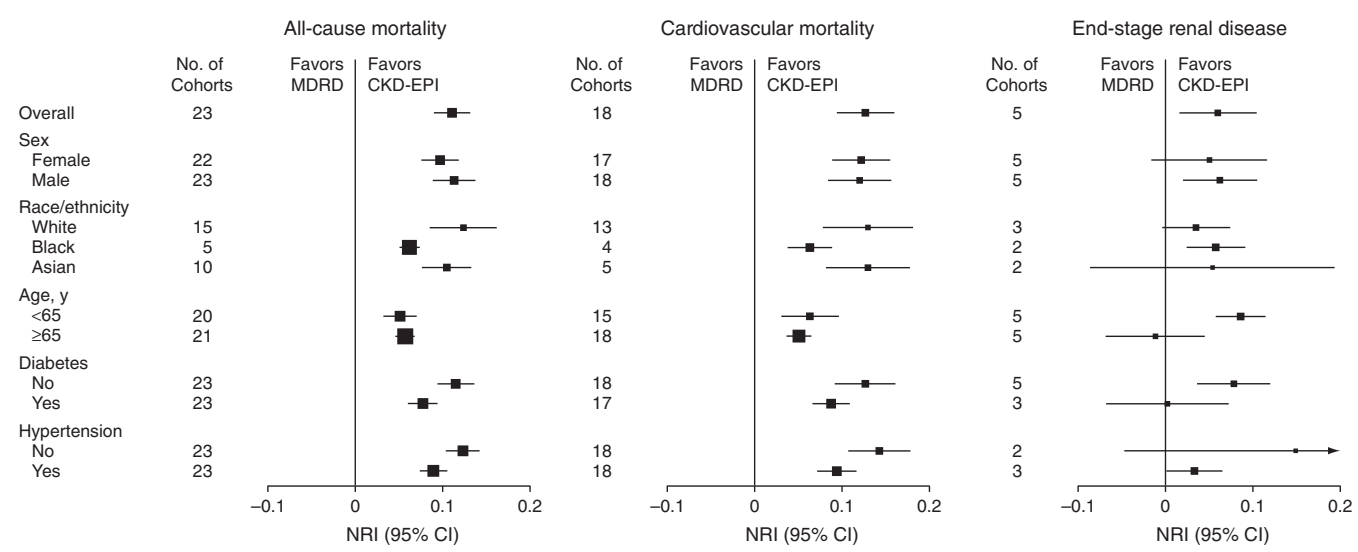

Figure 13 Meta-analysis of NRI for all-cause mortality, CVD mortality, and ESRD. NRI summarizes the risk of clinical outcomes among participants who are reclassified from one estimated GFR category using the MDRD Study equation to another estimated GFR category using the CKD-EPI equation compared with those who are not reclassified. NRI greater than zero favors the CKD-EPI equation. NRI less than zero favors the MDRD Study equation. The sizes of the data markers are proportional to the inverse of the variance of the NRIs. CKD-EPI, Chronic Kidney Disease Epidemiology Collaboration; CVD, cardiovascular disease; ESRD, end-stage renal disease; GFR, glomerular filtration rate; MDRD, Modification of Diet in Renal Disease; NRI, net reclassification improvements. Reprinted with permission from Matsushita K, Mahmoodi BK, Woodward M, et al. ${ }^{107}$ Comparison of risk prediction using the CKD-EPI equation and the MDRD Study equation for estimated glomerular filtration rate. JAMA 2012; 307(18): 1941-1951. Copyright (c) (2012) American Medical Association. All rights reserved.

markedly elevated risks for death, CVD, and ESRD end points compared with persons with eGFR creat $>60 \mathrm{ml} / \mathrm{min} / 1.73 \mathrm{~m}^{2}$. The Work Group therefore considers this group to have "confirmed CKD." In contrast, about one-third of those with eGFR creat $<60 \mathrm{ml} / \mathrm{min} / 1.73 \mathrm{~m}^{2}$ had $\mathrm{eGFR}_{\text {cys }}>60 \mathrm{ml} /$ $\mathrm{min} / 1.73 \mathrm{~m}^{2}$ and this group were similar in risk for adverse outcomes as persons with $\mathrm{eGFR}$ creat $>60 \mathrm{ml} / \mathrm{min} / 1.73 \mathrm{~m}^{2}$.

New data from CKD-EPI also showed improved accuracy in GFR estimation using both creatinine and cystatin C $($ eGFR creat-cys $)$ compared to either marker alone. In the subgroup with eGFR creat $45-59 \mathrm{ml} / \mathrm{min} / 1.73 \mathrm{~m}^{2}$, the combined equation correctly reclassified $16.8 \%$ of those with eGFR $45-59 \mathrm{ml} / \mathrm{min} / 1.73 \mathrm{~m}^{2}$ to measured GFR $\geq 60 \mathrm{ml} / \mathrm{min} /$ $1.73 \mathrm{~m}^{2}{ }^{113}$

The consensus of the Work Group was therefore that the large group of persons with $\mathrm{GGFR}_{\text {creat }} 45-59 \mathrm{ml} / \mathrm{min} / 1.73 \mathrm{~m}^{2}$ without markers of kidney damage, but with $\mathrm{eGFR}_{\text {cys }} /$ eGFR $_{\text {creat-cys }} \geq 60 \mathrm{ml} / \mathrm{min} / 1.73 \mathrm{~m}^{2}$ could be considered not to have CKD. The removal of the diagnosis and label of CKD may be reassuring to patients and may help clinicians to focus their efforts on higher risk CKD patients.

The guideline statement suggesting the use of eGFR $\mathrm{Fys}_{\text {c }}$ /

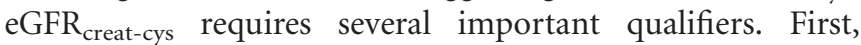
clinicians may not want or need to confirm the diagnosis of CKD in patients with $\mathrm{eGFR}_{\text {creat }} 45-59 \mathrm{ml} / \mathrm{min} / 1.73 \mathrm{~m}^{2}$ without markers of kidney damage, either because the likelihood of CKD is high because of the presence of risk factors for CKD or presence of complications of CKD. Second, cystatin $\mathrm{C}$ is not universally available, so it may not be practical for a clinician to request a cystatin $\mathrm{C}$ blood test. Third, in certain clinical settings, the cost of measuring cystatin C (US $\$ 1-5)$ may be prohibitive. For all these reasons, the guideline statement 1.4.3.5 is stated as a suggestion.

In addition to the population described above, $\mathrm{eGFR}_{\mathrm{cys}}$ may be useful as a confirmatory test in situations where either the eGFR $\mathrm{Great}_{\text {may }}$ be inaccurate or biased, or when the clinical scenario warrants a secondary test (Recommendation 1.4.3.2). In these clinical situations, a clearance measurement using an exogenous filtration marker may be optimal when it is available. The measurement of eGFR $\mathrm{cys}_{\mathrm{s}} / \mathrm{GFR}_{\text {creat-cys }}$ would be a relatively low-cost, feasible alternative when GFR measurement is not practical. The Work Group believed that measured urinary $\mathrm{CrCl}$ was an inferior confirmatory test relative to either GFR measurement or GFR estimation using both creatinine and cystatin C.

If cystatin $\mathrm{C}$ testing is desired, it is very important that clinicians understand principles of GFR estimation using cystatin C. As with creatinine, GFR should be estimated from cystatin C and an appropriate equation should be chosen for the specific clinical population (Recommendation 1.4.3.6), and an assay be chosen for measurement that is traceable to the international standard reference material (Recommendation 1.4.3.7).

\section{Pediatric Considerations}

The utility of this specific statement to pediatrics is unclear as the vast majority of children with significant reductions in GFR, e.g., below $60 \mathrm{ml} / \mathrm{min} / 1.73 \mathrm{~m}^{2}$, have either structural abnormalities or findings of renal damage as evidenced by urinary or serum abnormalities. It is very unlikely that isolated reduction in GFR would occur as in older adults. As such, the confirmation of CKD will be made on criteria beyond that of GFR alone.

1.4.3.6: If cystatin $C$ is measured, we suggest that health professionals $(2 C)$ :

- use a GFR estimating equation to derive GFR from serum cystatin $\mathrm{C}$ rather than relying on the serum cystatin $\mathrm{C}$ concentration alone.

- understand clinical settings in which eGFR cys $_{\text {s }}$ and eGFR $_{\text {creat-cys }}$ are less accurate. 


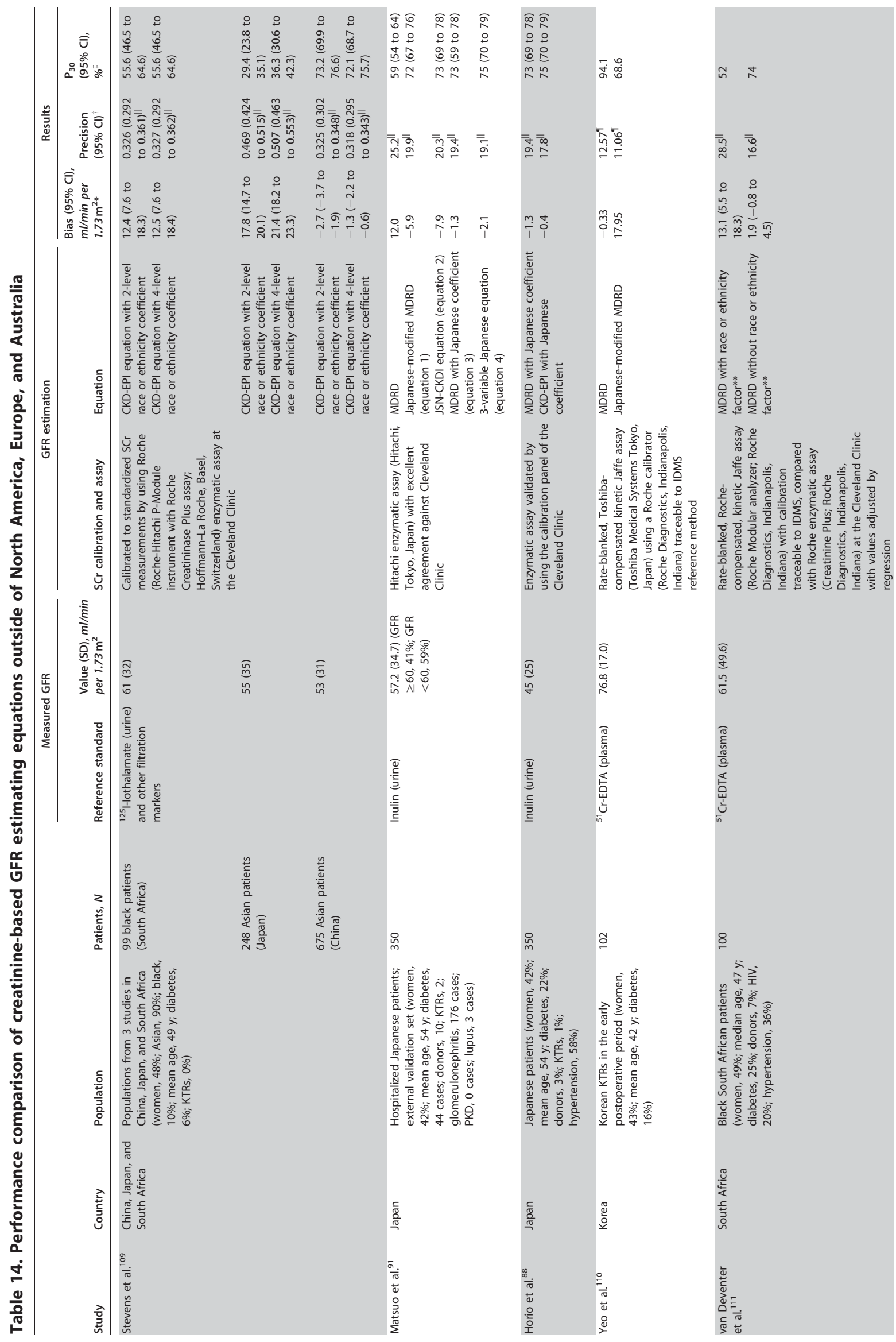




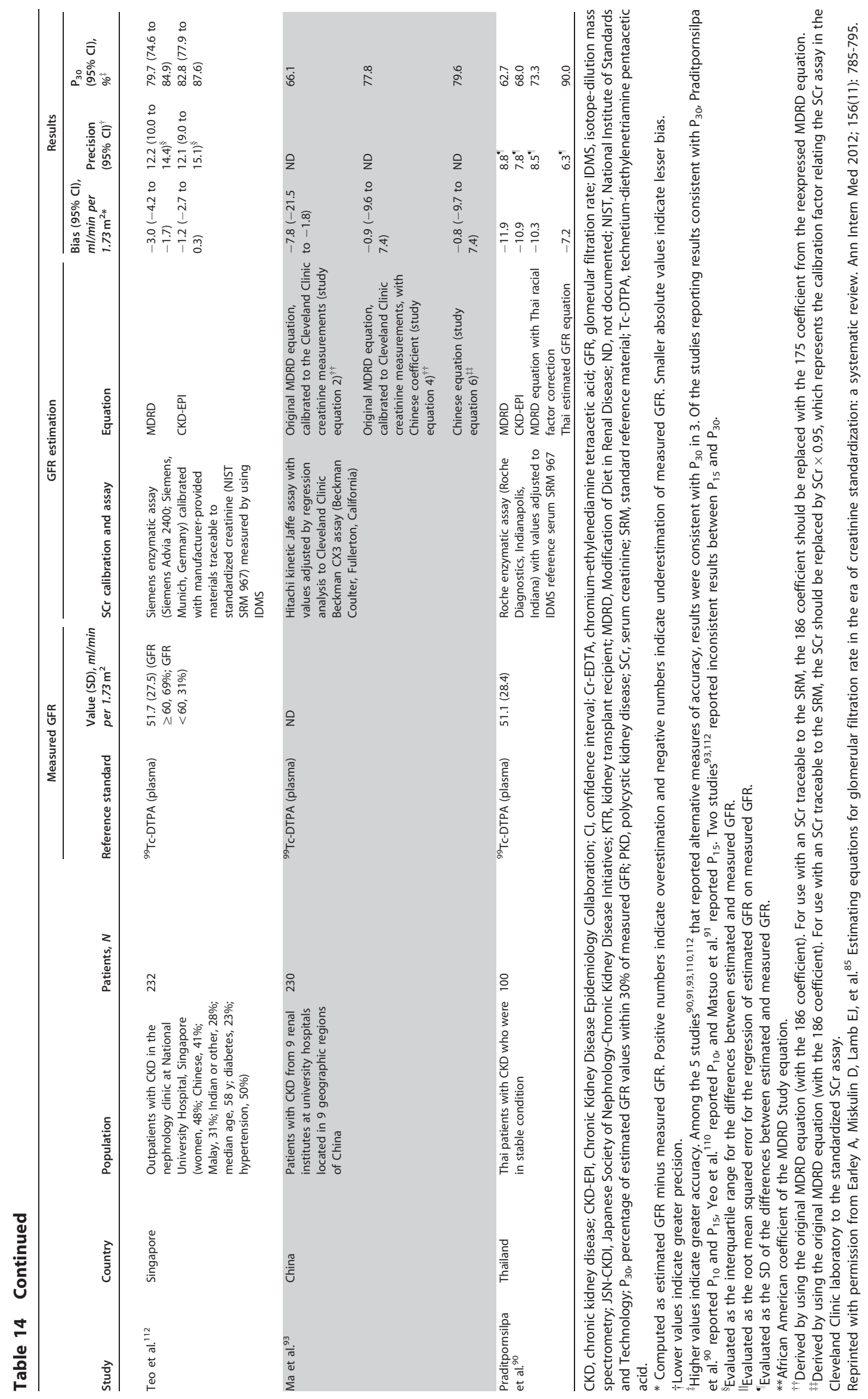



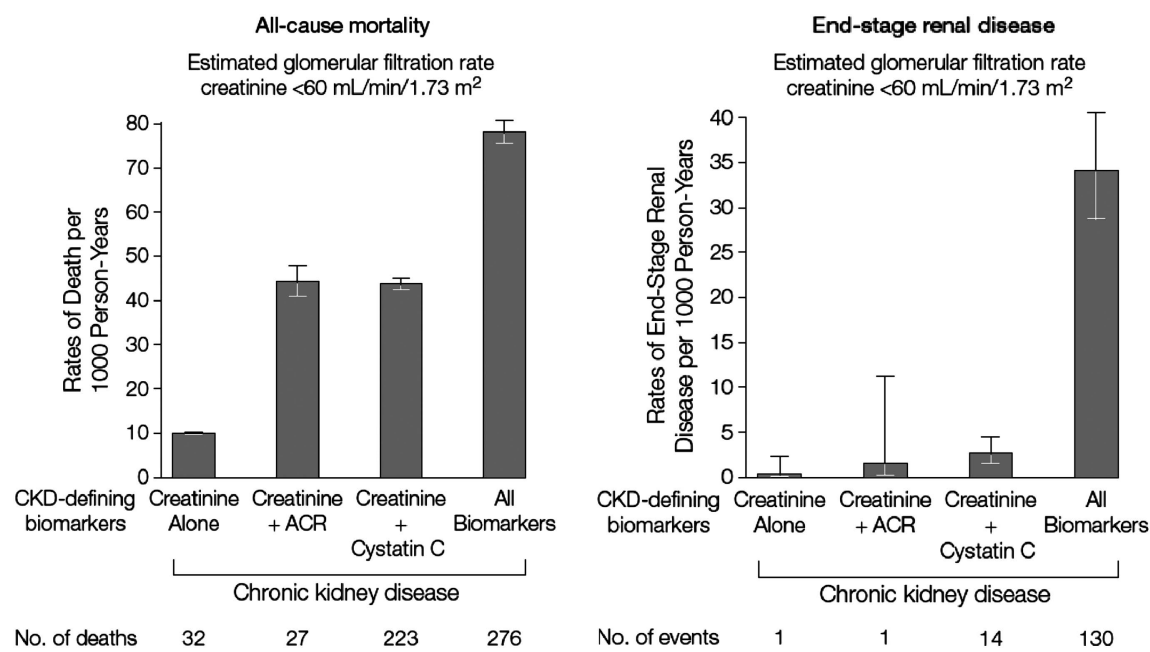

Figure $14 \mid$ Association of CKD definitions with all-cause mortality and ESRD. CKD, chronic kidney disease; ESRD, end-stage renal disease. Reprinted with permission from Peralta CA, Shlipak MG, Judd S, et al. ${ }^{114}$ Detection of chronic kidney disease with creatinine, cystatin $C$, and urine albumin-to-creatinine ratio and association with progression to end-stage renal disease and mortality. JAMA 2011; 305(15): 1545-1552. Copyright (c) (2011) American Medical Association. All rights reserved.

\section{Table 15 | Sources of error in GFR estimating using cystatin C}

\begin{tabular}{|c|c|}
\hline Source of error & Example \\
\hline Non-steady state & - $\mathrm{AKI}$ \\
\hline \multicolumn{2}{|c|}{$\begin{array}{l}\text { Non-GFR determinants of SCysC that differ from study populations in which } \\
\text { equations were developed }\end{array}$} \\
\hline Factors affecting extra-renal elimination of cystatin C & Increased by severe decrease in GFR \\
\hline Higher GFR & $\begin{array}{l}\text { - Higher biological variability in non-GFR determinants relative to } \\
\text { GFR } \\
\text { - Higher measurement error in SCysC and GFR }\end{array}$ \\
\hline Interference with cystatin C assay & - Heterophilic antibodies \\
\hline
\end{tabular}

Abbreviations: AKI, acute kidney injury; GFR, glomerular filtration rate, SCysC, serum cystatin C.

\section{RATIONALE}

Cystatin C is licensed for use in some countries in Europe and has been approved by the FDA as a measure of kidney function in the United States for nearly 10 years. In certain regions, notably Sweden and parts of China, eGFR is routinely estimated by both creatinine and cystatin C. As with creatinine, GFR estimates using cystatin $\mathrm{C}$ are more accurate in estimating measured GFR than the SCysC concentration alone. As with creatinine, sources of error in GFR estimation from SCysC concentration include non-steady state conditions, nonGFR determinants of SCysC, measurement error at higher GFR, and interferences with the cystatin C assays (Table 15).

\section{Pediatric Considerations}

For Recommendation 1.4.3.6, this guideline is fully applicable in pediatrics. See Recommendation 1.4.3.7 for details.

In terms of clinical settings where $\mathrm{GGFR}_{\text {cys }}$ might be less accurate, it should be noted that Schwartz et al. determined that the only variable that explained the outlier values of estimated GFR (in both univariate and multivariate formulas) was heavier weight; race, high blood pressure, albumin levels and use of steroids did not contribute. ${ }^{115}$ 
1.4.3.7: We recommend that clinical laboratories that measure cystatin $C$ should (1B):

- measure serum cystatin $C$ using an assay with calibration traceable to the international standard reference material.

- report eGFR from serum cystatin $\mathrm{C}$ in addition to the serum cystatin $C$ concentration in adults and specify the equation used whenever reporting eGFR $_{\text {cys }}$ and eGFR creat-cys. .

- report eGFR cys $_{\text {and }}$ a GFR $\mathrm{Great}_{\text {cys }}$ in adults using the 2012 CKD-EPI cystatin $\mathrm{C}$ and 2012 CKD-EPI creatinine-cystatin $C$ equations, respectively, or an alternative cystatin C-based GFR estimating equations if they have been shown to improve accuracy of GFR estimates compared to the 2012 CKDEPI cystatin C and 2012 CKD-EPI creatinine-cystatin $\mathrm{C}$ equations.

When reporting serum cystatin C:

- We recommend reporting serum cystatin $\mathrm{C}$ concentration rounded to the nearest $100^{\text {th }}$ of a whole number when expressed as conventional units $(\mathrm{mg} / \mathrm{l})$.

When reporting eGFR cys $_{\text {s }}$ and eGFR $_{\text {creat-cys: }}$ :

- We recommend that $\mathrm{eGFR}_{\mathrm{cys}}$ and eGFR $_{\text {creat-cys }}$ be reported and rounded to the nearest whole number and relative to a body surface area of $1.73 \mathrm{~m}^{2}$ in adults using the units $\mathrm{ml} / \mathrm{min} / 1.73 \mathrm{~m}^{2}$.

- We recommend eGFR cys $_{\text {and }}$ eGFR $_{\text {creat-cys }}$ levels less than $60 \mathrm{ml} / \mathrm{min} /$ $1.73 \mathrm{~m}^{2}$ should be reported as "decreased."

\section{RATIONALE}

As for SCr, reporting eGFR using cystatin $\mathrm{C}$ in addition to cystatin C will facilitate clinician's use of cystatin C for GFR estimation. It is important to acknowledge that calibration of assays is essential to interpretation of kidney function measures. Cystatin $\mathrm{C}$ is measured by a variety of immunoassays and, as for creatinine, there can be variation among methods in reported SCysC concentration but reported analytic variation appears less common than with creatinine. In June 2010 the Institute for Reference Materials and Measurements (IRMM) released a reference material (ERMDA471/IFCC) for cystatin C measurement. Reagent manufacturers are in the process of recalibrating their assays against this standard which will enable standardized reporting of cystatin $\mathrm{C}$ and eGFR results. This recommendation is directed to laboratories with the intent to clarify the details of such calibration and the use of specific equations so as to facilitate international standardization.

\section{Evidence Base}

Numerous equations have been developed to estimate GFR. Some equations include cystatin $\mathrm{C}$ as the only variable, while others include, age, sex, or race, but the magnitude of coefficients for these variables are smaller than in creatininebased equations, presumably reflecting less contribution of muscle to cystatin $\mathrm{C}$ generation than to creatinine generation. Equations without race are a potential advantage for cystatin C-based estimating equations in non-black, non-white populations.

For our review of GFR estimating equations, we only considered equations that were developed using assays that were traceable to the new reference methods and study populations in which SCysC concentration was measured using traceable assays. At this time, only the equations developed by CKD-EPI are expressed for use with standardized SCysC (Table 16), including equations developed in CKD populations in $2008^{116,117}$ and re-expressed for use with standardized cystatin C in 2011, and equations developed in diverse populations in 2012. ${ }^{113}$ Equations using assays that are not traceable to the the reference standard are listed in Supplemental Table 3.

The 2012 creatinine-cystatin C equation is more accurate than equations using creatinine or cystatin $\mathrm{C}$ separately (Figure 15), and more accurate than the 2008 creatinine-cystatin C equation (Table 17). The average of the GFR computed by the equations using creatinine and cystatin C separately is similar to the GFR computing using the creatinine-cystatin C equations. The 2012 cystatin C equation has similar accuracy to the 2009 creatinine equation described above but does not require use of race, and may be more accurate in non-black, non-white populations or in clinical conditions with variation in non-GFR determinants of SCr. We anticipate the development of additional equations using cystatin $\mathrm{C}$ in the future and recommend that they be compared with the CKD-EPI 2012 cystatin C and creatinine-cystatin C equations as well as with the CKD-EPI 2009 creatinine equation.

\section{Pediatric Considerations}

For Recommendation 1.4.3.7 this set of statements would need to be altered for application in pediatric practice in the following way:

- Measure SCysC using an immunonephelometrically determined method in which the assay is calibrated and traceable to the international standard reference material.

- Report eGFR $\mathrm{cys}_{\text {in }}$ addition to the SCysC concentration in children.

- Report $\mathrm{eGFR}_{\text {cys }}$ in children specifying the specific equation used.

Based on their recent work comparing particle-enhanced nephelometric to turbidometric immunoassays for cystatin C in a pediatric population with significant reduction in GFR (median GFR $\sim 45 \mathrm{ml} / \mathrm{min} / 1.73 \mathrm{~m}^{2}$ ), Schwartz et al. demonstrated less bias for the nepholometric value and that its reciprocal showed a substantially improved correlation to the iohexol GFR ( 0.87 versus 0.74 ) when compared to that of the 


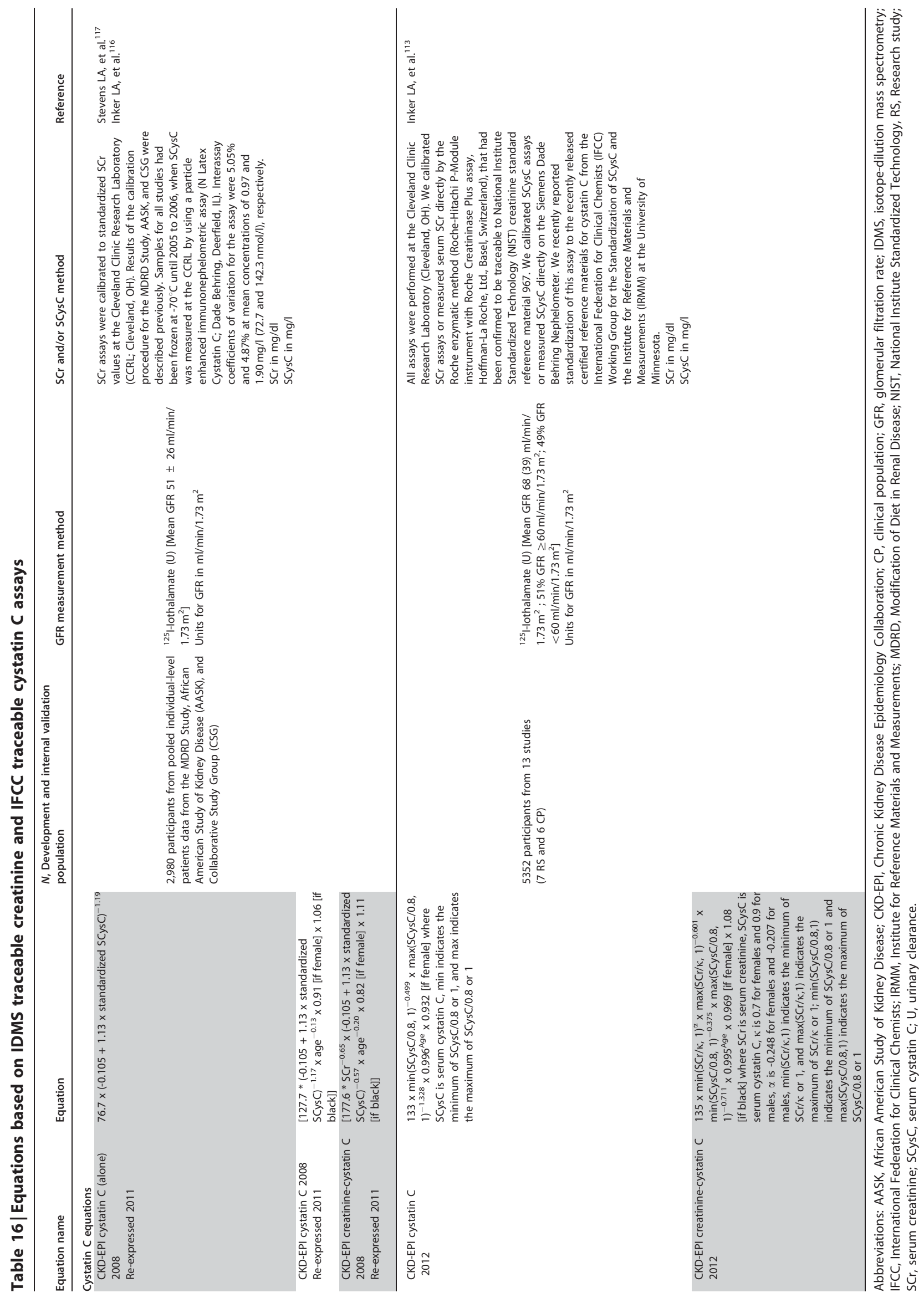



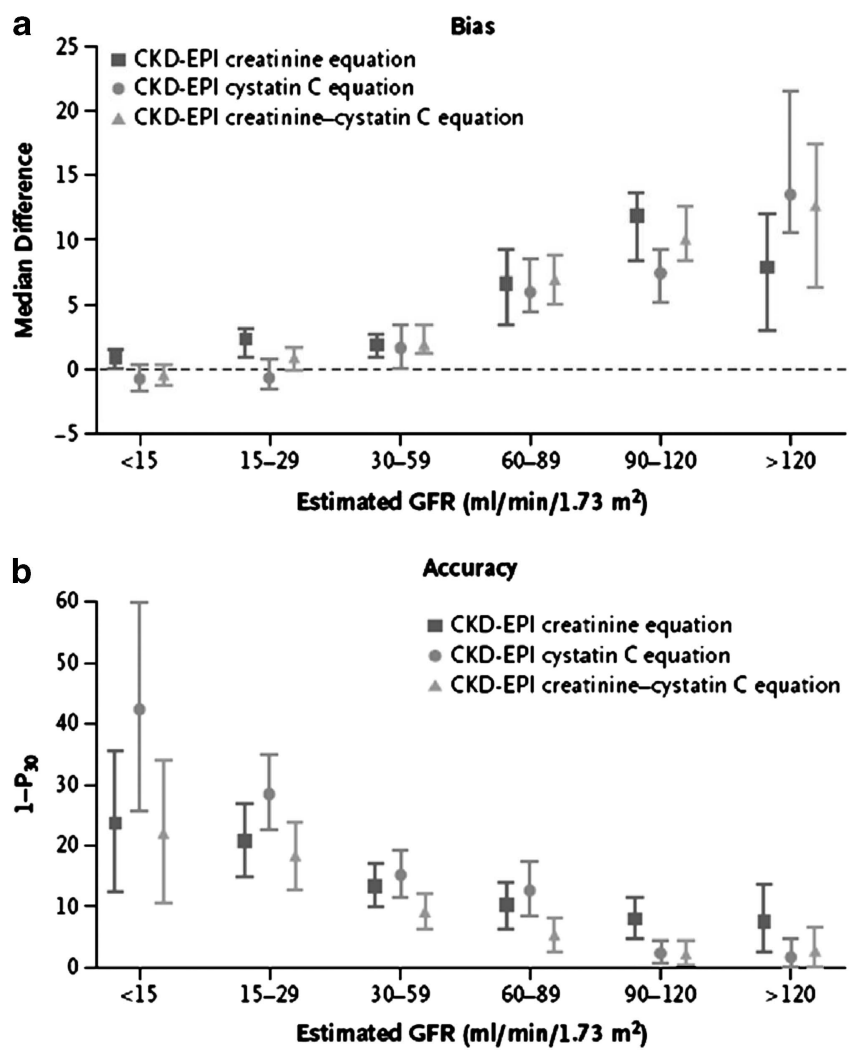

Figure 15 | Performance of three equations for estimating GFR. Panel a shows the median difference between measured and estimated GFR. The bias is similar with the equation using creatinine alone, the equation using cystatin $\mathrm{C}$ alone, and the combined creatinine-cystatin $C$ equation. Panel b shows the accuracy of the three equations with respect to the percentage of estimates that were greater than $30 \%$ of the measured GFR $\left(1-P_{30}\right)$. Whiskers indicate $95 \%$ confidence intervals. GFR, glomerular filtration rate; $P_{30}$, percentage of estimated GFR values within $30 \%$ of measured GFR. From N Engl J Med, Inker LA, Schmid $\mathrm{CH}$, Tighiouart $\mathrm{H}$, et al. ${ }^{113}$ Estimating glomerular filtration rate from serum creatinine and cystatin C. 367: 20-29. Copyright (c) 2012 Massachusetts Medical Society. Reprinted with permission from Massachusetts Medical Society.

turbidometric assay. ${ }^{115}$ This demonstrates the importance of using an assay calibrated and traceable to the international standard reference material.

Numerous pediatric specific and derived eGFR $\mathrm{cys}_{\text {s }}$ formulas have been published, the most current and recent by Schwartz ${ }^{115}$ who derives the newest available from a validation set from the CKiD study and compares those results to 3 other well-recognized formulas from the literature, namely Zapitelli et al., ${ }^{118}$ Filler and Lepage, ${ }^{119}$ and Hoek et al. ${ }^{120}$

Their results demonstrate that the newest univariate cystatin $\mathrm{C}$ formula derived from the $\mathrm{CKiD}$ cohort has excellent accuracy with $82.6 \%$ of eGFR cys $_{\text {within }} 30 \%$ of the true measured iohexol GFR and $37.6 \%$ within $10 \%$ of the true measured iohexol GFR. Likewise the bias of $0.3 \%$ and correlation of 0.85 are the best of all formulas reported to date. The formula they use to obtain these values is: $70.69 \times$

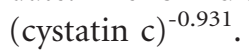

Of note, their final multivariate equation, when applied to the validation set and using height/SCr, nepholemetric cystatin C, BUN, sex, and an adjusted height term demonstrated the best accuracy reported in pediatric studies to date, $91 \%$ and $45 \%$ within $30 \%$ and $10 \%$ of the true GFR, respectively; with a bias of only -0.2 and correlation of 0.92 .

\subsubsection{8: We suggest measuring GFR using an exogenous filtration marker under circumstances where more accurate ascertainment of GFR will impact on treatment decisions. (2B)}

\section{RATIONALE}

In clinical practice, there may be a requirement to measure GFR when the need for a 'truer' more precise value is identified (such as for organ donation or for dosing of toxic drugs). The intention of this statement is to recognize that specialty centers for kidney disease, usually tertiary referral centers, should have the capacity to measure GFR using exogenous filtration markers as a recognized specialist service. We recognize that this ability does not currently constitute the definition of specialty kidney referral centers and that it may be problematic, but resources to ensure accurate measurement ought to be made available. Given that these specific measurements require levels of rigor and reproducibility similar to those of laboratory calibration issues, specialist centers would be the right place to suggest that these facilities be made available.

\section{Evidence Base}

GFR is measured as the clearance of an exogenous filtration marker. The "gold standard" method is the urinary clearance of inulin during a continuous intravenous infusion. To simplify the procedure there are a number of alternative clearance methods and alternative filtration markers, with minor differences among them. ${ }^{79}$ For all measurement methods, measured GFR should be reported as described for eGFR.

Table 18 summarizes the strengths and limitations of clearance methods and filtration markers for clearance measurements. Thus measured GFR may also be associated with error, and in evaluation of GFR estimating equations, random error in GFR measurement is a source of some of the imprecision in GFR estimating equations. ${ }^{27,121}$ In principle, the magnitude of random error in GFR measurements is likely to be smaller than errors in GFR estimation using creatinine and cystatin $\mathrm{C}$ due to conditions listed in Tables 11 and 15.

\section{International Relevance}

The calculation of eGFR using these equations usually requires computer programming and some processes for quality monitoring. Nonetheless the statements are here to serve as 'best practice' recommendations so that these can be 


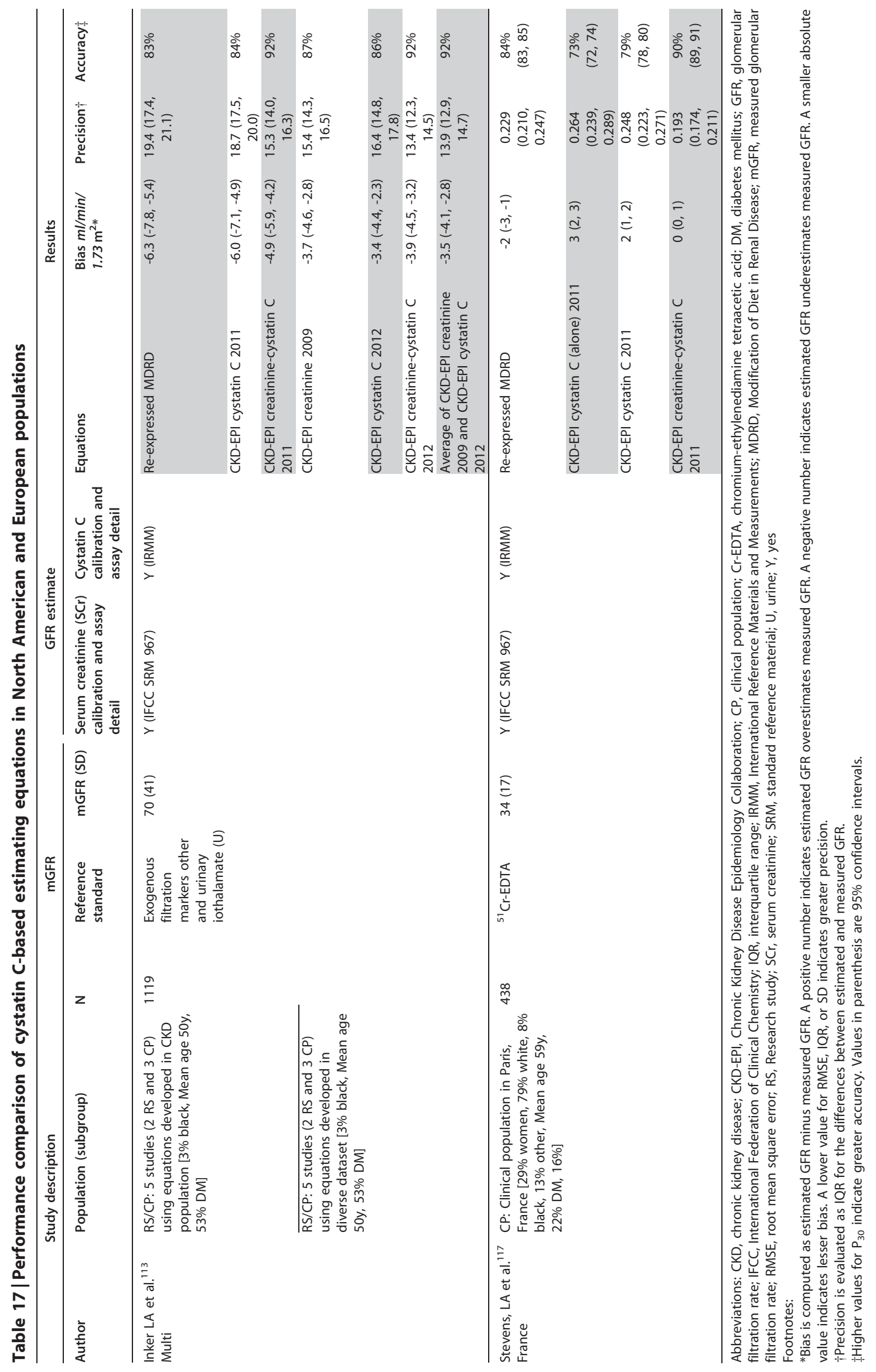


Table 18 |Strengths and limitations of GFR measurement methods and markers

\begin{tabular}{|c|c|c|}
\hline Approach & Strengths & Limitations \\
\hline \multicolumn{3}{|l|}{ Methods } \\
\hline \multicolumn{3}{|l|}{ Urinary clearance } \\
\hline $\begin{array}{l}\text { Bladder catheter and continuous } \\
\text { intravenous infusion of marker }\end{array}$ & - Gold standard method & - Invasive \\
\hline Spontaneous bladder emptying & $\begin{array}{l}\text { - Patient comfort } \\
\text { - Less invasive }\end{array}$ & $\begin{array}{l}\text { - Possibility of incomplete bladder emptying } \\
\text { - Low flow rates in people with low levels of GFR }\end{array}$ \\
\hline Bolus administration of marker & - Shorter duration & $\begin{array}{l}\text { - Rapidly declining plasma levels at high levels of GFR } \\
\text { - Longer equilibration time in extracellular volume expansion }\end{array}$ \\
\hline $24 \mathrm{~h}$ urinary collection & & $\begin{array}{l}\text { - Cumbersome } \\
\text { - Prone to error }\end{array}$ \\
\hline Plasma clearance & $\begin{array}{l}\text { - No urine collection required } \\
\text { - Potential for increased precision }\end{array}$ & $\begin{array}{l}\text { Overestimation of GFR in extracellular volume expansion } \\
\text { Inaccurate values with 1-sample technique, particularly at } \\
\text { lower GFR levels } \\
\text { - Longer duration of plasma sampling required for low GFR }\end{array}$ \\
\hline Nuclear imaging & $\begin{array}{l}\text { - No urine collection or repeated } \\
\text { blood samples required } \\
\text { - Relatively short duration }\end{array}$ & - Less accurate \\
\hline \multicolumn{3}{|l|}{ Markers } \\
\hline Inulin & $\begin{array}{l}\text { - Gold standard } \\
\text { - No side effects }\end{array}$ & $\begin{array}{l}\text { - Expensive } \\
\text { - Difficult to dissolve and maintain into solution } \\
\text { - Short supply }\end{array}$ \\
\hline Creatinine & $\begin{array}{l}\text { Endogenous marker, no need } \\
\text { for administration } \\
\text { Assay available in all clinical } \\
\text { laboratories }\end{array}$ & - Secretion can vary among and within individuals \\
\hline lothalamate & $\begin{array}{l}-\quad \text { Inexpensive } \\
\text { - Long half life }\end{array}$ & $\begin{array}{l}\text { - Probable tubular secretion } \\
\text { - Requirement for storage, administration, and disposal of } \\
\text { radioactive substances when }{ }^{125} \mathrm{I} \text { used as tracer } \\
\text { - Use of non-radioactive iothalamate requires expensive assay } \\
\text { - Cannot be used in patients with allergies to iodine }\end{array}$ \\
\hline lohexol & $\begin{array}{l}\text { - Not radioactive } \\
\text { - Inexpensive } \\
\text { - Sensitive assay allows for low dose }\end{array}$ & $\begin{array}{l}\text { - Possible tubular reabsorption or protein binding } \\
\text { - Use of low doses requires expensive assay } \\
\text { - Cannot be used in patients with allergies to iodine } \\
\text { - Nephrotoxicity and risk for allergic reactions at high doses }\end{array}$ \\
\hline EDTA & - Widely available in Europe & $\begin{array}{l}\text { - Probable tubular reabsorption } \\
\text { - Requirement for storage, administration, and disposal of } \\
\text { radioactive substances when }{ }^{51} \mathrm{Cr} \text { is used as tracer }\end{array}$ \\
\hline DTPA & $\begin{array}{l}\text { Widely available in the US } \\
\text { - New sensitive and easy to } \\
\text { use assay for gadolinium }\end{array}$ & $\begin{array}{l}\text { - Requirement for storage, administration, and disposal of } \\
\text { radioactive substances when }{ }^{99 \mathrm{~m}} \mathrm{Tc} \text { used as tracer } \\
\text { - Requires standardization for }{ }^{99 \mathrm{~m}} \mathrm{Tc} \\
\text { - Dissociation and protein binding of }{ }^{99 \mathrm{~m}} \mathrm{Tc} \\
\text { - Concern for NSF when gadolinium is used as the tracer }\end{array}$ \\
\hline
\end{tabular}

Abbreviations: DTPA, diethylenetriamine pentaacetic acid; EDTA, ethylenediamine tetraacetic acid; GFR, glomerular filtration rate; NSF, nephrogenic systemic fibrosis.

aspired to over time in those locations where these recommendations are currently not able to be implemented.

The Work Group appreciated that not all laboratories have capabilities to assay cystatin C. Different countries and regions will have different availabilities for measurement of GFR. The statement about GFR measurements mostly applies to countries with tertiary care services such as kidney transplantation and oncology.

\section{Implications for Clinical Practice and Public Policy}

It is important for clinicians to understand various methods for estimating and measuring kidney function and the situations in which specific methods may be superior in clinical decision making about treatment and referral.
Standardized assays and robust equations are important for epidemiological and planning purposes so that public policy can be informed by more accurate estimates of CKD, which may be possible with improved standardization of both assays and equations.

In different parts of the world, different assays are used and equations for estimating eGFR may differ. Thus, appreciating and understanding local standards is important for individual patients who may travel, and for comparative research across countries or regions.

In the event that a clinician requires measurement of GFR instead of an estimate, knowledge of these different tests and availability of them is important. Situations in which measurement would be required are likely quite infrequent 
but include donor evaluation in kidney transplantation and use of toxic drugs which have a narrow therapeutic range. We acknowledge that drug development and clinical observation programs may not define the various thresholds with sufficient granularity to require greater accuracy than is

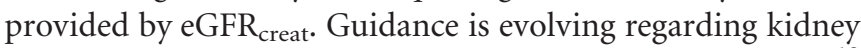
function evaluation during drug development programs. ${ }^{13}$ There are no direct implications for public policy for the statement about GFR measurement.

\section{Areas of Controversy, Confusion, or Non-consensus}

The Work Group recognizes that no single creatinine-based estimating equation will perform optimally in all clinical circumstances and that there may be changes in the performance of estimating equations over time and in different regions. However, for the purpose of eGFR reporting, it is important to select a single equation within a region or country. At the writing of this guideline, in North America, Europe, and Australia, the advantages of the CKD-EPI equation at higher GFR make it more applicable than the MDRD Study equation for general practice and public health.

While cystatin C offers some advantages over $\mathrm{SCr}$ as the basis of estimating equations, the cost of the assay and potential lack of standardization across laboratories for this 'newer' test limit our ability to recommend it as a preferred or even usual second test after creatinine. We recognize that these factors may lead to variations in implementation. The recommendation to consider confirmatory or additional testing if there is a need for more accurate determination of GFR is important. That there are other laboratory markers to estimate GFR (i.e., cystatin C) is stated here as there has been accumulating data to support its use in these situations. We have specifically mentioned cystatin $\mathrm{C}$ because of these data.

\section{Clarification of Issues and Key Points}

It is important for clinicians to appreciate the need for standardized assays and standardized equations for laboratory reporting of eGFR. Changes in laboratory assays or calculation methods should be reported to clinicians in order to avoid confusion when serially following individuals. This is because values in an individual might indicate a worsening or improvement in eGFR which may be attributable to different assays or calculation methods, rather than a reflection of true change.

When precise information about GFR is required, direct measurement using reliable methods should be pursued.

\section{Pediatric Considerations}

For Recommendation 1.4.3.8 this guideline is fully applicable in pediatrics.

\subsubsection{Evaluation of albuminuria}

1.4.4.1: We suggest using the following measurements for initial testing of proteinuria (in descending order of preference, in all cases an early morning urine sample is preferred) $(2 B)$ :

(1) urine albumin-to-creatinine ratio (ACR);

(2) urine protein-to-creatinine ratio (PCR);

(3) reagent strip urinalysis for total protein with automated reading;

(4) reagent strip urinalysis for total protein with manual reading.

1.4.4.2: We recommend that clinical laboratories report ACR and PCR in untimed urine samples in addition to albumin concentration or proteinuria concentrations rather than the concentrations alone. $(1 B)$

1.4.4.2.1: The term microalbuminuria should no longer be used by laboratories. (Not Graded)

1.4.4.3: Clinicians need to understand settings that may affect interpretation of measurements of albuminuria and order confirmatory tests as indicated (Not Graded):

- Confirm reagent strip positive albuminuria and proteinuria by quantitative laboratory measurement and express as a ratio to creatinine wherever possible.

- Confirm ACR $\geq 30 \mathrm{mg} / \mathrm{g}$ ( $\geq 3 \mathrm{mg} / \mathrm{mmol}$ ) on a random untimed urine with a subsequent early morning urine sample.

- If a more accurate estimate of albuminuria or total proteinuria is required, measure albumin excretion rate or total protein excretion rate in a timed urine sample.

\section{RATIONALE}

We recommend measurement of urinary albumin because it is relatively standardized and because it is the single most important protein lost in the urine in most chronic kidney diseases. Use of urinary albumin measurement as the preferred test for proteinuria detection will improve the sensitivity, quality, and consistency of approach to the early detection and management of kidney disease.

By contrast, laboratory tests purporting to measure urinary total protein are commonly flawed, often being standardized against, and predominantly sensitive to, albumin. They have poor precision at low concentrations and demonstrate poor between-laboratory agreement while being insensitive, non-specific, and susceptible to a range of falsepositive and false-negative problems. There may occasionally 


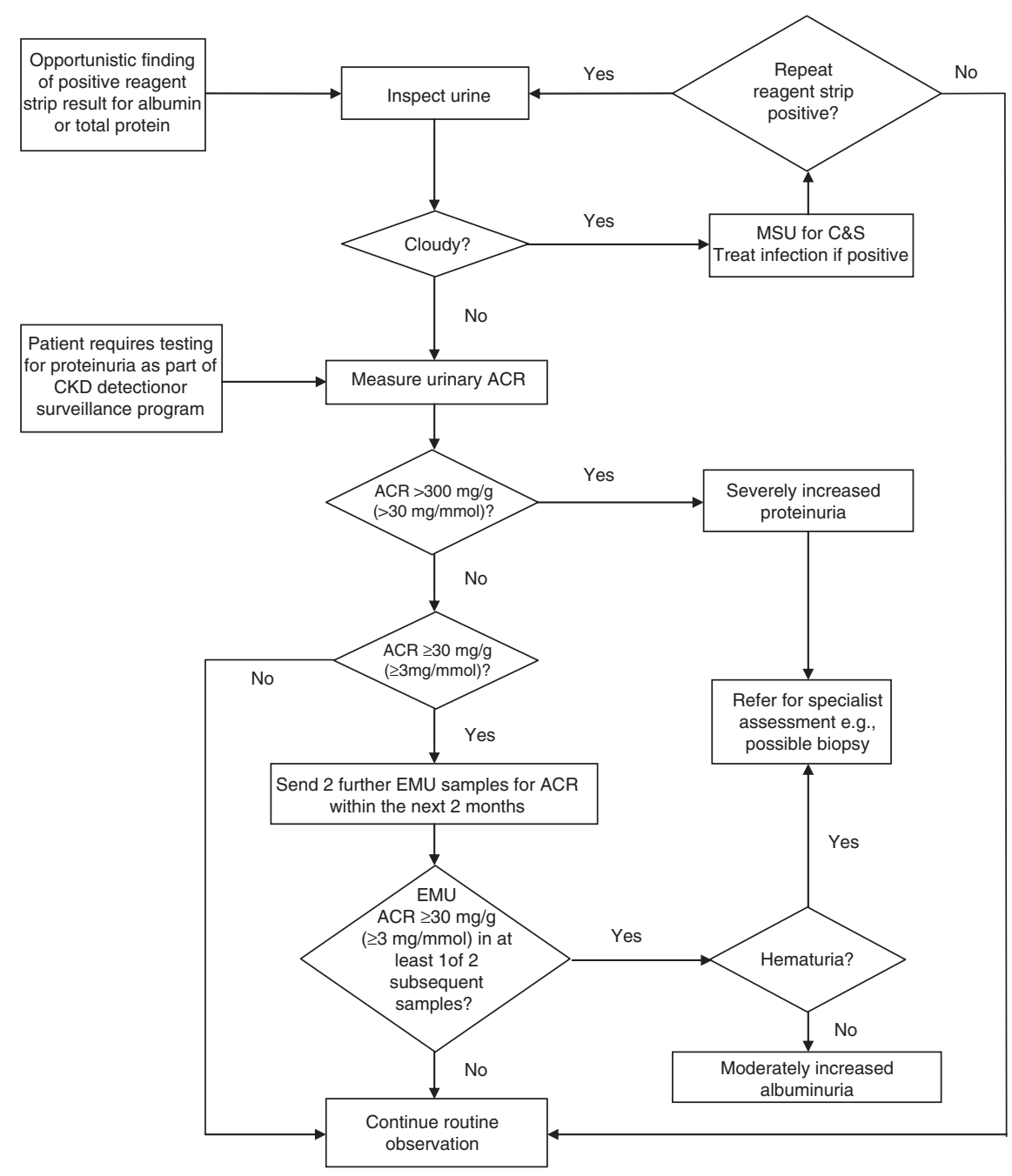

Figure 16|Suggested protocol for the further investigation of an individual demonstrating a positive reagent strip test for albuminuria/proteinuria or quantitative albuminuria/proteinuria test. Reagent strip device results should be confirmed using laboratory testing of the ACR on at least two further occasions. Patients with two or more positive ( $\geq 30 \mathrm{mg} / \mathrm{g}$ or $\geq 3 \mathrm{mg} / \mathrm{mmol}$ ) tests on early morning samples 1-2 weeks apart should be diagnosed as having persistent albuminuria. The possibility of postural proteinuria should be excluded by the examination of an EMU. PCR measurement can be substituted for the ACR but is insensitive in the detection of moderately increased albuminuria/proteinuria. Approximate PCR equivalent to an ACR of $30 \mathrm{mg} / \mathrm{mmol}$ is $50 \mathrm{mg} / \mathrm{mmol}$. ACR, albumin-tocreatinine ratio; $C \& S$, culture and sensitivity; CKD, chronic kidney disease; EMU, early morning urine; MSU, mid-stream urine; PCR, protein-tocreatinine ratio. ${ }^{a}$ Consider other causes of increased ACR (e.g., menstrual contamination, uncontrolled hypertension, symptomatic urinary tract infection, heart failure, other transitory illnesses, and strenuous exercise), especially in the case of type 1 diabetes present for less than 5 years. The presence of hematuria may indicate non-diabetic renal disease. This figure was published and adapted from Lamb EJ, Price CP. ${ }^{122}$ Kidney function tests, in Tietz Textbook of Clinical Chemistry and Molecular Diagnostics, eds Burtis CA, Ashwood E, Bruns DE, $5^{\text {th }}$ edition, pp 669-708, 2012. Copyright Elsevier.

be clinical reasons for a specialist to use PCR instead of ACR to quantify and monitor significant levels of proteinuria (e.g., in patients with monoclonal gammopathies).

Commonly used reagent strip devices measuring total protein are insufficiently sensitive for the reliable detection of proteinuria, do not adjust for urinary concentration, and are only semi-quantitative. Furthermore, there is no standardization between manufacturers. The use of such strips should be discouraged in favor of quantitative laboratory measurements of albuminuria or proteinuria. When used, reagent strip results should be confirmed by laboratory testing (Figure 16).

The combination of reagent strips with automated reader devices can improve inter-operator variability. More recently launched reagent strip devices capable of producing albumin or total protein results as a ratio to urinary creatinine require further evaluation to provide evidence that they have equivalent sensitivity and specificity to laboratory tests and are economically advantageous.

Although the reference point remains the accurately timed 24-hour specimen, it is widely accepted that this is a difficult procedure to control effectively and that inaccuracies in urinary collection may contribute to errors in estimation of protein losses. In practice, untimed urine samples are a reasonable first test for ascertainment of albuminuria. An EMU ('first pass') sample is preferred since it correlates well with 24-hour protein excretion, has relatively low 
intra-individual variability, and is required to exclude the diagnosis of orthostatic (postural) proteinuria. However, a random urine sample is acceptable if no EMU sample is available.

The concentration of protein or albumin in a urine sample will be affected by hydration (i.e., how diluted or concentrated a urine sample is). Creatinine excretion is considered to be fairly constant throughout the day and it has become customary to correct for urinary concentration by expressing either the protein or albumin concentrations as a ratio to the creatinine concentration in the same sample.

Timed urine collections may be used for confirmatory purposes but are not required except in circumstances in which untimed urine ACR is less accurate. It is worthwhile noting that albumin and protein excretion display considerable biological variability and may be increased by a variety of pathological and non-pathological factors. Consequently, confirmation of increased excretion rates is recommended.

\section{Evidence Base}

Why is albumin measurement being recommended instead of total protein? Urine albumin measurement provides a more specific and sensitive measure of changes in glomerular permeability than urinary total protein. ${ }^{123-125}$ There is substantial evidence linking increased albuminuria to outcomes of $\mathrm{CKD}^{4,30}$ (e.g., CKD Prognosis Consortium ${ }^{2-5}$, Nord-Trøndelag Health Study [HUNT 2] ${ }^{125 a}$, Prevention of Renal and Vascular Endstage Disease [PREVEND] ${ }^{125 b}$ ). There is also evidence that urinary albumin is a more sensitive test to enable detection of glomerular pathology associated with some other systemic diseases including diabetes, hypertension and systemic sclerosis. ${ }^{126-129}$

In health, relatively small amounts of albumin $(<30 \mathrm{mg} /$ 24 hours) are lost in the urine. Because of this, and additionally because total protein assays are imprecise and insensitive at low concentrations, relatively large increases in urine albumin excretion can occur without causing a significant measurable increase in urinary total protein. ${ }^{125}$

Total protein measurement is problematic in urine due to: large sample-to-sample variation in the amount and composition of proteins; high and variable concentrations of non-protein interfering substances relative to the protein concentration; and high inorganic ion content. All these factors affect the precision and accuracy of the various methods. Most laboratories currently use either turbidimetry or colorimetry ${ }^{130}$ to measure total protein and as with urine reagent strip analysis, these methods do not give equal analytical specificity and sensitivity for all proteins which can contribute to diverse estimates of proteinuria prevalence. ${ }^{131,132}$ Most methods tend to react more strongly with albumin than with globulin and other non-albumin proteins. $^{34,133-135}$ There are significant interferences causing falsely high results. ${ }^{136-138}$. There is no reference measurement procedure and no standardized reference material for urinary total protein listed by the JCTLM. The variety of methods and calibrants in use means that there is inevitably significant between-laboratory variation. ${ }^{139-141}$ Since a variable mixture of proteins is measured, it is difficult to define a standardized reference material.

\section{How should albumin be measured and reported?}

Albumin should be measured using immunological assays capable of specifically and precisely quantifying albumin at low concentrations and of producing quantitative results over the clinically relevant range. Currently urinary albumin is predominantly measured by diagnostic laboratories using turbidimetric assays. ${ }^{130}$ At present there is no reference measurement procedure or standardized reference material for urine albumin listed by the JCTLM, although the NKDEP and the International Federation of Clinical Chemistry and Laboratory Medicine have recently established a joint committee to address these issues. ${ }^{142,143}$ At present, most assays are standardized against a serum-based calibrant (CRM 470) distributed by the IRMM of the European Commission, as has previously been recommended by KDIGO. ${ }^{31}$

Albumin concentration should be reported as a ratio to urinary creatinine concentration $(\mathrm{mg} / \mathrm{mmol}$ or $\mathrm{mg} / \mathrm{g}$ ). ACR results should be expressed to one decimal place $(\mathrm{mg} / \mathrm{mmol})$ or whole numbers $(\mathrm{mg} / \mathrm{g})$. Both enzymatic and Jaffe assays are suitable for the measurement of creatinine in urine. We suggest that the term 'microalbuminuria' no longer be used because it can be misleading in suggesting that the albumin may be small or different in some way. The proposed albuminuria categories A1-3 are a more clinically meaningful way to express information about categories within the continuum of albumin excretion.

Reagent strip point-of-care testing devices capable of measuring low concentrations of albumin are also available producing both semi-quantitative and fully quantitative ACR results. Reasonable analytical ${ }^{144-147}$ and diagnostic performance has been demonstrated. ${ }^{148-150}$ While studies of these devices have been somewhat limited in size, they demonstrate their potential to play a significant role in the care pathway of patients suspected of having CKD.

\section{Why are reagent strip devices for protein measurement considered less accurate than laboratory measurement?} Reagent strip devices for proteinuria detection have been in use for more than 50 years. As discussed earlier, a positive reagent strip result is also associated with outcomes of CKD. Such devices have been used to support screening programs in some countries, ${ }^{151-153}$ although there appears to be no evidence supporting such screening of unselected populations. ${ }^{154}$

Although purporting to measure total protein, the reagent pad is most sensitive to albumin. ${ }^{155-157}$ There is evidence that strips from different manufacturers perform differently at the cutoff (' + ') concentration of $300 \mathrm{mg} / \mathrm{l}$ and degrees of 'plusness' between different manufacturers don't always correspond to the same nominal concentration of protein in urine. ${ }^{124}$ Concentrated urines may give a color change in the positive range of a reagent strip device even though protein loss remains normal and vice versa. False-positive results may occur if the urine is alkalinized (e.g., due to urinary tract 
infection) or in the presence of quaternary ammonium compounds that alter the $\mathrm{pH}$ of the urine. The performance of reagent strips is operator-dependent ${ }^{158}$ and affected by the presence of colored compounds such as bilirubin and certain drugs (e.g., ciprofloxacin, quinine, and chloroquine). ${ }^{159}$ Reagent strips cannot reliably distinguish between proteinuria categories ${ }^{124,157}$ and show relatively poor diagnostic accuracy for proteinuria detection. ${ }^{160,161}$ In the Australian Diabetes, Obesity and Lifestyle (AusDiab) study, a reagent strip reading of + or greater had $58 \%$ and $99 \%$ sensitivity for detecting ACR $\geq 30 \mathrm{mg} / \mathrm{g}$ ( $\geq 3 \mathrm{mg} / \mathrm{mmol}$ ) and $\geq 300 \mathrm{mg} /$ g $(\geq 30 \mathrm{mg} / \mathrm{mmol})$, respectively. $47 \%$ of individuals who tested + or greater had an ACR $\geq 30 \mathrm{mg} / \mathrm{g}(\geq 3 \mathrm{mg} / \mathrm{mmol})$ on laboratory testing. ${ }^{162}$

Automated devices capable of reading the color changes of reagent strips using reflectance spectrometry are available. These reduce inter-operator variability and improve diagnostic accuracy. ${ }^{150,158,163} \mathrm{~A}$ creatinine test pad has been added to some reagent strip systems to enable a PCR to be reported and so reduce the intra-individual variation seen with random urine collections. Such devices have been shown to be suitable for ruling out significant proteinuria ( $>300 \mathrm{mg} /$ 24 hours) in an outpatient setting. ${ }^{149}$

Correcting for urinary dilution. Since creatinine excretion in the urine is fairly constant throughout the 24-hour period, measurement of ACR (or PCR) allows correction for variations in urinary concentration. ${ }^{164,165} \mathrm{ACR}$ is a suitable alternative to timed measurement of urine albumin loss. ${ }^{143,166-170}$ PCR on random or early morning untimed samples shows good diagnostic performance and correlation with 24 -hour collection. ${ }^{160,163,171-177}$
Expressing albumin as a ratio to creatinine reduces intraindividual variability: lowest variability for the ACR has been reported in EMU samples as opposed to other untimed samples or timed collections. ${ }^{142,178}$ In one study albumin variability was reduced from $80 \%$ to $52 \%$ when expressed as an ACR rather than an albumin concentration. ${ }^{179}$ The within-subject biological variation for urinary ACR in an EMU has been reported to be $31 \%$, compared to $36 \%$ for urinary albumin concentration. ${ }^{180}$ The same study reported variability for ACR of $103 \%$ and $85 \%$ in random and timed 24-hour collections, respectively. ${ }^{180}$ Intra-individual variation for protein loss is also significantly reduced when reported as a PCR compared to protein concentration in random urine samples collected throughout the day (a mean reduction from $97 \%$ to $39 \%) .{ }^{179}$

Why and how should a finding of albuminuria be confirmed? Given the high biological variation and other pathological and physiological causes of albuminuria (Table 19), ${ }^{143}$ repeat testing to confirm albuminuria, ideally using an EMU and laboratory testing, is recommended (Figure 16).

There has been extensive discussion in the literature about the appropriate urine sample to use for the investigation of protein loss. It is generally recognized that a 24 -hour sample is the definitive means of demonstrating the presence of proteinuria. However, overnight, first void in the morning (i.e., EMU), second void in the morning, or random sample collections can also be used. In a systematic review random urine PCR was shown to have better performance as a test for ruling out significant proteinuria than as a "rule-in" test; the authors suggested that positive PCR results may still require

Table 19|Factors affecting urinary ACR

\begin{tabular}{|c|c|}
\hline Factor & Examples of effect \\
\hline \multicolumn{2}{|l|}{ Preanalytical factors } \\
\hline Transient elevation in albuminuria & $\begin{array}{l}\text { Menstrual blood contamination } \\
\text { Symptomatic UTI } \\
\text { Exercise }^{182} \\
\text { Upright posture (orthostatic proteinuria) } \\
\text { Other conditions increasing vascular permeability (e.g., septicemia) }\end{array}$ \\
\hline Intraindividual variability & $\begin{array}{l}\text { Intrinsic biological variability }{ }^{180} \\
\text { Genetic variability }^{184}\end{array}$ \\
\hline Preanalytical storage conditions & Degradation of albumin before analysis ${ }^{a}$ \\
\hline $\begin{array}{l}\text { Non-renal causes of variability in creatinine } \\
\text { excretion }\end{array}$ & $\begin{array}{l}\text { Age (lower in children and older people) } \\
\text { Race (lower in Caucasian than black people) } \\
\text { Muscle mass (e.g., lower in people with amputations, paraplegia, muscular dystrophy) } \\
\text { Gender (lower in women) }\end{array}$ \\
\hline
\end{tabular}

Analytical factors

Antigen excess ('prozone') effect

Samples with very high albumin concentrations may be falsely reported as low or normal using some assays $^{124}$

Abbreviations: ACR, albumin-to-creatinine ratio; AKI, acute kidney injury; UTI, urinary tract infection.

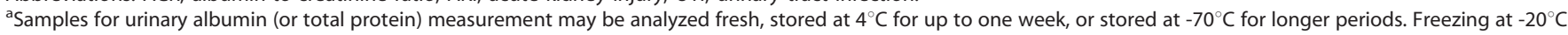
appears to result in loss of measurable albumin and is not recommended. When analyzing stored samples, they should be allowed to reach room temperature and thoroughly mixed prior to analysis. ${ }^{142}$ 
confirmation with a 24-hour collection. ${ }^{185}$ If an EMU is unavailable, subsequent samples can give a reliable indication of the 24-hour urine protein loss. ${ }^{174}$

\section{International Relevance}

The recommendation to replace urinary total protein with albumin as the test of choice in testing for proteinuria is consistent with most, ${ }^{1,31,130,186,187}$ but not all, ${ }^{188,189}$ current national and international guidance. It is accepted that cost pressures may affect implementation of this recommendation and may differ across the world.

Most international guidelines have also discouraged the use of reagent strip analysis for proteinuria detection. ${ }^{186,189-191}$ Nevertheless, in the present guideline we acknowledge that these devices may have a role, particularly in settings where access to laboratory services may be limited.

ACRs in North America tend to be reported in $\mathrm{mg} / \mathrm{g}$ whereas in other parts of the world usage of $\mathrm{mg} / \mathrm{mmol}$ predominates. This difference appears unlikely to be resolved in the foreseeable future. When publishing data authors should ensure either that both units are cited or that a conversion factor is provided.

There is increasing adoption of the term 'albuminuria' instead of microalbuminuria by international and national laboratory and some clinical organizations.

\section{Implications for Clinical Practice and Public Policy}

Direct reagent costs of total protein measurement are generally lower than those of albumin measurement, which requires antibody-based reagents. It is often considered that reagent strip analyses are a cheaper option. Therefore some health-care systems may struggle to justify the recommendations in this guideline.

Costs of diagnostic tests vary depending on local financial agreements between hospitals and suppliers. In England, the National Institute for Health and Clinical Excellence (NICE) sampled a small random number of laboratories and estimated the average cost of an ACR to be $£ 2.16$ whereas a PCR cost $£ 1.42 .{ }^{186}$ It is acknowledged that increased use of ACR testing may reduce the unit cost on the basis of economies of scale. In Canada, laboratory analysis costs (Canadian dollars) of $\$ 2.81$ for reagent strip, $\$ 11.67$ for PCR, and \$29.23 for ACR have been cited. ${ }^{192}$ In relation to albumin-specific reagent strips, a cost of approximately $\$ 4$ for a Micral test II (Roche Diagnostics) compared to $\$ 2$ for a laboratory ACR has been reported. ${ }^{193}$

The cost- and clinical-effectiveness of an approach utilizing reagent strip testing followed by laboratory measurement compared to an approach in which samples are submitted directly to the laboratory (for either albumin or protein measurement) has recently been evaluated in a health economics model. ${ }^{186}$ The model favored abandoning the use of reagent strips for identification of proteinuria.

\section{Areas of Controversy, Confusion, or Non-consensus}

Some data suggest that ACR is a poorer predictor of 24-hour total protein loss than $\mathrm{PCR}^{194}$ and has no advantage over
PCR as a predictor of renal outcomes and mortality in patients with CKD. ${ }^{195,196}$ In the prediction of future transplant rejection, PCR has been reported to have equal utility to ACR, ${ }^{192}$ although in a separate study ACR was found to be a better predictor. ${ }^{197}$

In the setting of preeclampsia, proteinuria is generally defined as $\geq 300 \mathrm{mg} / 24$ hours or a PCR $\geq 300 \mathrm{mg} / \mathrm{g}$ ( $\geq 30 \mathrm{mg} / \mathrm{mmol}) .{ }^{175}$ Currently, there is insufficient evidence to substitute urine albumin measurement for total protein in this setting. ${ }^{172}$

Creatinine excretion is affected by a variety of non-renal influences (Table 19) and it therefore follows that different cutoffs for ACR (and PCR) may be required in different individuals. ${ }^{194,198}$ While age-related cutoffs have not generally been applied in clinical practice, clinicians should bear this in mind when interpreting urine ACR data in older individuals or those with very low body mass, as these will impact the urine creatinine excretion.

While most guidelines agree that an ACR greater than approximately $3 \mathrm{mg} / \mathrm{mmol}(30 \mathrm{mg} / \mathrm{g})$ is pathological in the setting of diabetes, in the non-diabetic population a higher threshold has commonly been used to define proteinuria. In the NICE guideline in England and Wales, proteinuria in nondiabetic individuals was defined as $\geq 30 \mathrm{mg} / \mathrm{mmol}(\geq 300 \mathrm{mg} / \mathrm{g})$, with higher level proteinuria being $>70 \mathrm{mg} / \mathrm{mmol}(>700 \mathrm{mg} /$ g). ${ }^{186}$ Confirmation of results lying between 30 and $70 \mathrm{mg} / \mathrm{mmol}$ $(300-700 \mathrm{mg} / \mathrm{g})$ was recommended. ${ }^{186}$ The present guideline proposes a lower threshold definition for albuminuria for use in both diabetic and non-diabetic individuals.

A study from Italy in type 2 diabetes has reported that, although intra-individual biological variation of albuminuria is large, a single sample (either ACR or timed collection) can accurately classify patients into albuminuria categories, negating the need for multiple collections. ${ }^{178}$

Some data suggest that a significant proportion of albumin present in urine may be non-immunoreactive, ${ }^{199-202}$ although this finding has been questioned. ${ }^{203,204}$

There is a substantial existing literature using the term microalbuminuria and many existing guidelines use this term especially in the context of diabetes and cardiovascular risk, as its presence confers risk. Nonetheless, the Work Group believes that it is important for this international guideline to foster 'best practices' and clarity of communication, and since the risk of adverse events is continuous throughout the spectrum of albuminuria, we encourage adoption of the term 'albuminuria' with subsequent quantification of the level or amount.

\section{Pediatric Considerations}

For Recommendation 1.4.4.1, this set of statements would need to be altered for application in the pediatric practice as follows:

We suggest using the following measurements for initial testing of proteinuria in children (in descending order of preference):

(1) urine PCR, EMU sample preferred;

(2) urine ACR, EMU sample preferred; 
(3) reagent strip urinalysis for total protein with automated reading;

(4) reagent strip urinalysis for total protein with manual reading.

For Recommendations 1.4.4.2 and 1.4.4.3, this set of statements would need to be altered for application in the pediatric practice as follows:

Currently the urinary PCR should be favored over the urine ACR in children. Unlike in adults where powerful evidence exists in support of the use of measures of albumin rather than total protein to predict adverse outcomes, this level of evidence is currently lacking in children. ${ }^{205}$ However, current longitudinal trials such as $\mathrm{CKiD}^{55}$ and European $4 \mathrm{C}^{78}$ may eventually shed light on this issue.

In children the underlying conditions associated with the diagnosis of CKD are also important considerations as to which form of testing is most valuable. Unlike adults where the majority of patients with CKD are attributed to an underlying glomerular disease or hypertensive damage, the vast majority of children have underlying developmental abnormalities often referred to as CAKUT (congenital anomalies of the kidney and urinary tract). ${ }^{70}$ This relative paucity of glomerular conditions makes the use of albumin excretion a less sensitive test for diagnostic purposes as many children will have underlying tubular conditions and hence tend to excrete more Tamm-Horsfall protein and other lowmolecular-weight proteins that will not be captured by the albumin-to-creatinine (or formal albumin excretion) assay.

For Recommendations 1.4.4.2 and 1.4.4.2.1 this guideline is fully applicable in pediatrics. The recommendation that clinical laboratories report ACR and PCR in untimed urine samples in addition to albumin concentration or proteinuria concentrations rather than the concentrations alone is valid and useful in the pediatric population. As per Recommendation 1.2.4, however, note should be made that age-related normal values for urinary protein losses must be considered when laboratories choose to report either ACR or PCR.

Albuminuria in children, whether measured as an absolute value per day, an excretion rate, or as an albumin to creatinine ratio is fraught with more uncertainity than in adults as they are known to vary across categories of age, sex, height, weight, and Tanner staging. ${ }^{206}$

In two recent reviews by Rademacher ${ }^{206}$ and Tsioufis et al., ${ }^{205}$ both groups examined the results of all relevant studies on normative values of AER or ACR. Rademacher's paper in particular provides detailed information on the mean AER values (with $\mathrm{SD}$ ) across a variety of studies, ages, sex, and race, and provides a normative estimate for overnight AER of between $2-6 \mu \mathrm{g} /$ minute or a $95^{\text {th }}$ percentile value from $4.5-28 \mu \mathrm{g} /$ minute. Similarly, they summarize results for ACR in normal children and suggest that the mean for children older than 6 years would seem to fall between $8-10 \mathrm{mg} / \mathrm{g}(0.8-1.0 \mathrm{mg} / \mathrm{mmol})$.

For Recommendation 1.4.4.3, this guideline is fully applicable in pediatrics.
1.4.4.4: If significant non-albumin proteinuria is suspected, use assays for specific urine proteins (e.g., $\alpha_{1}$-microglobulin, monoclonal heavy or light chains, [known in some countries as "Bence Jones"proteins]). (Not Graded)

\section{RATIONALE}

Testing for tubular proteinuria using a total protein approach almost certainly has very poor sensitivity for detecting tubular disease. When an isolated tubular lesion is suspected (Table 3 ), this is probably best investigated by measuring a specific tubular protein (e.g., $\alpha_{1}$-microglobulin) using an immunoassay approach.

\section{Evidence Base}

There have been concerns that replacing urinary total protein measurement with albumin measurement may cause nonalbuminuric (effectively tubular and overproduction) proteinuria to be missed. Low-molecular-weight proteinuria is a defining feature in some uncommon kidney diseases (e.g., Dent's disease). ${ }^{207}$ However, for some of the reasons already discussed, total protein assays will also be poor at detecting tubular proteinuria. When investigating patients for tubular proteinuria, it is advisable to use assays targeted at specific tubular proteins.

In the AusDiab study, of those with proteinuria $(2.4 \%$ of the general population, defined as a PCR $>23 \mathrm{mg} / \mathrm{mmol}[230 \mathrm{mg} /$ g]) $92 \%$ had albuminuria (defined as an ACR $>3.4 \mathrm{mg} / \mathrm{mmol}$ [34 mg/g]); $8 \%$ had an ACR within the reference range. ${ }^{208}$ These individuals were less likely to have diabetes than those with both proteinuria and albuminuria, but no further information is available as to the nature of the proteinuria in these individuals or its likely significance. The authors speculate that these individuals could have had light chain proteinuria or interstitial nephropathies. Using albuminuria testing to identify proteinuria had a specificity of $95 \%$. The negative predictive value was $99.8 \%$ and the positive predictive value was $32.4 \%$. The authors concluded that testing for albuminuria rather than proteinuria was supported.

As discussed above, quite significant increases in urinary albumin loss have to occur before such an increase is detectable on the background of a total protein assay. The situation is even more extreme for tubular proteins which, in health, are present in urine at lower concentrations than albumin (e.g., normal daily losses of retinol binding protein, $\alpha_{1}$-microglobulin and $\beta_{2}$-microglobulin are $0.08,3.6$, and $0.1 \mathrm{mg} / \mathrm{d}$, respectively). ${ }^{209}$ This problem will be exacerbated by the fact that the recognition of tubular proteins by some total protein assays is poor. ${ }^{210}$

In disease states concentrations of tubular proteins, at least collectively, can reach levels detectable by total protein assays. For example, among patients with tubulointerstitial disease but without renal insufficiency, median concentrations of $\alpha_{1}$ microglobulin were $37 \mathrm{mg} / \mathrm{l}$, with concentrations up to $100 \mathrm{mg} / \mathrm{l}$ being observed; higher concentrations were seen in patients with decreased GFR. ${ }^{211}$ Among a group of patients with acute 
tubular necrosis requiring dialysis treatment, median $\alpha_{1}$ microglobulin concentration was $35 \mathrm{mg} / \mathrm{mmol}$ of creatinine. ${ }^{212}$ However, although tubular proteinuria is characterized by a relative increase in low-molecular-weight protein concentrations, generally albumin still remains a significant component of the total protein concentration. Indeed, it is thought that tubular disease results in an increase in albumin loss as a result of decreased tubular reabsorption of filtered albumin. For example, it has been estimated that when tubular absorption fails completely, $\beta_{2}$-microglobulin loss increases to $180 \mathrm{mg} / 24$ hours (approximately 1800-fold normal) but there will also be an increase in urinary albumin loss to about $360 \mathrm{mg} / 24$ hours (approximately 20 -fold normal). ${ }^{209}$ In a series of patients with Dent's disease, a classical tubular disorder, 21 of the 23 patients demonstrating increased urinary $\alpha_{1}$-microglobulin and $\beta_{2^{-}}$ microglobulin loss also had increased urinary albumin loss: those who did not had borderline increases in tubular protein losses that would not have been detectable using a total protein measurement approach. ${ }^{207}$ The authors comment that in those patients in whom proteinuria was marked $(>1 \mathrm{~g} / \mathrm{d})$, urinary albumin loss was also markedly increased. In some situations, however, tubular proteinuria in the absence of albuminuria has been reported (e.g., in some children with type 1 diabetes $^{213}$ and in kidney scarring in reflux nephropathy ${ }^{214}$ ).

\section{International Relevance}

There is no reason to believe that there are significant differences around the world with respect to incidence or prevalence of conditions in which measurement of nonalbumin proteins would be required. The availability of reliable tests for these alternative proteins, however, may be different in different regions.

\section{Implications for Clinical Practice and Public Policy}

The incidence and prevalence of tubular disorders will vary geographically with the clinical setting (e.g., adult or pediatric practice) and factors such as occupational exposure. Clinicians should agree with their local laboratories a suitable approach to the detection of tubular proteinuria and laboratories should be able to advise on suitable sample handling procedures. It is acknowledged that many laboratories do not currently offer assays of tubular proteins.

In patients with suspected myeloma, monoclonal heavy or light chains (known in some countries as Bence Jones) protein should be sought in concentrated urine using electrophoresis with immunofixation of any identified protein bands in accordance with current myeloma guidelines. ${ }^{215}$ Simultaneous albumin measurement is needed when the possibility of immunoglobulin light chain (AL) amyloid or light chain deposition disease is suspected.

Non-albumin proteinuria may also be suspected in patients with disorders of tubular function (see Table 3).

\section{Areas of Controversy, Confusion, or Non-consensus}

Testing for proteinuria using a urine albumin rather than total protein first-line approach may occasionally miss cases of tubular proteinuria but the significance of this problem is probably overestimated and should be the subject of further research.

Earlier guidance from KDOQI ${ }^{1}$ suggested that proteinuria in children should be detected with total protein rather than albumin assays due to the higher prevalence of nonglomerular diseases in this group of patients. For the reasons outlined above, we do not think total protein assays are suitable for this purpose and would ideally recommend testing for albumin and for specific tubular proteins when non-glomerular disease is suspected.

\section{Pediatric Considerations}

For Recommendation 1.4.4.4, this statement is fully applicable in pediatrics. In children the likelihood of any form of overflow proteinuria such as seen in conditions of heavy or light chain production is extremely low; however a significant number of underlying genetic tubular disorders do exist and protein electrophoresis can assist the practitioner in determining the presence of such a condition or the concurrent finding of severe tubular injury in addition to a glomerular condition.

\section{DISCLAIMER}

While every effort is made by the publishers, editorial board, and ISN to see that no inaccurate or misleading data, opinion or statement appears in this Journal, they wish to make it clear that the data and opinions appearing in the articles and advertisements herein are the responsibility of the contributor, copyright holder, or advertiser concerned. Accordingly, the publishers and the ISN, the editorial board and their respective employers, office and agents accept no liability whatsoever for the consequences of any such inaccurate or misleading data, opinion or statement. While every effort is made to ensure that drug doses and other quantities are presented accurately, readers are advised that new methods and techniques involving drug usage, and described within this Journal, should only be followed in conjunction with the drug manufacturer's own published literature.

\section{SUPPLEMENTARY MATERIAL}

Supplemental Table 1: Search strategy.

Supplemental Table 2: Equations based on serum creatinine assays in adults that are not traceable to the standard reference material.

Supplemental Table 3: Equations based on serum cystatin $C$ assays in adults that are not traceable to standard reference material.

Supplementary material is linked to the online version of the paper at http://www.kdigo.org/clinical_practice_guidelines/ckd.php 


\section{Chapter 2: Definition, identification, and prediction of CKD progression}

Kidney International Supplements (2013) 3, 63-72; doi:10.1038/kisup.2012.65

\section{1: DEFINITION AND IDENTIFICATION OF CKD PROGRESSION}

2.1.1: Assess GFR and albuminuria at least annually in people with CKD. Assess GFR and albuminuria more often for individuals at higher risk of progression, and/ or where measurement will impact therapeutic decisions (Figure 17). (Not Graded)

\section{RATIONALE}

The statement is worded this way to remind the practitioner to use both GFR and albuminuria in order to assess progression and is consistent with the definition offered in Chapter 1 regarding definitions of CKD which include both parameters. There is increasing evidence which supports that both parameters are valuable. Lower GFR and greater albuminuria are both associated with an increased rate of progression and are synergistic.

More frequent measures of eGFR and albuminuria should be considered in patients with a lower GFR and greater albuminuria as these people are more likely to progress. Frequency of measurement should also be individualized based on the patient history and underlying cause of kidney disease.

In specific conditions (e.g., GN or increased levels of albuminuria), frequent (every 1-3 months) assessment may guide therapeutic decisions. Regular monitoring of stable patients may include more frequent monitoring than annually, but will be dictated by underlying cause, history, and estimates of GFR and ACR values obtained previously.

\section{Evidence Base}

There is variability in the presence of or rate of decline of kidney function in those with CKD. The rate at which

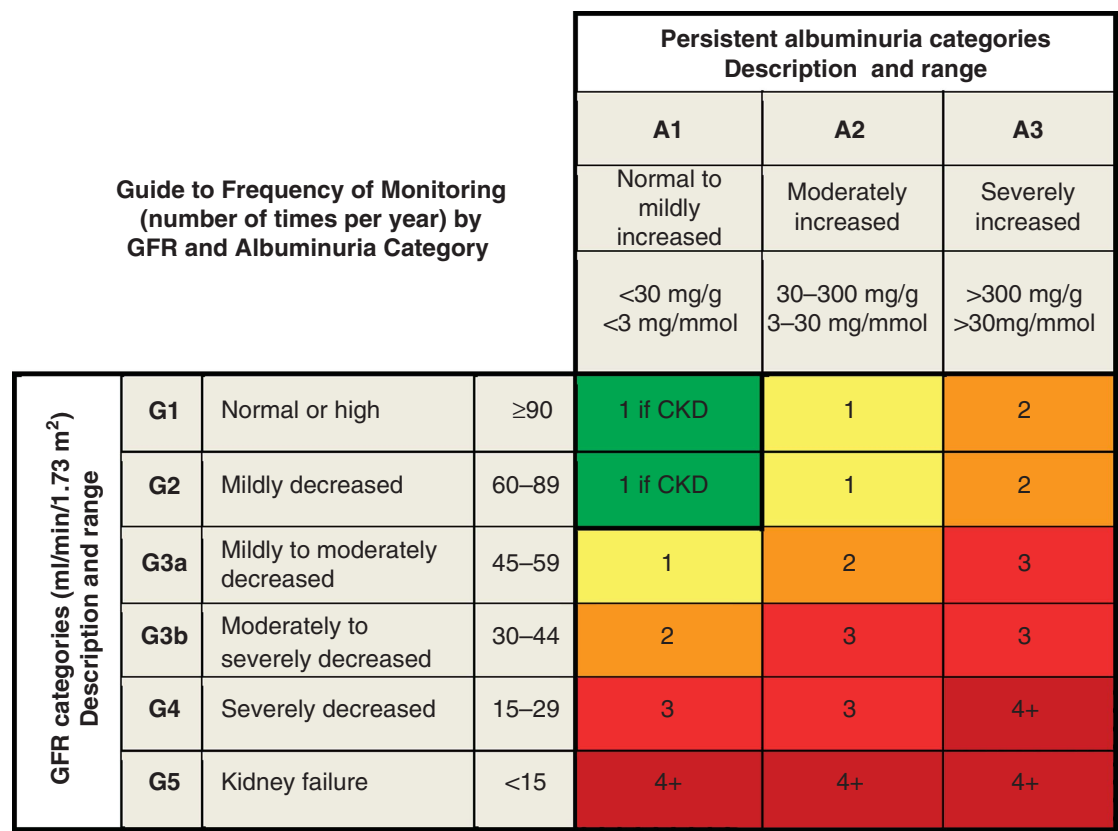

Figure $17 \mid$ GFR and albuminuria grid to reflect the risk of progression by intensity of coloring (green, yellow, orange, red, deep red). The numbers in the boxes are a guide to the frequency of monitoring (number of times per year). Green reflects stable disease, with follow-up measurements annually if CKD is present; yellow requires caution and measurements at least once per year; orange requires measurements twice per year; red requires measurements at 3 times per year while deep red may require closest monitoring approximately 4 times or more per year (at least every 1-3 months). These are general parameters only based on expert opinion and must take into account underlying comorbid conditions and disease state, as well as the likelihood of impacting a change in management for any individual patient. CKD, chronic kidney disease; GFR, glomerular filtration rate. Modified with permission from Macmillan Publishers Ltd: Kidney International. Levey AS, de Jong PE, Coresh J, et al. ${ }^{30}$ The definition, classification, and prognosis of chronic kidney disease: a KDIGO controversies conference report. Kidney Int 2011; 80: 17-28; accessed http://www.nature.com/ki/journal/v80/n1/full/ki2010483a.html 
Table 20|Decline in kidney function in various populations (longitudinal studies only)

\begin{tabular}{|c|c|c|c|}
\hline Reference & Population & $N$ & GFR decline \\
\hline & Healthy & & \\
\hline Slack TK & Healthy kidney donors & 141 & $0.40 \mathrm{ml} / \mathrm{min} /$ year \\
\hline Rowe JW et al. ${ }^{217}$ & Healthy males & 293 & $0.90 \mathrm{ml} / \mathrm{min} / 1.73 \mathrm{~m}^{2} /$ year $(\mathrm{CrCl})$ \\
\hline Lindeman $\mathrm{RD}^{218}$ & Healthy males & 254 & $0.75 \mathrm{ml} / \mathrm{min} /$ year $(\mathrm{CrCl})$ \\
\hline Halbesma $\mathrm{N}$ et al. 219 & PREVEND cohort (all participants) & 6894 & $0.55 \mathrm{ml} / \mathrm{min} / 1.73 \mathrm{~m}^{2} /$ year \\
\hline Imai E et al. ${ }^{220}$ & Annual health exam participants in Japan & 120,727 & $0.36 \mathrm{ml} / \mathrm{min} / 1.73 \mathrm{~m}^{2} /$ year \\
\hline Matsuchita $\mathrm{K}$ et al. $^{221}$ & Atherosclerosis Risk In Communities Cohort & 13,029 & 0.47\%/year (median) \\
\hline \multirow[t]{2}{*}{ Kronborg J et al. ${ }^{222}$} & Healthy adults from Norway & 4441 & $\begin{array}{l}1.21 \mathrm{ml} / \mathrm{min} / 1.73 \mathrm{~m}^{2} / \text { year (men) } \\
1.19 \mathrm{ml} / \mathrm{min} / 1.73 \mathrm{~m}^{2} / \text { year (women) }\end{array}$ \\
\hline & With comorbidity & & \\
\hline Lindeman $\mathrm{RD}^{218}$ & Males with renal/urinary tract disease & 118 & $1.10 \mathrm{ml} / \mathrm{min} /$ year $(\mathrm{CrCl})$ \\
\hline Lindeman $\mathrm{RD}^{218}$ & Males with hypertension & 74 & $0.92 \mathrm{ml} / \mathrm{min} /$ year $(\mathrm{CrCl})$ \\
\hline Halbesma $\mathrm{N}$ et al. ${ }^{219}$ & PREVEND cohort - adults with macroalbuminuria ( $>300 \mathrm{mg} / 24$ hours) & 86 & $1.71 \mathrm{ml} / \mathrm{min} / 1.73 \mathrm{~m}^{2} /$ year \\
\hline Halbesma $\mathrm{N}$ et al. ${ }^{219}$ & $\begin{array}{l}\text { PREVEND cohort - Adults with impaired renal function } \\
\text { (5\% lowest } \mathrm{CrCl} / \mathrm{MDRD} \text { GFR) }\end{array}$ & 68 & $0.05 \mathrm{ml} / \mathrm{min} / 1.73 \mathrm{~m}^{2} /$ year \\
\hline Imai $E$ et al. ${ }^{220}$ & Annual health exam participants in Japan with hypertension & 16,722 & 0.3 to $0.5 \mathrm{ml} / \mathrm{min} / 1.73 \mathrm{~m}^{2} /$ year \\
\hline Imai E et al. ${ }^{220}$ & $\begin{array}{l}\text { Annual health exam participants in Japan with proteinuria } \\
(\geqslant 1+\text { dipstick proteinuria) }\end{array}$ & 2054 & 0.6 to $0.9 \mathrm{ml} / \mathrm{min} / 1.73 \mathrm{~m}^{2} /$ year \\
\hline
\end{tabular}

\begin{tabular}{|c|c|c|c|}
\hline \multirow{2}{*}{ Hemmelgarn B et al. ${ }^{223}$} & \multicolumn{3}{|l|}{ Older adults } \\
\hline & Males age $>65$ with diabetes & 490 & $2.7 \mathrm{ml} / \mathrm{min} / 1.73 \mathrm{~m}^{2} /$ year \\
\hline Hemmelgarn B et al. ${ }^{223}$ & Males age $>65$ without diabetes & 2475 & $1.4 \mathrm{ml} / \mathrm{min} / 1.73 \mathrm{~m}^{2} /$ year \\
\hline Hemmelgarn B et al. $^{223}$ & Females age $>65$ with diabetes & 445 & $2.1 \mathrm{ml} / \mathrm{min} / 1.73 \mathrm{~m}^{2} /$ year \\
\hline Hemmelgarn B et al. ${ }^{223}$ & Females age $>65$ without diabetes & 3163 & $0.8 \mathrm{ml} / \mathrm{min} / 1.73 \mathrm{~m}^{2} /$ year \\
\hline Keller C et al. ${ }^{224}$ & Cardiovascular Health Study & 4128 & $\begin{array}{l}1.83 \mathrm{ml} / \mathrm{min} / 1.73 \mathrm{~m}^{2} / \text { year (based on } \\
\text { cystatin C-based eGFR) }\end{array}$ \\
\hline
\end{tabular}

Abbreviations: $\mathrm{CrCl}$, creatinine clearance; GFR, glomerular filtration rate; MDRD, Modification of Diet in Renal Disease; PREVEND, Prevention of Renal and Vascular End-Stage Disease.

this decline occurs also varies based on the underlying population, cause of CKD, presence of albuminuria/proteinuria, comorbidities and age. The Work Group searched the literature for longitudinal studies that evaluated decline in kidney function. As outlined in Table 20 the study populations included healthy adults, those with comorbidity, as well as a subgroup of adults aged 65 and older.

Data from the PREVEND study, a prospective, population-based cohort study, provides important information on decline in kidney function at the population level. ${ }^{219}$ The PREVEND study evaluated 6894 people over a 4 -year period and reported loss in eGFR of $2.3 \mathrm{ml} / \mathrm{min} / 1.73 \mathrm{~m}^{2} /$ 4 years in the whole population, $7.2 \mathrm{ml} / \mathrm{min} / 1.73 \mathrm{~m}^{2} / 4$ years in participants with macroalbuminuria ( $>300 \mathrm{mg} / 24$ hours) and $0.2 \mathrm{ml} / \mathrm{min} / 1.73 \mathrm{~m}^{2} / 4$ year in participants with impaired renal function. The yearly decline in eGFR among a Japanese general population over 10 years was slightly lower at $0.36 \mathrm{ml} / \mathrm{min} / 1.73 \mathrm{~m}^{2} /$ year. $^{220}$ The rate of eGFR decline was approximately two times higher in participants with proteinuria, and about 1.5 times higher among participants with hypertension. ${ }^{220}$ Among adults aged 65 and older, progression (median follow-up 2 years) varied by sex and presence of diabetes. ${ }^{223}$ In general these studies suggest progression rates of approximately 0.3 to $1 \mathrm{ml} / \mathrm{min} / 1.73 \mathrm{~m}^{2}$ / year among participants without proteinuria or comorbidity and rates of approximately two to three times higher among participants with proteinuria or comorbidity. The somewhat surprising finding that eGFR had low rates of progression among the group with impaired renal function at baseline ${ }^{219}$ has been shown in other studies ${ }^{225}$ and may relate to the statistical phenomenon of regression to the mean. There is also a concern that it is hard to maintain consistent calibration of the SCr assay over time and progression results are highly sensitive to drift in the creatinine assay.

Studies evaluating rate of decline in eGFR among populations with CKD specifically are outlined in Table 21 and typically demonstrate a slightly more rapid rate of decline in this subgroup, thus requiring more frequent assessment of GFR and albuminuria.

Data from the MDRD Study during a mean 2.2 year follow-up showed that the average rate of decline in GFR ranged from 2.3 to $4.5 \mathrm{ml} / \mathrm{min} /$ year, depending on the baseline GFR and protein/MAP treatment assignments. ${ }^{227}$ Similarly, a more recent study of 4231 patients with GFR categories G3a-G5 (GFR $\left.<60 \mathrm{ml} / \mathrm{min} / 1.73 \mathrm{~m}^{2}\right)$ referred to a nephrologist showed a mean decline in GFR of $2.65 \mathrm{ml} / \mathrm{min} /$ $1.73 \mathrm{~m}^{2} /$ year, ${ }^{231}$ and variability in the rates of decline in this referred cohort.

Note that there have been no studies which evaluate the utility of more or less frequent monitoring in CKD cohorts.

\section{International Relevance}

Frequency of measurements of GFR and albuminuria may vary by country and so are economic resources available to support such testing, and the ability to implement therapeutic strategies to address changes. Nonetheless, given the availability of simple monitoring tools like urine reagent 
Table 21 | Decline in kidney function in CKD populations

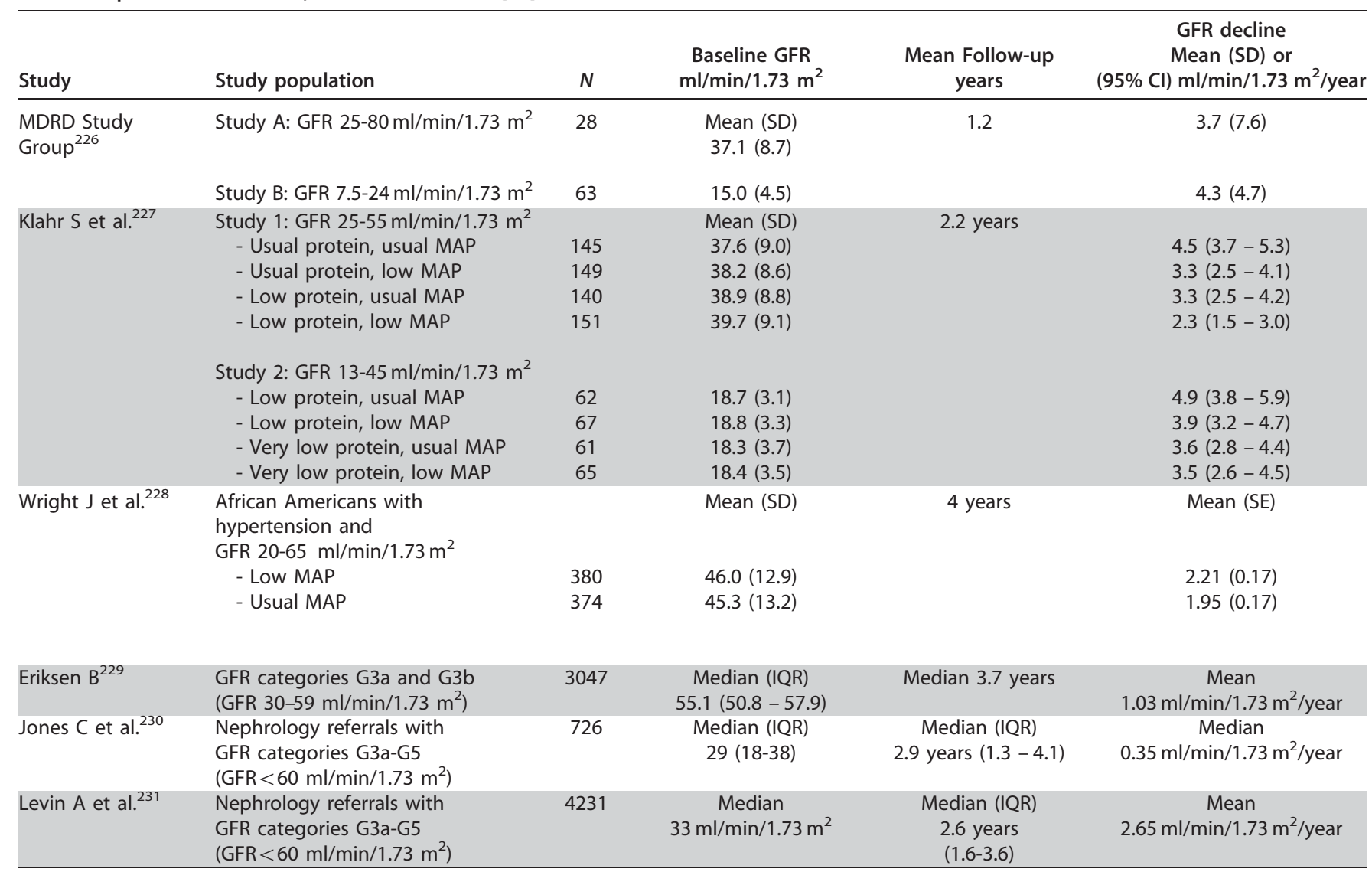

Abbreviations: $\mathrm{Cl}$, confidence interval; CKD, chronic kidney disease; GFR, glomerular filtration rate; IQR, interquartile range; MAP, mean arterial pressure; MDRD, Modification of Diet in Renal Disease; SD, standard deviation; SE, standard error.

strips, consideration may be given to implementation of this assessment in high-risk groups.

\section{Implications for Clinical Practice and Public Policy}

Practitioners must incorporate underlying category of GFR and albuminuria as well as cause of kidney disease and individual patient characteristics in determining the frequency of ongoing assessment. The implications for practice include the incorporation of regular monitoring of both GFR and albuminuria into clinical care for patients with CKD.

There are no immediate implications for public policy of this statement.

\section{Areas of Controversy, Confusion, or Non-consensus}

There are many who would like more definitive guidance on frequency of measurement according to specific categories of risk. However this is not possible at the current time given the lack of evidence to guide such statements and the extreme number of individual circumstances that would mitigate any proposed protocol.

We recommend further research to more accurately define the frequency with which GFR and albuminuria measure- ments should be performed based on their ability to inform strategies which prevent adverse outcomes (e.g., progression of kidney disease and death).

\section{Clarification of Issues and Key Points}

a) Assessment of both GFR and albuminuria should be undertaken to evaluate progression.

b) More frequent assessment is required as kidney disease progresses.

c) Not all individuals with CKD require close surveillance and monitoring; clinical context remains an important modifier for all recommendations.

d) While cause of CKD is an important predictor of progression, it is the values of GFR and albuminuria that are used to assess progression.

\section{Pediatric Considerations}

Currently there is no evidence as to the value of increasing the frequency of assessment of either GFR or proteinuria in children with CKD. Eventually more complete longitudinal data from the CKiD cohor ${ }^{55}$ and hence better granularity of individual and group rates of decline in GFR may provide the data required to strengthen proof of this guideline in children. 
As described in detail in the Pediatric Considerations for Recommendation 1.3.1, there is good observational evidence acknowledging the importance of level of GFR and proteinuria at baseline on predicting rates of progression and it may be that interventional opportunities would exist if closer monitoring and earlier recognition of worsening in these values for the individual were available. Future studies may examine this.

\subsection{2: Recognize that small fluctuations in GFR are common and are not necessarily indicative of progression. (Not Graded)}

2.1.3: Define CKD progression based on one of more of the following (Not Graded):

- Decline in GFR category ( $\geq 90$ [G1], 60-89 [G2], 45-59 [G3a], 30-44 [G3b], 15-29 [G4], $<15$ [G5] $\mathrm{ml} / \mathrm{min} / 1.73 \mathrm{~m}^{2}$ ). A certain drop in eGFR is defined as a drop in GFR category accompanied by a $25 \%$ or greater drop in eGFR from baseline.

- Rapid progression is defined as a sustained decline in eGFR of more than $5 \mathrm{ml} / \mathrm{min} / 1.73 \mathrm{~m}^{2} /$ year.

- The confidence in assessing progression is increased with increasing number of serum creatinine measurements and duration of follow-up.

\section{RATIONALE}

This statement serves to inform clinicians that some consistent definition of progression is required and should be implemented based on an appreciation of baseline values for an individual. There is considerable controversy as to what constitutes normal progression of CKD. The potential for biological and analytical variation associated with use of $\mathrm{SCr}$ measurements should be taken into account as they represent reversible fluctuations in GFR and are not necessarily indicative of progression. Further, it is important to recognize that the degree of precision with which progression is able to be estimated is highly dependent upon two factors: the number of SCr measurements used to define progression and the duration of follow-up. Estimating risk of ESRD based on extrapolation of the previous rate of change in GFR required substantial information ( $\geq 4$ measurements over $\geq 3$ years in most cases) to add to the risk information in the most recent GFR. Further, it should be recognized that some reno-protective treatments (e.g., BP lowering and RAAS antagonists) result in a slower rate of GFR decline longterm but often lower GFR in a stepwise fashion as a result of hemodynamic effects. Even substantial (5-25\%) reductions in GFR may be protective, complicating the interpretation of progression in these individuals. Finally, underlying disease activity should be considered when assessing patients for progression of kidney dysfunction.

The importance of determining the rate of decline in kidney function over time is to identify individuals who are progressing at a more rapid rate than anticipated, which is associated with increased morbidity and mortality. Individuals who are "rapid progressors" should be targeted to slow their progression and associated adverse outcomes. A progressive decline in kidney function is influenced by baseline GFR category and albuminuria category.

\section{Evidence Base}

Unfortunately few studies are available to guide us regarding the optimal definition of "rapid progression." Such studies require serial follow-up of patients to calculate change in GFR over time, with more frequent measurements and longer duration of follow-up providing more accurate estimates. The Work Group reviewed cohort studies of the general population that have evaluated rapid progression of kidney function (Table 22).

Approaches to define decline in kidney function included absolute rate of loss ${ }^{232,233,235}$ as well as percent change. ${ }^{221,234}$ Studies consistently demonstrate that a more rapid rate of loss of kidney function was associated with an increased risk of adverse clinical outcomes including death and vascular related events. These studies have been limited however by relatively few patients with GFR levels $<60 \mathrm{ml} / \mathrm{min} / 1.73 \mathrm{~m}^{2}$, few measurements of SCr, and relatively short duration of follow-up to obtain accurate estimates of the rate of decline in kidney function. The precision of the estimate of the slope depends on a number of factors including the number of measurements of kidney function, biological variability, measurement error, and duration of follow-up. In general at least three measures of kidney function are required to permit an estimate of slope. ${ }^{1}$

None of these studies assessed the impact of albuminuria on rate of "rapid decline" in kidney function. However as noted in Recommendation 2.1.1, the presence of proteinuria has been associated with a faster rate of kidney function decline compared with people without proteinuria. ${ }^{236-238}$ Two of the largest prospective cohort studies have shown an approximate two-fold increase in the rate of decline in GFR in the presence of proteinuria. ${ }^{219,220}$ Further evidence regarding the potential adverse effects of albuminuria on outcomes has been reported among patients with diabetes. The AER is one of the best indicators of diabetic nephropathy risk in both type 1 and type 2 diabetes, and patients with microalbuminuria have been reported to have 200 to $400 \%$ higher risk for progression to proteinuria than patients with normal albuminuria. ${ }^{239,240}$ Long-term follow-up studies have also demonstrated the increased risk of ESRD associated with albuminuria among patients with both type 1 and type 2 diabetes. $^{241,242}$

Given the recognized limitations in defining rapid progression, the Work Group aimed to provide options for determination of progression, based on their clinical utility, and ease of use. One approach included an assessment of change in GFR category, combined with a minimal percent change. A criterion requiring both a change in GFR category (e.g., change from G2 to G3a) and percent change would 


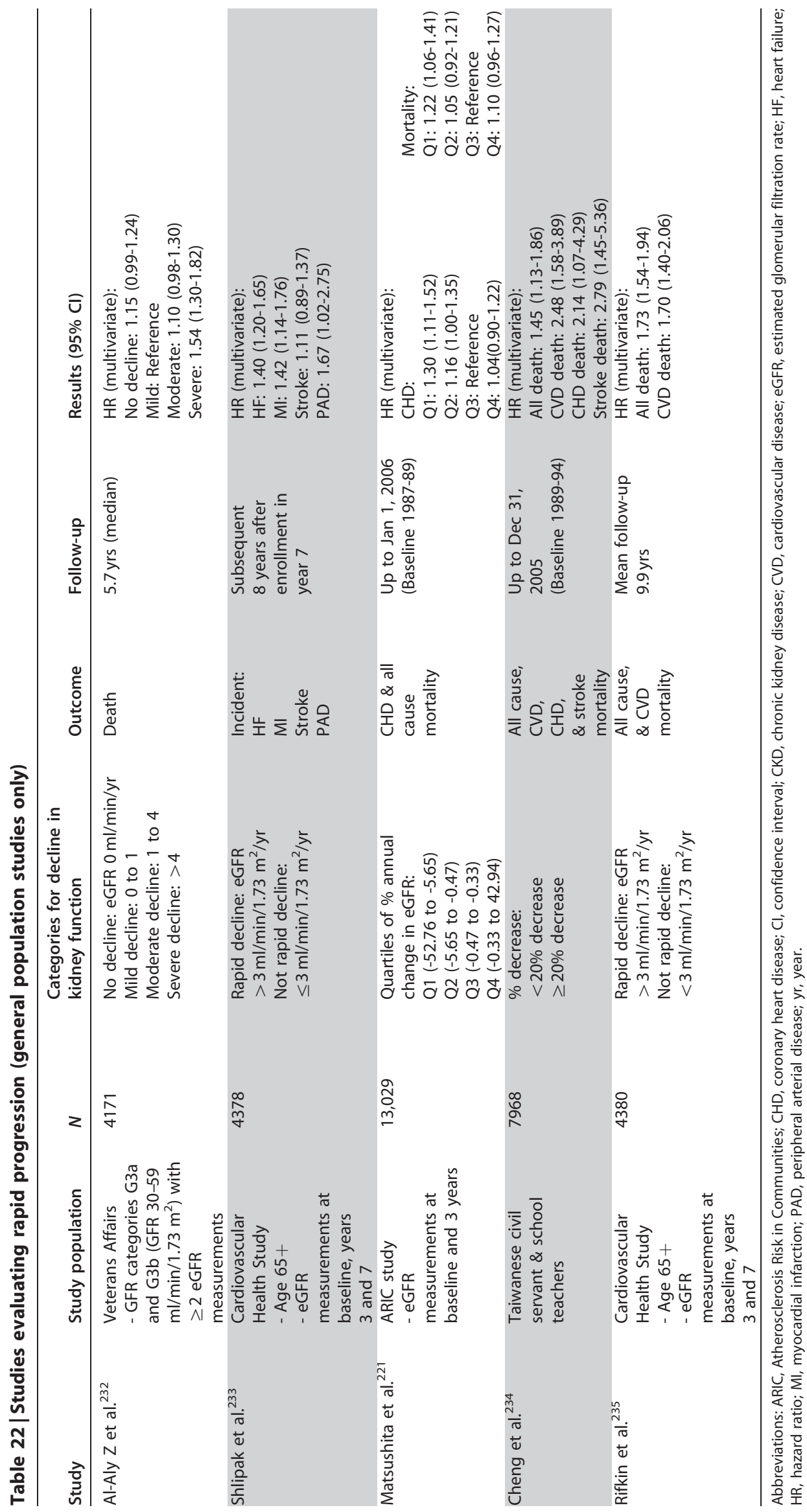


ensure that small changes GFR from 61 to $59 \mathrm{ml} / \mathrm{min} / 1.73 \mathrm{~m}^{2}$ for example, which represents a change in category but a minimal change in GFR, would not be misinterpreted to represent progression. A change of $<25 \%$ in a pair of GFR estimates may reflect physiologic variation rather than true progression.

Additional work to inform this definition has been undertaken, using data from the Alberta Kidney Disease Network (AKDN) ${ }^{243}$ In this analysis 598,397 adults with at least two out-patient measures of SCr spaced at least 6 months apart were included. Progression was defined as "certain" (rise or drop) if during the median follow-up time of 2.4 years there was a change in GFR category combined with a $25 \%$ or greater change in GFR from the baseline measurement (constituting a certain rise or a certain drop). Participants who changed GFR category but did not meet the criterion of $25 \%$ change in GFR were categorized as "uncertain" rise or drop. The reference group was comprised of participants who did not change GFR category over the follow-up period. As outlined in Table 23, compared to participants with stable eGFR, those with a certain drop had an almost two-fold increase in the risk of all-cause mortality

Table 23 | CKD progression and risk of all-cause mortality and ESRD using baseline (first) eGFR

\begin{tabular}{lcc}
\hline $\begin{array}{l}\text { Definition of } \\
\text { progression }\end{array}$ & $\begin{array}{c}\text { All-cause mortality } \\
\mathrm{HR}^{* *}(95 \% \mathrm{Cl})\end{array}$ & $\begin{array}{c}\mathrm{ESRD}^{*} \\
\mathrm{HR}^{* *}(95 \% \mathrm{Cl})\end{array}$ \\
\hline Certain rise & $1.51(1.46-1.56)$ & $0.33(0.26-0.42)$ \\
Uncertain rise & $1.12(1.08-1.16)$ & $0.39(0.30-0.51)$ \\
Stable (reference) & Ref & Ref \\
Uncertain drop & $0.98(0.95-1.01)$ & $2.13(1.84-2.47)$ \\
Certain drop & $1.89(1.83-1.95)$ & $5.11(4.56-5.71)$ \\
\hline
\end{tabular}

Abbreviations: $\mathrm{Cl}$, confidence interval; eGFR, glomerular filtration rate; ESRD, endstage renal disease; $\mathrm{HR}$, hazard ratio.

Data from Turin et al. ${ }^{244,245}$

*ESRD defined as requiring renal replacement therapy.

**Adjusted for age, gender, hypertension, diabetes, proteinuria, Charlson comorbidities and baseline (first) eGFR.
(HR 1.89; 95\% CI 1.83-1.95) ${ }^{244}$ and a five-fold increase in the risk of ESRD (HR 5.11; 95\% CI 4.56-5.71). ${ }^{245}$ Lesser degree of risk was present for those with an uncertain drop (reflecting a change in category only). It is worth noting that once progression occurs, the last eGFR which has a lower level often contains much of the information about risk of ESRD and extrapolation of progression using information from prior progression and the most recent eGFR is only useful if the information about progression is precise and the patient's trajectory is linear.

The second approach to define progression takes into account the rate of change in kidney function based on a slope analysis. In this approach the rate of loss is defined by both the absolute rate of change and the percent change, as determined among a cohort of 529,312 adults who had at least 3 outpatient SCr measurements over a four year period (AKDN databases). Two indices of change in eGFR were estimated: the absolute annual rate of change (categorized as: increase, stable and $-1,-2,-3,-4$, and $\geq-5 \mathrm{ml} / \mathrm{min} / 1.73 \mathrm{~m}^{2} /$ year decline); and the annual percentage change (categorized as: increase, stable, -1 to $-2,-3$ to $-4,-5$ to -6 , and $\geq-7$ percent decline/year). The adjusted ESRD risk associated with each category of change in eGFR was estimated, using stable eGFR (no change in eGFR) as the reference. The results were adjusted in two ways: for eGFR and covariates at the time of the first eGFR measurement, and at the time of the last eGFR measurement. As outlined in Table 24, the risk of ESRD increased almost two-fold for every $1 \mathrm{ml} / \mathrm{yr}$ decline in eGFR, when adjusted for covariates and eGFR at the time of the first eGFR measurement. The risk remained significant, but was less pronounced, when adjustments were performed at the time of the last eGFR measurement. This suggests that extrapolation of kidney function beyond the last measurement of eGFR is still informative, but identifies a lesser risk. Similar results were obtained when change in eGFR was defined by a percentage.

Table 24| Association between absolute and percentage change in kidney function and risk of ESRD*, based on adjustment for eGFR at the first and last measurement

\begin{tabular}{|c|c|c|}
\hline $\begin{array}{l}\text { Absolute rate of change } \\
\text { (over a median of } 3.5 \text { years) }\end{array}$ & $\begin{array}{l}\text { Adjusted for eGFR at first } \\
\text { creatinine measurement } \mathrm{HR}^{* *}(95 \% \mathrm{Cl})\end{array}$ & $\begin{array}{l}\text { Adjusted eGFR at last creatinine } \\
\text { measurement } \mathrm{HR}^{* *}(95 \% \mathrm{Cl})\end{array}$ \\
\hline Increasing eGFR & $0.64(0.48-0.86)$ & $1.20(0.90-1.61)$ \\
\hline Stable $\left(0 \mathrm{ml} / \mathrm{min} / 1.73 \mathrm{~m}^{2} /\right.$ year $)$ & Ref & Ref \\
\hline$-2 \mathrm{ml} / \mathrm{min} / 1.73 \mathrm{~m}^{2} /$ year & $2.71(2.08-3.53)$ & $1.58(1.21-2.06)$ \\
\hline$-3 \mathrm{ml} / \mathrm{min} / 1.73 \mathrm{~m}^{2} /$ year & $3.98(3.06-5.17)$ & $1.63(1.25-2.13)$ \\
\hline$-4 \mathrm{ml} / \mathrm{min} / 1.73 \mathrm{~m}^{2} /$ year & $5.82(4.45-7.61)$ & $1.90(1.45-2.48)$ \\
\hline Increasing & $0.76(0.55-1.07)$ & $1.11(0.80-1.55)$ \\
\hline Stable & Ref & Ref \\
\hline-1 to $-2 \% /$ year & $1.17(0.81-1.68)$ & $0.97(0.67-1.40)$ \\
\hline-3 to $-4 \% /$ year & $1.79(1.25-2.56)$ & $1.19(0.83-1.71)$ \\
\hline-5 to $-6 \% /$ year & $2.26(1.55-3.29)$ & $1.21(0.83-1.78)$ \\
\hline$-7 \% /$ year or more & $11.30(8.53-14.97)$ & $2.17(1.60-2.93)$ \\
\hline
\end{tabular}

Abbreviations: $\mathrm{Cl}$, confidence interval; eGFR, estimated glomerular filtration rate; ESRD, end-stge renal disease; HR, hazard ratio.

Data from the Alberta Kidney Disease Network.

*ESRD defined as requiring renal replacement therapy.

**Adjusted for age, gender hypertension, diabetes, proteinuria, Charlson comorbidities and eGFR at the first or last measurement. 

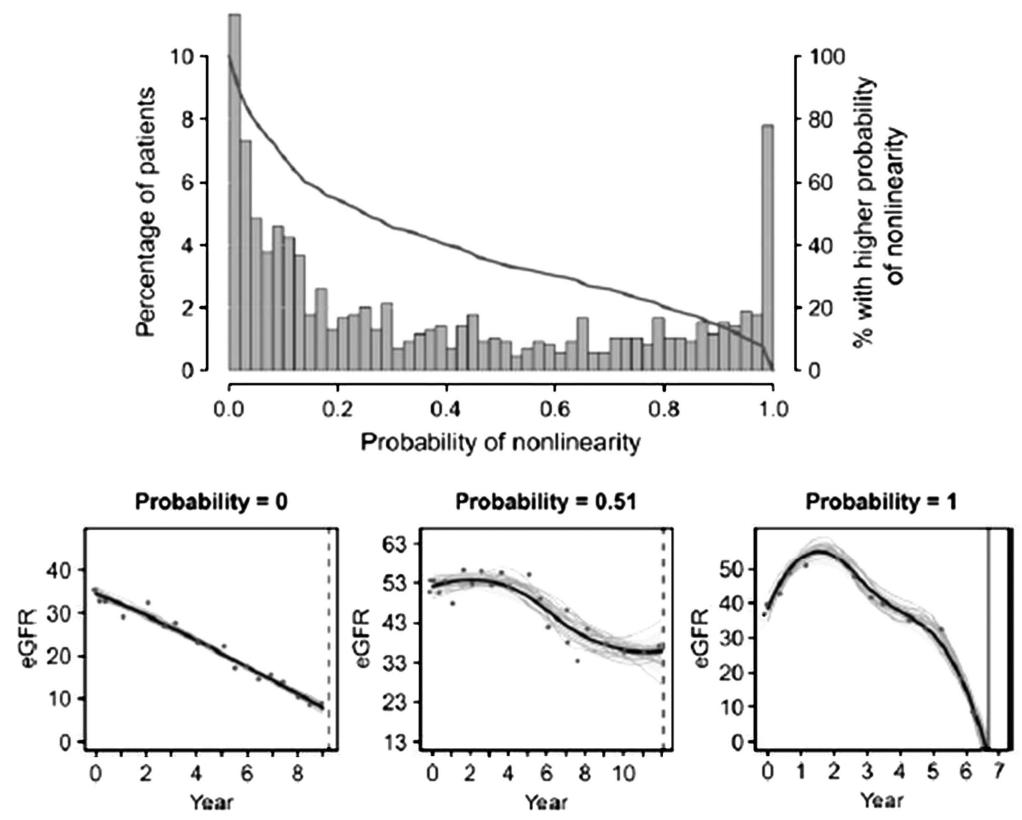

Figure 18 Distribution of the probability of nonlinearity (top) with three example trajectories demonstrating different probabilities of nonlinearity (bottom). Reprinted from Li L, Astor BC, Lewis J, et al. Longitudinal Progression Trajectory of GFR Among Patients With CKD. Am J Kidney Dis 2012; 59: 504-512 with permission from the National Kidney Foundation. ${ }^{247}$; accessed http://download.journals.elsevierhealth.com/pdfs/journals/0272-6386/PIIS0272638611017896.pdf

With respect to the impact of changes in albuminuria over time, a study from the Ongoing Telmisartan Alone and in Combination with Ramipril Global Endpoint trial (ONTARGET) investigators showed that a greater than or equal to twofold increase in albuminuria from baseline to 2 years associated with a nearly $50 \%$ higher mortality (HR 1.48; CI 1.32-1.66), while a greater than or equal to twofold decrease in albuminuria associated with $15 \%$ lower mortality (HR 0.85; CI 0.74-0.98) compared with those with lesser changes in albuminuria, after adjustment for baseline albuminuria, $\mathrm{BP}$, and other potential confounders. Increases in albuminuria also significantly associated with renal outcomes, defined as ESRD or doubling of SCr (HR 1.40; CI 1.11-1.78), while a decrease in albuminuria was associated with a decrease of the combined renal outcome (HR 0.73; CI 0.57-0.95)..$^{246}$ However, preliminary analysis of cohort studies is limited and suggests that further research is required to more accurately determine the change in albuminuria associated with an increased risk of kidney disease progression.

There is accumulating evidence that the trajectories of GFR decline are non-linear, and may take a number of different courses over time. The longer an individual is followed over time, the more likely they are to experience non-linear change in trajectory. ${ }^{247,248}$ The non-linearity of a trajectory may be due to intercurrent illness, changes in medication, intrinsic to the disease process, or any combination of these factors. Li et al. ${ }^{247}$ described individual GFR progression trajectories over twelve years of follow-up among participants in the African American Study of Kidney Disease (AASK) (Figure 18). The authors reported that $41.6 \%$ of patients exhibited a greater than $90 \%$ probability of having a non-linear trajectory; in $66.1 \%$ the probability of non-linearity was $>50 \%$.

\section{International Relevance}

Studies to date evaluating rapid progression of kidney disease have been limited to North American (White and African American), European, and Asian populations. Given the differences in the prevalence of CKD by ethnic group, there may also be variations in rate of progression by ethnicity, and in particular ethnic groups with high rates of comorbid conditions leading to ESRD such as the Aboriginal population. Thus, the definition of rapid progression may vary according to country or region. However, by using a general definition of progression, which includes change of category of eGFR or albuminuria or both, as well as a numeric change over an established period of time, we believe that the definition of progression can be used in all cohorts.

\section{Implications for Clinical Practice and Public Policy}

Practitioners should monitor the GFR and albuminuria at regular intervals to identify rates of decline which exceed that normally demonstrated. The rate of GFR decline may be relatively constant over time in an individual; however the rate of GFR decline is highly variable among people and over long periods of observation, within individuals. Thus clinicians are encouraged to evaluate changes in GFR or albuminuria in the context of multiple observations over time, and with attention to clinical events which may have impacted the change. A number of factors influence assessment of rate of progression including frequency and duration of GFR and albuminuria measurements as well as 
factors related to the patient (e.g., baseline GFR, comorbidities, age etc.).

There are no implications for public policy at this time.

\section{Areas of Controversy, Confusion, or Non-consensus}

The practical issue in clinical practice and clinical trials is how to define progression (as inferring true deterioration in kidney function) with meaningful thresholds that are easy to understand for the non-nephrologist. While changes in proteinuria may signify change in clinical condition, there are no data yet to suggest that change in proteinuria is itself reliably associated with progression of CKD per se. This may be confusing to practitioners, since a change in quantity of proteinuria is an indication for referral.

We recommend research to confirm rates which can be classified as slow, moderate, and rapid progression of kidney disease. The rate to define "rapid progression" may vary depending on the outcome considered, such as kidney failure versus mortality for example. It will be important for researchers to determine methods by which reproducible classification systems for describing rates of progression can be developed. There are increasing data to suggest the nonlinearity of progressive disease in many individuals. This makes extrapolation risky and warrants continued assessment of the slope on a regular basis.

\section{Clarification of Issues and Key Points}

Small fluctuations in GFR are common and should not be misinterpreted to represent progressive decline in kidney function. Many factors can cause a small change in GFR including medications, volume status, measurement error, and biological variability. Assessment of progressive decline in GFR needs to take into account the number of measurements considered and time period of assessment.

In pediatrics, information about utilty of serial creatinine measurements over periods of time during which growth (and muscle mass increase) is occurring, for the diagnosis of progression or regression, remains problematic.

\section{Pediatric Considerations}

Applying strict GFR criteria in order to develop cutoff values associated with 'true' progression in terms of any one individual child is not currently possible. Conceptually the movement from various levels of renal function downward, in particular if that movement is associated with increasing comorbidities or intensity of such, is a reasonable approach.

The most informative data available in children comes from the longitudinal GFR data from CKiD. ${ }^{72}$ Examination of the whole cohort reveals an annual decline in GFR of $-4.2 \%$; median GFR decline was $-1.8 \mathrm{ml} / \mathrm{min} / 1.73 \mathrm{~m}^{2}$ (interquartile range [IQR] -6.6 to 1.6 ); this can be expressed as a median absolute decline in GFR of $-4.3 \mathrm{ml} / \mathrm{min} / 1.73 \mathrm{~m}^{2}$ (IQR -11.9 to 1.1) and $-1.5 \mathrm{ml} / \mathrm{min} / 1.73 \mathrm{~m}^{2}$ (IQR -5.0 to 1.8 ).

Given that the lower IQR in each of the overall cohort and both sub-groups is equal to or exceeds the suggested decline of $5 \mathrm{ml} / \mathrm{min} / 1.73 \mathrm{~m}^{2}$ as stated here, we suggest that it is reasonable to adopt this definition at least for the purpose of classification as it relates to 'rapid' progression; note the above values all relate to measured GFR.

Increasing numbers of any given measurement of an event generally allow for greater precision and accuracy. However, the simple repeated measurements of creatinine over time are less likely to be valuable in children than in adults with CKD. Unlike adults with static muscle mass and hence expected stability in creatinine values, or adults with expected declining muscle mass and hence expectations of declining creatinine if renal function has remained stable, pediatric populations have a situation of increasing muscle mass with expectation of increasing creatinine in the otherwise normal child without CKD. In a child with CKD who is growing therefore, and in particular one going through puberty, the simple comparison of creatinine values over time will likely not be sufficient to presume CKD progression or regression has occurred. The two exceptions to this would be a) a series of creatinine measurements demonstrating significant increase over a short period of time wherein there is no demonstrable or expected gain of muscle mass; b) values of creatinine that over time demonstrate an increase to levels above that which is expected of the child's age and sex based on population normative value for the lab and method of measurement.

2.1.4: In people with CKD progression, as defined in Recommendation 2.1.3, review current management, examine for reversible causes of progression, and consider referral to a specialist. (Not Graded)

\section{RATIONALE}

This statement intends to reassure patients and practitioners that not all patients necessarily require referral to specialists, but that this should be considered in the event that the patient or clinician requires further guidance or prognostic information. CKD progression, contextualized for the individual circumstance, does not always require referral, and earlier guidelines may not have been so overt in stating this. Faster or unusual trajectories of progression should alert the patient and physician to assess for potentially reversible causes of progression.

Progressive kidney disease requires the need for more aggressive assessment and treatment, which may include referral to a nephrologist or specialist (if they are not currently being managed by a nephrologist).

\section{Evidence Base}

Decline in GFR may not be constant, with acute decline superimposed on CKD (see Chapter 2.2 for discussion of factors associated with progression of a more chronic nature). The most common risk factors identified for acute decline in GFR for patients with established CKD include: obstruction of the urinary tract; volume depletion; nonsteroidal anti-inflammatory drugs (NSAIDs), including cyclooxygenase-2 (COX 2) inhibitors; select antimicrobial agents 
such as aminoglycosides and amphotericin B; radiocontrast agents; and angiotensin-converting enzyme inhibitors (ACE-Is) and angiotensin-receptor blockers (ARBs). ${ }^{249-252}$ Readers are also referred to the KDIGO Clinical Practice Guideline for Acute Kidney Injury ${ }^{7}$ which contains additional relevant details regarding risk factors for rapid progression and management strategies.

Rapid sustained decline in GFR could also be considered an indication for referral once potentially reversible factors as outlined above have been assessed and treated. The principles guiding referral include additional input from a nephrologist into management of CKD and preparation for RRT, such as that which may be required with rapidly declining GFR. The NICE guidelines for CKD also recommend referral for "rapidly declining GFR" although the definition of rapidly declining is not provided. ${ }^{186}$ Most studies assessing nephrology referral have focused on early versus late referral, and not considered the impact or implications of nephrology referral in situations associated with rapid decline in GFR. ${ }^{253}$ The evidence that such referral will change outcomes is not clear but given that nephrologists often have access to education and specialized services, which are essential for optimal preparation for RRT, referral to a specialist is recommended. Additional discussion of when to consider a referral to a nephrologist can be found in Chapter 5 .

\section{International Relevance}

Risk factors for acute deterioration in kidney function may vary slightly by country of origin, although the common categories in general (e.g., medications, volume depletion and urinary tract obstruction) would be relevant irrespective of country. This set of statements serves to ensure that attention to changes in kidney function in those identified with kidney disease is part of the usual care of these individuals.

\section{Implications for Clinical Practice and Public Policy}

Practitioners should be aware of the common risk factors for acute-on-chronic kidney disease resulting in a rapid loss of kidney function. Acute rapid deterioration in kidney function should alert the practitioner to assess for these potentially reversible causes of progression. This assessment would include an evaluation for potential urinary tract obstruction as well as a volume assessment and detailed medication review.

\section{Areas of Controversy, Confusion, or Non-consensus}

Readers are referred to the KDIGO Clinical Practice Guideline for Acute Kidney Injury ${ }^{7}$ for a detailed discussion of issues related to definition, diagnosis, and treatment of rapid progression of kidney dysfunction, as defined by AKI.

\section{Clarification of Issues and Key Points}

There are no specific numeric values to define "rapid loss" as this varies within and between disease states, individuals, and populations. The key point for practitioners is to serially review individual trajectories of changes in kidney function so as to determine stability or not, identify changes in previous stable trajectories, and to be aware of the importance of circumstances where AKI changes the course of CKD. Further studies are required to determine the rate of loss of GFR that constitutes rapid loss of kidney function. In children, interpretation of serial changes in $\mathrm{SCr}$ requires understanding of normative value ranges and drivers of change in SCr which are different than in adults.

\section{2: PREDICTORS OF PROGRESSION}

2.2.1: Identify factors associated with CKD progression to inform prognosis. These include cause of CKD, level of GFR, level of albuminuria, age, sex, race/ethnicity, elevated $\mathrm{BP}$, hyperglycemia, dyslipidemia, smoking, obesity, history of cardiovascular disease, ongoing exposure to nephrotoxic agents, and others. (Not Graded)

\section{RATIONALE}

The rationale for estimating the risk of kidney failure is that it may inform referral, care plans, and other therapeutic strategies, including frequency of monitoring and follow-up. Previous guidelines have not been able to suggest risk equations or relative and absolute risks of specific outcomes but with the data currently available, this is now possible. There are several factors that influence the likelihood and rate of CKD progression including GFR and albuminuria category, the degree of albuminuria, the cause of kidney disease, ongoing exposure to nephrotoxic agents, obesity, hypertension, age, race/ethnicity and laboratory parameters such as $\mathrm{Hb}$ (hemoglobin), albumin, calcium, phosphate, and bicarbonate.

As some of these risk factors are modifiable they should be actively identified and, if present, be treated as they may impact long-term outcomes including cardiovascular conditions, QOL, and progression of CKD.

It is not yet clear what the relative weight of each of these factors is in predicting in an individual whether he/she will have progressive CKD.

\section{Evidence Base}

As progression of CKD is defined as either a progressive decrease in GFR or a progressive increase in albuminuria, we should consider separately whether different factors would predict these two components of CKD differently. Given the limited evidence, this will not be discussed separately. It is however clear that a subject with a lower GFR to start with will progress more rapidly to a GFR $<15 \mathrm{ml} / \mathrm{min} / 1.73 \mathrm{~m}^{2}$, just as a subject with already elevated albuminuria will progress more rapidly to an ACR $>300 \mathrm{mg} / \mathrm{g}$ (>30 mg/ mmol). Similarly, it is well-known that a subject with membranous glomerulopathy is more likely to progress to nephrotic syndrome, while a subject with adult polycystic kidney disease is more likely to progress to ESRD.

Although there are many cross-sectional studies that describe factors associated with a low GFR and factors associated with a high albuminuria, the number of studies evaluating which 
factors are associated with progressive decreases in GFR and progressive increases in albuminuria are more limited. In general, it can be argued that most of the above-mentioned factors are associated with a more progressive rise in albuminuria and a more progressive fall in GFR. Most recently, studies have focused on the development of risk scores for identifying progressive decreases in GFR and progressive increases in albuminuria. It has not yet been established which prediction formula could best be used. Some formulas use just simple demographic and clinical measures, while others also include laboratory tests. Some were developed for high-risk populations, such as people with known underlying CVD, ${ }^{254}$ with $\mathrm{CKD}$ in general, ${ }^{255}$ or with specific causes of CKD, such as IgA nephropathy, ${ }^{256}$ diabetic nephropathy, ${ }^{257}$ or renal artery stenosis. ${ }^{258}$ Others developed a risk prediction model in the general population. ${ }^{259}$ This latter model included age, race, gender, and in dichotomized version, the presence of anemia, hypertension, diabetes, and CVD history. More recently, two studies used more accurate laboratory parameters in addition to demographic characteristics. The first study was in patients with an eGFR of $15-60 \mathrm{ml} / \mathrm{min} / 1.73 \mathrm{~m}^{2} /$ year, and included age, gender, eGFR, albuminuria, and serum calcium, phosphate, bicarbonate and albumin. ${ }^{260}$ The second study was in subjects from the general population and included age, eGFR, albuminuria, measured levels of BP and C-reactive protein (CRP). ${ }^{261}$ The results from these predictive models require validation in future studies but they demonstrate the potential and the capabilities of developing clinically meaningful classification of risk for individual patients. Further research is required to establish whether prediction formulas may differ for different ethnicities.

\section{International Relevance}

Studies describing factors associated with lower GFR and higher ACR have been described from all over the world. In general, there is much overlap between these data. It may be that in different parts of the world the relative weight of each of the factors predicting progressive increases in albuminuria or decreases in GFR may substantially differ.

\section{Implications for Clinical Practice and Public Policy}

It is important to realize that some factors predicting progression of CKD are modifiable. This holds true for lifestyle measures such as cessation of smoking and prevention of obesity. It also subtends to lowering of BP, lowering of albuminuria and prevention of hyperglycemia. A further factor that may be modifiable is the underlying cause of CKD. As various causes may respond to targeted treatment, finding the cause of CKD is the starting point of the work-up of a subject with CKD. If this causal disease is modifiable, for example by immunosuppressive treatment, then such treatment is the first step to consider. Management of patients with CKD and delay of progression are dealt with in Chapter 3 and more fully in other guidelines (see KDIGO Clinical Practice Guideline for Glomerulonephritis ${ }^{8}$ and KDOQI Clinical Practice Guideline for Diabetes and CKD: 2012 Update. $^{262}$ )

\section{Areas of Controversy, Confusion, or Non-consensus}

While there are prediction equations available using combinations of eGFR, albuminuria, cause of CKD, and some or all of the laboratory abnormalities listed, they have not been used in clinical practice to guide therapy as yet. Furthermore, while the associated abnormalities clearly increase in severity with worsening kidney function, normalizing them in some instances has not changed progression to ESRD. The need for prediction equations to take into account changes over time (trajectories) and stability or instability of specific factors has been raised by many. Nonetheless, the ability to determine progression versus stability should be of value for patients and clinicians.

\section{Clarification of Issues and Key Points}

Clinicians should attempt to determine stability or progression of patients with CKD for the purposes of informing care. Further research is required to determine which formula best predicts who will have progressive increases in albuminuria and progressive decreases in GFR. The key components of prediction equations for ESRD may well be different than prediction equations for cardiovascular events or death.

\section{Pediatric Considerations}

A more complete discussion of the evidence in children supporting these factors as potentially related to risk of progression, in addition to the pediatric specific risk of growth/puberty, can be found in the Pediatric Considerations for Recommendation 1.3.1.

\section{DISCLAIMER}

While every effort is made by the publishers, editorial board, and ISN to see that no inaccurate or misleading data, opinion or statement appears in this Journal, they wish to make it clear that the data and opinions appearing in the articles and advertisements herein are the responsibility of the contributor, copyright holder, or advertiser concerned. Accordingly, the publishers and the ISN, the editorial board and their respective employers, office and agents accept no liability whatsoever for the consequences of any such inaccurate or misleading data, opinion or statement. While every effort is made to ensure that drug doses and other quantities are presented accurately, readers are advised that new methods and techniques involving drug usage, and described within this Journal, should only be followed in conjunction with the drug manufacturer's own published literature. 


\section{Chapter 3: Management of progression and complications of CKD}

Kidney International Supplements (2013) 3, 73-90; doi:10.1038/kisup.2012.66

\section{DELAYING PROGRESSION OF CKD}

The following section describes key recommendations and guidance for people with CKD with respect to delaying progression of CKD. General lifestyle recommendations are provided as well as caveats given for those with diabetes. Cardiovascular risk reduction including management of hypertension, dyslipidemia, and hyperuricemia is further addressed. Unless otherwise stated, the guidance is intended to apply to adults with CKD.

For the practicing clinician, ideally working with a team of health-care professionals, it is important to institute general lifestyle modification practices in people with CKD so that they may gain the benefit of these in addition to more kidney-specific strategies. Often these general measures are overlooked or disregarded in people with CKD, thus their utility is underscored here.

\subsection{PREVENTION OF CKD PROGRESSION}

The management of progression of CKD is aimed at addressing a multiplicity of factors known to be associated with progression. There are general measures which have been shown to address cardiovascular health and CKD together, or each separately. Addressing CVD risk factors may indirectly and directly impact CKD progression. Strategies include general lifestyle measures which improve cardiovascular health, BP control, and interruption of the RAAS. In addition, control of other metabolic parameters such as blood sugar, uric acid, acidosis, and dyslipidemia may also be important. This section deals with management of BP, RAAS interruption, glycemic control and dietary/lifestyle manipulations which have been examined in the context of delaying progression of CKD.

\section{$B P$ and RAAS interruption}

The following statements are excerpted and where necessary, condensed, from the KDIGO Clinical Practice Guideline for the Management of Blood Pressure in CKD. ${ }^{10}$

3.1.1: Individualize BP targets and agents according to age, coexistent cardiovascular disease and other comorbidities, risk of progression of $\mathrm{CKD}$, presence or absence of retinopathy (in CKD patients with diabetes), and tolerance of treatment as described in the KDIGO 2012 Blood Pressure Guideline. (Not Graded)
3.1.2: Inquire about postural dizziness and check for postural hypotension regularly when treating CKD patients with BP-lowering drugs. (Not Graded)

3.1.3: Tailor BP treatment regimens in elderly patients with CKD by carefully considering age, comorbidities and other therapies, with gradual escalation of treatment and close attention to adverse events related to BP treatment, including electrolyte disorders, acute deterioration in kidney function, orthostatic hypotension and drug side effects. (Not Graded)

3.1.4: We recommend that in both diabetic and nondiabetic adults with CKD and urine albumin excretion $<30 \mathrm{mg} / 24$ hours (or equivalent ${ }^{\star}$ ) whose office $\mathrm{BP}$ is consistently $>140 \mathrm{~mm} \mathrm{Hg}$ systolic or $>90 \mathrm{~mm} \mathrm{Hg}$ diastolic be treated with BP-lowering drugs to maintain a BP that is consistently $\leq 140 \mathrm{~mm}$ $\mathrm{Hg}$ systolic and $\leq 90 \mathrm{~mm} \mathrm{Hg}$ diastolic. (1B)

3.1.5: We suggest that in both diabetic and non-diabetic adults with CKD and with urine albumin excretion of $\geq 30 \mathrm{mg} / 24$ hours (or equivalent ${ }^{*}$ ) whose office $\mathrm{BP}$ is consistently $>130 \mathrm{~mm} \mathrm{Hg}$ systolic or $>80 \mathrm{~mm} \mathrm{Hg}$ diastolic be treated with BP-lowering drugs to maintain a $\mathrm{BP}$ that is consistently $\leq 130 \mathrm{~mm}$ $\mathrm{Hg}$ systolic and $\leq 80 \mathrm{~mm} \mathrm{Hg}$ diastolic. (2D)

3.1.6: We suggest that an ARB or ACE-I be used in diabetic adults with CKD and urine albumin excretion $30-300 \mathrm{mg} / 24$ hours (or equivalent ${ }^{\star}$ ). (2D)

3.1.7: We recommend that an ARB or ACE-I be used in both diabetic and non-diabetic adults with $\mathrm{CKD}$ and urine albumin excretion $>300 \mathrm{mg} / 24$ hours (or equivalent $\left.{ }^{*}\right)$. (1B)

3.1.8: There is insufficient evidence to recommend combining an ACE-I with ARBs to prevent progression of CKD. (Not Graded)

3.1.9: We recommend that in children with $C K D, B P-$ lowering treatment is started when $\mathrm{BP}$ is consistently above the $90^{\text {th }}$ percentile for age, sex, and height. (1C)

3.1.10: We suggest that in children with CKD (particularly those with proteinuria), BP is lowered to consistently achieve systolic and diastolic readings less than or equal to the $50^{\text {th }}$ percentile for age, sex, and height, unless achieving these targets is limited by signs or symptoms of hypotension. (2D)

\footnotetext{
${ }^{*}$ Approximate equivalents for albumin excretion rate per 24 hoursexpressed as protein excretion rate per 24 hours, albumin-to-creatinine ratio, protein-to-creatinine ratio, and protein reagent strip results- are given in Table 7, Chapter 1
} 
3.1.11: We suggest that an ARB or ACE-I be used in children with CKD in whom treatment with BP-lowering drugs is indicated, irrespective of the level of proteinuria. $(2 D)$

These statements are worded to maintain consistency with the KDIGO Clinical Practice Guideline for the Management of Blood Pressure in $C K D,{ }^{10}$ where the full rationale and evidence behind the statements may be found. In detailing BP targets, we recognize that we have not made recommendations or suggestions concerning lower limits of BP. The risks of overtreatment should be specifically considered when making decisions about BP lowering and this is encapsulated in the first two guideline statements.

\section{CKD and risk of $A K I$}

3.1.12: We recommend that all people with CKD are considered to be at increased risk of AKI. (1A)

3.1.12.1: In people with $\mathrm{CKD}$, the recommendations detailed in the KDIGO AKI Guideline should be followed for management of those at risk of AKI during intercurrent illness, or when undergoing investigation and procedures that are likely to increase the risk of AKI. (Not Graded)

\section{RATIONALE}

Observational data suggest a strong association between preexisting CKD and AKI. The appreciation that CKD patients may be more susceptible to AKI is the purpose of the above set of statements. However, methodological issues such as how CKD and AKI are defined in clinical studies and the statistical adjustments for non-uniformity of comorbidities among various studies may affect the validity of observed associations. These statements would be applicable in pediatrics, though the data are not available for this specific issue.

\section{Evidence Base}

CKD is designated as a risk factor for AKI because of the epidemiological association between the two. ${ }^{263,264}$ A number of studies in a variety of settings report an association between pre-existing CKD and AKI. ${ }^{265-271} \mathrm{CKD}$ is a potent predictor of acute decline in kidney function following exposure to radiocontrast, ${ }^{272}$ major surgery, ${ }^{273}$ and other medical conditions. ${ }^{274}$

Hsu et al. ${ }^{14}$ compared the pre-hospitalization MDRD GFR of 1764 adult members of the Kaiser Permanente Northern California health-care system who developed dialysis-requiring AKI during hospitalization with 600,820 individuals who did not. Compared with a reference baseline GFR of $\geq 60 \mathrm{ml} /$ $\min / 1.73 \mathrm{~m}^{2}$, a baseline GFR of $45-59 \mathrm{ml} / \mathrm{min} / 1.73 \mathrm{~m}^{2}$ was associated with an adjusted odds ratio (OR) of in-hospital AKI of 1.66 (95\% CI 1.40-1.97). For GFR values of 15-29 ml/ $\min / 1.73 \mathrm{~m}^{2}$, the adjusted OR for in-hospital AKI was 20.42 (95\% CI 17.40-23.96). The presence of diabetes, hypertension, and proteinuria increased the likelihood of developing in-hospital AKI, with adjusted OR of $1.99(95 \%$ CI 1.78-2.23), 1.55 (95\% CI 1.37-1.76) and 2.84 (95\% CI 2.52-3.19), respectively. The authors concluded that CKD is the main risk factor for AKI during hospitalization. A contrasting approach by Singh et al. defined AKI as dialysisrequiring acute renal failure. ${ }^{275}$ Because the clinical decision to dialyze a patient is frequently influenced by a higher overall SCr, presence of hemodialysis access, or consideration of inevitable progression to ESRD, this definition of AKI could bias toward capturing more AKI cases in CKD patients. Moreover, in patients with advanced CKD, the progression of CKD to ESRD may sometimes be difficult to separate from acute-on-chronic renal failure. A cohort study by Lafrance et al. followed a referred CKD population in British Columbia for a median of 19.4 months after achieving a GFR of $\leq 30 \mathrm{ml} / \mathrm{min} / 1.73 \mathrm{~m}^{2}$. Forty-five percent had at least one episode of AKI. ${ }^{276}$ In another cohort study of 920,985 adults in Alberta, Canada with at least one outpatient measurement of $\mathrm{SCr}$ and proteinuria and not requiring chronic dialysis, risk of admission with AKI increased with heavier proteinuria and reduced GFR. ${ }^{16}$

\section{International Relevance}

The incidence of AKI in CKD populations may be different around the world, or have different etiologies. It is not yet clear what the recovery rates from AKI are in the CKD population, and how these vary around the world dependent on cause and duration of AKI.

\section{Areas of Controversy, Confusion, or Non-consensus}

Interpretation of published data examining the influence of pre-existing CKD on the increased likelihood of AKI is potentially confounded by a number of issues. These include the comorbidities associated with CKD, influenced by repeated exposure to various nephrotoxic insults or in-hospital errors, ${ }^{57,277}$ or primarily due to the altered physiology in CKD. There are also methodological issues such as how CKD and AKI are defined in clinical studies and the varying statistical adjustments for comorbidities.

A further important issue to clarify is whether pre-existing CKD influences the outcome of AKI. Currently, there is no single biomarker that can differentiate 'acute' from 'chronic' kidney disease and help to address this issue. Several large observational and database studies report, surprisingly, lower in-hospital mortality in patients with AKI superimposed on CKD compared with controls. ${ }^{278-283}$ Data from the Program to Improve Care in Acute Renal Disease (PICARD) reveal lower in-patient mortality and median length of stay in intensive-care unit (ICU) subjects with acute-on-chronic renal injury compared with non-CKD subjects with AKI, though the post-discharge dialysis rates were higher in subjects with pre-existing CKD. ${ }^{284}$

\section{Clarification of Issues and Key Points}

AKI is relatively common in CKD populations and impacts progression adversely. Clinicans should attempt to minimize 
avoidable episodes of AKI (see Chapter 4 for more details) as part of a holistic approach to delaying progression.

\section{RESEARCH RECOMMENDATIONS}

Prospectively designed clinical studies with a clear and uniform definition of CKD and AKI and adjusted for comorbidities are needed to determine the:

- frequency of AKI events in a CKD population;

- outcome of AKI in patients with CKD;

- importance of proteinuria in addition to low GFR in the risk of AKI.

\section{Protein intake}

3.1.13: We suggest lowering protein intake to $0.8 \mathrm{~g} / \mathrm{kg} / \mathrm{day}$ in adults with diabetes $(2 C)$ or without diabetes (2B) and GFR $<30 \mathrm{ml} / \mathrm{min} / 1.73 \mathrm{~m}^{2}$ (GFR categories G4-G5), with appropriate education.

3.1.14: We suggest avoiding high protein intake $(>1.3 \mathrm{~g} /$ $\mathrm{kg}$ /day) in adults with CKD at risk of progression. $(2 C)$

\section{RATIONALE}

These statements are worded to reflect the potential benefits and dangers of varying dietary protein intake (DPI) in people with CKD. Excess dietary protein leads to the accumulation of uremic toxins, conversely insufficient protein intake may lead to loss of lean body mass, and malnutrition (the latter more frequent in the elderly). The benefits of dietary protein restriction include reduction of accumulation of metabolic waste products that may suppress the appetite and stimulate muscle protein wasting. The role of dietary protein restriction in slowing progression of CKD is more controversial and advanced CKD is associated with a protein wasting syndrome which is directly correlated with morbidity and mortality. Note that statements about reduction in dietary protein do not apply to pediatric populations given issues related to growth and nutrition.

\section{Evidence Base}

A number of systematic reviews and meta-analyses have pooled the available RCT data. ${ }^{285-289}$ Pedrini et al. ${ }^{288}$ compared a low-protein diet (LPD), defined as a DPI of 0.4 to $0.6 \mathrm{~g} / \mathrm{kg} / \mathrm{day}$, with a usual diet ( $5 \mathrm{RCTs}, \mathrm{N}=1413$ ) over a period of follow-up ranging between 18-36 months in people with non-diabetic CKD and GFR $<55 \mathrm{ml} / \mathrm{min} / 1.73 \mathrm{~m}^{2}$. Fouque et al. $^{285}$ updated this analysis to include 8 RCTs in people with non-diabetic CKD $(\mathrm{N}=1524)$. DPI in their low-protein group was between $0.3-0.6 \mathrm{~g} / \mathrm{kg} / \mathrm{day}$ and follow-up ranged from 12-24 months (5 of 8 studies were in people with GFR categories G4-G5 (GFR $<30 \mathrm{ml} / \mathrm{min} /$ $\left.1.73 \mathrm{~m}^{2}\right)$. Roberston et al. ${ }^{289}$ compared diabetic subjects (8 studies in type 1 diabetes, $\mathrm{N}=322 ; 1$ study in type 2 diabetes, $N=263$ ). DPI in the low-protein subjects was $0.3-0.8 \mathrm{~g} / \mathrm{kg} /$ day and usual protein intake ranged from 1-2 $\mathrm{g} / \mathrm{kg} /$ day. Mean follow-up ranged from 4.5 months to 4 years. In all studies, compliance with a low DPI was poor. There was no convincing or conclusive evidence that long-term protein restriction delayed the progression of CKD.

The largest RCT to date was the MDRD Study. ${ }^{227}$ The MDRD Study compared the effects of LPD and BP control on the progression of CKD in over 800 subjects split into 2 groups. Study A compared a DPI of $1.3 \mathrm{~g} / \mathrm{kg} /$ day (usual protein intake) with $0.58 \mathrm{~g} / \mathrm{kg} /$ day (LPD) in 585 subjects with a measured GFR of $25-55 \mathrm{ml} / \mathrm{min} / 1.73 \mathrm{~m}^{2}$ and the actual DPIs were 1.11 and $0.73 \mathrm{~g} / \mathrm{kg} /$ day, respectively. Study B randomized 255 patients with a measured GFR $13-24 \mathrm{ml} /$ $\mathrm{min} / 1.73 \mathrm{~m}^{2}$ to DPIs of $0.58 \mathrm{~g} / \mathrm{kg} /$ day (LPD) or $0.28 \mathrm{~g} / \mathrm{kg} / \mathrm{day}$ supplemented by keto-aminoacids (denoted by VLPD-KA), actual DPIs were 0.69 and $0.46 \mathrm{~g} / \mathrm{kg} / \mathrm{day}$, respectively. In each of the randomization groups ACE-Is were allowed and were used by $32-44 \%$ of patients. Mean follow-up was 2.2 years and the loss of GFR was estimated by the slope of ${ }^{125}$ I-iothalamate clearance measured over 2 years. There was no difference in GFR decline between groups in Study A and in Study B. Although there was a somewhat faster decline in GFR in the LPD group compared with the VLPD-KA group, this was not significant.

A follow-up study of the original MDRD Study followed those subjects recruited to Study B between 1989-1993 up until the year 2000. Median duration of follow-up until kidney failure, death, or administrative censoring was 3.2 years and median time to death was 10.6 years. ${ }^{290}$ The authors concluded that assignment to a very LPD did not delay progression to kidney failure, but appeared to increase the risk of death in the long-term. The chief limitation of this follow-up study was the lack of measurements of DPI and nutritional measurements during the course of the long-term follow-up period and it is therefore not known how many patients continued with the LPD or the VLPD-KA diets after the study concluded.

There is some evidence to suggest that higher protein diets above the recommended daily intake may accelerate renal functional decline in people with early CKD. In a study of 1624 women enrolled in the Nurses' Health Study, Knight et al. described the effect of protein intake over an 11 -year period in women with eGFR $\geq 80 \mathrm{ml} / \mathrm{min} / 1.73 \mathrm{~m}^{2}$ (normal renal function) at baseline and those with eGFR $55-80 \mathrm{ml} / \mathrm{min} / 1.73 \mathrm{~m}^{2} .{ }^{291}$ DPI was measured twice during the study period at intervals of 4 years using a semiquantitative food-frequency questionnaire that inquired about the average intake of specified foods and beverages during the previous year. In women with normal renal function at baseline high protein intake was not significantly associated with change in eGFR. However in those with eGFR $55-80 \mathrm{ml} / \mathrm{min} / 1.73 \mathrm{~m}^{2}$ at baseline, protein intake was significantly associated with a change in eGFR of $-1.69 \mathrm{ml} / \mathrm{min}$ per $1.73 \mathrm{~m}^{2}$ (95\% CI, -2.93 to $-0.45 \mathrm{ml} /$ min per $1.73 \mathrm{~m}^{2}$ ) per $10 \mathrm{~g}$ increase in protein intake. The effect was greatest in those with the highest intake of non-dairy animal protein.

Dietary protein restriction of $<0.80 \mathrm{~g} / \mathrm{kg} / \mathrm{day}$ appears to offer no advantage and any dietary protein restriction should 
include careful monitoring of clinical and biochemical markers of nutritional deficiencies. A high total protein intake, particularly high intake of non-dairy animal protein, may accelerate renal function decline in people with CKD and should be avoided.

\section{International Relevance}

Studies on protein restriction have not been widely tested in different ethnicities or within cultures with low baseline proten intake or purely vegetarian diets. Thus, the applicability of statements to all regions of the world is limited.

\section{Implications for Clinical Practice and Public Policy}

Clinicians should be aware of different sources of protein, and if lowering of protein is recommended, education and monitoring for malnutrition should be implemented. Appropriate dietary counseling for CKD patients may have healthcare resource implications, although as part of a combined strategy to manage obesity, salt intake, and diabetes may be considered cost-effective on a population basis in certain countries. Avoidance of malnutrition is important.

\section{Areas of Controversy, Confusion, or Non-consensus}

Data are mixed on the value of protein restriction, the values which are achievable in general populations, and the level of GFR at which they should be instituted. Nonetheless, the Work Group felt that on balance there is enough data to support a reduction in dietary protein in selected individuals. It is important to avoid this advice in those with evidence of or at risk of malnutrition.

\section{Pediatric Considerations}

A Cochrane review addresses this issue in children ${ }^{292}$ by examining two RCTs with a total of 250 children to determine the effect of protein restriction on a number of variables. The RR of progression to ESRD in the low-protein restricted versus normal group was 1.12 (95\% CI 0.54-2.33). At two years, progression of kidney disease was not significant as measured by change in $\mathrm{CrCl}$ : mean difference $1.47 \mathrm{ml} / \mathrm{min} / 1.73 \mathrm{~m}^{2}$ (95\% CI $\left.-1.19-4.14\right)$ or growth as measured by mean weight difference: $-0.13 \mathrm{~kg}$ (95\% CI $-1.10-0.84)$ or mean height difference: $-1.99 \mathrm{~cm}(95 \% \mathrm{CI}$ $-4.84-0.86$ ). The conclusion of the authors was that a lowprotein diet did not delay progression to kidney failure in children, but it may be detrimental to growth.

\section{Glycemic control}

Diabetes is the leading cause of CKD worldwide. Diabetic nephropathy occurs in $25-40 \%$ of patients with type 1 or type 2 diabetes within 20-25 years of disease onset and is an independent risk factor for early death due to CVD. The mortality rate in people with diabetes and urinary ACR $>30 \mathrm{mg} / \mathrm{g}(>3 \mathrm{mg} / \mathrm{mmol})$ is more than twice that in those with normal urinary albumin levels.

The National Kidney Foundation (NKF) KDOQI Clinical Practice Guideline for Diabetes and $C K D^{293}$ has been updated in 2012. The first three recommendations below are reproduced verbatim from this guideline.

3.1.15: We recommend a target hemoglobin $\mathrm{A}_{1 \mathrm{c}}\left(\mathrm{HbA}_{1 \mathrm{c}}\right)$ of $\sim 7.0 \%(53 \mathrm{mmol} / \mathrm{mol})$ to prevent or delay progression of the microvascular complications of diabetes, including diabetic kidney disease. (1A)

3.1.16: We recommend not treating to an $\mathrm{HbA}_{1 \mathrm{c}}$ target of $<7.0 \%(<53 \mathrm{mmol} / \mathrm{mol})$ in patients at risk of hypoglycemia. (1B)

3.1.17: We suggest that target $\mathrm{HbA}_{1 \mathrm{c}}$ be extended above $7.0 \%(53 \mathrm{mmol} / \mathrm{mol})$ in individuals with comorbidities or limited life expectancy and risk of hypoglycemia. $(2 C)$

3.1.18: In people with $\mathrm{CKD}$ and diabetes, glycemic control should be part of a multifactorial intervention strategy addressing blood pressure control and cardiovascular risk, promoting the use of angiotensin-converting enzyme inhibition or angiotensin receptor blockade, statins, and antiplatelet therapy where clinically indicated. (Not Graded)

\section{RATIONALE}

These statements are included to reflect the current evidence that achieving a hemoglobin $\mathrm{A}_{1 \mathrm{c}}\left(\mathrm{HbA}_{1 \mathrm{c}}\right)$ level of $\sim 7.0 \%$ $(53 \mathrm{mmol} / \mathrm{mol}$ ) is able to prevent the microvascular complications of diabetes, although recognizing that the major risk for patients attaining $\mathrm{HbA}_{1 \mathrm{c}}$ levels $<7.0 \%(<53 \mathrm{mmol} / \mathrm{mol})$ is hypoglycemia, and that this risk will be higher in people with lower levels of kidney function.

\section{Evidence Base}

The evidence base for these statements is reviewed in the NKF KDOQI Clinical Practice Guideline for Diabetes and CKD: 2012 Update $^{293}$ and will not be reiterated in full here. It should be noted that the evidence that intensive glycemic control reduces the microvascular complications of diabetes is based almost exclusively on prevention of development of albuminuria (ACR $>30 \mathrm{mg} / \mathrm{g}$ or $>3 \mathrm{mg} / \mathrm{mmol}$ ) and prevention of increasing albuminuria. Evidence from the three most recent studies, Action in Diabetes and Vascular Disease: Preterax and Diamicron Modified Release Controlled Evaluation (ADVANCE), ${ }^{294}$ Action to Control Cardiovascular Risk in Diabetes (ACCORD), ${ }^{295}$ and the Veterans Affairs Diabetes Trial (VADT), ${ }^{296}$ is summarized in Table 25.

ADVANCE, ACCORD, or VADT did not show significant benefits of more intensive glycemic control on creatininebased estimates of GFR. However, in the Diabetes Control and Complications Trial (DCCT)/Epidemiology of Diabetes Interventions and Complications (EDIC) follow-up study, $1.4 \%$ of participants in the previously intensive treatment group and $3.6 \%$ of those in the previously conventional treatment group developed SCr concentrations $>2.0 \mathrm{mg} / \mathrm{dl}$ $(177 \mu \mathrm{mol} / \mathrm{l}) \quad(\mathrm{P}=0.01)$ and $0.6 \%$ versus $1.9 \%$ required kidney replacement therapy $(\mathrm{P}<0.03) .{ }^{297}$ For patients with type 2 diabetes, intensive treatment in the United Kingdom 
Table 25 | Intensive versus normal glycemic control and albuminuria outcome

\begin{tabular}{|c|c|c|}
\hline Study & Intensified treatment versus normal treatment $\mathrm{HbA}_{1 \mathrm{c}}$ goals & Albuminuria outcome \\
\hline ADVANCE ${ }^{294}$ & $6.5 \%(48 \mathrm{mmol} / \mathrm{mol})$ versus $7.3 \%(56 \mathrm{mmol} / \mathrm{mol})$ & $\begin{array}{l}9 \% \downarrow \text { in new ACR } 3-30 \mathrm{mg} / \mathrm{mmol}(30-300 \mathrm{mg} / \mathrm{g}) \\
30 \% \downarrow \text { in ACR progression to }>30 \mathrm{mg} / \mathrm{mmol}(>300 \mathrm{mg} / \mathrm{g})\end{array}$ \\
\hline ACCORD $^{295}$ & $6.3 \%(45 \mathrm{mmol} / \mathrm{mol})$ versus $7.6 \%(60 \mathrm{mmol} / \mathrm{mol})$ & $\begin{array}{l}21 \% \downarrow \text { in new ACR } 3-30 \mathrm{mg} / \mathrm{mmol}(30-300 \mathrm{mg} / \mathrm{g}) \\
32 \% \downarrow \text { in ACR progression to }>30 \mathrm{mg} / \mathrm{mmol}(>300 \mathrm{mg} / \mathrm{g})\end{array}$ \\
\hline VADT $^{296}$ & $6.9 \%(52 \mathrm{mmol} / \mathrm{mol})$ versus $8.4 \%(68 \mathrm{mmol} / \mathrm{mol})$ & $\begin{array}{l}32 \% \downarrow \text { in new ACR } 3-30 \mathrm{mg} / \mathrm{mmol}(30-300 \mathrm{mg} / \mathrm{g}) \\
37 \% \downarrow \text { in ACR progression to }>30 \mathrm{mg} / \mathrm{mmol}(>300 \mathrm{mg} / \mathrm{g})\end{array}$ \\
\hline
\end{tabular}

Abbreviations: ACCORD, Action to Control Cardiovascular Risk in Diabetes; ACR, albumin-to-creatinine ratio; ADVANCE, Action in Diabetes and Vascular Disease: Preterax and Diamicron Modified Release Controlled Evaluation; $\mathrm{HbA}_{1 \mathrm{C}}$, hemoglobin $\mathrm{A}_{1 \mathrm{C}}$; VADT, Veterans Affairs Diabetes Trial.

Prospective Diabetes Study (UKPDS) was associated with a $67 \%$ risk reduction for a doubling of plasma creatinine levels at 9 years $(0.71 \%$ of the intensive group and $1.76 \%$ of the conventional group, $\mathrm{P}=0.027){ }^{298}$

\section{International Relevance}

The incidence and prevalence of diabetes is rising around the world and at rapid rates in developing countries. Glycemic control is therefore one of the most important strategies for delaying progression of $\mathrm{CKD}$, irrespective of region or country. It is recognized that not all hypoglycemic strategies or treatments are available in all countries.

\section{Implications for Clinical Practice and Public Policy}

Practitioners should encourage glycemic control in all people with CKD and diabetes, including referral to education sessions and diabetic clinics where available. Public health policies and diabetic strategies should include screening in high-risk populations such as those with diabetes, as the presence of CKD would confer higher risk of adverse events, and could represent an opportunity for intensified intervention with large implications for health-care.

\section{Areas of Controversy, Confusion, or Non-consensus}

Recommendations on the use of specific medications for glycemic control (e.g., metformin and glyburide) remain variable depending on different perspectives. These are covered more fully in Chapter 4 on medication dosing and AKI.

\section{Clarification of Issues and Key Points}

Glycemic control improves outcomes in people with diabetes, with or without CKD. Those with diabetes and CKD have higher risk of adverse outcomes and therefore presumed higher benefit from control. Many agents are renally excreted and therefore adjustments to doses may be necessary as GFR declines or if patients are acutely unwell (see Chapter 4 on medication dosing).

Caveats in measuring glycemic control with $\mathrm{HbA}_{1 \mathrm{C}}$ $H b A_{1 C}$ may not be reflective of glucose control in people with CKD who have reduced red cell life span, and thus should be interpreted with caution. Review of blood sugar daily logs may be more reliable.

Clinicians should be aware that $\mathrm{HbA}_{1 \mathrm{C}}$ measurements that inform glycemic control are based on an assumed red cell life span of 90 days. In people with CKD the RBC life span is shortened, even if receiving erythropoiesis-stimulating agents (ESAs). As such, measurement may only reflect glycemic control over a shorter time period than the presumed 3 months and thus, $\mathrm{HbA}_{1 \mathrm{C}}$ measurements may be falsely low. Awareness of this may alter clinicians' reliance on this measurement as a long-term measure. ${ }^{299-304}$ Ongoing research intitiatives comparing $\mathrm{HbA}_{1 \mathrm{C}}$ with glycated albumin using continuous glucose monitoring suggest that glycated albumin may provide a more reliable index of glycemic control in people with advanced CKD.

\section{Pediatric Considerations}

It is recommended that the Guidelines of the American Diabetes Association $^{305}$ or similar national guidelines for diabetes management in children and adolescents, be reviewed to address issues related to management of diabetic children either with, or at risk of, CKD.

\section{Salt intake}

3.1.19: We recommend lowering salt intake to $<90 \mathrm{mmol}$ $(<2 \mathrm{~g})$ per day of sodium (corresponding to $5 \mathrm{~g}$ of sodium chloride) in adults, unless contraindicated (see rationale). (1C)

3.1.19.1: We recommend restriction of sodium intake for children with CKD who have hypertension (systolic and/or diastolic blood pressure $>95^{\text {th }}$ percentile) or prehypertension (systolic and/or diastolic blood pressure $>90^{\text {th }}$ percentile and $<95^{\text {th }}$ percentile), following the age-based Recommended Daily Intake. (1C)

3.1.19.2: We recommend supplemental free water and sodium supplements for children with CKD and polyuria to avoid chronic intravascular depletion and to promote optimal growth. (1C)

\section{RATIONALE}

In subjects with CKD, impaired excretion of sodium is often present. High sodium intake increases BP and proteinuria, induces glomerular hyperfiltration and blunts the response to RAAS blockade. Lowering salt intake not only reduces BP, but also lowers albuminuria. The importance of salt intake in the general management of CKD patients cannot be overemphasized, hence the need for specific statements here. We appreciate that there are some conditions in which salt 
restriction may be harmful, and hence the qualifier "unless contraindicated.' These conditions include salt losing nephropathies and those prone to hypotension and volume contraction who do not have heart failure.

\section{Evidence Base}

A systematic review of 16 studies addressing salt intake and kidney disease set out to establish whether variations in dietary sodium consumption influence renal outcomes in people with $\mathrm{CKD}^{306}$ Despite marked heterogeneity, the review suggested that increased salt intake was associated with worsening albuminuria and an increased likelihood of reduction of GFR. Although the quality of the studies included was insufficient to support the authors' hypothesis that increased salt intake is nephrotoxic, the results were strong enough to suggest modest dietary avoidance of salt should be encouraged in people with CKD, especially those with hypertension and/or proteinuria. In a randomized, double-blind, placebo-controlled trial of salt reduction in 40 Afro-Caribbean hypertensives, a salt-restricted diet (approximately $5 \mathrm{~g}$ daily) significantly reduced 24-hour urinary protein excretion by $19 \%$ and led to a fall in systolic and diastolic $\mathrm{BP}$ of $8 \mathrm{~mm} \mathrm{Hg}$ and $3 \mathrm{~mm} \mathrm{Hg}$, respectively. ${ }^{307}$ The fall in urinary protein excretion correlated with a reduction in urinary sodium excretion, not with BP reduction. Individuals with metabolic syndrome may be especially sensitive to the effects of sodium intake. Hoffman and Cubeddu examined the role of salt intake in increased BP in 109 subjects with metabolic syndrome. ${ }^{308}$ Salt restriction from an average usual intake of $8.2 \mathrm{~g} /$ day to nearly $2.3 \mathrm{~g} /$ day reduced the percentage of hypertensive patients from $23.8 \%$ to $8.2 \%$. In a six-month prospective controlled trial, 110 patients with GFR categories G4 or G5 (GFR $<30 \mathrm{ml} / \mathrm{min} /$ $1.73 \mathrm{~m}^{2}$ ) followed a low-sodium (circa $1 \mathrm{~g} /$ day) diet as part of either a LPD $(0.6 \mathrm{~g} / \mathrm{kg} /$ day $)$ or very low-protein $\operatorname{diet}(\mathrm{VLPD})$ supplemented with essential amino acids $(0.35 \mathrm{~g} / \mathrm{kg} /$ day $)$, or a free diet. $^{309}$ BP fell significantly in the VLPD group from $143 \pm 19 / 84 \pm 10$ to $128 \pm 16 / 78 \pm 7 \mathrm{~mm} \mathrm{Hg} \quad(\mathrm{P}<0.0001)$, despite reduction of antihypertensive drugs. The improved BP correlated with decreased urinary sodium and the authors concluded that the antihypertensive effect was due to reduction of salt intake independent of actual protein intake. Finally, a randomized controlled crossover trial in 52 nondiabetic subjects with CKD compared the effects of a lowsodium diet (target $50 \mathrm{mmol}$ [ $1.15 \mathrm{~g}]$ sodium per day) versus a regular sodium diet (target $200 \mathrm{mmol}$ [ $4.60 \mathrm{~g}$ ] sodium per day) on lowering of proteinuria through RAAS blockade. ${ }^{310}$ The reduction of proteinuria by the addition of a low-sodium diet to angiotensin-converting enzyme inhibition was significantly larger $(\mathrm{P}<0.001)$ than the addition of angiotensin receptor blockade to angiotensin-converting enzyme inhibition and similarly the reduction of systolic BP by the addition of a low-sodium diet was significantly larger $(\mathrm{P}=$ 0.003 ) than the addition of angiotensin receptor blockade. The authors concluded that sodium restriction to a level recommended in guidelines was more effective than dual renin-angiotensin blockade for reduction of proteinuria and $\mathrm{BP}$ in non-diabetic nephropathy.

\section{International Relevance}

Salt intake has been identified as an important driver of high $\mathrm{BP}$ in all countries and in adults, thus this recommendation is of international relevance and applicability. The relevance and caveats to children in different situations around the world merits further review.

\section{Implications for Clinical Practice and Public Policy}

In most developed countries, a reduction in salt intake can be achieved by a gradual and sustained reduction in the amount of salt added to foods by the food industry. In other countries where most of the salt consumed comes from salt added during cooking or from sauces, a public health campaign is needed to encourage consumers to use less salt. The range of sodium intake associated with improved long-term outcomes is easily achievable and may have the potential to greatly improve health outcomes in patients with CKD around the world.

\section{Areas of Controversy, Confusion, or Non-consensus}

While salt restriction is of benefit on a population-basis, and in most people with hypertension and CKD, there may be individual conditions in which salt restriction might be detrimental (e.g., those with salt wasting tubular disorders; those either hypotensive in the absence of heart failure or those prone to volume contraction). Thus while this general statement applies to the majority of people, individualization is required based on clinical circumstance. This may be particularly relevant to children.

\section{Pediatric Considerations}

The suggested relative salt restrictions for children with CKD and/or hypertension are age-based with consideration given to both average intakes and upper limit values. The recommended upper limit for sodium intake from which a reduced intake could be derived is provided in Table 26.

It must also be recognized that children with CKD often have underlying tubular conditions that predispose to numerous electrolyte losses, including sodium. For these children a supplemental rather than restricted sodium intake will be required.

\section{Table 26| Recommended Daily Intake of sodium for healthy} children

\begin{tabular}{ll}
\hline Age & Upper Limit \\
\hline $0-6$ months & No data \\
$7-12$ months & No data \\
$1-3$ years & $1500 \mathrm{mg}$ \\
$4-8$ years & $1900 \mathrm{mg}$ \\
$9-13$ years & $2200 \mathrm{mg}$ \\
$14-18$ years & $2300 \mathrm{mg}$ \\
\hline
\end{tabular}




\section{Hyperuricemia}

3.1.20: There is insufficient evidence to support or refute the use of agents to lower serum uric acid concentrations in people with CKD and either symptomatic or asymptomatic hyperuricemia in order to delay progression of CKD. (Not Graded)

Hyperuricemia is common in people with CKD and is defined by urate concentrations above $7.0 \mathrm{mg} / \mathrm{dl}(420 \mu \mathrm{mol} / \mathrm{l})$ as measured by automated enzymatic (uricase) laboratory methods. Concentrations obtained with colorimetric methods are approximately $1 \mathrm{mg} / \mathrm{dl} \quad(60 \mu \mathrm{mol} / \mathrm{l})$ lower. The Work Group believe it important to acknowledge the accumulating body of evidence describing the association of hyperuricemia with CKD and adverse cardiovascular outcomes, and thus list hyperuricemia as a potential contributor to progression. However, at the time of current writing, there is not a reliable body of evidence from which to recommend treatment of hyperuricemia for the specific goal of delaying progression of CKD. Thus, the wording of this ungraded statement is very deliberate.

\section{RATIONALE}

Observational data had implicated uric acid in the progression of CKD suggesting that adverse outcomes in people with CKD may be improved by specific uric acid lowering therapy. Small studies using appropriate RCT design have shown reduced left ventricular mass, improved endothelial function, and reduced progression of CKD in people with either symptomatic or asymptomatic hyperuricemia and CKD. Thus, the specific statement is intended to ensure and foster RCTs to properly assess the risk and benefits of uric acid lowering strategies in people with CKD.

\section{Evidence Base}

Published data implicate elevated serum uric acid (SUA) concentrations in the progression of CKD. ${ }^{311-315}$ Reduction of SUA by allopurinol has been reported to delay progression of $\mathrm{CKD}$ in people with both diabetic and nondiabetic CKD. ${ }^{316,317}$ Treatment of asymptomatic hyperuricemia has also been reported to improve kidney function even in subjects with normal levels of GFR. ${ }^{318,319}$ Both GFR and endothelial function significantly improved in asymptomatic hyperuricemic subjects randomly assigned to $300 \mathrm{mg} /$ day of allopurinol in comparison to placebo. ${ }^{318}$ A separate doubleblind, placebo-controlled, parallel-group study in 67 people with CKD (GFR 30-60 ml/min/1.73 $\mathrm{m}^{2}$ ) and left ventricular hypertrophy (LVH) randomly assigned subjects to treatment with allopurinol $(300 \mathrm{mg} /$ day $)$ or placebo for 9 months. ${ }^{320}$ In comparison to placebo, the allopurinol-treated subjects had significant reductions in left ventricular mass assessed by magnetic resonance imaging (MRI), and improvements in endothelial function assessed by flow-mediated dilation of the brachial artery and in central arterial stiffness assessed by pulse-wave analysis. Another study randomized 70 subjects with known hyperuricemia or SUA concentra- tions $\geq 7.0 \mathrm{mg} / \mathrm{dl}(\geq 420 \mu \mathrm{mol} / \mathrm{l})$ to treatment with either allopurinol monotherapy (100-200 mg/day) or a combination of allopurinol and a citrate preparation $(3 \mathrm{~g} /$ day $) .{ }^{321}$ SUA concentrations were decreased in both groups but to a significantly lower level by combination treatment. GFR assessed by $\mathrm{CrCl}$ increased in the combination therapy group but remained unchanged in those treated with allopurinol alone. Other uric acid lowering agents have also been reported to improve outcomes in people with CKD. In an 8-week, placebo-controlled group comparison of rasburicase and placebo, a single $4.5 \mathrm{mg}$ dose of rasburicase significantly lowered SUA and resulted in a significant improvement in kidney function assessed by $\mathrm{CrCl}^{322}$ In a post hoc analysis of 1342 patients with type 2 diabetes mellitus and nephropathy participating in the Reduction of Endpoints in Non-InsulinDependent Diabetes Mellitus with the Angiotensin II Antagonist Losartan (RENAAL) trial, Miao et al. examined the relationship between change in SUA concentration after 6 months of treatment with losartan and doubling of SCr or ESRD. ${ }^{323}$ Baseline SUA was $6.7 \mathrm{mg} / \mathrm{dl}(400 \mu \mathrm{mol} / \mathrm{l})$ in placebo and losartan-treated subjects. During the first 6 months, losartan lowered SUA by $0.16 \mathrm{mg} / \mathrm{dl}(9.5 \mu \mathrm{mol} / \mathrm{l})$ [95\% CI $0.30-0.01 ; \mathrm{P}=0.031$ ] as compared with placebo. The risk of doubling of SCr or ESRD was decreased by $6 \%$ (95\% CI 10\%$3 \%)$ per $0.5-\mathrm{mg} / \mathrm{dl}(30-\mu \mathrm{mol} / \mathrm{l})$ decrement in SUA during the first 6 months. This effect was independent of other risk markers, including albuminuria.

\section{International Relevance}

There are no data to support or refute the importance of hyperuricemia in different geographical reasons or ethnic groups. Further study is needed.

\section{Implications for Clinical Practice and Public Policy}

There is insufficient evidence to recommend the use of uric acid lowering agents in asymptomatic individuals for the specific purpose of delaying CKD progression. Further large trials are required to better understand the potential benefit of uric acid lowering for this purpose.

\section{Lifestyle}

3.1.21: We recommend that people with CKD be encouraged to undertake physical activity compatible with cardiovascular health and tolerance (aiming for at least 30 minutes 5 times per week), achieve a healthy weight (BMI 20 to 25 , according to country specific demographics), and stop smoking. (1D)

\section{RATIONALE}

People with CKD have self-reported reduced physical functioning and are not as aerobically fit as the general population. Frailty, impaired physical performance, disability, and geriatric syndromes are common among older adults even with mild kidney disease. Reduced physical functioning and inactivity are associated with increased mortality and poor QOL. Obesity is associated with increased morbidity, 
mortality, and reduction in life expectancy and leads to an increase in the incidence of diabetes, hypertension, and dyslipidemia. Associations between smoking and CKD suggest that smoking increases the risk of kidney failure and that smoking cessation decreases that risk. Thus, this statement reflects the importance of ensuring lifestyle recommendations.

\section{Evidence Base}

CKD patients have a reduced exercise capacity and impaired physical functioning. ${ }^{324-326}$ Moreover, reduced physical activity is associated with increased mortality and poor QOL in people with CKD. ${ }^{327-329}$ Regular exercise leads to increased exercise capacity, decreased morbidity, and improved health-related QOL. ${ }^{330-332}$ Exercise may reduce cardiovascular risk through its beneficial effects on BP, triglycerides, high-density lipoprotein cholesterol (HDL-C), insulin resistance, and glycemic control. In ESRD, exercise has been shown to improve arterial stiffness, BP, cardiorespiratory function, and QOL. ${ }^{333-339}$ Less data are available on the beneficial effects of exercise on early CKD. However, as cardiovascular risk gradually increases with both a lower GFR and a higher ACR, it is expected that exercise will also help to prevent progressive CVD in less severe CKD. Indeed, in subjects with GFR categories G3a-G4 (GFR $15-59 \mathrm{ml} / \mathrm{min} /$ $1.73 \mathrm{~m}^{2}$ ), long-term exercise training improved physical impairment, arterial stiffness, and health-related QOL. ${ }^{340}$ It has therefore been argued that exercise training is imperative in CKD patients ${ }^{341}$ and that support programs including selfmonitoring, verbal reinforcement, and motivation should be applied $^{342-344}$ in an attempt to prevent the high cardiovascular risk in CKD. A prospective study compared the benefits of 6 months of regular walking in 40 predialysis patients with GFR categories G4-G5 (GFR $\left.<30 \mathrm{ml} / \mathrm{min} / 1.73 \mathrm{~m}^{2}\right)(20$ in an exercising group and 20 patients who continued with usual physical activity for comparison). Improvements noted after 1 month were sustained to 6 months in the 18 of 20 who completed the exercise study. These included improvements in exercise tolerance (reduced exertion to achieve the same activity), weight loss, improved cardiovascular reactivity, avoiding an increase in BP medication and improvements in quality of health and life and uremic symptom scores as assessed by questionnaire. ${ }^{345}$

In the absence of diabetes, hypertension or other cardiovascular risk factors there has been a lack of evidence to support a causal link between obesity and CKD. Observational studies suggest that obesity is an independent risk factor for CKD. ${ }^{346-348}$ The evidence in population studies is conflicting; some studies have failed to link obesity with decreased GFR, ${ }^{349,350}$ possibly because BMI in isolation is a poor measure, while others suggest that $\mathrm{CKD}$ is independently associated with BMI. ${ }^{351}$ It has been known for some time that obesity is associated with secondary focal and segmental glomerulosclerosis, ${ }^{352}$ yet significant associations between obesity and CKD in large observational studies such as The Framingham Heart Study disappear after adjustment for age, gender, and cardiovascular risk factors. ${ }^{353}$ However, systematic review and meta-analysis of weight loss interventions in CKD have shown weight loss to be associated with significant decrease in proteinuria and systolic BP with no further decrease in GFR in people with CKD during a mean follow-up of 7.4 months. ${ }^{354}$ A further systematic review drew very similar conclusions. Weight loss interventions were associated with decreased proteinuria and albuminuria by $1.7 \mathrm{~g}$ (95\% CI $0.7-2.6 \mathrm{~g})$ and $14 \mathrm{mg}$ (95\% CI $11-17 \mathrm{mg}$ ), respectively $(\mathrm{P}<0.05) .{ }^{355}$ Each $1-\mathrm{kg}$ weight loss was associated with $110 \mathrm{mg}$ (95\% CI 60-160 mg; $\mathrm{P}<0.001)$ decrease in proteinuria and $1.1 \mathrm{mg}$ ( $95 \% \mathrm{CI} 0.5-2.4 \mathrm{mg} ; \mathrm{P}=0.011$ ) decrease in albuminuria, respectively, independent of reduction in BP.

Multiple studies document a clear association between smoking and renal damage in the general population, patients with diabetes, and hypertensive patients. ${ }^{356}$ Smoking is causally linked to cardiovascular events in the general population and is also associated with an increased risk for cardiovascular events in patients with CKD. ${ }^{357-360}$ Studies investigating the beneficial effects of smoking cessation on kidney function have all been positive. ${ }^{361-365}$

\section{International Relevance}

Exercise, weight loss and smoking cessation in CKD are equally important in all countries and thus this recommendation is of international relevance and applicability.

\section{Implications for Clinical Practice and Public Policy}

Implementation of this recommendation has no public health cost but has the potential to make far-reaching public health gains both in terms of population health and healthcare economics.

\section{Additional dietary advice}

3.1.22: We recommend that individuals with CKD receive expert dietary advice and information in the context of an education program, tailored to severity of CKD and the need to intervene on salt, phosphate, potassium, and protein intake where indicated. (1B)

\section{RATIONALE}

\section{International Relevance}

The importance of lifestyle recommendations and dietary counseling cannot be overstated. It is however recognized that within the context of different countries, health-care systems and jurisdictions, the degree to which these can be implemented is variable. These recommendations serve as a 'best practice' suggestion.

\subsection{COMPLICATIONS ASSOCIATED WITH LOSS OF KIDNEY FUNCTION}

People with CKD are prone to develop a variety of complications which reflect loss of endocrine or exocrine function of 
Table 27| Prevalence of CKD complications by GFR category* derived from CKD cohorts

\begin{tabular}{lrrrrrr}
\hline \multirow{2}{*}{ Complication } & \multicolumn{4}{c}{ GFR category $(\mathrm{ml} / \mathrm{min} / 1.73$} & $\left.\mathrm{m}^{2}\right)$ & \multirow{2}{*}{ Reference } \\
\cline { 2 - 6 } & $\geq 90$ & $60-89$ & $\mathbf{4 5 - 5 9}$ & $30-44$ & $<30$ & \\
\hline Anemia $^{1}$ & $4.0 \%$ & $4.7 \%$ & $12.3 \%$ & $22.7 \%$ & $51.5 \%$ & 366 \\
Hypertension $^{2}$ & $18.3 \%$ & $41.0 \%$ & $71.8 \%$ & $78.3 \%$ & $82.1 \%$ & 366 \\
25(OH) Vit D deficiency $^{3}$ & $14.1 \%$ & $9.1 \%$ & $10.7 \%$ & $27.2 \%$ & 367 \\
Acidosis $^{4}$ & $11.2 \%$ & $8.4 \%$ & $9.4 \%$ & $18.1 \%$ & $31.5 \%$ & 366 \\
Hyperphosphatemia $^{5}$ & $7.2 \%$ & $7.4 \%$ & $9.2 \%$ & $9.3 \%$ & $23.0 \%$ & 366 \\
Hypoalbuminemia $^{6}$ & $1.0 \%$ & $1.3 \%$ & $2.8 \%$ & $9.0 \%$ & $7.5 \%$ & 366 \\
Hyperparathyroidism $^{7}$ & $5.5 \%$ & $9.4 \%$ & $23.0 \%$ & $44.0 \%$ & $72.5 \%$ & 366 \\
\hline
\end{tabular}

Abbreviations: CKD, chronic kidney disease; GFR, glomerular filtration rate.

* Note that modification of prevalence according to albuminuria categories data is not yet available to inform this table adequately, though there are limited data to suggest increasing prevalence of hypoalbuminemia, hypertension, anemia, and acidosis as albuminuria category increases

${ }^{1}$ Defined as hemoglobin levels $<12 \mathrm{~g} / \mathrm{dl}(120 \mathrm{~g} / \mathrm{l})$ for women; $<13.5 \mathrm{~g} / \mathrm{dl}(135 \mathrm{~g} / \mathrm{l})$ for men

${ }^{2}$ Defined as a systolic blood pressure $\geq 140 \mathrm{~mm} \mathrm{Hg}$, diastolic blood pressure $\geq 90 \mathrm{~mm} \mathrm{Hg}$, or self-reported use of antihypertensive medication

${ }^{3}$ Less than $15 \mathrm{ng} / \mathrm{ml}$ [37 nmol/l] (as defined in Study for the Evaluation of Early Kidney disease [SEEK])

${ }^{4}$ Defined as serum bicarbonate less than $21 \mathrm{mEq} / \mathrm{l}$

${ }^{5}$ Defined as serum phosphate $\geq 4.5 \mathrm{mg} / \mathrm{dl}$ ( $\left.\geq 1.5 \mathrm{mmol} / \mathrm{l}\right)$

${ }^{6}$ Defined as serum albumin less than $3.5 \mathrm{~g} / \mathrm{dl}(35 \mathrm{~g} / \mathrm{l})$

${ }^{7}$ Defined as PTH levels $\geq 70 \mathrm{pg} / \mathrm{ml}$; $(\geq 7.4 \mathrm{pmol} / \mathrm{l})$

the kidneys. The incidence and prevalence of these complications increase with severity of CKD as defined predominantly by GFR categories (Table 27).

It is beyond the scope of this guideline to describe each of the complications and the proposed treatment options for them in detail as guidance for these conditions can be found in other documents. However, for the purpose of completeness, the key complications and management recommendations for people with CKD are addressed in this section.

In addition to these complications, we have described strategies to delay progression of CKD which are in part predicated on the identification and management of the clinical, metabolic, and hematologic complications. Note that not all people with CKD will have all of the complications and complications may not occur at the same rate or to the same degree in individuals with the same categories of GFR or albuminuria. Nonetheless knowledge of the common complications and treatment options is important in the care of CKD.

\section{Management of Complications}

Anemia in CKD. Anemia is an important complication of CKD because it contributes significantly to the heavy symptom burden of CKD. It has a major impact on the lives of people with CKD but it is potentially reversible with appropriate treatment. The guideline statements included here are those we consider to be the key considerations for people with non-dialysis CKD. Interested readers are referred to the KDIGO Clinical Practice Guideline for Anemia in $C K D^{11}$ for comprehensive guidance on this topic.
Table 28 | Hemoglobin thresholds used to define anemia

\begin{tabular}{ll}
\hline Age or gender group & Hemoglobin threshold: $\mathbf{g} / \mathbf{d l}(\mathbf{g} / \mathbf{l})$ \\
\hline Children & \\
6 mo-5 yr & $11.0(110)$ \\
$5-12 \mathrm{yr}$ & $11.5(115)$ \\
$12-15 \mathrm{yr}$ & $12.0(120)$ \\
Non-pregnant females $>15 \mathrm{yr}$ & $12.0(120)$ \\
Pregnant females $>15 \mathrm{yr}$ & $11.0(110)$ \\
Men $>15 \mathrm{yr}$ & $13.0(130)$ \\
\hline
\end{tabular}

Abbreviations: mo, month; yr, year.

Reproduced with permission from World Health Organization. Worldwide prevalence of anaemia 1993-2005: WHO global database on anaemia. ${ }^{368}$ In: de Benoist B, McLean E, Egli I and Cogswell M (eds), 2008; accessed: http://whqlibdoc.who.int/ publications/2008/9789241596657_eng.pdf.

\section{Definition and identification of anemia in CKD}

3.2.1: Diagnose anemia in adults and children $>15$ years with CKD when the $\mathrm{Hb}$ concentration is $<13.0 \mathrm{~g} /$ $\mathrm{dl}(<130 \mathrm{~g} / \mathrm{l})$ in males and $<12.0 \mathrm{~g} / \mathrm{dl}(<120 \mathrm{~g} / \mathrm{l})$ in females. (Not Graded)

3.2.2: Diagnose anemia in children with $\mathrm{CKD}$ if $\mathrm{Hb}$ concentration is $<11.0 \mathrm{~g} / \mathrm{dl}(<110 \mathrm{~g} / \mathrm{l})$ in children $0.5-5$ years, $<11.5 \mathrm{~g} / \mathrm{dl}(115 \mathrm{~g} / \mathrm{l})$ in children $5-12$ years, and $<12.0 \mathrm{~g} / \mathrm{dl}(120 \mathrm{~g} / \mathrm{l})$ in children $12-15$ years. (Not Graded)

\section{RATIONALE}

These statements reflect the need to measure $\mathrm{Hb}$ in people with CKD in order to detect if anemia is present, given its association with poor outcomes and its use in prediction models. Anemia is a common occurrence in CKD patients, though variable in its time of presentation and severity within individuals. Thus, the guidelines stress that evaluation and treatment of anemia in people CKD should be undertaken as in other individuals, and emphasize that anemia due to CKD is a diagnosis of exclusion. Furthermore, the guidelines stress that laboratory values used for diagnosis do not imply therapeutic thresholds or targets. For details, consult KDIGO Clinical Practice Guideline for Anemia in $C K D{ }^{11}$

\section{Evidence Base}

Conventionally anemia is defined as a $\mathrm{Hb}$ concentration lower than the established cutoff defined by the WHO. ${ }^{368}$ Different biological groups have different cutoff $\mathrm{Hb}$ values below which anemia is said to be present, ranging from $11 \mathrm{~g} / \mathrm{dl}(110 \mathrm{~g} / \mathrm{l})$ for pregnant women and for children between 6 months and 5 years of age, to $12 \mathrm{~g} / \mathrm{dl}(120 \mathrm{~g} / \mathrm{l})$ for non-pregnant women, and to $13 \mathrm{~g} / \mathrm{dl}(130 \mathrm{~g} / \mathrm{l})$ for men (Table 28).

\section{International Relevance}

Globally the prevalence of anemia is $24.8 \%$ of the population, much of this is linked to nutritional deficiency exacerbated by 
infectious disease. However, altitude, race, and smoking will each also have an impact on $\mathrm{Hb}$ concentration. $\mathrm{Hb}$ concentration can be expected to increase by about $0.6 \mathrm{~g} / \mathrm{dl}$ $(6 \mathrm{~g} / \mathrm{l})$ in women and $0.9 \mathrm{~g} / \mathrm{dl}(9 \mathrm{~g} / \mathrm{l})$ in men for each $1000 \mathrm{~m}$ of altitude above sea level. ${ }^{369} \mathrm{Hb}$ concentrations also vary between races, with African-American individuals consistently showing concentrations 0.5 to $0.9 \mathrm{~g} / \mathrm{dl}$ ( 5 to $9 \mathrm{~g} / \mathrm{l}$ ) lower than those of whites or Asian populations. ${ }^{370-372}$ Smoking is associated with elevated carboxyhemoglobin levels, which are associated with a compensatory increase in total $\mathrm{Hb}$ concentration. Hence, the US Centers for Disease Control and Prevention have recommended a downwards adjustment of $0.3 \mathrm{~g} / \mathrm{dl}(3 \mathrm{~g} / \mathrm{l})$ for smokers. ${ }^{373}$ Thus, specific $\mathrm{Hb}$ levels expected for different levels of eGFR should be contextualized within the normal distribution of the population.

\section{Implications for Clinical Practice and Public Policy}

The recommended thresholds for diagnosis and evaluation of anemia should not be interpreted as being thresholds for treatment of anemia but simply for the identification of this complication. Practice preferences with respect to treatment strategies should be directed according to local resources.

\section{Evaluation of anemia in people with CKD}

3.2.3: To identify anemia in people with CKD measure $\mathrm{Hb}$ concentration (Not Graded):

- when clinically indicated in people with GFR $\geq 60 \mathrm{ml} / \mathrm{min} / 1.73 \mathrm{~m}^{2}$ (GFR categories G1-G2);

- at least annually in people with GFR $30-59 \mathrm{ml} /$ $\mathrm{min} / 1.73 \mathrm{~m}^{2}$ (GFR categories G3a-G3b);

- at least twice per year in people with GFR $<30 \mathrm{ml} / \mathrm{min} / 1.73 \mathrm{~m}^{2}$ (GFR categories G4-G5).

\section{RATIONALE}

The recommendation that patients be evaluated at least annually rests on observations from clinical trials that (in the absence of ESA therapy) the natural history of anemia in patients with CKD is a gradual decline in $\mathrm{Hb}$ concentration over time. ${ }^{374-376}$ The exact frequency of monitoring of $\mathrm{Hb}$ concentration will be influenced by kidney function, underlying disease process, the initial $\mathrm{Hb}$ concentration, and the rate of change in $\mathrm{Hb}$ concentration. The latter will also be influenced by whether or not anemia is being treated and what type of treatment is undertaken. The statements specifically address the need to measure $\mathrm{Hb}$ concentrations at a minimum and are not intended to deter the clinician from more frequent measurements as required for individual circumstances.

The initial evaluation of anemia in CKD is geared towards the exclusion of causes other than those directly related to kidney disease (relative iron and erythropoietin deficiency); see KDIGO Clinical Practice Guideline for Anemia in $C K D^{11}$ for details.

\section{Evidence Base}

Anemia as defined above is found in people with CKD in increasing proportions as GFR declines (Table 27). This is due to a number of reasons, including loss of erythropoietic hormone efficacy, production, substrate deficiency (most notably iron), and other conditions which may contribute to the lack of effective erythropoiesis. For a full description of the evidence behind this statement please refer to the KDIGO Clinical Practice Guideline for Anemia in CKD. ${ }^{11}$

\section{International Relevance}

Frequency of $\mathrm{Hb}$ concentration monitoring and the initial evaluation of anemia should not differ by country, except that causes of anemia other than those related to CKD do differ. Nutritional deficiencies in resource-poor areas are common, particularly iron deficiency, which is frequently exacerbated by infectious diseases.

\section{Implications for Clinical Practice and Public Policy}

Anemia is associated with increased morbidity, mortality, and consumption of health-care resources. The major health consequences include poor pregnancy outcome, impaired physical and cognitive development, increased risk of morbidity in children, and reduced work productivity in adults.

Treatment of anemia in CKD. The last 30 years have seen a major transition in approach to the treatment of anemia in people with CKD beginning with the introduction of erythropoietin therapy into clinical practice and the subsequent resurgence of interest in iron therapies. The promise of early intervention with anemia treatment in non-dialysis CKD suggested by observational study has been tempered with the reality of risks of adverse cardiovascular outcomes in RCTs. Nevertheless treatment of anemia with iron and ESAs has an important positive role to play in the lives of people with CKD. For the treatment of anemia in people with CKD, we suggest clinicians refer to the KDIGO Clinical Practice Guideline for Anemia in CKD for further details. ${ }^{11}$

The key points for practitioners to remember include the following:

1. Work-up for anemia in CKD should include assessment of secondary causes including iron deficiency.

2. Iron replacement is often effective in anemia of CKD as initial therapy and routes of administration (intravenous or oral) will be determined by clinicians, patient preferences, and local available resources.

3. ESA therapy is not recommended in those with active malignancy, or recent history of malignancy.

4. In most people with CKD, ESAs should not be used to intentionally increase the $\mathrm{Hb}$ concentration above 11.5 $\mathrm{g} / \mathrm{dl}(115 \mathrm{~g} / \mathrm{l})$

5. For pediatric patients, the selection of $\mathrm{Hb}$ concentration at which ESA therapy is initiated should be individualized after taking into account the potential benefits (e.g., improvement in QOL, school 
attendance/performance, and avoidance of transfusion) and potential harms.

\subsection{CKD METABOLIC BONE DISEASE INCLUDING LABORATORY ABNORMALITIES}

Changes in bone mineral metabolism and alterations in calcium and phosphate homeostasis occur early in the course of CKD and progress as kidney function declines (Table 27). These changes are grouped under the umbrella term Chronic Kidney Disease-Mineral and Bone Disorder (CKD-MBD) which includes renal osteodystrophy and extraskeletal (vascular) calcification related to abnormalities of bone mineral metabolism. Renal osteodystrophy is the component of CKD-MBD that is identified and quantified through bone biopsy histomorphometry and includes osteitis fibrosa (hyperparathyroidism), osteomalacia, and adynamic bone disease.

The evidence on which existing recommended guideline treatment targets for serum concentrations of calcium, phosphate, and parathyroid hormone (PTH), and the strategies to achieve these targets, is exclusively observational and thus problematic for that reason. Furthermore, very little of the evidence is derived from patients with non-dialysis CKD. Nevertheless we feel it is important to include here some of the key statements relating to mineral metabolism abnormalities in patients with non-dialysis CKD from the KDIGO Clinical Practice Guideline for the Diagnosis, Evaluation, Prevention, and Treatment of Chronic Kidney Disease-Mineral and Bone Disorder (CKD-MBD) published in 2009. ${ }^{9}$ These statements will be qualified where new data since the publication of the CKD-MBD guidance have become available.

\subsection{1: We recommend measuring serum levels of calcium, phosphate, PTH, and alkaline phosphatase activity at least once in adults with GFR $<45 \mathrm{ml} / \mathrm{min} /$ $1.73 \mathrm{~m}^{2}$ (GFR categories G3b-G5) in order to determine baseline values and inform prediction equations if used. (1C)}

\section{RATIONALE}

As kidney function declines, abnormalities of serum calcium, phosphate, and circulating hormones related to CKD-MBD progress. These include PTH; 25-hydroxyvitamin D $(25(\mathrm{OH}) \mathrm{D}), 1,25$-dihydroxyvitamin $\mathrm{D}\left(1,25(\mathrm{OH})_{2} \mathrm{D}\right)$, and other vitamin D metabolites; fibroblast growth factor-23 (FGF-23); and growth hormone. At the tissue level there is down regulation of vitamin $\mathrm{D}$ receptors and resistance to the actions of PTH. Immunohistochemical abnormalities in bone also occur early and generally precede changes in mineral homeostasis. Extraskeletal calcification may result from deranged mineral and bone metabolism and from the therapies used in an attempt to correct these abnormalities. Associations between disorders of mineral metabolism and CVD have widened the focus of CKD-MBD to include abnormal mineral metabolism, abnormal bone, and extra- skeletal calcification. Once baseline values have been obtained, the subsequent frequency of testing will be determined on an individual basis by the actual value and any intervention that may be introduced.

\section{Evidence Base}

There are observational studies which describe the abnormalities of each of these parameters at relatively high values of eGFR in general and high-risk population cohorts. ${ }^{367,377,378}$ Importantly, abnormalities of calcium and phosphate appear to occur relatively later in the course of CKD than do abnormalities in values of $1,25(\mathrm{OH})_{2} \mathrm{D}, 25(\mathrm{OH}) \mathrm{D}$, and PTH. Thus, the recommendation is to evaluate these parameters relatively early in the trajectory of CKD, as an assessment of burden of illness. In dialysis patients, the highest risks for mortality have been reported with combinations of high serum phosphate and calcium together with either high PTH (RR 3.71; 95\% CI 1.53-9.03; P = 0.004) or low PTH (RR 4.30; 95\% CI 2.01-9.22; $\mathrm{P}<0.001$ ) compared with the combination of high PTH with normal serum calcium and phosphate which had the lowest mortality and was used as the index category. ${ }^{379}$ The importance of examining combinations of parameters of mineral metabolism is likely to be no different in patients with less severe CKD, but this has not been tested in non-dialysis populations.

There are also racial differences in the parameters of mineral metabolism. In a multicenter cohort of 227 black and 1633 non-black patients with early CKD, blacks had similar $1,25(\mathrm{OH})_{2} \mathrm{D}$ levels compared with non-blacks but significantly lower levels of 25(OH)D with higher levels of calcium, phosphate, and $\mathrm{PTH}$, and were significantly more likely to have hyperphosphatemia than non-blacks. ${ }^{380}$ In multivariable analyses adjusted for age, gender, eGFR, BMI, and diabetes, blacks had significantly lower $25(\mathrm{OH}) \mathrm{D}$ and higher PTH levels than non-blacks. Examining the relationships between $25(\mathrm{OH}) \mathrm{D}$ and PTH in 8415 adult participants $(25 \%$ black and 24\% Mexican-American) in NHANES 2003-2004 and 2005-2006, and the relationship between 25(OH)D and bone mineral density (BMD) in 4206 adult participants (24\% black and 24\% Mexican-American) in the 2003-2004 NHANES sample, Guitierrez et al. found significant racial differences. ${ }^{381}$ Blacks and Mexican-Americans had significantly lower $25(\mathrm{OH}) \mathrm{D}$ and higher PTH concentrations than whites $(\mathrm{P}<0.01$ for both). Bone mineral density significantly decreased $(\mathrm{P}<0.01)$ as serum $25(\mathrm{OH}) \mathrm{D}$ and calcium intake declined among whites and Mexican-Americans, but not among blacks $(\mathrm{P}=0.2)$.

\section{International Relevance}

The association between black race and Hispanics and secondary hyperparathyroidism, independent of known risk factors, suggests that novel mechanisms may contribute to secondary hyperparathyroidism in non-whites with CKD. Testing for these parameters would therefore be informed by the demographics of the population. In different countries and regions, the ability to measure these parameters may 
vary, thus the authors of the guideline statements appreciate that implementation of regular measurements of all these parameters may not be possible in all jurisdictions.

\section{Implications for Clinical Practice and Public Policy}

Given there remains no clear recommendation as to 'expected' values in CKD nor consensus on thresholds regarding treatment, the testing of $\mathrm{PTH}$, and vitamin D parameters would lead to substantial costs to the health-care system. Abnormal values lead to repeat testing. There are no data to suggest how effective or useful repeated monitoring of abnormal values is, nor what an acceptable interval of monitoring should be to inform care. Laboratory testing for phosphate and calcium is relatively inexpensive, but treatment and ongoing monitoring may be expensive. At the current time, recommendations for testing frequency may be problematic for clinical practice.

\section{Areas of Controversy, Confusion, or Non-consensus}

The inter-relationship of calcium, phosphate, and PTH, and the potential impact of vitamin D on these mineral metabolites and extraskeletal calcification remains an area of research and debate among clinicians. Newer research examining the role of FGF-23, an important molecule in phosphate, $\mathrm{PTH}$, and vitamin D homeostasis, has caused many to question the previous focus on PTH values as misplaced. The questions of whether vitamin $\mathrm{D}$ therapies are toxic in some or all patients and what values of phosphate are pathologic have yet to be resolved.

\section{Pediatric Considerations}

Application of guidelines related to bone health, growth, and CKD in children is extremely complex. Numerous issues arise, including age-related variation in normative values, comparisons across age, sex, size, and the need to account for pubertal changes etc., when one considers the options and targets for evaluation and treatment.

It is recommended that in the application of any of these specific guidelines, the reader carefully reviews the publications as they relate to pediatrics, starting with the following two documents and then accessing the most currently available pediatric CKD resources for the topic(s).

Recommended primary pediatric CKD-MBD resources:

- KDOQI Clinical Practice Guideline for Nutrition in Children with CKD: 2008 Update $^{382}$

- KDIGO Clinical Practice Guidelines for the Diagnosis, Evaluation, Prevention, and Treatment of $C K D-M B D^{9}$

3.3.2: We suggest not to perform bone mineral density testing routinely in those with eGFR $<45 \mathrm{ml} / \mathrm{min} /$ $1.73 \mathrm{~m}^{2}$ (GFR categories G3b-G5), as information may be misleading or unhelpful. (2B)

\section{RATIONALE}

While there is an appreciation that BMD is measured in many elderly, this statement is intended to highlight for the clinician the fact that the information gained from BMD in those with reduced GFR may be false, leading to either under- or over-treatment. Although fractures rates and fracture-related mortality are elevated in CKD, bone densitometry does not reliably predict fracture risk in patients with GFR $<45 \mathrm{ml} / \mathrm{min} / 1.73 \mathrm{~m}^{2}$ and neither does it predict the type of renal osteodystrophy. Thus, BMD measurements do not provide the information usually sought from such testing, which is usually the basis of interventions.

\section{Evidence Base}

Decreased bone mass and changes in bone microarchitecture occur early in CKD and worsen with progression of disease such that patients with CKD are at increased risk of bone fracture. ${ }^{383}$ Bone strength is determined by the density and quality of the bone. Dual-energy $\mathrm{x}$-ray absorptiometry (DXA) scanning measures density of bone but is not able to determine bone quality (cortical and trabecular microarchitecture). Studies using high-resolution peripheral quantitative computed tomography (HR-pQCT) demonstrate abnormalities in the cortical and trabecular microarchitecture of patients with early CKD compared with healthy control subjects. ${ }^{384}$ Although abnormalities of both DXA and HR-pQCT associate with fractures in patients with CKD, receiver operator characteristic curve analysis suggests that neither technique is predictive of fracture (area under the curve $<0.75$ ), although this improved for patients with longer duration of $\mathrm{CKD}^{385}$ In a cross-sectional study, the combination of these imaging techniques with markers of bone turnover improved prediction of fracture. ${ }^{386}$

\section{Implications for Clinical Practice and Public Policy}

A major component of fracture risk is related to fall risk, thus reduction in fall risk may be achieved through establishment of falls prevention programs. Such programs include medication review; prevention of postural hypotension; cardiac pacing, where appropriate; home hazard assessment and modifications; muscle strengthening and retraining; and treatment of vitamin D deficiency.

\section{Areas of Controversy, Confusion, or Non-consensus}

The combination of measurements of bone thickness, BMD of femoral neck, and a history of fracture may be useful to identify CKD patients who might benefit from fracture prevention strategies. Prospective studies are needed to assess the utility of these parameters for fracture prediction in the CKD population.

\section{Treatment of CKD-MBD}

Disturbances of calcium, phosphate, vitamin D, and PTH develop early during the course of CKD and are associated with adverse outcomes. Studies of these and other markers of bone mineral metabolism have improved our understanding of disease mechanisms governing adverse outcomes of CKDMBD but clinical studies have yet to indicate whether or not manipulation of these markers improves patient-level 
outcomes. In making recommendations for therapeutic targets for mineral metabolism abnormalities, we have been careful not to reach beyond the evidence.

3.3.3: In people with GFR $<45 \mathrm{ml} / \mathrm{min} / 1.73 \mathrm{~m}^{2}$ (GFR categories G3b-G5), we suggest maintaining serum phosphate concentrations in the normal range according to local laboratory reference values. (2C)

3.3.4: In people with GFR $<45 \mathrm{ml} / \mathrm{min} / 1.73 \mathrm{~m}^{2}$ (GFR categories G3b-G5) the optimal PTH level is not known. We suggest that people with levels of intact PTH above the upper normal limit of the assay are first evaluated for hyperphosphatemia, hypocalcemia, and vitamin $\mathrm{D}$ deficiency. $(2 C)$

\section{RATIONALE}

Higher serum phosphate concentrations are associated with mortality and experimental data suggests that serum phosphate concentration is directly related to bone disease, vascular calcification and CVD. Serum phosphate, calcium, and PTH concentrations are all inter-related in patients with CKD. Randomized studies linking manipulation of these parameters to clinical outcomes are lacking but systematic review indicates that earlier phosphate control may help reduce the early clinical consequences of CKD-MBD. Similarly there is insufficient evidence that any specific phosphate binder significantly impacts patient-level outcomes.

\section{Evidence Base}

Systematic review of serum concentrations of calcium, phosphate, and PTH and the risk of death and CVD in people with CKD showed that the risk of death increased $18 \%$ for every $1 \mathrm{mg} / \mathrm{dl}(0.33 \mathrm{mmol} / \mathrm{l})$ increase in serum phosphate concentration (RR 1.18; 95\% CI 1.12-1.25). ${ }^{387}$ There was no association seen with either PTH or serum calcium and all-cause mortality (Figure 19). Of the 327,644 subjects included in the review only 16,247 were not receiving dialysis and of these only 8990 were people with GFR $<60 \mathrm{ml} / \mathrm{min} / 1.73 \mathrm{~m}^{2}$ not receiving RRT. In these subjects the risk of all-cause mortality for each $1 \mathrm{mg} / \mathrm{dl}(0.33 \mathrm{mmol} / \mathrm{l})$ increase in serum phosphate concentration was very similar (RR 1.29; 95\% CI 1.12-1.48). As with all subjects included there was no evidence of an association between serum calcium concentration and all-cause mortality in people with CKD not receiving RRT (RR 1.02; 95\% CI 0.81-1.29). Data for associations of calcium, phosphate, and PTH with cardiovascular death were only available in one of the studies included.

In the Multi-Ethnic Study of Atherosclerosis (MESA) study, the associations of serum phosphate concentrations with vascular and valvular calcification were examined in 439 people with GFR $<60 \mathrm{ml} / \mathrm{min} / 1.73 \mathrm{~m}^{2}$. Each $1 \mathrm{mg} / \mathrm{dl}$ $(0.33 \mathrm{mmol} / \mathrm{l})$ increase in serum phosphate concentration was associated with a $21 \%(\mathrm{P}=0.002), 33 \%(\mathrm{P}=0.001), 25 \%$ $(\mathrm{P}=0.16)$, and $62 \%(\mathrm{P}=0.007)$ greater prevalence of coronary artery, thoracic, aortic valve, and mitral valve calcification, respectively. ${ }^{388}$ The strength of the associations did not differ by age, race, or diabetes. Adjustment for serum concentrations of PTH and $1,25(\mathrm{OH})_{2} \mathrm{D}$ did not alter the strength of the associations.

Factors affecting gastrointestinal phosphate absorption include $1,25(\mathrm{OH})_{2} \mathrm{D}$, food content, phosphate bioavailability and phosphate binders (natural and prescribed). Sources of dietary phosphate are protein-rich foods, including dairy products, meat, and fish as well as legumes, nuts, chocolates and inorganic phosphate additives such as those found in carbonated drinks. In a non-vegetarian Western diet, over half the dietary intake of phosphate comes from animal protein. Although the phosphate content of plant-derived phosphate is higher than animal derived, its bioavailability in terms of gastrointestinal absorption is lower. ${ }^{389}$ Inorganic phosphate additives have the highest bioavailability. A number of clinical studies detail benefit from dietary phosphate and protein control in terms of secondary hyperparathyroidism and progression of CKD in people with moderate CKD. ${ }^{390}$ Few studies have evaluated the impact of dietary phosphate restriction on bone disease or vascular calcification and only one has addressed survival. In people on hemodialysis, a post hoc analysis suggested that more restrictive prescribed dietary phosphate was associated with poorer indices of nutritional status and a greater need for nutritional supplementation. ${ }^{391}$ There was a stepwise trend toward greater survival with more liberal phosphate prescription, which reached statistical significance among subjects prescribed 1001 to $2000 \mathrm{mg} / \mathrm{d}$ and those with no specified phosphate restriction, raising concerns about protein energy malnutrition with dietary phosphate restriction. The means used to achieve phosphate restriction may therefore be important.

Table 29 details the relative cost comparisons of phosphate binders currently in clinical use for which there is observational or study trial data demonstrating their efficacy. Data concerning comparative patient-level outcomes such as mortality are not available.

There are a number of agents available for phosphate binding which are listed in the table ranked in order of relative cost, appreciating that both availability and specific costs are country- and era-specific.

A Cochrane meta-analysis considered 60 RCTs or quasiRCTs (7631 participants) that assessed the effects of various phosphate binders in adults with CKD. ${ }^{392}$ The authors concluded that all available phosphate-binders reduced serum phosphate concentrations in comparison to placebo but that data to date do not support superiority of novel non-calcium binding agents for patient-level outcomes such as all-cause mortality and cardiovascular end points in CKD.

\section{International Relevance}

Availability of different phosphate binders differs around the globe. Thus, recommendations as to specific agents are not possible within the context of these statements. Similarly, dietary phosphate intake may be different around the world, 


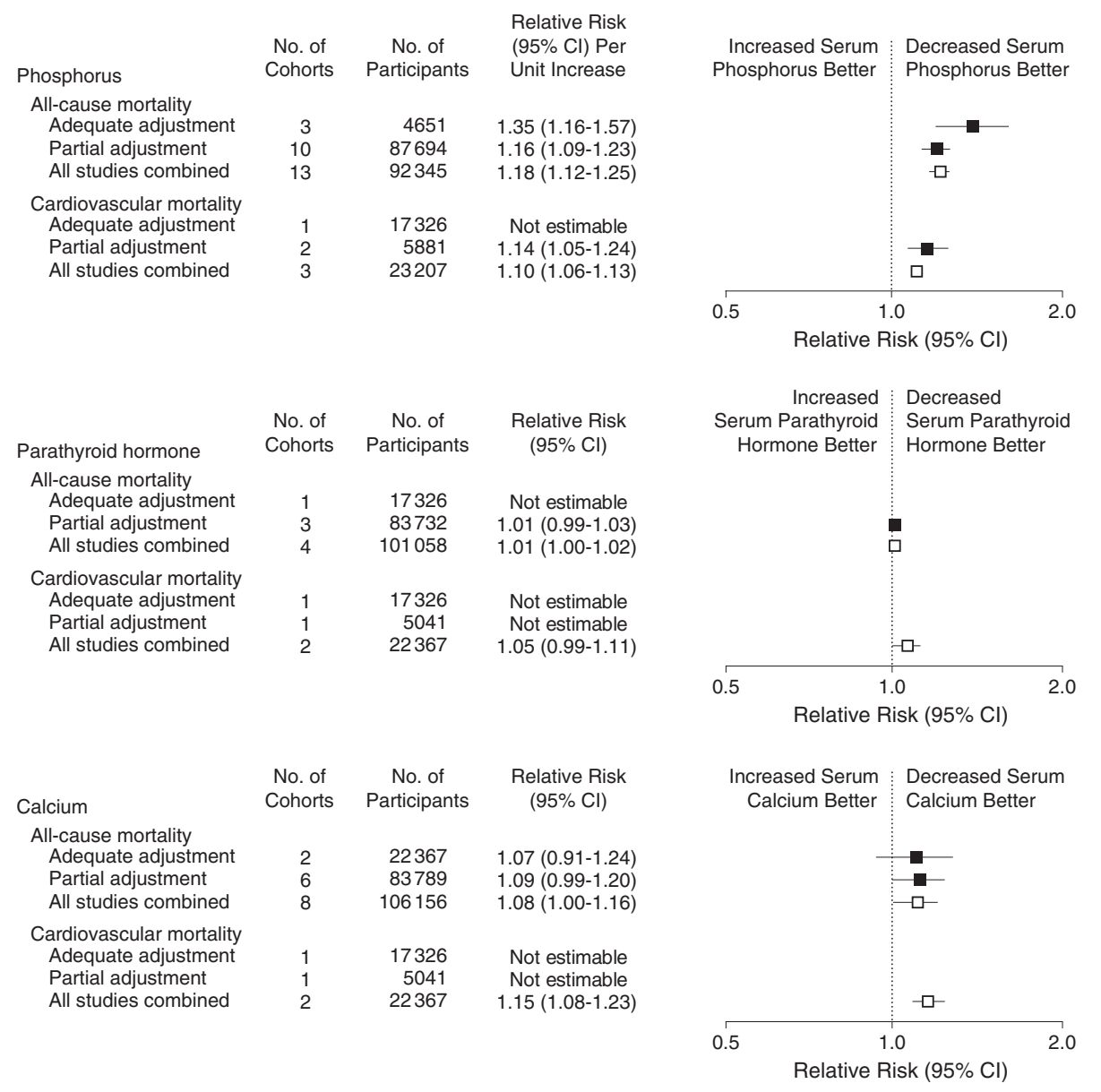

Risks of all-cause mortality, cardiovascular mortality, and nonfatal cardiovascular events are shown per 1-mg/dL increase in serum levels of phosphorus, $100-\mathrm{pg} / \mathrm{mL}$ increase in serum parathyroid hormone, and 1-mg/dL increase in serum calcium. Summary estimates are not reported when only a single cohort contributed data. $\mathrm{Cl}$ indicates confidence interval.

Figure 19|Summary estimates for risks of all-cause mortality and cardiovascular mortality associated with levels of serum phosphorus, PTH, and calcium. PTH, parathyroid hormone. Reprinted with permission from Palmer SC, Hayen A, Macaskill P, et al. Serum levels of phosphorus, parathyroid hormone, and calcium and risks of death and cardiovascular disease in individuals with chronic kidney disease: a systematic review and meta-analysis. JAMA 2011; 305(11): 1119-1127. ${ }^{387}$ Copyright ( ) (2011) American Medical Association. All rights reserved. Accessed http://jama.jamanetwork.com/data/Journals/JAMA/18301/jrv15003_1119_1127.pdf

rendering this problem of greater or lesser significance in different jurisdictions. Measurement of specific hormones $(\mathrm{PTH}$, vitamin $\mathrm{D})$ is expensive and may not inform care sufficiently to warrant the expense at this time.

\section{Implications for Clinical Practice and Public Policy}

Existing data support prevention of hyperphosphatemia and associated secondary hyperparathyroidism in CKD. In the absence of hypercalcemia, there is no indication to prescribe phosphate-binders that are less cost-effective than calcium-based agents. Current data are insufficient to make recommendations about target levels of serum calcium or PTH concentrations that should be achieved in order to reduce mortality or cardiovascular morbidity in people with CKD not requiring dialysis. Assay variability of PTH and vitamin $\mathrm{D}$ remains problematic and this issue is beyond the scope of this document. The practitioner and health-care administrators are advised to appreciate this problem in developing targets for care or thresholds for treatment.

\section{Areas of Controversy, Confusion, or Non-consensus}

As per comments above, the data to support levels of laboratory values for interventions, types of interventions, and target values remain problematic. Thus recommendations for therapy remain similarly problematic and practice varies depending on location and resource availability. Likely correlation of symptoms with blood values and addressing laboratory abnormalities within that context is a pragmatic approach at the current time. The non-specialist is asked to seek advice from local experts for best advice for specific individuals.

The KDIGO guidelines on this subject have not been updated at the time of the writing of this CKD guideline. 
Table $29 \mid$ Phosphate binding agents in routine clinical practice and their ranked cost

\begin{tabular}{|c|c|c|c|}
\hline Agent & Dose/day & Clinical experience and evidence base & $\begin{array}{c}\text { Ranked } \\
\text { cost* }^{*}\end{array}$ \\
\hline Aluminium hydroxide & $1.425-2.85 \mathrm{~g}$ & $\begin{array}{l}\text { Extensive clinical experience in CKD and ESRD, no RCT comparison versus placebo. } \\
\text { Aluminium accumulates in bone and neural tissue with long-term use, avoids } \\
\text { calcium }\end{array}$ & 1 \\
\hline Calcium citrate & $1.5-3 \mathrm{~g}$ & $\begin{array}{l}\text { Limited trial evidence in ESRD. Reduction in phosphate and elevation in calcium } \\
\text { dose-dependent }\end{array}$ & 2 \\
\hline Magnesium carbonate & $\begin{array}{l}0.7-1.4 \mathrm{~g} \text { (plus calcium } \\
\text { carbonate } 0.33-0.66 \mathrm{~g} \text { ) }\end{array}$ & Short-term RCT evidence in ESRD, less hypercalcemia & 3 \\
\hline $\begin{array}{l}\text { Calcium acetate and } \\
\text { Magnesium carbonate } \\
\text { combination }\end{array}$ & $\begin{array}{l}\text { Calcium acetate } 435 \mathrm{mg} \\
\text { plus } \\
\text { Magnesium carbonate } \\
235 \mathrm{mg}, 3-10 \text { tablets daily }\end{array}$ & Short-term RCT evidence in ESRD, less hypercalcemia & 3 \\
\hline Calcium carbonate & $3-6 \mathrm{~g}$ & $\begin{array}{l}\text { Extensive clinical experience in CKD and ESRD, limited RCT evidence versus placebo. } \\
\text { Reduction in phosphate and elevation in calcium both dose-dependent }\end{array}$ & 4 \\
\hline Calcium acetate & $3-6 \mathrm{~g}$ & $\begin{array}{l}\text { Extensive clinical experience in ESRD; RCT evidence comparing to other binders. } \\
\text { Reduction in phosphate and elevation in calcium dose-dependent but less than with } \\
\text { calcium carbonate }\end{array}$ & 4 \\
\hline Lanthanum carbonate & $3 \mathrm{~g}$ & $\begin{array}{l}\text { Extensive prospective cohort evidence, RCT evidence compared to other phosphate } \\
\text { binders. Potential for accumulation in bone and other tissues, avoids calcium }\end{array}$ & 5 \\
\hline Sevelamer-HCl & $4.8-9.6 \mathrm{~g}$ & $\begin{array}{l}\text { Extensive prospective cohort evidence in ESRD; RCT evidence compared to other } \\
\text { phosphate binders; surrogate and patient-centered outcomes, avoids calcium }\end{array}$ & 6 \\
\hline Sevelamer carbonate & $4.8-9.6 \mathrm{~g}$ & $\begin{array}{l}\text { RCT evidence compared to other phosphate binders; equivalency studies compared } \\
\text { to sevelamer- } \mathrm{HCl} \text {, avoids calcium }\end{array}$ & 6 \\
\hline
\end{tabular}

Abbreviations: CKD, chronic kidney disease; ESRD, end-stage renal disease; RCT, randomized controlled trial.

*The average annual cost of aluminium hydroxide in the UK, for example, is $£ 51 /$ year equivalent to US\$84/year. The cost of lanthanum and sevelamer in the UK is $38-42$ times higher and the cost of calcium and magnesium-based binders 5-7 times higher than aluminium hydroxide (All drug costs derived from 2011 British National Formulary list prices).

We have attempted to balance current knowledge with published guidance statements in non-CKD populations.

\section{Clarification of Issues and Key points}

Clinical trials comparing strategies such as vitamin D replacement, dietary phosphate restriction, phosphate binders, and calcimimetics with placebo are needed to address patient-level outcomes such as mortality and cardiovascular morbidity in people with CKD.

Measurement of vitamin D levels is problematic and expensive and is not advocated here.

\section{Vitamin $D$ supplementation and bisphosphonates in people with CKD}

\subsection{5: We suggest not to routinely prescribe vitamin D} supplements or vitamin $D$ analogs, in the absence of suspected or documented deficiency, to suppress elevated PTH concentrations in people with CKD not on dialysis. $(2 B)$

\section{RATIONALE}

This statement is intended to highlight the lack of robust data to support either the measurement or the treatment of vitamin $\mathrm{D}$ deficiency in non-dialysis CKD populations. The statement asks the clinician to more fully evaluate the individual situation. The internationally accepted definition of vitamin D deficiency is a blood concentration $<20 \mathrm{ng} / \mathrm{ml}$ $(<50 \mathrm{nmol} / \mathrm{l})$. Low $25(\mathrm{OH}) \mathrm{D}$ levels are common in patients with non-dialysis dependent CKD; concentrations of $<15 \mathrm{ng} / \mathrm{ml}(<37 \mathrm{nmol} / \mathrm{l})$ occur in at least $12-15 \%$ of patients with CKD and are more prevalent at lower GFR levels, in institutionalized subjects, at extremes of age, and in certain racial groups. Deficiency of $25(\mathrm{OH}) \mathrm{D}$ increases fracture risk and is associated with increased mortality. As CKD progresses, levels of $1,25(\mathrm{OH})_{2} \mathrm{D}$ progressively fall and are closely associated with increasing PTH concentrations. In vitamin D-deficient subjects supplementation with vitamin D increases BMD and muscle strength, reduces risk for fractures and falls, and reduces PTH. In the absence of deficiency, treatment with vitamin $\mathrm{D}$ and related compounds has not been shown to improve either mortality or cardiovascular outcomes.

\section{Evidence Base}

There is a substantial amount of data to support $25(\mathrm{OH}) \mathrm{D}$ deficiency in general and CKD populations ${ }^{367,393}$ which is likely multifactorial. In addition to $25(\mathrm{OH}) \mathrm{D}$ deficiency, note has been made that there is a progressive increase in prevalence of $1,25(\mathrm{OH})_{2} \mathrm{D}$ deficiency with lower GFR category, which occurs earlier than $25(\mathrm{OH}) \mathrm{D}$ deficiency (Figure 20).

No relationship between $25(\mathrm{OH}) \mathrm{D}$ levels and $1,25(\mathrm{OH})_{2} \mathrm{D}$ levels was apparent but there was a strong association between $1,25(\mathrm{OH})_{2} \mathrm{D}$ deficiency and PTH concentration. Of particular note, a higher urinary ACR was associated with lower levels of $1,25(\mathrm{OH})_{2} \mathrm{D}$ at GFR values of $<60 \mathrm{ml} / \mathrm{min} /$ $1.73 \mathrm{~m}^{2}$. 


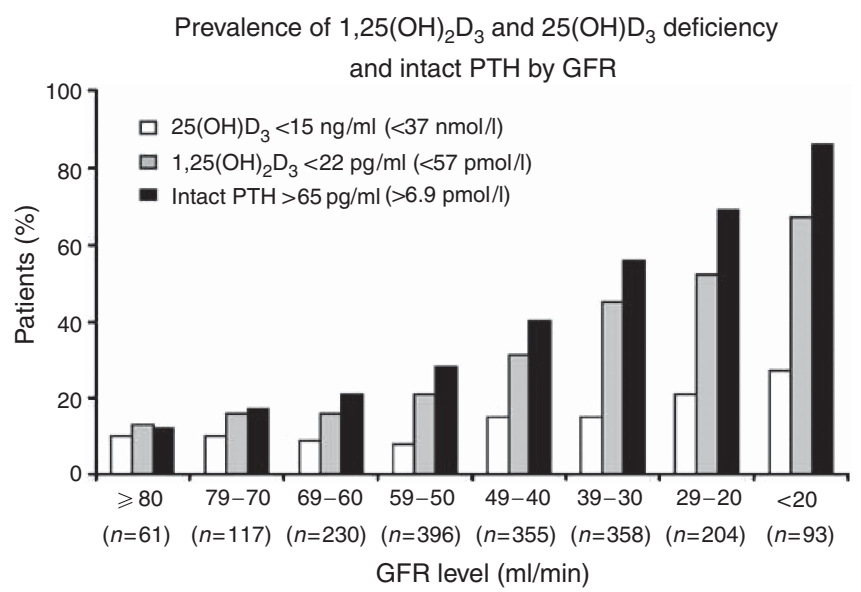

Figure $20 \mid$ Prevalence of deficiency of $1,25(\mathrm{OH})_{2} \mathrm{D}_{3}, 25(\mathrm{OH}) \mathrm{D}_{3}$, and secondary hyperparathyroidism by GFR intervals. GFR, glomerular filtration rate; PTH, parathyroid hormone. Adapted by permission from Macmillan Publishers Ltd: Kidney International. Levin A, Bakris GL, Molitch $M$, et al. ${ }^{367}$ Prevalence of abnormal serum vitamin D, PTH, calcium, and phosphorus in patients with chronic kidney disease: results of the study to evaluate early kidney disease. Kidney Int 2007; 71: 31-38; accessed http:// www.nature.com/ki/journal/v71/n1/pdf/5002009a.pdf

The potential importance of these deficiencies has been illustrated in a number of studies examining the relationship between low levels of $25(\mathrm{OH}) \mathrm{D}$ and $1,25(\mathrm{OH})_{2} \mathrm{D}$ and all-cause and cardiovascular mortality in a different cohorts. ${ }^{393,394}$

Despite the associations with mortality, systematic review of published data to date on vitamin $\mathrm{D}$ supplementation in patients with CKD not on dialysis has only shown an improvement in biochemical end points. A series of publications ${ }^{395,396}$ have attempted to summarize the efficacy of vitamin D therapy on biochemical, bone, cardiovascular, and mortality outcomes in people with CKD and not requiring dialysis. No formulation, route, or schedule of vitamin D compound was found to alter the mortality risk or need for dialysis although vitamin D compounds significantly lowered serum PTH concentrations. None of the studies assessed reported outcomes related to CVD, bone disease, or mortality.

\section{International Relevance}

The importance of vitamin D deficiency has been addressed in both general and CKD populations. Specific populations have been identified as more likely to be vitamin $\mathrm{D}$ deficient depending on cultural and environmental factors; estimates of worldwide prevalence of vitamin D deficiency range from $25-60 \%$. CKD populations within high-risk areas may be particularly vulnerable. The interplay between loss of kidney function and exacerbation of vitamin D deficiency is not known.

\section{Implications for Clinical Practice and Public Policy}

Vitamin D supplementation improves biochemical end points similar to active vitamin $\mathrm{D}$ analogs with a lower burden of costs and side effects. Measurement of serum $25(\mathrm{OH}) \mathrm{D}$ is expensive and in the CKD population with vitamin $\mathrm{D}$ deficiency, simple vitamin $\mathrm{D}$ replenishment is all that is indicated until new evidence becomes available. Except for education or research purposes, there is no need to measure vitamin D levels in general practice.

\section{Areas of Controversy, Confusion, or Non-consensus}

The appropriate dose and formulation of vitamin D, target range for treatment, frequency, route of administration, and safety at different severities of CKD remain to be determined.

\subsection{6: We suggest not to prescribe bisphosphonate treat-} ment in people with GFR $<30 \mathrm{ml} / \mathrm{min} / 1.73 \mathrm{~m}^{2}$ (GFR categories G4-G5) without a strong clinical rationale. $(2 B)$

\section{RATIONALE}

The risk-benefit ratio of bisphosphonates has not been well studied in CKD populations. Indications for bisphosphonate therapy include osteoporosis, corticosteroid therapy, malignant disease and Paget's disease. In people with CKD and GFR categories $>60 \mathrm{ml} / \mathrm{min} / 1.73 \mathrm{~m}^{2}$ with osteoporosis and/ or at high risk of fractures, and in people with GFRs between $30-60 \mathrm{ml} / \mathrm{min} / 1.73 \mathrm{~m}^{2}$ with normal PTH, osteoporosis and/or at high risk of fracture, treatment should be the same as for the general population (although dose modification may be necessary, see Table 30 ).

In people with CKD at lower categories of GFR $\left(<30-35 \mathrm{ml} / \mathrm{min} / 1.73 \mathrm{~m}^{2}\right)$, the correct diagnosis of osteoporosis becomes increasingly complex and other forms of renal osteodystrophy requiring alternative management strategies to osteoporosis require exclusion before treatment with bisphosphonates is considered. In people with adynamic renal bone disease, more likely at lower GFR categories, there is a lack of evidence of either harm or benefit of bisphosphonates on bone strength or vascular calcification.

\section{Evidence Base}

Bisphosphonates increase BMD, reduce bone turnover, and reduce the risk of fragility fractures. The bioavailability of intravenous bisphosphonate formulations is $100 \%$ but the bioavailability of oral formulations is only 1-5\%. Approximately $50-80 \%$ of available bisphosphonate is taken up by bone and the remaining $20-50 \%$ is excreted in urine without being metabolized. Although oral bisphosphonates have not been shown to adversely affect kidney function in people with GFRs as low as $15 \mathrm{ml} / \mathrm{min} / 1.73 \mathrm{~m}^{2}$, in post hoc analysis of clinical trial data their safety and efficacy below GFRs of $30 \mathrm{ml} / \mathrm{min} / 1.73 \mathrm{~m}^{2}$ have not been well-validated and intravenous bisphosphonates have been implicated in nephrotoxicity, especially when rapidly infused (Table 30). ${ }^{9,397,398}$ Thus, from a patient's safety perspective, the statement serves to limit exposure of those with abnormal kidney function to these agents.

\section{International Relevance}

Given cost and clinical practice variation, the use of bisphosphonates varies around the world. Thus, this statement may be less applicable in different jurisdictions. 
Table 30 |Summary data for bisphosphonates and CKD

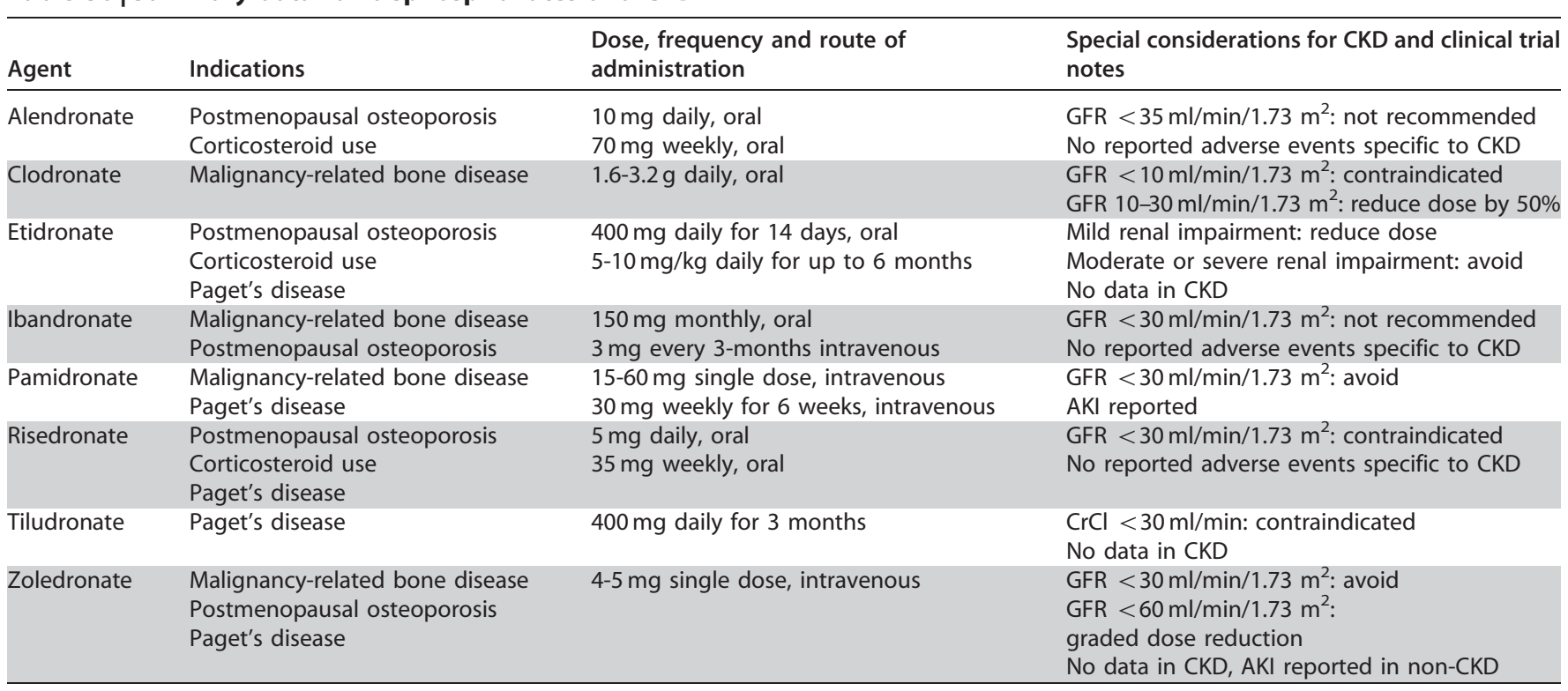

Abbreviations: AKI, acute kidney injury; CKD, chronic kidney disease; $\mathrm{CrCl}$, creatinine clearance; GFR, glomerular filtration rate.

\section{Implications for Clinical Practice and Public Policy}

Given the widespread use of bisphosphonates in developed countries, especially in older women who are also likely to have some degree of kidney dysfunction, the cessation of bisphosphonates in that group may be problematic. There is a need to monitor clinical practice and understand the implications of this recommendation for large populations, who may or may not be deriving benefit from these agents.

\section{Areas of Controversy, Confusion, or Non-consensus}

Despite these concerns, primary care data from the USA suggest that prescription of bisphosphonates is common in people with CKD. Even in the subset with GFR $<30 \mathrm{ml} / \mathrm{min} / 1.73 \mathrm{~m}^{2}$, bisphosphonate use was no less prevalent than in the non-CKD population. ${ }^{399}$ People with CKD in this study were nearly seven times more likely to receive a bisphosphonate than active vitamin D. Similar uncertainty about the efficacy and safety of such treatment for people with CKD was demonstrated in a study of people under the care of a UK renal unit. ${ }^{400}$ Half of all people prescribed bisphosphonates had a GFR $<30 \mathrm{ml} / \mathrm{min} /$ $1.73 \mathrm{~m}^{2}$ and yet $50 \%$ of people with higher GFRs and documented osteoporosis were not prescribed bisphosphonates.

\section{Clarification of Issues and Key Points}

Further study is required to elucidate whether or not bisphosphonates, through reduction of bone turnover in people with pre-existing low bone turnover states, would be beneficial or harmful both for bone and vascular calcification.

\subsection{ACIDOSIS}

The prevalence and severity of metabolic acidosis in people with CKD progressively rises as GFR falls (Table 27).
Adaptations in acid excretion by the kidneys initially prevent a fall in serum bicarbonate concentration but as GFR continues to decline below $40 \mathrm{ml} / \mathrm{min} / 1.73 \mathrm{~m}^{2}$, metabolic acidosis commonly develops.

3.4.1: We suggest that in people with CKD and serum bicarbonate concentrations $<22 \mathrm{mmol} / \mathrm{l}$ treatment with oral bicarbonate supplementation be given to maintain serum bicarbonate within the normal range, unless contraindicated. $(2 B)$

\section{RATIONALE}

Serum bicarbonate concentrations less than $22 \mathrm{mmol} / \mathrm{l}$ are associated with increased risk of CKD progression and increased risk of death. Conversely, high serum bicarbonate concentrations greater than $32 \mathrm{mmol} / \mathrm{l}$ are associated with increased risk of death irrespective of the level of kidney function. Small studies of alkali supplementation have shown reduction in progression of CKD and improved nutritional status in people with CKD. This statement is therefore worded to reflect the potential benefits of oral bicarbonate supplementation to maintain serum bicarbonate concentrations within the normal range, but the word 'suggest' in the statement reflects the lack of robust evidence base on which to support this statement.

\section{Evidence Base}

Chronic metabolic acidosis is associated with increased protein catabolism, uremic bone disease, muscle wasting, chronic inflammation, impaired glucose homeostasis, impaired cardiac function, progression of $\mathrm{CKD}$, and increased mortality. ${ }^{401-410}$ The suggestion that treatment of acidosis may be beneficial was first made by Richard Bright, ${ }^{411}$ later 
taken up enthusiastically by Arthur Osman ${ }^{412}$ and further explored by Lyon et al. who conducted a form of crossover study of supplementation with bicarbonate in 17 people with moderate kidney failure and suggested that treatment with alkali might preserve kidney function. ${ }^{413}$ Rustom et al. described a reduction in proximal renal tubular catabolism and markers of tubular injury after oral $\mathrm{NaHCO}_{3}$ in 11 people with mild/moderate renal impairment (mean ${ }^{51}$ Cr-EDTA GFR $46.2 \pm 6.0 \mathrm{ml} / \mathrm{min} / 1.73 \mathrm{~m}^{2}$ ) and proteinuria $(3.2 \pm 0.8 \mathrm{~g} / 24$ hours $) .{ }^{414}$ They suggested that oral sodium bicarbonate may protect the proximal renal tubule and help delay kidney disease progression. Mathur et al. randomized 40 subjects with mild to moderate CKD to oral bicarbonate $(1.2 \mathrm{mEq} / \mathrm{kg}$ of body weight) or placebo for 3 months and suggested that correction of metabolic acidosis significantly attenuates the rise in blood urea. ${ }^{415} \mathrm{~A}$ larger study randomly assigned 134 adults with moderate to severe $\mathrm{CKD}(\mathrm{CrCl} 15$ to $30 \mathrm{ml} / \mathrm{min} / 1.73 \mathrm{~m}^{2}$ ) and serum bicarbonate 16 to $20 \mathrm{mmol} / \mathrm{l}$ to either supplementation with oral sodium bicarbonate $(1.82 \pm 0.80 \mathrm{~g} /$ day $)$ or standard care for 2 years. ${ }^{416}$ Primary end points were rate of kidney function decline, the proportion of subjects with rapid decline of kidney function $\left(>3 \mathrm{ml} / \mathrm{min} / 1.73 \mathrm{~m}^{2} / \mathrm{yr}\right)$, and ESRD $(\mathrm{CrCl}<10 \mathrm{ml} / \mathrm{min}$ $[<0.17 \mathrm{ml} / \mathrm{s}]$ ). Secondary end points were DPI, normalized protein nitrogen appearance, serum albumin, and mid-arm muscle circumference. Serum bicarbonate increased significantly in the bicarbonate group but despite the associated sodium load there was no difference in BP control, prescription of antihypertensives and loop diuretics, or hospitalization for heart failure compared with the control group. The decline in $\mathrm{CrCl}$ was significantly slowed in the bicarbonate group compared with controls (5.93 versus $\left.1.88 \mathrm{ml} / \mathrm{min} / 1.73 \mathrm{~m}^{2}, \mathrm{P}<0.0001\right)$, rapid progression was less prevalent ( 9 versus $45 \%$, RR 0.15 ; $95 \%$ CI $0.06-0.40$; $\mathrm{P}<0.0001)$, and fewer subjects developed ESRD (6.5 versus $33 \%$, RR 0.13; 95\% CI 0.04-0.40; P<0.001). Nutritional parameters also improved significantly with bicarbonate supplementation. In a non-randomized study the effects of supplementation of $1 \mathrm{mmol}$ of bicarbonate equivalent per $\mathrm{kg}$ body weight per day for 2 years using oral sodium citrate in 30 subjects with hypertensive nephropathy was compared with 29 control subjects. ${ }^{417}$ There were no statistically significant differences in baseline demographic data, systolic $\mathrm{BP}$, venous bicarbonate levels, or parameters of kidney function between the two groups. All subjects were receiving angiotensin-converting enzyme inhibition throughout the study and BP was controlled to similar levels in both groups in a 6-month run-in period prior start of study. In those treated with oral sodium citrate, urine endothelin-1 excretion and $\mathrm{N}$-acetyl- $\beta-\mathrm{D}$ glucosaminidase were each significantly lower while the rate of GFR decline was significantly slower after 24 months of treatment compared to the control group.

\section{International Relevance}

Alkali supplementation appears to be a promising low-cost, high-benefit adjunct treatment for patients with CKD and may be accessible to all populations. It is not known how prevalent acidosis is among different communities or countries around the world.

\section{Implications for Clinical Practice and Public Policy}

The use of sodium bicarbonate or calcium carbonate as a source of alkali should not present financial hardships in most practices or jurisdictions.

\section{Areas of Controversy, Confusion, or Non-consensus}

A potential concern regarding treatment of patients with CKD with sodium bicarbonate or sodium citrate is the possible associated sodium loading. A crossover study of $200 \mathrm{mmol} /$ day (17 g/day) of sodium bicarbonate versus $200 \mathrm{mmol} /$ day ( $12 \mathrm{~g} /$ day) of sodium chloride in 10 subjects with severe $\mathrm{CKD}(\mathrm{CrCl} 2.5-16.8 \mathrm{ml} / \mathrm{min}[0.04-0.28 \mathrm{ml} / \mathrm{s}])$ on an extremely low-sodium diet showed that sodium chloride but not sodium bicarbonate produced an increase in weight and $\mathrm{BP}^{418}$ A subsequent follow-up study suggested that if dietary sodium chloride intakes are near maximum tolerance, then sodium bicarbonate supplementation should be accompanied by reductions in sodium chloride intake to maintain sodium balance. ${ }^{419}$ Given the lack of large and long-term trials, many are still not convinced that this is a reasonable recommendation, and is thus controversial. We would like to ensure that individual clinicians are aware of the controversy rather than making no statement at all.

\section{DISCLAIMER}

While every effort is made by the publishers, editorial board, and ISN to see that no inaccurate or misleading data, opinion or statement appears in this Journal, they wish to make it clear that the data and opinions appearing in the articles and advertisements herein are the responsibility of the contributor, copyright holder, or advertiser concerned. Accordingly, the publishers and the ISN, the editorial board and their respective employers, office and agents accept no liability whatsoever for the consequences of any such inaccurate or misleading data, opinion or statement. While every effort is made to ensure that drug doses and other quantities are presented accurately, readers are advised that new methods and techniques involving drug usage, and described within this Journal, should only be followed in conjunction with the drug manufacturer's own published literature. 


\section{Chapter 4: Other complications of CKD: CVD, medication dosage, patient safety, infections, hospitalizations, and caveats for investigating complications of CKD}

Kidney International Supplements (2013) 3, 91-111; doi:10.1038/kisup.2012.67

This section addresses a series of important topics which impact the outcomes of people with CKD. Specifically CVD, interpretation of tests for CVD, infections, vaccinations and hospitalizations, medications, and patient safety are addressed. This section is designed to help the practitioner appreciate the specific nuances related to testing and drug administration in people with CKD and to alert all clinicians to the fact that many common tests may not be as useful in people with CKD to diagnose or evaluate therapies. It was beyond the scope of the guideline and evidence review to thoroughly evaluate all tests with respect to sensitivity and specificity in different groups of people with CKD but future research may consider this as a useful direction.

\subsection{CKD and CVD}

Population-based studies have demonstrated an increased risk of death and cardiovascular mortality as GFR falls below 60 $\mathrm{ml} / \mathrm{min} / 1.73 \mathrm{~m}^{2}$ or when albumin is detected on urinalysis. This is not explained by an increase in traditional risk factors. There are CKD-specific risk factors associated with more advanced CKD which drive the high rates of mortality and morbidity even at young ages. People with CKD are more likely to experience a cardiovascular event than to progress to ESRD, have a worse prognosis with higher mortality after acute myocardial infarction (MI), and have a higher risk of recurrent MI, heart failure and sudden cardiac death. Management of modifiable cardiovascular risk factors, such as improved BP and diabetes control, also reduces CKD progression.

4.1.1: We recommend that all people with CKD be considered at increased risk for cardiovascular disease. $(1 A)$

\section{RATIONALE}

This statement is worded in this way to reflect the strong and independent associations between GFR and albuminuria categories and risk of CVD in people with CKD and applies to both adult and pediatric populations.

\section{Evidence Base}

Large cohort studies have demonstrated the strong and independent associations between CVD (acute coronary syndrome
[ACS], stroke, heart failure and sudden cardiac death) and CKD by category of eGFR, after adjusting for known CVD risk factors, history of CVD events, and proteinuria. In those with an eGFR of $45-59 \mathrm{ml} / \mathrm{min} / 1.73 \mathrm{~m}^{2}$, risk is increased by $43 \%$ and in those with eGFR below $15 \mathrm{ml} / \mathrm{min} / 1.73 \mathrm{~m}^{2}$, risk is increased by $343 \%{ }^{58}$ Although people with GFR category G5 $\left(\mathrm{GFR}<15 \mathrm{ml} / \mathrm{min} / 1.73 \mathrm{~m}^{2}\right.$ ) are at the highest risk of a CVD event, there will be more events in people with GFR categories G3a-G3b (GFR $30-59 \mathrm{ml} / \mathrm{min} / 1.73 \mathrm{~m}^{2}$ ) because of the much higher prevalence at these categories. ${ }^{420}$ These events occur at a younger age in people with CKD suggesting that CKD promotes CVD at an accelerated rate. ${ }^{421}$ The prognosis after an acute event is related to level of GFR with a significant rise in mortality when eGFR falls below $45 \mathrm{ml} / \mathrm{min} / 1.73 \mathrm{~m}^{2}$. ${ }^{422-424}$

Albuminuria is associated with duration and severity of hypertension; an adverse lipid profile with higher levels of total cholesterol, triglycerides and lipoprotein(a) and low HDL-C levels; ${ }^{425}$ and abnormalities of coagulation. The presence of higher levels of proteinuria increases the risk of mortality and MI independently of level of eGFR. ${ }^{426}$ Many studies have demonstrated low levels of urinary albumin to be associated with the increased risk of CVD in people with diabetes independent of renal function; however population studies of non-diabetic individuals have confirmed that even small amounts of albuminuria are associated with increased CVD risk. In the Third Copenhagen study, in people with microalbuminuria, risk of coronary heart disease was increased independently of age, sex, renal function, diabetes, hypertension, and plasma lipids. ${ }^{427}$ The Chronic Kidney Disease Prognosis Consortium demonstrated that in general practice cohorts there was an increase in cardiovascular mortality when ACR is higher than $30 \mathrm{mg} / \mathrm{g}(3 \mathrm{mg} / \mathrm{mmol}){ }^{4}$ Analysis of data from the Heart Outcomes Prevention Evaluation (HOPE) study demonstrated that any degree of albuminuria is a risk factor for cardiovascular events in individuals with or without diabetes. ${ }^{428}$ The lack of a threshold of albuminuria for cardiac risk was also confirmed in the HUNT 2 Study $^{429}$ and the Losartan Intervention For Endpoint Reduction in Hypertension (LIFE) study in patients with $\mathrm{LVH}^{430}$ Albuminuria and low eGFR were synergistic cardiovascular mortality risk factors in the HUNT 2 study and using both ACR and eGFR improved 
cardiovascular risk stratification at all age levels, but particularly in persons 70 years and over. ${ }^{429}$

In the MDRD Study cohort of patients with GFR categories G3a-G4 (GFR 15-59 ml/min/1.73 $\mathrm{m}^{2}$ ), cystatin C level was strongly associated with all-cause and cardiovascular mortality particularly in the elderly. ${ }^{431}$ Analysis of data from MESA and the Cardiovascular Health Study (CHS) demonstrated that the worst prognosis of CVD, heart failure, and progressive CKD was found in those subjects with CKD diagnosed using the cystatin C-based equation. ${ }^{432}$

$\mathrm{LVH}$ is common with CKD, is known to reflect target organ damage, and is associated with increased cardiovascular mortality in CKD. ${ }^{360}$ It is also important to consider the role of CKD-specific risk factors particularly in patients with more severe CKD (GFR $<30 \mathrm{ml} / \mathrm{min} / 1.73 \mathrm{~m}^{2}$ ) and correct them where possible. Anemia has been thought to have a particular role in the early development of CVD in people with CKD. Although treatment of anemia is associated with improved well-being and greater exercise capacity, the results of several RCTs of anemia correction have suggested that complete correction of anemia is not advisable in people with lower GFR $\left(<60 \mathrm{ml} / \mathrm{min} / 1.73 \mathrm{~m}^{2}\right)$. A meta-analysis of 9 RCTs comparing different target $\mathrm{Hb}$ levels suggests an increase in mortality and worse BP control with the higher treatment targets, independent of the GFR category. ${ }^{433,434}$ Those patients with $\mathrm{LVH}$ at baseline, a sign of target organ damage and which was present in $47 \%$ of the Cardiovascular Risk Reduction by Early Anemia Treatment with Epoetin Beta Trial (CREATE) cohort, had significantly worse cardiovascular outcomes when treated to the higher targets. ${ }^{435}$

Abnormalities of mineral metabolism with low 1,25dihydroxyvitamin D and PTH were found to occur early in CKD in the Study for the Evaluation of Early Kidney disease [SEEK] study, however serum calcium and phosphate were usually normal until eGFR fell below $40 \mathrm{ml} / \mathrm{min} / 1.73 \mathrm{~m}^{2}{ }^{367}$ An association between elevated serum phosphate and cardiovascular mortality was demonstrated in a prospective study of people with CKD and this was also thought to be associated with low vitamin D levels. ${ }^{436}$

\section{Pediatric Considerations}

While the majority of data for, and evidence of, the high risk of cardiovascular morbidity and mortality in the pediatric renal population stems from the ESRD (dialysis and transplant) populations, ${ }^{437-440}$ there is recognition that many if not all of the factors responsible for such outcomes will develop much earlier in this course of CKD. In fact, the current American Heart Association's guidelines for reduction of cardiovascular risk in pediatrics stratified the child with CKD to the highest risk category. ${ }^{441}$

Based on data from the CKiD trial, Wilson et al. ${ }^{442}$ demonstrated extremely high prevalence rates for four traditional cardiovascular risk factors (hypertension, dyslipidemia, abnormal glucose metabolism, and obesity) in a subgroup of this cohort of 586 children with iohexol GFR proven mild to moderate CKD. In this cross-sectional analysis of 250 children who had data on all variables of interest, median GFR of $45.2 \mathrm{ml} / \mathrm{min} / 1.73 \mathrm{~m}^{2}$ (IQR 34.658.2 ), the prevalence of hypertension was $46 \%$, dyslipidemia was $44 \%$, abnormal glucose metabolism was present in $21 \%$, and obesity in $15 \%$. Hypertriglyceridemia was common, seen in $33 \%$. In aggregate, only $26 \%$ of the cohort had no risk factors present, $39 \%$ had at least one and $22 \%$ had two, $11 \%$ had three and $2 \%$ had all four risk factors present. The prevalence of all these factors remained elevated even when the population was restricted to those defined as lean, BMI $<85^{\text {th }}$. In this group, the presence of 2 or 3 cardiovascular risk factors was still seen in $20 \%$ and $2 \%$, respectively. Within the lean group 12\% demonstrated abnormal glucose metabolism. Finally, multivariate regression analysis of their data demonstrated that glomerular disease as a cause of CKD and the presence of nephrotic range proteinuria were associated with much higher odds of having cardiovascular risk factors, 1.96 (95\% CI 1.04-3.72) and 2.04 (95\% CI 0.944.43), respectively.

It is also well-recognized that carotid intimal media thickness and pulse wave velocity changes, as potential surrogate measures for vascular damage in children, can be increased in in children with CKD. ${ }^{443,444}$

For further detailed discussions of the pathophysiology and complexity of CVD in the pediatric CKD population, two recent review papers from Mitsnefes ${ }^{445}$ and Shroff et al. ${ }^{446}$ are suggested. Both papers identify and discuss the pathophysiology of both traditional and CKDspecific cardiovascular risk factors as seen in this population.

\subsection{2: We recommend that the level of care for ischemic heart disease offered to people with CKD should not be prejudiced by their CKD. (1A) \\ 4.1.3: We suggest that adults with $C K D$ at risk for atherosclerotic events be offered treatment with antiplatelet agents unless there is an increased bleeding risk that needs to be balanced against the possible cardiovascular benefits. $(2 B)$}

\section{RATIONALE}

These statements are worded in this way to reflect the growing body of evidence that suggests that treatment of traditional risk factors in CKD patients is of benefit, and that attempts to modify or preclude usual investigations and therapies are not necessary in CKD patients. Inasmuch as CKD populations have been understudied, but likely inadvertently included in larger population studies, the rationale for risk factor modification is similar to that in the general population. Modification of risk factors includes:

1. Smoking cessation

2. Exercise

3. Weight reduction to optimal targets

4. Lipid modification recognizing that the risk reduction associated with statin therapy in adults with CKD is 
relatively constant across a broad range of baseline lowdensity lipoprotein cholesterol (LDL-C) levels

5. Optimal diabetes control $\mathrm{HbA}_{1 \mathrm{C}}<7 \%(53 \mathrm{mmol} / \mathrm{mol})$

6. Optimal BP control to $<140 / 90 \mathrm{~mm} \mathrm{Hg}$ or $<130 / 80$ $\mathrm{mm} \mathrm{Hg}$ in those with CKD and depending on the degree of proteinuria (see Recommendations 3.1.4 and 3.1.5)

7. Aspirin is indicated for secondary prevention but not primary prevention

8. Correction of anemia to individualized targets (see KDIGO Clinical Practice Guideline for Anemia in $C K D^{11}$ )

\section{Evidence Base}

The maintenance of cardiovascular health ultimately impacts 'kidney health' given the links between cardiac function, renal perfusion, atherosclerosis, and glomerulosclerois. The details of this complex biology are beyond the scope of these guidelines, but can be found in key references below.

There is evidence in the general population that smoking cessation is associated with a reduction in cardiovascular risk. In CKD there is evidence that smoking is associated with progression of renal disease, though no specific data exist that support cessation of smoking and delay of progression. ${ }^{447}$

People with CKD have reduced levels of muscle strength and aerobic activity. In those not on dialysis, exercise training is shown to improve functional capacity and BP. ${ }^{448}$

Weight loss in obese CKD patients can reduce rate of decline, proteinuria, and $\mathrm{BP}^{354}$

The Study of Heart and Renal Protection (SHARP) ${ }^{449}$ is the largest RCT in people with CKD to date. The results demonstrate that a lipid-lowering strategy which included fixed dose simvastatin and ezetimibe resulted in a $17 \%$ reduction in atherosclerotic events, as compared to placebo. The cohort enrolled included those with eGFR under $60 \mathrm{ml} /$ $\min / 1.73 \mathrm{~m}^{2}$, age greater than 40 years, and was an international study including more than 9000 subjects from around the globe. The lipid-lowering strategy was effective and safe. Lipid targets have not been established specifically for people with CKD. Treatment strategies should be implemented in accordance with current recommendations for high-risk populations.

At the time of this writing, the KDIGO Clinical Practice Guideline for Lipid Management in Chronic Kidney Disease was under preparation for public review. In brief, the key aspects of the draft recommendations include treating those at high risk for atherosclerotic disease with lipid-lowering therapies, regardless of LDL levels, in those 50 years of age and above. Since this Guideline has not yet been finalized, interested readers should refer to the final document when it is formally released in 2013.

The benefits of aspirin in people with CKD and hypertension was demonstrated in a post hoc analysis of the Hypertension Optimal Treatment (HOT) trial. ${ }^{450}$ Jardine et al. reported that among every 1000 persons with eGFR $<45 \mathrm{ml} / \mathrm{min} / 1.73 \mathrm{~m}^{2}$ treated for 3.8 years, 76 major cardiovascular events and 54 all-cause deaths will be prevented while 27 excess major bleeds will occur. They concluded that an increased risk of major bleeding appears to be outweighed by the substantial benefits. Clopidogrel is used as an alternative to aspirin but CKD has been shown to be associated with an increase in platelet reactivity and there is resistance to clopidogrel in people with $\mathrm{CKD}$, diabetes, and CVD. ${ }^{451}$ The Clopidogrel for Reduction of Events During Observation (CREDO) trial concluded that clopidogrel in mild or moderate CKD may not have the same beneficial effect as it does in people without CKD. Subjects with normal renal function who received 1 year of clopidogrel had a marked reduction in death, MI, or stroke compared with those who received placebo $(10.4 \%$ versus $4.4 \%, \mathrm{P}<0.001)$, whereas those with mild and moderate CKD did not have a significant difference in outcomes with clopidogrel therapy versus placebo (mild: $12.8 \%$ versus $10.3 \%, \mathrm{P}=0.30$; moderate: $13.1 \%$ versus $17.8 \%, \mathrm{P}=0.24$ ). Clopidogrel use was associated with an increased RR of major or minor bleeding, but this increased risk was not different based on renal function. ${ }^{452}$

\section{International Relevance}

Although it is clear and mentioned in many guidelines that CKD is associated with an increase in CVD risk, it is not included in many assessment tools and there is a deficiency in ethnicity and regional specific tools.

The assessment tool from US Third Report of the National Cholesterol Education Program (NCEP III) does not include CKD. The Fourth Joint Task Force of the European Society of Cardiology suggest that both an eGFR of less than $60 \mathrm{ml} /$ $\min / 1.73 \mathrm{~m}^{2}$ and albuminuria increase risk but do not quantify the risk or include CKD in their Systematic COronary Risk Evaluation (SCORE) assessment tool. ${ }^{453}$ In the UK, the QRISK ${ }^{\circledR} 2$ online tool includes CKD as 'yes' or 'no' and therefore does not allow for level of eGFR or proteinuria. ${ }^{454}$ The Joint British Societies Guidelines regards proteinuria as a sign of target organ damage, which conveys a risk of at least $20 \%$ in 10 years. ${ }^{455}$

A study of cardiovascular risk estimation in Chinese adults in the USA-People's Republic of China Collaborative Study of Cardiovascular and Cardiopulmonary Epidemiology (USAPRC Study) did not include CKD as a risk factor at all. ${ }^{456}$ It is also important to remember that in this population stroke was the predominant CVD. A review of 25 risk assessment tools identified only 2 derived from an Asian population. ${ }^{457}$ However, links between GFR categories and CVD events are evident in the Asian population, for example in the Japanese Gonryo study. ${ }^{458}$ A prospective study in a general Japanese population demonstrated links between lower GFR, high creatinine levels, and proteinuria with cardiovascular mortality particularly stroke. ${ }^{459}$ In Chinese patients who were at least 50 years old and either had existing CVD or were at high risk, $34 \%$ had an eGFR $<60 \mathrm{ml} / \mathrm{min} / 1.73 \mathrm{~m}^{2}$ and eGFR $<45$ $\mathrm{ml} / \mathrm{min} / 1.73 \mathrm{~m}^{2}$ was found to be an independent predictor of all-cause and cardiovascular death. ${ }^{460}$ In a population-based study of 2353 people aged over 40 years in Beijing, an eGFR 
$<90 \mathrm{ml} / \mathrm{min} / 1.73 \mathrm{~m}^{2}$ was associated with increased CVD risk, and for each CKD category stroke was more prevalent than MI. ${ }^{461}$

In India the increasing prevalence of type 2 diabetes is driving an increase in CKD and both are associated with increased cardiovascular risk. In CKD patients attending one clinic in North India, $28 \%$ had diabetes, $27 \%$ were overweight, and $92 \%$ had hypertension. Metabolic syndrome as defined by the International Diabetes Federation (IDF) 2007 guidelines was present in $39 \%$ and more frequent in women. ${ }^{462}$

\section{Implications for Clinical Practice and Public Policy}

A full CVD risk assessment in a person with CKD should include an estimate of GFR and a quantitative assessment of albuminuria. Cystatin $\mathrm{C}$ may be helpful at risk stratifying those at intermediate risk.

The key aspect of these recommendations is to ensure that people with CKD are not deprived of treatment strategies known to be effective in general populations.

\section{Areas of Controversy, Confusion, or Non-consensus}

Many of the traditional cardiovascular risk assessment tools do not adjust for the presence of early CKD. Any tool should also adjust for level of eGFR because of increasing risk associated with lower levels of kidney function. The Framingham risk equation underestimates true events in a CKD population, but no validated different tools exist at present which better quantify cardiovascular or mortality risk in CKD populations.

Proteinuria is considered to be a sign of target organ damage and thus associated with high cardiovascular risk. However, assessment of risk should also include the presence of albuminuria. Few studies exist which demonstrate that targeting lowering of proteinuria results in reduced cardiovascular risk, though a number of studies which have targeted interruption of the RAAS (and in which lowering of urine protein has also been shown) have demonstrated a benefit.

An appropriate risk assessment tool should be available to assess risk in people with CKD. Addition of the presence of $\mathrm{CKD}$ to conventional risk factors in the Reykjavik population increased discrimination but did not increase risk to the same degree as smoking or diabetes. ${ }^{463}$ In the Framingham Heart Study, GFR category $3 b$ (GFR $30-44 \mathrm{ml} / \mathrm{min} / 1.73 \mathrm{~m}^{2}$ ) was associated with CVD but not equivalent to previous CVD. ${ }^{464}$

Lifestyle modification has not been studied in large trials in people with CKD but smoking cessation, achievement of optimum weight, regular exercise, and salt restriction should be seen as reasonable aims.

\section{Pediatric Considerations}

It is presently unclear whether children with CKD and elevated lipid levels will benefit from lipid-lowering strategies with no prospective trials ongoing in this area. It is hoped that data from both $\mathrm{CKiD}^{55}$ and the $4 \mathrm{C}$ trials $^{78}$ may be able to address these shortcomings.
However, given that these children demonstrably have elevated risk of cardiovascular and atherosclerotic disease (see Recommendation 4.1.1), that there are data supporting the use of a number of statins in the pediatric population, ${ }^{465}$ and that the adult evidence for benefit of statins in a subset of the SHARP trial ${ }^{449}$ with a GFR $<60 \mathrm{ml} / \mathrm{min} / 1.73 \mathrm{~m}^{2}$ exist, it would seem reasonable to consider the use of such drugs in children with CKD and elevated lipids.

Recommendations from $2006^{441}$ for use of dietary modifications, followed by statins in children older than 8 years of age with persistent elevation in LDL-C levels, have been endorsed by the American Academy of Pediatrics. However, at the time of publication of these guidelines, the KDIGO Dyslipidemia Work Group has suggested that statin therapy may not be appropriate.

Age- and renal function-adjusted doses of such drugs should be carefully considered prior to any therapy being initiated (see Recommendation 4.4.1).

Regarding diabetes control, treatment in keeping with national and international diabetes recommendations is prudent. Note is made that there are specific caveats with respect to drugs and side effects that are important (see Recommendations 3.1.15-3.1.18).

There is no literature in the area of antiplatelet agents in atherosclerotic disease in the subset of children with CKD and the suggestion to offer antiplatelet agents does not apply to pediatric practice.

4.1.4: We suggest that the level of care for heart failure offered to people with CKD should be the same as is offered to those without CKD. $(2 A)$

4.1.5: In people with CKD and heart failure, any escalation in therapy and/or clinical deterioration should prompt monitoring of eGFR and serum potassium concentration. (Not Graded)

\section{RATIONALE}

This statement is worded this way to make clinicians aware that people with CKD and heart failure should receive at least equal benefit from heart failure therapy as those without $\mathrm{CKD}$, but that heart failure therapies (in particular increase of RAAS blockade and diuretic therapy) may lead to significant changes in GFR and serum potassium concentrations. This does not imply that such therapy should be avoided but only that clinicians are cognizant of this possibility, monitor it, and understand it in the context of individual risks and benefits.

Heart failure is a complex clinical syndrome which can be caused by any structural or functional cardiac disorder that impairs the pump function of the heart and has a high mortality. Within the general population, the commonest causes of heart failure are ischemic heart disease causing left ventricular systolic dysfunction, and hypertensive heart disease with left ventricular hypertrophy and diastolic dysfunction. Thus people with CKD are at increased risk of both. Evidence-based medication for heart failure impacts on 
GFR and prescribers need to be alert to this. Changes in GFR are often transient and the downward trajectory GFR is not sustained if all other aspects of care and clinical status are stable. Monitoring and close follow-up are required.

\section{Evidence Base}

In an outpatient setting, normal GFR is unusual in heart failure. A study from the Alberta Heart Function Clinic found that in people with an ejection fraction of $\leq 35 \%$, GFR categories G3a and G3b (GFR 30-59 ml/min/1.73 $\mathrm{m}^{2}$ ) were present in $40 \%$ and $16 \%$ of people were GFR categories G4 or G5 $\left(\right.$ GFR $\left.<30 \mathrm{ml} / \mathrm{min} / 1.73 \mathrm{~m}^{2}\right) .{ }^{466}$ This study demonstrated a $1 \%$ increase in mortality associated with each $1 \mathrm{ml} / \mathrm{min}$ fall in $\mathrm{CrCl}$ demonstrating the clear link between kidney function and outcome for people with CKD and heart failure. Metaanalysis of 16 studies and over 80,000 people with heart failure by Smith et al. showed that renal impairment was present in $63 \%$ and was associated with increased mortality across the range of kidney function, with an adjusted HR of 1.56 (95\% CI 1.53-1.60). ${ }^{467}$ In the Candesartan in Heart Failure-Assessment of Reduction in Mortality and Morbidity (CHARM) Program with candesartan in heart failure, $36 \%$ of subjects had eGFR $<60 \mathrm{ml} / \mathrm{min} / 1.73 \mathrm{~m}^{2}$ which was associated with increased mortality and hospital admission regardless of ejection fraction. ${ }^{468}$ The Digitalis Intervention Group (DIG) identified a GFR of $50 \mathrm{ml} / \mathrm{min}$ as a risk threshold for increased mortality risk in heart failure. ${ }^{469}$ Smith et al. demonstrated that 1-year mortality after admission for heart failure was $29 \%$ in people with normal kidney function but $52 \%$ in those with moderate to severe renal impairment. ${ }^{470}$ Anand et al. also demonstrated an independent association between the presence of positive reagent strip proteinuria present in $8 \%$ of subjects and increased mortality in heart failure. ${ }^{471}$

In the Alberta Provincial Project for Outcome Assessment in Coronary Heart Disease (APPROACH) study of people with heart failure related to coronary artery disease $(\mathrm{CAD})$, $39 \%$ had $\mathrm{CrCl}<60 \mathrm{ml} / \mathrm{min}(<1 \mathrm{ml} / \mathrm{s})$. This study also demonstrated that the level of kidney function was a better indicator of poor outcome than cardiac anatomy. ${ }^{472}$ In people admitted to hospital with heart failure, increasing $\mathrm{SCr}$ concentration is associated with a longer hospital stay and worse outcome. ${ }^{473}$

$\mathrm{LVH}$ is present in $40 \%$ of people with $\mathrm{CKD},{ }^{474}$ progressively increasing as kidney function declines. ${ }^{475}$ $\mathrm{Ha}$ et al. demonstrated LVH in $87 \%$ of people with predialysis CKD. ${ }^{476}$ Parfrey et al. in a study of 432 people who were predialysis, found that only $16 \%$ had a normal echocardiogram while $41 \%$ had concentric LVH, $16 \%$ systolic dysfunction, and $28 \%$ left ventricular dilatation. ${ }^{477} \mathrm{LVH}$ is related to increased work load caused by arterial stiffness and hypertension, and at lower levels of GFR, volume overload. Alterations in electrolyte balance, anemia, bone metabolism, uremia, oxidative stress, inflammation, and other inflammatory mechanisms all play a role. ${ }^{478}$

In the majority of people with $\mathrm{CKD}$, the myocardium develops concentric hypertrophy ${ }^{476}$ with interstitial fibrosis and CKD-associated cardiomyopathy, leading to left ventricular stiffness. ${ }^{474}$ This results in diastolic heart failure; people with CKD have higher mortality from diastolic heart failure than systolic heart failure. ${ }^{479}$ Diastolic heart failure is more common in people with CKD who are older females with hypertension and/or diabetes. ${ }^{480}$

Left ventricular hypertrophy is particularly associated with anemia and a fall in $\mathrm{Hb}$ to less than $12.8 \mathrm{~g} / \mathrm{dl}(128 \mathrm{~g} / \mathrm{l})$ is associated with left ventricular growth in people with early CKD. ${ }^{481}$ Lower $\mathrm{Hb}$ levels are associated with increased risk of death and hospitalization in heart failure. ${ }^{482}$ Other cardiovascular conditions, for example MI and atrial fibrillation, are more common in CKD and may exacerbate heart failure.

It is important to note that in a study of an older population (age $>64$ years), heart failure was an independent predictor of rapid kidney function decline. ${ }^{483}$

Standard therapy for the management of systolic heart failure includes an ACE-I or ARB and a beta-blocker licensed for heart failure such as bisoprolol or carvedilol. Metaanalysis of 25 trials of RAAS blockade (ACE-I or ARB) in people with CKD or proteinuria, demonstrated a reduction in the risk for heart failure and reduction in cardiovascular outcomes. ${ }^{484}$ Subgroup analysis from the Valsartan Heart Failure Trial (Val-HeFT) demonstrated the beneficial effect of valsartan in people with CKD and that valsartan reduced the eGFR by the same amount in people with and without CKD. ${ }^{471}$ Candesartan was also clinically effective in the CHARM study regardless of underlying kidney function ${ }^{468}$ while irbesartan was associated with a $23 \%$ reduction in hospital admissions related to heart failure in people with nephropathy due to type 2 diabetes. ${ }^{485}$ Analysis of data on bisoprolol from the Cardiac Insufficiency Bisoprolol Study II (CIBIS II) $^{486}$ identified $32 \%$ of subjects with eGFR $<60 \mathrm{ml} /$ $\mathrm{min} / 1.73 \mathrm{~m}^{2}$. These subjects were more likely to die or be admitted to hospital than those with eGFR $\geq 60 \mathrm{ml} / \mathrm{min} / 1.73$ $\mathrm{m}^{2}$. However, they showed a similar benefit from bisoprolol treatment. A post hoc analysis of data on carvedilol from the Carvedilol Post-Infarct Survival Control in Left Ventricular Dysfunction (CAPRICORN) and Carvedilol Prospective Randomized Cumulative Survival (COPERNICUS) trials identified $60.8 \%$ of participants with eGFR $<60 \mathrm{ml} / \mathrm{min} /$ $1.73 \mathrm{~m}^{2}{ }^{487}$ Carvedilol was well tolerated by subjects with $\mathrm{CKD}$, and decreased all-cause, cardiovascular, and heart failure mortality. However, doubts were raised over the benefits when eGFR $<45 \mathrm{ml} / \mathrm{min} / 1.73 \mathrm{~m}^{2}$.

Aldosterone antagonists are included in standard heart failure management, usually in addition to an ACE-I or ARB, leading to concerns relating to risk of hyperkalemia, particularly in people with lower GFRs. In a study of spironolactone therapy in severe heart failure, Pitt et al. ${ }^{488}$ included subjects with SCr concentrations up to $2.5 \mathrm{mg} / \mathrm{dl}$ $(221 \mu \mathrm{mol} / \mathrm{l})$. There was a $30 \%$ reduction in mortality with spironolactone and the incidence of hyperkalemia was low. However, a review of real-world practice demonstrated a significant increase in hyperkalemia with the combination of RAAS blockade and an aldosterone antagonist, highlighting 
the need for close monitoring with the introduction of combination therapy. ${ }^{489}$

In the APPROACH study on the specialist management of heart failure, subjects with $\mathrm{CAD}$ and $\mathrm{CKD}$, despite being at high risk, were less likely to be taking ACE-Is, beta-blockers, statin, and aspirin. This study demonstrated that those subjects taking beta blockers had lower mortality, however ACE-Is were only of benefit when eGFR was $>60 \mathrm{ml} / \mathrm{min} / 1.73 \mathrm{~m}^{2} .{ }^{472}$

\section{International Relevance}

The treatment of heart failure is similar around the globe. The ability to closely monitor kidney function or to offer conventional therapies may differ, however. Irrespective, this statement should hold true internationally. The National Heart Care Project of community subjects admitted to hospital with heart failure studied the differences between people of black and white ethnic groups. People with worse renal function were more likely to be black, older and female, and black people had a greater prevalence of hypertension and diabetes but less ischemic heart disease. Black people had a lower risk of mortality at every level of creatinine, for every $0.5 \mathrm{mg} / \mathrm{dl}$ (44.2 $\mu \mathrm{mol} / \mathrm{l})$ increase in creatinine, 1-year death risk increased $10 \%$ in black people and $15 \%$ in white people. ${ }^{470}$

\section{Implications for Clinical Practice and Public Policy}

Cardiorenal syndrome, an impairment of kidney function in the presence of heart disease, is a marker of worse prognosis. In addition to GFR, the presence and severity of albuminuria and anemia aid in prognostication and management. ${ }^{490}$

Where the link between LVH and heart failure in CKD is so strong and the outcome poor for people with LVH, efforts should be made to optimize care particularly BP. There is a lack of robust evidence for the optimal medical management of heart failure specifically in people with CKD; however, the prevalence of people with early CKD is so high in heart failure trials that we can apply standard treatment to these people. For those people with eGFR $<45 \mathrm{ml} / \mathrm{min} / 1.73 \mathrm{~m}^{2}$, the situation is less clear. ${ }^{487}$

\section{Areas of Controversy, Confusion, or Non-consensus}

There are difficulties in making the diagnosis of heart failure in people with $\mathrm{CKD}$, especially diastolic heart failure. Biomarkers such as B-type natriuretic peptide (BNP) may be abnormally elevated in CKD, but may or may not be as responsive to treatment or diagnostically accurate in people with CKD (see Chapter 4.2). There is little evidence to guide the management of left ventricular systolic dysfunction in people with CKD and evidence for the management of diastolic heart failure is generally lacking. However, there is evidence that medications to improve the outcome of people with heart failure are underused in people with CKD.

Appropriate management of the metabolic complications of kidney disease which can exacerbate heart failure needs to be clarified. Clinical studies are required on the role of device therapy in people with CKD (e.g., pacemakers, defibrillators, etc.).

\subsection{CAVEATS WHEN INTERPRETING TESTS FOR CVD IN PEOPLE WITH CKD}

\section{$B N P / N$-terminal-proBNP (NT-proBNP)}

As GFR diminishes, the prevalence and severity of cardiovascular abnormalities increase and are accepted as major causes of morbidity and mortality in people with CKD. The presence of congestive heart failure (CHF) in people with CKD conveys a worse prognosis than either condition alone. Therefore, early diagnosis and aggressive management of CHF are both highly desirable. A number of cardiac biomarkers (i.e., BNPs and cardiac troponins) appear to have both increasing clinical importance and increasing facility for detection and stratification of CHF. The stimulus for secretion of these biomarkers is the hemodynamic load (i.e., myocardial stretch) and their secretion is associated with the severity of CHF and the degree of left ventricular dysfunction. They are thus useful markers for diagnosis, management, and prognosis in people with normal renal function. However, when the eGFR is less than $60 \mathrm{ml} / \mathrm{min} /$ $1.73 \mathrm{~m}^{2}$, the accuracy of plasma BNP and NT-proBNP levels for detection and stratification of CHF becomes unreliable and the degree of responsiveness to treatment is not known.

4.2.1: In people with GFR $<60 \mathrm{ml} / \mathrm{min} / 1.73 \mathrm{~m}^{2}$ (GFR categories G3a-G5), we recommend that serum concentrations of BNP/NT-proBNP be interpreted with caution and in relation to GFR with respect to diagnosis of heart failure and assessment of volume status. (1B)

\section{RATIONALE}

This statement is intended to remind clinicians that although the prevalence of fluid overload and heart failure increases with lower GFR categories, BNP becomes less reliable as a predictor of fluid overload and heart failure at lower GFRs. While it is associated with worse outcomes, cutoff values indicative of heart failure in general populations may or may not be appropriate and changes in values with treatment may or may not have the same meaning.

\section{Evidence Base}

Natriuretic peptides belong to a family of circulatory peptide hormones from either myocardial cell origin (atrial natriuretic peptide [ANP] and BNP) or endothelial cell origin (C-type) ${ }^{491,492}$ They play an important role in regulating BP and body fluid volume by their natriuretic and diuretic actions, arterial dilatation, and inhibition of the RAAS. ${ }^{493}$ Natriuretic peptides for the diagnosis of CHF have been a diagnostic breakthrough in cardiology where determination of their concentration in serum can help identify patients with left ventricular systolic dysfunction. ${ }^{494,495}$

Concentrations of natriuretic peptides increase in people with $\mathrm{CHF}$ and other CVDs as a consequence of 
pressure and volume overload. ${ }^{496,497}$ Among people with progressive LVH, concentrations of NT-proBNP are typically elevated in proportion to the degree of left ventricular mass increase.

Overall, BNP levels provide a better index of left ventricular mass and load than do ANP levels and BNP has emerged as a superior biomarker to ANP for clinical applications involving CHF and left ventricular dysfunction. ${ }^{491,498}$ NT-proBNP may have analytical advantages over BNP because of greater stability due to a longer half-life. The cause of an elevated BNP level is multifactorial in origin and may reflect cardiac dysfunction, and/or changes in renal function. ${ }^{499}$ Since renal dysfunction intrinsically was shown to affect BNP levels in some studies, the diagnostic value of BNP levels in the presence of $\mathrm{CKD}$ has been questioned. ${ }^{500,501}$

Earlier research hypothesized that measurement of natriuretic peptides could be of use in monitoring excess fluid volume and dry weight in people requiring dialysis, ${ }^{502,503}$ but this remains unproven and the significance of plasma natriuretic peptides and their clinical role in dialysis remains unclear. ${ }^{491}$

Although several studies revealed higher BNP levels as GFR declined, outcomes of other studies suggest that the relationship between renal function and BNP levels may be most strongly dependent on cardiac and volume-related factors. ${ }^{504-506}$ To test this hypothesis, Tagore et al. studied BNP levels in a cohort of 143 clinically euvolemic subjects with CKD in whom absence of heart disease was clinically validated and found that plasma BNP levels were independent of GFR. ${ }^{504}$ Suresh and Farrington ${ }^{507}$ studied people on dialysis and concluded that BNP levels were predictive of presence of left ventricular dysfunction, cardiac events, and survival in the presence of ESRD, suggesting that BNP levels may be informative across the full range of renal function and even in its absence. As previously established by several studies, the level of NT-proBNP is a strong prognostic marker in both the general population ${ }^{508}$ and in various disease states, e.g., acute and $\mathrm{CHF}^{509,510}$ coronary heart disease, ${ }^{511,512}$ and hypertension. ${ }^{513}$

\section{International Relevance}

The issue of how to differentially interpret BNP in the presence or absence of CHF and within the context of declining renal function has received considerable attention but remains incompletely resolved from a clinical perspective. Changes in either renal or cardiac function could potentially impact levels of natriuretic peptides and incomplete knowledge of an individual's clinical status relative to the degree of cardiac pathology or renal dysfunction could confound interpretation of BNP levels. Given the cost of biomarkers assays and the uncertainties related to interpretation of serum levels of BNP/NT-proBNP in people with $\mathrm{CKD}$, competing priorities for health-care resource may dictate reliance on clinical evaluation and local practices of care in some areas of the world. Until better data are available, clinicians are asked to use this laboratory measurement with caution as a diagnostic test for heart failure in those with CKD.

\section{Implications for Clinical Practice and Public Policy}

Since people with ESRD with elevated levels of BNP have a higher RR of death, this biomarker could be used as an individual risk marker with the aim of identifying those at high risk. Clinical correlation is of utmost importance to ensure accurate diagnosis and appropriate therapy.

Studies to understand the variability of BNP by categories of eGFR and urine ACR, within and between individuals over time, and the response to therapy should be undertaken so as to better inform clinicians.

\section{Areas of Controversy, Confusion, or Non-consensus}

The relationship between renal function and BNP, the role of natriuretic peptides as markers of fluid overload and as predictors of mortality, and the utility of serial monitoring of BNP/NT-proBNP levels in people with CKD, and responsiveness to therapy remains unknown.

\section{Clarification of issues and key points}

Natriuretic peptides may be surrogate markers for prediction of mortality in people with CKD reflecting the association of mortality with left ventricular dysfunction and LVH. Before routine measurements of these biomarkers are recommended, their utility in guiding or changing clinical practice should be assessed.

\section{Pediatric Considerations}

There is robust literature in both children and neonates regarding both the normative values of $\mathrm{BNP}^{514}$ and its value in predicting outcomes of pediatric heart failure. ${ }^{515}$ To date no studies in children specifically address the issue of reduced GFR in this population. However, application of similar principles would likely apply.

\section{Troponins}

In recent years newer markers of myocardial injury have been introduced into clinical practice. ${ }^{516,517}$ Among these, cardiac troponins have proven to be specific markers of myocardial damage. ${ }^{518,519}$ Cardiac troponins have been considered the gold standard biochemical test for diagnosis of myocardial damage because they have nearly absolute myocardial tissue specificity, as well as high sensitivity, thereby reflecting even microscopic zones of myocardial necrosis. ${ }^{517,520}$ Furthermore, increasing evidence indicates that abnormal troponin measurements identify a subgroup of patients who have an increased risk of major cardiac events ${ }^{519,521-525}$ and that measurement of cardiac troponin $\mathrm{T}$ ( $\mathrm{cTnT}$ ) prior to commencing renal replacement is a significant independent predictor of survival. ${ }^{526}$ However, these markers are renally excreted and are often found to be elevated in people with CKD irrespective of specific symptomatology. Thus, there is uncertainty as to the clinical importance of their different 
magnitudes of elevation in CKD. There are no data on 'expected' values of these markers in the context of CKD, or at different categories of GFR and albuminuria.

4.2.2: In people with GFR $<60 \mathrm{ml} / \mathrm{min} / 1.73 \mathrm{~m}^{2}$ (GFR categories G3a-G5), we recommend that serum concentrations of troponin be interpreted with caution with respect to diagnosis of acute coronary syndrome. (1B)

\section{RATIONALE}

This statement is intended to remind clinicians that isolated elevated serum concentrations of troponin (in the absence of symptoms or electrocardiographic changes) may not necessarily portend a diagnosis of ACS at lower GFR categories. While there are data linking troponin elevations with poor outcomes, the specific implications for an individual in clinical practice are not clear. Hence, this statement simply cautions the clinician with respect to interpretation but does not suggest that the laboratory values are not of significance. In a patient with clinical symptoms suggestive for ischemic heart disease, an elevated cardiac troponin I (cTnI) is suggestive for ACS.

\section{Evidence Base}

cTnT and cTnI are low-molecular-weight proteins that form part of the troponin complex and are integral components of the myofibrillar contractile apparatus of the heart. ${ }^{527}$ Loss of integrity of cardiac myocyte membranes causes release of cardiac troponins into the circulation, which can be detected by highly sensitive assays developed for $\mathrm{cTnT}$ and cTnI to diagnose ACS. $^{521} \mathrm{~A}$ potent stimulus for the release or production of natriuretic peptides is mechanical stretching, ${ }^{528}$ whereas plasma cTnT specifically and sensitively reflects myocardial injury and is considered to be an index of irreversible myocardial change. ${ }^{521}$ In addition, cTnT levels can predict multi-vessel CAD in people requiring dialysis. ${ }^{529}$ However, the significance of screening people with CKD for $\mathrm{CAD}$ using these cardiac biomarkers is questionable ${ }^{530}$ since increases in serum cardiac troponin concentration can occur in the absence of an ACS. ${ }^{531}$

Most studies ${ }^{517,532-545}$ have focused on people with ESRD where increases in serum cTnT concentrations have been observed in $20 \%-90 \%$ of subjects ${ }^{540}$ but generally much lower when $\mathrm{cTnI}$ was measured. Information about cardiac troponins and their relationship with comorbidity is sparse in people with CKD who are not receiving dialysis treatment. ${ }^{526,527,546,547}$ Increased cardiac troponin concentrations may occur early in CKD, including GFR categories $30-59 \mathrm{ml} / \mathrm{min} / 1.73 \mathrm{~m}^{2}$, and more commonly as CKD advances.

Powerful prognostic data emerging from the dialysis population demonstrate that increased concentrations of cardiac troponins reflect either subclinical myocardial damage caused by silent ischemia or myocardial remodeling in the development of $\mathrm{LVH}^{539,542}$

Increased levels of cTnT measured with the highly sensitive assay, well below the detection range of standard assays, have been shown to be associated with cardiac structural abnormalities including LVH (both left ventricular wall thickening and dilation) and left ventricular systolic dysfunction, ${ }^{548}$ and to have a graded association with allcause and CVD mortality independent of traditional risk factors, renal function, and levels of other biomarkers such as high sensitivity CRP and NT-proBNP.

These findings suggest that chronic elevation of troponin levels may be mediated to a greater extent by indices of heart failure (such as higher left ventricular mass, left ventricular dysfunction, or increased NT-proBNP levels) than indices of atherosclerosis or ischemia. ${ }^{548}$

\section{International Relevance}

The costs of these tests, the differing sensitivity of the assays available, the paucity of data in people with CKD, and the poor correlation of serum troponin levels with ACS in people with CKD should be weighed against competing local healthcare priorities and resource before their introduction into routine clinical practice. Emphasis should be placed on the clinical context and local standard practices of care.

\section{Implications for Clinical Practice and Public Policy}

Troponin elevation may result from repeated episodes of clinically silent $\mathrm{MI},{ }^{52,549}$ although CAD frequently underlies troponin elevation and increased cTnT is also frequently encountered in patients at low risk for CAD. Troponin elevations are as frequent in subjects without $\mathrm{CAD}$ as in those with known $C A D^{517,550-552}$ and in people with $C K D$, serial increasing cTn levels may be of greater utility for diagnosis of ACS. In the context of symptoms and signs of ACS, elevations of troponins should not be ignored nor incorrectly attributed to simply 'CKD.' Thus the importance of ordering the test for specific indications for those with CKD should be emphasized.

\section{Areas of Controversy, Confusion, or Non-consensus}

Information about cardiac troponins and their relationship with comorbidity is sparse in people with CKD who are not receiving dialysis treatment. Although elevated values for $\mathrm{TnT}$ are common in people with low GFR in the absence of ACS or CHF, the prevelance of elevated TnI in the absence of ACS or CHF in people with low GFR may be only $0.4-6 \%$ depending on the cutoff value chosen. ${ }^{532}$

\section{Clarification of Issues and Key Points}

As pointed out by Roberts et al., ${ }^{553}$ although management strategies in people without CKD based on these biomarkers are quite clear, in people with CKD it is not the case despite the strong data on the prognostic implications. These biochemical markers can only be useful if they are interpreted in the context of the clinical history and examination with consideration given as to what management is appropriate for each individual, and if a cardiovascular therapy that is effective in this population is identified or developed. ${ }^{553}$ The importance of understanding and contextualizing elevations 
in troponin values in CKD populations cannot be overstated: under-treatment in symptomatic individuals should be avoided, and better understanding of treatment strategies in those asymptomatic individuals is the focus of ongoing study.

\section{Pediatric Considerations}

While normative values exist for troponin in children, ${ }^{554}$ its utility as a general screening marker for myocardial ischemia in pediatrics has recently been called into question, ${ }^{555}$ and no data exist relating reduced renal function and troponin levels in children.

\section{Non-invasive testing}

Most studies of cardiac evaluation in people with CKD have involved candidates for kidney transplantation in order to identify existing cardiac conditions amenable to risk modification and to exclude people with short expected survival due to cardiac morbidity. Among the tests for cardiovascular assessment, coronary angiography is considered invasive, expensive, and associated with risks such as contrast-induced nephropathy and cholesterol embolism. When trying to detect CAD or predict future cardiovascular events in people with CKD, nuclear imaging modalities using thallium tracers have shown conflicting results, especially in the context of small vessel disease. Clinicians should be aware of the limitations of radionuclear testing as a screening tool for significant CAD given the limitations of the test. Exercisedependent techniques are limited by poor exercise tolerance of many subjects and people with CKD frequently have cardiac evaluation performed with pharmacological agents. ${ }^{556,557}$

\subsection{3: We recommend that people with CKD presenting with} chest pain should be investigated for underlying cardiac disease and other disorders according to the same local practice for people without CKD (and subsequent treatment should be initiated similarly). (1B)

4.2.4: We suggest that clinicians are familiar with the limitations of non-invasive cardiac tests (e.g., exercise electrocardiography [ECG], nuclear imaging, echocardiography, etc.) in adults with CKD and interpret the results accordingly. (2B)

\section{RATIONALE}

These statements are worded in this way firstly because although people with CKD are at increased risk from cardiovascular events, observational study has shown that they are frequently discriminated against in terms of investigation and management of such events. Secondly, non-invasive cardiac tests have known additional limitations in people with CKD and such limitations and their consequences need to be clearly understood by health professionals caring for people with CKD.

\section{Evidence Base}

Noninvasive stress testing appears to be the most common first approach to cardiac evaluation of asymptomatic patients, prompted by diabetes, age and risk-factor burden. These tests include myocardial perfusion studies, stress echocardiography, and most recently, cardiac computed tomographic angiography. However, people with CKD are underrepresented in studies evaluating the diagnostic sensitivities and specificities of noninvasive tests. Exercise ECG is limited by lack of specificity of the ST-segment response and by inability of many people with CKD to exercise to a diagnostic workload.

These tests have imperfect sensitivity and specificity in people with $\mathrm{CKD}$ or, in the case of tomographic angiography, evaluation in this population is as yet unpublished. ${ }^{556} \mathrm{CAD}$ has been detected in a high proportion of people on long-term dialysis therapy. Studies describing associations of angiographic coronary stenosis with subsequent clinical events in people with ESRD, including those undergoing transplant evaluations, have reached inconsistent conclusions. ${ }^{558-562}$

Abnormalities on myocardial perfusion studies correlate well with the presence of CAD in the general population, with mean weighted sensitivity of $88 \%$ and mean weighted specificity of $74 \% .{ }^{563}$ The performance of myocardial perfusion studies in identifying CAD in people with CKD is more variable, with reported sensitivities and specificities in people with ESRD ranging from $37 \%$ to $90 \%$ and $40 \%$ to $90 \%$, respectively. ${ }^{564-567}$ Nonetheless, results from myocardial perfusion studies have prognostic value for cardiac events and mortality. ${ }^{568,569}$ In a meta-analysis of 12 studies involving thallium-201 scintigraphy and dobutamine stress echocardiography, people with ESRD with inducible ischemia had approximately 6 times the risk of MI and 4 times the risk of cardiac death as those without inducible defects. ${ }^{570}$ Moreover, subjects with fixed defects also had nearly 5 times the risk of cardiac death.

The prognostic value of myocardial perfusion studies has been shown with other perfusion tracers. For example, in a study of 126 people with ESRD who underwent technetium$99 \mathrm{~m}$ myocardial perfusion studies as part of their pretransplant assessment, the presence of a reversible defect was associated with 3 times the risk of post-transplant cardiac events (HR 3.1; 95\% CI 1.1-18.2) and nearly twice the risk of death (HR 1.92; 95\% CI 1.1-4.4) compared with normal test results. ${ }^{571}$

De Lima et al. ${ }^{572}$ prospectively studied 126 renal transplant candidates clinically classified as moderate (age $>50$ years) or high (diabetes, extra cardiac vascular disease, or known CAD) coronary risk with myocardial perfusion studies, dobutamine stress echocardiography, and coronary angiography. Significant $\mathrm{CAD}$, defined as greater than $70 \%$ stenosis in 1 or more major epicardial artery on angiography was found in $42 \%$ of the sample. After a median follow-up of 46 months, clinical risk stratification and coronary angiography predicted major cardiac events, but myocardial perfusion studies and dobutamine stress echocardiography did not.

Newer imaging techniques are under current evaluation but a review here is beyond the scope of this guideline; these 
techniques include: stress myocardial perfusion single photon emission computed tomography (SPECT); $5{ }^{57,573-576}$ ${ }^{18} \mathrm{~F}$-BMS, a novel fluorine-based myocardial perfusion tracer; ${ }^{557,577}$ and gated imaging using radio-labelled ammonia $\left({ }^{13} \mathrm{NH}_{3}\right)$.

\section{International Relevance}

People with CKD who are being investigated prior to transplantation should be assessed according to the national and international cardiology guidelines and taking into account local practice and availability of resource.

\section{Implications for Clinical Practice and Public Policy}

There is still a need for a safe, noninvasive, diagnostic modality that will allow stratification of cardiovascular risk among people with CKD. With the development of new imaging markers, it becomes difficult for clinicians to choose the imaging tests that can best aid clinical decisions for a given patient. Imaging methods should provide accuracy and precision at acceptable cost. Until advanced imaging techniques (e.g., cardiac MRI, positron emission tomography, cardiac computed tomography) are standardized, clinicians should rely on the most reliable and familiar techniques in their environment. ${ }^{578}$

\section{Areas of Controversy, Confusion, or Non-consensus}

Clinical and cost-effectiveness could be improved; cardiac evaluation in people with CKD should be based on data from clinical trials.

\section{Clarification of Issues and Key Points}

If cardiac evaluation is at present predominantly used for risk stratification and prognosis, in the future it may guide optimal therapy and monitor clinical progress. ${ }^{557}$

\subsection{CKD AND PERIPHERAL ARTERIAL DISEASE}

There is a strong link between $\mathrm{CKD}$ and peripheral arterial disease (PAD). Symptoms of PAD may only be present in a minority of people who have clinical evidence of PAD. ${ }^{579}$ It is therefore important to measure ankle-brachial index and perform regular systematic assessment of the lower limbs of people at high risk of PAD to identify bruits, loss of pulses, cool pale extremities, delay in venous filling, and skin ulceration. ${ }^{580}$

\subsection{1: We recommend that adults with CKD be regularly examined for signs of peripheral arterial disease and be considered for usual approaches to therapy. (1B) \\ 4.3.2: We suggest that adults with CKD and diabetes are offered regular podiatric assessment. (2A)}

\section{RATIONALE}

These statements are worded in this way in order to highlight the increased risk of PAD in people with CKD, particularly those with CKD and diabetes.

\section{Evidence Base}

The prevalence of PAD is common in people with CKD and it increases with lower levels of GFR (Table 31). PAD can be

Table 31 | Peripheral arterial disease and CKD

\begin{tabular}{|c|c|c|c|}
\hline Study & Population & PAD definition & Outcome of interest \\
\hline $\mathrm{O}^{\prime}$ Hare et al. ${ }^{585}$ & NHANES age $40+$ & $\mathrm{ABI}<0.9$ & $\begin{array}{l}24 \% \text { prevalence in people with } \mathrm{CKD} \text { and } \mathrm{arCl} \text { of } \\
<60 \mathrm{ml} / \mathrm{min}(<1 \mathrm{ml} / \mathrm{s}) \text { versus } 3.7 \% \text { in those with } \\
\text { normal kidney function }\end{array}$ \\
\hline O'Hare et al. ${ }^{586}$ & $\begin{array}{l}\text { Cardiovascular Health Study, adults } \\
\text { age } 65+\end{array}$ & $\begin{array}{l}\text { Lower-extremity PAD procedure } \\
\text { (bypass surgery, angioplasty, or } \\
\text { amputation) }\end{array}$ & $\begin{array}{l}\text { HR for PAD procedure } 2.5(95 \% \mathrm{Cl} 1.2-5.1) \text { for } \\
\text { highest quintile of cystatin } \mathrm{C}(\geq 1.28 \mathrm{mg} / \mathrm{l}) \text { versus } \\
\text { lowest }(\leq 0.9 \mathrm{mg} / \mathrm{l})\end{array}$ \\
\hline De Vinuesa et al. ${ }^{582}$ & $\begin{array}{l}102 \text { adults in a CKD clinic, mean age } \\
70 \pm 11 \text { years, GFR } 15-60 \mathrm{ml} / \mathrm{min} / \\
1.73 \mathrm{~m}^{2}\end{array}$ & $\mathrm{ABI}<0.9$ & $\begin{array}{l}17 \% \text { signs and symptoms of PAD, which had } \\
\text { passed unnoticed; } 32 \% \text { had } A B I<0.9 \text { (mean } \\
0.64 \pm 0.25 \text { ) }\end{array}$ \\
\hline Liew et al. ${ }^{584}$ & $\begin{array}{l}\text { 6-year follow up of } 1027 \text { subjects (ABI } \\
\text { index recording and GFR measured } \\
\text { within } 90 \mathrm{~d} \text { ) }\end{array}$ & $\mathrm{ABI}<0.9$ & $\begin{array}{l}\text { 6-year mortality rate for CKD and PAD } 45 \% \text { versus } \\
28 \% \text { CKD alone, 26\% PAD alone, and } 18 \% \text { for } \\
\text { neither condition }\end{array}$ \\
\hline Bello et al. ${ }^{581}$ & $\begin{array}{l}920,985 \text { subjects with GFR and } \\
\text { proteinuria assessment, median } \\
\text { follow-up } 35 \text { months (IQR 22-44) }\end{array}$ & $\begin{array}{l}\text { Time to first hospitalization with } \\
\text { PAD }\end{array}$ & $\begin{array}{l}1891 \text { of subjects }(0.2 \%) \text { hospitalized at least once } \\
\text { for PVD, adjusted rates increased with lower GFR }\end{array}$ \\
\hline
\end{tabular}

Abbreviations: $\mathrm{ABI}$, ankle-brachial index; $\mathrm{CHD}$, coronary heart disease; $\mathrm{Cl}$, confidence interval; $\mathrm{CKD}$, chronic kidney disease; $\mathrm{CrCl}$, creatinine clearance; d, day; GFR, glomerular filtration rate; HERS, Heart and Estrogen/Progestin Replacement Study; HR, hazard ratio; IQR, interquartile range; NHANES, National Health and Nutrition Examination Survey; PAD, peripheral arterial disease; PVD, peripheral vascular disease. 
attributed to the greater prevalence of traditional risk factors such as diabetes, hypertension, dyslipidemia, advanced age, and renal-specific factors. ${ }^{581-588}$

Diabetes is the leading cause of CKD globally and the risk of diabetic foot ulceration and lower extremity amputation in subjects with diabetes increases dramatically with lower GFR. In a cohort of 90,617 individuals with diabetes over a median observation time of 2.4 years, the HR for amputation increased from 2.08 (95\% CI 1.68-2.58) for those with GFR $30-59 \mathrm{ml} / \mathrm{min} / 1.73 \mathrm{~m}^{2}$ to 7.71 (95\% CI 5.29-11.26) for those with GFR $<30 \mathrm{ml} / \mathrm{min} / 1.73 \mathrm{~m}^{2}$ compared to reference subjects with GFR $>60 \mathrm{ml} / \mathrm{min} / 1.73 \mathrm{~m}^{2}$. 589

Screening for PAD is recommended for adults in the general population based on age and number of risk factors. ${ }^{50}$ Although an ankle-brachial index of $<0.9$ is generally considered evidence of PAD, the utility of this measure is unproven in CKD because of the greater prevalence of vessel calcification. Evidence-based medical therapies for PAD in people with CKD are similarly lacking. Smoking cessation is mandatory. Aspirin may be beneficial for prevention of cardiovascular events. Clopidogrel has not been studied in people with CKD and PAD. No RCTs evaluating percutaneous versus surgical revascularization techniques have been conducted in people with CKD and PAD but outcome studies all suggest that CKD confers an increased risk of adverse outcome, regardless of the revascularization technique employed. ${ }^{591,592}$ These statements do not apply to pediatric practice.

\section{International Relevance}

There is no evidence to suggest that the international approach to identification and management of PAD in people with CKD should differ.

\section{Implications for Clinical Practice and Public Policy}

People with CKD are more at risk of PAD and need regular assessment and monitoring. The role of ankle-brachial index versus other diagnostic techniques may have implications for future practice. Prospective data on non-surgical therapies and data regarding percutaneous versus surgical revascularization are required to inform policy and recommended procedure.

\section{Areas of Controversy, Confusion, or Non-consensus}

There remains ongoing debate as to the timing and use of specific diagnostic criteria for identification of PAD in people with CKD and no studies have examined the utility of conventional methods in early detection of PAD in CKD. The use of specific medical and surgical management strategies in people with CKD and PAD has not been evaluated. There is no reason to believe that treatment strategies should differ, though risks of diagnostic testing (such as angiography) remain real.

\subsection{MEDICATION MANAGEMENT AND PATIENT SAFETY IN CKD}

Many drugs and investigative pharmaceuticals are renally excreted and their dosage may need to be reduced in patients with CKD in order to avoid toxicity. As many people with CKD are elderly, there should also be consideration of chronological age.

It is beyond the scope of this guideline to list all the agents that may need dose adjustment in CKD or which should be avoided due to potential nephrotoxicity. This information is widely available in documents which may exist at local, regional, or national bodies (e.g., British National Formulary: www.bnf.org), and other textbooks of pharmacology. ${ }^{533,594}$ However key classes of commonly prescribed drugs in people with CKD will be mentioned together with suggestions for dose adjustment. Much of this guidance is based upon our understanding of pharmacology and pharmacokinetics rather than randomized control trial evidence.

The statements presented here are intended to inform clinicians caring for those with CKD or at risk for CKD with respect to common clinical situations or exposures which may put people at risk for AKI or progression of CKD.

4.4.1: We recommend that prescribers should take GFR into account when drug dosing. (1A)

4.4.2: Where precision is required for dosing (due to narrow therapeutic or toxic range) and/or estimates may be unreliable (e.g., due to low muscle mass), we recommend methods based upon cystatin $C$ or direct measurement of GFR. (1C)

4.4.3: We recommend temporary discontinuation of potentially nephrotoxic and renally excreted drugs in people with a GFR $<60 \mathrm{ml} / \mathrm{min} / 1.73 \mathrm{~m}^{2}$ (GFR categories G3a-G5) who have serious intercurrent illness that increases the risk of AKI. These agents include, but are not limited to: RAAS blockers (including ACE-Is, ARBs, aldosterone inhibitors, direct renin inhibitors), diuretics, NSAIDs, metformin, lithium, and digoxin. (1C)

4.4.4: We recommend that adults with CKD seek medical or pharmacist advice before using over-the-counter medicines or nutritional protein supplements. (1B)

4.4.5: We recommend not using herbal remedies in people with CKD. (1B)

4.4.6: We recommend that metformin be continued in people with GFR $\geq 45 \mathrm{ml} / \mathrm{min} / 1.73 \mathrm{~m}^{2}$ (GFR categories G1-G3a); its use should be reviewed in those with GFR $30-44 \mathrm{ml} / \mathrm{min} / 1.73 \mathrm{~m}^{2}$ (GFR category G3b); and it should be discontinued in people with GFR $<30 \mathrm{ml} / \mathrm{min} / 1.73 \mathrm{~m}^{2}$ (GFR categories G4-G5). (1C)

4.4.7: We recommend that all people taking potentially nephrotoxic agents such as lithium and calcineurin inhibitors should have their GFR, electrolytes and drug levels regularly monitored. $(1 A)$

4.4.8: People with CKD should not be denied therapies for other conditions such as cancer but there should be appropriate dose adjustment of cytotoxic drugs according to knowledge of GFR. (Not Graded) 


\section{RATIONALE}

These statements are worded in this way in order to inform appropriate prescribing and management of medical conditions in people with CKD. Recommendation 4.4.1 is specifically worded to ensure that clinicians remember that in those circumstances where accurate GFR is required (narrow therapeutic or toxic window), a direct measurement, not an estimate of GFR should be undertaken.

\section{Evidence Base}

Potential problems associated with use of medication in people with CKD include:

a) Reduced ability to excrete drugs and/or their metabolites

b) Increased sensitivity to medications (e.g., those bound to albumin in hypoalbuminemic states such as nephrotic syndrome)

c) Diminished tolerance of side effects, particularly in the elderly

d) Loss of efficacy ${ }^{13}$

Wherever possible, people with CKD should receive the same treatment as those with normal renal function. However, the dosages may need adjustment according to GFR.

There are medicines whose toxicity is worsened in acute illness particularly in a setting of dehydration such as diarrhea and vomiting. General advice about appropriate dosing and when to restart these agents should be given to people taking these drugs during intercurrent illness, together with a recommendation for consultation with a health-care professional as soon as possible.

Use of herbal and over-the-counter medicines is very common worldwide and some (such as those containing aristolochic acid $)^{595,596}$ are known to be nephrotoxic. There is no good quality safety or efficacy data for many of these compounds.

Table 32 details specific advice for various classes of drugs where CKD may be an issue. These include RAAS blocking agents, ${ }^{262}$ beta-blockers, analgesics, antibiotics, lithium, ${ }^{597}$ hypoglycemic agents, ${ }^{598-600}$ lipid-lowering agents, ${ }^{449,601-604}$ certain chemotherapeutic agents, and anticoagulants. ${ }^{605}$

\section{International Relevance}

This guidance is based upon knowledge of pharmacology that has universal relevance. The main international implication is centered on costs of some newer therapies compared to the older ones. Somewhat paradoxically, as the weight of evidence resides mainly with agents that have been available for longer, they have the advantage of being less costly, and have the side effects that are well documented; thus many of these older agents are preferred.

\section{Pediatric Considerations}

No specific recommendation for or against the use of a BSA adjusted eGFR in relation to drug dosing can be made in children with CKD as the majority of drug studies have been performed in adult males and extrapolated to children without consideration for BSA and/or pediatric renal function or clearance.

The exception to this rule would be that for any drug where pediatric pharmacokinetic or pharmacodynamic studies exist, the method used in calculating the effect of renal function should be used when estimating the need for dose adjustments or modifications for the individual patient.

Numerous resources for dose adjustments for pediatric CKD and ESRD patients exist, including those found in numerous textbooks ${ }^{606,607}$ and online resources. ${ }^{608}$

An additional value of dedicated pediatric renal multidisciplinary teams, in terms of caring for children with CKD and ESRD, is their ability to provide anticipatory guidance for the patients and families. All such teams should at the least provide written or online information to their patients and families directing them to seek advice in situations where they may be prescribed medications from other providers or may be seeking over-the-counter drugs or supplements. The presence of trained pediatric pharmacists as part of such teams can be invaluable in achieving such support and education for both families as well as the CKD health-care providers and community resources such as local pharmacists.

\section{LIMITATIONS}

People with CKD are often excluded or not identified from trials of medications for non-renal disease. Recommendations are partly based upon knowledge of pharmacology rather than controlled trials in carefully defined populations.

\section{RESEARCH RECOMMENDATIONS}

National and international research groups, and those with CKD-focus organizations (International Society of Nephrology, International Federation of Kidney Foundations, and other national bodies) should ensure adequate representation of people with CKD in clinical trials, leading to an improved understanding of pharmacodynamics of those with CKD.

\section{5: IMAGING STUDIES}

\subsection{1: Balance the risk of acute impairment in kidney function due to contrast agent use against the diagnostic value and therapeutic implications of the investigation. (Not Graded)}

\section{RATIONALE}

Use of iodinated radiocontrast media has been associated with AKI with reported rates of $0-11 \%$ depending on the population under study, the type of agent that is used, and the definition of nephrotoxicity. ${ }^{609}$ The following recommendations are concordant with those from the American College of Radiology (ACR), ${ }^{610}$ the European Society of Urogenital Radiology (ESUR) ${ }^{611}$ and the KDIGO Clinical Practice Guideine for Acute Kidney Injury. ${ }^{7}$ 
Table 32 | Cautionary notes for prescribing in people with CKD

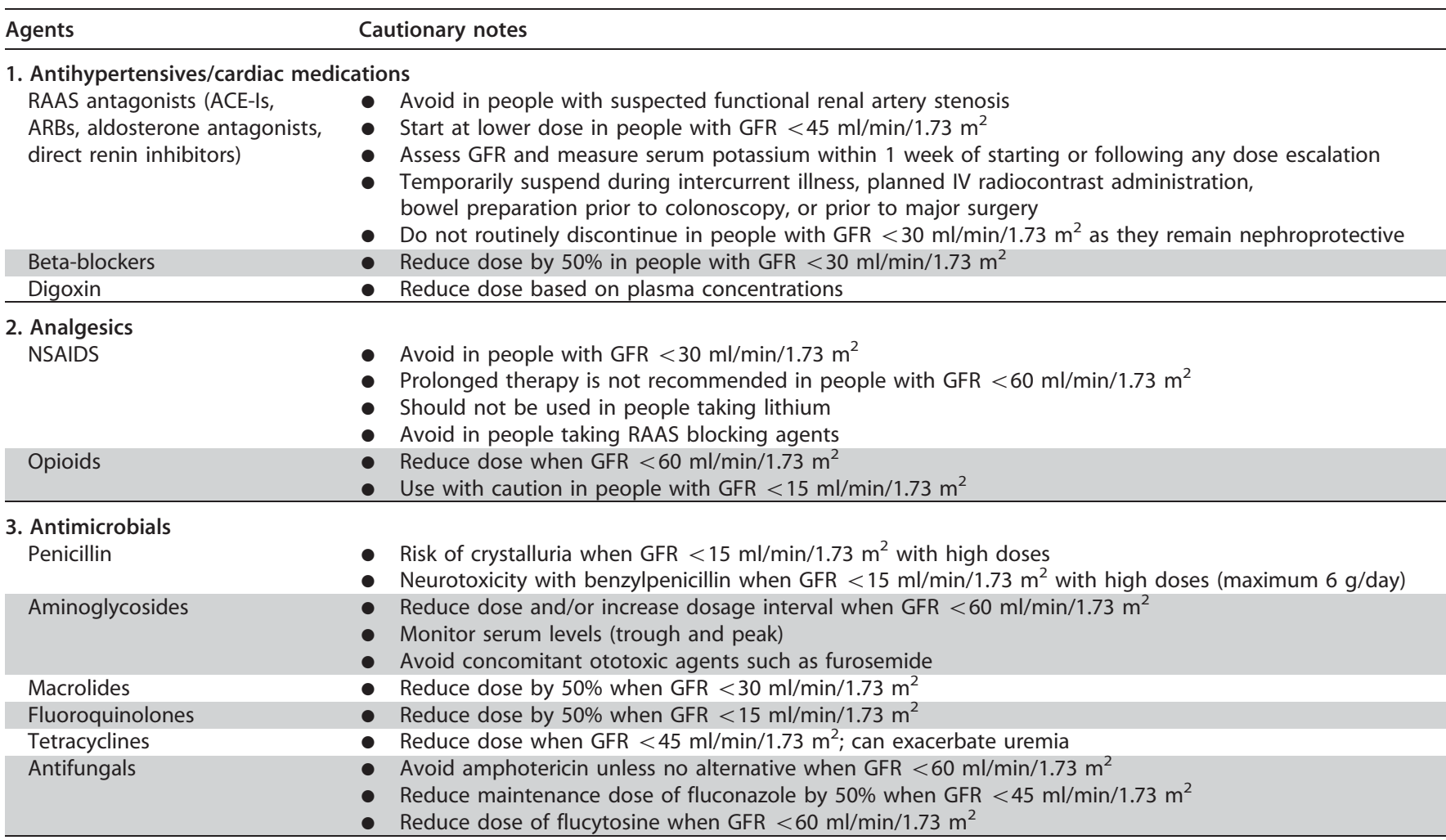

\section{Hypoglycemics Sulfonylureas}

Insulin

Metformin
- Avoid agents that are mainly renally excreted (e.g., glyburide/ glibenclamide)

- Other agents that are mainly metabolized in the liver may need reduced dose when GFR $<30 \mathrm{ml} / \mathrm{min} / 1.73 \mathrm{~m}^{2}$ (e.g., gliclazide, gliquidone)

- Partly renally excreted and may need reduced dose when GFR $<30 \mathrm{ml} / \mathrm{min} / 1.73 \mathrm{~m}^{2}$

- Suggest avoid when GFR $<30 \mathrm{ml} / \mathrm{min} / 1.73 \mathrm{~m}^{2}$, but consider risk-benefit if GFR is stable

- Review use when GFR $<45 \mathrm{ml} / \mathrm{min} / 1.73 \mathrm{~m}^{2}$

- Probably safe when GFR $\geqslant 45 \mathrm{ml} / \mathrm{min} / 1.73 \mathrm{~m}^{2}$

- Suspend in people who become acutely unwell

\section{Lipid-lowering \\ Statins}

- No increase in toxicity for simvastatin dosed at $20 \mathrm{mg}$ per day or simvastatin $20 \mathrm{mg}$ /ezetimide $10 \mathrm{mg}$ combinations per day in people with GFR $<30 \mathrm{ml} / \mathrm{min} / 1.73 \mathrm{~m}^{2}$ or on dialysis ${ }^{449}$

- Other trials of statins in people with GFR $<15 \mathrm{ml} / \mathrm{min} / 1.73 \mathrm{~m}^{2}$ or on dialysis also showed no excess toxicity - Increases SCr by approximately $0.13 \mathrm{mg} / \mathrm{dl}(12 \mu \mathrm{mol} / \mathrm{l})$

Fenofibrate

- Reduce dose when GFR $<60 \mathrm{ml} / \mathrm{min} / 1.73 \mathrm{~m}^{2}$

6. Chemotherapeutic Cisplatin

- Avoid when GFR $<30 \mathrm{ml} / \mathrm{min} / 1.73 \mathrm{~m}^{2}$

- Reduce dose when GFR $<60 \mathrm{ml} / \mathrm{min} / 1.73 \mathrm{~m}^{2}$

- Reduce dose when GFR $<60 \mathrm{ml} / \mathrm{min} / 1.73 \mathrm{~m}^{2}$

- Avoid if possible when GFR $<15 \mathrm{ml} / \mathrm{min} / 1.73 \mathrm{~m}^{2}$
- Halve the dose when GFR $<30 \mathrm{ml} / \mathrm{min} / 1.73 \mathrm{~m}^{2}$

- Consider switch to conventional heparin or alternatively monitor plasma anti-factor Xa in those at high risk for bleeding

- Increased risk of bleeding when GFR $<30 \mathrm{ml} / \mathrm{min} / 1.73 \mathrm{~m}^{2}$

- Use lower doses and monitor closely when GFR $<30 \mathrm{ml} / \mathrm{min} / 1.73 \mathrm{~m}^{2}$

\section{Miscellaneous}

Lithium
- Nephrotoxic and may cause renal tubular dysfunction with prolonged use even at therapeutic levels

- Monitor GFR, electrolytes, and lithium levels 6 monthly or more frequently if the dose changes or the patient is acutely unwell

- Avoid using concomitant NSAIDs

- Maintain hydration during intercurrent illness

- Risk-benefit of drug in specific situation must be weighed

Abbreviations: ACE-I, angiotensin-converting enzyme inhibitor; ARB, angiotensin-receptor blocker; CKD, chronic kidney disease; GFR, glomerular filtration rate; NSAIDs, nonsteroidal anti-inflammatory drugs; RAAS, renin-angiotensin-aldosterone system; $\mathrm{SCr}$, serum creatinine. 


\section{Radiocontrast}

4.5.2: We recommend that all people with GFR $<60 \mathrm{ml} /$ $\mathrm{min} / 1.73 \mathrm{~m}^{2}$ (GFR categories G3a-G5) undergoing elective investigation involving the intravascular administration of iodinated radiocontrast media should be managed according to the KDIGO Clinical Practice Guideline for AKI including:

- Avoidance of high osmolar agents (1B);

- Use of lowest possible radiocontrast dose (Not Graded);

- Withdrawal of potentially nephrotoxic agents before and after the procedure $(1 C)$;

- Adequate hydration with saline before, during, and after the procedure $(1 A)$;

- Measurement of GFR 48-96 hours after the procedure $(1 C)$.

\section{RATIONALE}

These statements have been worded in order to inform safe radiological investigation of people with CKD and to avoid potential nephrotoxcity that can be associated with radiological imaging.

\section{Evidence Base}

Radiocontrast media associated AKI is a largely preventable cause of morbidity and mortality. There is no internationally agreed definition but most studies use an increase in SCr of $>0.5 \mathrm{mg} / \mathrm{dl}(44 \mu \mathrm{mol} / \mathrm{l})$ and/or a $25 \%$ increase from baseline SCr within 3 days of the procedure. ${ }^{7,609-611}$ Epidemiological studies and case series have identified the following risk factors for AKI:

a) GFR $<60 \mathrm{ml} / \mathrm{min} / 1.73 \mathrm{~m}^{2}$ (particularly if $<30 \mathrm{ml} / \mathrm{min} /$ $1.73 \mathrm{~m}^{2}$ )

b) Diabetes

c) Concurrent dehydration

d) $\mathrm{CHF}$

e) $>70$ years of age

f) Concurrent use of known nephrotoxic agents such as NSAIDs

g) Use of high osmolality agents (especially in those with GFR $<60 \mathrm{ml} / \mathrm{min} / 1.73 \mathrm{~m}^{2}$ )

h) Use of large doses of radiocontrast media

i) Intra-arterial injection

j) Gout (hyperuricemia)

Numerous studies of preventative strategies have been performed with the following conclusions:

a) High osmolar agents pose a greater risk of AKI in people with CKD. 609

b) Iso-osmolar agents compared to low-osmolar agents are associated with lower rates of AKI in some but not all studies. Wherever possible iso-osmolar agents should be used in people with CKD at high risk for AKI (although these tend to be more expensive). ${ }^{612}$ c) Although risk for AKI increases with GFR $<60 \mathrm{ml} / \mathrm{min} /$ $1.73 \mathrm{~m}^{2}$, the rates are particularly high $(7.8 \%$ in one study) when GFR is $<30 \mathrm{ml} / \mathrm{min} / 1.73 \mathrm{~m}^{2}$. Implementing preventative strategies for all with a GFR $<60 \mathrm{ml} / \mathrm{min} /$ $1.73 \mathrm{~m}^{2}$ may not be practical but a graded risk assessment taking into account all factors may be more realistic. Some guidelines such as American College Radiology $^{610}$ and ESUR $^{611}$ provide a checklist as a means of identifying patients at risk of AKI before investigation.

d) Extracellular volume expansion is widely recommended although there are few good quality trials on which to base an ideal protocol. ${ }^{609} 0.9 \%$ saline given by continuous infusion appears superior to $0.45 \%$ saline or bolus injection and there is no demonstration of consistent superiority of sodium bicarbonate over saline. Current guidance suggests either infusion of $1 \mathrm{ml} / \mathrm{kg}$ body weight/ hour for 3-12 hours before and after the procedure or $100 \mathrm{ml} / \mathrm{hr}$, beginning 6 to 12 hours before and continuing 4 to 12 hours after intravascular iodinated contrast medium administration. ${ }^{609,610}$

e) Use of $\mathrm{N}$-acetylcysteine or ascorbic acid as preventative measures has not been shown to be a consistent benefit.

\section{International Relevance}

This guidance has universal relevance although there are cost implications as the iso-osmolar contrast media are more expensive.

\section{LIMITATIONS}

Some of the guidance is based upon limited evidence and there is a need for more research into simple preventative measure such as pre-investigation rehydration (see below). There has not previously been a universal definition for AKI following administration of contrast media. However, recommendations from the KDIGO AKI Guideline suggest that the same general AKI definition and staging be used for changes in kidney function, irrespective of etiology.

\section{RESEARCH RECOMMENDATIONS}

Prospective studies using direct measures of GFR before and after administration of radiological contrast media are required to help define the incidence of AKI. Such studies would also be able to validate creatinine or other estimates of GFR in people undergoing radiological investigation.

Prospective controlled trials of rehydration using different fluids (saline, bicarbonate, Hartmann's) and validated estimates of GFR are urgently required.

Definitive studies of $\mathrm{N}$-acetylcysteine and other antioxidants would help determine their usefulness or otherwise.

\section{Gadolinium-containing contrast media}

Gadolinium is a rare earth element that is naturally highly toxic. When bound to proprietary chelating agents, it is essentially biologically inert in people with normal renal function and provides excellent contrast during MRI. These 
chelates are excreted unchanged by the kidneys by glomerular filtration and have much lower direct nephrotoxicity than conventional radioiodine contrast media. ${ }^{613}$ However over 200 cases of a scleroderma-like condition now termed nephrogenic systemic fibrosis (NSF) following gadolinium use in patients with CKD have been reported. ${ }^{613,614}$

4.5.3: We recommend not using gadolinium-containing contrast media in people with GFR $<15 \mathrm{ml} / \mathrm{min} /$ $1.73 \mathrm{~m}^{2}$ (GFR category G5) unless there is no alternative appropriate test. (1B)

4.5.4: We suggest that people with a GFR $<30 \mathrm{ml} / \mathrm{min} /$ $1.73 \mathrm{~m}^{2}$ (GFR categories G4-G5) who require gadolinium-containing contrast media are preferentially offered a macrocyclic chelate preparation. $(2 B)$

\section{RATIONALE}

These statements have been worded in order to enable safe administration of gadolinium to people with CKD. As with all tests requested in the CKD population, clinicians should be aware of the risk-benefit ratio of the use of gadolinium in people with GFR $15-29 \mathrm{ml} / \mathrm{min} / 1.73 \mathrm{~m}^{2}$.

\section{Evidence Base}

NSF is an untreatable and sometimes fatal condition that complicates the use of gadolinium-containing contrast media in people with CKD. Prevention is therefore the best approach with avoidance of gadolinium exposure unless clinically indicated and to use the lowest risk agent at the lowest dose. ${ }^{613,615}$

Although the risk of AKI with iodinated contrast media is much greater than that for NSF with gadolinium, the former is treatable with dialysis whereas the latter is not. Thus, consideration of more conventional imaging techniques should be undertaken in all with a GFR $<30 \mathrm{ml} / \mathrm{min} /$ $1.73 \mathrm{~m}^{2}{ }^{2}$. $10,613-615$

A recent meta-analysis and review have highlighted those patients most at risk and quantified an OR for NSF of between $20-50$ for those with a GFR $<15 \mathrm{ml} / \mathrm{min} / 1.73 \mathrm{~m}^{2}{ }^{2}{ }^{614}$

While there is general agreement that patients with a GFR $>15 \mathrm{ml} / \mathrm{min} / 1.73 \mathrm{~m}^{2}$ are at increased risk, it is not possible to derive a precise estimate. No patients with a GFR $>30 \mathrm{ml} /$ $\min / 1.73 \mathrm{~m}^{2}$ have developed NSF without concomitant liver failure. ${ }^{614}$

There is some suggestion that the type of gadolinium preparation plays a role: ${ }^{614,615}$ Linear chelated preparations such as gadodiamide may be more likely to cause NSF and should be avoided when GFR is $<30 \mathrm{ml} / \mathrm{min} / 1.73 \mathrm{~m}^{2}$. Gadoteridol, gadobutrol, or gadoterate should be considered if MRI with contrast is clinically essential. ${ }^{610,613,615}$ Moreover, as gadolinium is freely dialysed, ${ }^{616}$ most guidelines recommend dialysis in patients with a GFR $<15 \mathrm{ml} / \mathrm{min} / 1.73 \mathrm{~m}^{2}$ or for those already on dialysis immediately after (and perhaps repeated 24 hours later) completion of the procedure. ${ }^{610,615}$ The role of dialysis in people with GFR above $15 \mathrm{ml} / \mathrm{min} / 1.73 \mathrm{~m}^{2}$ is uncertain.

\section{International Relevance}

This guidance has universal relevance although there are cost implications as the non-linear chelated preparations are more expensive.

\section{Pediatric Considerations}

Regarding both Recommendations 4.5.3 and 4.5.4, a number of considerations specific to the use of gadolinium preparations in young children and neonates must also be considered in addition to the general admonishments against their use in situations of GFR $<30 \mathrm{ml} / \mathrm{min} / 1.73 \mathrm{~m}^{2}$. In particular, the FDA currently does not license any gadolinium-based contrast agent (GBCA) product for use in children less than 2 years of age; and likewise the European Medicines Agency cautions against the use of any GBCA in a child less than 1 year of age.

In addition, the ability to accurately estimate the relative value of a neonate or young infant's GFR leads to great difficulty in terms of assigning risk of GBCA exposure if one bases this on renal clearance. In a recent paper by Meng and colleagues, ${ }^{617}$ they surveyed a worldwide group of both cardiologists and radiologists with respect to their use of gadolinium in their pediatric MRI practice. While $93 \%$ of the respondents did evaluate renal function in some or all of their patients, only a slight majority (54\%) used an estimating formula, most often the Schwartz equation, with the remainder relying on $\mathrm{SCr}$ alone $(31 \%)$ or urine output $(6 \%)$. Perhaps of most concern was that renal function was assessed in only $33 \%$ and $31 \%$, respectively, of patients classified as 'all neonates' or 'all patients $<1$ week of age.' Equally concerning was the fact that $13 \%$ of the respondents would give gadolinium to some of these children in the face of a GFR $<30 \mathrm{ml} / \mathrm{min} / 1.73 \mathrm{~m}^{2}$.

In recognition of our inability to accurately measure GFR in the neonate and, by extension, the clearance of compounds such as gadolinium, all nephrologists and radiologists must exercise caution in terms of use of GBCA in this potentially high-risk population, and all other imaging modalities should be considered prior to choosing one requiring gadolinium exposure.

\section{LIMITATIONS}

The evidence is largely based upon case series rather than prospective studies or RCTs.

\section{RESEARCH RECOMMENDATIONS}

As for iodinated contrast media, a prospective study of people with CKD undergoing nuclear MRI with gadolinium contrast would help define change in GFR and validate estimators. Because NSF is such a serious condition, an RCT of dialysis in people with GFR $<30 \mathrm{ml} / \mathrm{min} / 1.73 \mathrm{~m}^{2}$ would help to determine risk-benefit in these patients, though recruitment may be difficult due to potential ethical concerns.

\section{Bowel preparation}

The increasing use of colonoscopy as a screening tool for bowel cancer has resulted in many people undergoing bowel 
preparation with oral sodium phosphate-containing preparations. Case reports of acute and late irreversible renal failure with biopsy-proven phosphate deposition have led to a new disease entity termed acute phosphate nephropathy. ${ }^{618,619}$ Less than 40 definite cases have been reported and these have been extensively reviewed.

4.5.5: We recommend not to use oral phosphate-containing bowel preparations in people with a GFR $<60$ $\mathrm{ml} / \mathrm{min} / 1.73 \mathrm{~m}^{2}$ (GFR categories G3a-G5) or in those known to be at risk of phosphate nephropathy. (1A)

\section{RATIONALE}

This statement has been worded in order to enable safe bowel preparation in people with CKD who need to undergo investigation of bowel disease.

\section{Evidence Base}

Electrolyte disturbances that are sometimes severe and include hyperphosphatemia, hypocalcemia, hypo- and hypernatremia, and hypokalemia have been reported in normal volunteers undergoing oral phosphate bowel preparation. ${ }^{620}$ Renal injury has been reported in a small number of people although the condition is likely to be underrecorded. A recent study from Iceland estimated the incidence to be around 1 per thousand doses ${ }^{621}$ but others would suggest incidence rates of between $1 \%$ and $4 \% .{ }^{618,619}$

Two broad patterns of renal injury have been described. An early symptomatic response associated with severe hyperphosphatemia and hypocalcemia and a later (days to months) irreversible kidney injury associated with a specific tubulointerstitial calcium phosphate deposition. ${ }^{618,619}$

The following people are said to be at particular risk although the link to kidney injury is associative in many cases and firm evidence is lacking:
a) GFR $<60 \mathrm{ml} / \mathrm{min} / 1.73 \mathrm{~m}^{2}$
b) $>60$ years of age
c) Female
d) Hypertension
e) Diabetes
f) $\mathrm{CHF}$
g) Dehydration
h) Active colitis
i) Concurrent use of RAAS blocking agents, diuretics, lithium, NSAIDs
j) Large and/or repeat dosing of oral phosphate prepara- tions
k) Hypoparathyroidism

Although the FDA has banned oral phosphate solutions, there is no qualitative difference with tablet preparations. Both should be avoided in people at risk and this is the current recommendation of the American Society for Gastrointestinal Endoscopy. ${ }^{622}$
There is some debate as to whether the cause of the kidney injury is entirely due to dehydration rather than phosphate use per se. ${ }^{623}$ However, it is hard to understand why calcium phosphate deposits occur and cause late renal impairment if dehydration was the only factor.

There is a single RCT of phosphate versus non-phosphate preparations but assessment of renal function was limited to change in SCr and GFR at baseline is not reported. ${ }^{624}$ Notwithstanding these limitations, there were greater changes in serum potassium, calcium, and phosphate in those given sodium phosphate-containing preparations.

As there are non-phosphate-containing bowel preparations available, these should be used in all the above groups (and arguably in all people given the biochemical abnormalities observed in normal volunteers). As for radiocontrast media, rehydration with saline may be required in the frail and ill irrespective of the bowel preparation that is used.

\section{International Relevance}

This guidance has universal relevance but non-phosphatecontaining bowel preparations are more expensive, so the use of these agents may differ around the world.

\section{LIMITATIONS}

The data are based upon case series with a limited number of affected individuals and retrospective population studies.

\section{RESEARCH RECOMMENDATIONS}

Prospective study in people with normal renal function and those with different severities of CKD are urgently required in order to define the acute biochemical and metabolic effects of phosphate-containing bowel preparations. There is also a need for a study of all people undergoing bowel preparation with whatever preparation in order to explore the effects on GFR on the incidence of this complication. More definitive exploration of rehydration therapy (type and volume) in people with CKD undergoing bowel preparation is urgently needed.

\subsection{CKD AND RISKS FOR INFECTIONS, AKI, HOSPITALIZATIONS, AND MORTALITY}

The following section gives guidance for the care of people with CKD given that they are at increased risk for a number of events such as infections, AKI, CVD, hospitalizations, and mortality. Appreciating that increased risk and implementing some of the recommendations below may result in improved outcomes for people. It will be important to develop policies and robust research agendas to address areas which do not have a substantial evidentiary base.

\section{CKD and risk of infections}

CKD is associated with significant major infectious complications, which occur at rates 3 to 4 times the general population. Infection is an important cause of morbidity and mortality among patients with kidney failure and is the 
second leading cause of death following CVD. CKD may be a risk-multiplier for acute infectious disease-associated mortality, as it is for CVD. Despite a less effective response to vaccination, there are data suggesting benefit from immunization among people with CKD as in the general population.

4.6.1: We recommend that all adults with CKD are offered annual vaccination with influenza vaccine, unless contraindicated. $(1 B)$

4.6.2: We recommend that all adults with eGFR $<30 \mathrm{ml} /$ $\min / 1.73 \mathrm{~m}^{2}$ (GFR categories G4-G5) and those at high risk of pneumococcal infection (e.g., nephrotic syndrome, diabetes, or those receiving immunosuppression) receive vaccination with polyvalent pneumococcal vaccine unless contraindicated. (1B)

4.6.3: We recommend that all adults with CKD who have received pneumococcal vaccination are offered revaccination within 5 years. $(1 B)$

4.6.4: We recommend that all adults who are at high risk of progression of CKD and have GFR $<30 \mathrm{ml} / \mathrm{min} /$ $1.73 \mathrm{~m}^{2}$ (GFR categories G4-G5) be immunized against hepatitis $B$ and the response confirmed by appropriate serological testing. (1B)

4.6.5: Consideration of live vaccine should include an appreciation of the patient's immune status and should be in line with recommendations from official or governmental bodies. (Not Graded)

4.6.6: Pediatric immunization schedules should be followed according to official international and regional recommedations for children with CKD. (Not Graded)

\section{RATIONALE}

CKD is associated with alterations in primary host defense mechanisms and increases the risk of bacterial infections (Table 33). Epidemiological study suggests that the 3 most commonly seen infectious complications in the CKD population are: urinary tract infection, pneumonia, and sepsis. In the general population, there is strong evidence that preventive measures are effective in adults and there are data suggesting benefit from immunization in people with CKD.

\section{Evidence Base}

Previous investigations have firmly established ESRD as a strong risk factor for infectious complications. ${ }^{625-628}$ However, few epidemiologic reports have addressed the risk of infections in people with CKD not treated with dialysis. ${ }^{629,630}$ Data from the US Renal Data System (USRDS) suggest that higher rates of hospital admission because of septicemia are noted in people with CKD compared with those without CKD. ${ }^{631}$ Comprehensive studies of the absolute rates, risk factors, clinical course, and outcomes of different types of clinically relevant infections across the spectrum of CKD are not available.

Among people with CKD, very few studies have examined the incidence or prevalence of infections and no published data describe GFR category-specific infection rates. Among
Table 33 | Risk factors for infection in people with CKD

Advanced age
High burden of coexisting illnesses such as diabetes
Hypoalbuminemia $^{625}$
Immunosuppressive therapy $^{628}$
Nephrotic syndrome
Uremia
Anemia and malnutrition
High prevalence of functional disabilities

Abbreviation: CKD, chronic kidney disese

Medicare beneficiaries aged more than $66 \mathrm{yr}$, people with diagnosed CKD seem to have substantially higher rates of hospitalization for diagnoses of pneumonia and sepsis compared with people without diagnosed $\mathrm{CKD}^{628}$ In addition to an increased incidence of being hospitalized with infections, people with CKD have longer lengths of hospital stay during infection-related admissions compared with people without $\mathrm{CKD}{ }^{632}$

Among USRDS data admission rates for all causes, CVD and infection, are $38-46 \%$ higher for those with CKD than for those without. ${ }^{633}$ Rates for pneumonia in CKD were nearly three times higher than those for non-CKD. Hospitalizations for bacteremia/septicemia, were nearly four times higher for people with CKD compared to those without. Hospitalization rates for urinary tract infections were three-fold higher for people with CKD.

In people with ESRD, the function of polymorphonuclear white blood cells, lymphocytes, and monocytes is altered, resulting in an impaired host response to infection. ${ }^{636-638}$ However, these issues have not been adequately investigated in people with CKD.

There is a growing body of evidence in the general population for reduction in infections, hospitalizations, and mortality as a result of immunizations. Current data suggest that vaccination is an underused prevention strategy in the CKD and ESRD populations. ${ }^{639}$ Potential barriers to immunization that are specific to the CKD populations have not been systematically examined. Although lower vaccine responsiveness has been widely recognized in the ESRD population, to what extent moderate to advanced CKD modifies vaccine responsiveness remains unclear. ${ }^{640-642}$ Studies that have examined vaccination in the setting of CKD or ESRD have been limited by small study size, variable follow-up, and ascertainment of surrogates for vaccine effectiveness such as antibody response and rate of antibody decline after vaccination as opposed to vaccine efficacy for preventing infection. ${ }^{643}$

Influenza $A$ and $B$ vaccine. Despite potentially impaired antibody responses, a 2-year analysis of US Medicare claims data found that people vaccinated against influenza A and B on dialysis had a substantially lower chance of any-cause hospital admission and any-cause death than those patients not vaccinated on dialysis. ${ }^{644}$ This finding might indicate clinical effectiveness of vaccinating this population but its observational design might also reflect differences in the underlying clinical status among people vaccinated and not 
vaccinated. No unique adverse events related to influenza vaccine have been identified in people on dialysis.

Pneumococcal vaccine. People with kidney disease vaccinated with the pneumococcal vaccine seem to develop different serotype-specific titers, develop lower levels of antibody titers, and have a more rapid loss of antibody titers as compared with healthy control subjects. ${ }^{639,645,646}$ Practitioners should be aware of the impact of specific vaccines on responsiveness and duration of responsiveness. Revaccination practices will be dictated by that knowledge.

Hepatitis B vaccine. Widespread hepatitis B virus (HBV) vaccination at the onset of dialysis has led to a marked reduction of HBV infections in people with ESRD, although improved screening of blood products and dissemination of recommendations for reducing the spread of HBV infections in dialysis units have also likely contributed. ${ }^{647}$ Among people with moderate to advanced CKD, hepatitis B vaccination responsiveness has been shown to range from approximately 60 to $80 \%$ depending on the dosage, number of administered vaccines, and study population. Although findings have been inconsistent as to whether the level of GFR affects vaccine responsiveness in people with $\mathrm{CKD}^{640,642}$ those with higher GFR are more likely to respond with seroconversion, independent of other factors. ${ }^{640}$

S. aureus vaccine. StaphVAX has not been shown to be efficacious in reducing the risk for Staphylococcus aureus bacteremia in people on hemodialysis. ${ }^{648,649}$ No data are published in people with CKD.

Live vaccines. Due to the fact that people with CKD are often immunocompromised, live vaccines should only be used with caution on an individual basis.

In summary, although some vaccines (like influenza) in usual doses provide protection, other vaccines (HBV and pneumococcal) require more frequent dosing or larger doses to achieve and maintain protective antibody titers. Frequency and type of vaccination will vary according to local circumstances and prevalence of disease.

\section{International Relevance}

The availability of different vaccinations may vary worldwide, as does the prevalence of specific bacterial, viral, and other infections. It is reasonable to offer individuals appropriate immunization according to local practices.

\section{Implications for Clinical Practice and Public Policy}

Vaccines for influenza, hepatitis B, and pneumococcus are currently recommended for people with CKD by the local, regional, or national advisory committees on immunization practices from most countries.

Current recommendations are to:

- Provide influenza vaccination annually to people with CKD.

- Provide pneumococcal vaccine with a single booster dose 5 years after the initial dose.
- Provide HBV vaccine to people with CKD who are likely to require RRT. Although the recommendation is to give the $\mathrm{HBV}$ vaccine during more severe CKD (GFR $<15 \mathrm{ml} /$ $\min / 1.73 \mathrm{~m}^{2}$ ), it may be preferable to give this earlier to maximize the chances of achieving immunity; there are data to support this practice. ${ }^{640}$ This would also ensure that all patients are immunized against HBV before receiving a transplant. As protective antibody levels may fall, this should be checked (possibly annually) with booster doses given if appropriate.

\section{Areas of Controversy, Confusion, or Non-consensus}

Much remains to be understood concerning impaired host response to infection in patients with CKD.

- Studies should be undertaken to determine the absolute rates, risk factors, and clinical course of different types of clinically relevant infections across the spectrum of CKD, by GFR and albuminuria category, and by cause.

- The outcomes across the range of acute infections in CKD population need to be ascertained.

- Studies should be undertaken to assess the rate of decline of antibody titers post-vaccination and the efficacy of immunization in people with CKD.

\section{Pediatric Considerations}

Current immunization schedules for children are regularly updated by both the US Centers for Disease Control and Prevention ${ }^{650}$ and American Academy of Pediatrics. ${ }^{651}$

Current and comprehensive immunization recommendations for children with CKD have been published by Neu in $2012 .{ }^{652}$ The paper addresses key issues regarding the use of vaccines in CKD pediatric populations who are receiving concomitant immunosuppression and in those awaiting transplantation. The need for, and interpretation of, protective antibody levels for those vaccines where this is indicated is described.

An oversimplified summary of the recommendations would be to provide all recommended childhood vaccines to every child with CKD with the exception of any live viral vaccine in a child receiving immunosuppressive medications. Likewise children on dialysis should not receive the live attenuated influenza vaccine although the inactivated version can and should be given to all children with CKD on an annual basis otherwise. Pneumococcal vaccination is particularly important in children with nephrotic syndrome and those with CKD, and current vaccination schedules and products should be carefully reviewed to ensure proper serotype coverage is being provided. Hepatitis B status and vaccination are of extreme importance in all children who may go onto dialysis - and specific recommendations for ongoing monitoring and interpretation of antibody levels should be carefully reviewed.

\section{$C K D$ and risk of $A K I$}

Due to the epidemiological association between CKD and AKI and the number of observational studies reporting an association between pre-existing CKD and AKI, CKD is 
considered the most consistent pre-existing condition associated with a high risk of AKI. However the potential linkage between patients with AKI, CKD, and ESRD has been inadequately studied to date and remains ill defined. This section describes AKI as a complication which needs to be managed in those with CKD. Given its association with progression, it is also described in that section.

4.6.7: We recommend that all people with $C K D$ are considered to be at increased risk of AKI. (1A)

4.6.7.1: In people with $\mathrm{CKD}$, the recommendations detailed in the KDIGO AKI Guideline should be followed for management of those at risk of AKI during intercurrent illness, or when undergoing investigation and procedures that are likely to increase the risk of AKI. (Not Graded)

\section{RATIONALE}

Observational data suggest a strong association between preexisting CKD and AKI. The appreciation that CKD patients may be more susceptible to AKI is the purpose of the above set of statements. However, methodological issues such as how CKD and AKI are defined in clinical studies and the statistical adjustments for non-uniformity of comorbidities among various studies may affect the validity of observed associations.

\section{Evidence Base}

CKD is designated as a risk factor for AKI because of the epidemiological association between the two. ${ }^{263,264} \mathrm{~A}$ number of studies in a variety of settings report an association between pre-existing CKD and AKI. ${ }^{265-271} \mathrm{CKD}$ is a potent predictor of acute decline in kidney function following exposure to radiocontrast, ${ }^{272}$ major surgery, ${ }^{273}$ and other medical conditions. ${ }^{274}$

Hsu et al. ${ }^{14}$ compared the pre-hospitalization MDRD GFR of 1764 adult members of the Kaiser Permanente Northern California health-care system who developed dialysis-requiring AKI during hospitalization with 600,820 individuals who did not. Compared with a reference baseline GFR of $\geq 60 \mathrm{ml} /$ $\mathrm{min} / 1.73 \mathrm{~m}^{2}$, a baseline GFR of $45-59 \mathrm{ml} / \mathrm{min} / 1.73 \mathrm{~m}^{2}$ was associated with an adjusted OR of in-hospital AKI of 1.66 (95\% CI 1.40-1.97). For GFR values of $15-29 \mathrm{ml} / \mathrm{min} / 1.73 \mathrm{~m}^{2}$, the adjusted OR for in-hospital AKI was 20.42 (95\% CI 17.40-23.96). The presence of diabetes, hypertension, and proteinuria increased the likelihood of developing in-hospital AKI, with adjusted ORs of 1.99 (95\% CI 1.78-2.23), 1.55 (95\% CI 1.37-1.76) and 2.84 (95\% CI 2.52-3.19), respectively. The authors concluded that $\mathrm{CKD}$ is the main risk factor for AKI during hospitalization. A contrasting approach by Singh et al. defined AKI as dialysis-requiring acute renal failure. ${ }^{275}$ Because the clinical decision to dialyze a patient is frequently influenced by a higher overall SCr, presence of hemodialysis access, or consideration of inevitable progression to ESRD, this definition of AKI could bias toward capturing more AKI cases in CKD patients. Moreover, in patients with advanced
CKD, the progression of CKD to ESRD may sometimes be difficult to separate from acute-on-chronic renal failure. A cohort study by Lafrance et al. followed a referred CKD population in British Columbia for a median of 19.4 months after achieving a GFR of $\leq 30 \mathrm{ml} / \mathrm{min} / 1.73 \mathrm{~m}^{2}$. Forty-five percent had at least one episode of AKI. ${ }^{276}$ In another cohort study of 920,985 adults in Alberta, Canada with at least one outpatient measurement of SCr and proteinuria and not requiring chronic dialysis, risk of admission with AKI increased with heavier proteinuria and reduced GFR. ${ }^{16}$

\section{International Relevance}

These guidelines about AKI have relevance around the world. While the causes of AKI may differ by region, country, socioeconomic status, and age, the consequences remain the similar. Where there are no facilitities to support AKI or $\mathrm{CKD}$, people will die.

\section{Areas of Controversy, Confusion, or Non-consensus}

Interpretation of published data examining the influence of pre-existing CKD on the increased likelihood of AKI is potentially confounded by a number of issues. These include the comorbidities associated with $\mathrm{CKD}$, influenced by repeated exposure to various nephrotoxic insults or inhospital errors, ${ }^{57,277}$ or primarily due to the altered physiology in CKD. There are also methodological issues such as how CKD and AKI are defined in clinical studies and the varying statistical adjustments for comorbidities which may affect the validity of observed associations.

A further important issue to clarify is whether pre-existing CKD influences the outcome of AKI. Currently, there is no single biomarker that can differentiate 'acute' from 'chronic' kidney disease and help to address this issue. Several large observational and database studies report, surprisingly, lower in-hospital mortality in patients with AKI superimposed on CKD compared with controls. ${ }^{278-283}$ Data from PICARD reveal lower in-patient mortality and median length of stay in ICU subjects with acute-on-chronic renal injury compared with non-CKD subjects with AKI, though the post-discharge dialysis rates were higher in subjects with pre-existing CKD. ${ }^{284}$

\section{Pediatric Considerations}

The relative paucity of pediatric specific guidelines (due to lack of high-quality studies) in the KDIGO AKI guideline would suggest that the use of pediatric data and review papers as well as relevant pediatric nephrology texts would be of benefit to the practitioner interested in reviewing this topic in greater detail and applying pediatric data to their practice. $^{653-658}$

\section{RESEARCH RECOMMENDATIONS}

Prospectively designed clinical studies with a clear and uniform definition of CKD and AKI and adjusted for comorbidities are needed to determine:

- the frequency of AKI events in a CKD population

- the outcome of AKI in patients with CKD condition 
- the importance of proteinuria in addition to low GFR in the risk of AKI

\section{CKD and risk of hospitalization and mortality}

Regardless of the method used to estimate GFR, hospitalization and mortality rates are higher in people with CKD. Exact rates vary with comorbidity and severity of CKD, and are not well-defined. Selection of interventions that could reduce hospitalizations, morbidity, mortality, and costs in people with CKD is not well-studied.

4.6.8: CKD disease management programs should be developed in order to optimize the community management of people with CKD and reduce the risk of hospital admission. (Not Graded)

4.6.9: Interventions to reduce hospitalization and mortality for people with CKD should pay close attention to the management of associated comorbid conditions and cardiovascular disease in particular. (Not Graded)

\section{RATIONALE}

There are observational and database studies reporting an association between pre-existing CKD and hospitalizations and mortality. A better understanding of the rates, causes, and risk factors for hospitalization among people with CKD would allow estimates of the economic burden of CKD and identification of those at risk for increased resource utilization. People with CKD are an ideal target for interventions aimed at reduction of morbidity, hospitalization, mortality, and costs. These statements suggest that a coordinated approach to the identification and management would result in better outcomes and are intended as 'best practices' statements, recognizing the difficulty in developing an evidence base while addressing issues related to resource allocations (Table 34).

\section{Evidence Base}

Mortality rates remain high (16-22\%) with the use of dialysis, with more than half of all deaths related to CVD. Less is known about mortality and CVD rates, and resource use among persons with a reduced GFR who are not yet receiving maintenance dialysis. Few studies have

\section{Table 34|Components of community CKD management} programs

\section{Disease monitoring}

Integration with other chronic disease management programs including

diabetes, hypertension and heart failure

Medication management and dietary advice

Anemia management programs

Vaccination programs

Information and psychosocial support

Renal replacement therapy (dialysis and transplant) education

Advanced care planning and end-of-life care (where appropriate)

Abbreviation: CKD, chronic kidney disease. investigated the association between CKD and the risk of hospitalization.

Data from the USRDS reveal that hospitalization rates vary with comorbidity and interact with degrees of CKD. Adjusted rates are 38\% higher in people with CKD and 19\% greater in people with CKD and GFR under $60 \mathrm{ml} / \mathrm{min} /$ $1.73 \mathrm{~m}^{2}$ than in those with GFR $>60 \mathrm{ml} / \mathrm{min} / 1.73 \mathrm{~m}^{2}$, who in turn are $20 \%$ higher than in people without CKDillustrating the graded impact of advancing kidney disease. Not surprisingly, rates of cardiovascular hospitalization are greater for people with CKD, particularly those with increasing severity of CKD. ${ }^{659}$ In both the CKD and nonCKD populations, adjusted rates of hospitalization increase with greater comorbidity. In 2008, for example, the rate for people with CKD with both diabetes and CHF was 726 per 1000 person-years at risk $-85 \%$ greater than the rate of 393 among people with neither diagnosis. Rates of admission for CVD increase even more in the higher categories of GFR. Among Medicare subjects, the rate of 141 admissions per 1000 person-years for those with GFR $<60 \mathrm{ml} / \mathrm{min} / 1.73 \mathrm{~m}^{2}$ is $26 \%$ higher than the rate of 112 reported for those with CKD and GFR $\geq 60 \mathrm{ml} / \mathrm{min} / 1.73 \mathrm{~m}^{2}$. The admission rates of 101 and 90 reported for MarketScan and Ingenix i3 subjects with GFR $<60 \mathrm{ml} / \mathrm{min} / 1.73 \mathrm{~m}^{2}$ are 48 and $16 \%$ greater, respectively, than those occurring in people with higher GFRs. ${ }^{633}$ Adjusted rates of mortality in USRDS 2008 increased with age, and were highest in people with advanced categories of GFR: $31-72 \%$ higher, for example, in people with GFR $<60 \mathrm{ml} /$ $\min / 1.73 \mathrm{~m}^{2}$ compared to those with no CKD. By gender, rates in men with CKD were 91.8 per 1000 person-years at risk compared to 85.6 in women. Rates for people with CKD overall were similar in whites and African Americans, but in people with GFR $<60 \mathrm{ml} / \mathrm{min} / 1.73 \mathrm{~m}^{2}$, rates for African Americans were $18 \%$ higher than those for whites, at 95.0 and 80.5 per 1000 person-years, respectively. ${ }^{633}$

Khan et al. ${ }^{660}$ confirmed that hospital utilization among people with CKD is high. During a median follow-up of 11.4 months, $47 \%$ of subjects had at least one hospitalization and there were on average 0.96 hospitalizations, 6.6 hospital days, and 4.0 outpatient nephrology visits per person-year at risk. Cardiac disease/hypertension was the most common primary diagnosis of hospitalizations and progression of $\mathrm{CKD}$ /acute kidney failure was the most common secondary cause of hospitalization. The authors had previously shown that the dialysis population at their institution had 2.2 hospitalizations and 14.8 hospital days per person-year at risk, ${ }^{659}$ which was similar to that among US hemodialysis patients between 1996 and 1998, who had 1.9 hospitalizations and 14 hospital days per person-year at risk. ${ }^{661}$ In the general population, there were 0.31 hospitalizations and 1.9 hospital days per person in $1998 .{ }^{662}$

Go et $a .^{58}$ reported an independent, graded association between GFR and the risk of death, cardiovascular events, and hospitalization in 1,120,295 adults within a large, integrated system of health-care delivery in whom SCr had been measured between 1996 and 2000 and who had not 
undergone dialysis or kidney transplantation. These risks were evident at GFR $<60 \mathrm{ml} / \mathrm{min} / 1.73 \mathrm{~m}^{2}$ and substantially increased at GFR $<45 \mathrm{ml} / \mathrm{min} / 1.73 \mathrm{~m}^{2}$.

The higher hospital utilization among people with CKD compared to the general population, and the similarity in the comorbid conditions and the causes of hospitalization between people with CKD and ESRD, confirm the hypothesis that the complications and comorbidity observed in ESRD are manifest earlier in the disease process. Previous studies have demonstrated an association between age, gender, race, cardiac disease, peripheral vascular disease, serum albumin and hematocrit levels, and resource utilization among people on dialysis. ${ }^{659,663,664}$ Holland et al. ${ }^{665}$ identified baseline predictors of first non-elective hospitalization among a retrospective cohort of 362 predialysis subjects. Multivariate analysis, adjusted for baseline creatinine concentration, selected advanced age (RR 1.02; 95\% CI 1.01-1.03), angina (RR 1.9; CI 1.37-2.61), peripheral vascular disease (RR 1.55, CI 1.05-2.27), and $\mathrm{Hb}$ concentration (RR 0.99, CI 0.94-0.98) as independent predictors of hospitalization. These comorbid conditions progressively worsen with advancing kidney disease and result in a substantial proportion of people having severe complications by the time they come to RRT.

Interventions to reduce hospitalizations and mortality for people with CKD should pay close attention to the management of associated comorbid conditions and CVD. ${ }^{666}$ Closer attention to the management of heart disease in this population could substantially improve outcomes. ${ }^{667}$

The influence of correction of anemia on hospitalization is controversial. Drüeke et al. ${ }^{668}$ randomly assigned 603 subjects with GFR 15-35 ml/min/1.73 $\mathrm{m}^{2}$ and mild-to-moderate anemia (Hb level: 11.0-12.5 g/dl [110-125 g/l]) to a target $\mathrm{Hb}$ value in the normal range $(13.0-15.0 \mathrm{~g} / \mathrm{dl}[130-150 \mathrm{~g} / \mathrm{l}])$ or the subnormal range $(10.5-11.5 \mathrm{~g} / \mathrm{dl}[105-115 \mathrm{~g} / \mathrm{l}])$. There were no significant differences between the two groups in the incidence of hospital admission (61\% and 59\%, respectively) or mean duration of hospitalization for cardiovascular reasons (33.0 and 28.2 days, respectively). Singh et al. ${ }^{669}$ studied 1432 people with CKD, 715 of whom were randomly assigned to receive a dose of epoetin alfa targeted to achieve a $\mathrm{Hb}$ level of $13.5 \mathrm{~g} / \mathrm{dl}(135 \mathrm{~g} / \mathrm{l})$ and 717 of whom were assigned to receive a dose targeted to achieve a level of $11.3 \mathrm{~g} / \mathrm{dl}(113 \mathrm{~g} / \mathrm{l})$. The median study duration was
16 months. The primary end point was a composite of death, MI, hospitalization for CHF (without RRT), and stroke. They observed an increased risk of the primary composite end point in the high $\mathrm{Hb}$ group as compared with the low $\mathrm{Hb}$ group. Death and hospitalization for CHF accounted for $74.8 \%$ of the composite events. The Trial to Reduce Cardiovascular Events with Aranesp Therapy (TREAT) $)^{375}$ study involved 4038 people with diabetes, CKD, and anemia. Subjects were randomly assigned to treatment with darbepoetin alfa to achieve a $\mathrm{Hb}$ level of approximately $13 \mathrm{~g} / \mathrm{dl}$ $(130 \mathrm{~g} / \mathrm{l})$ or to placebo, with rescue darbepoetin alfa when the $\mathrm{Hb}$ level was less than $9.0 \mathrm{~g} / \mathrm{dl}(90 \mathrm{~g} / \mathrm{l})$. The primary end points were the composite outcomes of death or a cardiovascular event (nonfatal MI, CHF, stroke, or hospitalization for myocardial ischemia) and of death or ESRD. Again there were no significant between-group differences in the outcomes of interest.

\section{International Relevance}

It is of benefit for all jurisdictions to appreciate the increase in resource utilization by CKD populations. Identification of people at risk for increased resource utilization and economic burden of CKD should result in strategies to attenuate that risk or address the resource implications. Selection of interventions that could reduce hospitalizations, morbidity, mortality, and costs in these populations should be evaluated.

\section{DISCLAIMER}

While every effort is made by the publishers, editorial board, and ISN to see that no inaccurate or misleading data, opinion or statement appears in this Journal, they wish to make it clear that the data and opinions appearing in the articles and advertisements herein are the responsibility of the contributor, copyright holder, or advertiser concerned. Accordingly, the publishers and the ISN, the editorial board and their respective employers, office and agents accept no liability whatsoever for the consequences of any such inaccurate or misleading data, opinion or statement. While every effort is made to ensure that drug doses and other quantities are presented accurately, readers are advised that new methods and techniques involving drug usage, and described within this Journal, should only be followed in conjunction with the drug manufacturer's own published literature. 


\section{Chapter 5: Referral to specialists and models of care}

Kidney International Supplements (2013) 3, 112-119; doi:10.1038/kisup.2012.68

Early identification and referral of people with CKD has the potential to reverse, delay, or prevent progression of disease and is a key focus of international initiatives in the area of kidney disease. The goals of early identification and referral are several-fold and include:

1. Provision of specific therapy based on diagnosis

2. Slowing/arresting CKD progression

3. Evaluation and management of comorbid conditions

4. Prevention and management of CVD

5. Identification, prevention, and management of CKDspecific complications (e.g., malnutrition, anemia, bone disease, acidosis)

6. Planning and preparation for RRT (e.g., choice of modality, access-placement and care, preemptive transplantation)

7. Psychosocial support and provision of conservative care and palliative care options where required

\section{1: REFERRAL TO SPECIALIST SERVICES}

5.1.1: We recommend referral to specialist kidney care services for people with CKD in the following circumstances $(1 B)$ :

- AKI or abrupt sustained fall in GFR;

- GFR $<30 \mathrm{ml} / \mathrm{min} / 1.73 \mathrm{~m}^{2}$ (GFR categories G4-G5)*;

- a consistent finding of significant albuminuria (ACR $\geq 300 \mathrm{mg} / \mathrm{g}[\geq 30 \mathrm{mg} / \mathrm{mmol}]$ or AER $\geq 300 \mathrm{mg} / 24$ hours, approximately equivalent to $\mathrm{PCR} \geq 500 \mathrm{mg} / \mathrm{g}$ [ $\geq 50 \mathrm{mg} / \mathrm{mmol}]$ or PER $\geq 500 \mathrm{mg} / 24$ hours);

- progression of CKD (see Recommendation 2.1.3 for definition);

- urinary red cell casts, $\mathrm{RBC}>20$ per high power field sustained and not readily explained;

- CKD and hypertension refractory to treatment with 4 or more antihypertensive agents;

- persistent abnormalities of serum potassium;

- recurrent or extensive nephrolithiasis;

- hereditary kidney disease.

5.1.2: We recommend timely referral for planning renal replacement therapy (RRT) in people with progressive CKD in whom the risk of kidney failure within 1 year is $10-20 \%$ or higher ${ }^{\dagger}$, as determined by validated risk prediction tools. (1B)

\section{RATIONALE}

This statement reminds the practitioner that there is a need for timely referral for RRT planning in order to ensure good decision making and outcomes. The use of the word 'timely' is vague as this is not yet determined, and is based on patient and system factors. The actual amount of time required at a minimum is at least 1 year to ensure appropriate education, understanding and referrals to other practitioners (e.g., vascular access surgeons, transplant teams, etc). The second part of the statement refers to the fact that those who are progressing (versus those who are stable) are the ones who will benefit from this referral. Hence, there is a need to apply prediction tools to help identify the risk of progression. We have not stated which prediction tool is preferred as these may differ depending on information available in any individual or local experience. Examples of prediction tools can be found in recent publications. ${ }^{257,260,261}$

The scope of nephrology practice includes a wide variety of conditions including not only ESRD but also acute and chronic primary and systemic diseases involving individual elements of the kidney, resistant hypertension, and biochemical derangements. There are thus more potential benefits of nephrology referral than those widely recognized such as identification of reversible causes of $\mathrm{CKD}$, provision of treatment that may slow progression of CKD, management of the metabolic complications of advanced CKD, and preparation for dialysis and transplantation.

In certain people, such as those with diabetes, transition to a severe reduction in GFR and kidney failure may progress rapidly. In such individuals early nephrology referral is the watchword but when an individual's kidney function is relatively stable (rate of decline in GFR $<5 \mathrm{ml} / \mathrm{min} / 1.73 \mathrm{~m}^{2} /$ year), we suggest using the grid as a guide (Figure 21). Where refer is marked by an asterisk, referring clinicians may wish to discuss with their nephrology service depending on local arrangements.

\section{Evidence Base}

Although referral recommendations in the literature are inconsistent, criteria for nephrology referral include SCr or GFR, proteinuria, hematuria, $\mathrm{BP}$, and electrolyte derange-

\footnotetext{
${ }^{*}$ If this is a stable isolated finding, formal referral (i.e., formal consultation and ongoing care management) may not be necessary and advice from specialist services may be all that is required to facilitate best care for the patients. This will be health-care system dependent.

${ }^{\dagger}$ The aim is to avoid late referral, defined here as referral to specialist services less than 1 year before start of RRT.
} 


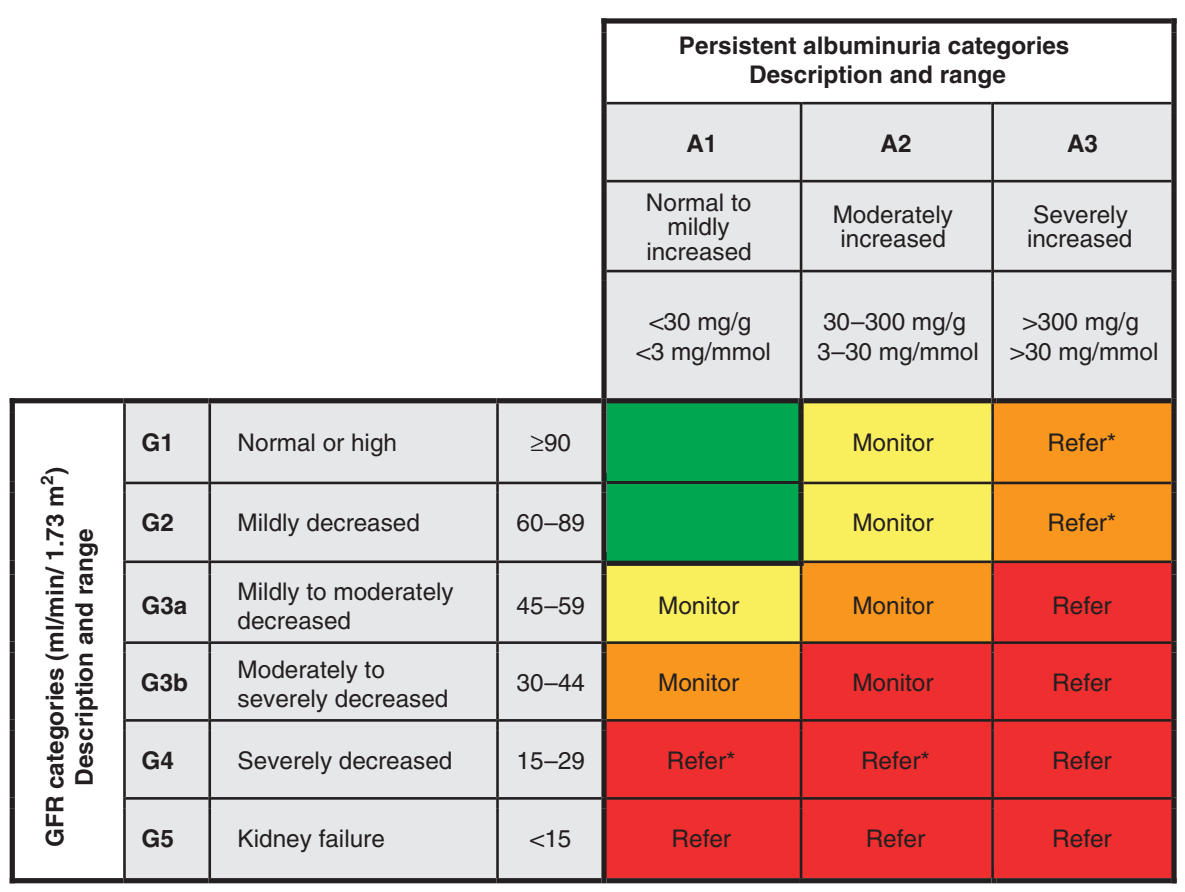

Figure 21 | Referral decision making by GFR and albuminuria. *Referring clinicians may wish to discuss with their nephrology service depending on local arrangements regarding monitoring or referring. GFR, glomerular filtration rate. Modified with permission from Macmillan Publishers Ltd: Kidney International. Levey AS, de Jong PE, Coresh J, et al. ${ }^{30}$ The definition, classification, and prognosis of chronic kidney disease: a KDIGO controversies conference report. Kidney Int 2011; 80: 17-28; accessed http://www.nature.com/ki/journal/v80/n1/ full/ki2010483a.html

ment. ${ }^{670}$ Data relating to referral for those with glomerular disease, hypertension, AKI, and diabetes may be found in the relevant guidelines. ${ }^{7,8,10,262}$

In this section we will briefly consider summaries of the evidence relating to timely referral for planning RRT in people with progressive CKD. In this aspect the literature concerning late referral in the last quarter of a century has been remarkably consistent; both studies and narrative reviews identifying a number of adverse consequences of late referral and related benefits of early referral (Table 35).

Patients who are aged $>75$ years, female, non-Caucasian, uninsured, of lower socioeconomic or educational status, or have multiple comorbidities are most at risk for non-referral for CKD care. ${ }^{671,672}$ Patients with kidney disease have never been randomized to early or late referral to nephrology services and the definition of late referral in the published studies varies; three months is probably less than the absolute minimum amount of time required for assessment, education, preparation for RRT and creation of access but is the most frequently employed definition. Overall there are more than 50 studies in the published literature and a meta-analysis of 22 of these studies from 10 different countries serves to underline some of the key messages (Table 36), giving an indication of the size of the differences in mortality and hospital length of stay and also highlighting the significantly lower serum albumin level in late referred patients. ${ }^{673}$

A systematic review considered twenty-seven longitudinal cohort studies providing data on 17,646 participants of whom 11,734 were referred early and 5912 (33\%) were referred late. ${ }^{674} \mathrm{OR}$ for mortality reductions in patients referred early were evident at 3 months (OR 0.51; 95\% CI $0.44-0.59 ; \mathrm{P}<0.0001)$ and remained significant at 5 years (OR 0.45; 95\% CI 0.38-0.53; P<0.0001). Initial hospitalization was 8.8 days shorter with early referral $(95 \% \mathrm{CI}-10.7$ to -7.0 days; $\mathrm{P}<0.0001)$. Differences in mortality and hospitalization data between the 2 groups were not explained by differences in prevalence of diabetes mellitus, previous CAD, BP control, serum phosphate, and serum albumin. Early referral was associated with better preparation and earlier placement of dialysis access and better uptake of peritoneal dialysis.

Over a decade ago McLaughlin et al. evaluated the cost implications of early versus late referral. ${ }^{675}$ Outcomes of interest were total cost of patient care, patient life-years, patient life-years free of RRT and hospital length of stay. Mean total costs per patient over five years were US $\$ 87,711$ and US $\$ 110,056$ for early and late referrals, respectively. The mean patient life-years were 3.53 and 3.36 years, respectively, and the patient life-years free of RRT were 2.18 and 1.76 years, respectively. Those patients referred early spent significantly less time in hospital (length of stay 25 days versus 41 days). Klebe et al. subsequently investigated the annualized cost of implementation of referral guidelines for CKD ${ }^{676}$ Although CKD guideline implementation resulted in significant increases in nephrology referral and additional investigation, they estimated that the associated costs could 
Table 35 | Early versus late referral: consequences and benefits

\begin{tabular}{ll}
\hline Consequences of late referral & Benefits of early referral \\
\hline Anemia and bone disease & Delay need to initiate RRT \\
Severe hypertension and fluid overload & Increased proportion with permanent access \\
Low prevalence of permanent access & Greater choice of treatment options \\
Delayed referral for transplant & Reduced need for urgent dialysis \\
Higher initial hospitalization rate & Reduced hospital length of stay and costs \\
Higher 1-year mortality rate & Improved nutritional status \\
Less patient choice of RRT modality & Better management of CVD and comorbid conditions \\
Worse psychosocial adjustment & Improved patient survival \\
\hline
\end{tabular}

Abbreviations: CVD, cardiovascular disease; RRT, renal replacement therapy.

Table 36| Outcomes of early versus late referral

\begin{tabular}{lccc}
\hline Variable & Early referral mean (SD) & Late referral mean (SD) & P value \\
\hline Overall mortality, \% & $11(3)$ & $23(4)$ & $29(5)$ \\
1-year mortality, \% & $13(4)$ & $25.3(3.8)$ & 0.0001 \\
Hospital length of stay, days & $13.5(2.2)$ & $3.40(0.03)[34.0(0.3)]$ & 0.0007 \\
Serum albumin at RRT start, g/dl [g/l] & $3.62(0.05)[36.2(0.5)]$ & $29.71(0.10)$ & 0.001 \\
Hematocrit at RRT start, \% & $30.54(0.18)$ & 0.013 \\
\hline
\end{tabular}

Abbreviation: RRT, renal replacement therapy.

Adapted from Am J Med, Chan MR, Dall AT, Fletcher KE, et al. ${ }^{673}$ Outcomes in patients with chronic kidney disease referred late to nephrologists: a meta-analysis. 120: 1063-1070, 2007, with permission from Elsevier; accessed http://download.journals.elsevierhealth.com/pdfs/journals/0002-9343/PIIS000293430700664X.pdf

be recouped by delaying dialysis requirement by 1 year in one individual per 10,000 patients managed according to guidelines.

\section{International Relevance}

Local practice and resource will dictate local referral practice but regardless of the health-care system, delay or prevention of progression of both CKD and complications associated with CKD will add value. Local organizations will determine the best methods of communication and interaction between patients, specialists, and primary care physicians.

\section{Implications for Clinical Practice and Public Policy}

Implementation of referral guidelines will inevitably lead to an increased workload for specialist nephrology services. However, introduction of local initiatives in conjunction with primary care providers can improve the appropriateness and quality of the referral. Local initiatives combined with national policy and practice changes can lead to an improvement in the outcomes of CKD patients regardless of the level of resource available.

\section{Pediatric Considerations}

Current pediatric practice in most areas of the world would suggest a higher level of kidney function for referral than that for adults, though the principles remain the same. Much of the pediatric nephrologist's consultation occurs from infants, or even antenatal sources, where the identification of the child as being 'at-risk' may be apparent from radiographic studies performed in utero. The relatively non-specific signs and symptoms of most forms of renal disease in the young child mandates a higher level of suspicion in the referring physician, and a lower threshold of acceptance of the consult in the subspecialty clinic accepting these referrals.

Attempts to develop universal guidelines for referral of children to pediatric nephrology services would be dependent on local resources (as is the case for adults) and it is of value to consider in broad categories the types of conditions for which referral to a pediatric nephrologist would be expected to provide benefit to the referring physician and patient/family.

In a recent review Barakat ${ }^{677}$ attempts to address a number of these issues by outlining the most common presentations of a child with significant renal disorders. Barakat and Chesney also suggest a number of specific areas in terms of initial investigations, management, and follow-up where the primary care physician can legitimately play a role in the care of the child with renal disease and provides a list of suggested referral triggers. ${ }^{678}$

While there will be variation in referral triggers, referral would be recommended for the following: acute or chronic reduction in renal function, poorly treated or severe hypertension, severe electrolyte abnormalities, the finding of significant abnormalities in urinary tract structure, or the presence of systemic diseases likely to produce renal effects. Similarly, the need for education in progressive conditions, performance of and interpretation of renal biopsies, and allaying parental/patient anxiety would also be acceptable reasons for referral.

There is no 'minimum acceptable value' of renal dysfunction below which one can be certain to see significant abnormalities in clearance, electrolyte, or other side effects associated with progressive renal disease in children such as growth failure or neurocognitive issues. However, the 
KDOQI CKD Guidelines ${ }^{1}$ stated that while a child with a eGFR $<30 \mathrm{ml} / \mathrm{min} / 1.73 \mathrm{~m}^{2}$ warranted referral to a pediatric nephrologist in all cases, that in fact it was also reasonable to consider any child with evidence of CKD- and in particular those with eGFR $<60 \mathrm{ml} / \mathrm{min} / 1.73 \mathrm{~m}^{2}$ - for referral to a pediatric nephrologist regarding evaluation and management. ${ }^{43}$

It is reasonable to presume that, as in adults, the concept of referral 'early' or 'above' some minimum level of function should allow for numerous benefits to the patient and family, but again there are few data for this in children. The most informative information on both of these issues can be derived from two papers examining the issue of late referrals of children who eventually required ESRD care. Kennedy et al. $^{679}$ demonstrated that at the time of referral to their center, the children $>1$ year of age had a median Schwartz eGFR of only 27 (IQR 9-52) $\mathrm{ml} / \mathrm{min} / 1.73 \mathrm{~m}^{2}$, and in fact $55 \%$ of these children over the age of 1 year were referred with an eGFR $<30 \mathrm{ml} / \mathrm{min} / 1.73 \mathrm{~m}^{2}$, meeting one of two definitions of a late referral. When they considered their second definition of initiation of RRT within 90 days of referral, commonly used in the literature for this particular topic, $30 \%$ of the eligible cohort required RRT within that 90-day window and hence were considered late referrals on this basis. The potential effect on the child's health could be ascribed to such a delay in referral: those children had lower mean $\mathrm{Hb}(8.7 \pm 0.6 \mathrm{~g} / \mathrm{dl}[87 \pm 6 \mathrm{~g} / \mathrm{l}])$ versus $(12.8 \pm$ $0.6 \mathrm{~g} / \mathrm{dl}[128 \pm 6 \mathrm{~g} / \mathrm{l}])$ and higher median urea (34 (IQR $5-14) \mathrm{mmol} / \mathrm{l}[203$ (IQR 30-84) $\mathrm{mg} / \mathrm{dl}]$ versus 6 (IQR 5-14) $\mathrm{mmol} / \mathrm{l}[36(\mathrm{IQR} 30-84) \mathrm{mg} / \mathrm{dl}], \mathrm{P}<0.001$ ) than those who presented with eGFR $>30 \mathrm{ml} / \mathrm{min} / 1.73 \mathrm{~m}^{2}$. Secondary analysis confirmed this was also true in the groups if one looked at those above or below an eGFR of $60 \mathrm{ml} / \mathrm{min} /$ $1.73 \mathrm{~m}^{2}$. The second paper by Boehm and colleagues ${ }^{680}$ was a retrospective single pediatric center report that demonstrated that over $\sim 30$ years of referrals $(\mathrm{N}=111), 24 \%$ of them could be considered as late referrals, as defined by need for RRT within 90 days of referral. In this late referral group, the eGFR (Schwartz) was significantly lower than those presenting later, (14.9 versus $\left.34.2 \mathrm{ml} / \mathrm{min} / 1.73 \mathrm{~m}^{2}, \mathrm{P}<0.001\right)$ as was the $\mathrm{Hb}$ at presentation $(8.0 \mathrm{~g} / \mathrm{dl}[80 \mathrm{~g} / \mathrm{l}]$ versus $10.5 \pm 2.3 \mathrm{~g} /$ dl $[105 \pm 23 \mathrm{~g} / \mathrm{l}] ; \mathrm{P}<0.001)$. Importantly the $\mathrm{Hb}$ deficit persisted at the time of RRT initiation, with the $\mathrm{Hb}$ in the late referral group being $8.5 \mathrm{~g} / \mathrm{dl}[85 \mathrm{~g} / \mathrm{l}]$ versus $9.8 \pm 1.9 \mathrm{~g} / \mathrm{dl}[98$ $\pm 19 \mathrm{~g} / \mathrm{l}]$ in the earlier referred patients, $\mathrm{P}<0.01$. The other metric of interest chosen by these authors to evaluate the possible detrimental effect of late referral was the likelihood of a given child having a pre-emptive transplant, i.e., prior to dialysis initiation. While this association was not confirmed in Kennedy's paper, Boehm and his group demonstrated that in their patient population children who were referred late only $11 \%$ were preemptively transplanted as compared to $40 \%$ of children who presented to care more than 90 days prior to the need for some form of RRT. Of further interest, although somewhat counterintuitive, the proportion of patients in the Boehm study who initiated hemodialysis was not statistically different between those in the late versus early referral groups, $62 \%$ versus $67 \%, \mathrm{P}<0.05$ respectively.

The underlying concept of referral to a pediatric nephrologist in the face of rapid progression of renal failure is of course applicable in full. However, no current validated risk of progression tool exists in pediatric nephrology. As to what level of functional decline might be considered 'concerning,' the best evidence is derived from the longitudinal iohexol GFR data as accrued in the CKiD trial. Data from that study related to the annualized rate of decline in renal function for children with glomerular conditions is $-10.5 \%$ as compared to those with a non-glomerular causes in whom the annualized rate of change is only $-3.9 \%{ }^{71}$ Any child whose rate of decline exceeded these two values would at least warrant much closer follow-up and/or investigation for modifiable factors to slow progression. Note this is not to say these rates of decline should be considered to be 'normal' for either category. All efforts to slow decline in renal function would still be of vital importance in every individual.

\section{2: CARE OF THE PATIENT WITH PROGRESSIVE CKD}

The following section describes recommended structures and key milestones for people with progressive CKD. The recommendations are intended to model best practices but it is appreciated that different health-care systems, geographical issues, and economic considerations will have variable abilities to implement these recommendations.

Key aspects of caring for people with progressive CKD, as they approach end of life or RRT options are addressed in this section.

5.2.1: We suggest that people with progressive CKD should be managed in a multidisciplinary care setting. (2B)

5.2.2: The multidisciplinary team should include or have access to dietary counseling, education and counseling about different RRT modalities, transplant options, vascular access surgery, and ethical, psychological, and social care. (Not Graded)

\section{RATIONALE}

Optimal care is that care which leads to the best outcomes for the individual, the population, and society. The model of care varies according to $\mathrm{CKD}$ severity, which will determine the target population and goals. These statements are worded to predominantly encompass those people likely to progress to ESRD. CKD models of care follow the same principles embodied in the chronic disease model of care (Figure 22). The specific components for CKD models of care include: protocols for laboratory and clinic visits; attention to cardiovascular comorbidities and CKD-associated comorbidities such as anemia; a vaccination program (see Recommendation 4.6.1-4.6.6); an education program which includes both general CKD and RRT education (including conservative management where appropriate); self-management; lifestyle modification including diet, exercise, and smoking 


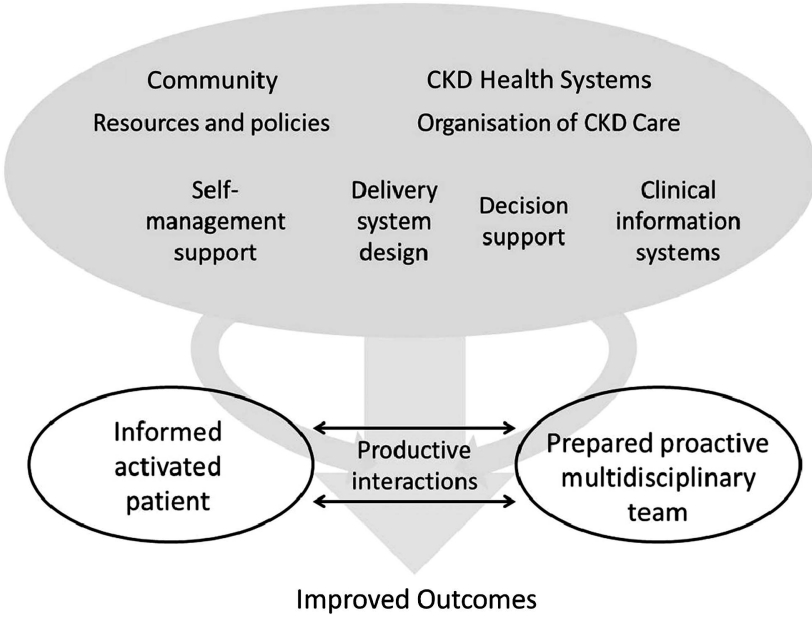

Figure 22 | The CKD chronic care model. CKD, chronic kidney disease. Adapted by permission from BMJ Publishing Group Limited. Improving the quality of health-care for chronic conditions. Epping-Jordan JE, Pruitt SD, Bengoa R, et al. ${ }^{681}$ Qual Saf Health Care. 13: 299-305, 2004; accessed http://qualitysafety.bmj.com/content/13/4/299.full.pdf+html

cessation; and counseling and support for factors such as social bereavement, depression, and anxiety.

\section{International Relevance}

Standardized and culturally appropriate protocols should be considered. While it is recognized that resources may vary across and within jurisdictions, recommendations here are based on principles of care, which should be relevant across the globe.

\section{Implications for Clinical Practice and Public Policy}

$\mathrm{CKD}$ is a complex condition and co-exists with many other conditions. Therefore models of care should be developed that integrate the complexity of the clinical conditions involved, patient-centered philosophies, and the health-care environment. The principles of care are universal but implementation may be customized to specific circumstances.

\section{Pediatric Considerations}

Papers addressing the need for and effectiveness in the utility of multidisciplinary clinics for management of pediatric CKD patients have been published. In 2012, Ajarmeh et al. ${ }^{682}$ described a retrospective comparison of two cohorts of children aged 0-18 years of age and followed at a single Canadian tertiary care referral center over a 1-year time period either before or following the full implementation of a multidisciplinary CKD clinic which included the services of dedicated pediatric nephrologists, renal nursing, pharmacy, dietitians, social workers, and a renal database manager. In 2009, Menon et al. ${ }^{683}$ published data from a large American pediatric referral center and compared the outcomes of children from one of two 5-year cohorts in the period immediately preceding or following the initiation of a specific Chronic Renal Insufficiency clinic which was staffed by pediatric nephrologists, nurse clinicians, transplant coordinators, dietitians, social workers, and a psychologist.

Ajarmeh et al. ${ }^{682}$ compared the two cohorts in a number of areas during their year of follow-up whereas Menon et al. ${ }^{683}$ chose to look at each individual patient in the 12 months immediately prior to initiating RRT. While the studies had different variables of interest and used slightly different approaches for comparison of results between their groups, both demonstrated a reduction in the rate of decline in estimated renal function, improved $\mathrm{Hb}$ levels, and improvement in at least some aspect of bone mineral metabolism control in their respective cohorts cared for in the multidisciplinary era. In addition, both studies also demonstrated a decrease in resource utilization by patients in the multidisciplinary clinic as measured by median length of stay ${ }^{682}$ and number of unplanned admissions per patient per year. ${ }^{683}$ Also of note, Menon et al. demonstrated that in the patients who initiated dialysis (which was how the cohort was chosen), more patients who started hemodialysis did so with a functioning arteriovenous fistula or arteriovenous graft at the time of initiation compared to those from the general nephrology clinic era ( 85.7 versus $20 \%, P=0.02)$.

\section{3: TIMING THE INITIATION OF RRT}

5.3.1: We suggest that dialysis be initiated when one or more of the following are present: symptoms or signs attributable to kidney failure (serositis, acidbase or electrolyte abnormalities, pruritus); inability to control volume status or blood pressure; a progressive deterioration in nutritional status refractory to dietary intervention; or cognitive impairment. This often but not invariably occurs in the GFR range between 5 and $10 \mathrm{ml} / \mathrm{min} / 1.73 \mathrm{~m}^{2}$. (2B)

5.3.2: Living donor preemptive renal transplantation in adults should be considered when the GFR is $<20 \mathrm{ml} / \mathrm{min} / 1.73 \mathrm{~m}^{2}$, and there is evidence of progressive and irreversible CKD over the preceding 6-12 months. (Not Graded)

\section{RATIONALE}

The statement is worded very precisely to highlight the need for RRT to address symptoms and to avoid the institution of dialysis therapy at an arbitrary number representing the degree of residual renal function. Given the risks and benefits of RRT, as well as the potential imprecision of measurements, patients need to be treated according to symptoms and signs, not simply based on a laboratory value.

Data from national registries has shown a consistent rise in GFR at initiation of RRT. This rise has been driven partly by a desire to improve nutritional status and also by earlier observational data suggesting adverse outcomes associated with RRT initiation at lower GFR. These early data were subject to much confounding. RCT data, supported by large registry-based studies corrected for confounding, show no survival advantage to early start dialysis (Initiating Dialysis 
Early and Late [IDEAL] study, see Evidence Base Below). Thus, the statement as written should help the clinician to balance symptoms with laboratory values in decision making.

The statement regarding living donor transplantation is intended to ensure that practitioners think about this option in the context of the totality of RRT options. It is worded to be concordant with local living donation transplant policies. It aims to reflect the need for risk-benefit assessment of preemptive renal transplantation in all individuals. Note that the statement asks clinicians to consider this option, but does not state at which level of GFR the preemptive transplant would occur as this is a matter of local practice and patientspecific considerations.

\section{Evidence Base}

The perceived survival advantage of early start of dialysis was questioned by a study from the Netherlands Cooperative Study on the Adequacy of Dialysis Study Group (NECOSAD). ${ }^{684}$ Of 253 patients with new ESRD, 94 (37\%) started dialysis late (GFR $4.9 \pm 1.7 \mathrm{ml} / \mathrm{min} / 1.73 \mathrm{~m}^{2}$ ) and 157 started in a timely fashion (GFR $7.1 \pm 2.4 \mathrm{ml} / \mathrm{min} / 1.73 \mathrm{~m}^{2}$ ). Although there was a small gain in survival time over 3 years after start of dialysis for the timely start group (2.5 months) there was no significant difference in survival between the 2 groups and the gain in survival time was offset by an estimated lead-time bias of between 4.1 and 8.3 months. In a study eliminating the effect of lead-time bias Traynor et al. found no benefit in survival in those initiating dialysis early. ${ }^{685}$ Subjects starting dialysis early $(\mathrm{N}=119)$ had a median IQR $\mathrm{CrCl}$ of 10.4 (9.1-11.9) $\mathrm{ml} / \mathrm{min}$ versus 6.7 (5.67.5) $\mathrm{ml} / \mathrm{min}$ in 116 subjects starting dialysis late. After correction for multiple confounders, they found an $11 \%$ greater hazard for mortality with each $1 \mathrm{ml} / \mathrm{min}$ increase in $\mathrm{CrCl}$ at start of dialysis (HR 1.11; 95\% CI 1.01-1.21; $\mathrm{P}=0.024$ ). Subsequent observational studies, including large dialysis registry studies from around the globe, have all suggested increased mortality when dialysis is started early. ${ }^{686-696}$ These studies encountered multiple difficulties in drawing conclusions from this observational approach including lead-time bias, problems with estimating GFR from $\mathrm{SCr}$ in people with low muscle mass and/or fluid overload, the fact that people with symptoms and/or increased comorbidity are more likely to start dialysis early, and finally survivor bias in studies including people only when they start dialysis (and therefore excluding those who die prior to starting diaysis). These confounders were largely addressed by a multicenter controlled trial in which 828 adults with progressive CKD and $\mathrm{CrCl} 10-15 \mathrm{ml} / \mathrm{min} / 1.73 \mathrm{~m}^{2}$ were randomly assigned to early $(\mathrm{CrCl} \quad 10-14 \mathrm{ml} / \mathrm{min}[0.17-$ $0.23 \mathrm{ml} / \mathrm{s}])$ or late $(\mathrm{CrCl} 5-7 \mathrm{ml} / \mathrm{min}[0.08-0.12 \mathrm{ml} / \mathrm{s}])$ initiation of dialysis. ${ }^{697}$ The study protocol allowed an earlier start where deemed clinically necessary by investigators and although the difference in GFR at dialysis initiation between the 2 groups was significant (early start $\mathrm{CrCl} 12.0$ versus late start $9.8 \mathrm{ml} / \mathrm{min}$, MDRD GFR 9.0 versus $7.2 \mathrm{ml} / \mathrm{min} /$ $1.73 \mathrm{~m}^{2}$ ), it was less than planned because $19 \%$ of early starters started late and $76 \%$ of late starters started early. There was no difference in mortality between the early and late start groups and no difference in the study's secondary outcomes (cardiovascular events, infectious events and complications of dialysis) between the 2 groups. Finally, a further observational study sought to examine the effect of early initiation of dialysis on survival in a 'healthy' group of 81,176 subjects with ESRD aged 20-64 years, without diabetes, and with no comorbidity other than hypertension. ${ }^{698}$ The unadjusted 1-year mortality by MDRD GFR at dialysis initiation ranged from $6.8 \%$ in the reference group (GFR $<5.0 \mathrm{ml} / \mathrm{min} / 1.73 \mathrm{~m}^{2}$ ) to $20.1 \%$ in the highest GFR group $\left(\geq 15.0 \mathrm{ml} / \mathrm{min} / 1.73 \mathrm{~m}^{2}\right)$. In an even healthier subset of 35,665 subjects with serum albumin concentrations of $3.5 \mathrm{~g} / \mathrm{dl}$ or higher prior to hemodialysis initiation, the 1 -year mortality was $4.7 \%$. In this group the adjusted HR for mortality was 1.27 for GFR $5.0-9.9 \mathrm{ml} / \mathrm{min} /$ $1.73 \mathrm{~m}^{2}, 1.53$ for GFR $10.0-14.9 \mathrm{ml} / \mathrm{min} / 1.73 \mathrm{~m}^{2}$, and 2.18 for GFR $\geq 15.0 \mathrm{ml} / \mathrm{min} / 1.73 \mathrm{~m}^{2}$ compared with the reference group of GFR $<5.0 \mathrm{ml} / \mathrm{min} / 1.73 \mathrm{~m}^{2}$.

\section{International Relevance}

The availability of resources for formal multidisciplinary teams, educational materials, and access to specialized counseling for diet, advance directives, access planning, and pre-emptive transplantation varies around the world. These statements are proposed so that 'best practices' can be documented or aspired to. The need for education, planning, and appropriate expertise for the management of this patient group is internationally relevant. The methods, frequency, and tools with which this can be accomplished will be region specific.

\section{Implications for Clinical Practice and Public Policy}

There is a need to focus on regular symptom assessment as part of CKD review in those with lower eGFR values. Individual assessment and availability of resources will dictate specific timing of therapies.

Clinicians should be aware of the impact of early dialysis start on $\mathrm{QOL}^{699}$ before recommending this strategy to patients.

\section{Pediatric Consideration}

Timing of initiation of dialysis. As might be expected, information as to the proper timing for initiation of dialysis does not exist for children. Thus, it would seem reasonable to follow the guidelines as set out for the adults. A review by Greenbaum and Schaefer ${ }^{700}$ provides the reader with generally agreed upon absolute and relative indications for initiation of dialysis in the child. The absolute indications listed are those of neurologic consequences attributable to uremia; hypertension that fails to respond to antihypertensive therapy; pulmonary edema unresponsive to diuretics; pericarditis; bleeding tendency; and refractory nausea or vomiting. However, the authors clearly state that there is no current consensus as to the validity of the relative indications and specifically the level at which each engenders the need for 
dialysis. Relative indications to commence dialysis include less severe uremic symptoms, hyperkalemia, hyperphosphatemia, malnutrition, and growth failure. The authors point out as well that the answer to 'early' versus 'late' dialysis initiation cannot be addressed in any meaningful way in children as there is currently lack of data; and the unique factors to consider in a child such as growth, psychosocial development, likely renal transplant, and need for extended time on both dialysis and in transplant make the probability of a clinical trial to address this issue nearly impossible.

Timing of living donor transplant. There is no direct evidence to guide the pediatric nephrologist as to the 'best' timing for a living donor transplant in children. In each individual case the relative likelihood of improvement in presumed uremic symptoms and burden of disease as related to management of CKD will need to be balanced against the risks of transplant surgery itself and the attendant medication risks with prolonged immunosuppression. While there is little doubt as to the benefit in overall lifespan accrued by a child who is transplanted as opposed to one maintained on standard dialysis therapies during their childhood (average life expectancy of 63 versus 38 years respectively), ${ }^{701}$ the issue as to what level of residual function is sufficient to justify delay or conversely proceeding to transplant is simply not readily available.

It should also be noted that reliance on GFR as the sole marker of disease intensity is unlikely to be sufficient for making such a decision, and patient symptoms and/or unique family factors may play a significant role in the decision as to timing of a living donor transplant (e.g., a young grandparent available and healthy to donate at age 60 years may not be eligible at age 63 years and so an earlier transplant would be favored).

\section{4: STRUCTURE AND PROCESS OF COMPREHENSIVE CONSERVATIVE MANAGEMENT}

5.4.1: Conservative management should be an option in people who choose not to pursue RRT and this should be supported by a comprehensive management program. (Not Graded)

5.4.2: All CKD programs and care providers should be able to deliver advance care planning for people with a recognized need for end-of-life care, including those people undergoing conservative kidney care. (Not Graded)

5.4.3: Coordinated end-of-life care should be available to people and families through either primary care or specialist care as local circumstances dictate. (Not Graded)

5.4.4: The comprehensive conservative management program should include protocols for symptom and pain management, psychological care, spiritual care, and culturally sensitive care for the dying patient and their family (whether at home, in a hospice or a hospital setting), followed by the provision of culturally appropriate bereavement support. (Not Graded)

\section{RATIONALE}

These statements are intended to highlight the need for comprehensive conservative care processes and resources in the care of this complex patient group. There is increasing recognition that provision of organized care to those who are dying or choose to not pursue dialysis and transplant care is of value to the patients and their families. Clinicians involved in caring for these patients should be alerted to this need. These statements are positioned so as to overtly state this need.

In different societies or cultural areas, the form and structure of this care may vary tremendously and families or religious organizations may be able to deliver expert and sensitive care. The details here are listed not to be prescriptive but rather to articulate the 'best practices' in communities where resources may be available and to serve a construct to review in those locations where resources are more limited.

\section{Evidence Base}

The evidence base for these statements is limited to documents describing the burden of illness and unmet needs in patients with CKD as they decide either not to pursue dialysis or to withdraw from RRT. As the readers will appreciate, there are neither RCTs nor even large cohort studies to inform these statements within the CKD populations. Analogies with oncology have been drawn and literature from there has been used to justify the concepts above.

Patients with advanced CKD have extensive palliative care needs for years before death. Throughout their illness trajectory, symptom burden is high. ${ }^{702-707}$ The number and severity of physical and emotional symptoms are similar to those of cancer patients hospitalized in palliative care settings. ${ }^{703,708}$ Advanced CKD patients managed without dialysis are equally symptomatic. ${ }^{706}$ An increasing number of dialysis patients die after withdrawal of dialysis $(10-15 \%$ in 1990, 20\% in 2004), primarily due to poor QOL, representing the second leading cause of death after CVD.

Conservative care focuses on slowing the decline in renal function, actively managing symptoms, advance care planning, and the provision of appropriate palliative care. In oncology, receiving early palliative care is associated with better QOL, fewer emergency department visits and hospitalizations, less aggressive care at the end of life, and surprisingly, longer survival. ${ }^{709}$ These observations suggest that development of clinical models that integrate appropriate palliative care, including the creation of conservative care pathways, are likely to be hugely beneficial and would help avoid harmful dialysis to those patients unlikely to realize benefit. Such conservative care programs are slowly being developed, primarily in the UK and Canada. 
The literature describing experiences of specific programs and symptom burden in CKD, as well as advocating for better care, has only become more prevalent since $2005 .^{709-716}$

Poor quality, hospitalized deaths began to improve for patients dying with cancer as a result of an increasing emphasis on palliative care teams providing attention to symptom control, psychosocial needs, and options for location of care. The literature suggests that current models of palliative care for cancer patients that deinstitutionalized health-care services have not only improved patient outcomes and quality of care $\mathrm{c}^{717-720}$ but have been costneutral. $^{721}$

\section{International Relevance}

All countries have people with CKD who withdraw either voluntarily or involuntarily from dialysis services. Best care for those patients will obviously need to respect cultural and religious values, but would necessarily be based on the same philosophical grounds of maintaining dignity of the individual. Appreciating the need for and articulating conservative care pathways overtly would be internationally applicable.

\section{Implications for Clinical Practice and Public Policy}

There is a need to ensure appropriate access to services and education surrounding quality care during terminal stages of a chronic condition. There is increasing attention to this in many societies but not in all. Appreciating the variability in the resources required and their availability to different groups of patients is important for implementation. Furthermore, involvement of religious and cultural leaders in recognition of these care practices can be enhanced if they are overtly recognized as part of the care continuum.

There is a need for robust assessment of best practices in CKD and other chronic conditions so that we may provide best care throughout the continuum of life. Researchers around the world are actively pursuing this so that we may have better tools, programs, and ultimately, better outcomes for our patients.

\section{Pediatric Consideration}

Despite the lack of published evidence, there is no reason to believe that children and families faced with the need to consider conservative care or a palliative approach to their impending ESRD would not benefit from all of the above recommendations. It would seem reasonable for pediatric nephrology centers to liaise with their pediatric oncology or palliative care teams, ethicists, and pastoral care providers to assist them in developing, defining, and/or identifying locally available resources for situations where a child is not deemed a candidate for RRT prior to their occurrence.

\section{DISCLAIMER}

While every effort is made by the publishers, editorial board, and ISN to see that no inaccurate or misleading data, opinion or statement appears in this Journal, they wish to make it clear that the data and opinions appearing in the articles and advertisements herein are the responsibility of the contributor, copyright holder, or advertiser concerned. Accordingly, the publishers and the ISN, the editorial board and their respective employers, office and agents accept no liability whatsoever for the consequences of any such inaccurate or misleading data, opinion or statement. While every effort is made to ensure that drug doses and other quantities are presented accurately, readers are advised that new methods and techniques involving drug usage, and described within this Journal, should only be followed in conjunction with the drug manufacturer's own published literature. 


\section{Methods for guideline development}

Kidney International Supplements (2013) 3, 120-127; doi:10.1038/kisup.2012.69

\section{AIM}

The overall aim of this project was to develop an evidencebased clinical practice guideline for evaluation and management of CKD. The guideline consists of recommendation statements, rationales, and a summary of systematically generated evidence on relevant pre-defined clinical topics. To a large extent the guideline builds on the output of the KDIGO Controversies Conference in 2009, ${ }^{30}$ which generated epidemiological data to support a revision of the classification and staging system. The vision for this KDIGO guideline is that it would endorse the current CKD definition as an imperfect convention for describing a state of function, revise classification based on risk, revise risk states, and revise and update action plans in view of the revised classifications. Additional systematic evidence review focused on specific topics.

\section{OVERVIEW PROCESS}

The guideline development process included the following steps:

- Appointing Work Group members and the ERT

- Discussing process, methods, and results

- Developing and refining topics

- Identifying populations, interventions or predictors, and outcomes of interest

- Selecting topics for systematic evidence review

- Standardizing quality assessment methodology

- Developing and implementing literature-search strategies

- Screening abstracts and retrieving full text articles on the basis of predefined eligibility criteria

- Creating data extraction forms

- Extracting data and performing critical appraisal of the literature

- Grading the methodology and outcomes in individual studies

- Tabulating data from individual studies into summary tables

- Grading the strength of recommendations on the basis of the quality of evidence and other considerations

- Finalizing guideline recommendations and supporting rationales

- Sending the guideline draft for peer review to the KDIGO Board of Directors in January 2012 and for public review in May 2012

- Publishing the final version of the guideline

\section{Collaboration Among Participants}

The KDIGO Co-Chairs appointed the Work Group Co-Chairs, who then assembled the Work Group of domain experts, including individuals with expertise in internal medicine, adult and pediatric nephrology, diabetology/ endocrinology, clinical chemistry, and epidemiology. The Tufts Center for Kidney Disease Guideline Development and Implementation at Tufts Medical Center in Boston, Massachusetts, USA, was contracted to conduct systematic evidence review and provide expertise in guideline development methodology. The ERT consisted of physician-methodologists with expertise in nephrology, a project coordinator, a research assistant, and a medical writer-editor. The ERT instructed and advised Work Group members in all steps of literature review, critical literature appraisal, and guideline development. The Work Group and the ERT collaborated closely throughout the project.

The Work Group and its Chairs, KDIGO Co-chairs, ERT, and KDIGO support staff met for three 2-day meetings for training in the guideline development process, topic discussion, and consensus development.

Throughout the project, the ERT offered suggestions for guideline development and led discussions on systematic review, literature searches, data extraction, assessment of quality and applicability of articles, evidence synthesis, grading of evidence and guideline recommendations, and consensus development. The Work Group took the primary role of writing the recommendation statements and rationales and retained final responsibility for their content.

\section{Defining Scope and Topics}

This KDIGO CKD guideline was set out to update the KDOQI Clinical Practice Guidelines for CKD: Evaluation, Classification, and Stratification ${ }^{1}$ in 2002, which spans many topics related to the diagnosis, classification, stratification, and management of CKD. ${ }^{1}$

The Work Group Co-Chairs prepared the first draft of the scope of work document as a series of open-ended questions to be considered by Work Group members. At their first 2day meeting, members added further questions until the initial working document included all topics of interest to the Work Group. The inclusive, combined set of questions formed the basis for the deliberation and discussion that followed. The Work Group strove to ensure that all topics deemed clinically relevant and worthy of review were identified and addressed.

Updating the topics of definitions and classification was based on the output from the KDIGO Controversies Conference and the CKD Prognosis Consortium. ${ }^{4,30}$

Additional topics that relate to explicit selection of diagnostic tests or interventions were chosen to undergo systematic review of the best available evidence. Systematic 
evidence review entails a priori question formulation, specification of important outcomes for the review, systematic searches, data extraction, tabulation, analysis, and synthesis of evidence and is described in detail for each of the specific questions. The process followed for each evidence review topic (a total of four non-treatment topics and four treatment topics) is detailed below.

The eight topics for which the ERT conducted searches and evidence review are shown in Table 37. For the systematic review topics, the Work Group and ERT further developed and refined each topic and specified screening criteria, literature search strategies, and data extraction forms.

Many other topics were not suitable to be addressed by indepth evidence review. When the anticipated outcome of an extensive literature search was unlikely to yield evidence that directly informs practice choices, the approach chosen was that of a narrative review.

\section{APPROACH TO EVIDENCE REVIEW TOPICS Formulating Questions of Interest}

Questions of interest were formulated according to the PICODD (Population, Intervention or Predictor, Comparator, Outcome, study Design, and Duration of follow-up) criteria. Details of the PICODD criteria are presented in Table 37.

\section{Literature Searches and Article Selection for Evidence Review Topics}

Search strategies were developed by the ERT, with input from the Work Group, for each topic of interest (whether treatment or non-treatment topics). The ERT performed literature searches and conducted abstract and article screening. The ERT also coordinated the methodological and analytic processes, data extraction, and summarizing of the evidence. Before initiating our own de novo systematic review, we searched for existing systematic reviews that could be used. The searches and search terms are provided in Supplemental Table 1 and the search dates and yields for all topics are presented in Table 38 .

\section{Selection of Outcomes of Interest}

The Work Group selected outcomes of interest on the basis of their importance for informing clinical decision making. Importance of mortality and ESRD was considered to be critical; the importance of progression of CKD and categorical or continuous measures of kidney function was considered to be high; and the importance of QOL, BP, gout attacks, and proteinuria was considered to be moderate.

\section{Data Extraction}

Text articles were extracted by the ERT onto forms customized to capture data on design, methodology, baseline characteristics, interventions or predictors, comparators, outcomes, results, and limitations of individual studies. Study methodology and risk of bias were also systematically graded for each outcome and recorded.

\section{Summary Tables}

Pertinent information for systematic review topics was tabulated in summary tables. Summary tables list outcomes of interest as well as relevant population characteristics, descriptions of interventions and comparators, results, and quality grades for each outcome. Categorical and continuous outcomes were summarized separately. Work Group members reviewed all summary table data and quality grades.

\section{Evidence Profiles}

Evidence profiles are usually constructed as a means to assess the quality and record quality grades and descriptions of effect for each outcome across studies, as well as the quality grades and description of net benefits or harms of the intervention or comparator across studies. These profiles aim to make the evidence synthesis process transparent. However, since no treatment or non-treatment topic had more than one study in a summary table for which the quality was graded, no evidence profiles were generated, and the information in the summary table shows the highest level of synthesis.

\section{Grading of Quality of Evidence for Outcomes of Individual Studies}

Methodological quality. Methodological quality (internal validity) refers to the design, conduct, and reporting of outcomes of a clinical study. A previously devised three-level classification system for quality assessment was used to grade the overall study quality and quality of all relevant outcomes in the study (Table 39). Variations of this system have been used in most KDOQI and all KDIGO guidelines and have been recommended for the US Agency for Healthcare Research and Quality Evidence-based Practice Center program. ${ }^{722}$

Each study was given an overall quality grade on the basis of its design, methodology (randomization, allocation, blinding, definition of outcomes, appropriate use of statistical methods, etc.), conduct (drop-out percentage, outcome assessment methodologies, etc.), and reporting (internal consistency, clarity, thoroughness, and precision, etc.). Each reported outcome was then evaluated and given an individual grade depending on the quality of reporting and methodological issues specific to that outcome. However, the quality grade of an individual outcome could not exceed the quality grade for the overall study.

\section{Grading the Quality of Evidence and the Strength of Guideline Recommendations}

A structured approach, based on the GRADE approach, ${ }^{723-725}$ was used to grade the quality of the overall evidence and the strength of recommendations for each topic. This grading scheme-with two levels for the strength of a recommendation together with four levels of grading for the quality of the evidence, as well as the option of an ungraded statement for general guidance-was adopted by the KDIGO Board in December 2008. 


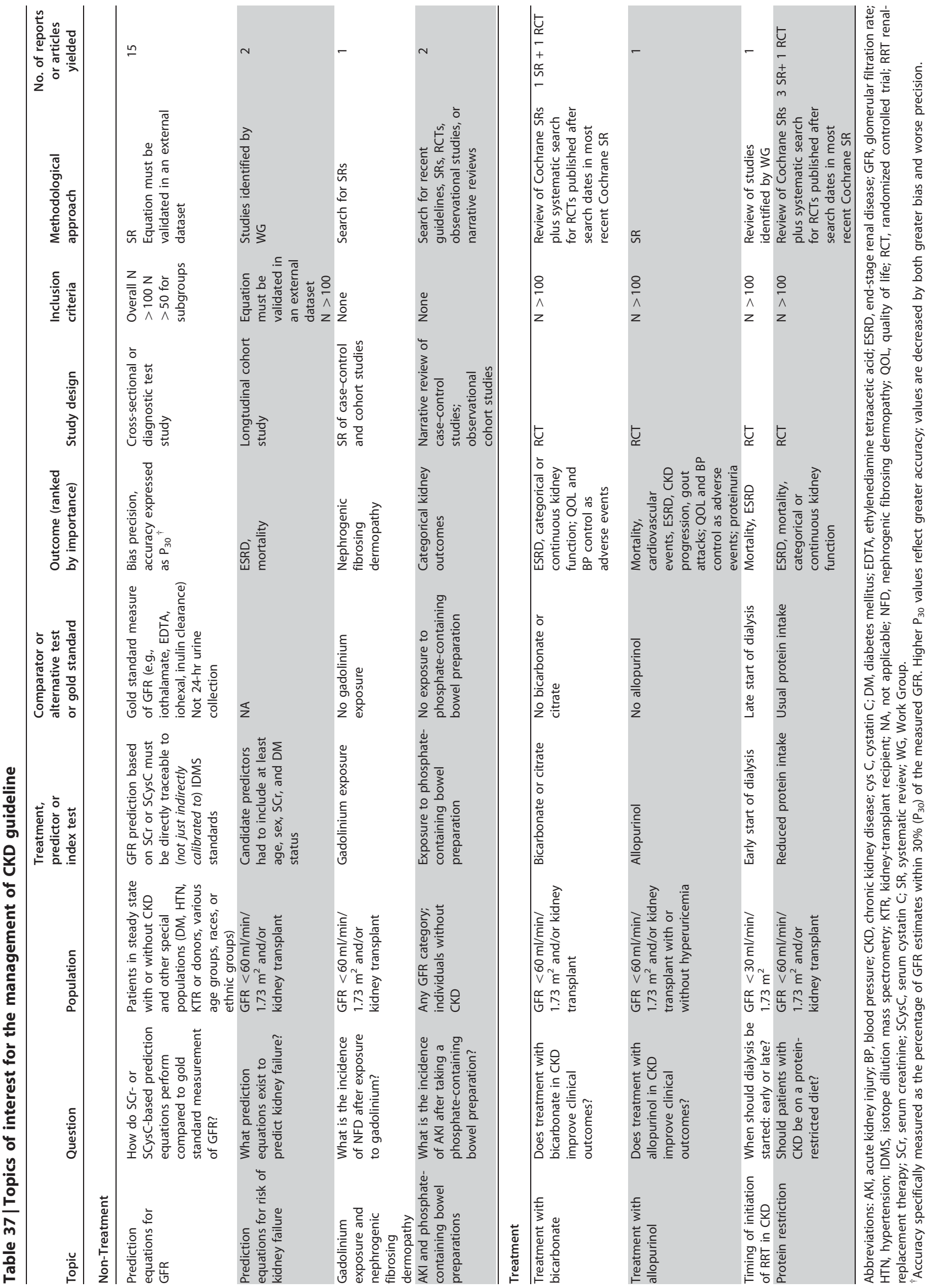


Table 38 | Literature yield of primary articles for all topics

\begin{tabular}{|c|c|c|c|c|c|c|}
\hline Topic & $\begin{array}{l}\text { Systematic } \\
\text { review } \\
\text { performed }\end{array}$ & Search dates & Search terms & $\begin{array}{l}\text { Articles } \\
\text { retrieved }\end{array}$ & $\begin{array}{l}\text { Articles } \\
\text { with full } \\
\text { text } \\
\text { screened }\end{array}$ & $\begin{array}{l}\text { Articles } \\
\text { used in } \\
\text { tables }\end{array}$ \\
\hline \multicolumn{7}{|l|}{ Non-treatment topics } \\
\hline Prediction equations for eGFR & $\mathrm{Y}$ & $\begin{array}{l}\text { MEDLINE search from } 2006 \text { until } \\
\text { 2011, with an update in June } 2011\end{array}$ & $\begin{array}{l}\text { "kidney," and "prediction," } \\
\text { "equation," "formula," or } \\
\text { "regression analysis" }\end{array}$ & 2921 & 86 & 15 \\
\hline $\begin{array}{l}\text { Prediction equations for risk of } \\
\text { kidney failure or death }\end{array}$ & $\mathrm{N}$ & nd & nd & $10^{*}$ & 10 & 2 \\
\hline $\begin{array}{l}\text { Risk from gadolinium for } \\
\text { nephrogenic fibrosing } \\
\text { dermopathy }\end{array}$ & $\mathrm{Y}$ & $\begin{array}{l}\text { MEDLINE search from } 1996 \text { until } \\
2011 \text { was run in March } 2011\end{array}$ & $\begin{array}{l}\text { "kidney," and "gadolinium," } \\
\text { "nephrogenic fibrosing } \\
\text { dermopathy," or "nephrogenic } \\
\text { systemic fibrosis," and systematic } \\
\text { review }\end{array}$ & $1^{\dagger}$ & 0 & 0 \\
\hline $\begin{array}{l}\text { Risk for nephropathy from } \\
\text { phosphate-containing bowel } \\
\text { preparations }\end{array}$ & $\mathrm{N}$ & $\begin{array}{l}\text { MEDLINE search for recent (2008- } \\
\text { 2011) narrative reviews was run in } \\
\text { March } 2011\end{array}$ & "Phosphate nephropathy" & 2 & 2 & 2 \\
\hline \multicolumn{7}{|l|}{ Treatment topics } \\
\hline Treatment with bicarbonate & $\mathrm{Y}$ & $\begin{array}{l}\text { MEDLINE search from } 2005 \text { until } \\
\text { 2011, with an update in May } 2011\end{array}$ & $\begin{array}{l}\text { "randomized controlled trial," and } \\
\text { "kidney," or "metabolic acidosis," } \\
\text { and "bicarbonate" }\end{array}$ & 3981 & 6 & 1 \\
\hline Treatment with allopurinol & Y & $\begin{array}{l}\text { MEDLINE search from } 1996 \text { until } \\
\text { 2011, with an update in May } 2011\end{array}$ & $\begin{array}{l}\text { "randomized controlled trial," and } \\
\text { "kidney," and "allopurinol" }\end{array}$ & 393 & 4 & 1 \\
\hline $\begin{array}{l}\text { Earlier versus later initiation of } \\
\text { RRT in CKD }\end{array}$ & $\mathrm{N}$ & nd & nd & $8^{\ddagger}$ & 8 & 1 \\
\hline $\begin{array}{l}\text { Treatment with protein } \\
\text { restriction }\end{array}$ & $\mathrm{Y}$ & $\begin{array}{l}\text { Three Cochrane systematic reviews } \\
\text { were identified and used instead of } \\
\text { a de novo systematic review. } \\
\text { MEDLINE search from 1996-2011, } \\
\text { with an update in March } 2011\end{array}$ & $\begin{array}{l}\text { "randomized controlled trial," and } \\
\text { "kidney," and "diet, protein- } \\
\text { restricted" }\end{array}$ & 561 & 1 & 1 \\
\hline
\end{tabular}

Abbreviations: CKD, chronic kidney disease; eGFR, estimated GFR; nd, not done; RRT, renal replacement therapy.

*References were provided by Work Group members.

Systematic review provided to Work Group members.

${ }^{\ddagger}$ References were provided by Work Group members.

The strength of a recommendation indicates the extent to which one can be confident that adherence to the recommendation will do more good than harm. The quality of a body of evidence refers to the extent to which our confidence in an estimate of effect is sufficient to support a particular recommendation. ${ }^{724}$ The process of transparently grading evidence and recommendations for treatment topics is described below in further detail. However, the approach had to be adapted for the main topics of the KDIGO CKD guideline because they were not treatment-related topics.

\section{Grading the Quality of Evidence for Each Outcome Across Studies}

Following the GRADE approach, the quality of a body of evidence pertaining to a particular outcome of interest was initially categorized on the basis of study design (Table 40). For questions of interventions, the initial quality grade was high if the body of evidence consisted of RCTs, low if it consisted of observational studies, and very low if it consisted of studies of other designs. For questions of interventions, the Work Group decided to use only RCTs. The grade for the quality of evidence for each intervention-outcome pair was then lowered if there were serious limitations to the methodological quality of the aggregate of studies, if there was thought to be a high likelihood of bias, if there were

\section{Table 39 | Classification of study quality}

\begin{tabular}{ll}
\hline $\begin{array}{l}\text { Good } \\
\text { quality }\end{array}$ & $\begin{array}{l}\text { Low risk of bias and no obvious reporting errors, complete } \\
\text { reporting of data. Must be prospective. If study of } \\
\text { intervention, must be randomized controlled study. }\end{array}$ \\
$\begin{array}{l}\text { Fair } \\
\text { quality }\end{array}$ & $\begin{array}{l}\text { Moderate risk of bias, but problems with study or paper are } \\
\text { unlikely to cause major bias. If study of intervention, must be } \\
\text { prospective. }\end{array}$ \\
$\begin{array}{l}\text { Poor } \\
\text { quality risk of bias or cannot exclude possible significant biases. }\end{array}$ & $\begin{array}{l}\text { Poor methods, incomplete data, reporting errors. Prospective } \\
\text { or retrospective. }\end{array}$ \\
\hline
\end{tabular}

important inconsistencies in the results across studies, if there was uncertainty about the directness of evidence (including limited applicability of the findings to the population of interest), if the data were sparse (for example if there was only one study or if the results include just a few events or observations and were uninformative) or imprecise (for example the CI spans a range greater than 1 or confidence limits are $<0.5$ to $>2.0$ ). The final grade for the quality of the evidence for an intervention-outcome pair was then assigned as high, moderate, low, or very low (Table 40).

\section{Grading the Overall Quality of Evidence}

The quality of the overall body of evidence was then determined on the basis of the quality grades for all 
Table 40|GRADE system for grading quality of evidence

\begin{tabular}{|c|c|c|c|}
\hline $\begin{array}{l}\text { Step 1: Starting grade for } \\
\text { quality of evidence based } \\
\text { on study design }\end{array}$ & Step 2: Reduce grade & Step 3: Raise grade & Final grade for quality of evidence and definition \\
\hline \multirow[t]{3}{*}{ Randomized trials = High } & $\begin{array}{l}\text { Study quality } \\
-1 \text { level if serious limitations } \\
-2 \text { levels if very serious limitations }\end{array}$ & \multirow{5}{*}{$\begin{array}{l}\text { Strength of association } \\
+1 \text { level is strong }{ }^{\mathrm{a}} \text {, no plausible } \\
\text { confounders } \\
+2 \text { levels if very strong }{ }^{\mathrm{b}} \text {, no major } \\
\text { threats to validity } \\
\text { Other } \\
+1 \text { level if evidence of a } \\
\text { dose-response gradient } \\
+1 \text { level if all residual plausible } \\
\text { confounders would have } \\
\text { reduced the observed effect }\end{array}$} & $\begin{array}{l}\text { High = Further research is unlikely to change confidence } \\
\text { in the estimate of the effect }\end{array}$ \\
\hline & $\begin{array}{l}\text { Consistency } \\
-1 \text { level if important inconsistency }\end{array}$ & & \multirow[t]{2}{*}{$\begin{array}{l}\text { Moderate = Further research is likely to have an } \\
\text { important impact on confidence in the estimate of effect, } \\
\text { and may change the estimate }\end{array}$} \\
\hline & & & \\
\hline Udservational stuay = Low & $\begin{array}{l}\text { Directness } \\
-1 \text { level if some uncertainty } \\
-2 \text { levels if major uncertainty }\end{array}$ & & $\begin{array}{l}\text { Low }=\text { Further research is very likely to have an important } \\
\text { impact on confidence in the estimate and may change } \\
\text { the estimate }\end{array}$ \\
\hline $\begin{array}{l}\text { Any other evidence = Very } \\
\text { low }\end{array}$ & $\begin{array}{l}\text { Other: } \\
-1 \text { level if sparse or imprecise data }{ }^{c} \\
-1 \text { level if high probability of } \\
\text { reporting bias }\end{array}$ & & Very low = Any estimate of effect is very uncertain \\
\hline \multicolumn{4}{|c|}{$\begin{array}{l}\text { Abbreviation: GRADE, Grading of Recommendations Assessment, Development, and Evaluation. } \\
\text { a'Strong evidence of association is defined as 'significant relative risk of }>2(<0.5) \text { ' based on consistent evidence from two or more observational studies, with no plausible } \\
\text { confounders. } \\
\text { b Very strong evidence of association is defined as 'significant relative risk of }>5(<0.2) \text { ' based on direct evidence with no major threats to validity. } \\
\text { 'Sparse if there was only one study or if the results include just a few events or observations and were uninformative. Imprecise if the confidence interval spans a range } \\
\text { greater than } 1 \text { or confidence limits are }<0.5 \text { to }>2.0 \\
\text { Adapted by permission from Macmillan Publishers Ltd: Kidney International. Uhlig K, Macleod A, Craig J et al. }{ }^{725} \text { Grading evidence and recommendations for clinical practice } \\
\text { guidelines in nephrology. A position statement from Kidney Disease: Improving Global Outcomes (KDIGO). Kidney Int 2006; 70: } 2058-2065 \text {; accessed http://www.nature.com/ } \\
\text { ki/journal/v70/n12/pdf/5001875a.pdf }\end{array}$} \\
\hline
\end{tabular}

Table 41 | Final grade for overall quality of evidence

\begin{tabular}{lll}
\hline Grade $\begin{array}{l}\text { Quality of } \\
\text { evidence }\end{array}$ & Meaning \\
\hline A & High & $\begin{array}{l}\text { We are confident that the true effect lies close to that of } \\
\text { the estimate of the effect. }\end{array}$ \\
B & Moderate & $\begin{array}{l}\text { The true effect is likely to be close to the estimate of the } \\
\text { effect, but there is a possibility that it is substantially } \\
\text { different. }\end{array}$ \\
C & Low & $\begin{array}{l}\text { The true effect may be substantially different from the } \\
\text { estimate of the effect. } \\
\text { The estimate of effect is very uncertain, and often will be } \\
\text { far from the truth. }\end{array}$ \\
D & Very low &
\end{tabular}

outcomes of interest, taking into account explicit judgments about the relative importance of each outcome. The resulting four final categories for the quality of overall evidence were A, B, C, and D (Table 41).

\section{Assessment of the Net Health Benefit Across All Important Clinical Outcomes}

The net health benefit was determined on the basis of the anticipated balance of benefits and harms across all clinically important outcomes (Table 42). The assessment of net benefit also involved the judgment of the Work Group and the ERT.

\section{Grading the Strength of the Recommendations}

The strength of a recommendation is graded as level 1 or level 2. Table 43 shows the KDIGO nomenclature for grading the strength of a recommendation and the implications of each level for patients, clinicians, and policy makers. Recommendations can be for or against doing something. Table 44 shows that the strength of a recommendation is determined

\section{Table 42 | Balance of benefits and harm}

When there was evidence to determine the balance of medical benefits and harm of an intervention to a patient, conclusions were categorized as follows:

- For statistically significant benefit/harm, report as 'benefit [or harm] of drug $X^{\prime}$.

- For non-statistically significant benefit/harm, report as 'possible benefit [or harm] of drug $X^{\prime}$.

- In instances where studies are inconsistent, report as 'possible benefit [or harm] of drug $X^{\prime}$.

- 'No difference' can only be reported if a study is not imprecise.

- 'Insufficient evidence' is reported if imprecision is a factor.

not only by the quality of the evidence, but also by other, often complex judgments regarding the size of the net medical benefit, values and preferences, and costs. Formal decision analyses including cost analysis were not conducted.

\section{Ungraded Statements}

This category was designed to allow the Work Group to issue general advice. Typically an ungraded statement meets the following criteria: it provides guidance that is based on common sense, it provides reminders of the obvious, and it is not sufficiently specific to allow for application of evidence to the issue and therefore it is not based on systematic evidence review. The GRADE system is best suited to evaluate evidence on comparative effectiveness. Some of our most important guideline topics involve diagnosis and staging within CKD, about which the Work Group chose to provide ungraded statements. These statements are indirectly supported by evidence on risk relationships and are the consensus of the Work Group. Thus, we believe that ungraded statements should not be viewed as weaker than graded recommendations. 
Table 43 | KDIGO nomenclature and description for grading recommendations

\begin{tabular}{|c|c|c|c|}
\hline \multirow[b]{2}{*}{ Grade* } & \multicolumn{3}{|c|}{ Implications } \\
\hline & Patients & Clinicians & Policy \\
\hline $\begin{array}{l}\text { Level } 1 \\
\text { 'We recommend' }\end{array}$ & $\begin{array}{l}\text { Most people in your situation would } \\
\text { want the recommended course of action } \\
\text { and only a small proportion would not. }\end{array}$ & $\begin{array}{l}\text { Most patients should receive the } \\
\text { recommended course of action. }\end{array}$ & $\begin{array}{l}\text { The recommendation can be evaluated as } \\
\text { a candidate for developing a policy or a } \\
\text { performance measure. }\end{array}$ \\
\hline $\begin{array}{l}\text { Level } 2 \\
\text { 'We suggest' }\end{array}$ & $\begin{array}{l}\text { The majority of people in your situation } \\
\text { would want the recommended course of } \\
\text { action, but many would not. }\end{array}$ & $\begin{array}{l}\text { Different choices will be appropriate for } \\
\text { different patients. Each patient needs help } \\
\text { to arrive at a management decision } \\
\text { consistent with her or his values and } \\
\text { preferences. }\end{array}$ & $\begin{array}{l}\text { The recommendation is likely to require } \\
\text { substantial debate and involvement of } \\
\text { stakeholders before policy can be } \\
\text { determined. }\end{array}$ \\
\hline
\end{tabular}

Abbreviation: KDIGO, Kidney Disease: Improving Global Outcomes.

*The additional category "Not Graded" was used, typically, to provide guidance based on common sense or where the topic does not allow adequate application of evidence. The most common examples include recommendations regarding monitoring intervals, counseling, and referral to other clinical specialists. The ungraded recommendations are generally written as declarative statements, but are not meant to be interpreted as being stronger recommendations than Level 1 or 2 recommendations.

\section{Table 44 | Determinants of strength of recommendation}

\begin{tabular}{|c|c|}
\hline Factor & Comment \\
\hline $\begin{array}{l}\text { Balance between desirable and } \\
\text { undesirable effects }\end{array}$ & $\begin{array}{l}\text { The larger the difference between the desirable and undesirable effects, the more likely a strong recommendation } \\
\text { is warranted. The narrower the gradient, the more likely a weak recommendation is warranted. }\end{array}$ \\
\hline Quality of the evidence & The higher the quality of evidence, the more likely a strong recommendation is warranted. \\
\hline Values and preferences & $\begin{array}{l}\text { The more variability in values and preferences, or more uncertainty in values and preferences, the more likely a } \\
\text { weak recommendation is warranted. }\end{array}$ \\
\hline Costs (resource allocation) & $\begin{array}{l}\text { The higher the costs of an intervention-that is, the more resources consumed-the less likely a strong } \\
\text { recommendation is warranted. }\end{array}$ \\
\hline
\end{tabular}

Reproduced from Going from evidence to recommendations. Guyatt GH, Oxman AD, Kunz R, et al. ${ }^{724}$ BMJ 336: 1049-1051, 2008 with permission from BMJ Publishing Group Ltd.; accessed http://www.ncbi.nlm.nih.gov/pmc/articles/PMC2376019/pdf/bmj-336-7652-analysis-01049.pdf

\section{Table 45 | The Conference on Guideline Standardization ${ }^{727}$ checklist for reporting clinical practice guidelines}

Topic Description Discussed in KDIGO CKD Guideline

$\begin{array}{ll}\text { 1. Overview material } & \text { Provide a structured abstract that includes the } \\ \text { guideline's release date, status (original, revised, }\end{array}$

2. Focus
Describe the primary disease/condition and
intervention/service/technology that the
guideline addresses. Indicate any alternative
preventative, diagnostic, or therapeutic
interventions that were considered during
development.
Describe the goal that following the guideline is
expected to achieve, including the rationale for
development of a guideline on this topic.

4. User/setting

Describe the intended users of the guideline (e.g., provider types, patients) and the settings in which the guideline is intended to be used.

5. Target population Describe the patient population eligible for guideline recommendations and list any exclusion criteria.

6. Developer
$\begin{aligned} & \text { Identify the organization(s) responsible for } \\ & \text { guideline development and the names/ } \\ & \text { credentials/potential conflicts of interest of } \\ & \text { individuals involved in the guideline's } \\ & \text { development. }\end{aligned}$
$\begin{aligned} & \text { Identify the funding source/sponsor and describe } \\ & \text { its role in developing and/or reporting the } \\ & \text { source/sponsor }\end{aligned}$
guideline. Disclose potential conflict of interest.

Evaluation and management of adults and children with CKD.

This clinical practice guideline is intended to assist the practitioner caring for patients with CKD and to prevent deaths, cardiovascular disease events and progression to kidney failure while optimizing patients' quality of life.

Providers: Nephrologists (adult and pediatric), Dialysis providers (including nurses), Internists, and Pediatricians.

Patients: Adult and pediatric individuals at risk for or with CKD. Policy Makers: Those in related health fields. Individuals at risk for or with CKD.

Organization: KDIGO.

Refer to Biographic and Disclosure Information section.

KDIGO is supported by the following consortium of sponsors: Abbott, Amgen, Bayer Schering Pharma, Belo Foundation, BristolMyers Squibb, Chugai Pharmaceutical, Coca-Cola Company, Dole Food Company, Fresenius Medical Care, Genzyme, HoffmannLaRoche, JC Penney, Kyowa Hakko Kirin, NATCO-The Organization for Transplant Professionals, NKF-Board of Directors, Novartis, Pharmacosmos, PUMC Pharmaceutical, Robert and Jane Cizik 


\section{Table 45|Continued}

\begin{tabular}{lll}
\hline Topic & Description & Discussed in KDIGO CKD Guideline
\end{tabular}

Foundation, Shire, Takeda Pharmaceutical, Transwestern Commercial Services, Vifor Pharma, and Wyeth.

No funding is accepted for the development or reporting of specific guidelines.

All stakeholders could participate in open review.

8. Evidence collection Describe the methods used to search the scientific literature, including the range of dates and databases searched, and criteria applied to filter the retrieved evidence.

9. Recommendation grading criteria

Describe the criteria used to rate the quality of evidence that supports the recommendations and the system for describing the strength of the recommendations. Recommendation strength communicates the importance of adherence to a recommendation and is based on both the quality of the evidence and the magnitude of anticipated benefits and harms.

10. Method for synthesizing evidence Describe how evidence was used to create recommendations, e.g., evidence tables, metaanalysis, decision analysis.

11. Prerelease review

12. Update plan

State whether or not there is a plan to update the guideline and, if applicable, expiration date for this version of the guideline.

Screening criteria are outlined in the methods chapter. The search was updated through June 2011 and supplemented by articles identified by Work Group members through November 2012. We also searched for pertinent existing guidelines and systematic reviews.

Quality of individual studies was graded in a three-tiered grading system (see Table 39). Quality of evidence (Table 40) was graded following the GRADE approach. Strength of the recommendation was graded in a two-level grading system which was adapted from GRADE for KDIGO with the quality of overall evidence graded on a four-tiered system (Tables 41 and 43).

The Work Group could provide general guidance in ungraded statements.

For systematic review topics, summary tables and evidence profiles were generated.

For recommendations on treatment interventions, the steps outlined by GRADE were followed.

The guideline had undergone internal review by the KDIGO Board of Directors in January 2012 and external review in May 2012. Public review comments were compiled and fed back to the Work Group, which considered comments in its revision of the guideline.

There is no date set for updating this entire guideline. The need for updating of the guideline will depend on the publication of new evidence that would change the quality of the evidence or the estimates for the benefits and harms. Results from registered ongoing studies and other publications will be reviewed periodically to evaluate their potential to impact on the recommendations in this guideline. Specific sections may be updated separately from the entire guideline within the next 3-5 years depending on the evidence base.

Acronyms and Abbreviations.

\section{Definitions}

14. Recommendations and rationale

15. Potential benefits and harm

16. Patient preferences

17. Algorithm

18. Implementation considerations
Define unfamiliar terms and those critical to correct application of the guideline that might be subject to misinterpretation.

State the recommended action precisely and the specific circumstances under which to perform it. Justify each recommendation by describing the linkage between the recommendation and its supporting evidence. Indicate the quality of evidence and the recommendation strength, based on the criteria described in Topic 9. Describe anticipated benefits and potential risks associated with implementation of guideline recommendations.

Describe the role of patient preferences when a recommendation involves a substantial element of personal choice or values.

Provide (when appropriate) a graphical description of the stages and decisions in clinical care described by the guideline.

Describe anticipated barriers to application of the recommendations. Provide reference to any auxiliary documents for providers or patients that are intended to facilitate implementation. Suggest review criteria for measuring changes in care when the guideline is implemented.
Each guideline chapter contains recommendations for evaluation and management of CKD patients. Each recommendation builds on a supporting rationale with evidence tables if available. The strength of the recommendation and the quality of evidence are provided in parenthesis within each recommendation.

The benefits and harm for each comparison of interventions is provided in summary tables and summarized in evidence profiles. The estimated balance between potential benefits and harm was considered when formulating the recommendations. Many recommendations are ungraded which indicates a greater need to help each patient arrive at a management decision consistent with her or his values and preferences. Algorithm for proteinuria/albuminuria testing in Chapter 1.

These recommendations are global and the Work Group acknowledges the importance of local application. Review criteria were not suggested because implementation with prioritization and development of review criteria must proceed locally. Furthermore, most recommendations are discretionary, requiring substantial discussion among stakeholders before they can be considered for adoptions as review criteria.

Suggestions were provided for future research.

Abbreviations: CKD, chronic kidney disease; COGS, Conference on Guideline Standardization; GRADE, Grading of Recommendations Assessment, Development, and Evaluation; KDIGO, Kidney Disease: Improving Global Outcomes; NKF, National Kidney Foundation.

Adapted with permission from Shiffman RN, Shekelle P, Overhage JM, et al ${ }^{727}$ Standardized reporting of clinical practice guidelines: a proposal from the Conference on Guideline Standardization. Ann Intern Med 2003; 139(6): 493-498; accessed http://annals.org/data/Journals/AIM/20049/0000605-200309160-00013.pdf 
The Work Group took on the primary role of writing the recommendations and rationale statements and retained final responsibility for the content of the guideline statements and the accompanying narrative. The ERT reviewed draft recommendations and grades for consistency with the conclusions of the evidence review.

\section{Format for Guideline Recommendations}

Each chapter contains one or more specific recommendations. Within each recommendation, the strength of recommendation is indicated as level 1 or level 2 and the quality of the supporting evidence is shown as A, B, C or D. The recommendation statements and grades are followed by the rationale and clarification of the wording of the statement, a brief background with relevant definitions of terms, and then a chain of logic which summarizes the key points of the evidence base and the judgments supporting the recommendation. Some sections also contain research recommendations in variable degrees of detail, suggesting future research to resolve current uncertainties.

\section{Limitations of Approach}

Although the literature searches were intended to be comprehensive, they were not exhaustive. MEDLINE was the only database searched. Hand searches of journals were not performed, and review articles and textbook chapters were not systematically searched. However, important studies known to domain experts that were missed by the electronic literature searches were added to the retrieved articles and reviewed by the Work Group.

\section{Summary of the Review Process}

Several tools and checklists have been developed to assess the quality of the methodological process for systematic review and guideline development. These include the Appraisal of Guidelines for Research and Evaluation (AGREE) criteria, ${ }^{726}$ the Conference on Guideline Standardization (COGS) checklist, ${ }^{727}$ and the Institute of Medicine's recent Standards for Systematic Reviews ${ }^{728}$ and Clinical Practice Guidelines We Can Trust. ${ }^{729}$ Table 45 and Appendix 1 demonstrate the level of concurrence to the COGS criteria and the Institute of Medicine standards, respectively.

\section{SUPPLEMENTARY MATERIAL}

Supplemental Table 1: Search strategy.

Appendix 1: Concurrence with Institute of Medicine standards for systematic reviews and for guidelines.

Supplementary material is linked to the online version of the paper at http://www.kdigo.org/clinical_practice_guidelines/ckd.php 


\section{Biographic and Disclosure Information}

Kidney International Supplements (2013) 3, 128-133; doi:10.1038/kisup.2012.70

Adeera Levin, MD, FRCPC (Work Group Co-Chair), is Professor of Medicine, Division Head of Nephrology at the University of British Columbia, and Consultant Nephrologist at Providence Health Care/St Paul's Hospital, in Vancouver Canada.

Dr Levin is the Executive Director of the BC Renal Agency, which oversees the care, planning, and budgets for kidney services in the province of British Columbia. In this capacity, she has leveraged her epidemiological training, clinical knowledge, and health outcomes research to develop an evidence-based transparent system which enhances the care of patients across the continuum of care (from identification of CKD to dialysis, transplant, and death).

She is active in international activities across the spectrum of kidney activities including her appointment as Secretary General of the International Society of Nephrology (ISN), founding executive committee member of KDIGO, and as coChair of the Declaration of Istanbul Custodian group (DICG). Dr Levin has a long-standing clinical and research interest in CKD and CVD, and is Founding Director of the Kidney Function Clinic at St Paul's Hospital.

As an active researcher and mentor, she is involved in a national curriculum development for clinician scientists interested in kidney disease (KRESCENT), and mentors students, residents, and nephrology fellows in Vancouver as well. She has over 210 peer-reviewed publications, numerous book chapters, and is co-editor of a textbook Chronic Kidney Disease: A Practical Guide to Understanding and Management. Her major research areas of interest include non-traditional risk factors for CVD in CKD patients, with particular focus on anemia, phosphate and vitamin $\mathrm{D}$, and progression of CKD variability.

\section{Advisor/Consultant: Merck; Otsuka}

Grant/Research Support: Abbott; Amgen; Canadian Institutes of Health Research; Genzyme, Kidney Foundation of Canada; Merck; Ortho

Paul E Stevens, MB, FRCP (Work Group Co-Chair), is Consultant Nephrologist and Associate Medical Director, East Kent Hospitals University NHS Foundation Trust, Kent and Canterbury Hospital in the UK. He is a member of the Department of Health's Renal Advisory Group and Renal Pathology Group and immediate past-President of the British Renal Society. Dr Stevens established the Kent Kidney Care Centre, one of the largest general hospital renal units in the UK and served as a clinical advisor on NICE publications Anaemia Management in People with Chronic Kidney Disease and Chronic Kidney Disease: National Clinical Guideline for Early Identification and Management in Adults in Primary and Secondary Care. He is also Chair of NICE Quality Standards in Chronic Kidney Disease.

His main interests include the epidemiology of CKD and AKI, prospective evaluation of a new method of assessing adequacy in peritoneal dialysis, evaluation of anemia in unreferred patients with CKD and in patients with diabetes, collaborative research with the elderly CKD care team, and the development of information technology-based solutions in the referral and follow-up of patients with kidney disease. Over the last several years, his program has also developed a clinical decision support system to aid management of CKD in primary care and this system is currently running live in a population of over 300,000 primary care patients. Dr Stevens has authored over 90 publications in peerreviewed journals and textbooks, and has given over 100 presentations and 65 guest lectures in national and international society meetings.

\section{Dr Stevens reported no relevant financial relationships}

Rudy W Bilous, MD, is Professor of Clinical Medicine at Newcastle University and Honorary Consultant Physician at the James Cook University Hospital, Middlesbrough, UK. He studied medicine at Guy's Hospital and completed his clinical training at various hospitals in London. He was an National Institutes of Health (NIH) Fellow at the Unit for Metabolic Medicine at Guy's from 1980-1982 working for Professor Harry Keen and with Drs John Pickup and Giancarlo Viberti examining the effects of metabolic control on microvascular complications. From 1985-1987 he was Juvenile Diabetes Research Foundation fellow at the University of Minnesota working for Drs Mike Steffes and Mike Mauer. He was awarded his MD from the University of London in 1987 and elected Fellow of the Royal College of Physicians of London in 1991. He served as Secretary and Chair of the Specialty Advisory Committee for Endocrinology and Diabetes and Chairman of the Professional section of Diabetes UK. He is currently Chairman of the workgroup reviewing the NICE guideline for Diabetes in Pregnancy and President of the European Diabetic Nephropathy Study Group of the European Association for the Study of Diabetes.

Advisor/Consultant: Abbott; Boehringer Ingelheim; Novartis; Roche

Speaker: Boehringer Ingelheim; Novo Nordisk; Roche 
Josef Coresh, MD, PhD, MHS, is Professor, Department of Epidemiology, Medicine, and Biostatistics at Johns Hopkins Bloomberg School of Public Health and School of Medicine where he also received his medical degree and $\mathrm{PhD}$ in epidemiology, and a master's in biostatistics. $\mathrm{He}$ is also presently the Director of the George W Comstock Center for Public Health Research and Prevention, and the Cardiovascular Epidemiology Program and Training Grant at Johns Hopkins Bloomberg School of Public Health.

Dr Coresh's primary research interest has been the investigation of risk factors for CVD in CKD and their role in mediating the excess risk among patients with various degrees of CKD severity. With the use of innovative epidemiologic methods and statistical analyses, his recent work uncovered several novel genetic susceptibility loci for gout as well as a susceptibility locus (MYH9/APOL1) which accounts for much of the excess risk of non-diabetic kidney disease among African-Americans. Since 2000, he has focused his attention on enhancing CKD classification, management and prognostication by improving the estimation of kidney function. In 2002, he served as Co-Chair on the KDOQI Clinical Practice Guidelines for CKD which produced the CKD definition and classification system that have been adopted widely today. More recently, his research led to the development of the 2009 CKD-EPI equation for estimating kidney function which is rapidly become the new standard for GFR estimation globally. In 2010, he became Steering Committee Chair of Chronic Kidney Disease Prognosis Consortium (CKD-PC) which analyzes data from two million study participants in order to provide better estimation on CKD prognosis. Dr Coresh has authored close to 300 publications and has mentored over 60 students. In recognition of his contributions, he was awarded the NKF's Garabed Eknoyan Award (2010), American Heart Association's 2010 Epidemiology and Prevention Mentoring Award and served as plenary speaker at the American Association for Clinical Chemistry 2011 Annual Meeting.

\section{Advisor/Consultant: Merck}

Grant/Research Support: Amgen; National Institutes of Health; National Kidney Foundation

Speaker: Abbott; Amgen

Angel LM de Francisco, MD, PhD, is Head of Dialysis Unit and Nephrology Section, Hospital Universitario Valdecilla, Santander (Spain), and Full Professor of Nephrology at Faculty of Medicine in Cantabria University. He completed his medical residency and nephrology fellowship at Hospital Puerta de Hierro in Madrid, and received his Doctor of Medicine degree from University of Cantabria (Spain). Prof de Francisco is an active member of several professional societies, an Executive member of the KDIGO Board, and was President of the Spanish Society of Nephrology from 2002 to 2008 . He has been a frequently invited lecturer, speaking extensively on advancements in treatment of chronic kidney diseases and comorbid conditions such as metabolic bone disease, anemia, dialysis, and kidney transplantation. Prof de Francisco has authored or coauthored over 150 publications, including original peerreviewed articles and book chapters. His primary focus has been on improving outcomes in patients receiving hemodialysis, and on issues surrounding organ transplantation and the impact of treatment guidelines on clinical practice.

\section{Advisor/Consultant: Amgen \\ Speaker: Abbott; Fresenius}

Paul E de Jong, MD, PhD, was Professor of Nephrology and Head, Division of Nephrology of the Groningen University Medical Center in Groningen, the Netherlands from 1992 to 2011. His main research interest is prevention of CKD. From 1980 to 2000, he focused most of his attention on the mechanisms of urinary protein loss in CKD patients and options to lower proteinuria with ACE-I. His studies had indeed shown that such treatment prevented ESRD progression. In 1996, he initiated the PREVEND study which consisted of a large cohort of 8592 subjects followed for the development of kidney disease and CVD. This prospective longitudinal study demonstrated the impact of albuminuria, even in the ranges of microalbuminuria and below, in predicting future cardiovascular and renal events. As former Director of the Groningen Institute of Kidney Diseases, his research program encompasses the studying of underlying susceptibility to kidney damage in health subjects and the identification of common pathophysiological pathways between CKD and CVD. Currently, he serves on the board of KDIGO, CKD-PC, and European Kidney Health Alliance. In 2010, he was the recipient of International Distinguished Medal from the US National Kidney Foundation and was awarded the Officer in the Order of Orange-Nassau for his lifetime achievement by Her Majesty Queen Beatrix of the Netherlands in 2011.

\section{Dr de Jong reported no relevant financial relationships}

Kathryn E Griffith, BM, BS, MSc, MRCP, MRCGP, is a general practitioner affiliated with The University Health Centre, York University and York Hospital Foundation Trust. Dr Griffith has a long-standing interest in the relationship between CVD and renal disease from a primary care physician's perspective. In this vein, she has served as a panel member for the Royal College of Physicians (RCP) Edinburgh Consensus Conference on CKD and was involved in the development of the 18-week Commissioning Pathway to CKD with the Department of Health. She is presently a member of the Renal Advisory Group at the Department of Health and a member of both UK Renal Association and British Renal Society. Dr Griffith has also been involved in the education of general practitioners and practice nurses, and most recently she has lectured extensively on various topics such as atrial fibrillation, heart failure, coronary heart 
disease, hypertension, and stroke. For the past two years, she has worked with Yorkshire Cardiac and Stroke Networks on a guide to stroke prevention in atrial fibrillation, and contributed to the RCP Edinburgh Consensus on atrial fibrillation management and European Society of Cardiology's clinical practice guideline on management of dyslipidemias.

\section{Advisor/Consultant: Boehringer Ingelheim}

Honoraria: Pfizer

Travel Stipend: Merck Sharp \& Dohme

Grant/Research Support: Roche

Speaker: Boehringer Ingelheim

Brenda R Hemmelgarn, $\mathrm{MD}, \operatorname{PhD}, \operatorname{FRCP}(\mathbf{C})$, is Associate Professor in the Departments of Medicine and Community Health Sciences, University of Calgary, Calgary, Alberta. She is a clinician scientist with clinical training in nephrology as well as a PhD in Epidemiology and Biostatistics. Dr Hemmelgarn's research interests are in the study of chronic medical conditions including CKD, diabetes, hypertension and CVD, as well as health services research aimed at improving management of these chronic conditions. Dr Hemmelgarn holds provincial and national salary awards and operating grants, and is the recipient of the Roy and Vi Baay Chair in Kidney Research. She is also actively involved in Clinical Practice Guideline development both nationally through the Canadian Society of Nephrology and internationally through KDIGO, and has over 180 peer-reviewed publications.

\section{Grant/Research Support: Roche}

Kunitoshi Iseki, MD, is currently affiliated with University Hospital of the Ryukyus in Okinawa, Japan. Dr Iseki received his medical training at Kyushu University in Fukuoka, Japan and completed his fellowship at University of Southern California under Professors Shaul Massry and Vito Campese. He was Senior Assistant Professor of Medicine at Ryukyu University Hospital and later became its Director of Dialysis Unit. Dr Iseki is a member of numerous professional organizations including the Japanese Society of Nephrology and Japanese Society for Dialysis Therapy. He is also an organizing member for Asian Forum of Chronic Kidney Disease Initiatives (AFCKDI) since 2007 and an ISN-COMGAN Educational Ambassador since 2009. Dr. Iseki has also participated and contributed data to the CKD-PC whose collaborative meta-analyses were conducted to study CKD prognosis. He has previously served as Editorial board member for American Journal Kidney Disease and is presently an editorial board member of Nephrology, Nephron Clinical Practice and Nephrology Dialysis Transplantation.
Edmund J Lamb, PhD, FRCPath, is a Consultant Clinical Scientist and Head of Clinical Biochemistry at East Kent Hospitals University NHS Foundation Trust, Canterbury, Kent, UK. He has a special interest in kidney disease and undertook his $\mathrm{PhD}$ in the Renal Research Laboratory at St Bartholomew's Hospital, London. He has 30 years experience as a clinical biochemist and his research interests relate to the use of biochemical markers to diagnose and monitor kidney disease, including the assessment of kidney function using eGFR, the evaluation of renal bone disease, the use and role of cystatin $\mathrm{C}$, and the significance and use of cardiac biomarkers in kidney disease. Dr Lamb has authored or co-authored more than 60 full peer-reviewed papers in this area. He is a member of the UK Kidney Research Consortium Clinical Study Group on CKD-MBD and he is the lead investigator on two National Institute for Health Research-Research for Patient Benefit funded studies investigating aspects of kidney function testing. He has been involved in national and international committees and guidelines (e.g., Royal College of Physicians UK CKD Guidelines Group, NICE CKD Guideline Development Group, KDIGO Chronic Kidney Disease Guideline Work Group) and was recently involved in the Department of Health initiative to roll-out eGFR across England. He is Editor-in-Chief of Annals of Clinical Biochemistry.

\section{Grant/Research Support: Siemens}

Andrew S Levey, MD, is Dr Gerald J and Dorothy R Friedman Professor of Medicine at Tufts University School of Medicine, Chief of the William B Schwartz, MD Division of Nephrology at Tufts Medical Center, Senior Scientist at the US Department of Agriculture Human Nutrition Research Center on Aging at Tufts University, and Professor, Clinical Research at the Sackler School of Graduate Biomedical Sciences at Tufts University. His research is mainly in the areas of epidemiology of CKD and CVD in CKD, controlled trials to slow the progression of CKD, clinical assessment of kidney function, assessment and improvement of outcomes in dialysis and transplantation, and clinical practice guideline development and implementation. Dr Levey is currently Program Director for an NIDDK-funded clinical research training program, "Clinical Trials, Epidemiology and Outcomes Research in Nephrology." He is also the Director of the Tufts Center for Guideline Development and Implementation and Editor of American Journal of Kidney Disease. Dr Levey is past Chair of the NKF's Task Force on Cardiovascular Disease in Chronic Renal Disease and served on KDOQI Work Group on Chronic Kidney Disease: Evaluation, Classification and Stratification, KDOQI Work Group on Hypertension and Antihypertensive Agents in Chronic Kidney Disease, and KDIGO Work Group on Acute Kidney Injury Guideline.

Dr Levey reported no relevant financial relationships 
Miguel C Riella, MD, PhD, FACP, is a graduate of Federal University of Parana in Curitiba, Brazil and has undertaken his post-graduate training in the United States, first in Internal Medicine Residency at Mount Sinai Hospital in New York City and then Research Renal Fellowship at the University of Washington in Seattle, USA under the leadership of Prof Belding $\mathrm{H}$ Scribner, pioneer of chronic hemodialysis. Dr Riella's main interest has been on dialysis, particularly peritoneal dialysis and nutrition in uremia. More recently his research work has concentrated on the relationship of inflammation, uremia, nutrition and cardiovascular complications, and interventional nephrology. As part of his new Renal, Diabetes and Hypertension Research Center, he has recently established the Islet Cell Laboratory at Catholic University of Parana in Curitiba, Brazil for cell transplant in diabetic patients. Dr Riella has published several books in Portuguese and Spanish language in the area of nephrology and nutrition. His book Principles of Nephrology and Electrolyte Disturbances (Guanabara Koogan Group Gen, 2010) is on its $5^{\text {th }}$ Edition. He has more than 150 publications and has given more than 600 lectures locally and internationally. He is a past Board member and executive committee member of the ISN and is currently the Chairman of the ISN-Interventional Nephrology Committee. Dr Riella is a member of the editorial board of several medical journals, including as Associate Editor of Hemodialysis International and International Editor of Seminar in Dialysis for Interventional Nephrology. He is also President and founder of Pro-Renal Foundation of Brazil (www.pro-renal.org.br), a non-for-profit organization dedicated to patient assistance, CKD prevention and education, and supporting research in kidney and metabolic diseases. The Foundation provides free medical care to more than 1800 renal patients through a multidisciplinary team of physicians, nurses, psychologists, social workers, and dietitians. The Foundation is maintained by donations of the community and receives no public funds. Dr Riella is the past-President of the International Federation of Kidney Foundations (IFKF) 2009-2011. $\mathrm{He}$ is also the co-chairman of World Kidney Day, a joint project of IFKF and and ISN. He is a member of the Executive Committee of KDIGO, National Academy of Medicine in Brazil, and the Academy of Medicine of Parana, Brazil. He has received many awards such as the International Distinguished Medal from the US NKF and Honorary Citizenship of the city of Curitiba and of the State of Parana in Brazil.

\section{Advisor/Consultant: Abbott}

Michael G Shlipak, MD, MPH, is Professor of Medicine, Epidemiology, and Biostatistics at University of California, San Francisco. Dr Shlipak obtained his MD degree from Harvard Medical School and his MPH from Harvard School of Public Health. His research program has been dedicated to understanding the causes and consequences of age-related kidney disease and to utilize novel biomarkers for the early detection of kidney damage and reduced kidney function. Along with his colleagues from the Cardiovascular Health
Study and Health Aging and Body Composition study, Professor Shlipak has conducted the pioneering work to demonstrate with cystatin $\mathrm{C}$ the remarkable prognostic importance of impaired kidney function in elders. He coined the entity of "preclinical kidney disease" to describe the large population of elders with elevated cystatin C levels, but normal creatinine-based estimates of kidney function, who are at high risk for death and CVD.

The recent work from his research group has been dedicated to finding early markers of kidney injury in the urine that will precede any detectable loss in kidney function. They will study these urine biomarkers in several populations, including the ambulatory elderly, HIV-infected men and women, and in population-based studies. The group believes that these injury markers hold promise both for understanding how various exposures impact the kidney, and also to forecast future declines in kidney failure.

\section{Dr Shlipak reported no relevant financial relationships}

Haiyan Wang, MD, is Professor of Medicine at Peking University First Hospital. She is the current President of Peking University Institute of Nephrology and the Vice Director, Academic Committee, Peking University Health Science Center and Member, Academic Degree Committee, Peking University Health Science Center.

Professor Wang is very active in the academic area of national and international nephrology and internal medicine. She was the Vice President of Chinese Medical Association (1999-2009) and the President of Chinese Society of Internal Medicine (2000-2008), as well as the President of Chinese Society of Nephrology (1992-1998). She has also served as the ISN Councilor member (2004-2008), Executive Councilor of ISN (2006-2008) and is now Chair, East Asia committee of ISN GO (2004-present).

\section{Dr Wang reported no relevant financial relationships}

Colin T White, MD, FRCPC, completed his medical degree at the University of Ottawa, Canada and Pediatric Residency at the University of Western Ontario, Canada. His pediatric nephrology training took place at the University of Western Ontario and University of British Columbia. He is a Clinical Associate Professor in Pediatrics/Nephrology at the University of British Columbia and has a clinical appointment at BC Children's Hospital where in addition to an interest and participation in the CKD clinic, he is the Director of the Dialysis Program and is the Fellowship Program Director. Dr White was a work group member for the 2006 and 2007 Updated KDOQI Anemia Guidelines, and for the Canadian Society of Nephrology's Clinical Practice Guidelines for Anemia Management in 2007-2008; he is currently on the 2011-2012 CSN Workgroup revisiting these guidelines. He is the site Principal Investigator for the North American multicenter NIH-sponsored CKiDs trial and has particular research interests in hemodialysis and peritoneal dialysis as well as CKD management. His particular 
areas of research interest include estimation of clearance, anemia protocol development, and translation of evidence into functional guidelines. He is active at the undergraduate medical school level and has coordinated the UBC Renal and Genitourinary Course for 1st year medical students for nearly a decade. He is currently the President of the Western Society of Pediatric Research and an invited member of the Renal Work Group for the World Health Organization's ICD-11 Code Revision.

\section{Advisor/Consultant: Baxter Healthcare}

Christopher G Winearls, MB, DPhil, FRCP, graduated in 1973 from University of Cape Town and as a Rhodes Scholar completed a $\mathrm{PhD}$ from University of Oxford in transplant immunology. Dr Winearls later pursued training in nephrology at the Hammersmith Hospital where he was part of the team that investigated the first clinical use of erythropoietin. Dr Winearls was appointed consultant in Oxford in 1988 and was Clinical Director 1995 to 2009. He has been Secretary and later Clinical Vice President of The Renal Association and an Editor of the Oxford Textbook of Clinical Nephrology. His research interests include myeloma kidney and autosomal dominant polycystic kidney disease, and has recently taken an interest in the new classification of CKD.

\section{Dr Winearls reported no relevant financial relationships}

\section{KDIGO CHAIRS}

Bertram L Kasiske, MD, is Professor of Medicine at the University of Minnesota, USA. He received his medical degree from the University of Iowa and completed his Internal Medicine residency and fellowship training in Nephrology at Hennepin County Medical Center where he is currently Director of Nephrology.

Dr Kasiske is former Deputy Director of the United States Renal Data System and former Editor-in-Chief of American Journal of Kidney Diseases. He has served as Secretary/ Treasurer and on the Board of Directors of the American Society of Transplantation, and on the Organ Procurement and Transplantation Network/United Network of Organ Sharing Board of Directors, and the Scientific Advisory Board of the US NKF. He is currently serving on the Board of Councilors of the ISN and he is the Principal Investigator for a National Institutes of Health-sponsored, multicenter study of long term outcomes after kidney donation. Dr Kasiske is the Director of the Scientific Registry of Transplant Recipients and has over 160 scientific publications in major peer-reviewed journals, and 230 review articles, editorials and textbook chapters. Dr Kasiske is also a recipient of the US NKF's Garabed Eknoyan Award in 2003.

\section{Advisor/Consultant: Litholink}

Grant/Research Support: Bristol-Myers Squibb; Merck (Schering Plough)
David C Wheeler, MD, FRCP, holds an academic position in Nephrology (Reader) at University College London, UK and is an Honorary Consultant Nephrologist at the Royal Free Hospital. His research is focused on the cardiovascular complications of CKD and the role of vascular risk factors in progression of kidney damage. Dr Wheeler is a member of the International Steering Committee of the Study of Heart and Renal Protection (SHARP) and was UK National Coordinator for the trial. He is involved in several other randomized trials and observational studies involving patients with CKD.

Dr Wheeler is Co-Chair of KDIGO, having served previously on its Executive Committee and Board. He received an International Distinguished Medal from the US NKF in recognition of his contribution to guideline development. In the UK, he has been elected President of the Renal Association for the term 2012-2014.

Dr Wheeler has served on the editorial boards of American Journal of Kidney Diseases and Journal of the American Society of Nephrology and is presently Co-Editor for Nephrology Dialysis Transplantation.

\section{Advisor/Consultant: Amgen}

Honoraria: Abbott; Amgen; Fresenius; Otsuka; Shire

Travel Stipend: Amgen; Merck Sharp \& Dohme; Shire

Grant/Research Support: Abbott; Genzyme

\section{EVIDENCE REVIEW TEAM}

Katrin Uhlig, MD, MS, is the Director, Guideline Development at the Tufts Center for Kidney Disease Guideline Development and Implementation, Boston, MA, USA, Associate Professor of Medicine at Tufts University School of Medicine, and a staff nephrologist at Tufts Medical Center. Dr Uhlig completed her training in internal medicine, nephrology, and rheumatology in Germany (Aachen University Hospital and Munich University Hospital) and the USA (Georgetown University Medical Center and Tufts Medical Center). Since 2001, she has been participating in or directing the evidence review for KDOQI and KDIGO guidelines. As Director of Guideline Development, Dr Uhlig has a substantial role in coordinating the ERT. She orchestrates and supervises the guideline development process and didactic curriculum that provides Work Group members with formal instruction on topics related to guideline development. As project director on individual guidelines, she directs and supervises the collection, evaluation, grading, and synthesis of evidence and the drafting and revisions of the final evidence report. She provides methodological guidance and training to Work Group members at meetings regarding topic refinement, key question formulation, data extraction, study assessment, evidence grading, and recommendation formulation. She provides nephrology expertise in the interpretation and review of guideline recommendations and evidence reports. In this capacity, 
Dr Uhlig possesses unique knowledge as a methods expert in evidence synthesis and critical literature appraisal in the domain of nephrology.

In 2005, she co-chaired the KDIGO Evidence Rating Group to develop a consensus on grading of KDIGO guidelines and also co-chaired the KDIGO Consensus Conference on Guideline Methodology in October 2007. From 2006 to 2007, she served as Co-Editor of American Journal of Kidney Diseases. Her teaching and research focus includes evidence-based medicine, systematic review, clinical practice guideline development, and critical literature appraisal. Dr Uhlig lectures on guideline topics and is a co-instructor of an annual course on meta-analysis in the Master of Science Program at the Sackler School of Graduate Biomedical Sciences at Tufts University.

\section{Dr Uhlig reported no relevant financial relationships}

Dana C Miskulin, MD, MS, is Assistant Professor of Medicine at Tufts University School of Medicine, Boston, MA, USA. She completed a fellowship in Clinical Care Research and participated in the conduct of systematic reviews and critical literature appraisals for this guideline. Her primary research interests are in comparative effectiveness research in dialysis patients, BP treatment in dialysis patients, and autosomal dominant polycystic kidney disease.

\section{Dr Miskulin reported no relevant financial relationships}

Amy Earley, BS, is a project coordinator at the Tufts Center for Kidney Disease Guideline Development and Implementation in Boston, MA, USA. She is key in coordinating the guideline development activities within the ERT, especially in the development of the evidence reports for all guidelines. Ms Earley also heads the actual evidence review, which includes running searches, screening, data extraction, drafting of tables and methods sections, proofing of guideline drafts, and critical literature appraisals. She participates in the conduct of research projects at the Center and actively collaborates with other members of the Center on independent research topics and manuscript submissions.

\section{Ms. Earley reported no relevant financial relationships}

Shana Haynes, MS, DHSc, is a research assistant at the Tufts Center for Kidney Disease Guideline Development and Implementation in Boston, MA, USA. She participates in all aspects of evidence review and guideline development. She screens abstracts and articles, extracts data, and assists in the drafting and editing of evidence tables. Dr Haynes also assists in the development of clinical practice guidelines and conducts systematic reviews and critical literature appraisals.

\section{Dr Haynes reported no relevant financial relationships}

Jenny Lamont, MS, is a project manager and medical writer at the Tufts Center for Kidney Disease Guideline Development and Implementation in Boston, MA, USA. She participates in all aspects of evidence review and guideline development, assists in the preparation of talks and manuscripts, and edits KDIGO draft guidelines currently in progress.

Ms. Lamont reported no relevant financial relationships 


\section{Acknowledgments}

Kidney International Supplements (2013) 3, 134-135; doi:10.1038/kisup.2012.71

A special debt of gratitude is owed to the KDIGO Co-Chairs Kai-Uwe Eckardt, Bertram Kasiske, David Wheeler, and the KDIGO Board for their invaluable guidance throughout the development of this guideline. In particular, we thank the ERT members: Katrin Uhlig, Dana Miskulin, Amy Earley, Shana Haynes, and Jenny Lamont for their substantial contribution to the rigorous assessment of the available evidence. We are also especially grateful to the Work Group members for their expertise throughout the entire process of literature review, data extraction, meeting participation, the critical writing and editing of the statements and rationale, which made the publication of this guideline possible. The generous gift of their time and dedication is greatly appreciated. The entire Work Group and its Co-Chairs would also like to extend a special debt of gratitude to Michael Cheung, who has committed time and energy above and beyond, to the formatting and proof reading of the completed manuscript. Without his help, we would have been hard pushed to complete this task. Finally, and on behalf of the Work Group, we gratefully acknowledge the careful assessment of the draft guideline by external reviewers. The Work Group considered all of the valuable comments made and where appropriate, suggested changes were incorporated into the final publication. The following individuals provided feedback during the public review of the draft guideline:

Mario Abbud-Filho; Hugo Abensur; Patricia Abreu; Matias Abuchanab; Nasrulla Abutaleb; Anil K Agarwal; Ahmed Akl; Mona Al-Rukhaimi; Fernando A Almeida; Altun Bulent; Maria Almerinda Ribeiro Alves; Pablo Amair; Richard Amerling; Alessandro Amore; Ryoichi Ando; Rajeev Annigeri; Ramadan Arafa; Mustafa Arici; Mariano Arriola; Ferruh Artunc; Suheir Assady; Marcelo Rodrigues Bacci; Ashraf AM Bakr; Breda Peèovnik Balon; Rashad S Barsoum; Don Batisky; Dawlat Belal; Mohammed Benyahia; Murray Berall; Anatole Besarab; Premila Bhat; Mar Biechy; Patrick Biggar; Brenda Brewer; Inga Arune Bumblyte; Jian-Fang Cai; Rafael Burgos Calderon; Bernard Canaud; Jorge B CannataAndía; Antonio Carlos Duarte Cardoso; Laurence Carroll; Sue Cary; Luis Castillo; Arlene Chabanuk; Philippe Chauveau; Jiang-Hua Chen; Kathleen Claes; Peter Clausen; Jean Colaneri; Mario Cozzolino; Andrew Crannage; Michael Cunningham; Jane S Davis; Kimberly Davis; Rodrigo Daza; Julián Segura de la Morena; Luca De Nicola; Rodrigo Bueno de Oliveira; Rogerio Baumgratz de Paula; Goffredo Del Rosso; David DeMicco; Sheila Deziel; Bruno Didier; Guillermo J Rosa Diez; Ian Dittmer; Walter Guillermo Douthat; Reham Eid Elsayed; Montserrat Mercedes Díaz
Encarnación; Tracy Evans-Walker; Joyce Ezaki-Yamaguchi; Jarraya Faical; Bonita Falkner; Sandro Feriozzi; Manuel Ferreira; Charles Ferro; Joseph T Flynn; Patricia Folk; Lui G Forni; Costas Fourtounas; Roberto Jorge da Silva Franco; Ping Fu; Naohiko Fujii; Susan L Furth; Suzanne Gagne; Sishir Gang; Colin C Geddes; Rozina Ghazalli; Karla Giles; Rodney Gilkey; Cheryl Gilmartin; Matthias Girndt; Richard J Glassock; Carlo Francisco S Gochuico; Eliezer Golan; David J Goldsmith; Fatima Ramirez Gonzalez; Suzanne Gore; Sílvia Gràcia-Garcia; Martin Haas; Lisa Hall; Karen Hamacher; Kathy Schiro Harvey; Niwrutti Hase; Domingo Hernandez; Elisabeth M Hodson; Ronald J Hogg; Hallvard Holdaas; Michael F Holick; Noriyuki Homma; Lai Seong Hooi; Mark Houser; Christian Hugo; Salwa Ibrahim; Enyu Imai; Jula Inrig; Sanjay Jain; Matthew James; Alan G Jardine; Andrzej Jaroszynski; Simon Jenkins; Chandra Mauli Jha; Kazibek Joldoshov; Graham Jones; Kamyar Kalantar-Zadeh; Nada Kanaan; Gülçin Kantarci; Frederick J Kaskel; Cecelia Kasnick; Hasan Kayabasi; James S Kennedy; Johannes Kessel; Bryce A Kiberd; Vera Koch; Daisuke Koya; Peter Krisper; Batya Kristal; Stefan Krivoshiev; Dirk R J Kuypers; Francisco Lacordelle; Craig B Langman; Roberta Lattes; Rakesh Lattupalli; Keith Lau; Edgar V Lerma; Visnja Lezaic; Hao Li; Yuan Liang; Robert Liebl; Jimmy A Light; Petrica Ligia; Tuta Liliana; Annabelle Sy Lim; Jelka Lindic; Zhangsuo Liu; Attilio Losito; Richard Lund; Kelvin Lynn; Linlin Ma; Janette Mansour; Abeera Mansur; Judith Marin; Alberto MartinezCastelao; Ken Massey; Timothy Mathew; Michael Mauer; Sandro Mazzaferro; Peter A McCullough; Amanda Medland; Gong Mengchun; Enisa Mesic; Sergio A Mezzano; Marius Miglinas; Tanuja Mishra; Takehiko Miyaji; Rosario Montañés-Bermúdez; Assad Monzer; José M Morales; Eugen Mota; Ricardo Mouzo; Cesar Loza Munarriz; Mohsen Nafar; Judit Nagy; Ramesh Naik; Takahiro Nakayama; Lavinia Negrea; Armando Luis Negri; Robert G Nelson; Marcus Vinícius de Pádua Netto; Alicia M Neu; Eezsafryna Azalin Nordin; Maurizio Nordio; Diego Novo; Thomas Oates; Yun Kyu Oh; Raymond Oliva; Patricia de Sequera Ortiz; Messaoud Ouziala; Antonino Paglialunga; Sonia Pasquali; Ioan Mihai Patiu; Saime Paydas; Glenda Payne; Erling Bjerregaard Pedersen; Adriana Peñalba; Graciela Pennacchiotti; Harry Brian Peppiatt; Gerson Marques Pereira Junior; Virginia Pernas; Todd E Pesavento; Frida Liane Plavnik; Claudio Ponticelli; Roberto Pontremoli; Amilee Poucher; Kearkiat Praditpornsilpa; Anastasia Ptinopoulou; Wajeh Qunibi; Rashida Rahman; Venkataraman Ramanathan; Roberto Ramirez; Harun ur Rashid; Hugh Rayner; Leonardo Marin Restrepo; Enrique Andrés Ribes; Peter Ricci; 
Troels Ring; A Adibul Rizvi; Nicolas Roberto Robles; Karen Rochelle; Hector J Rodriguez; Sara Rotaru; Jacques Rottembourg; Luis M Ruilope; Domenico Russo; Karen E Ryals; Jordi Bover Sanjuan; Francisco Santa-Cruz; Rafael Santamaria; Bento C Santos; Sergio Santos; Kenneth R Say; Sharon Schatz; Francesco Paolo Schena; Adalbert Schiller; Bernhard Schmekal; Rebecca Schmidt; Ammar Serawan; Mehmet Sever; Deepak Sharma; Robert Shay; Mitesh B Sheth; Alexander Shutov; Aleksandar Sikole; Fernando Simal; Dhavee Sirivongs; Itzchak Slotki; Yves Smets; Kimberly Smith; Laura Sola; Yalcin Solak; David Spiegel; Maria Stasios; Pavai Sthaneshwar; Gere Sunder-Plassmann; Charles Swanepoel; Mihály Tapolyai; Natalia Tomilina; Rezan
Topaloglu; Robert D Toto; Giorgio Triolo; Kriang Tungsanga; Katherine R Tuttle; Anitha Vijayan; Rowan Walker; Marc R Weber; Clare Weekes; Talia Weinstein; Matthew R Weir; Kerstin Westman; Jack F Wetzels; Sara Wolfson; Mai-Szu Wu; Yanhua Wu; Li Xuemei; Jerry Yee; Elena Zakharova; Kostyantyn Zakon; Weiming Zhang; Carmine Zoccali; Alessandro Zuccalà.

Participation in the review does not necessarily constitute endorsement of the content of this report by the above individuals, or the organization or institution they represent.

Adeera Levin, MD, FRCPC, Work Group Co-Chair Paul E Stevens, MB, FRCP, Work Group Co-Chair 


\section{References}

Kidney International Supplements (2013) 3, 136-150; doi:10.1038/kisup.2012.72
1. National Kidney Foundation. K/DOQI clinical practice guidelines for chronic kidney disease: evaluation, classification, and stratification. Am J Kidney Dis 2002; 39: S1-266.

2. Astor BC, Matsushita K, Gansevoort RT et al. Lower estimated glomerular filtration rate and higher albuminuria are associated with mortality and end-stage renal disease. A collaborative meta-analysis of kidney disease population cohorts. Kidney Int 2011; 79: 1331-1340.

3. Gansevoort RT, Matsushita K, van der Velde M et al. Lower estimated GFR and higher albuminuria are associated with adverse kidney outcomes. A collaborative meta-analysis of general and high-risk population cohorts. Kidney Int 2011; 80: 93-104.

4. Matsushita $\mathrm{K}$, van der Velde $\mathrm{M}, \mathrm{Astor} B C$ et al. Association of estimated glomerular filtration rate and albuminuria with all-cause and cardiovascular mortality in general population cohorts: a collaborative metaanalysis. Lancet 2010; 375: 2073-2081.

5. van der Velde $M$, Matsushita K, Coresh J et al. Lower estimated glomerular filtration rate and higher albuminuria are associated with allcause and cardiovascular mortality. A collaborative meta-analysis of high-risk population cohorts. Kidney Int 2011; 79: 1341-1352.

6. Levey AS, Stevens LA, Coresh J. Conceptual model of CKD: applications and implications. Am J Kidney Dis 2009; 53: S4-16.

7. KDIGO AKI Work Group. KDIGO clinical practice guideline for acute kidney injury. Kidney inter., Suppl. 2012; 2: 1-138.

8. KDIGO GN Work Group. KDIGO clinical practice guideline for glomerulonephritis. Kidney inter., Suppl. 2012; 2: 139-274.

9. KDIGO CKD-MBD Work Group. KDIGO clinical practice guideline for the diagnosis, evaluation, prevention, and treatment of Chronic Kidney DiseaseMineral and Bone Disorder (CKD-MBD). Kidney Int Suppl 2009; 76(Suppl 113): S1-130.

10. KDIGO BP Work Group. KDIGO clinical practice guideline for the management of blood pressure in chronic kidney disease. Kidney inter., Suppl. 2012; 2: 337-414.

11. KDIGO Anemia Work Group. KDIGO clinical practice guideline for anemia in chronic kidney disease. Kidney inter., Suppl. 2012; 2: 279-335.

12. Herzog CA, Asinger RW, Berger AK et al. Cardiovascular disease in chronic kidney disease. A clinical update from Kidney Disease: Improving Global Outcomes (KDIGO). Kidney Int 2011; 80: 572-586.

13. Matzke GR, Aronoff GR, Atkinson AJ, Jr. et al. Drug dosing consideration in patients with acute and chronic kidney disease-a clinical update from Kidney Disease: Improving Global Outcomes (KDIGO). Kidney Int 2011; 80: 1122-1137.

14. Hsu CY, Ordonez JD, Chertow GM et al. The risk of acute renal failure in patients with chronic kidney disease. Kidney Int 2008; 74: 101-107.

15. Hailpern SM, Melamed ML, Cohen HW et al. Moderate chronic kidney disease and cognitive function in adults 20 to 59 years of age: Third National Health and Nutrition Examination Survey (NHANES III). J Am Soc Nephrol 2007; 18: 2205-2213.

16. James $\mathrm{MT}$, Hemmelgarn $\mathrm{BR}$, Wiebe $\mathrm{N}$ et al. Glomerular filtration rate, proteinuria, and the incidence and consequences of acute kidney injury: a cohort study. Lancet 2010; 376: 2096-2103.

17. James MT, Quan $\mathrm{H}$, Tonelli $\mathrm{M}$ et al. CKD and risk of hospitalization and death with pneumonia. Am J Kidney Dis 2009; 54: 24-32.

18. Wilhelm-Leen ER, Hall YN, M KT et al. Frailty and chronic kidney disease: the Third National Health and Nutrition Evaluation Survey. Am J Med 2009; 122: 664-671 e662.

19. Levey AS, Coresh J. Chronic kidney disease. Lancet 2012; 379: 165-180.

20. Wesson L. Physiology of the human kidney. Grune \& Stratton: New York, 1969.

21. Rowe JW, Andres R, Tobin JD. Letter: Age-adjusted standards for creatinine clearance. Ann Intern Med 1976; 84: 567-569.

22. Poggio ED, Rule AD, Tanchanco $R$ et al. Demographic and clinical characteristics associated with glomerular filtration rates in living kidney donors. Kidney Int 2009; 75: 1079-1087.

23. Rule AD, Amer $\mathrm{H}$, Cornell LD et al. The association between age and nephrosclerosis on renal biopsy among healthy adults. Ann Intern Med 2010; 152: 561-567.

24. Barai S, Gambhir S, Prasad N et al. Levels of GFR and protein-induced hyperfiltration in kidney donors: a single-center experience in India. Am J Kidney Dis 2008; 51: 407-414.
25. Eastwood JB, Kerry SM, Plange-Rhule J et al. Assessment of GFR by four methods in adults in Ashanti, Ghana: the need for an eGFR equation for lean African populations. Nephrol Dial Transplant 2010; 25: 2178-2187.

26. Jafar $T H$, Islam $M$, Jessani $S$ et al. Level and determinants of kidney function in a South Asian population in Pakistan. Am J Kidney Dis 2011; 58: 764-772.

27. Stevens LA, Coresh J, Greene $T$ et al. Assessing kidney functionmeasured and estimated glomerular filtration rate. N Engl J Med 2006; 354: 2473-2483.

28. Remuzzi G, Benigni A, Remuzzi A. Mechanisms of progression and regression of renal lesions of chronic nephropathies and diabetes. $J$ Clin Invest 2006; 116: 288-296.

29. KDIGO Transplant Work Group. KDIGO clinical practice guideline for the care of kidney transplant recipients. Am J Transplant 2009; 9 (Suppl 3): S1-155.

30. Levey AS, de Jong PE, Coresh J et al. The definition, classification, and prognosis of chronic kidney disease: a KDIGO Controversies Conference report. Kidney Int 2011; 80: 17-28.

31. Levey AS, Eckardt KU, Tsukamoto $\mathrm{Y}$ et al. Definition and classification of chronic kidney disease: a position statement from Kidney Disease: Improving Global Outcomes (KDIGO). Kidney Int 2005; 67: 2089-2100.

32. Eckardt KU, Berns JS, Rocco MV et al. Definition and classification of CKD the debate should be about patient prognosis-a position statement from KDOQI and KDIGO. Am J Kidney Dis 2009; 53: 915-920.

33. Eknoyan G. Chronic kidney disease definition and classification: no need for a rush to judgment. Kidney Int 2009; 75: 1015-1018.

34. El Nahas M. Cardio-Kidney-Damage: a unifying concept. Kidney Int 2010; 78: $14-18$

35. Levey AS, Astor BC, Stevens LA et al. Chronic kidney disease, diabetes, and hypertension: what's in a name? Kidney Int 2010; 78: 19-22.

36. Winearls CG, Glassock RJ. Dissecting and refining the staging of chronic kidney disease. Kidney Int 2009; 75: 1009-1014.

37. Silva FG. The aging kidney: a review - part I. Int Urol Nephrol 2005; 37: 185-205.

38. Silva FG. The aging kidney: a review-part II. Int Urol Nephrol 2005; 37: 419-432.

39. Weinstein JR, Anderson S. The aging kidney: physiological changes. Adv Chronic Kidney Dis 2010; 17: 302-307.

40. King AJ, Levey AS. Dietary protein and renal function. J Am Soc Nephrol 1993; 3: 1723-1737.

41. Vehaskari VM. Orthostatic proteinuria. Arch Dis Child 1982; 57: 729-730.

42. Seikaly MG, Ho PL, Emmett $L$ et al. Chronic renal insufficiency in children: the 2001 Annual Report of the NAPRTCS. Pediatr Nephrol 2003; 18: $796-804$.

43. Hogg RJ, Furth S, Lemley KV et al. National Kidney Foundation's Kidney Disease Outcomes Quality Initiative clinical practice guidelines for chronic kidney disease in children and adolescents: evaluation, classification, and stratification. Pediatrics 2003; 111: 1416-1421.

44. Schwartz GJ, Brion LP, Spitzer A. The use of plasma creatinine concentration for estimating glomerular filtration rate in infants, children, and adolescents. Pediatr Clin North Am 1987; 34: 571-590.

45. Aperia A, Broberger $\mathrm{O}$, Elinder $\mathrm{G}$ et al. Postnatal development of renal function in pre-term and full-term infants. Acta Paediatr Scand 1981; 70: 183-187.

46. Bueva A, Guignard JP. Renal function in preterm neonates. Pediatr Res 1994; 36: 572-577.

47. Fetterman GH, Shuplock NA, Philipp FJ et al. The Growth and Maturation of Human Glomeruli and Proximal Convolutions from Term to Adulthood: Studies by Microdissection. Pediatrics 1965; 35: 601-619.

48. Guignard JP, Torrado A, Da Cunha $O$ et al. Glomerular filtration rate in the first three weeks of life. J Pediatr 1975; 87: 268-272.

49. Haycock GB. Development of glomerular filtration and tubular sodium reabsorption in the human fetus and newborn. Br J Urol 1998; 81 (Suppl 2): 33-38.

50. Gallini F, Maggio L, Romagnoli $C$ et al. Progression of renal function in preterm neonates with gestational age $<$ or $=32$ weeks. Pediatr Nephrol 2000; 15: 119-124. 
51. Vieux R, Hascoet JM, Merdariu D et al. Glomerular filtration rate reference values in very preterm infants. Pediatrics 2010; 125: e1186-1192.

52. Schwartz GJ, Furth SL. Glomerular filtration rate measurement and estimation in chronic kidney disease. Pediatr Nephrol 2007; 22: 1839-1848.

53. Waters AM. Chapter 6, Part 2: Functional development of the nephron. In: Geary DF, Schaefer F (eds). Comprehensive Pediatric Nephrology, Mosby Elsevier: Philadelphia, PA, 2008, pp 111-129.

54. Langlois V. Chapter 2: Laboratory evaluation at different ages. In: Geary DF, Schaefer F (eds) Comprehensive Pediatric Nephrology, Mosby Elsevier: Philadelphia, PA, 2008, pp 39-54.

55. Furth SL, Cole SR, Moxey-Mims $M$ et al. Design and methods of the Chronic Kidney Disease in Children (CKiD) prospective cohort study. Clin J Am Soc Nephrol 2006; 1: 1006-1015.

56. Copelovitch L, Warady BA, Furth SL. Insights from the Chronic Kidney Disease in Children (CKiD) study. Clin J Am Soc Nephrol 2011; 6: 2047-2053.

57. Seliger SL, Zhan M, Hsu VD et al. Chronic kidney disease adversely influences patient safety. J Am Soc Nephrol 2008; 19: 2414-2419.

58. Go AS, Chertow GM, Fan D et al. Chronic kidney disease and the risks of death, cardiovascular events, and hospitalization. N Engl J Med 2004; 351: 1296-1305.

59. Coresh J, Astor BC, Greene T et al. Prevalence of chronic kidney disease and decreased kidney function in the adult US population: Third National Health and Nutrition Examination Survey. Am J Kidney Dis 2003; 41: 1-12.

60. Burgert TS, Dziura J, Yeckel C et al. Microalbuminuria in pediatric obesity: prevalence and relation to other cardiovascular risk factors. Int J Obes (Lond) 2006; 30: 273-280.

61. Csernus K, Lanyi E, Erhardt E et al. Effect of childhood obesity and obesity-related cardiovascular risk factors on glomerular and tubular protein excretion. Eur J Pediatr 2005; 164: 44-49.

62. Houser MT, Jahn MF, Kobayashi A et al. Assessment of urinary protein excretion in the adolescent: effect of body position and exercise. J Pediatr 1986; 109: 556-561.

63. Trachtenberg F, Barregard L. The effect of age, sex, and race on urinary markers of kidney damage in children. Am J Kidney Dis 2007; 50: 938-945.

64. Brem AS. Neonatal hematuria and proteinuria. Clin Perinatol 1981; 8: 321-332.

65. Hogg RJ, Portman RJ, Milliner D et al. Evaluation and management of proteinuria and nephrotic syndrome in children: recommendations from a pediatric nephrology panel established at the National Kidney Foundation conference on proteinuria, albuminuria, risk, assessment, detection, and elimination (PARADE). Pediatrics 2000; 105: 1242-1249.

66. Jones $\mathrm{CA}$, Francis ME, Eberhardt MS et al. Microalbuminuria in the US population: third National Health and Nutrition Examination Survey. Am J Kidney Dis 2002; 39: 445-459.

67. Levey AS, Coresh J. Should the K/DOQI definition of chronic kidney disease be changed? Am J Kidney Dis 2003; 42: 626-630.

68. Uhlig K, Levey AS. Developing guidelines for chronic kidney disease: we should include all of the outcomes. Ann Intern Med 2012; 156: 599-601.

69. North American Pediatric Renal Trials and Collaborative Studies NAPRTCS 2008 Annual Report. (https://web.emmes.com/study/ped/ annlrept/Annual\%20Report\%20-2008.pdf). Accessed September 7, 2012.

70. Ardissino G, Dacco V, Testa S et al. Epidemiology of chronic renal failure in children: data from the ItalKid project. Pediatrics 2003; 111: e382-387.

71. Pierce CB, Cox C, Saland JM et al. Methods for characterizing differences in longitudinal glomerular filtration rate changes between children with glomerular chronic kidney disease and those with nonglomerular chronic kidney disease. Am J Epidemiol 2011; 174: 604-612.

72. Furth SL, Abraham AG, Jerry-Fluker J et al. Metabolic abnormalities, cardiovascular disease risk factors, and GFR decline in children with chronic kidney disease. Clin J Am Soc Nephrol 2011; 6: 2132-2140.

73. Wingen AM, Fabian-Bach $C$, Schaefer $F$ et al. Randomised multicentre study of a low-protein diet on the progression of chronic renal failure in children. European Study Group of Nutritional Treatment of Chronic Renal Failure in Childhood. Lancet 1997; 349: 1117-1123.

74. Staples AO, Greenbaum LA, Smith JM et al. Association between clinical risk factors and progression of chronic kidney disease in children. Clin J Am Soc Nephrol 2010; 5: 2172-2179.

75. Ardissino G, Testa S, Dacco V et al. Proteinuria as a predictor of disease progression in children with hypodysplastic nephropathy. Data from the Ital Kid Project. Pediatr Nephrol 2004; 19: 172-177.

76. Wong CS, Pierce CB, Cole SR et al. Association of proteinuria with race, cause of chronic kidney disease, and glomerular filtration rate in the chronic kidney disease in children study. Clin J Am Soc Nephrol 2009; 4: 812-819.

77. Ardissino G, Testa S, Dacco V et al. Puberty is associated with increased deterioration of renal function in patients with CKD: data from the ItalKid Project. Arch Dis Child 2012; 97: 885-888.

78. Querfeld U, Anarat A, Bayazit AK et al. The Cardiovascular Comorbidity in Children with Chronic Kidney Disease (4C) study: objectives, design, and methodology. Clin J Am Soc Nephrol 2010; 5: 1642-1648.

79. Stevens LA, Levey AS. Measured GFR as a confirmatory test for estimated GFR. J Am Soc Nephrol 2009; 20: 2305-2313.

80. Schwartz GJ, Munoz A, Schneider MF et al. New equations to estimate GFR in children with CKD. J Am Soc Nephrol 2009; 20: 629-637.

81. Myers GL, Miller WG, Coresh J et al. Recommendations for improving serum creatinine measurement: a report from the Laboratory Working Group of the National Kidney Disease Education Program. Clin Chem 2006; 52: 5-18.

82. Miller WG. Estimating glomerular filtration rate. Clin Chem Lab Med 2009; 47: 1017-1019.

83. Kilpatrick ES, Verrill H. A national audit of estimated glomerular filtration rate and proteinuria reporting in the UK. Ann Clin Biochem 2011; 48: 558-561.

84. Mclntosh JF, Moller E, Van Slyke DD. Studies of urea excretion. III: The influence of body size on urea output. J Clin Invest 1928; 6: 467-483.

85. Earley A, Miskulin D, Lamb EJ et al. Estimating equations for glomerular filtration rate in the era of creatinine standardization: a systematic review. Ann Intern Med 2012; 156: 785-795.

86. Levey AS, Coresh J, Greene T et al. Using standardized serum creatinine values in the modification of diet in renal disease study equation for estimating glomerular filtration rate. Ann Intern Med 2006; 145: 247-254.

87. Levey $\mathrm{AS}$, Stevens LA, Schmid $\mathrm{CH}$ et al. A new equation to estimate glomerular filtration rate. Ann Intern Med 2009; 150: 604-612.

88. Horio M, Imai E, Yasuda $Y$ et al. Modification of the CKD epidemiology collaboration (CKD-EPI) equation for Japanese: accuracy and use for population estimates. Am J Kidney Dis 2010; 56: 32-38.

89. Imai $\mathrm{E}$, Horio $\mathrm{M}$, Nitta $\mathrm{K}$ et al. Estimation of glomerular filtration rate by the MDRD study equation modified for Japanese patients with chronic kidney disease. Clin Exp Nephrol 2007; 11: 41-50.

90. Praditpornsilpa K, Townamchai N, Chaiwatanarat $\mathrm{T}$ et al. The need for robust validation for MDRD-based glomerular filtration rate estimation in various CKD populations. Nephrol Dial Transplant 2011; 26: 2780-2785.

91. Matsuo S, Imai E, Horio $M$ et al. Revised equations for estimated GFR from serum creatinine in Japan. Am J Kidney Dis 2009; 53: 982-992.

92. Levey AS, Greene T, Kusek J et al. A simplified equation to predict glomerular filtration rate from serum creatinine. J Am Soc Nephrol 2000; 11: $155 \mathrm{~A}$.

93. Ma YC, Zuo L, Chen JH et al. Modified glomerular filtration rate estimating equation for Chinese patients with chronic kidney disease. J Am Soc Nephrol 2006; 17: 2937-2944.

94. Levey AS, Bosch JP, Lewis JB et al. A more accurate method to estimate glomerular filtration rate from serum creatinine: a new prediction equation. Modification of Diet in Renal Disease Study Group. Ann Intern Med 1999; 130: 461-470.

95. Murata K, Baumann NA, Saenger AK et al. Relative performance of the MDRD and CKD-EPI equations for estimating glomerular filtration rate among patients with varied clinical presentations. Clin J Am Soc Nephrol 2011; 6: 1963-1972.

96. Lane BR, Demirjian S, Weight CJ et al. Performance of the chronic kidney disease-epidemiology study equations for estimating glomerular filtration rate before and after nephrectomy. J Urol 2010; 183: 896-901.

97. Michels WM, Grootendorst DC, Verduijn $M$ et al. Performance of the Cockcroft-Gault, MDRD, and new CKD-EPI formulas in relation to GFR, age, and body size. Clin J Am Soc Nephrol 2010; 5: 1003-1009.

98. Tent $H$, Rook $M$, Stevens LA et al. Renal function equations before and after living kidney donation: a within-individual comparison of performance at different levels of renal function. Clin J Am Soc Nephrol 2010; 5: 1960-1968.

99. Kukla A, El-Shahawi Y, Leister E et al. GFR-estimating models in kidney transplant recipients on a steroid-free regimen. Nephrol Dial Transplant 2010; 25: 1653-1661.

100. White CA, Akbari A, Doucette $S$ et al. Estimating glomerular filtration rate in kidney transplantation: is the new chronic kidney disease epidemiology collaboration equation any better? Clin Chem 2010; 56: 474-477.

101. Poge U, Gerhardt T, Stoffel-Wagner B et al. Validation of the CKD-EPI formula in patients after renal transplantation. Nephrol Dial Transplant 2011; 26: 4104-4108. 
102. Jones GR, Imam SK. Validation of the revised MDRD formula and the original Cockcroft and Gault formula for estimation of the glomerular filtration rate using Australian data. Pathology 2009; 41: 379-382.

103. Jones GR. Use of the CKD-EPI equation for estimation of GFR in an Australian cohort. Pathology 2010; 42: 487-488.

104. Cirillo M, Lombardi C, Luciano MG et al. Estimation of GFR: a comparison of new and established equations. Am J Kidney Dis 2010; 56: 802-804.

105. Eriksen BO, Mathisen UD, Melsom T et al. Cystatin C is not a better estimator of GFR than plasma creatinine in the general population. Kidney Int 2010; 78: 1305-1311.

106. Redal-Baigorri B, Stokholm KH, Rasmussen K et al. Estimation of kidney function in cancer patients. Dan Med Bull 2011; 58: A4236.

107. Matsushita K, Mahmoodi BK, Woodward M et al. Comparison of risk prediction using the CKD-EPI equation and the MDRD study equation for estimated glomerular filtration rate. JAMA 2012; 307: 1941-1951.

108. Rule AD, Teo BW. GFR estimation in Japan and China: what accounts for the difference? Am J Kidney Dis 2009; 53: 932-935.

109. Stevens LA, Claybon MA, Schmid CH et al. Evaluation of the Chronic Kidney Disease Epidemiology Collaboration equation for estimating the glomerular filtration rate in multiple ethnicities. Kidney Int 2011; 79: 555-562.

110. Yeo Y, Han DJ, Moon DH et al. Suitability of the IDMS-traceable MDRD equation method to estimate GFR in early postoperative renal transplant recipients. Nephron Clin Pract 2010; 114: c108-117.

111. van Deventer HE, George JA, Paiker JE et al. Estimating glomerular filtration rate in black South Africans by use of the modification of diet in renal disease and Cockcroft-Gault equations. Clin Chem 2008; 54: 1197-1202.

112. Teo BW, Xu H, Wang D et al. GFR estimating equations in a multiethnic Asian population. Am J Kidney Dis 2011; 58: 56-63.

113. Inker LA, Schmid $\mathrm{CH}$, Tighiouart $\mathrm{H}$ et al. Estimating glomerular filtration rate from serum creatinine and cystatin C. N Engl J Med 2012; 367: 20-29.

114. Peralta CA, Shlipak MG, Judd S et al. Detection of chronic kidney disease with creatinine, cystatin $C$, and urine albumin-to-creatinine ratio and association with progression to end-stage renal disease and mortality. JAMA 2011; 305: 1545-1552.

115. Schwartz GJ, Schneider MF, Maier PS et al. Improved equations estimating GFR in children with chronic kidney disease using an immunonephelometric determination of cystatin C. Kidney Int 2012; 82: 445-453.

116. Inker LA, Eckfeldt J, Levey AS et al. Expressing the CKD-EPI (Chronic Kidney Disease Epidemiology Collaboration) cystatin $C$ equations for estimating GFR with standardized serum cystatin C values. Am J Kidney Dis 2011; 58: 682-684.

117. Stevens LA, Coresh J, Schmid CH et al. Estimating GFR using serum cystatin $C$ alone and in combination with serum creatinine: a pooled analysis of 3,418 individuals with CKD. Am J Kidney Dis 2008; 51: 395-406.

118. Zappitelli $M$, Parvex $P$, Joseph $L$ et al. Derivation and validation of cystatin C-based prediction equations for GFR in children. Am J Kidney Dis 2006; 48: 221-230.

119. Filler G, Lepage N. Should the Schwartz formula for estimation of GFR be replaced by cystatin C formula? Pediatr Nephrol 2003; 18: 981-985.

120. Hoek FJ, Kemperman FA, Krediet RT. A comparison between cystatin C plasma creatinine and the Cockcroft and Gault formula for the estimation of glomerular filtration rate. Nephrol Dial Transplant 2003; 18: 2024-2031.

121. Kwong YT, Stevens LA, Selvin E et al. Imprecision of urinary iothalamate clearance as a gold-standard measure of GFR decreases the diagnostic accuracy of kidney function estimating equations. Am J Kidney Dis 2010 56: $39-49$.

122. Lamb EJ, Price CP. Kidney function tests. In: Burtis CA, Ashwood E, (eds.) Bruns DE. Tietz Textbook of Clinical Chemistry and Molecular Diagnostics, $5^{\text {th }}$ edition, Elsevier, 2012, pp 669-708.

123. Ballantyne FC, Gibbons J, O'Reilly DS. Urine albumin should replace total protein for the assessment of glomerular proteinuria. Ann Clin Biochem 1993; 30 (Pt 1): 101-103.

124. Lamb EJ, MacKenzie F, Stevens PE. How should proteinuria be detected and measured? Ann Clin Biochem 2009; 46: 205-217.

125. Newman DJ, Thakkar $H$, Medcalf EA et al. Use of urine albumin measurement as a replacement for total protein. Clin Nephrol 1995; 43 104-109.

125a. Hallan SI, Ritz E, Lydersen S et al. Combining GFR and albuminuria to classify CKD improves prediction of ESRD. J Am Soc Nephrol 2009; 20: 1069-1077.

125b. Brantsma $\mathrm{AH}$, Bakker SJ, Hillege $\mathrm{HL}$ et al. Cardiovascular and renal outcome in subjects with $\mathrm{K} / \mathrm{DOQ}$ I stage 1-3 chronic kidney disease: the importance of urinary albumin excretion. Nephrol Dial Transplant 2008; 23: 3851-3858.

126. Dawnay A, Wilson AG, Lamb $E$ et al. Microalbuminuria in systemic sclerosis. Ann Rheum Dis 1992; 51: 384-388.
127. Gross JL, de Azevedo MJ, Silveiro SP et al. Diabetic nephropathy: diagnosis, prevention, and treatment. Diabetes Care 2005; 28: 164-176.

128. Ninomiya T, Perkovic V, de Galan BE et al. Albuminuria and kidney function independently predict cardiovascular and renal outcomes in diabetes. J Am Soc Nephrol 2009; 20: 1813-1821.

129. Shihabi ZK, Konen JC, O'Connor ML. Albuminuria vs urinary total protein for detecting chronic renal disorders. Clin Chem 1991; 37: 621-624.

130. Martin H. Laboratory measurement of urine albumin and urine total protein in screening for proteinuria in chronic kidney disease. Clin Biochem Rev 2011; 32: 97-102.

131. Waugh J, Bell SC, Kilby $M$ et al. Effect of concentration and biochemical assay on the accuracy of urine dipsticks in hypertensive pregnancies. Hypertens Pregnancy 2001; 20: 205-217.

132. Waugh J, Bell SC, Kilby MD et al. Urine protein estimation in hypertensive pregnancy: which thresholds and laboratory assay best predict clinical outcome? Hypertens Pregnancy 2005; 24: 291-302.

133. McElderry LA, Tarbit IF, Cassells-Smith AJ. Six methods for urinary protein compared. Clin Chem 1982; 28: 356-360.

134. Nishi HH, Elin RJ. Three turbidimetric methods for determining total protein compared. Clin Chem 1985; 31: 1377-1380.

135. Sedmak JJ, Grossberg SE. A rapid, sensitive, and versatile assay for protein using Coomassie brilliant blue G250. Anal Biochem 1977; 79: 544-552.

136. de Keijzer MH, Klasen IS, Branten AJ et al. Infusion of plasma expanders may lead to unexpected results in urinary protein assays. Scand J Clin Lab Invest 1999; 59: 133-137.

137. Marshall T, Williams KM. Extent of aminoglycoside interference in the pyrogallol red-molybdate protein assay depends on the concentration of sodium oxalate in the dye reagent. Clin Chem 2004; 50: 934-935.

138. Yilmaz FM, Yucel D. Effect of addition of hemolysate on urine and cerebrospinal fluid assays for protein. Clin Chem 2006; 52: 152-153.

139. Chambers RE, Bullock DG, Whicher JT. External quality assessment of total urinary protein estimation in the United Kingdom. Ann Clin Biochem 1991; 28 (Pt 5): 467-473.

140. Heick HM, Begin-Heick N, Acharya C et al. Automated determination of urine and cerebrospinal fluid proteins with Coomassie Brilliant Blue and the Abbott ABA-100. Clin Biochem 1980; 13: 81-83.

141. Marshall T, Williams KM. Total protein determination in urine: elimination of a differential response between the coomassie blue and pyrogallol red protein dye-binding assays. Clin Chem 2000; 46: 392-398.

142. Miller WG. Urine albumin: Recommendations for standardization. Scand J Clin Lab Invest Suppl 2008; 241: 71-72.

143. Miller WG, Bruns DE, Hortin GL et al. Current issues in measurement and reporting of urinary albumin excretion. Clin Chem 2009; 55: 24-38.

144. Medicines and Healthcare products Regulatory Agency. MHRA 04086 Point of care devices for the quantitation of microalbuminuria. 2004.

145. Medicines and Healthcare products Regulatory Agency. MHRA 04098 Point of care devices for the detection and semi-quantitation of microalbuminuria. 2004

146. Parsons $M$, Newman DJ, Pugia $M$ et al. Performance of a reagent strip device for quantitation of the urine albumin: creatinine ratio in a point of care setting. Clin Nephrol 1999; 51: 220-227.

147. Parsons MP, Newman DJ, Newall RG et al. Validation of a point-of-care assay for the urinary albumin:creatinine ratio. Clin Chem 1999; 45: 414-417.

148. Graziani MS, Gambaro G, Mantovani L et al. Diagnostic accuracy of a reagent strip for assessing urinary albumin excretion in the general population. Nephrol Dial Transplant 2009; 24: 1490-1494.

149. Guy M, Newall R, Borzomato J et al. Diagnostic accuracy of the urinary albumin: creatinine ratio determined by the CLINITEK Microalbumin and DCA $2000+$ for the rule-out of albuminuria in chronic kidney disease. Clin Chim Acta 2009; 399: 54-58.

150. Waugh JJ, Bell SC, Kilby MD et al. Optimal bedside urinalysis for the detection of proteinuria in hypertensive pregnancy: a study of diagnostic accuracy. BJOG 2005; 112: 412-417.

151. Iseki K, Iseki C, Ikemiya $Y$ et al. Risk of developing end-stage renal disease in a cohort of mass screening. Kidney Int 1996; 49: 800-805.

152. Kaplan RE, Springate JE, Feld LG. Screening dipstick urinalysis: a time to change. Pediatrics 1997; 100: 919-921.

153. Kitagawa T. Lessons learned from the Japanese nephritis screening study. Pediatr Nephrol 1988; 2: 256-263.

154. Boulware LE, Jaar BG, Tarver-Carr ME et al. Screening for proteinuria in US adults: a cost-effectiveness analysis. JAMA 2003; 290: 3101-3114.

155. Bowie L, Smith S, Gochman N. Characteristics of binding between reagent-strip indicators and urinary proteins. Clin Chem 1977; 23: 128-130.

156. Gyure WL. Comparison of several methods for semiquantitative determination of urinary protein. Clin Chem 1977; 23: 876-879. 
157. James GP, Bee DE, Fuller JB. Proteinuria: accuracy and precision of laboratory diagnosis by dip-stick analysis. Clin Chem 1978; 24: 1934-1939.

158. Rumley A. Urine dipstick testing: comparison of results obtained by visual reading and with the Bayer CLINITEK 50. Ann Clin Biochem 2000; 37 (Pt 2): 220-221.

159. Scotti da Silva-Colombeli A, Falkenberg M. Analytical interferences of drugs in the chemical examination of urinary protein. Clin Biochem 2007; 40: 1074-1076.

160. Ralston SH, Caine $\mathrm{N}$, Richards I et al. Screening for proteinuria in a rheumatology clinic: comparison of dipstick testing, $24 \mathrm{~h}$ urine quantitative protein, and protein/creatinine ratio in random urine samples. Ann Rheum Dis 1988; 47: 759-763.

161. Waugh JJ, Clark TJ, Divakaran TG et al. Accuracy of urinalysis dipstick techniques in predicting significant proteinuria in pregnancy. Obstet Gynecol 2004; 103: 769-777.

162. White SL, Yu R, Craig JC et al. Diagnostic accuracy of urine dipsticks for detection of albuminuria in the general community. Am J Kidney Dis 2011; 58: 19-28.

163. Saudan PJ, Brown MA, Farrell T et al. Improved methods of assessing proteinuria in hypertensive pregnancy. Br J Obstet Gynaecol 1997; 104: 1159-1164.

164. Beetham R, Cattell WR. Proteinuria: pathophysiology, significance and recommendations for measurement in clinical practice. Ann Clin Biochem 1993; 30 (Pt 5): 425-434.

165. Keane WF, Eknoyan G. Proteinuria, albuminuria, risk, assessment, detection, elimination (PARADE): a position paper of the National Kidney Foundation. Am J Kidney Dis 1999; 33: 1004-1010.

166. Claudi T, Cooper JG. Comparison of urinary albumin excretion rate in overnight urine and albumin creatinine ratio in spot urine in diabetic patients in general practice. Scand J Prim Health Care 2001; 19: 247-248.

167. Gatling W, Knight C, Mullee MA et al. Microalbuminuria in diabetes: a population study of the prevalence and an assessment of three screening tests. Diabet Med 1988; 5: 343-347.

168. Hutchison AS, O'Reilly DS, MacCuish AC. Albumin excretion rate, albumin concentration, and albumin/creatinine ratio compared for screening diabetics for slight albuminuria. Clin Chem 1988; 34: 2019-2021.

169. Marshall SM. Screening for microalbuminuria: which measurement? Diabet Med 1991; 8: 706-711.

170. Marshall SM, Alberti KG. Screening for early diabetic nephropathy. Ann Clin Biochem 1986; 23 (Pt 2): 195-197.

171. Chitalia VC, Kothari J, Wells EJ et al. Cost-benefit analysis and prediction of 24-hour proteinuria from the spot urine protein-creatinine ratio. Clin Nephrol 2001; 55: 436-447.

172. Cote AM, Brown MA, Lam E et al. Diagnostic accuracy of urinary spot protein:creatinine ratio for proteinuria in hypertensive pregnant women: systematic review. BMJ 2008; 336: 1003-1006.

173. Dyson EH, Will EJ, Davison AM et al. Use of the urinary protein creatinine index to assess proteinuria in renal transplant patients. Nephrol Dial Transplant 1992; 7: 450-452.

174. Ginsberg JM, Chang BS, Matarese RA et al. Use of single voided urine samples to estimate quantitative proteinuria. N Engl J Med 1983; 309: 1543-1546.

175. Leanos-Miranda A, Marquez-Acosta J, Romero-Arauz F et al. Protein:creatinine ratio in random urine samples is a reliable marker of increased 24-hour protein excretion in hospitalized women with hypertensive disorders of pregnancy. Clin Chem 2007; 53: 1623-1628.

176. Lemann J, Jr., Doumas BT. Proteinuria in health and disease assessed by measuring the urinary protein/creatinine ratio. Clin Chem 1987; 33: 297-299.

177. Ruggenenti $P$, Gaspari F, Perna A et al. Cross sectional longitudinal study of spot morning urine protein:creatinine ratio, $24 \mathrm{~h}$ urine protein excretion rate, glomerular filtration rate, and end stage renal failure in chronic renal disease in patients without diabetes. BMJ 1998; 316: 504-509.

178. Pugliese G, Solini A, Fondelli $C$ et al. Reproducibility of albuminuria in type 2 diabetic subjects. Findings from the Renal Insufficiency And Cardiovascular Events (RIACE) study. Nephrol Dial Transplant 2011; 26: 3950-3954.

179. Newman DJ, Pugia MJ, Lott JA et al. Urinary protein and albumin excretion corrected by creatinine and specific gravity. Clin Chim Acta 2000; 294: 139-155.

180. Howey JE, Browning MC, Fraser CG. Selecting the optimum specimen for assessing slight albuminuria, and a strategy for clinical investigation: novel uses of data on biological variation. Clin Chem 1987; 33: 2034-2038.

181. Carter JL, Tomson CR, Stevens PE et al. Does urinary tract infection cause proteinuria or microalbuminuria? A systematic review. Nephrol Dial Transplant 2006; 21: 3031-3037.
182. Heathcote $\mathrm{KL}$, Wilson MP, Quest DW et al. Prevalence and duration of exercise induced albuminuria in healthy people. Clin Invest Med 2009; 32: E261-265.

183. Leung AK, Wong AH. Proteinuria in children. Am Fam Physician 2010; 82: 645-651.

184. Boger $\mathrm{CA}$, Chen $\mathrm{MH}$, Tin A et al. CUBN is a gene locus for albuminuria. J Am Soc Nephrol 2011; 22: 555-570.

185. Price CP, Newall RG, Boyd JC. Use of protein:creatinine ratio measurements on random urine samples for prediction of significant proteinuria: a systematic review. Clin Chem 2005; 51: 1577-1586.

186. National Institute for Health and Clinical Excellence. NICE clinica guideline 73. Chronic kidney disease: early identification and management of chronic kidney disease in adults in primary and secondary care. 2008.

187. Montanes Bermudez R, Gracia Garcia S, Perez Surribas D et al. Consensus document. Recommendations on assessing proteinuria during the diagnosis and follow-up of chronic kidney disease. Nefrologia 2011; 31: 331-345.

188. Johnson DW, Jones GR, Mathew TH et al. Chronic kidney disease and measurement of albuminuria or proteinuria: a position statement. Med $\mathrm{J}$ Aust 2012; 197: 224-225.

189. Scottish Intercollegiate Guidelines Network. Guideline 103. Diagnosis and management of chronic kidney disease. 2008.

190. Caring for Australasians with Renal Impairment. http://www.cari.org.au/ guidelines.php

191. Clarke W, Frost SJ, Kraus E et al. Renal function testing. In: Nichols JH (ed) Evidence-based Practice for Point-of-Care Testing. National Academy of Clinical Biochemistry, 2006, pp 126-134.

192. Panek R, Lawen T, Kiberd BA. Screening for proteinuria in kidney transplant recipients. Nephrol Dial Transplant 2011; 26: 1385-1387.

193. Incerti J, Zelmanovitz T, Camargo JL et al. Evaluation of tests for microalbuminuria screening in patients with diabetes. Nephrol Dial Transplant 2005; 20: 2402-2407.

194. Methven S, MacGregor MS, Traynor JP et al. Assessing proteinuria in chronic kidney disease: protein-creatinine ratio versus albumin-creatinine ratio. Nephrol Dial Transplant 2010; 25: 2991-2996.

195. Methven S, MacGregor MS, Traynor JP et al. Comparison of urinary albumin and urinary total protein as predictors of patient outcomes in CKD. Am J Kidney Dis 2011; 57: 21-28.

196. Methven S, Traynor JP, Hair MD et al. Stratifying risk in chronic kidney disease: an observational study of UK guidelines for measuring total proteinuria and albuminuria. QJM 2011; 104: 663-670.

197. Nauta FL, Bakker SJ, van Oeveren $W$ et al. Albuminuria, proteinuria, and novel urine biomarkers as predictors of long-term allograft outcomes in kidney transplant recipients. Am J Kidney Dis 2011; 57: 733-743.

198. Ellam TJ. Albumin:creatinine ratio-a flawed measure? The merits of estimated albuminuria reporting. Nephron Clin Pract 2011; 118: c324-330.

199. Comper WD, Osicka TM, Clark $M$ et al. Earlier detection of microalbuminuria in diabetic patients using a new urinary albumin assay. Kidney Int 2004; 65: 1850-1855.

200. Comper WD, Osicka TM, Jerums G. High prevalence of immunounreactive intact albumin in urine of diabetic patients. Am J Kidney Dis 2003; 41: 336-342.

201. Magliano DJ, Polkinghorne KR, Barr EL et al. HPLC-detected albuminuria predicts mortality. J Am Soc Nephrol 2007; 18: 3171-3176.

202. Osicka TM, Comper WD. Characterization of immunochemically nonreactive urinary albumin. Clin Chem 2004; 50: 2286-2291.

203. Sviridov D, Drake SK, Hortin GL. Reactivity of urinary albumin (microalbumin) assays with fragmented or modified albumin. Clin Chem 2008; 54: 61-68.

204. Sviridov D, Meilinger B, Drake SK et al. Coelution of other proteins with albumin during size-exclusion HPLC: Implications for analysis of urinary albumin. Clin Chem 2006; 52: 389-397.

205. Tsioufis C, Mazaraki A, Dimitriadis $\mathrm{K}$ et al. Microalbuminuria in the paediatric age: current knowledge and emerging questions. Acta Paediatr 2011; 100: 1180-1184.

206. Rademacher ER, Sinaiko AR. Albuminuria in children. Curr Opin Nephrol Hypertens 2009; 18: 246-251.

207. Wrong OM, Norden AG, Feest TG. Dent's disease; a familial proximal renal tubular syndrome with low-molecular-weight proteinuria, hypercalciuria, nephrocalcinosis, metabolic bone disease, progressive renal failure and a marked male predominance. QJM 1994; 87: 473-493.

208. Atkins RC, Briganti EM, Zimmet PZ et al. Association between albuminuria and proteinuria in the general population: the AusDiab Study. Nephrol Dial Transplant 2003; 18: 2170-2174. 
209. Gosling P. Proteinuria. In: Marshall WJ, Bangert SK (eds). Clinical Biochemistry: Metabolic and Clinical Aspects, 2nd Ed. Elsevier, 2008, pp 156-173.

210. Goren MP, Li JT. The Coomassie Brilliant Blue method underestimates drug-induced tubular proteinuria. Clin Chem 1986; 32: 386-388.

211. Weber $\mathrm{MH}$, Verwiebe R. Alpha 1-microglobulin (protein $\mathrm{HC}$ ): features of a promising indicator of proximal tubular dysfunction. Eur J Clin Chem Clin Biochem 1992; 30: 683-691.

212. Herget-Rosenthal S, Poppen D, Husing J et al. Prognostic value of tubular proteinuria and enzymuria in nonoliguric acute tubular necrosis. Clin Chem 2004; 50: 552-558.

213. Ginevri F, Piccotti $E$, Alinovi $R$ et al. Reversible tubular proteinuria precedes microalbuminuria and correlates with the metabolic status in diabetic children. Pediatr Nephrol 1993; 7: 23-26.

214. Tomlinson PA, Smellie JM, Prescod N et al. Differential excretion of urinary proteins in children with vesicoureteric reflux and reflux nephropathy. Pediatr Nephrol 1994; 8: 21-25.

215. Bird JM, Owen RG, D'Sa S et al. Guidelines for the diagnosis and management of multiple myeloma 2011. Br J Haematol 2011; 154: 32-75.

216. Slack TK, Wilson DM. Normal renal function: CIN and CPAH in healthy donors before and after nephrectomy. Mayo Clinic Proc 1976; 51: 296-300.

217. Rowe JW, Andres R, Tobin JD et al. The effect of age on creatinine clearance in men: a cross-sectional and longitudinal study. J Gerontol 1976; 31: 155-163.

218. Lindeman RD, Tobin JD, Shock NW. Association between blood pressure and the rate of decline in renal function with age. Kidney Int 1984; 26: 861-868.

219. Halbesma N, Kuiken DS, Brantsma AH et al. Macroalbuminuria is a better risk marker than low estimated GFR to identify individuals at risk for accelerated GFR loss in population screening. J Am Soc Nephrol 2006; 17: 2582-2590.

220. Imai $\mathrm{E}$, Horio $\mathrm{M}$, Yamagata $\mathrm{K}$ et al. Slower decline of glomerular filtration rate in the Japanese general population: a longitudinal 10-year follow-up study. Hypertens Res 2008; 31: 433-441.

221. Matsushita K, Selvin E, Bash LD et al. Change in estimated GFR associates with coronary heart disease and mortality. J Am Soc Nephrol 2009; 20: 2617-2624.

222. Kronborg J, Solbu M, Njolstad I et al. Predictors of change in estimated GFR: a population-based 7-year follow-up from the Tromso study. Nephrol Dial Transplant 2008; 23: 2818-2826.

223. Hemmelgarn BR, Zhang J, Manns BJ et al. Progression of kidney dysfunction in the community-dwelling elderly. Kidney Int 2006; 69: 2155-2161.

224. Keller C, Katz R, Sarnak MJ et al. Inflammatory biomarkers and decline in kidney function in the elderly: the Cardiovascular Health Study. Nephrol Dial Transplant 2010; 25: 119-124.

225. John $\mathrm{R}$, Webb $M$, Young $A$ et al. Unreferred chronic kidney disease: a longitudinal study. Am J Kidney Dis 2004; 43: 825-835.

226. Levey AS, Gassman JJ, Hall PM et al. Assessing the progression of renal disease in clinical studies: effects of duration of follow-up and regression to the mean. Modification of Diet in Renal Disease (MDRD) Study Group. J Am Soc Nephrol 1991; 1: 1087-1094.

227. Klahr S, Levey AS, Beck GJ et al. The effects of dietary protein restriction and blood-pressure control on the progression of chronic renal disease. Modification of Diet in Renal Disease Study Group. N Engl J Med 1994; 330: $877-884$

228. Wright JT, Jr., Bakris G, Greene $T$ et al. Effect of blood pressure lowering and antihypertensive drug class on progression of hypertensive kidney disease: results from the AASK trial. JAMA 2002; 288: 2421-2431.

229. Eriksen BO, Ingebretsen OC. The progression of chronic kidney disease: a 10-year population-based study of the effects of gender and age. Kidney Int 2006; 69: 375-382.

230. Jones $C$, Roderick $P$, Harris $S$ et al. Decline in kidney function before and after nephrology referral and the effect on survival in moderate to advanced chronic kidney disease. Nephrol Dial Transplant 2006; 21: 2133-2143.

231. Levin A, Djurdjev O, Beaulieu M et al. Variability and risk factors for kidney disease progression and death following attainment of stage 4 CKD in a referred cohort. Am J Kidney Dis 2008; 52: 661-671.

232. Al-Aly Z, Zeringue A, Fu J et al. Rate of kidney function decline associates with mortality. J Am Soc Nephrol 2010; 21: 1961-1969.

233. Shlipak MG, Katz R, Kestenbaum B et al. Rapid decline of kidney function increases cardiovascular risk in the elderly. J Am Soc Nephrol 2009; 20: 2625-2630.
234. Cheng TY, Wen SF, Astor BC et al. Mortality risks for all causes and cardiovascular diseases and reduced GFR in a middle-aged working population in Taiwan. Am J Kidney Dis 2008; 52: 1051-1060.

235. Rifkin DE, Shlipak MG, Katz $R$ et al. Rapid kidney function decline and mortality risk in older adults. Arch Intern Med 2008; 168: 2212-2218.

236. Appel LJ, Wright JT, Jr., Greene T et al. Intensive blood-pressure contro in hypertensive chronic kidney disease. N Engl J Med 2010; 363: 918-929.

237. Hunsicker LG, Adler S, Caggiula A et al. Predictors of the progression of renal disease in the Modification of Diet in Renal Disease Study. Kidney Int 1997; 51: 1908-1919.

238. Menon V, Wang X, Sarnak MJ et al. Long-term outcomes in nondiabetic chronic kidney disease. Kidney Int 2008; 73: 1310-1315.

239. Adler Al, Stevens RJ, Manley SE et al. Development and progression of nephropathy in type 2 diabetes: the United Kingdom Prospective Diabetes Study (UKPDS 64). Kidney Int 2003; 63: 225-232.

240. Caramori ML, Fioretto $P$, Mauer $M$. Enhancing the predictive value of urinary albumin for diabetic nephropathy. J Am Soc Nephrol 2006; 17: 339-352.

241. Hoefield RA, Kalra PA, Baker PG et al. The use of eGFR and ACR to predict decline in renal function in people with diabetes. Nephrol Dial Transplant 2011; 26: 887-892

242. Rosolowsky ET, Skupien J, Smiles AM et al. Risk for ESRD in type 1 diabetes remains high despite renoprotection. J Am Soc Nephrol 2011; 22: $545-553$.

243. Hemmelgarn BR, Clement F, Manns BJ et al. Overview of the Alberta Kidney Disease Network. BMC Nephrol 2009; 10: 30.

244. Turin TC, Coresh J, Tonelli M et al. One-year change in kidney function is associated with an increased mortality risk. Am J Nephrol 2012; 36: 41-49.

245. Turin TC, Coresh J, Tonelli M et al. Short-term change in kidney function and risk of end-stage renal disease. Nephrol Dial Transplant 2012; 27: 3835-3843.

246. Schmieder RE, Mann JF, Schumacher $\mathrm{H}$ et al. Changes in albuminuria predict mortality and morbidity in patients with vascular disease. J Am Soc Nephrol 2011; 22: 1353-1364.

247. Li L, Astor BC, Lewis J et al. Longitudinal Progression Trajectory of GFR Among Patients With CKD. Am J Kidney Dis 2012; 59: 504-512.

248. O'Hare AM, Batten A, Burrows NR et al. Trajectories of Kidney Function Decline in the 2 Years Before Initiation of Long-term Dialysis. Am J Kidney Dis 2012; 59: 513-522.

249. Leblanc M, Kellum JA, Gibney RT et al. Risk factors for acute renal failure: inherent and modifiable risks. Curr Opin Crit Care 2005; 11: 533-536.

250. Naughton CA. Drug-induced nephrotoxicity. Am Fam Physician 2008; 78: 743-750.

251. Pannu N, Nadim MK. An overview of drug-induced acute kidney injury. Crit Care Med 2008; 36: S216-223.

252. Solomon R, Dauerman HL. Contrast-induced acute kidney injury. Circulation 2010; 122: 2451-2455.

253. Black C, Sharma P, Scotland G et al. Early referral strategies for management of people with markers of renal disease: a systematic review of the evidence of clinical effectiveness, cost-effectiveness and economic analysis. Health Technol Assess 2010; 14: 1-184.

254. Bang $\mathrm{H}$, Mazumdar M, Newman $\mathrm{G}$ et al. Screening for kidney disease in vascular patients: SCreening for Occult REnal Disease (SCORED) experience. Nephrol Dial Transplant 2009; 24: 2452-2457.

255. Johnson ES, Smith DH, Thorp ML et al. Predicting the risk of end-stage renal disease in the population-based setting: a retrospective casecontrol study. BMC Nephrol 2011; 12: 17.

256. Wakai K, Kawamura T, Endoh $M$ et al. A scoring system to predict renal outcome in IgA nephropathy: from a nationwide prospective study. Nephrol Dial Transplant 2006; 21: 2800-2808.

257. Keane WF, Zhang Z, Lyle PA et al. Risk scores for predicting outcomes in patients with type 2 diabetes and nephropathy: the RENAAL study. Clin $J$ Am Soc Nephrol 2006; 1: 761-767.

258. Fine EJ, Blaufox MD. Prediction rule for renal artery stenosis. Ann Intern Med 1999; 131: 227-228.

259. Kshirsagar AV, Bang $H$, Bomback AS et al. A simple algorithm to predict incident kidney disease. Arch Intern Med 2008; 168: 2466-2473.

260. Tangri N, Stevens LA, Griffith J et al. A predictive model for progression of chronic kidney disease to kidney failure. JAMA 2011; 305: 1553-1559.

261. Halbesma N, Jansen DF, Heymans MW et al. Development and validation of a general population renal risk score. Clin J Am Soc Nephrol 2011; 6: 1731-1738.

262. National Kidney Foundation. KDOQI Clinical Practice Guideline for Diabetes and CKD: 2012 Update. Am J Kidney Dis 2012; 60: 850-886. 
263. Mittalhenkle A, Stehman-Breen CO, Shlipak MG et al. Cardiovascular risk factors and incident acute renal failure in older adults: the cardiovascular health study. Clin J Am Soc Nephrol 2008; 3: 450-456.

264. Uchino $S$, Kellum JA, Bellomo $R$ et al. Acute renal failure in critically ill patients: a multinational, multicenter study. JAMA 2005; 294: 813-818.

265. Hoste $\mathrm{EA}$, Lameire $\mathrm{NH}$, Vanholder $\mathrm{RC}$ et al. Acute renal failure in patients with sepsis in a surgical ICU: predictive factors, incidence, comorbidity, and outcome. J Am Soc Nephrol 2003; 14: 1022-1030.

266. McCullough PA, Wolyn R, Rocher LL et al. Acute renal failure after coronary intervention: incidence, risk factors, and relationship to mortality. Am J Med 1997; 103: 368-375.

267. Mehran R, Aymong ED, Nikolsky E et al. A simple risk score for prediction of contrast-induced nephropathy after percutaneous coronary intervention: development and initial validation. J Am Coll Cardiol 2004; 44: 1393-1399.

268. Mehta RH, Grab JD, O'Brien SM et al. Bedside tool for predicting the risk of postoperative dialysis in patients undergoing cardiac surgery. Circulation 2006; 114: 2208-2216.

269. Thakar CV, Worley S, Arrigain S et al. Influence of renal dysfunction on mortality after cardiac surgery: modifying effect of preoperative renal function. Kidney Int 2005; 67: 1112-1119.

270. Waikar SS, Liu KD, Chertow GM. Diagnosis, epidemiology and outcomes of acute kidney injury. Clin J Am Soc Nephrol 2008; 3: 844-861.

271. Yegenaga I, Hoste E, Van Biesen $W$ et al. Clinical characteristics of patients developing ARF due to sepsis/systemic inflammatory response syndrome: results of a prospective study. Am J Kidney Dis 2004; 43: 817-824.

272. Parfrey PS, Griffiths SM, Barrett BJ et al. Contrast material-induced renal failure in patients with diabetes mellitus, renal insufficiency, or both. A prospective controlled study. N Engl J Med 1989; 320: 143-149.

273. Browner WS, Li J, Mangano DT. In-hospital and long-term mortality in male veterans following noncardiac surgery. The Study of Perioperative Ischemia Research Group. JAMA 1992; 268: 228-232.

274. Hou SH, Bushinsky DA, Wish JB et al. Hospital-acquired renal insufficiency: a prospective study. Am J Med 1983; 74: 243-248.

275. Singh P, Rifkin DE, Blantz RC. Chronic kidney disease: an inherent risk factor for acute kidney injury? Clin J Am Soc Nephrol 2010; 5: 1690-1695.

276. Lafrance JP, Djurdjev O, Levin A. Incidence and outcomes of acute kidney injury in a referred chronic kidney disease cohort. Nephrol Dial Transplant 2010; 25: 2203-2209.

277. Chapin E, Zhan M, Hsu VD et al. Adverse safety events in chronic kidney disease: the frequency of "multiple hits". Clin J Am Soc Nephrol 2010; 5: 95-101.

278. Chertow GM, Christiansen CL, Cleary PD et al. Prognostic stratification in critically ill patients with acute renal failure requiring dialysis. Arch Intern Med 1995; 155: 1505-1511.

279. Chertow GM, Soroko SH, Paganini EP et al. Mortality after acute renal failure: models for prognostic stratification and risk adjustment. Kidney Int 2006; 70: 1120-1126.

280. Mehta RL, Pascual MT, Gruta CG et al. Refining predictive models in critically ill patients with acute renal failure. J Am Soc Nephrol 2002; 13: 1350-1357.

281. Paganini EP, Larive B, Kanagasundaram NS. Severity scores and outcomes with acute renal failure in the ICU setting. Contrib Nephrol 2001: 181-195.

282. Uchino $\mathrm{S}$, Bellomo $\mathrm{R}$, Morimatsu $\mathrm{H}$ et al. External validation of severity scoring systems for acute renal failure using a multinational database. Crit Care Med 2005; 33: 1961-1967.

283. Waikar SS, Curhan GC, Wald R et al. Declining mortality in patients with acute renal failure, 1988 to 2002. J Am Soc Nephrol 2006; 17: 1143-1150.

284. Khosla N, Soroko SB, Chertow GM et al. Preexisting chronic kidney disease: a potential for improved outcomes from acute kidney injury. Clin J Am Soc Nephrol 2009; 4: 1914-1919.

285. Fouque $D$, Laville M. Low protein diets for chronic kidney disease in non diabetic adults. Cochrane Database Syst Rev 2009: CD001892.

286. Fouque D, Laville M, Boissel JP et al. Controlled low protein diets in chronic renal insufficiency: meta-analysis. BMJ 1992; 304: 216-220.

287. Kasiske BL, Lakatua JD, Ma JZ et al. A meta-analysis of the effects of dietary protein restriction on the rate of decline in renal function. Am J Kidney Dis 1998; 31: 954-961.

288. Pedrini MT, Levey AS, Lau J et al. The effect of dietary protein restriction on the progression of diabetic and nondiabetic renal diseases: a metaanalysis. Ann Intern Med 1996; 124: 627-632.

289. Robertson L, Waugh N, Robertson A. Protein restriction for diabetic renal disease. Cochrane Database Syst Rev 2009: CD002181.
290. Menon V, Kopple JD, Wang X et al. Effect of a very low-protein diet on outcomes: long-term follow-up of the Modification of Diet in Renal Disease (MDRD) Study. Am J Kidney Dis 2009; 53: 208-217.

291. Knight EL, Stampfer MJ, Hankinson SE et al. The impact of protein intake on renal function decline in women with normal renal function or mild renal insufficiency. Ann Intern Med 2003; 138: 460-467.

292. Chaturvedi S, Jones C. Protein restriction for children with chronic renal failure. Cochrane Database Syst Rev 2007: CD006863.

293. National Kidney Foundation. KDOQI clinical practice guideline for diabetes and chronic kidney disease: 2012 Update. Am J Kidney Dis 2012; 60: 850-886.

294. Patel A, MacMahon S, Chalmers J et al. Intensive blood glucose control and vascular outcomes in patients with type 2 diabetes. $N$ Engl J Med 2008; 358: 2560-2572.

295. Ismail-Beigi F, Craven T, Banerji MA et al. Effect of intensive treatment of hyperglycaemia on microvascular outcomes in type 2 diabetes: an analysis of the ACCORD randomised trial. Lancet 2010; 376: 419-430.

296. Duckworth W, Abraira C, Moritz T et al. Glucose control and vascular complications in veterans with type 2 diabetes. N Engl J Med 2009; 360: 129-139.

297. Nathan DM, Zinman B, Cleary PA et al. Modern-day clinical course of type 1 diabetes mellitus after 30 years' duration: the diabetes control and complications trial/epidemiology of diabetes interventions and complications and Pittsburgh epidemiology of diabetes complications experience (1983-2005). Arch Intern Med 2009; 169: 1307-1316.

298. UK Prospective Diabetes Study (UKPDS) Group. Intensive blood-glucose control with sulphonylureas or insulin compared with conventional treatment and risk of complications in patients with type 2 diabetes (UKPDS 33). Lancet 1998; 352: 837-853.

299. Ichikawa $\mathrm{H}$, Nagake $\mathrm{Y}$, Takahashi $\mathrm{M}$ et al. What is the best index of glycemic control in patients with diabetes mellitus on hemodialysis? Nihon Jinzo Gakkai Shi 1996; 38: 305-308.

300. Joy MS, Cefalu WT, Hogan SL et al. Long-term glycemic control measurements in diabetic patients receiving hemodialysis. Am J Kidney Dis 2002; 39: 297-307.

301. Nakao T, Matsumoto H, Okada $T$ et al. Influence of erythropoietin treatment on hemoglobin A1c levels in patients with chronic renal failure on hemodialysis. Intern Med 1998; 37: 826-830.

302. $\mathrm{Ng} \mathrm{JM}$, Cooke $\mathrm{M}$, Bhandari $\mathrm{S}$ et al. The effect of iron and erythropoietin treatment on the $\mathrm{A} 1 \mathrm{C}$ of patients with diabetes and chronic kidney disease. Diabetes Care 2010; 33: 2310-2313.

303. Shima K, Chujo K, Yamada $\mathrm{M}$ et al. Lower value of glycated haemoglobin relative to glycaemic control in diabetic patients with end-stage renal disease not on haemodialysis. Ann Clin Biochem 2012; 49: 68-74.

304. Vos FE, Schollum JB, Coulter CV et al. Assessment of markers of glycaemic control in diabetic patients with chronic kidney disease using continuous glucose monitoring. Nephrology (Carlton) 2012; 17: 182-188.

305. American Diabetes Association. Executive summary: Standards of medical care in diabetes-2012. Diabetes Care 2012; 35 (Suppl 1): S4-S10.

306. Jones-Burton C, Mishra SI, Fink JC et al. An in-depth review of the evidence linking dietary salt intake and progression of chronic kidney disease. Am J Nephrol 2006; 26: 268-275.

307. Swift PA, Markandu ND, Sagnella GA et al. Modest salt reduction reduces blood pressure and urine protein excretion in black hypertensives: a randomized control trial. Hypertension 2005; 46: 308-312.

308. Hoffmann IS, Cubeddu LX. Increased blood pressure reactivity to dietary salt in patients with the metabolic syndrome. J Hum Hypertens 2007; 21: 438-444.

309. Bellizzi V, Di lorio BR, De Nicola L et al. Very low protein diet supplemented with ketoanalogs improves blood pressure control in chronic kidney disease. Kidney Int 2007; 71: 245-251.

310. Slagman MC, Waanders F, Hemmelder MH et al. Moderate dietary sodium restriction added to angiotensin converting enzyme inhibition compared with dual blockade in lowering proteinuria and blood pressure: randomised controlled trial. BMJ 2011; 343: d4366.

311. Bellomo G, Venanzi $S$, Verdura $C$ et al. Association of uric acid with change in kidney function in healthy normotensive individuals. $\mathrm{Am} J$ Kidney Dis 2010; 56: 264-272.

312. Iseki K, Ikemiya $Y$, Inoue $T$ et al. Significance of hyperuricemia as a risk factor for developing ESRD in a screened cohort. Am J Kidney Dis 2004; 44: $642-650$

313. Mok Y, Lee SJ, Kim MS et al. Serum uric acid and chronic kidney disease: the Severance cohort study. Nephrol Dial Transplant 2012; 27: 1831-1835.

314. Wen CP, David Cheng TY, Chan HT et al. Is high serum uric acid a risk marker or a target for treatment? Examination of its independent effect 
in a large cohort with low cardiovascular risk. Am J Kidney Dis 2010; 56: 273-288.

315. Yamada T, Fukatsu M, Suzuki S et al. Elevated serum uric acid predicts chronic kidney disease. Am J Med Sci 2011; 342: 461-466.

316. Goicoechea M, de Vinuesa SG, Verdalles U et al. Effect of allopurinol in chronic kidney disease progression and cardiovascular risk. Clin J Am Soc Nephrol 2010; 5: 1388-1393.

317. Siu YP, Leung KT, Tong MK et al. Use of allopurinol in slowing the progression of renal disease through its ability to lower serum uric acid level. Am J Kidney Dis 2006; 47: 51-59.

318. Kanbay $M$, Huddam $B$, Azak A et al. A randomized study of allopurinol on endothelial function and estimated glomular filtration rate in asymptomatic hyperuricemic subjects with normal renal function. Clin J Am Soc Nephrol 2011; 6: 1887-1894.

319. Kanbay M, Ozkara A, Selcoki Y et al. Effect of treatment of hyperuricemia with allopurinol on blood pressure, creatinine clearence, and proteinuria in patients with normal renal functions. Int Urol Nephrol 2007; 39: 1227-1233.

320. Kao MP, Ang DS, Gandy SJ et al. Allopurinol benefits left ventricular mass and endothelial dysfunction in chronic kidney disease. J Am Soc Nephrol 2011; 22: 1382-1389.

321. Saito J, Matsuzawa $\mathrm{Y}$, Ito $\mathrm{H}$ et al. The alkalizer citrate reduces serum uric Acid levels and improves renal function in hyperuricemic patients treated with the xanthine oxidase inhibitor allopurinol. Endocr Res 2010; 35: 145-154.

322. Malaguarnera $M$, Vacante $M$, Russo $C$ et al. A single dose of rasburicase in elderly patients with hyperuricaemia reduces serum uric acid levels and improves renal function. Expert Opin Pharmacother 2009; 10: 737-742.

323. Miao Y, Ottenbros SA, Laverman GD et al. Effect of a reduction in uric acid on renal outcomes during losartan treatment: a post hoc analysis of the reduction of endpoints in non-insulin-dependent diabetes mellitus with the Angiotensin II Antagonist Losartan Trial. Hypertension 2011; 58: 2-7.

324. Johansen KL. Exercise and chronic kidney disease: current recommendations. Sports Med 2005; 35: 485-499.

325. Johansen KL, Chertow GM, Ng AV et al. Physical activity levels in patients on hemodialysis and healthy sedentary controls. Kidney Int 2000; 57: 2564-2570.

326. Padilla J, Krasnoff J, Da Silva M et al. Physical functioning in patients with chronic kidney disease. J Nephrol 2008; 21: 550-559.

327. Beddhu S, Baird BC, Zitterkoph J et al. Physical activity and mortality in chronic kidney disease (NHANES III). Clin J Am Soc Nephrol 2009; 4: 1901-1906.

328. Churchill DN, Torrance GW, Taylor DW et al. Measurement of quality of life in end-stage renal disease: the time trade-off approach. Clin Invest Med 1987; 10: 14-20.

329. DeOreo PB. Hemodialysis patient-assessed functional health status predicts continued survival, hospitalization, and dialysis-attendance compliance. Am J Kidney Dis 1997; 30: 204-212.

330. Booth FW, Gordon SE, Carlson CJ et al. Waging war on modern chronic diseases: primary prevention through exercise biology. J Appl Physiol 2000; 88: 774-787.

331. Dickinson HO, Mason JM, Nicolson DJ et al. Lifestyle interventions to reduce raised blood pressure: a systematic review of randomized controlled trials. J Hypertens 2006; 24: 215-233.

332. Stewart KJ. Exercise training and the cardiovascular consequences of type 2 diabetes and hypertension: plausible mechanisms for improving cardiovascular health. JAMA 2002; 288: 1622-1631.

333. Daul AE, Schafers RF, Daul $\mathrm{K}$ et al. Exercise during hemodialysis. Clin Nephrol 2004; 61 (Suppl 1): S26-30.

334. Deligiannis A. Cardiac adaptations following exercise training in hemodialysis patients. Clin Nephrol 2004; 61 (Suppl 1): S39-45.

335. Liu SH, C. LC, Yeh SH et al. Effect of exercise training on hemodialysis. Journal of the Formosan Medical Association 2002; 6: 129-142.

336. Mustata S, Chan C, Lai V et al. Impact of an exercise program on arterial stiffness and insulin resistance in hemodialysis patients. J Am Soc Nephrol 2004; 15: 2713-2718.

337. Ouzouni S, Kouidi E, Sioulis A et al. Effects of intradialytic exercise training on health-related quality of life indices in haemodialysis patients. Clin Rehabil 2009; 23: 53-63.

338. Storer TW, Casaburi R, Sawelson $\mathrm{S}$ et al. Endurance exercise training during haemodialysis improves strength, power, fatigability and physical performance in maintenance haemodialysis patients. Nephrol Dial Transplant 2005; 20: 1429-1437.

339. Vaitkevicius PV, Fleg JL, Engel JH et al. Effects of age and aerobic capacity on arterial stiffness in healthy adults. Circulation 1993; 88: 1456-1462.
340. Mustata S, Groeneveld S, Davidson W et al. Effects of exercise training on physical impairment, arterial stiffness and health-related quality of life in patients with chronic kidney disease: a pilot study. Int Urol Nephrol 2011; 43: 1133-1141.

341. Szromba C, Thies MA, Ossman SS. Advancing chronic kidney disease care: new imperatives for recognition and intervention. Nephrol Nurs $J$ 2002; 29: 547-559.

342. Chen PY, Huang YC, Kao YH et al. Effects of an exercise program on blood biochemical values and exercise stage of chronic kidney disease patients. J Nurs Res 2010; 18: 98-107.

343. Tobita I, Suzuki S, Kobayashi $\mathrm{T}$ et al. A programme to encourage participation of haemodialysis patients in an exercise regimen. $J$ Ren Care 2009; 35: 48-53.

344. van Vilsteren MC, de Greef MH, Huisman RM. The effects of a low-tomoderate intensity pre-conditioning exercise programme linked with exercise counselling for sedentary haemodialysis patients in The Netherlands: results of a randomized clinical trial. Nephrol Dial Transplant 2005; 20: 141-146.

345. Kosmadakis GC, John SG, Clapp EL et al. Benefits of regular walking exercise in advanced pre-dialysis chronic kidney disease. Nephrol Dial Transplant 2012; 27: 997-1004.

346. Hall JE, Crook ED, Jones DW et al. Mechanisms of obesity-associated cardiovascular and renal disease. Am J Med Sci 2002; 324: 127-137.

347. Wahba IM, Mak RH. Obesity and obesity-initiated metabolic syndrome: mechanistic links to chronic kidney disease. Clin J Am Soc Nephrol 2007; 2: $550-562$.

348. Wang $Y$, Chen $X$, Song $Y$ et al. Association between obesity and kidney disease: a systematic review and meta-analysis. Kidney Int 2008; 73: 19-33.

349. Hobbs H, Farmer C, Irving J et al. Is high body mass index independently associated with diminished glomerular filtration rate? An epidemiological study. J Ren Care 2011; 37: 148-154.

350. Mohsen A, Brown R, Hoefield $R$ et al. Body mass index has no effect on rate of progression of chronic kidney disease in subjects with type 2 diabetes mellitus. J Nephrol 2012: 25: 384-393.

351. Burton JO, Gray LJ, Webb DR et al. Association of anthropometric obesity measures with chronic kidney disease risk in a non-diabetic patient population. Nephrol Dial Transplant 2012; 27: 1860-1866.

352. Kasiske BL, Napier J. Glomerular sclerosis in patients with massive obesity. Am J Nephrol 1985; 5: 45-50.

353. Hsu CY, McCulloch CE, Iribarren $C$ et al. Body mass index and risk for endstage renal disease. Ann Intern Med 2006; 144: 21-28.

354. Navaneethan SD, Yehnert H, Moustarah F et al. Weight loss interventions in chronic kidney disease: a systematic review and meta-analysis. Clin $J$ Am Soc Nephrol 2009; 4: 1565-1574.

355. Afshinnia F, Wilt TJ, Duval S et al. Weight loss and proteinuria: systematic review of clinical trials and comparative cohorts. Nephrol Dial Transplant 2010; 25: 1173-1183.

356. Orth SR, Hallan SI. Smoking: a risk factor for progression of chronic kidney disease and for cardiovascular morbidity and mortality in renal patients-absence of evidence or evidence of absence? Clin J Am Soc Nephrol 2008; 3: 226-236.

357. Jungers $P$, Massy ZA, Nguyen Khoa T et al. Incidence and risk factors of atherosclerotic cardiovascular accidents in predialysis chronic renal failure patients: a prospective study. Nephrol Dial Transplant 1997; 12: 2597-2602.

358. Muntner $\mathrm{P}, \mathrm{He} \mathrm{J}$, Astor $\mathrm{BC}$ et al. Traditional and nontraditional risk factors predict coronary heart disease in chronic kidney disease: results from the atherosclerosis risk in communities study. J Am Soc Nephrol 2005; 16: 529-538.

359. Myllymaki J, Syrjanen J, Helin $\mathrm{H}$ et al. Vascular diseases and their risk factors in IgA nephropathy. Nephrol Dial Transplant 2006; 21: 1876-1882.

360. Shlipak MG, Fried LF, Cushman $M$ et al. Cardiovascular mortality risk in chronic kidney disease: comparison of traditional and novel risk factors. JAMA 2005; 293: 1737-1745.

361. Chase HP, Garg SK, Marshall G et al. Cigarette smoking increases the risk of albuminuria among subjects with type I diabetes. JAMA 1991; 265: 614-617.

362. Gambaro G, Bax G, Fusaro M et al. Cigarette smoking is a risk factor for nephropathy and its progression in type 2 diabetes mellitus. Diabetes Nutr Metab 2001; 14: 337-342.

363. Hallan SI, Orth SR. Smoking is a risk factor in the progression to kidney failure. Kidney Int 2011; 80: 516-523.

364. Sawicki PT, Didjurgeit U, Muhlhauser I et al. Smoking is associated with progression of diabetic nephropathy. Diabetes Care 1994; 17: 126-131. 
365. Sung RS, Althoen M, Howell TA et al. Excess risk of renal allograft loss associated with cigarette smoking. Transplantation 2001; 71: 1752-1757.

366. Inker LA, Coresh J, Levey AS et al. Estimated GFR, albuminuria, and complications of chronic kidney disease. J Am Soc Nephrol 2011; 22: 2322-2331.

367. Levin $A$, Bakris $G L$, Molitch $M$ et al. Prevalence of abnormal serum vitamin D, PTH, calcium, and phosphorus in patients with chronic kidney disease: results of the study to evaluate early kidney disease. Kidney Int 2007; 71: 31-38.

368. World Health Organization. Worldwide prevalence of anaemia 1993-2005: WHO global database on anaemia. de Benoist B, McLean E, Egli I, and Cogswell M (eds), 2008.

369. Beall CM, Goldstein MC. Hemoglobin concentration of pastoral nomads permanently resident at 4,850-5,450 meters in Tibet. Am J Phys Anthropol 1987; 73: 433-438.

370. Cresanta JL, Croft JB, Webber LS et al. Racial difference in hemoglobin concentration of young adults. Prev Med 1987; 16: 659-669.

371. Meyers LD, Habicht JP, Johnson CL. Components of the difference in hemoglobin concentrations in blood between black and white women in the United States. Am J Epidemiol 1979; 109: 539-549.

372. Pan WH, Habicht JP. The non-iron-deficiency-related difference in hemoglobin concentration distribution between blacks and whites and between men and women. Am J Epidemiol 1991; 134: 1410-1416.

373. Centers for Disease Control and Prevention. CDC criteria for anemia in children and childbearing-aged women. MMWR Morb Mortal Wkly Rep 1989; 38: 400-404.

374. Levin A, Djurdjev O, Thompson $\mathrm{C}$ et al. Canadian randomized trial of hemoglobin maintenance to prevent or delay left ventricular mass growth in patients with CKD. Am J Kidney Dis 2005; 46: 799-811.

375. Pfeffer MA, Burdmann EA, Chen CY et al. A trial of darbepoetin alfa in type 2 diabetes and chronic kidney disease. N Engl J Med 2009; 361: 2019-2032.

376. Roger SD, McMahon LP, Clarkson A et al. Effects of early and late intervention with epoetin alpha on left ventricular mass among patients with chronic kidney disease (stage 3 or 4): results of a randomized clinical trial. J Am Soc Nephrol 2004; 15: 148-156.

377. Moranne O, Froissart M, Rossert J et al. Timing of onset of CKD-related metabolic complications. J Am Soc Nephrol 2009; 20: 164-171.

378. Vassalotti JA, Uribarri J, Chen SC et al. Trends in mineral metabolism: Kidney Early Evaluation Program (KEEP) and the National Health and Nutrition Examination Survey (NHANES) 1999-2004. Am J Kidney Dis 2008; 51: S56-68.

379. Stevens LA, Djurdjev O, Cardew S et al. Calcium, phosphate, and parathyroid hormone levels in combination and as a function of dialysis duration predict mortality: evidence for the complexity of the association between mineral metabolism and outcomes. J Am Soc Nephrol 2004; 15: 770-779.

380. Gutierrez OM, Isakova T, Andress DL et al. Prevalence and severity of disordered mineral metabolism in Blacks with chronic kidney disease. Kidney Int 2008; 73: 956-962.

381. Gutierrez OM, Farwell WR, Kermah D et al. Racial differences in the relationship between vitamin $D$, bone mineral density, and parathyroid hormone in the National Health and Nutrition Examination Survey. Osteoporos Int 2011; 22: 1745-1753.

382. National Kidney Foundation. KDOQI clinical practice guideline for nutrition in children with CKD: 2008 update. Am J Kidney Dis 2009; 53: S1-124.

383. Nickolas TL, Leonard MB, Shane E. Chronic kidney disease and bone fracture: a growing concern. Kidney Int 2008; 74: 721-731.

384. Bacchetta J, Boutroy S, Vilayphiou $\mathrm{N}$ et al. Early impairment of trabecular microarchitecture assessed with HR-pQCT in patients with stage II-IV chronic kidney disease. J Bone Miner Res 2010; 25: 849-857.

385. Nickolas TL, Stein E, Cohen A et al. Bone mass and microarchitecture in CKD patients with fracture. J Am Soc Nephrol 2010; 21: 1371-1380.

386. Nickolas TL, Cremers S, Zhang A et al. Discriminants of prevalent fractures in chronic kidney disease. J Am Soc Nephrol 2011; 22: 1560-1572.

387. Palmer SC, Hayen A, Macaskill P et al. Serum levels of phosphorus, parathyroid hormone, and calcium and risks of death and cardiovascular disease in individuals with chronic kidney disease: a systematic review and meta-analysis. JAMA 2011; 305: 1119-1127.

388. Adeney KL, Siscovick DS, Ix JH et al. Association of serum phosphate with vascular and valvular calcification in moderate CKD. J Am Soc Nephrol 2009; 20: 381-387.
389. Moe SM, Zidehsarai MP, Chambers MA et al. Vegetarian compared with meat dietary protein source and phosphorus homeostasis in chronic kidney disease. Clin J Am Soc Nephrol 2011; 6: 257-264.

390. Sigrist MK, Chiarelli G, Lim L et al. Early initiation of phosphate lowering dietary therapy in non-dialysis chronic kidney disease: a critical review. J Ren Care 2009; 35 (Suppl 1): 71-78.

391. Lynch KE, Lynch R, Curhan GC et al. Prescribed dietary phosphate restriction and survival among hemodialysis patients. Clin $J$ Am Soc Nephrol 2011; 6: 620-629.

392. Navaneethan SD, Palmer SC, Vecchio $M$ et al. Phosphate binders for preventing and treating bone disease in chronic kidney disease patients. Cochrane Database Syst Rev 2011: CD006023.

393. Mehrotra R, Kermah D, Budoff $M$ et al. Hypovitaminosis D in chronic kidney disease. Clin J Am Soc Nephrol 2008; 3: 1144-1151.

394. Navaneethan SD, Schold JD, Arrigain S et al. Low 25-hydroxyvitamin D levels and mortality in non-dialysis-dependent CKD. Am J Kidney Dis 2011; 58: 536-543.

395. Kandula P, Dobre M, Schold JD et al. Vitamin D supplementation in chronic kidney disease: a systematic review and meta-analysis of observational studies and randomized controlled trials. Clin J Am Soc Nephrol 2011; 6: 50-62.

396. Palmer SC, McGregor DO, Craig JC et al. Vitamin D compounds for people with chronic kidney disease not requiring dialysis. Cochrane Database Syst Rev 2009: CD008175.

397. Miller PD. The kidney and bisphosphonates. Bone 2011; 49: 77-81.

398. Torregrosa JV, Ramos AM. [Use of bisphosphonates in chronic kidney disease]. Nefrologia 2010; 30: 288-296.

399. Bhan I, Dubey A, Wolf M. Diagnosis and management of mineral metabolism in CKD. J Gen Intern Med 2010; 25: 710-716.

400. Courtney $A E$, Leonard $N$, McCloskey $M C$ et al. Bisphosphonate prescribing in chronic kidney disease. J R Coll Physicians Edinb 2009; 39: 4-9.

401. Ayus JC, Krothapalli RK. Effect of bicarbonate administration on cardiac function. Am J Med 1989; 87: 5-6.

402. Bailey JL, Wang $X$, England BK et al. The acidosis of chronic renal failure activates muscle proteolysis in rats by augmenting transcription of genes encoding proteins of the ATP-dependent ubiquitin-proteasome pathway. J Clin Invest 1996; 97: 1447-1453.

403. Domrongkitchaiporn S, Pongskul C, Sirikulchayanonta V et al. Bone histology and bone mineral density after correction of acidosis in distal renal tubular acidosis. Kidney Int 2002; 62: 2160-2166.

404. Kovesdy CP, Anderson JE, Kalantar-Zadeh K. Association of serum bicarbonate levels with mortality in patients with non-dialysis-dependent CKD. Nephrol Dial Transplant 2009; 24: 1232-1237.

405. Mak RH. Effect of metabolic acidosis on insulin action and secretion in uremia. Kidney Int 1998; 54: 603-607.

406. Menon V, Tighiouart $H$, Vaughn NS et al. Serum bicarbonate and long-term outcomes in CKD. Am J Kidney Dis 2010; 56: 907-914.

407. Mitch WE, Price SR. Mechanisms activated by kidney disease and the loss of muscle mass. Am J Kidney Dis 2001; 38: 1337-1342.

408. Navaneethan SD, Schold JD, Arrigain S et al. Serum bicarbonate and mortality in stage 3 and stage 4 chronic kidney disease. Clin J Am Soc Nephrol 2011; 6: 2395-2402.

409. Raphael KL, Wei G, Baird BC et al. Higher serum bicarbonate levels within the normal range are associated with better survival and renal outcomes in African Americans. Kidney Int 2011; 79: 356-362.

410. Shah SN, Abramowitz M, Hostetter TH et al. Serum bicarbonate levels and the progression of kidney disease: a cohort study. Am J Kidney Dis 2009; 54: 270-277.

411. Bright R. Reports of Medical Cases, Selected with a View of Illustrating the Symptoms and Cure of Diseases by a Reference to Morbid Anatomy, Volume 1. London: Brown \& Green. 1827.

412. Osman AA. The value of alkalis in the treatment of chronic nephritis. Lancet 1930; 2: 945-959.

413. Lyon DM, Dunlop DM, Stewart CP. The alkaline treatment of chronic nephritis. Lancet 1931; 2: 1009-1013.

414. Rustom R, Grime JS, Costigan M et al. Oral sodium bicarbonate reduces proximal renal tubular peptide catabolism, ammoniogenesis, and tubular damage in renal patients. Ren Fail 1998; 20: 371-382.

415. Mathur RP, Dash SC, Gupta N et al. Effects of correction of metabolic acidosis on blood urea and bone metabolism in patients with mild to moderate chronic kidney disease: a prospective randomized single blind controlled trial. Ren Fail 2006; 28: 1-5.

416. de Brito-Ashurst I, Varagunam $M$, Raftery $M J$ et al. Bicarbonate supplementation slows progression of CKD and improves nutritional status. J Am Soc Nephrol 2009; 20: 2075-2084. 
417. Phisitkul S, Khanna A, Simoni J et al. Amelioration of metabolic acidosis in patients with low GFR reduced kidney endothelin production and kidney injury, and better preserved GFR. Kidney Int 2010; 77: 617-623.

418. Husted FC, Nolph KD, Maher JF. NaHCO3 and $\mathrm{NaCl}$ tolerance in chronic renal failure. J Clin Invest 1975; 56: 414-419.

419. Husted FC, Nolph KD. $\mathrm{NaHCO}_{3}$ and $\mathrm{NaCl}$ tolerance in chronic renal failure II. Clin Nephrol 1977; 7: 21-25.

420. Hallan SI, Dahl K, Oien CM et al. Screening strategies for chronic kidney disease in the general population: follow-up of cross sectional health survey. BMJ 2006; 333: 1047.

421. McCullough PA, Li S, Jurkovitz CT et al. Chronic kidney disease, prevalence of premature cardiovascular disease, and relationship to short-term mortality. Am Heart J 2008; 156: 277-283.

422. Anavekar NS, McMurray JJ, Velazquez EJ et al. Relation between renal dysfunction and cardiovascular outcomes after myocardial infarction. N Engl J Med 2004; 351: 1285-1295.

423. Shlipak MG, Heidenreich PA, Noguchi $\mathrm{H}$ et al. Association of renal insufficiency with treatment and outcomes after myocardial infarction in elderly patients. Ann Intern Med 2002; 137: 555-562.

424. Wright RS, Reeder GS, Herzog CA et al. Acute myocardial infarction and renal dysfunction: a high-risk combination. Ann Intern Med 2002; 137: 563-570.

425. Sarnak MJ. Cardiovascular complications in chronic kidney disease. Am J Kidney Dis 2003; 41: 11-17.

426. Hemmelgarn BR, Manns BJ, Lloyd A et al. Relation between kidney function, proteinuria, and adverse outcomes. JAMA 2010; 303: 423-429.

427. Klausen K, Borch-Johnsen K, Feldt-Rasmussen B et al. Very low levels of microalbuminuria are associated with increased risk of coronary heart disease and death independently of renal function, hypertension, and diabetes. Circulation 2004; 110: 32-35.

428. Gerstein HC, Mann JF, Yi Q et al. Albuminuria and risk of cardiovascular events, death, and heart failure in diabetic and nondiabetic individuals. JAMA 2001; 286: 421-426.

429. Hallan S, Astor B, Romundstad S et al. Association of kidney function and albuminuria with cardiovascular mortality in older vs younger individuals: The HUNT II Study. Arch Intern Med 2007; 167: 2490-2496.

430. Ibsen $\mathrm{H}$, Wachtell $\mathrm{K}$, Olsen $\mathrm{MH}$ et al. Albuminuria and cardiovascular risk in hypertensive patients with left ventricular hypertrophy: the LIFE Study. Kidney Int Suppl 2004; 92: S56-58.

431. Menon V, Shlipak MG, Wang $X$ et al. Cystatin $C$ as a risk factor for outcomes in chronic kidney disease. Ann Intern Med 2007; 147: 19-27.

432. Peralta CA, Katz R, Sarnak MJ et al. Cystatin C identifies chronic kidney disease patients at higher risk for complications. J Am Soc Nephrol 2011; 22: 147-155.

433. Phrommintikul A, Haas SJ, Elsik $\mathrm{M}$ et al. Mortality and target haemoglobin concentrations in anaemic patients with chronic kidney disease treated with erythropoietin: a meta-analysis. Lancet 2007; 369: 381-388.

434. Strippoli GF, Tognoni G, Navaneethan SD et al. Haemoglobin targets: we were wrong, time to move on. Lancet 2007; 369: 346-350.

435. Eckardt KU, Scherhag A, Macdougall IC et al. Left ventricular geometry predicts cardiovascular outcomes associated with anemia correction in CKD. J Am Soc Nephrol 2009; 20: 2651-2660.

436. Kestenbaum B, Sampson JN, Rudser KD et al. Serum phosphate levels and mortality risk among people with chronic kidney disease. J Am Soc Nephrol 2005; 16: 520-528.

437. Groothoff JW, Gruppen MP, Offringa M et al. Mortality and causes of death of end-stage renal disease in children: a Dutch cohort study. Kidney Int 2002; 61: 621-629.

438. McDonald SP, Craig JC. Long-term survival of children with end-stage renal disease. N Engl J Med 2004; 350: 2654-2662.

439. Oh J, Wunsch R, Turzer $M$ et al. Advanced coronary and carotid arteriopathy in young adults with childhood-onset chronic renal failure. Circulation 2002; 106: 100-105.

440. Parekh RS, Carroll CE, Wolfe RA et al. Cardiovascular mortality in children and young adults with end-stage kidney disease. J Pediatr 2002; 141: 191-197.

441. Kavey RE, Allada V, Daniels SR et al. Cardiovascular risk reduction in highrisk pediatric patients: a scientific statement from the American Heart Association Expert Panel on Population and Prevention Science; the Councils on Cardiovascular Disease in the Young, Epidemiology and Prevention, Nutrition, Physical Activity and Metabolism, High Blood Pressure Research, Cardiovascular Nursing, and the Kidney in Heart Disease; and the Interdisciplinary Working Group on Quality of Care and Outcomes Research: endorsed by the American Academy of Pediatrics. Circulation 2006; 114: 2710-2738.
442. Wilson AC, Schneider MF, Cox C et al. Prevalence and correlates of multiple cardiovascular risk factors in children with chronic kidney disease. Clin J Am Soc Nephrol 2011; 6: 2759-2765.

443. Litwin $M$, Wuhl $E_{1}$ Jourdan $C$ et al. Altered morphologic properties of large arteries in children with chronic renal failure and after renal transplantation. J Am Soc Nephrol 2005; 16: 1494-1500.

444. Mitsnefes MM, Kimball TR, Kartal J et al. Cardiac and vascular adaptation in pediatric patients with chronic kidney disease: role of calciumphosphorus metabolism. J Am Soc Nephrol 2005; 16: 2796-2803.

445. Mitsnefes MM. Cardiovascular disease in children with chronic kidney disease. J Am Soc Nephrol 2012; 23: 578-585.

446. Shroff R, Weaver DJ, Jr., Mitsnefes MM. Cardiovascular complications in children with chronic kidney disease. Nat Rev Nephrol 2011; 7: 642-649.

447. Halimi JM, Giraudeau B, Vol S et al. Effects of current smoking and smoking discontinuation on renal function and proteinuria in the general population. Kidney Int 2000; 58: 1285-1292.

448. Boyce ML, Robergs RA, Avasthi PS et al. Exercise training by individuals with predialysis renal failure: cardiorespiratory endurance, hypertension, and renal function. Am J Kidney Dis 1997; 30: 180-192.

449. Baigent C, Landray MJ, Reith $C$ et al. The effects of lowering LDL cholesterol with simvastatin plus ezetimibe in patients with chronic kidney disease (Study of Heart and Renal Protection): a randomised placebo-controlled trial. Lancet 2011; 377: 2181-2192.

450. Jardine MJ, Ninomiya T, Perkovic $V$ et al. Aspirin is beneficial in hypertensive patients with chronic kidney disease: a post-hoc subgroup analysis of a randomized controlled trial. J Am Coll Cardiol 2010; 56: 956-965.

451. Angiolillo DJ, Bernardo E, Capodanno D et al. Impact of chronic kidney disease on platelet function profiles in diabetes mellitus patients with coronary artery disease taking dual antiplatelet therapy. J Am Coll Cardiol 2010; 55: 1139-1146.

452. Best PJ, Steinhubl SR, Berger PB et al. The efficacy and safety of shortand long-term dual antiplatelet therapy in patients with mild or moderate chronic kidney disease: results from the Clopidogrel for the Reduction of Events During Observation (CREDO) trial. Am Heart J 2008; 155: 687-693.

453. Graham I, Atar D, Borch-Johnsen K et al. European guidelines on cardiovascular disease prevention in clinical practice: executive summary. Fourth Joint Task Force of the European Society of Cardiology and other societies on cardiovascular disease prevention in clinical practice (constituted by representatives of nine societies and by invited experts). Eur J Cardiovasc Prev Rehabil 2007; 14 (Suppl 2): E1-40.

454. Hippisley-Cox J, Coupland C, Vinogradova Y et al. Predicting cardiovascular risk in England and Wales: prospective derivation and validation of QRISK2. BMJ 2008; 336: 1475-1482.

455. Joint British Societies. JBS 2: Joint British Societies' guidelines on prevention of cardiovascular disease in clinical practice. Heart 2005; 91 (Suppl 5): v1-52.

456. Wu Y, Liu X, Li X et al. Estimation of 10-year risk of fatal and nonfatal ischemic cardiovascular diseases in Chinese adults. Circulation 2006; 114: 2217-2225

457. Liau SY, Izham MI, Hassali MA et al. A literature review of the cardiovascular risk-assessment tools: applicability among Asian population. Heart Asia 2010; 2: 15-18.

458. Nakayama M, Sato T, Sato $\mathrm{H}$ et al. Different clinical outcomes for cardiovascular events and mortality in chronic kidney disease according to underlying renal disease: the Gonryo study. Clin Exp Nephrol 2010; 14: 333-339.

459. Irie $\mathrm{F}$, Iso $\mathrm{H}$, Sairenchi $\mathrm{T}$ et al. The relationships of proteinuria, serum creatinine, glomerular filtration rate with cardiovascular disease mortality in Japanese general population. Kidney Int 2006; 69: 1264-1271.

460. Yang JG, Li J, Lu C et al. Chronic kidney disease, all-cause mortality and cardiovascular mortality among Chinese patients with established cardiovascular disease. J Atheroscler Thromb 2010; 17: 395-401.

461. Zhang L, Zuo L, Wang F et al. Cardiovascular disease in early stages of chronic kidney disease in a Chinese population. J Am Soc Nephrol 2006; 17: 2617-2621.

462. Banerjee D, Chitalia N, Raja R et al. Metabolic syndrome in chronic kidney disease and renal transplant patients in North India. Int Urol Nephrol 2012; 44: 937-943.

463. Di Angelantonio E, Chowdhury R, Sarwar N et al. Chronic kidney disease and risk of major cardiovascular disease and non-vascular mortality: prospective population based cohort study. BMJ 2010; 341: c4986.

464. Parikh NI, Hwang SJ, Larson MG et al. Chronic kidney disease as a predictor of cardiovascular disease (from the Framingham Heart Study). Am J Cardiol 2008; 102: 47-53. 
465. Eiland LS, Luttrell PK. Use of statins for dyslipidemia in the pediatric population. J Pediatr Pharmacol Ther 2010; 15: 160-172.

466. McAlister FA, Ezekowitz J, Tonelli $M$ et al. Renal insufficiency and heart failure: prognostic and therapeutic implications from a prospective cohort study. Circulation 2004; 109: 1004-1009.

467. Smith GL, Lichtman JH, Bracken MB et al. Renal impairment and outcomes in heart failure: systematic review and meta-analysis. J Am Coll Cardiol 2006; 47: 1987-1996.

468. Hillege $\mathrm{HL}$, Nitsch D, Pfeffer MA et al. Renal function as a predictor of outcome in a broad spectrum of patients with heart failure. Circulation 2006; 113: 671-678.

469. Shlipak MG, Smith GL, Rathore SS et al. Renal function, digoxin therapy, and heart failure outcomes: evidence from the digoxin intervention group trial. J Am Soc Nephrol 2004; 15: 2195-2203.

470. Smith GL, Shlipak MG, Havranek EP et al. Race and renal impairment in heart failure: mortality in blacks versus whites. Circulation 2005; 111: 1270-1277.

471. Anand IS, Bishu K, Rector TS et al. Proteinuria, chronic kidney disease, and the effect of an angiotensin receptor blocker in addition to an angiotensin-converting enzyme inhibitor in patients with moderate to severe heart failure. Circulation 2009; 120: 1577-1584.

472. Ezekowitz J, McAlister FA, Humphries KH et al. The association among renal insufficiency, pharmacotherapy, and outcomes in 6,427 patients with heart failure and coronary artery disease. J Am Coll Cardiol 2004; 44: 1587-1592.

473. Gottlieb SS, Abraham W, Butler J et al. The prognostic importance of different definitions of worsening renal function in congestive heart failure. J Card Fail 2002; 8: 136-141.

474. Middleton RJ, Parfrey PS, Foley RN. Left ventricular hypertrophy in the renal patient. J Am Soc Nephrol 2001; 12: 1079-1084.

475. Levin A, Singer J, Thompson CR et al. Prevalent left ventricular hypertrophy in the predialysis population: identifying opportunities for intervention. Am J Kidney Dis 1996; 27: 347-354.

476. Ha SK, Park HS, Kim SJ et al. Prevalence and patterns of left ventricular hypertrophy in patients with predialysis chronic renal failure. $J$ Korean Med Sci 1998; 13: 488-494.

477. Parfrey PS, Foley RN, Harnett JD et al. Outcome and risk factors for left ventricular disorders in chronic uraemia. Nephrol Dial Transplant 1996; 11: 1277-1285.

478. Cerasola G, Nardi E, Palermo A et al. Epidemiology and pathophysiology of left ventricular abnormalities in chronic kidney disease: a review. J Nephrol 2011; 24: 1-10.

479. Ahmed A, Rich MW, Sanders PW et al. Chronic kidney disease associated mortality in diastolic versus systolic heart failure: a propensity matched study. Am J Cardiol 2007; 99: 393-398.

480. Lazzeri C, Valente S, Tarquini R et al. Cardiorenal syndrome caused by heart failure with preserved ejection fraction. Int J Nephrol 2011; 634903.

481. Levin A, Thompson CR, Ethier J et al. Left ventricular mass index increase in early renal disease: impact of decline in hemoglobin. Am J Kidney Dis 1999; 34: 125-134.

482. Go AS, Yang J, Ackerson LM et al. Hemoglobin level, chronic kidney disease, and the risks of death and hospitalization in adults with chronic heart failure: the Anemia in Chronic Heart Failure: Outcomes and Resource Utilization (ANCHOR) Study. Circulation 2006; 113: 2713-2723.

483. Shlipak MG, Katz R, Kestenbaum B et al. Clinical and subclinical cardiovascular disease and kidney function decline in the elderly. Atherosclerosis 2009; 204: 298-303.

484. Balamuthusamy S, Srinivasan L, Verma M et al. Renin angiotensin system blockade and cardiovascular outcomes in patients with chronic kidney disease and proteinuria: a meta-analysis. Am Heart J 2008; 155: 791-805.

485. Lewis EJ, Hunsicker LG, Clarke WR et al. Renoprotective effect of the angiotensin-receptor antagonist irbesartan in patients with nephropathy due to type 2 diabetes. $N$ Engl J Med 2001; 345: 851-860.

486. Erdmann $E$, Lechat $P$, Verkenne $P$ et al. Results from post-hoc analyses of the CIBIS II trial: effect of bisoprolol in high-risk patient groups with chronic heart failure. Eur J Heart Fail 2001; 3: 469-479.

487. Wali RK, lyengar M, Beck GJ et al. Efficacy and safety of carvedilol in treatment of heart failure with chronic kidney disease: a meta-analysis of randomized trials. Circ Heart Fail 2011; 4: 18-26.

488. Pitt B, Zannad F, Remme WJ et al. The effect of spironolactone on morbidity and mortality in patients with severe heart failure. Randomized Aldactone Evaluation Study Investigators. N Engl J Med 1999; 341: 709-717.

489. Juurlink DN, Mamdani MM, Lee DS et al. Rates of hyperkalemia after publication of the Randomized Aldactone Evaluation Study. N Engl J Med 2004; 351: 543-551.
490. Abdel-Qadir HM, Chugh S, Lee DS. Improving prognosis estimation in patients with heart failure and the cardiorenal syndrome. Int J Nephrol 2011; 351672.

491. Levin ER, Gardner DG, Samson WK. Natriuretic peptides. N Engl J Med 1998; 339: 321-328.

492. Wiley $\mathrm{CL}$, Switzer SP, Berg RL et al. Association of B-type natriuretic Peptide levels with estimated glomerular filtration rate and congestive heart failure. Clin Med Res 2010; 8: 7-12.

493. Madsen $\mathrm{LH}$, Ladefoged $\mathrm{S}$, Corell $\mathrm{P}$ et al. $\mathrm{N}$-terminal pro brain natriuretic peptide predicts mortality in patients with end-stage renal disease in hemodialysis. Kidney Int 2007; 71: 548-554.

494. Gustafsson F, Steensgaard-Hansen F, Badskjaer J et al. Diagnostic and prognostic performance of $\mathrm{N}$-terminal ProBNP in primary care patients with suspected heart failure. J Card Fail 2005; 11: S15-20.

495. McCullough PA, Omland T, Maisel AS. B-type natriuretic peptides: a diagnostic breakthrough for clinicians. Rev Cardiovasc Med 2003; 4: 72-80.

496. Maisel A, Mehra MR. Understanding B-type natriuretic peptide and its role in diagnosing and monitoring congestive heart failure. Clin Cornerstone 2005; 7 (Suppl 1): S7-17.

497. Wright SP, Doughty RN, Pearl A et al. Plasma amino-terminal pro-brain natriuretic peptide and accuracy of heart-failure diagnosis in primary care: a randomized, controlled trial. J Am Coll Cardiol 2003; 42: 1793-1800.

498. Yamamoto K, Burnett JC, Jr., Jougasaki $M$ et al. Superiority of brain natriuretic peptide as a hormonal marker of ventricular systolic and diastolic dysfunction and ventricular hypertrophy. Hypertension 1996; 28: 988-994.

499. Burnett JC, Jr., Kao PC, Hu DC et al. Atrial natriuretic peptide elevation in congestive heart failure in the human. Science 1986; 231: 1145-1147.

500. Mueller C, Laule-Kilian K, Scholer A et al. B-type natriuretic peptide for acute dyspnea in patients with kidney disease: insights from a randomized comparison. Kidney Int 2005; 67: 278-284.

501. Vickery S, Price CP, John Rl et al. B-type natriuretic peptide (BNP) and amino-terminal proBNP in patients with CKD: relationship to renal function and left ventricular hypertrophy. Am J Kidney Dis 2005; 46: 610-620.

502. Ishibe S, Peixoto AJ. Methods of assessment of volume status and intercompartmental fluid shifts in hemodialysis patients: implications in clinical practice. Semin Dial 2004; 17: 37-43.

503. Takahashi $\mathrm{M}$, Nagake $\mathrm{Y}$, Ichikawa $\mathrm{H}$ et al. Plasma concentrations of natriuretic peptides in patients on hemodialysis. Res Commun Mol Pathol Pharmacol 1996; 92: 19-30.

504. Tagore $\mathrm{R}$, Ling $\mathrm{LH}$, Yang $\mathrm{H}$ et al. Natriuretic peptides in chronic kidney disease. Clin J Am Soc Nephrol 2008; 3: 1644-1651.

505. Takami $Y$, Horio $T$, Iwashima $Y$ et al. Diagnostic and prognostic value of plasma brain natriuretic peptide in non-dialysis-dependent CRF. Am J Kidney Dis 2004; 44: 420-428.

506. Zoccali C, Mallamaci F, Benedetto FA et al. Cardiac natriuretic peptides are related to left ventricular mass and function and predict mortality in dialysis patients. J Am Soc Nephrol 2001; 12: 1508-1515.

507. Suresh M, Farrington K. Natriuretic peptides and the dialysis patient. Semin Dial 2005; 18: 409-419.

508. Kistorp C, Raymond I, Pedersen F et al. N-terminal pro-brain natriuretic peptide, C-reactive protein, and urinary albumin levels as predictors of mortality and cardiovascular events in older adults. JAMA 2005; 293: 1609-1616.

509. Bettencourt P, Azevedo A, Pimenta J et al. N-terminal-pro-brain natriuretic peptide predicts outcome after hospital discharge in heart failure patients. Circulation 2004; 110: 2168-2174.

510. Gardner RS, Ozalp F, Murday AJ et al. N-terminal pro-brain natriuretic peptide. A new gold standard in predicting mortality in patients with advanced heart failure. Eur Heart J 2003; 24: 1735-1743.

511. Kragelund $C B$, Gronning $B A$, Kober $L$ et al. [Prognostic value of $\mathrm{N}$-terminal pro-BNP-type natriuretic peptide in patients with stable coronary heart disease-secondary publication]. Ugeskr Laeger 2006; 168: 697-700.

512. Omland $\mathrm{T}$, Persson $\mathrm{A}, \mathrm{Ng} \mathrm{L}$ et al. $\mathrm{N}$-terminal pro-B-type natriuretic peptide and long-term mortality in acute coronary syndromes. Circulation 2002; 106: 2913-2918.

513. Olsen $M H$, Wachtell $K$, Tuxen $C$ et al. $\mathrm{N}$-terminal pro-brain natriuretic peptide predicts cardiovascular events in patients with hypertension and left ventricular hypertrophy: a LIFE study. J Hypertens 2004; 22: 1597-1604.

514. Koch A, Singer H. Normal values of B type natriuretic peptide in infants, children, and adolescents. Heart 2003; 89: 875-878. 
515. Auerbach SR, Richmond ME, Lamour JM et al. BNP levels predict outcome in pediatric heart failure patients: post hoc analysis of the Pediatric Carvedilol Trial. Circ Heart Fail 2010; 3: 606-611.

516. Barret BJ, Culleton B. Reducing the burden of cardiovascular disease in patients on dialysis. Dial Transplant 2002; 31: 155-163.

517. Beciani M, Tedesco A, Violante A et al. Cardiac troponin I (2nd generation assay) in chronic haemodialysis patients: prevalence and prognostic value. Nephrol Dial Transplant 2003; 18: 942-946.

518. Apple FS, Wu AH. Myocardial infarction redefined: role of cardiac troponin testing. Clin Chem 2001; 47: 377-379.

519. McLaurin MD, Apple FS, Voss EM et al. Cardiac troponin I, cardiac troponin $\mathrm{T}$, and creatine kinase $\mathrm{MB}$ in dialysis patients without ischemic heart disease: evidence of cardiac troponin $\mathrm{T}$ expression in skeletal muscle. Clin Chem 1997; 43: 976-982.

520. Musso P, Cox I, Vidano E et al. Cardiac troponin elevations in chronic renal failure: prevalence and clinical significance. Clin Biochem 1999; 32: 125-130.

521. Alpert JS, Thygesen K, Antman E et al. Myocardial infarction redefined-a consensus document of The Joint European Society of Cardiology/ American College of Cardiology Committee for the redefinition of myocardial infarction. J Am Coll Cardiol 2000; 36: 959-969.

522. Collinson PO, Boa FG, Gaze DC. Measurement of cardiac troponins. Ann Clin Biochem 2001; 38: 423-449.

523. Lindahl B, Venge $P$, Wallentin L. Troponin $T$ identifies patients with unstable coronary artery disease who benefit from long-term antithrombotic protection. Fragmin in Unstable Coronary Artery Disease (FRISC) Study Group. J Am Coll Cardiol 1997; 29: 43-48.

524. Rabbani LE. Acute coronary syndromes-beyond myocyte necrosis. $N$ Engl J Med 2001; 345: 1057-1059.

525. Tun A, Khan IA, Win MT et al. Specificity of cardiac troponin I and creatine kinase-MB isoenzyme in asymptomatic long-term hemodialysis patients and effect of hemodialysis on these cardiac markers. Cardiology 1998; 90: 280-285.

526. Wood GN, Keevil B, Gupta J et al. Serum troponin T measurement in patients with chronic renal impairment predicts survival and vascular disease: a 2 year prospective study. Nephrol Dial Transplant 2003; 18: 1610-1615.

527. Abbas NA, John RI, Webb MC et al. Cardiac troponins and renal function in nondialysis patients with chronic kidney disease. Clin Chem 2005; 51: 2059-2066.

528. Kinnunen $\mathrm{P}$, Vuolteenaho $\mathrm{O}$, Ruskoaho $\mathrm{H}$. Mechanisms of atrial and brain natriuretic peptide release from rat ventricular myocardium: effect of stretching. Endocrinology 1993; 132: 1961-1970.

529. deFilippi C, Wasserman S, Rosanio S et al. Cardiac troponin $\mathrm{T}$ and C-reactive protein for predicting prognosis, coronary atherosclerosis, and cardiomyopathy in patients undergoing long-term hemodialysis. JAMA 2003; 290: 353-359.

530. Hayashi T, Obi Y, Kimura $T$ et al. Cardiac troponin T predicts occult coronary artery stenosis in patients with chronic kidney disease at the start of renal replacement therapy. Nephrol Dial Transplant 2008; 23: 2936-2942.

531. Roongsritong C, Warraich I, Bradley C. Common causes of troponin elevations in the absence of acute myocardial infarction: incidence and clinical significance. Chest 2004; 125: 1877-1884.

532. Apple FS, Murakami MM, Pearce LA et al. Multi-biomarker risk stratification of $\mathrm{N}$-terminal pro-B-type natriuretic peptide, high-sensitivity C-reactive protein, and cardiac troponin $\mathrm{T}$ and $\mathrm{I}$ in end-stage renal disease for all-cause death. Clin Chem 2004; 50: 2279-2285.

533. De Zoysa JR. Cardiac troponins and renal disease. Nephrology (Carlton) 2004; 9: 83-88.

534. Deegan PB, Lafferty ME, Blumsohn A et al. Prognostic value of troponin T in hemodialysis patients is independent of comorbidity. Kidney Int 2001; 60: 2399-2405.

535. Dierkes J, Domrose U, Westphal S et al. Cardiac troponin T predicts mortality in patients with end-stage renal disease. Circulation 2000; 102: 1964-1969.

536. Fehr T, Knoflach A, Ammann P et al. Differential use of cardiac troponin $\mathrm{T}$ versus I in hemodialysis patients. Clin Nephrol 2003; 59: 35-39.

537. Francis GS, Tang WH. Cardiac troponins in renal insufficiency and other non-ischemic cardiac conditions. Prog Cardiovasc Dis 2004; 47: 196-206.

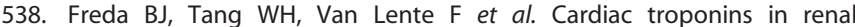
insufficiency: review and clinical implications. J Am Coll Cardiol 2002; 40: 2065-2071.

539. Hiou MC, Fumeron C, Benoit MO et al. Factors associated with increased serum levels of cardiac troponins $\mathrm{T}$ and $\mathrm{I}$ in chronic haemodialysis patients: Chronic Haemodialysis And New Cardiac Markers Evaluation (CHANCE) study. Nephrol Dial Transplant 2001; 16: 1452-1458.
540. Lamb EJ, Webb MC, Abbas NA. The significance of serum troponin T in patients with kidney disease: a review of the literature. Ann Clin Biochem 2004; 41: 1-9.

541. Lowbeer C, Ottosson-Seeberger A, Gustafsson SA et al. Increased cardiac troponin $\mathrm{T}$ and endothelin-1 concentrations in dialysis patients may indicate heart disease. Nephrol Dial Transplant 1999; 14: 1948-1955.

542. Mallamaci F, Zoccali C, Parlongo $\mathrm{S}$ et al. Troponin is related to left ventricular mass and predicts all-cause and cardiovascular mortality in hemodialysis patients. Am J Kidney Dis 2002; 40: 68-75.

543. Needham DM, Shufelt KA, Tomlinson $G$ et al. Troponin I and T levels in renal failure patients without acute coronary syndrome: a systematic review of the literature. Can J Cardiol 2004; 20: 1212-1218.

544. Ooi DS, Zimmerman D, Graham J et al. Cardiac troponin T predicts longterm outcomes in hemodialysis patients. Clin Chem 2001; 47: 412-417.

545. Roberts MA, Fernando D, Macmillan $N$ et al. Single and serial measurements of cardiac troponin I in asymptomatic patients on chronic hemodialysis. Clin Nephrol 2004; 61: 40-46.

546. Collinson PO, Hadcocks L, Foo $\mathrm{Y}$ et al. Cardiac troponins in patients with renal dysfunction. Ann Clin Biochem 1998; 35 (Pt 3): 380-386.

547. Roppolo LP, Fitzgerald R, Dillow J et al. A comparison of troponin $T$ and troponin I as predictors of cardiac events in patients undergoing chronic dialysis at a Veteran's Hospital: a pilot study. J Am Coll Cardiol 1999; 34: $448-454$.

548. de Lemos JA, Drazner $\mathrm{MH}$, Omland $\mathrm{T}$ et al. Association of troponin $T$ detected with a highly sensitive assay and cardiac structure and mortality risk in the general population. JAMA 2010; 304: 2503-2512.

549. Haller C, Zehelein J, Remppis A et al. Cardiac troponin T in patients with end-stage renal disease: absence of expression in truncal skeletal muscle. Clin Chem 1998; 44: 930-938.

550. Abaci A, Ekici $E$, Oguzhan A et al. Cardiac troponins $T$ and I in patients with end-stage renal disease: the relation with left ventricular mass and their prognostic value. Clin Cardiol 2004; 27: 704-709.

551. Ooi DS, House AA. Cardiac troponin $T$ in hemodialyzed patients. Clin Chem 1998; 44: 1410-1416.

552. Parfrey PS, Foley RN. The clinical epidemiology of cardiac disease in chronic renal failure. J Am Soc Nephrol 1999; 10: 1606-1615.

553. Roberts MA, Hedley AJ, lerino FL. Understanding cardiac biomarkers in end-stage kidney disease: Frequently asked questions and the promise of clinical application. Nephrology (Carlton) 2011; 16: 251-260.

554. Hirsch R, Landt Y, Porter S et al. Cardiac troponin I in pediatrics: normal values and potential use in the assessment of cardiac injury. $J$ Pediatr 1997; 130: 872-877.

555. Liesemer K, Casper TC, Korgenski $\mathrm{K}$ et al. Use and misuse of serum troponin assays in pediatric practice. Am J Cardiol 2012; 110: 284-289.

556. Lentine $\mathrm{KL}$, Hurst FP, Jindal RM et al. Cardiovascular risk assessment among potential kidney transplant candidates: approaches and controversies. Am J Kidney Dis 2010; 55: 152-167.

557. Mclntyre CW, Odudu A, Eldehni MT. Cardiac assessment in chronic kidney disease. Curr Opin Nephrol Hypertens 2009; 18: 501-506.

558. Bennett WM, Kloster F, Rosch J et al. Natural history of asymptomatic coronary arteriographic lesions in diabetic patients with end-stage renal disease. Am J Med 1978; 65: 779-784.

559. Braun WE, Phillips DF, Vidt DG et al. Coronary artery disease in 100 diabetics with end-stage renal failure. Transplant Proc 1984; 16: 603-607.

560. Lorber MI, Van Buren CT, Flechner SM et al. Pretransplant coronary arteriography for diabetic renal transplant recipients. Transplant Proc 1987; 19: 1539-1541.

561. Manske $\mathrm{CL}$, Wilson RF, Wang $\mathrm{Y}$ et al. Prevalence of, and risk factors for, angiographically determined coronary artery disease in type I-diabetic patients with nephropathy. Arch Intern Med 1992; 152: 2450-2455.

562. Weinrauch L, D'Elia JA, Healy RW et al. Asymptomatic coronary artery disease: angiographic assessment of diabetics evaluated for renal transplantation. Circulation 1978; 58: 1184-1190.

563. Klocke FJ, Baird MG, Lorell BH et al. ACC/AHA/ASNC guidelines for the clinical use of cardiac radionuclide imaging-executive summary: a report of the American College of Cardiology/American Heart Association Task Force on Practice Guidelines (ACC/AHA/ASNC Committee to Revise the 1995 Guidelines for the Clinical Use of Cardiac Radionuclide Imaging). J Am Coll Cardiol 2003; 42: 1318-1333.

564. Dahan M, Viron BM, Faraggi $M$ et al. Diagnostic accuracy and prognostic value of combined dipyridamole-exercise thallium imaging in hemodialysis patients. Kidney Int 1998; 54: 255-262.

565. Koistinen MJ, Huikuri HV, Pirttiaho $\mathrm{H}$ et al. Evaluation of exercise electrocardiography and thallium tomographic imaging in detecting asymptomatic coronary artery disease in diabetic patients. $\mathrm{Br}$ Heart $J$ 1990; 63: 7-11. 
566. Marwick TH, Steinmuller DR, Underwood DA et al. Ineffectiveness of dipyridamole SPECT thallium imaging as a screening technique for coronary artery disease in patients with end-stage renal failure. Transplantation 1990; 49: 100-103.

567. Schmidt A, Stefenelli T, Schuster E et al. Informational contribution of noninvasive screening tests for coronary artery disease in patients on chronic renal replacement therapy. Am J Kidney Dis 2001; 37: $56-63$

568. Morrow CE, Schwartz JS, Sutherland DE et al. Predictive value of thallium stress testing for coronary and cardiovascular events in uremic diabetic patients before renal transplantation. Am J Surg 1983; 146: 331-335.

569. Patel AD, Abo-Auda WS, Davis JM et al. Prognostic value of myocardial perfusion imaging in predicting outcome after renal transplantation. Am J Cardiol 2003; 92: 146-151.

570. Rabbat CG, Treleaven DJ, Russell JD et al. Prognostic value of myocardial perfusion studies in patients with end-stage renal disease assessed for kidney or kidney-pancreas transplantation: a meta-analysis. J Am Soc Nephrol 2003; 14: 431-439.

571. Wong CF, Little MA, Vinjamuri S et al. Technetium myocardial perfusion scanning in prerenal transplant evaluation in the United kingdom. Transplant Proc 2008; 40: 1324-1328.

572. De Lima JJ, Sabbaga E, Vieira ML et al. Coronary angiography is the best predictor of events in renal transplant candidates compared with noninvasive testing. Hypertension 2003; 42: 263-268.

573. Hatta T, Nishimura S, Nishimura T. Prognostic risk stratification of myocardial ischaemia evaluated by gated myocardial perfusion SPECT in patients with chronic kidney disease. Eur J Nucl Med Mol Imaging 2009; 36: 1835-1841.

574. Kusuoka H, Nishimura S, Yamashina A et al. Surveillance study for creating the national clinical database related to ECG-gated myocardial perfusion SPECT of ischemic heart disease: J-ACCESS study design. Ann Nucl Med 2006; 20: 195-202.

575. Momose $M$, Babazono $T$, Kondo $C$ et al. Prognostic significance of stress myocardial ECG-gated perfusion imaging in asymptomatic patients with diabetic chronic kidney disease on initiation of haemodialysis. Eur J Nucl Med Mol Imaging 2009; 36: 1315-1321.

576. Nakajima K, Nishimura T. Inter-institution preference-based variability of ejection fraction and volumes using quantitative gated SPECT with 99mTc-tetrofosmin: a multicentre study involving 106 hospitals. Eur $J$ Nucl Med Mol Imaging 2006; 33: 127-133.

577. Nekolla SG, Reder S, Saraste A et al. Evaluation of the novel myocardial perfusion positron-emission tomography tracer 18F-BMS-747158-02: comparison to $13 \mathrm{~N}$-ammonia and validation with microspheres in a pig model. Circulation 2009; 119: 2333-2342.

578. Biomarkers Definitions Working Group. Biomarkers and surrogate endpoints: preferred definitions and conceptual framework. Clin Pharmacol Ther 2001; 69: 89-95.

579. Ix JH, Criqui $\mathrm{MH}$. Epidemiology and diagnosis of peripheral arterial disease in patients with chronic kidney disease. Adv Chronic Kidney Dis 2008; 15: 378-383.

580. Lau JF, Weinberg MD, Olin JW. Peripheral artery disease. Part 1: clinical evaluation and noninvasive diagnosis. Nat Rev Cardiol 2011; 8: 405-418.

581. Bello AK, Hemmelgarn B, Lloyd A et al. Associations among estimated glomerular filtration rate, proteinuria, and adverse cardiovascular outcomes. Clin J Am Soc Nephrol 2011; 6: 1418-1426.

582. de Vinuesa SG, Ortega $M$, Martinez $P$ et al. Subclinical peripheral arterial disease in patients with chronic kidney disease: prevalence and related risk factors. Kidney Int Suppl 2005; 93: S44-47.

583. Lash JP, Go AS, Appel LJ et al. Chronic Renal Insufficiency Cohort (CRIC) Study: baseline characteristics and associations with kidney function. Clin J Am Soc Nephrol 2009; 4: 1302-1311.

584. Liew YP, Bartholomew JR, Demirjian $S$ et al. Combined effect of chronic kidney disease and peripheral arterial disease on all-cause mortality in a high-risk population. Clin J Am Soc Nephrol 2008; 3: 1084-1089.

585. O'Hare AM, Glidden DV, Fox CS et al. High prevalence of peripheral arterial disease in persons with renal insufficiency: results from the National Health and Nutrition Examination Survey 1999-2000. Circulation 2004; 109: 320-323.

586. O'Hare AM, Newman AB, Katz $R$ et al. Cystatin $C$ and incident peripheral arterial disease events in the elderly: results from the Cardiovascular Health Study. Arch Intern Med 2005; 165: 2666-2670.

587. O'Hare AM, Vittinghoff E, Hsia J et al. Renal insufficiency and the risk of lower extremity peripheral arterial disease: results from the Heart and Estrogen/Progestin Replacement Study (HERS). J Am Soc Nephrol 2004; 15: 1046-1051.
588. Wattanakit K, Folsom AR, Criqui MH et al. Albuminuria and peripheral arterial disease: results from the multi-ethnic study of atherosclerosis (MESA). Atherosclerosis 2008; 201: 212-216.

589. Margolis DJ, Hofstad O, Feldman HI. Association between renal failure and foot ulcer or lower-extremity amputation in patients with diabetes. Diabetes Care 2008; 31: 1331-1336.

590. Fuster V, Ryden LE, Cannom DS et al. ACC/AHA/ESC 2006 Guidelines for the Management of Patients with Atrial Fibrillation: a report of the American College of Cardiology/American Heart Association Task Force on Practice Guidelines and the European Society of Cardiology Committee for Practice Guidelines (Writing Committee to Revise the 2001 Guidelines for the Management of Patients With Atrial Fibrillation): developed in collaboration with the European Heart Rhythm Association and the Heart Rhythm Society. Circulation 2006; 114: e257-354.

591. Abbas AE, Goodman LM, Timmis $R$ et al. Predictors of poor outcome in female patients undergoing endovascular intervention. J Interv Cardiol 2010; 23: 401-410.

592. O'Hare AM, Bertenthal D, Sidawy AN et al. Renal insufficiency and use of revascularization among a national cohort of men with advanced lower extremity peripheral arterial disease. Clin J Am Soc Nephrol 2006; 1: 297-304.

593. Goodman and Gilman's Pharmacological Basis of Therapeutics, 12th Edn. Brunston LL, Chabner BA, Knollman BA (eds). McGraw-Hill: New York, 2011

594. Martindale: The Complete Drug Reference, 37th Edn. Sweetman SC (ed). Royal Pharmaceutical Society, London, 2011.

595. Gokmen MR, Lord GM. Aristolochic acid nephropathy. BMJ 2012; 344: e4000.

596. Su T, Zhang L, Li X et al. Regular use of nephrotoxic medications is an independent risk factor for chronic kidney disease-results from a Chinese population study. Nephrol Dial Transplant 2011; 26: 1916-1923.

597. National Institute for Health and Clinical Excellence. NICE Clinical Guideline 38: Bipolar Disorder. 2006.

598. Lipska KJ, Bailey CJ, Inzucchi SE. Use of metformin in the setting of mildto-moderate renal insufficiency. Diabetes Care 2011; 34: 1431-1437.

599. Rachmani R, Slavachevski I, Levi Z et al. Metformin in patients with type 2 diabetes mellitus: reconsideration of traditional contraindications. Eur $J$ Intern Med 2002; 13: 428.

600. Salpeter S, Greyber E, Pasternak G et al. Risk of fatal and nonfatal lactic acidosis with metformin use in type 2 diabetes mellitus. Cochrane Database Syst Rev 2010: CD002967.

601. Fellstrom BC, Jardine AG, Schmieder RE et al. Rosuvastatin and cardiovascular events in patients undergoing hemodialysis. $N$ Engl $J$ Med 2009; 360: 1395-1407.

602. Ginsberg HN, Elam MB, Lovato LC et al. Effects of combination lipid therapy in type 2 diabetes mellitus. $N$ Engl J Med 2010; 362: 1563-1574.

603. Keech A, Simes RJ, Barter P et al. Effects of long-term fenofibrate therapy on cardiovascular events in 9795 people with type 2 diabetes mellitus (the FIELD study): randomised controlled trial. Lancet 2005; 366: 1849-1861.

604. Wanner C, Krane V, Marz W et al. Atorvastatin in patients with type 2 diabetes mellitus undergoing hemodialysis. $N$ Engl J Med 2005; 353: 238-248.

605. Limdi NA, Beasley TM, Baird MF et al. Kidney function influences warfarin responsiveness and hemorrhagic complications. J Am Soc Nephrol 2009; 20: $912-921$

606. Wahba IM, Olyaei AJ, Rozansky D et al. Handling of drugs in children with abnormal renal function, in Pediatric Nephrology, eds. Avner ED, Harmon WE, Niaudet P, Yoshikawa, N. Springer-Verlag: 2009, pp 1693-1711.

607. Blowey DL. Chapter 64: Drug Use and Dosage in Renal Failure, in Comprehensive Pediatric Nephrology, eds. Geary DF, Schaefer F. Mosby Elsevier: Philadelphia, 2008, pp 991-1002.

608. Drug prescribing in renal failure. http://www.kdp-baptist.louisville.edu/ renalbook/.

609. Goldfarb S, McCullough PA, McDermott J et al. Contrast-induced acute kidney injury: specialty-specific protocols for interventional radiology, diagnostic computed tomography radiology, and interventional cardiology. Mayo Clin Proc 2009; 84: 170-179.

610. American College Radiology Guidelines. Manual on Contrast Media Version 8. http://www.acr.org/ /media/ACR/Documents/PDF/Quality Safety/Resources/Contrast\%20Manual/Contrast\%20Nephrotoxicity.pdf. Accessed October 12, 2012.

611. European Society for Urological Radiology (ESUR). http://www.esur.org/ Contrast-media.51.0.html, Accessed October 9, 2012. 
612. Heinrich MC, Haberle L, Muller V et al. Nephrotoxicity of iso-osmolar iodixanol compared with nonionic low-osmolar contrast media: metaanalysis of randomized controlled trials. Radiology 2009; 250: 68-86.

613. Perazella MA. Current status of gadolinium toxicity in patients with kidney disease. Clin J Am Soc Nephrol 2009; 4: 461-469.

614. Agarwal R, Brunelli SM, Williams K et al. Gadolinium-based contrast agents and nephrogenic systemic fibrosis: a systematic review and metaanalysis. Nephrol Dial Transplant 2009; 24: 856-863.

615. American College Radiology Guidelines. Manual on Contrast Media Version 8. http://www.acr.org/ /media/ACR/Documents/PDF/QualitySafety/Resources/Contrast\%20Manual/Nephrogenic\%20Systemic\%20Fibrosis.pdf. Accessed October 12, 2012.

616. Saitoh T, Hayasaka K, Tanaka Y et al. Dialyzability of gadodiamide in hemodialysis patients. Radiat Med 2006; 24: 445-451.

617. Meng H, Grosse-Wortmann L. Gadolinium in pediatric cardiovascular magnetic resonance: what we know and how we practice. J Cardiovasc Magn Reson 2012; 14: 56.

618. Lien YH. Is bowel preparation before colonoscopy a risky business for the kidney? Nat Clin Pract Nephrol 2008; 4: 606-614.

619. Markowitz GS, Perazella MA. Acute phosphate nephropathy. Kidney Int 2009; 76: 1027-1034.

620. Heher EC, Thier SO, Rennke $\mathrm{H}$ et al. Adverse renal and metabolic effects associated with oral sodium phosphate bowel preparation. Clin J Am Soc Nephrol 2008; 3: 1494-1503.

621. Palmadottir VK, Gudmundsson H, Hardarson S et al. Incidence and outcome of acute phosphate nephropathy in Iceland. PLoS One 2010; 5: e13484.

622. Wexner SD, Beck DE, Baron TH et al. A consensus document on bowel preparation before colonoscopy: prepared by a task force from the American Society of Colon and Rectal Surgeons (ASCRS), the American Society for Gastrointestinal Endoscopy (ASGE), and the Society of American Gastrointestinal and Endoscopic Surgeons (SAGES). Gastrointest Endosc 2006; 63: 894-909.

623. Brunelli SM. Association between oral sodium phosphate bowel preparations and kidney injury: a systematic review and meta-analysis. Am J Kidney Dis 2009; 53: 448-456.

624. Johanson JF, Popp JW, Jr., Cohen LB et al. A randomized, multicenter study comparing the safety and efficacy of sodium phosphate tablets with $2 \mathrm{~L}$ polyethylene glycol solution plus bisacodyl tablets for colon cleansing. Am J Gastroenterol 2007; 102: 2238-2246.

625. Foley RN, Guo H, Snyder JJ et al. Septicemia in the United States dialysis population, 1991 to 1999. J Am Soc Nephrol 2004; 15: 1038-1045.

626. Hoen B, Paul-Dauphin A, Hestin D et al. EPIBACDIAL: a multicenter prospective study of risk factors for bacteremia in chronic hemodialysis patients. J Am Soc Nephrol 1998; 9: 869-876.

627. Ishani A, Collins AJ, Herzog CA et al. Septicemia, access and cardiovascular disease in dialysis patients: the USRDS Wave 2 study. Kidney Int 2005; 68: 311-318.

628. Sarnak MJ, Jaber BL. Mortality caused by sepsis in patients with endstage renal disease compared with the general population. Kidney Int 2000; 58: 1758-1764.

629. Fried LF, Katz R, Sarnak MJ et al. Kidney function as a predictor of noncardiovascular mortality. J Am Soc Nephrol 2005; 16: 3728-3735.

630. Nissenson AR, Collins AJ, Hurley J et al. Opportunities for improving the care of patients with chronic renal insufficiency: current practice patterns. J Am Soc Nephrol 2001; 12: 1713-1720.

631. US Renal Data System. Atlas of Chronic Kidney Disease in the United States. Bethesda, MD: National Institutes of Diabetes and Digestive and Kidney Diseases, National Institutes of Health. 2007.

632. Naqvi SB, Collins AJ. Infectious complications in chronic kidney disease. Adv Chronic Kidney Dis 2006; 13: 199-204.

633. US Renal Data System. Atlas of Chronic Kidney Disease in the United States. Bethesda, MD: National Institutes of Diabetes and Digestive and Kidney Diseases, National Institutes of Health. 2010.

634. Mclntyre $P$, Craig JC. Prevention of serious bacterial infection in children with nephrotic syndrome. J Paediatr Child Health 1998; 34: 314-317.

635. Vanholder R, Ringoir S. Infectious morbidity and defects of phagocytic function in end-stage renal disease: a review. J Am Soc Nephrol 1993; 3: 1541-1554.

636. Cohen G, Haag-Weber M, Horl WH. Immune dysfunction in uremia. Kidney Int Suppl 1997; 62: S79-82.

637. Minnaganti VR, Cunha BA. Infections associated with uremia and dialysis. Infect Dis Clin North Am 2001; 15: 385-406.

638. Pesanti EL. Immunologic defects and vaccination in patients with chronic renal failure. Infect Dis Clin North Am 2001; 15: 813-832.
639. Kausz AT, Guo H, Pereira BJ et al. General medical care among patients with chronic kidney disease: opportunities for improving outcomes. J Am Soc Nephrol 2005; 16: 3092-3101.

640. DaRoza G, Loewen A, Djurdjev O et al. Stage of chronic kidney disease predicts seroconversion after hepatitis $B$ immunization: earlier is better. Am J Kidney Dis 2003; 42: 1184-1192.

641. Kausz AT, Gilbertson DT. Overview of vaccination in chronic kidney disease. Adv Chronic Kidney Dis 2006; 13: 209-214

642. McNulty CA, Bowen JK, Williams AJ. Hepatitis B vaccination in predialysis chronic renal failure patients a comparison of two vaccination schedules. Vaccine 2005; 23: 4142-4147.

643. Robinson J. Efficacy of pneumococcal immunization in patients with renal disease-what is the data? Am J Nephrol 2004; 24: 402-409.

644. Gilbertson DT, Unruh M, McBean AM et al. Influenza vaccine delivery and effectiveness in end-stage renal disease. Kidney Int 2003; 63: 738-743.

645. Dinits-Pensy M, Forrest GN, Cross AS et al. The use of vaccines in adult patients with renal disease. Am J Kidney Dis 2005; 46: 997-1011.

646. Nikoskelainen J, Koskela M, Forsstrom J et al. Persistence of antibodies to pneumococcal vaccine in patients with chronic renal failure. Kidney Int 1985; 28: 672-677.

647. Miller ER, Alter MJ, Tokars Jl. Protective effect of hepatitis B vaccine in chronic hemodialysis patients. Am J Kidney Dis 1999; 33: 356-360.

648. Fattom A, Fuller S, Propst $M$ et al. Safety and immunogenicity of a booster dose of Staphylococcus aureus types 5 and 8 capsular polysaccharide conjugate vaccine (StaphVAX) in hemodialysis patients. Vaccine 2004; 23: 656-663.

649. Shinefield H, Black S, Fattom A et al. Use of a Staphylococcus aureus conjugate vaccine in patients receiving hemodialysis. N Engl J Med 2002; 346: $491-496$

650. CDC immunization schedules. http://www.cdc.gov/vaccines/schedules/ index.html.

651. American Academy of Pediatrics. Immunization: http://www2.aap.org/ immunization/.

652. Neu AM. Immunizations in children with chronic kidney disease. Pediatr Nephrol 2012; 27: 1257-1263.

653. Goldstein SL. Acute kidney injury in children: prevention, treatment and rehabilitation. Contrib Nephrol 2011; 174: 163-172.

654. Goldstein SL. Acute kidney injury in children and its potential consequences in adulthood. Blood Purif 2012; 33: 131-137.

655. Zappitelli M, Goldstein SL. Acute kidney failure in children, In: Jorres A, Ronco C, Kellum JA (eds.). Management of Acute Kidney Problems, Springer, 2010, pp 459-467.

656. Andreoli SP. Clinical evaluation of acute kidney injury in children. In: Avner ED, Harmon WE, Niaudet P, Yoshikawa N (eds.). Pediatric Nephrology, (6th edn.). Springer, 2009, pp 1603-1618.

657. Zappitelli M, Goldstein SL. Management of acute kidney failure, In: Avner ED, Harmon WE, Niaudet P, Yoshikawa N (eds.). Pediatric Nephrology (6th edn), Springer, 2009, pp 1619-1628.

658. Zappitelli M, Goldstein SL. Acute kidney injury: General aspects. In: Kiessling SG, Goebel J, Somers MJG (eds.). Pediatric Nephrology in the ICU, Springer, 2009, pp 85-97.

659. Arora P, Kausz AT, Obrador GT et al. Hospital utilization among chronic dialysis patients. J Am Soc Nephrol 2000; 11: 740-746.

660. Khan SS, Kazmi WH, Abichandani R et al. Health care utilization among patients with chronic kidney disease. Kidney Int 2002; 62: 229-236.

661. Becker BN, Coomer RW, Fotiadis C et al. Risk factors for hospitalization in well-dialyzed chronic hemodialysis patients. Am J Nephrol 1999; 19: 565-570.

662. Popovic JR, Kozak $\sqcup$. National hospital discharge survey: annual summary, 1998. Vital Health Stat 13 2000: 1-194.

663. Rocco MV, Soucie JM, Reboussin DM et al. Risk factors for hospital utilization in chronic dialysis patients. Southeastern Kidney Council (Network 6). J Am Soc Nephrol 1996; 7: 889-896.

664. Thamer M, Ray NF, Fehrenbach SN et al. Relative risk and economic consequences of inpatient care among patients with renal failure. J Am Soc Nephrol 1996; 7: 751-762.

665. Holland DC, Lam M. Predictors of hospitalization and death among predialysis patients: a retrospective cohort study. Nephrol Dial Transplant 2000; 15: 650-658.

666. Culleton BF, Larson MG, Wilson PW et al. Cardiovascular disease and mortality in a community-based cohort with mild renal insufficiency. Kidney Int 1999; 56: 2214-2219.

667. Meyer KB, Levey AS. Controlling the epidemic of cardiovascular disease in chronic renal disease: report from the National Kidney Foundation Task Force on cardiovascular disease. J Am Soc Nephrol 1998; 9: S31-42. 
668. Drüeke TB, Locatelli F, Clyne N et al. Normalization of hemoglobin level in patients with chronic kidney disease and anemia. N Engl J Med 2006; 355: 2071-2084.

669. Singh AK, Szczech L, Tang KL et al. Correction of anemia with epoetin alfa in chronic kidney disease. $N$ Engl J Med 2006; 355: 2085-2098.

670. De Coster C, McLaughlin K, Noseworthy TW. Criteria for referring patients with renal disease for nephrology consultation: a review of the literature. J Nephrol 2010; 23: 399-407.

671. Navaneethan SD, Kandula P, Jeevanantham $V$ et al. Referral patterns of primary care physicians for chronic kidney disease in general population and geriatric patients. Clin Nephrol 2010; 73: 260-267.

672. Navaneethan SD, Nigwekar S, Sengodan M et al. Referral to nephrologists for chronic kidney disease care: is non-diabetic kidney disease ignored? Nephron Clin Pract 2007; 106: c113-118.

673. Chan MR, Dall AT, Fletcher KE et al. Outcomes in patients with chronic kidney disease referred late to nephrologists: a meta-analysis. Am J Med 2007; 120: 1063-1070.

674. Smart NA, Titus TT. Outcomes of early versus late nephrology referral in chronic kidney disease: a systematic review. Am J Med 2011; 124: 1073-1080.

675. McLaughlin K, Manns B, Culleton B et al. An economic evaluation of early versus late referral of patients with progressive renal insufficiency. Am J Kidney Dis 2001; 38: 1122-1128.

676. Klebe B, Irving J, Stevens PE et al. The cost of implementing UK guidelines for the management of chronic kidney disease. Nephrol Dial Transplant 2007; 22: 2504-2512.

677. Barakat AJ. Presentation of the child with renal disease and guidelines for referral to the pediatric nephrologist. Int J Pediatr 2012; 978673.

678. Barakat AJ, Chesney RW (eds.). Pediatric Nephrology for Primary Care. American Academy of Pediatrics: Illinois, 2009.

679. Kennedy SE, Bailey R, Kainer G. Causes and outcome of late referral of children who develop end-stage kidney disease. J Paediatr Child Health 2012; 48: 253-258.

680. Boehm M, Winkelmayer WC, Arbeiter $\mathrm{K}$ et al. Late referral to paediatric renal failure service impairs access to pre-emptive kidney transplantation in children. Arch Dis Child 2010; 95: 634-638.

681. Epping-Jordan JE, Pruitt SD, Bengoa R et al. Improving the quality of health care for chronic conditions. Qual Saf Health Care 2004; 13: 299-305.

682. Ajarmeh S, Er L, Brin G et al. The effect of a multidisciplinary care clinic on the outcomes in pediatric chronic kidney disease. Pediatr Nephrol 2012; 27: 1921-1927.

683. Menon S, Valentini RP, Kapur G et al. Effectiveness of a multidisciplinary clinic in managing children with chronic kidney disease. Clin J Am Soc Nephrol 2009; 4: 1170-1175.

684. Korevaar JC, Jansen MA, Dekker FW et al. When to initiate dialysis: effect of proposed US guidelines on survival. Lancet 2001; 358: 1046-1050.

685. Traynor JP, Simpson K, Geddes CC et al. Early initiation of dialysis fails to prolong survival in patients with end-stage renal failure. J Am Soc Nephrol 2002; 13: 2125-2132.

686. Beddhu S, Samore MH, Roberts MS et al. Impact of timing of initiation of dialysis on mortality. J Am Soc Nephrol 2003; 14: 2305-2312.

687. Clark WF, Na Y, Rosansky SJ et al. Association between estimated glomerular filtration rate at initiation of dialysis and mortality. CMAJ 2011; 183: 47-53.

688. Hwang SJ, Yang WC, Lin MY et al. Impact of the clinical conditions at dialysis initiation on mortality in incident haemodialysis patients: a national cohort study in Taiwan. Nephrol Dial Transplant 2010; 25: 2616-2624.

689. Kazmi WH, Gilbertson DT, Obrador GT et al. Effect of comorbidity on the increased mortality associated with early initiation of dialysis. Am Kidney Dis 2005; 46: 887-896.

690. Lassalle M, Labeeuw M, Frimat L et al. Age and comorbidity may explain the paradoxical association of an early dialysis start with poor survival. Kidney Int 2010; 77: 700-707.

691. Sawhney S, Djurdjev O, Simpson K et al. Survival and dialysis initiation: comparing British Columbia and Scotland registries. Nephrol Dial Transplant 2009; 24: 3186-3192.

692. Shiao CC, Huang JW, Chien KL et al. Early initiation of dialysis and late implantation of catheters adversely affect outcomes of patients on chronic peritoneal dialysis. Perit Dial Int 2008; 28: 73-81.

693. Stel VS, Dekker FW, Ansell D et al. Residual renal function at the start of dialysis and clinical outcomes. Nephrol Dial Transplant 2009; 24: 3175-3182.
694. Tang SC, Ho YW, Tang AW et al. Delaying initiation of dialysis till symptomatic uraemia-is it too late? Nephrol Dial Transplant 2007; 22: 1926-1932.

695. Wilson B, Harwood L, Locking-Cusolito $\mathrm{H}$ et al. Optimal timing of initiation of chronic hemodialysis? Hemodial Int 2007; 11: 263-269.

696. Wright S, Klausner D, Baird B et al. Timing of dialysis initiation and survival in ESRD. Clin J Am Soc Nephrol 2010; 5: 1828-1835.

697. Cooper BA, Branley P, Bulfone L et al. A randomized, controlled trial of early versus late initiation of dialysis. N Engl J Med 2010; 363: 609-619.

698. Rosansky SJ, Eggers $\mathrm{P}$, Jackson $\mathrm{K}$ et al. Early start of hemodialysis may be harmful. Arch Intern Med 2011; 171: 396-403.

699. Harris A, Cooper BA, Li JJ et al. Cost-effectiveness of initiating dialysis early: a randomized controlled trial. Am J Kidney Dis 2011; 57: 707-715.

700. Greenbaum LA, Schaefer F. The decision to initiate dialysis in a pediatric patient. In: Warady BA, Schaefer F, Alexander SR (eds). Pediatric Dialysis, Springer: New York, 2012, pp 85-100.

701. Kramer A, Stel VS, Tizard J et al. Characteristics and survival of young adults who started renal replacement therapy during childhood. Nephrol Dial Transplant 2009; 24: 926-933.

702. Davison SN. Pain in hemodialysis patients: prevalence, cause, severity, and management. Am J Kidney Dis 2003; 42: 1239-1247.

703. Davison SN, Jhangri GS, Johnson JA. Cross-sectional validity of a modified Edmonton symptom assessment system in dialysis patients: a simple assessment of symptom burden. Kidney Int 2006; 69: 1621-1625.

704. Davison SN, Jhangri GS, Johnson JA. Longitudinal validation of a modified Edmonton symptom assessment system (ESAS) in haemodialysis patients. Nephrol Dial Transplant 2006; 21: 3189-3195.

705. Murphy EL, Murtagh FE, Carey I et al. Understanding symptoms in patients with advanced chronic kidney disease managed without dialysis: use of a short patient-completed assessment tool. Nephron Clin Pract 2009; 111: c74-80.

706. Murtagh FE, Addington-Hall JM, Donohoe $P$ et al. Symptom management in patients with established renal failure managed without dialysis. EDTNA ERCA J 2006; 32: 93-98.

707. Murtagh FE, Addington-Hall JM, Edmonds PM et al. Symptoms in advanced renal disease: a cross-sectional survey of symptom prevalence in stage 5 chronic kidney disease managed without dialysis. J Palliat Med 2007; 10: 1266-1276.

708. Saini T, Murtagh FE, Dupont PJ et al. Comparative pilot study of symptoms and quality of life in cancer patients and patients with end stage renal disease. Palliat Med 2006; 20: 631-636.

709. Temel JS, Greer JA, Muzikansky A et al. Early palliative care for patients with metastatic non-small-cell lung cancer. N Engl J Med 2010; 363: 733-742.

710. Chandna SM, Da Silva-Gane M, Marshall C et al. Survival of elderly patients with stage 5 CKD: comparison of conservative management and renal replacement therapy. Nephrol Dial Transplant 2011; 26: 1608-1614.

711. Davison SN. End-of-life care preferences and needs: perceptions of patients with chronic kidney disease. Clin J Am Soc Nephrol 2010; 5: 195-204.

712. Davison SN, Murtagh FE, Higginson IJ. Methodological considerations for end-of-life research in patients with chronic kidney disease. J Nephrol 2008; 21: 268-282

713. De Biase V, Tobaldini O, Boaretti $C$ et al. Prolonged conservative treatment for frail elderly patients with end-stage renal disease: the Verona experience. Nephrol Dial Transplant 2008; 23: 1313-1317.

714. Ellam T, El-Kossi M, Prasanth KC et al. Conservatively managed patients with stage 5 chronic kidney disease-outcomes from a single center experience. QJM 2009; 102: 547-554.

715. Germain MJ, Kurella Tamura M, Davison SN. Palliative care in CKD: the earlier the better. Am J Kidney Dis 2011; 57: 378-380.

716. Murray AM, Arko C, Chen SC et al. Use of hospice in the United States dialysis population. Clin J Am Soc Nephrol 2006; 1: 1248-1255.

717. Hearn J, Higginson IJ. Do specialist palliative care teams improve outcomes for cancer patients? A systematic literature review. Palliat Med 1998; 12: 317-332.

718. Higginson IJ, Wade AM, McCarthy M. Effectiveness of two palliative support teams. J Public Health Med 1992; 14: 50-56.

719. Ventafridda V, De Conno F, Vigano A et al. Comparison of home and hospital care of advanced cancer patients. Tumori 1989; 75: 619-625.

720. Wallston KA, Burger C, Smith RA et al. Comparing the quality of death for hospice and non-hospice cancer patients. Med Care 1988; 26: 177-182. 
721. Fassbender K, Smythe JG, Carson M, et al. Report of the Institute for Public Economics Health Research Group to Alberta Health and Wellness: cost and utilization of health care services at end of life in Alberta, 1999-2002. Edmonton, AB: University of Alberta. 2006.

722. Owens DK, Lohr KN, Atkins D et al. AHRQ series paper 5: grading the strength of a body of evidence when comparing medical interventionsagency for healthcare research and quality and the effective health-care program. J Clin Epidemiol 2010; 63: 513-523.

723. Atkins D, Best D, Briss PA et al. Grading quality of evidence and strength of recommendations. BMJ 2004; 328: 1490.

724. Guyatt $\mathrm{GH}$, Oxman AD, Kunz $\mathrm{R}$ et al. Going from evidence to recommendations. BMJ 2008; 336: 1049-1051.

725. Uhlig K, Macleod A, Craig J et al. Grading evidence and recommendations for clinical practice guidelines in nephrology. A position statement from Kidney Disease: Improving Global Outcomes (KDIGO). Kidney Int 2006; 70: 2058-2065.

726. The AGREE Collaboration. Development and validation of an international appraisal instrument for assessing the quality of clinical practice guidelines: the AGREE project. Qual Saf Health Care 2003; 12: 18-23.

727. Shiffman RN, Shekelle P, Overhage JM et al. Standardized reporting of clinical practice guidelines: a proposal from the Conference on Guideline Standardization. Ann Intern Med 2003; 139: 493-498.

728. Institute of Medicine. Finding What Works in Health Care: Standards for Systematic Reviews. The National Academies Press: Washington, DC, 2011.

729. Institute of Medicine. Clinical Practice Guidelines We Can Trust. The National Academies Press: Washington, DC, 2011. 